\title{
Rottermann, Benno
}

\section{Sozialisation von Jugendlichen in geschlechtsuntypischen Berufslehren}

\author{
Opladen; Berlin; Toronto : Budrich UniPress Ltd. 2017, 243 S.
}

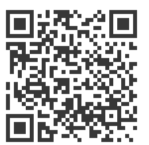

Quellenangabe/ Reference:

Rottermann, Benno: Sozialisation von Jugendlichen in geschlechtsuntypischen Berufslehren. Opladen; Berlin; Toronto : Budrich UniPress Ltd. 2017, 243 S. - URN: urn:nbn:de:0111-pedocs-170908 - DOI: $10.25656 / 01: 17090$

https://nbn-resolving.org/urn:nbn:de:0111-pedocs-170908

https://doi.org/10.25656/01:17090

in Kooperation mit / in cooperation with:

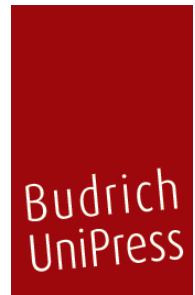

\section{Nutzungsbedingungen}

Dieses Dokument steht unter folgender Creative Commons-Lizenz: http://creativecommons.org/licenses/by-sa/4.0/deed.de - Sie dürfen das Werk bzw. den Inhalt vervielfältigen, verbreiten und öffentlich zugänglich machen sowie Abwandlungen und Bearbeitungen des Werkes bzw. Inhaltes anfertigen, solange sie den Namen des Autors/Rechteinhabers in der von inm festgelegten Weise nennen und die daraufhin neu entstandenen Werke bzw. Inhalte nur unter Verwendung von Lizenzbedingungen weitergeben, die mit denen dieses Lizenzvertrags identisch, vergleichbar oder kompatibel sind.

Mit der Verwendung dieses Dokuments erkennen Sie die Nutzungsbedingungen an.

\section{Terms of use}

This document is published under following Creative Commons-License: http://creativecommons.org/licenses/by-sa/4.0/deed.en - You may copy, distribute and transmit, adapt or exhibit the work or its contents in public and alter, transform, or change this work as long as you attribute the work in the manner specified by the author or licensor. New resulting works or contents must be distributed pursuant to this license or an identical or comparable license.

By using this particular document, you accept the above-stated conditions of use.

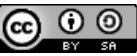

\section{Kontakt / Contact:}

peDOcs

DIPF | Leibniz-Institut für Bildungsforschung und Bildungsinformation Informationszentrum (IZ) Bildung

E-Mail: pedocs@dipf.de

Internet: www.pedocs.de

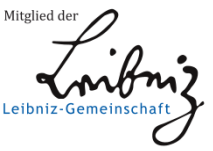


Benno Rottermann

Sozialisation von

Jugendlichen in

geschlechtsuntypischen

Berufslehren 


\section{Benno Rottermann}

Sozialisation von Jugendlichen in

geschlechtsuntypischen Berufslehren 
Benno Rottermann

Sozialisation von

Jugendlichen in

geschlechtsuntypischen

Berufslehren

Budrich UniPress Ltd.

Opladen • Berlin • Toronto 2017 
Bibliografische Information der Deutschen Nationalbibliothek

Die Deutsche Nationalbibliothek verzeichnet diese Publikation in der Deutschen

Nationalbibliografie; detaillierte bibliografische Daten sind im Internet über

http://dnb.d-nb.de abrufbar.

(C) 2017 Dieses Werk ist beim Verlag Barbara Budrich erschienen und steht unter der Creative Commons Lizenz Attribution-ShareAlike 4.0 International (CC BY-SA 4.0): https://creativecommons.org/licenses/by-sa/4.0/

Diese Lizenz erlaubt die Verbreitung, Speicherung, Vervielfältigung und Bearbeitung bei Verwendung der gleichen CC-BY-SA 4.0-Lizenz und unter Angabe der UrheberInnen, Rechte, Änderungen und verwendeten Lizenz.

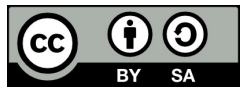

Dieses Buch steht im Open-Access-Bereich der Verlagsseite zum kostenlosen Download bereit (https://doi.org/10.3224/86388759).

Eine kostenpflichtige Druckversion kann über den Verlag bezogen werden. Die Seitenzahlen in der Druck- und Onlineversion sind identisch.

$\begin{array}{ll}\text { ISBN } & 978-3-86388-759-9 \\ \text { eISBN } & 978-3-86388-319-5 \\ \text { DOI } & 10.3224 / 86388759\end{array}$

Umschlaggestaltung: Bettina Lehfeldt, Kleinmachnow http://www.lehfeldtgraphic.de

Typografisches Lektorat: Anja Borkam, Jena 


\section{Inhaltsverzeichnis}

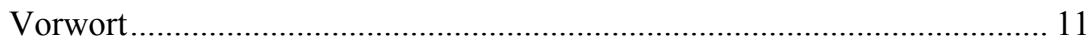

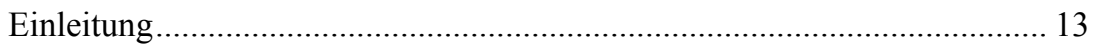

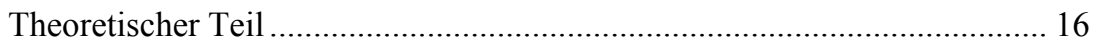

1 Beruf und berufliche Grundbildung ................................................ 18

1.1 Geschlechtliche Segregation des Arbeitsmarkts .................................. 20

1.1.1 Individuumzentrierter Erklärungsansatz ............................... 21

1.1.2 Institutionenzentrierter Erklärungsansatz ............................... 22

1.1.3 Erklärungsansatz Geschlechternormen und Stereotype ............ 24

1.1.4 Erklärungsansatz soziale Schließung....................................... 24

1.1.5 Erklärungsansatz Geschlechtshabitus ..................................... 25

1.2 Berufliche Sozialisation ................................................................. 27

1.2.1 Familiäre und schulische Sozialisation vor dem Beruf............. 29

1.2.2 Sozialisation durch den Beruf (für den und im Beruf).............. 29

1.3 Berufsfindung und Berufseinmündung ............................................. 30

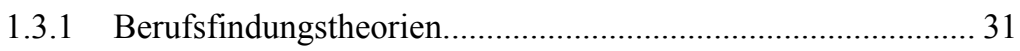

1.3.2 Berufsfindung als Abfolge von Kompromissen....................... 33

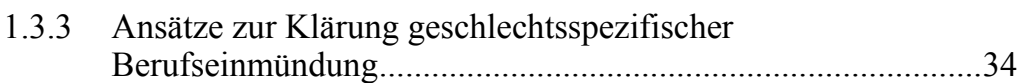

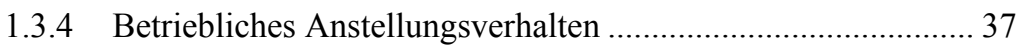

1.4 Berufliche Grundbildung in der Schweiz.......................................... 40

1.5 Erfolg und Misserfolg während der Berufsausbildung ........................ 42

1.5.1 Indikatoren für den Erfolg in der Berufslehre........................... 44

1.5.2 Erfolgsfaktor: Sich während der Berufslehre bewähren ........... 47

1.5.3 Erfolgsfaktoren im Selbstbericht ........................................... 50

1.5.4 Erfolgsfaktor: Lehrabschlussprüfung bestehen........................ 53

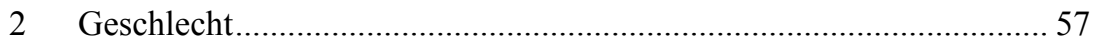

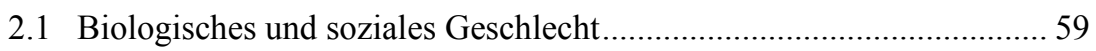

2.2 Erklärungsansätze der Geschlechtsunterschiede .................................. 60 
2.2.1 Evolutionspsychologische Erklärungsansätze .......................... 62

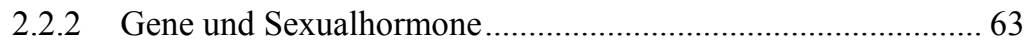

2.2.3 Hirnbeschaffenheit und Ausprägung der Hemisphären ............. 64

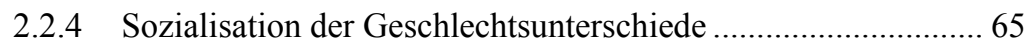

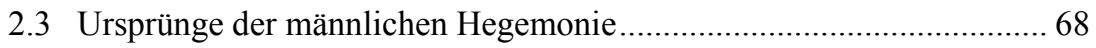

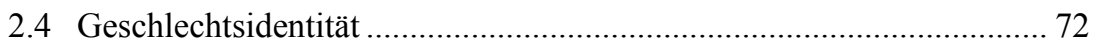

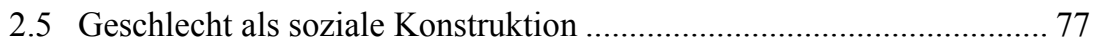

2.6 Geschlechterstereotype und Geschlechtsrollen .................................... 81

3 Sozialisation in geschlechtsuntypischen Berufen................................ 84

3.1 Beschreibung der geschlechtsuntypischen Berufssituation.................. 85

3.1.1 Befunde zur geschlechtsuntypischen Berufsfindungsphase...... 85

3.1.2 Befunde zu Frauen in männerreichen Berufen ........................ 86

3.1.3 Befunde zu Männern in frauenreichen Berufen......................... 88

3.2 Auswirkungen der geschlechtsuntypischen Berufssituation ................. 91

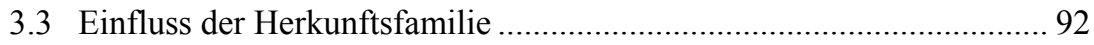

3.4 Modelle und Theorien zu geschlechtsuntypischen Berufen .................. 93

3.4.1 Modell der geschlechtsuntypischen Berufsfindung ................. 94

3.4.2 Rahmenmodell zur geschlechtsuntypischen Laufbahn ............ 95

3.4.3 Williams Glaslift-Theorie …................................................. 96

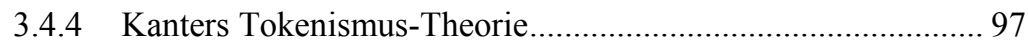

4 Modell der geschlechtsuntypischen Berufssozialisation ..................... 100

4.1 Definitionen grundlegender Begriffe des Modells .............................. 101

4.1.1 Berufliche Sozialisation...................................................... 102

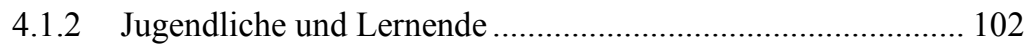

4.1.3 Beruf und Berufslehre.......................................................... 103

4.1.4 Geschlecht und Geschlechtsrolle ......................................... 103

4.1.5 Berufliche Segregation nach Geschlecht .............................. 104

4.1.6 Lernende in geschlechtsuntypischen Berufslehren................ 105

4.2 Beschreibung des Modells der geschlechtsuntypischen

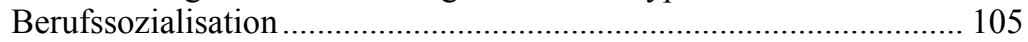

4.2.1 Geschlechtsrollenerwartungen - berufliche Segregation nach Geschlecht. 108 
4.2.2 Geschlechtsuntypische Berufslehre ...................................... 109

4.2.3 Irritation des sozialen Umfelds ............................................ 110

4.2.4 Reaktion und Bewältigung .................................................. 111

4.2.5 Verbleib in der geschlechtsuntypischen Berufslehre .............. 113

4.2.6 Austritt aus der geschlechtsuntypischen Berufslehre.............. 114

4.3 Forschungsfrage und Hypothesen ................................................... 115

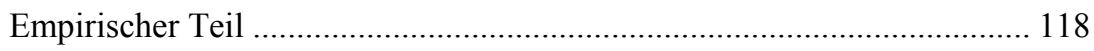

5 Analyse I: Lehrvertragsauflösungen (LVA)....................................... 119

5.1 Fragestellung der Analyse und Hypothesen........................................ 119

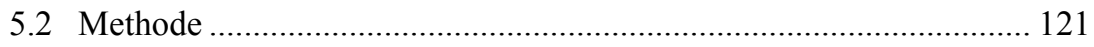

5.2.1 Beschreibung der Datenstruktur .......................................... 121

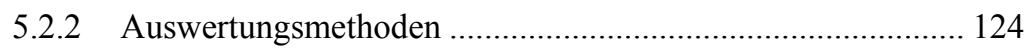

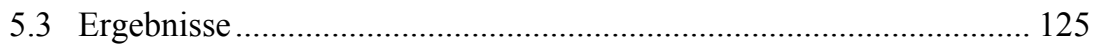

5.3.1 Stabilitäten der Frauenanteile und der LVA-Quoten .............. 125

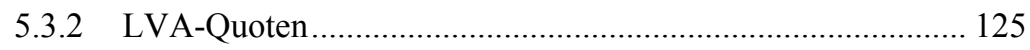

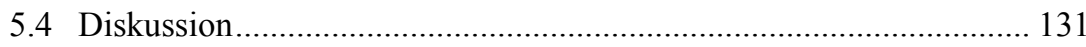

6 Analyse II: Erfolgsquoten bei Lehrabschlussprüfungen (LAP) .......... 134

6.1 Fragestellung der Analyse und Hypothesen..................................... 135

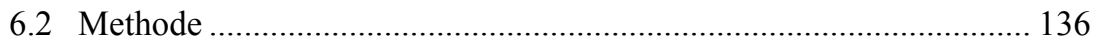

6.2.1 Beschreibung der Datenstruktur .......................................... 136

6.2.2 Auswertungsmethoden ..................................................... 137

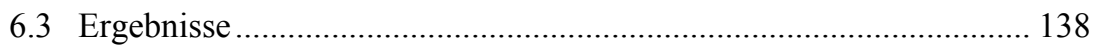

6.3.1 Stabilitäten der Frauenanteile und der Misserfolgsquoten...... 138

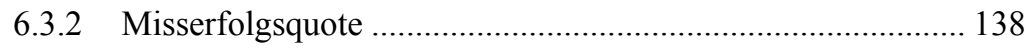

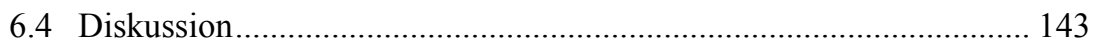

7 Analyse III: Berufserfolge im Selbstbericht - FASE B-Daten............ 147

7.1 Fragestellung der Analyse und Hypothesen....................................... 147

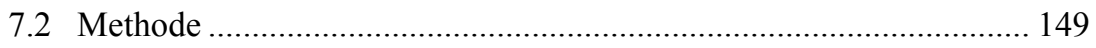

7.2.1 Forschungsprojekt Familie Schule Beruf - FASE B .............. 149

7.2.2 Auswertungsstrategie......................................................... 150 


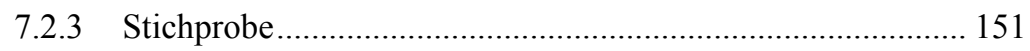

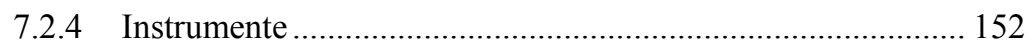

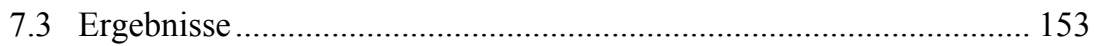

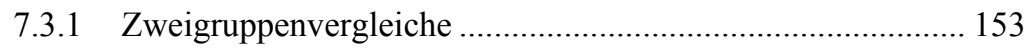

7.3.2 Viergruppenvergleiche ..................................................... 154

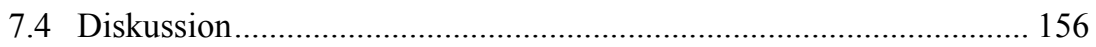

8 Analyse IV: Einfluss des sozialen Umfelds - TREE-Daten ............... 158

8.1 Fragestellung der Analyse und Hypothesen..................................... 158

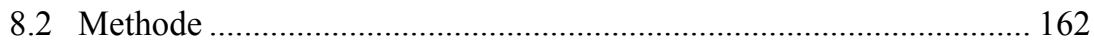

8.2.1 Forschungsprojekt TREE.................................................... 162

8.2.2 Auswertungsstrategie ........................................................ 163

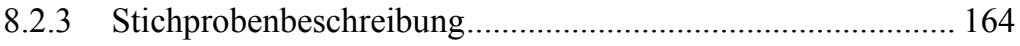

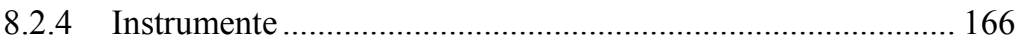

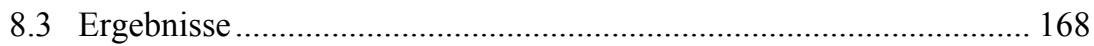

8.3.1 Mediation durch die Reaktion des sozialen Umfelds.............. 168

8.3.2 Verlaufsanalysen ............................................................. 171

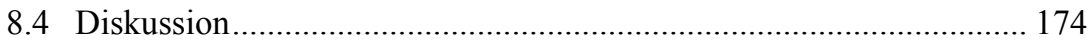

9 Interviewstudie: Belastungen und Ressourcen................................. 177

9.1 Fragestellung der Analyse............................................................... 177

9.2 Forschungsvorgehen und Methode ................................................... 178

9.2.1 Interviewleitfaden ............................................................ 178

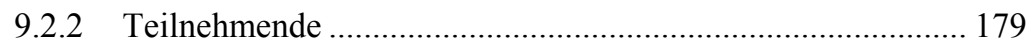

9.2.3 Datenaufbereitung und -auswertung ..................................... 180

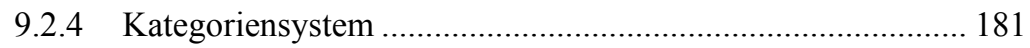

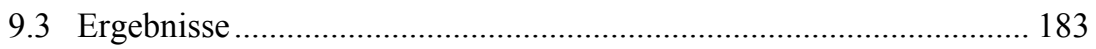

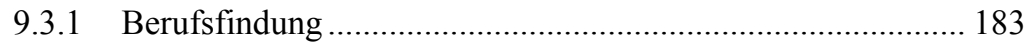

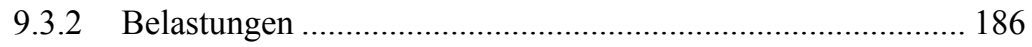

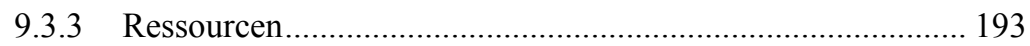

9.3.4 Doing Gender .................................................................. 204

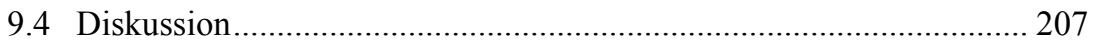




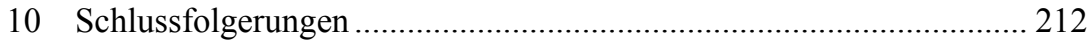

10.1 Zusammenfassung der empirischen Befunde................................... 212

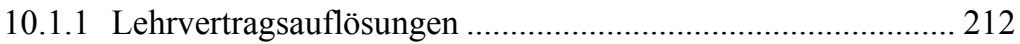

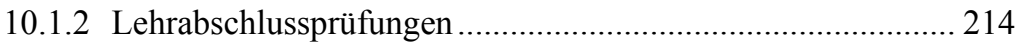

10.1.3 Berufliche Erfolge im Selbstbericht .................................... 215

10.1.4 Einfluss des sozialen Umfelds ............................................. 215

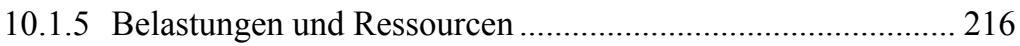

10.2 Reflexion zur Sozialisation in geschlechtsuntypischen

Berufslehren......................................................................... 218

10.3 Perspektiven geschlechtsuntypischer Sozialisationsprozesse ............ 221

10.4 Limitationen und Ausblick............................................................... 224

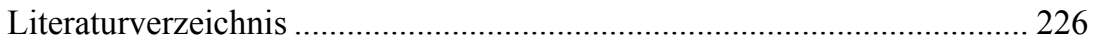

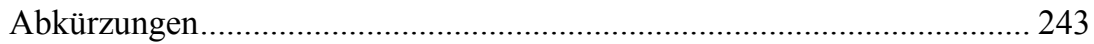




\section{Online-Anhang}

Den Online-Anhang finden Sie auf der Webseite zum Buch unter https://shop.budrich-academic.de/produkt (DOI 10.3224/86388759A).

Anhang I Tabelle Lehrvertragsauflösungen............................................2

Anhang II Tabelle Fachrichtungen im Detailhandel und bei Kaufleuten ....5

Anhang III Tabelle Übersicht Lehrverträge und LVA aller

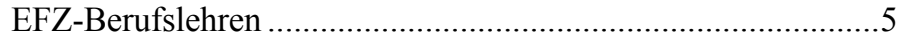

Anhang IV Tabelle Stabilitäten der Frauenanteile und der LVA-Quoten .....5

Anhang V Gründe der Lehrvertragsauflösungen........................................6

Anhang VI Tabelle Stabilitäten der Frauenanteile und der

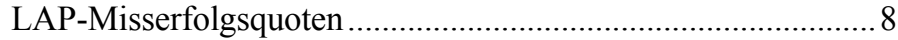

Anhang VII Tabelle Misserfolgsquote Lehrabschlussprüfungen ...................9

Anhang VIII Tabelle Anzahl Lernende nach Geschlechtstypik und

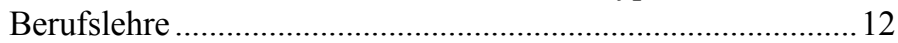

Anhang IX Abbildung Mittelwerte im 2. und 3. Lehrjahr........................... 13

Anhang X Tabelle Wilcoxon-Test bei verbundenen Stichproben............... 13

Anhang XI Tabelle Anzahl Lernende geschlechtstypischer und -untypischer Berufslehren ........................................................ 14

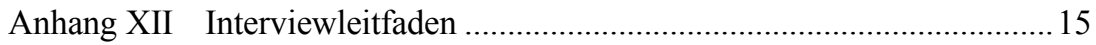

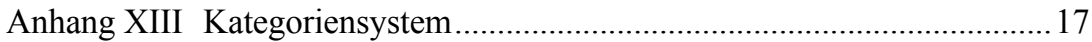




\section{Vorwort}

Bei der Analyse von Daten der Lehrabschlussprüfungen aus dem Jahr 2008 bemerkte ich, dass sich die Erfolgsquoten der frauen- von denjenigen der männertypischen Berufslehren unterschieden. In weiteren Berechnungen zeigte sich ein Muster: Lernende in geschlechtsuntypischen Berufslehren fielen öfter durch die Lehrabschlussprüfung als Lernende in geschlechtstypischen bzw. -gemischten Berufslehren. Dieser Befund ist der Ausgangspunkt der vorliegenden Arbeit. Sie stellt das Modell der geschlechtsuntypischen Berufssozialisation in den Rahmen der Berufsbildung und belegt seine Aussagen mittels quantitativen und qualitativen Methoden. Sie wurde an der Universität Basel als Dissertation angenommen.

Während der statistischen Auswertungen blieben mir zwei Presseartikel besonders in Erinnerung. Sie heben als augenfällige Überspitzung hervor, wie komplex und doppelbödig die Auseinandersetzung mit der Beziehung von Beruf und Geschlecht abläuft. So berichtet der „Tages-Anzeiger“ vom 13.10.2011 in seiner Analyse „Die schönste Frau ist ein Mann“ über den damals zwanzigjährigen Andrej Pejic, der für Jean-Paul Gaultier Frauenkleider vorführte. Darin offenbart sich mir die Widersprüchlichkeit. Einerseits lässt sich daraus eine Erweiterung der Geschlechterrolle ablesen: Männer können in Frauenberufen bestehen. Man könnte dies als Errungenschaft betrachten, die Vorbildcharakter für eine neue, aufgeklärte Generation von Männern mit weniger Hemmungen und Berührungsängsten in femininen Domänen hat und sich selbstverständlich darin bewegt. Andererseits kristallisiert sich an diesem Beispiel das Phänomen des postmodernes Patriarchats: Mann entwirft, produziert und verkauft nicht nur, was Frau tragen sollen, sondern demonstriert mittels eines jungen Männerkörpers, wie Frau darin auszusehen hat. Was bedeutet es, wenn ein Mann den Schönheitsidealen der zeitgenössischen Damenmode perfekt entspricht, ohne jegliche weibliche Rundungen, quasi als perfektionierte Reinkarnation der 80er-JahreAndrogynität? Oder ist es harmloses Marketing, das mit dem Irritationsmoment spielt?

Ein zweites Beispiel verdeutlicht, dass der Fokus, der auf Frauen in Männerberufen liegt, oft wenig mit der Tätigkeit, sondern mehr mit Äußerlichkeiten zu tun hat. „Hillary Clintons hundert Frisuren“ titelt die „NZZ am Sonntag" vom 11.12.2011. Die Auftritte der damaligen US-Außenministerin werden zwar mit dem Prädikat ,mächtigste Frau der Welt" geadelt, jedoch ebenso schnell wieder reduziert auf die Banalität der Form ihres Haarschnitts. Eine Darstellung, die sich der Berichterstattung über institutionelle Monarchien annähert. Die Frage bleibt, was Medienschaffende bezwecken, wenn sie über einen vermeintlichen Zusammenhang zwischen bildhaft festgehaltenen Momentaufnahmen von Haartrachten und den hineininterpretieren emo- 
tionalen Befindlichkeiten einer (weiblichen) Politikerin schreiben, die seit Jahrzehnten das einflussreichste Land der Welt mitgestaltet?

An diesen beiden Beispielen verdeutlicht sich pointiert der Vergeschlechtlichungsprozess der Arbeitswelt. Lernende haben jeweils vor kurzem den Schritt in diese Arbeitswelt vollzogen. Anhand der ersten Sozialisationsjahre im Beruf soll gezeigt werden, wie dieser Prozess für Jugendliche und junge Erwachsene im geschlechtsuntypischen Berufsumfeld abläuft. Aufgrund der relativ starken Segregation der Berufswelt sind Frauen, die sich in Männerdomänen bzw. Männer, die sich in Frauendomänen vorwagen, rar. Dies führte mich zu mancher spezifischen Herausforderung: Wo setze ich die Grenze zwischen geschlechtsuntypischen, -gemischten und -typischen Berufslehren? Wie erhalte ich eine genügend große Anzahl an Fällen für statistische Analysen? Wie finde ich die seltenen Interviewteilnehmenden? Wie bilde ich die hohe Dynamik des Untersuchungsumfelds ab: Berufe ändern ihre Bezeichnung oder ihr Geschlecht, Personen wechseln die Lehrstelle oder den Beruf. Und nicht zuletzt, wie habe ich mir Geschlecht vorzustellen? Im Verlauf der Arbeit wurde mir bewusst, dass ich Geschlecht nicht kategorial denken kann, sondern sowohl das biologische als auch das soziale Geschlecht als ein Kontinuum und vor allem als ein soziales Konstrukt.

Mein ganz herzlicher Dank geht an Prof. Dr. Markus P. Neuenschwander, der mir in der Funktion als Vorgesetzter und Betreuer/Gutachter Mentor, Ausbildner und Vorbild war, unermüdlich darin, zu lernen und Wissen weiterzugeben; Prof. Dr. Hans-Ulrich Grunder als Betreuer/Gutachter für die Unterstützung und die vielen wichtigen Tipps; Dr. Marc Baumgartner, Dr. Eva-Christina Edinger und Sandra Grizelj als intellektuelle und sportliche Sparring-Partnerinnen bzw. -Partner; Özlem Altin, Peter Ammann, Alexandra Bernoulli, Sandra Grizelj, Gerit Schütz und meine DissertationsSchreibgruppe fürs Gegenlesen und die unzähligen Verbesserungsvorschläge; meine Kolleginnen und Kollegen der Dissertationskolloquien Stephan Rösselet, Edith Niederbacher, Gerit Schütz, Astrid Marty, Simone Frey und Jennifer Fräulin für die wertvollen inhaltlichen Rückmeldungen; meine Eltern Margrit und Ruedi Rottermann für Mut, Trost, Durchhalteparolen, Zuversicht und den unerschütterlichen Glauben an mich; mein soziales Umfeld: Freundinnen und Freunde, Wohngenossenschaften und Arbeitskolleginnen und -kollegen für die vielen alltäglichen Unterstützungsleistungen.

Mein besonderer Dank geht an all die Personen, die mich bei der Datenbeschaffung unterstützten: die Interviewees und ihre Ausbildenden, das Bundesamt für Statistik, die Stadt Zürich, die TREE- und FASE B-Forschungsgruppe sowie ihre Teilnehmenden. Der PH FHNW und der Universität Basel danke ich für die Dissertationsentlastung und das produktive, forschungsfreundliche Umfeld. 


\section{Einleitung}

Die berufliche Sozialisation von Lernenden vollzieht sich auf dem Hintergrund eines nach Geschlecht segregierten Arbeits- bzw. Lehrstellenmarkts (Abraham/Arpagaus 2008, Charles/Grusky 2004, Gauthier/Gianettoni 2013, Leemann/Keck 2005). Für Lernende in geschlechtsuntypischen Berufslehren bedeutet dies, dass sie aufgrund ihres Geschlechts in der Minderheit sind, je nach Beruf und Lehrbetrieb die einzige weibliche bzw. männliche Person im Büro, auf der Baustelle, im Salon, im Geschäft oder in der Berufsfachschule. Dieses Alleinstellungsmerkmal generiert ein spezifisches Sozialisationsumfeld. Minderheiten haben eine hohe Gefahr, von der Mehrheit pauschalisiert und stereotypisiert zu werden (Erb/Bohner 2006, Kanter 1977). Da das Geschlecht eines der wichtigsten sozialen Kategorisierungssysteme ist (Eagly/Karau 2002), sind jene Personen besonders davon betroffen, die sich entgegen den gängigen Rollenerwartungen verhalten.

Die vorliegende Arbeit möchte einen Beitrag zum Verständnis der Situation von Jugendlichen in geschlechtsuntypischen Berufslehren leisten. Folgende übergeordnete Forschungsfrage gilt es deshalb im Hinblick auf die Überprüfung des postulierten Modells der geschlechtsuntypischen Berufssozialisation $\mathrm{zu}$ klären: Inwiefern sind Lernende in geschlechtsuntypischen Berufslehren benachteiligt?

Dazu habe ich auf der Grundlage von bestehenden Theorien und Forschungsergebnissen das ,Modell der geschlechtsuntypischen Berufssozialisation' entwickelt. Dieses Modell stelle ich in Kapitel 4 vor und überprüfe gezielt Teilaspekte in den fünf empirischen Kapiteln des zweiten Teils der vorliegenden Arbeit. Das Modell beschreibt, dass Lernende aufgrund ihres geschlechtsuntypischen Berufs in ihrem sozialen Umfeld (Familie, Schule und Beruf) Irritationen auslösen. Eltern, Lehrpersonen, Ausbildende, Mitarbeitende, Kolleginnen und Kollegen etc. reagieren mitunter dann ablehnend und missbilligend auf Berufswünsche, -ambitionen oder -entscheidungen, wenn diese nicht dem tradierten Geschlechtsrollenbild entsprechen. Die Eignungen, die Fähigkeiten sowie die Geschlechtsidentität dieser Jugendlichen werden vom sozialen Umfeld in Frage gestellt oder gar lächerlich gemacht (Simpson 2005). Dies und weitere Diskriminierungen müssen von den Jugendlichen verarbeitet und bewältigt werden. Diese zusätzlichen Belastungen führen dazu, dass Lernende in geschlechtsuntypischen Berufslehren häufiger von Lehrvertragsauflösung (Kapitel 5) und von Misserfolg an der Lehrabschlussprüfung (Kapitel 6) betroffen sind. Sie haben mehr Absenzen und leiden häufiger an psychosomatischen Beschwerden (Kapitel 8). Dennoch gelingt Lernenden, die bis zum Ende ihrer Lehrzeit durchhalten, die berufliche Integration und der Aufbau eines beruflichen Habitus (Kapitel 7).

Im theoretischen Teil der vorliegenden Arbeit beschreibe ich die einschlägigen Themen Beruf, Geschlecht, geschlechtsuntypische Berufe sowie 
mein postuliertes Modell der geschlechtsuntypischen Berufssozialisation. In Kapitel 1 ,Beruf und berufliche Grundbildung' bespreche ich fünf Erklärungsansätze zur geschlechtlichen Segregation des Arbeitsmarkts. Die Auslegeordnung möchte einen Beitrag zum Verständnis der Wirkmechanismen und vielschichtigen Restriktionen des Arbeitsumfelds in Bezug auf das Geschlecht bieten, in das die Lernenden hineinsozialisiert werden. Theorien zur beruflichen Sozialisation und zur Berufsfindung bilden den allgemeinen Teil, die Beschreibung der schweizerischen Berufsbildung den speziellen, um die Erfolge bzw. Misserfolge während der Berufsbildung einschätzen zu können. Die Indikatoren des beruflichen Erfolgs dienen der Validierung des Modells, indem überprüft wird, ob sich die Diskriminierungserfahrungen negativ auf die Erfolgsaussichten der Lernenden in geschlechtsuntypischen Berufslehren auswirken.

Dem umfassenden und beinahe allgegenwärtigen Thema Geschlecht nähere ich mich in Kapitel 2 über die Diskussion der Unterscheidung von biologischem vs. sozialem Geschlecht an. Ich werde aufzeigen, dass Geschlecht eine mentale Repräsentation oder ein Konstrukt ist, was uns gemeinhin wenig bewusst ist, und dass die Unterschiede zwischen den Geschlechtern oft erst hergestellt werden müssen (vgl. Doing Gender, Wetterer 2010). Dies zeigt sich auch darin, dass im Durchschnitt die meisten Unterschiede zwischen den Geschlechtern kleiner sind als die Bandbreite innerhalb des jeweiligen Geschlechts (vgl. Bischof-Köhler 2004). Die Übersicht der Erklärungsansätze der Geschlechtsunterschiede belegen, dass oft nicht die körperlichen Unterschiede (primäre und sekundäre Geschlechtsmerkmale) prägend sind, sondern wie wir als Gesellschaft die Unterschiede zwischen den Geschlechtern hervorheben und mit Verhalten sowie Persönlichkeitseigenschaften in Verbindung bringen. Dies führt zu einer Betrachtung der tradierten Rollen-, Arbeits- und Machtverteilungen in unserer Gesellschaft. Trotz zahlreicher Veränderungen sind nach wie vor die meisten außerfamiliären Entscheidungstragenden Männer. Dies prägt Geschlechtsidentität, Geschlechterstereotype und Geschlechtsrollenbilder.

Dem Forschungsstand zu Personen in geschlechtsuntypischen Berufen wird in Kapitel 3 Rechnungen getragen. Eine der ersten Forscherinnen, die dazu bereits 1977 eine Theorie entwickelt haben, ist Elisabeth Moss Kanter. Die Tokenismus-Theorie besagt, dass Frauen in geschlechtsuntypischen Positionen aufgrund ihrer leichten Sichtbarkeit stereotypisiert, ihre Leistungen jedoch selten anerkannt werden (Kanter 1977). Sie beschreibt damit das Phänomen, dass die Frauen in Führungspositionen ab einer gewissen Position an eine ,gläserne Decke' stoßen und ihre Karriere damit ins Stocken gerät. Das Gegenteil widerfährt Männern in frauentypischen Berufen, sie werden schnell in eine männertypische Position befördert. Dies wird von Christine L. Williams 1997 in der, Glaslift-Theorie‘ beschrieben. 
In einem integrativen Unterfangen, die geschlechtsuntypische Situation für Frauen und Männer zu erklären, entwickelte ich das Modell der geschlechtsuntypischen Berufssozialisation und prüfe es anhand des Erfolgs bzw. Misserfolgs von Lernenden in geschlechtstypischen, -gemischten und -untypischen Berufslehren. Dabei vergleiche ich im zweiten Teil der vorliegenden Arbeit die Quoten der Lehrvertragsauflösungen und Lehrabschlussprüfungen von 2008 bis 2012 aller Lernenden in der Schweiz. Die Reanalyse der Daten aus den Forschungsprojekten ,Familie Schule Beruf FASE B' und ,Transitionen von der Erstausbildung ins Erwerbsleben TREE ${ }^{\circ}$ geben weitere Aufschlüsse zur Validierung des Modells.

In der Interviewstudie (Kapitel 9) kommen schließlich die Lernenden in geschlechtsuntypischen Berufslehren selbst zu Wort. Die mit der qualitativen Inhaltsanalyse nach Mayring (2005) eruierten Befunde belegen insbesondere die zahlreichen und situationsspezifischen Belastungen. Eindrücklich schildern die Jugendlichen, wie sie mit den Diskriminierungserlebnissen aufgrund ihrer Minderheitsstatus umzugehen lernen und wie sie dabei auf persönliche sowie soziale Ressourcen zählen können.

Der dritte Teil der vorliegenden Arbeit umfasst die Integration und $\mathrm{Zu}$ sammenführung der theoretischen und empirischen Teile. In Kapitel 10 fasse ich die empirischen Befunde zusammen, diskutiere sie anschließend und ziehe Schlussfolgerungen. 


\section{Theoretischer Teil}

Beruf und Geschlecht sind miteinander verwoben. Berufe sind nicht geschlechtsneutral, sie haben ein Geschlecht (Schmid-Thomae 2012). Es bestehen starke geschlechtsspezifische Stereotype für die meisten Berufe: die Putzfrau, die Kleinkindbetreuerin, der Kranführer oder der Klempner. Ein weiteres Merkmal ist die geschlechtsspezifische Paarbildung von frauen- und männertypischen Berufen, bei denen die Tätigkeiten zwar im gleichen Umfeld erbracht werden, jedoch hoch spezialisiert und getrennt voneinander sind: die Krankenpflegerin und der Arzt, die Assistentin und der Manager, die Flugbegleiterin und der Pilot, die Serviceangestellte und der Koch.

Die Berufsbezeichnungen beschreiben lediglich einen Teil der Tätigkeiten, die ausgeführt werden, und rücken diesen Teil mit mehr oder weniger großen Alleinstellungsmerkmalen in den Vordergrund. Da die Namen der Berufe einen Einfluss darauf haben, ob eher Frauen oder eher Männer angesprochen werden, haben in den letzten Jahrzehnten viele Berufe ein geschlechtsneutralere Bezeichnung erhalten (etwa Fachangestellte/-r Betreuung; vgl. Berufsbildungsgesetz 2004). Dennoch ist die Arbeitswelt heute nach Geschlecht segregiert. Beispielsweise war der Frauenanteil 2010 im Berufsfeld ,Gesundheitswesen' 93 Prozent und im Berufsfeld ,Ingenieurswesen und technische Berufe 6 Prozent (BFS 2011). Sogar wenn in einem Berufsfeld beide Geschlechter zu gleichen Teilen vertreten sind, finden sich nach Geschlecht separierte Untergruppen, exemplarisch ,Detailhandelsfachmann/ -frau' in der Fachrichtung, Parfümerie' bzw. ,Autoteile-Logistik' (vgl. Online-Anhang I). Doch nicht nur im Verkauf macht sich diese Aufteilung der Arbeit zwischen den Geschlechtern bemerkbar, sondern ebenso in vielen anderen Bereichen des gesellschaftlichen Lebens. Eine amtliche Mitteilung des Eidgenössischen Büros für die Gleichstellung von Frau und Mann vom 27.06.2013 verdeutlicht dies:

So gleicht sich das Bildungsniveau von Frauen und Männern zwar tendenziell an, wenig hat sich hingegen in der geschlechtsspezifischen Berufs- und Studienfachwahl geändert. Die Erwerbsbeteiligung der Frauen ist deutlich gestiegen, doch nur vier von zehn Frauen arbeiten Vollzeit. Bei den Männern arbeitet nur einer von zehn Teilzeit $(13,8 \%)$. Unter den Arbeitnehmenden in Führungspositionen machen Frauen nur einen Drittel aus. Auf dem Weg in die nationalen und kantonalen Parlamente zeichnet sich eine Stagnation des langjährigen Vormarsches der Frauen ab: Der Frauenanteil unter den Kandidierenden beträgt $33 \%$, unter den Gewählten liegt er bei $29,0 \%$ im Nationalrat bzw. bei nur 19,6\% im Ständerat (2011). ${ }^{1}$

Die Arbeitsteilung nach Geschlecht im Familien- und Berufsalltag prägt die Sozialisation von Kindern und Jugendlichen, ihren Berufsfindungsprozess und die Entwicklung eines Berufshabitus. Nur sehr wenige Jugendliche ent-

1 Schweizerische Bundeskanzlei [https://www.admin.ch/gov/de/start/dokumentation/medienmitteilungen.msg-id-49198.html; Zugriff: 22.03.2017]. 
scheiden sich, einen Beruf zu erlernen, in dem sie in der geschlechtlichen Minderheit sind (Charles 2005, Wehner et al. 2016). Um zu verstehen, inwiefern Lernende in geschlechtsuntypischen Berufslehren diskriminiert werden und geringere Erfolgsaussichten haben, befassen sich die vier Kapitel des theoretischen Teils - wie bereits in der Einleitung angekündigt - mit den Rahmenbedingungen zum Beruf, zum Geschlecht und geschlechtsuntypischen Berufen.

Die zwei zentralen Themen in Kapitel 1 ,Beruf und berufliche Grundbildung' sind zum einen die geschlechtliche Segregation und zum anderen die Erfolgs- und Misserfolgsfaktoren während der Berufslehre. Die Erklärungsansätze der geschlechtlichen Segregation helfen zu verstehen, warum die Arbeitsteilung so stabil ist. Mit den Erkenntnissen zu Erfolgsfaktoren soll die Forschungsfrage der vorliegenden Arbeit geklärt werden. Weitere wichtige Themen zum Verständnis der Rahmenbedingungen liefern die Unterkapitel zur beruflichen Sozialisation und zu den Berufsfindungstheorien sowie die Beschreibung der beruflichen Grundbildung in der Schweiz.

Dem anderen großen Themenkreis der vorliegenden Arbeit ist das Kapitel 2 ,Geschlecht" gewidmet. Obwohl das biologische Geschlecht erst bei der Geburt explizit wird, gibt es Unterschiede zwischen den Geschlechtern bereits in der pränatalen Entwicklungsphase. Dennoch benötigen Menschen Jahre, um ihre Geschlechtsidentität auszubilden. Dabei interagieren individuelle und gesellschaftliche Prozesse. Die Sozialisation ist kein einseitiger Vorgang. Das Individuum ist selbst aktiv und konstruiert in Abhängigkeit von den Umweltbedingungen seine Realität. Die theoretischen Ausführungen zum Geschlecht in der vorliegenden Arbeit können nur wenige Aspekte aufgreifen. Die Auswahl ist so getroffen, dass die prägenden geschlechterstereotypisierten Erwartungen an die Jugendlichen beschrieben werden können.

Das Kapitel 3 ,Sozialisation in geschlechtsuntypische Berufe' fasst Theorien, Modelle und Befunde zum Forschungsstand der geschlechtsuntypischen Berufe zusammen. Dabei ist vor allem darauf Rücksicht zu nehmen, dass die Situation für Frauen und Männer in geschlechtsuntypischen Berufen unterschiedlich ist. Während einerseits Frauen sich öfter in geschlechtsuntypische Umfelder wagen und dabei zwar mehr verdienen als Frauen in geschlechtstypischen Berufen, jedoch weniger schnell Karriere machen können als ihre Kollegen, haben Männer andererseits andere Vor- und Nachteile in geschlechtsuntypischen Berufen: Beispielsweise weniger Einkommen, dafür bessere Laufbahnaussichten (Simpson 2005).

In Kapitel 4 ,Modell der geschlechtsuntypischen Berufssozialisation “ postuliere ich auf den oben genannten Grundlagen mein Modell. Lernende in geschlechtsuntypischen Berufslehren entsprechen nicht den Geschlechtsrollenerwartungen und werden deshalb von ihrem Umfeld diskriminiert, was wiederum ihre beruflichen Erfolge gefährden kann. 


\section{Beruf und berufliche Grundbildung}

,Arbeit' und ,zu arbeiten' ist in unserer Gesellschaft selbstverständlich und dient dem Gelderwerb (Rosenstiel 2001). Im Blickfeld liegt hier im engeren Sinn die Erwerbsarbeit, viele andere Tätigkeiten, wie Hausarbeiten oder ehrenamtliche Tätigkeiten, werden ausgeschlossen. Semmer und Udris (1995) definieren ,Arbeit' offener und zählen Tätigkeiten dazu, die nicht direkt mit monetärer Entschädigung verbunden sind.

Arbeit ist zielgerichtete menschliche Tätigkeit zum Zwecke der Transformation und Aneignung von Umwelt aufgrund selbst- oder fremddefinierter Aufgaben, mit gesellschaftlicher, materieller oder ideeller Bewertung, zur Realisierung oder Weiterentwicklung individueller oder kollektiver Bedürfnisse, Ansprüche und Kompetenzen. (Semmer/Udris 1995: 134)

Je geordneter und vielschichtiger die Arbeit im gesellschaftlichen Gefüge abläuft, desto ausgeprägter ist die Arbeitsteilung und desto größer ist die zeitliche Investition in den Lernprozess, um die Tätigkeiten ausführen zu können. Damit sind komplexere Aufgabenerfüllungen gemeint, die Fähigkeiten und Fertigkeiten voraussetzen. Diese wiederum müssen erworben werden und manifestieren sich als institutionalisiertes Kapital in einem Beruf (vgl. Bourdieu 1983). Im Wort „Beruf' sind eine Reihe von gesellschaftlichen Vorstellungen zusammengefasst. Mit der Zuschreibung eines Berufs zu einer Person ist die Erwartung verbunden, dass diese Person eine bestimmte Tätigkeit ausführen kann und für den Aufwand entschädigt wird. Berufe weisen nach Weber (1980) drei Hauptmerkmale auf: 1) Berufe vereinigen Leistung und Kompetenz eines Individuums in Form von Kenntnissen, Fertigkeiten, Fähigkeiten, Orientierungen, Verhaltens- und Handlungsmustern. Diese Kompetenzen erwirbt sich das Individuum in speziell hierfür ausgerichteten Ausbildungsgängen, deren erfolgreiche Absolvierung mit einem Zertifikat attestiert wird. 2) Berufe eröffnen Erwerbsmöglichkeiten und damit das Bestreiten des Lebensunterhalts. 3) Berufe sind von überdauerndem Bestand, wenngleich sie heute einem starken Wandel unterliegen und die erworbenen Kompetenzen nicht mehr ein Leben lang anwendbar bleiben. Die drei Merkmale von Weber (1980) finden sich ebenfalls in der kompakteren Definition von Beruf auf Wikipedia ${ }^{2}$. Dort wird Beruf definiert als „die im Rahmen einer arbeitsteiligen Wirtschaftsordnung aufgrund besonderer Eignung und Neigung systematisch erlernte und mit Qualifikationsnachweis versehene, dauerhaft gegen Entgelt ausgeübte spezialisierte Betätigung eines Menschen". Zentral für die vorliegende Arbeit sind damit folgende Merkmale, die den Beruf auszeichnen: Der Beruf ist eine komplexe, erlernte Tätigkeit zum Gelderwerb, die von den Organisationen der Arbeitswelt strukturiert wird 
und mit einem spezifischen Prestige verbunden ist. Der Beruf strahlt damit eine Reihe von Attributen aus, mit den sich das Individuum selbst ausdrücken kann.

Der Beruf und die mit ihm notwendigerweise verbundene Ausbildung haben also einerseits Auswirkungen auf die Selbstwahrnehmung des Individuums (Oerter/Dreher 1998), und andererseits hat er eine Signalwirkung, die von Personen im Umfeld des Individuums wahrgenommen und reflektiert wird. Insofern bildet der Beruf eine Identifikationsbasis und verleiht Prestige. Er wird damit durch die soziale Umgebung konstruiert.

Lempert (2002) versteht den Berufsverlauf oder die Berufsbiografie als eine Abfolge von Ereignissen im Berufsleben eines Individuums bzw. als Beschreibung dieser Ereignisse. Berufe bestimmen in unserer Gesellschaft „nach wie vor weitgehend das soziale Ansehen und das Selbstbewusstsein von Personen“ (Lempert 2002: 179). Der Beruf verortet eine Person in der Hierarchie der Gesellschaft. Die Person erhält damit eine Stellung und einen Status, aufgrund der Indikatoren ,Bildung', ,Beruf ${ }^{6}$ und ,Einkommen' (Hoffmeyer-Zlotnik/Geis 2003). Internationale Berufsklassifikationen wie ISCO (vgl. Kapitel 7) machen sich dies zunutze, um eine vergleichbare Einschätzung für den sozioökonomischen Status zu operationalisieren.

Die vorliegende Arbeit fokussiert auf Jugendliche, die am Anfang ihres beruflichen Werdegangs stehen und in diese komplex verzahnte Arbeitswelt hineinsozialisiert werden. Auf vier zentrale Aspekte in diesem sozialisierenden Umfeld geht dieses Kapitel im Folgenden ein. Erstens ist die geschlechtliche Segregation des Arbeitsmarkts eine zentrale Grundkonstante, die nicht nur, aber vor allem Lernende in geschlechtsuntypischen Berufslehren vor Herausforderungen stellt. Mit dem Ziel, herauszuarbeiten, warum die geschlechtliche Segregation des Arbeitsmarkts sich so dauerhaft festgesetzt hat, stelle ich fünf Erklärungsansätze vor (Unterkapitel 1.1, Geschlechtliche Segregation des Arbeitsmarkts'). Im Kontext des segregierten Arbeitsmarkts findet die Sozialisation im beruflichen Umfeld statt (Unterkapitel 1.2 ,Berufliche Sozialisation') und eng damit verbunden die Berufsfindung und die Berufslehre, die in Unterkapitel 1.3 ,Berufsfindung und Berufseinmündung“ behandelt werden. Der kurze Abriss zur dualen Berufsbildung in der Schweiz (Unterkapitel 1.4 ,Berufliche Grundbildung in der Schweiz') bildet den Kontext, in dem in der vorliegenden Arbeit ,Erfolg und Misserfolg während der Berufsbildung' (Unterkapitel 1.5) von Lernenden in geschlechtsuntypischen Berufsbildungen stattfindet. 


\subsection{Geschlechtliche Segregation des Arbeitsmarkts}

In der Schweiz stehen Frauen und Männern die Türen zu allen Berufen offen. Es gibt weder gesetzliche Bestimmungen noch institutionelle Vorgaben, die die Berufsfindung einschränken. Zahlreiche Bestrebungen, Berufe für beide Geschlechter attraktiv zu machen (z.B. Zukunftstag, Namensänderungen der Berufe), wurden in den letzten vier Jahrzehnten unternommen (Leemann/Keck 2005). Dennoch sind sowohl der Arbeitsmarkt (Leemann/ Keck 2005) als auch der Lehrstellenmarkt (Abraham/Arpagaus 2008) nach Geschlecht segregiert. In der Schweiz ist die geschlechtliche Segregation noch stärker ausgeprägt als in anderen Ländern (Charles/Grusky 2004, Gauthier/Gianettoni 2013). Dies veranschaulicht der Dissimilaritätsindex, der besagt, wie viel Prozent der Frauen und Männer ihren Beruf wechseln müssten, damit das Geschlechterverhältnis ausgeglichen wäre. Für den Schweizer Arbeitsmarkt wiesen Heintz et al. (1997) für 1990 einen Dissimilaritätsindex von über 60 Prozent aus. Aktuellere Daten sind gemäß Bundesamt für Statistik BFS aufgrund vieler fehlender Werte nicht verfügbar ${ }^{3}$. Der Dissimilaritätsindex für die Berufslehren lag 1998 in der Schweiz bei ebenfalls bei rund 60 Prozent (Borkowsky 2000). Bemerkenswert ist, dass er in den 1980erJahren von rund 68 Prozent auf rund 60 Prozent abnahm und dann bis 1998 auf diesem Niveau verharrte.

Der Arbeitsmarkt in Deutschland hat einen vergleichbar hohen Dissimilaritätsindex wie derjenige der Schweiz: 2010 hätten 58 Prozent der Frauen und Männer ihren Beruf wechseln müssen, um einen Ausgleich zu erreichen (Hausmann/Kleinert 2014). Bei der genaueren Analyse dieser Daten wird deutlich, dass der steigende Frauenanteil vor allem im gemischtgeschlechtlichen Dienstleistungsbereich für die Abnahme des Indexes um rund 8 Prozent seit 1976 verantwortlich gemacht werden konnte. Die ausgeprägten geschlechtstypischen Bereiche blieben jedoch konstant.

Der Dissimilaritätsindex ist mit einiger Vorsicht zu interpretieren. Er unterschätzt die Situation, da innerberufliche Segregationen auftreten, die statistisch nicht erfasst werden (Rodari/Anderführen 2011, Schwiter et al. 2014, Wetterer 1993). Beispielsweise tendieren im kaufmännischen Bereich Männer dazu, Kundenbesuche und Führungsaufgaben zu übernehmen, während Frauen öfter administrative Tätigkeiten ausführen (Heintz et al. 1997).

Neben der oben berichteten horizontalen Segregation (Geschlechterverteilung in den Berufen), führt die vertikale Segregation (Geschlechterverteilung in den Hierarchiestufen) zu einer Verstärkung der negativen Effekte zulasten der Frauen. Für Personen in frauenreichen Berufen bedeutet dies, verglichen mit Personen in männerreichen ${ }^{4}$ Berufen, Einbußen beim Lohn, bei den Auf-

3 BFS [http://www.bfs.admin.ch; Zugriff: 22.03.2017].

4 Erklärung der Wortneuschöpfung frauen-/männerreich in Abschnitt 4.1.6. 
stiegs- und Weiterbildungsmöglichkeiten, beim Sozialstatus und bei den Arbeitsbedingungen (Buchmann/Kriesi 2009, Bühler/Heye 2005, Charles 2005, Cornelissen et al. 2002, Gauthier/Gianettoni 2013, Schwiter et al. 2014).

Angesichts dieser Benachteiligungen und der Stabilität dieser Segregation trotz jahrelanger Maßnahmen dagegen wirkt die vermeintlich naheliegende Erklärung, Frauen und Männer interessierten sich nicht für die gleichen Berufe, vereinfacht. Eine Metaanalyse von Su et al. (2009) legt jedoch eben diese Erklärung nahe. Die Autoren fassen zusammen, dass sich Frauen für Menschen und Männer für Sachen interessieren. Sie schließen aus ihrer Studie, dass die unterschiedlichen Interessen von Frauen und Männern eine kritische Rolle bei der geschlechtstypischen Berufs- und Studienwahl spielen. Die geschlechtliche Segregation lässt sich jedoch nicht durch einen einzelnen individuellen Faktor erklären. Zusätzlich müsste in diesem Zusammenhang gefragt werden, wie das geschlechtsspezifische Interesse entsteht. Die berufliche Segregation bildet die Ausgangsbedingung, ohne sie gäbe es keine Personen, die einem geschlechtsuntypischen Beruf nachgehen könnten. Dieses Unterkapitel stellt darum Ansätze vor, die die Beständigkeit der beruflichen Segregation erklären. Einige dieser Ansätze fokussieren auf das Individuum und seine Entscheidungen (Abschnitt 1.1.1), andere auf strukturelle Mechanismen (Abschnitt 1.1.2), oder sie benennen Stereotype und Geschlechternormen (Abschnitt 1.1.3) sowie den Einfluss von sozialen SchlieBungsprozessen (Abschnitt 1.1.4). Eine Integration dieser unterschiedlichen Wirkebenen, die die Verschränkungen und Interaktionen der Phänomene berücksichtigt, beschreiben Schwiter et al. (2014) mit Bourdieus (2005) Geschlechtshabitus. Mit dem Geschlechtshabitus schließe ich die Betrachtungen der geschlechtlichen Segregation ab (Abschnitt 1.1.5).

\subsubsection{Individuumzentrierter Erklärungsansatz}

Als erste mögliche Erklärung für einen nach Geschlecht segregierten Arbeitsmarkt gelten individuelle Präferenzen, Interessen und Fähigkeiten, die zu einer eigenständigen individuellen Berufsfindung führen. Beispielsweise ist die Berufsfindung nach der Humankapitaltheorie eine rationale Entscheidung eines umfassend informierten Individuums (Polachek 1981). Angesichts der unterschiedlichen Investitionen von Frauen und Männern in der Haus- bzw. der Erwerbsarbeit können Frauen ihr akkumuliertes Humankapital weniger stark erhöhen als Männer. Aufgrund der erwarteten Erwerbsunterbrüche der Frauen wegen familiärer Verpflichtungen erscheint es rational, einen Beruf zu wählen, der weniger von einer zeitlichen Kompetenzentwertung betroffen ist. Unerklärt bleibt dabei, warum gerade die Pflegeberufe, die stetige Wei- 
terbildungen erfordern, sich so hartnäckig als typische Frauenberufe etabliert haben. Zweifel an diesem Erklärungsansatz sind angebracht (Mürner 2004).

Eine zweite individuumzentrierte Theorie greift auf das Konzept des weiblichen Arbeitsvermögens und damit auf die weibliche Sozialisation in der Familie und ihre gesellschaftliche Rolle als Hausfrau und Mutter zurück (Ostner 1990). Die Theorie besagt, dass Frauen Berufe suchen, die häuslichen und erzieherischen Tätigkeiten nahestehen, und dass sich solche Berufe herausgebildet haben, um der weiblichen Affinität zu entsprechen. Der empirische Nachweis ist allerdings gering (Buchmann et al. 2002). Heintz et al. (1997) kritisieren, dass Frauen zu stark als homogene Gruppen gesehen würden, sich in der Theorie die patriarchale Denkweise des 19. Jahrhunderts manifestiere und sich vermutlich Präferenzen besser formen ließen als die Möglichkeiten des Arbeitsmarkts (Mürner 2004). Schwiter et al. (2011: 21) bemängeln die einseitige Sicht auf ,individuelle (vergeschlechtlichte) Präferenzen" und heben weiter hervor, dass Geschlecht und Geschlechterdifferenzen als ,sozialisatorische Bedingtheit und gesellschaftliche Konstruiertheit" weitgehend ausgeblendet werden, wenn sie als Ursache und direkte Konsequenz unterschiedlicher Bevorzugungen verstanden werden.

Nach der individuellen Sichtweise entscheidet sich die Person gemäß ihren Interessen und Neigungen für einen Beruf. Die Erklärungsansätze aus der Humankapitaltheorie und der Theorie des weiblichen Arbeitsvermögens können jedoch nicht hinreichend aufzeigen, warum sich die geschlechtliche Segregation der Berufe ausgebildet und warum sie sich kaum verändert hat. Dies lässt vermuten, dass die individuellen Interessen und Entscheidung nicht unabhängig von der Umgebung und den strukturellen Bedingungen getroffen werden.

\subsubsection{Institutionenzentrierter Erklärungsansatz}

$\mathrm{Zu}$ den institutionellen Mechanismen, die zu einer ausgeprägten Segregation des Arbeitsmarkts führen, zählen die Berufsbildungssysteme, Einstellungsverhalten der Unternehmen, die Arbeitsverhältnisse und die Ausgestaltung von familienfreundlichen Strukturen wie Krippenplätzen, Ganztagesschulen und Blockzeiten (Schwiter et al. 2011). Die Berufslehren tragen insofern zur Segregation bei, als dass sie, ursprünglich den Männern vorbehalten, die tradierten Geschlechtsrollenbilder sowie die ihr zugrunde liegende Arbeitsteilung weiterführen und immer wieder herstellen (Krüger 2001). Die historisch gewachsene breite Differenzierung von männlich dominierten Berufen im handwerklichen Bereich trägt dazu bei, dass die wenigen Frauen, die sich in einen männerreichen Bereich vorwagen, zusätzlich marginalisiert sind. Im Gegensatz dazu sind die frauenreichen Berufe nicht so stark unterteilt. Dies führt zur Feststellung, dass der Großteil der Frauen in wenigen Berufen arbei- 
tet (Charles 2005). Ein weiterer Grund hierfür besteht darin, dass die vielen neugeschaffenen Arbeitsmöglichkeiten in der Pflege und der Kinderbetreuung eine Auslagerung von vormals häuslichen Frauenarbeiten sind, was wiederum Frauen für diese Arbeit prädestiniert. So weisen gerade jene Länder mit einem ausgeprägten Sozialangebot, wie die skandinavischen Staaten, eine Verfestigung der Segregation auf (Mürner 2004). In den letzten 15 bis 20 Jahren wurden die Zugänge zu diversen Berufen der Bereiche Pflege, Gesundheit, Pädagogik, Soziales um eine Bildungsstufe angehoben (von Sekundarstufe II auf Tertiärstufe, z.B. Pflegefachmann/-frau HF, Kindergarten- und Primarlehrperson). Ein langer, selektierender Bildungsweg, der eine abgeschlossene Grundbildung oder eine Mittelschule bedingt, ist nicht nur für Personen aus bildungsfernen Familien, sondern auch für männliche Jugendliche ein Grund, einen anderen Weg einzuschlagen (Faulstich-Wieland 2016b). Der Anteil junger Männer an den Mittelschulen ist tiefer als derjenige junger Frauen (BFS 20165). Die Zugänge zu besagten Berufen sind deshalb für junge Männer erschwert.

Die Theorie der statistischen Diskriminierung (Bielby/Baron 1986) beschreibt einen strukturalistischen Mechanismus, bei dem Arbeitgebende die Stelle eher an einen Mann vergeben als an eine Frau, da sie erwarten, dadurch die Produktivität der Unternehmung zu sichern. Die Einstellung von Personal geschieht aufgrund von unvollständigen Informationen, was dazu verleitet, auf Alltagserfahrungen zurückzugreifen. Das Vorurteil besteht darin, dass Frauen verallgemeinernd unterstellt wird, dass sie sich aus familiären Gründen eher zurückziehen, weniger Weiterbildungsbereitschaft haben und sich weniger mit der Berufstätigkeit identifizieren. Bielby und Baron (1986) konnten beweisen, dass diese Alltagshypothesen empirisch falsch sind. Frauen sind nicht weniger produktiv als Männer. Dennoch waren Frauen in ihrer Studie dort untervertreten, wo eine längere Einarbeitungszeit benötigt wird, dies im Gegensatz zur Postulierung des Humankapitalansatzes im vorangegangenen Abschnitt 1.1.1.

Die Theorie der statistischen Diskriminierung verdeutlicht sich anhand der Theorie des dreigeteilten Arbeitsmarkts (Mürner 2004). Der Arbeitsmarkt lässt sich nach Buchmann et al. (2002) in drei Teilarbeitsmärkte gliedern: den betriebsinternen, den fachspezifischen und den unstrukturierten Arbeitsmarkt. Während der unstrukturierte Arbeitsmarkt von Niedriglöhnen und Arbeitskräften ohne besondere Qualifikation gekennzeichnet ist, sind für die anderen beiden Arbeitsmärkte lange Einarbeitungszeiten und Fachwissen notwendig und sie sind mit guten Verdienstmöglichkeiten und einer relativen Sicherheit des Arbeitsplatzes verbunden. In diesen privilegierteren Teilarbeitsmärkten können sich Männer aufgrund der Vorurteile gegenüber Frauen besser etablieren.

5 BFS (2016). Lernende nach Bildungsstufe und Bildungstyp 2013/14 [http://www.bfs.admin .ch/bfs/portal/de/index/themen/15/04/00/blank/uebersicht.html; Zugriff: 03.08.2016]. 
Während Vorurteile im institutionenzentrierten Erklärungsansatz über Strukturen ihren Einfluss ausüben, wirken sich Geschlechternormen und Stereotype außerdem direkt auf das Verhalten von Menschen aus. Sie passen sowohl zu den individuum- als auch zu den institutionenzentrierten Erklärungsansätzen. Da sie zusätzlich einen unabhängigen Aspekt haben, werden sie hier im nachfolgenden Abschnitt separat beschrieben.

\subsubsection{Erklärungsansatz Geschlechternormen und Stereotype}

Tradierte Vorstellungen und kulturell geprägte Bilder, die Tätigkeiten und Berufe für Frauen oder Männer angemessen erscheinen lassen, gelten ebenfalls als Ursache für die geschlechtliche Segregation im Arbeitsmarkt. Traumberufe von Kindern weisen bereits diese Zweiteilung auf (Cornelissen/Gille 2005, Herzog et al. 2006). Nicht nur die Erwartungen, Wünsche und Interessen der Jugendlichen sind bei der Berufsfindung durch die Geschlechterstereotype beeinflusst, sondern auch die Attributionen und Ratschläge des sozialen Umfelds (Buchmann/Kriesi 2009, Cornelissen 2009, Imdorf 2004, Maihofer 2002, Ostendorf 2005, Schwiter 2011). Nach Cornelissen (2009) befürchten männliche Jugendliche, ein frauentypischer Beruf könnte ihre Männlichkeit gefährden, während sich weibliche Jugendliche fragen, ob sie den Herausforderungen in einem männerreichen Beruf gewachsen sind. Junge Erwachsene lassen sich ebenfalls von Geschlechtsrollenbildern leiten, wenn sie Beruf und Familie in Übereinstimmung bringen müssen (Schwiter 2011). Junge Erwachsene, die von einem geschlechtsuntypischen in einen -typischen Beruf gewechselt haben, geben an, nun ihrer Rolle als Ernährer (Verdienstmöglichkeiten) bzw. Mutter (Arbeitszeiten) besser gerecht zu werden (Schwiter et al. 2014).

Geschlechterstereotype führen einerseits zu einer Selbstselektion bei der Berufsfindung, andererseits bei der Fremdselektion zu Ausschlussverhalten von Arbeitgebenden bzw. Ausbildungsverantwortlichen bei der Einstellung von neuen Mitarbeitenden bzw. Lernenden. Dies wird im Folgenden an der Theorie der sozialen Schließung beschrieben.

\subsubsection{Erklärungsansatz soziale Schließung}

Bei der Theorie der sozialen Schließung handelt es sich um eine Fortführung von Max Webers Konzept offener und geschlossener sozialer Beziehungen (Weber/Winckelmann 1985). Die Theorie der sozialen Schließung erklärt „Prozesse, in denen soziale Akteure den Versuch unternehmen, Ressourcen, Privilegien, Macht und Prestige zu monopolisieren und andere Akteure davon auszuschließen“ (Mackert 2004: 11). In modernen Gesellschaften werden unterschiedliche Kriterien zur sozialen Ausgrenzung verwendet: Nationalität, 
Alter, soziale Schicht, Geschlecht, Ethnie, Religion, Sprache, Gesundheitszustand, Wohnort, sexuelle Orientierung, Familiensituation (z.B. Alleinerziehende vs. Zweielternfamilie), Diplome usw. Dabei ist das Kriterium nicht beliebig, sondern muss für die Privilegierten legitim und konsensfähig sein. Nach Cyba (1993) wäre im Zusammenhang mit geschlechtlicher Segregation dann von einer sozialen Schließung zu sprechen, wenn Männer in Führungspositionen ihre Konkurrenzvorteile sichern, indem sie Frauen nicht auf höhere Positionen gelangen lassen. Ausschlussmechanismen sind jedoch nicht nur bei der Besetzung von Kaderstellen auszumachen, sondern, wie Wilz (2004) feststellt, in so manchen Tätigkeiten, die zwischen den Geschlechtern differenzieren. Zusätzlich stellt sie neben der Trennung auch die Ungleichbehandlung der Geschlechter fest:

Die alltägliche Grenzziehung zwischen Männer- und Frauenbereichen (wie informeller Arbeitsteilung, Kooperationsformen oder symbolischer Darstellung) führt dann nicht nur zum wechselseitigen Ausschluss, sondern ist häufig auch mit einer Abwertung der ,weiblichen Bereiche“ verbunden. (Wilz 2004: 226)

Vorbehalte gegenüber der Theorie der sozialen Schließung ergeben sich einerseits aus der Frage, ob es sich um verselbstständigte Mechanismen handelt oder ob zwingend Personen (Akteurinnen und Akteure) den Ausschluss intendieren und aktiv umsetzen. Andererseits ist die Abgrenzung zum Beispiel zur Ausbeutung im Falle von ungelernten Arbeitskräften in Billiglohnsektoren oder in der geschlechtlichen Segregation zum Nachteil der Frauen nicht klar (Wilz 2004).

Nachdem ich die Segregation aus vier Blickwinkeln - Individuum, Institution, Geschlechternormen und Stereotype sowie soziale Schließung - zu erklären versucht habe, schließe ich die Betrachtungen mit der Darstellung eines integrativen Ansatzes ab. Dieser nimmt sich der in der Gesellschaft ablaufenden Prozesse an und beschreibt, wie sich das Individuum in einer vergeschlechtlichten Welt orientiert, verhält und behauptet.

\subsubsection{Erklärungsansatz Geschlechtshabitus}

Schwiter et al. (2011) gelingt mit dem Konzept des Geschlechtshabitus nach Bourdieu $(2005,2012)$ eine Integration von Erklärungsansätzen zur beruflichen Segregation. Sie verstehen berufliche Segregation nach Geschlecht als „komplexe Verschränkung von verschiedenen Einflussfaktoren auf subjektiver/individueller und institutioneller/struktureller Ebene" (Schwiter et al. 2014: 405). Der Geschlechtshabitus einer Person ist die Verinnerlichung von vergeschlechtlichten Wahrnehmungs-, Denk- und Handlungsschemata auf der Folie von gesellschaftlichen Strukturen (vgl. Bourdieu 2012, Jäger et al. 2015). Bourdieu (2005) erklärt in seiner Theorie der männlichen Herrschaft, wie die bürgerlich-kapitalistische Gesellschaftsordnung sich stetig reprodu- 
ziert. Frauen und Männer inkorporieren die binär-hierarchische heteronormative Geschlechtsordnung unbewusst. Die Geschlechterhierarchie erscheint selbstverständlich, naturgegeben und bedarf keiner Rechtfertigung. Die Einteilung des Kosmos in weiblich und männlich ist omnipräsent, nicht nur in der geschlechtlichen Segregation des Arbeitsmarkts und der Aufgabenteilung in der Familie, sondern auch in der Zuschreibung von Gegenständen, Räumen des Hauses, Emotionen etc. Beide Geschlechter unterstehen dem Zwang zur Vergeschlechtlichung. Bourdieu (2005) versteht diese als immerwährende Sozialisationsarbeit, in der die Differenzen zwischen Frauen und Männern stetig herausgearbeitet werden müssen. Männer müssen ihre Männlichkeit täglich aktiv beweisen. Sie sind darum ebenso verletzlich wie Frauen, obwohl sie von der Hierarchie mehr profitieren als diese und über mehr symbolische Macht verfügen. Sie sind stets mit der Angst des Verlusts der Männlichkeit konfrontiert, abhängig von der Bestätigung in ihrer Männlichkeit durch andere Männer und schlussendlich ebenso beherrscht von ihrer Herrschaft. Frauen sind den strukturellen Zwängen stärker unterworfen, da sie in eine passive Rolle gedrängt werden und von vornherein als ,symbolische Objekte“ konstituiert werden, wodurch sie ,,in einen andauernden Zustand körperlicher Verunsicherung oder, besser, symbolischer Abhängigkeit versetzt werden: Sie existieren zuallererst für und durch die Blicke der anderen" (Bourdieu 2005: 117, vgl. Jäger et al. 2015).

Aus der Perspektive von Bourdieu (2005) hält sich berufliche Segregation nach Geschlecht darum aufrecht, weil Kinder und Jugendliche bereits vergeschlechtlicht in die Schule kommen und so den Berufsfindungsprozess angehen. Es ist daher nicht verwunderlich, dass die Segregation trotz aller Veränderungen und Aufweichungen sowie Aufklärungsarbeiten und Öffnungsmaßnahmen derart ausgeprägt bleibt. Bourdieu (2005) sieht denn auch die „Überwindung der bestehenden Gesellschafts- und Geschlechterordnung nur durch eine dauerhafte Umwandlung der symbolischen Geschlechterordnung und der mit ihr verbunden inkorporierten Dispositionen" (Jäger et al. 2015: 32).

Kinder und Jugendliche wachsen in einem Umfeld auf, in dem das Geschlecht eine wichtige Rolle spielt, insbesondere wenn das Geschlecht dadurch salient wird, dass eine Person etwas tut, was implizit oder explizit dem anderen Geschlecht vorbehalten ist. Wie oben beschrieben, laufen viele dieser Prozesse unbewusst ab und werden im Älterwerden angeeignet, ohne dass sich das Individuum aktiv damit auseinandergesetzt hat. In den Sozialwissenschaften wird der Umstand des Hineinwachsens in und Aneignens von Umwelt mit dem Wort Vergesellschaftung bzw. hier Vergeschlechtlichung umschrieben. In der Psychologie findet sich eher der Terminus Sozialisation, wie im nachfolgenden Unterkapitel 1.2 beschrieben wird. Bei der Sozialisation entwickelt das Individuum Verhaltensweisen, die sowohl den eigenen Wünschen und Bedürfnissen entsprechen als auch den Wünschen und Be- 
dürfnissen der anderen nachkommen (vgl. Fröhlich 2000). In Bezug auf das erwünschte berufliche Verhalten von Männern und Frauen erfüllen Lernende in geschlechtsuntypischen Berufen die gesellschaftlichen Normen nicht. Die berufliche Sozialisation dieser Lernenden erscheint damit in einem zentralen Aspekt - dem Geschlecht - erschwert.

\subsection{Berufliche Sozialisation}

,Sozialisation' bezeichnet den allgemeinen und umfassenden Prozess, in dem das Individuum im Umgang mit anderen Menschen aktiv und passiv in impliziten und expliziten Lernprozessen gesellschaftliche und kulturelle Normen erwirbt und sich sozial relevante Erlebnis- und Verhaltensweisen aneignet (vgl. Fröhlich 2000). Entwicklung geschieht verstärkt bei Übergängen, weil das Individuum herausgefordert wird, sich an neue Anforderungen und Bedingungen anzupassen. Sozialisation ist also ein andauernder Prozess der Akkulturation, Enkulturation, Personalisation und Vergesellschaftung. In diesem Prozess eignet sich das Individuum im wechselseitigen Austausch mit seiner sozialen Umwelt Verhaltensweisen und Normen eben dieser Umwelt an. Dieser Verlauf ist im Sinn von Durkin (2002: 55) zu verstehen: „Sozialisation ist der Vorgang, bei dem sich Menschen die Verhaltensregeln und die Überzeugungs- und Einstellungssysteme aneignen, die einer Person ein Funktionieren als vollwertiges Mitglied der Gesellschaft erlauben". Nach Montada (1998: 57) erfolgt die Sozialisation ,durch Anleitung und Anforderung, Information und Belehrung, durch Beobachtung und Nachahmung von Vorbildern, durch Strafen und Belohnungen usw.“

Feij (1998) definiert die ,berufliche Sozialisation“ als einen Lernprozess, im Verlauf dessen Lernende Fähigkeiten, Wissen, Einstellungen, Werte und Verhaltensweisen erwerben, die sie als integrierte Berufstätige auszeichnen und zu einem wertschöpfenden Mitglied der Unternehmung machen. Lempert (2002) versteht unter beruflicher Sozialisation die „Entwicklung, das heißt die Entfaltung, Verfestigung und Veränderung individueller Persönlichkeitsstrukturen in Prozessen der direkten und indirekten Auseinandersetzung (Interaktion) mit sozialen und sozial geprägten Merkmalen beruflicher und betrieblicher Umweltstrukturen, die dadurch selbst reproduziert, aber auch transformiert werden können“ (Lempert 2002: 186). Dafür gilt die Anpassungsfähigkeit als Voraussetzung für eine gelungene Sozialisation (vgl. Wang et al. 2011). Sternberg (1997) ordnet diese Anpassungsfähigkeit nicht etwa einer passiven Eigenschaft zu, sondern bezeichnet sie als Intelligenzleistung in den neuen Situationen. Sich an neue Situationen anzupassen und rasch Automatismen zu entwickeln, bedeutet wiederum, sich vom Anpassungsdruck zu entlasten. 
Zwei wichtige Bereiche der beruflichen Sozialisation sind die soziale Integration in die Arbeitsgruppe des Lehrbetriebs und die Bewältigung der betrieblichen Anforderungen (vgl. Kammeyer-Mueller/Wanberg 2003, Neuenschwander/Gerber 2014). Zum einen resultieren aus einer gelungenen sozialen Integration Gefühle von Zugehörigkeit und tragfähige Beziehungen zwischen den Arbeitsgruppenmitgliedern. Zum andern stützt sich die subjektive Wahrnehmung der Aufgabenbewältigung auf das Feedback der Mitarbeitenden und Ausbildungsverantwortlichen. Neuenschwander und Gerber (2014) heben hervor, dass ein hoher Zusammenhang zwischen sozialer Integration und Aufgabenbewältigung im Lehrbetrieb besteht und dass die berufliche Sozialisation bereits in der obligatorischen Schule vorbereitet wird. Sowohl die soziale Integration als auch die Aufgabenbewältigung können mit der Passung der Lernenden zu ihrer Berufslehre, der Zufriedenheit mit der Berufslehre und der Intention, die Berufslehre abzuschließen, vorhergesagt werden. Die hohe Korrelation zwischen der sozialen Integration in den Lehrbetrieb und den drei postulierten Erfolgsfaktoren, Passung, Zufriedenheit und Lehrabschlussintention, belegt die ,hohe Bedeutung von sozialen Beziehungen für die Berufsausbildung“" (Neuenschwander/Gerber 2014: 257).

Eine unmittelbare Auseinandersetzung mit neuen Inhalten findet nach Lempert (2002) in Aus- und Fortbildungsgängen statt. Dabei unterstützt und motiviert der Wunsch, ein anerkanntes Mitglied der sozialen Einheit zu werden. Nach ihm lösen nicht nur Personenkontakte Sozialisationsprozesse aus, sondern auch der Umgang mit Werkzeugen und technischen Einrichtungen, da sie Produkte menschlicher Arbeit verkörpern und individuelle Handlungen sowie soziale und sozialisierende Interaktionen strukturieren.

Der Terminus ,Sozialisation“ kann weit gefasst werden und alle menschlichen Einflüsse beinhalten, die aus sozialen Interaktionen resultieren. In der vorliegenden Arbeit bezieht sich der Sozialisationsbegriff auf Erziehungs-, Bildungs- und Ausbildungsprozesse. Ein gelungener Sozialisationsprozess ermöglicht dem Individuum die Mitgliedschaft in der gewünschten sozialen Einheit. Der Wunsch, als vollwertiges Mitglied anerkannt zu werden und dazuzugehören, motiviert Jugendliche, diejenigen Fähigkeiten und Fertigkeiten zu erwerben, die eine Mitgliedschaft ermöglichen (vgl. Lempert 2002).

Bammé et al. (1983) unterscheiden drei Etappen der beruflichen Sozialisation: vor dem, für den und im Beruf. Während nach Heinz (1995) bei der Sozialisation vor dem Beruf besonders Elternhaus, Schule, Gleichaltrige und Berufsberatung auf Jugendliche im Berufsfindungsprozess den größten Einfluss ausüben (Abschnitt 1.2.1), gilt die Berufsbildung im Lehrbetrieb und in der Berufsfachschule als Sozialisation für den Beruf und die Erwerbstätigkeit als Sozialisation im Beruf (Abschnitt 1.2.2). 


\subsubsection{Familiäre und schulische Sozialisation vor dem Beruf}

Die vorberufliche, antizipatorische Sozialisation geschieht hauptsächlich in der Familie und in der Schule. Die Familie übt durch ihren sozioökonomischen Status, ihr Anregungsniveau und die Prägung von Wertvorstellungen Einfluss auf die Berufsfindung aus. Die Schule wiederum stellt Weichen durch Berufswahlvorbereitungsunterricht in Form von Auseinandersetzung mit den individuellen Möglichkeiten, Kennenlernen von Berufen, Schnupperlehren, Berufsberatung und nicht zuletzt mittels Benotung und Selektion (Bammé et al. 1983). Die geschlechts- und schichtspezifische Sozialisation in der Familie und in der Schule fördert und begrenzt jedoch den Erwerb von Fähigkeiten, Interessen und Wertvorstellungen für bestimmte Berufe und reproduziert sowohl die Ungleichheit in der Gesellschaft wie auch die geschlechtliche Segregation (Hurrelmann 2002).

\subsubsection{Sozialisation durch den Beruf (für den und im Beruf)}

Für Imdorf (2005) beginnt die berufliche Sozialisation von Jugendlichen mit dem Erhalt einer Lehrstelle. Voraussetzungen für das Zustandekommen eines Lehrvertrags ist, dass sich die Jugendlichen für eine bestimmte Berufsausbildung entscheiden und die Zusage eines Betriebs für einen Ausbildungsplatz erhalten, was das Vertrauen ausdrückt, dass die Person mit den beruflichen und betrieblichen Anforderungen zusammenpasst (Neuenschwander/Gerber 2014, Neuenschwander et al. 2012). Die berufliche Ausbildung, die als Sozialisation für den Beruf bezeichnet werden kann, ist wesentlich durch die vorangegangen Etappen beeinflusst und wirkt sich wiederum auf die folgenden Etappen aus. Es ist eine Übergangsphase, die die meisten in der Jugend durchlaufen und die durch viele andere Entwicklungsaufgaben geprägt ist (Bammé et al. 1983). Die Sozialisation im Beruf beginnt mit dem Eintritt in die Erwerbstätigkeit und ist ein lebenslanger Prozess der Persönlichkeitsentwicklung, der durch geschlechts-, schicht- und berufsspezifische Selektions-, Bildungs- und Laufbahnmuster geprägt ist. Damit spiegelt die individuelle Entwicklung die hierarchische Gliederung der Gesellschaft wider (Bammé et al. 1983).

Die Berufsbildung im Sinn einer Sozialisation befähigt eine Person in einem bestimmten Berufssegment und manifestiert sich in Form von Diplomen und Zertifikaten. Dies bedeutet jedoch auch eine Eingrenzung. Lempert (2002: 186f) beschreibt die Folgen derart, dass Ungleichheiten erhalten bleiben und nicht abnehmen: ,Indem sie Menschen nicht nur für verschiedenartige berufliche Leistungen ertüchtigen und für andere eher untauglich machen, sondern ihnen zugleich unterschiedlich bewertete betriebliche und gesellschaftliche Karrieren eröffnen und andere verschließen, tragen all diese Pro- 
zesse eher zur Stabilisierung als zum Abbau sozialer Ungleichheit bei“. Wenn Berufsbildung und berufliche Sozialisation derart den individuellen Werdegang und die gesellschaftlichen Bedingungen strukturieren, lohnt es sich, einen Blick auf den wichtigen Prozess der Berufsfindung zu werfen und die Frage zu stellen, ob sich in der Berufsfindung ebenso Erklärungen für die geschlechtliche Segregation des Arbeitsmarkts finden lassen.

\subsection{Berufsfindung und Berufseinmündung}

Jugendliche in der Schweiz haben stabile geschlechtsspezifische Berufsvorstellungen (Cornelissen/Gille 2005, Müller 2009). Diese Vorstellungen zu künftigen Berufen verinnerlichten die Jugendlichen während ihrer Sozialisation in der Kindheit, und sie manifestieren sich als Traumberufe. Während Mädchen gemäß Herzog et al. (2006) eher in einem pflegerischen oder erzieherischen Beruf tätig sein wollen (Lehrerin, Tierärztin, Krankenschwester, Kindergärtnerin), wählen Jungen dem männlichen Stereotyp entsprechend Berufe, die nach außen orientiert sind sowie Technik, Macht, Kraft und Geschwindigkeit implizieren (Pilot, Polizist, Buschauffeur, Lokomotivführer). Als Grundlage für den beruflichen Orientierungsprozess schränken die Traumberufe den Horizont möglicher Berufe ein. So befinden sich nach Analysen von Müller (2006b) lediglich 4.6 Prozent der Lernenden in einem der 14 Berufslehren, die unter 210 untersuchten als nicht segregierte Berufslehren identifiziert worden waren. Die Mehrheit der Lernenden absolviert die Berufslehre demnach in einem nach Geschlecht segregierten Beruf und die meisten in einem geschlechtstypischen Berufsumfeld. Zusätzlich unterstützt eine geschlechtstypische Berufsfindung die Entwicklung der eigenen Geschlechtsidentität (Unterkapitel 2.4). So gesehen ist der Berufsentscheid eine Möglichkeit, sich als Frau bzw. Mann zu präsentieren. Damit verorten sich die Jugendlichen im System der binär kodierten Geschlechterwelt (vgl. Brandt/Cornelissen 2004, vgl. Unterkapitel 2.5).

Im Folgenden stelle ich in Abschnitt 1.3.1 drei klassische Berufsfindungstheorien vor, die das Geschlecht als Einflussgröße berücksichtigen, und in Abschnitt 1.3.2 die Berufsfindung als Abfolge von Kompromissen unter Bezugnahme von Gottfredsons (1981) Theorie der beruflichen Ambitionen. In Abschnitt 1.3.3 beschreibe ich Ansätze, die die geschlechtsspezifische Berufseinmündung erklären. In Abschnitt 1.3.4 schließt ein Perspektivenwechsel hin zum Einstellungsverhalten von Betrieben die Betrachtung zur Berufsfindung und -einmündung ab. 


\subsubsection{Berufsfindungstheorien}

Jugendliche, die eine duale Ausbildung beginnen, haben einen Prozess hinter sich, der von ihnen äußerst individuell erlebt wird. Dennoch ist der Berufsfindungsprozess von Personen, Strukturen und Bedingungen beeinflusst. Für die vorliegende Arbeit sollen insbesondere Aspekte beleuchtet werden, die Erklärungen für die spezifische Berufseinmündungssituation von Jugendlichen anbieten, die eine geschlechtsuntypische Berufsausbildung wählen. ${ }^{6}$ Herzog et al. (2006) schlagen auf der Grundlage einer differenzierten Beschreibung des Berufsfindungsprozesses aus sechs unterschiedlichen Perspektiven ein Gesamtmodell vor:

Die verschiedenen Aspekte der Berufswahl lassen sich zu einem Gesamtmodell zusammenfügen, das ein aktives Subjekt supponiert, das unter Einsatz seiner persönlichen und unter Nutzung seiner sozialen Ressourcen, in Kenntnis seiner Fähigkeiten, Interessen und Werthaltungen sowie in Abwägung seiner Chancen, eingeschränkt durch äußere Bedingungen (wie Arbeitsmarkt und Berufsangebot), aber auch gestützt durch institutionelle Vorgaben eine Serie von Entscheidungen trifft, die seiner Situation und im Rahmen eines Übergangsprozesses in eine berufliche Ausbildung führen. (Herzog et al. 2006: $25 f$ )

Das Zitat macht das vielschichtige Gewebe von intra- und interindividuellen sowie institutionellen Prozessen deutlich, die während der Berufsfindung ablaufen, und integriert diverse Betrachtungsweisen. Im Folgenden werden diese Perspektiven einzeln beleuchtet. Die Berufsfindung ist aus entwicklungspsychologischer Perspektive ein lang andauernder Prozess der Anpassung der Berufsvorstellungen und -träume an die real existierenden Arbeitsmarktbedingungen. Diesen Prozess der Anpassung in Form von Kompromissfindung beschreibt Gottfredson (1981). Sie räumt dem Geschlecht eine zentrale Rolle ein, was auch für die vorliegende Arbeit wichtig ist. Deshalb wird diese Theorie im Abschnitt 1.3.2 etwas ausführlicher beschrieben. Die in diesem Unterkapitel zuerst beschriebenen Berufsfindungstheorien beziehen das Geschlecht als Einflussfaktor in ihre Überlegungen ebenfalls, wenngleich weniger explizit, mit ein.

Die entwicklungspsychologische Berufsfindungstheorie von Super (1992, 1994) teilt die Lebensspanne in fünf berufsrelevante Phasen: ,Wachstum “ (Geburt bis ca. 14 Jahre), ,Exploration' (ca. 14 bis 25 Jahre), ,Etablierung “ (ca. 25 bis 45 Jahre), ,Erhaltung' (ca. 45 bis 65 Jahre) und ,Abbau' (ab ca. 65 Jahren). Die Berufsfindungsphase im engeren Sinn ist in der Explorationsphase verortet, während der die Ausdifferenzierung des Selbstkonzepts und die Persönlichkeitsentwicklung ebenfalls wichtig sind. Super (1994) nahm eine Systematisierung von Laufbahnbiografien vor und entwickelte nach

6 Für einen Gesamtüberblick über die vielschichtigen Berufswahl-/Berufsfindungstheorien sei auf Busshoff (1989), Brown und Brooks (1994), Zihlmann (2009) und Brüggemann und Rahn (2013) hingewiesen. 
Geschlecht getrennte idealtypische Berufskarrieren. Für Frauen fand er sieben Laufbahnmuster (häusliche, übliche, zweigleisige, stetige, unterbrochene, unstetige und extrem unstetige), für Männer sind es deren vier (stetige, übliche, unstetige, extrem unstetige). Kritisch betrachtet sind diese Laufbahnmuster jedoch eher als Beschreibung denn als Erklärungsansatz zu verstehen und bleiben in ihrer Vorhersagekraft eingeschränkt (Herzog et al. 2006). Für die Beschreibung von geschlechtsuntypischen vs. -typischen Berufsbiografien eignen sich die Konzepte eher weniger.

Krumboltz' Theorie der Berufsentscheidung als sozialem Lernprozess (vgl. Mitchell/Krumboltz 1994) kennt vier Faktorengruppen, die den beruflichen Entscheidungsweg beeinflussen: 1) genetische Eigenschaften (unter anderem das Geschlecht) und besondere Begabungen, 2) Umweltbedingungen und Ereignisse, 3) Lernerfahrungen sowie 4) Aufgaben- und Problemlösefähigkeit. Diese Faktoren wirken zusammen mit generalisierten Selbstbeobachtungen (über Aufgabenwirksamkeit, Interesse und eigene Wertvorstellungen) sowie generalisierten Weltanschauungen. Obwohl diese Prozesse unabhängig vom Geschlecht ablaufen, sind die spezifischen Lernerfahrungen und die konkreten Umweltbedingungen stark vom Geschlecht abhängig. Dabei spielt es beispielsweise eine Rolle, wie die gesellschaftliche Akzeptanz von Männern in einem bestimmten frauentypischen Beruf ist (McLean/Kalin 1994, Simpson 2005).

Die sozial-kognitive Berufsfindungstheorie nach Hackett und Betz (1981) basiert auf Banduras (1977a) Theorie der Selbstwirksamkeit und bezieht sich darauf, inwieweit eine Person die Überzeugung entwickelt, sie könne eine bestimmte Aufgabe erfolgreich bewältigen. So sind geschlechtsspezifische Unterschiede im Berufsverhalten häufig auf unterschiedliche Sozialisationsprozesse zurückzuführen. Jugendliche in geschlechtsuntypischen Berufslehren erleben demnach andere Aspekte der Sozialisation (Loughrey 2008).

Die drei oben beschriebenen Berufsfindungstheorien erklären die spezifische Situation für Jugendliche in geschlechtsuntypischen Berufslehren lediglich am Rand oder indirekt. In Bezug auf die vorliegende Arbeit bedürfte es einer Erweiterung dieser Theorien. Gegebenenfalls können Gendertheorien oder soziale Lerntheorien eine ergänzende Funktion übernehmen. Beispielsweise legen Herzog et al. (2006) dar, dass mit Bezug auf die Geschlechtsspezifik der Berufsentscheidung weniger die Geschlechtsrollenorientierung als vielmehr die Traumberufe und die beruflichen Werte und Interessen in einem starken Zusammenhang stehen. Während für weibliche Jugendliche soziale Orientierung und eine soziale Tätigkeit wichtig sind, legen männliche Jugendliche mehr Wert auf beruflichen Erfolg und haben mehr Interesse an handwerklichen Tätigkeiten. Eine frühe Einschränkung des Berufsspektrums durch die Traumberufe und die Werthaltung der Jugendlichen wirkt sich stark auf die Wahl eines geschlechtstypischen Berufs aus. Die sozialisationstheoretische Perspektive von Gottfredsons Kompromiss-Ansatz (1981) stellt die 
Berufswahl in den Kontext gesellschaftlicher Bedingungen. Sie nimmt Bezug auf die Segregation der Arbeitswelt und die Zweigeschlechtlichkeit, was für die Betrachtung von Lernenden in geschlechtsuntypischen Berufslehren besonders relevant ist.

\subsubsection{Berufsfindung als Abfolge von Kompromissen}

Die sozialisationstheoretische Berufsfindungstheorie der beruflichen Ambitionen nach Gottfredson $(1981,1996,2005)$ erklärt die Geschlechtsunterschiede bei beruflichen Interessen als ein Produkt der geschlechtsrollenspezifischen Sozialisation. Das Geschlecht ist bei der Entwicklung des Selbstkonzepts im Alter von 6 bis 8 Jahren wichtig und somit ein wirksames Selektionskriterium im Vorfeld der eigentlichen Berufsfindung. Gottfredson (1981) beschreibt den Berufsfindungsprozess als eine Reihe von Kompromissen und damit als eine gestufte Abkühlung der beruflichen Aspirationen. Neben dem ,Geschlecht' bilden ,Status` und ,Interessen/Fähigkeiten“ die drei Kompromissbereiche. Die Kompromisse werden in drei Stufen durchlaufen und sind in der Regel irreversibel. 1) Im Alter von 6 bis 8 Jahren schließen Kinder diejenigen Berufe aus, die ihren geschlechtstypischen Vorstellungen widersprechen. 2) Mit 9 bis 13 Jahren erfolgt die Auswahl entsprechend dem sozialen Status bzw. den verinnerlichten Prestigevorstellungen. 3) Die dritte Restriktion orientiert sich im Alter von 14 bis 16 Jahren an den persönlichen Interessen und Fähigkeiten. Aufgrund der Orientierung an den Geschlechterstereotypen früh im Berufsfindungsprozess ziehen Jugendliche später geschlechtsuntypische Berufe nicht mehr in Erwägung. Wenn Kompromisse gemacht werden müssen, weil sich der Traumberuf nicht verwirklichen lässt, machen Jugendliche zuerst Eingeständnisse zugunsten ihrer Interessen und Fähigkeiten. Als Zweites geben sie ihre Ambitionen im Bereich des Berufsprestiges auf, und erst als Letztes erwägen sie einen Beruf, der nicht dem Geschlechterstereotyp entspricht (Gottfredson 1981). Diese restriktive Beschreibung konnte eine Langzeitstudie von Junk und Armstrong (2010) nicht erhärten. Sie stellen fest, dass die berufliche Aspiration in den beiden anderen von Gottfredson beschriebenen Kompromiss-Dimensionen ,Status' bzw. ,Interessen/Fähigkeiten“ stabiler war als in der Dimension ,Geschlecht'. Eine teilweise erfolgreiche Bestätigung der Theorie gelang Helwig (2001). Demnach achten Kinder eher auf das ,Prestige' des Berufs als auf ihre ,Interessen/Fähigkeiten'. Zu bedenken ist, ob an der Reihenfolge festgehalten werden muss oder ob Gottfredsons (1981) Theorie vor allem eines ins Zentrum der Berufsfindung von Kindern und Jugendlichen stellt: die Abhängigkeit von Geschlecht neben derjenigen von familialen und lokalen Bedingungen (Hartung 2005). 
Für die Schweiz liegen zwei empirische Arbeiten vor, die sich eingehend mit der Theorie von Gottfredson (1981, 1996, 2005) auseinandergesetzt haben. Zum einen kann Imdorf (2005) belegen, dass Frauen, männliche Migranten sowie Schülerinnen und Schüler aus Schulniveaus mit Grundansprüchen $\mathrm{zu}$ stärkeren Kompromissleistungen gezwungen sind. Zum anderen weist Müller (2009: 184) nach, dass Schülerinnen kompromissbereit bezüglich typischer Männerberufe sein müssen: „Der Kompromisszwang - besonders auch im Bereich der Geschlechtstypik - geschieht primär in Abhängigkeit der schulischen Voraussetzungen und nicht in Abhängigkeit der Geschlechtszugehörigkeit“". Diese Befunde lassen also an der Abfolge der Stufen in Gottfredsons (1981) Theorie zweifeln. Eine Relativierung der Stufung und der Reihenfolge ergibt sich durch die Überlagerung und das Überspringen von Stufen, die in der Realität auftreten können (Busshoff 2009). Ein weiterer Kritikpunkt an dieser Theorie ist das nativistisch orientierte Entwicklungskonzept zur Erklärung des Kompromissverhaltens. Nach Müller (2009) würde es genügen, ,darauf zu verweisen, dass die geschlechtsspezifischen Sozialisations- und Lernprozesse sich an traditionellen Frauen- und Männerbildern orientieren und dass diese beruflichen Frauen- und Männerbilder sowohl in den Jugendlichen als auch in den Vorstellungen von Lehrbetriebsverantwortlichen hartnäckig Bestand haben“ (Müller 2009: 184). Dieser Kritik ist entgegenzuhalten, dass nicht erst Jugendliche, sondern bereits Kinder von diesen gesellschaftlichen Rollenerwartungen beeinflusst werden und sich nicht nur daran orientieren, sondern sich selbst beschränken. Diese Einschränkung ist im Sinn der Minoritäten-Stress-Theorie (Testa et al. 2015) als eine Art antizipierter Stress zu verstehen, dem sich ein Individuum aussetzt, wenn es sich entgegen der Geschlechtsrollenerwartung für einen geschlechtsuntypischen Beruf entscheiden würde. Der Gewinn der Theorie von Gottfredson (1981) liegt zusätzlich in der heuristischen Funktion als „Such- und Interpretationsschemata für die Aneignung von Realität" (Busshoff 2009: 14).

Die vorgestellten traditionellen Berufsfindungstheorien haben verdeutlicht, wie sich Jugendliche in ihrem Umfeld geschlechtsspezifisch verhalten. Die Gründe für dieses Verhalten werden im nachfolgenden Abschnitt vorgestellt.

\subsubsection{Ansätze zur Klärung geschlechtsspezifischer Berufseinmündung}

Zur Orientierung in diesem Abschnitt folge ich dem Vorschlag von Brandt und Cornelissen (2004) und teile diese Erklärungsansätze den strukturorientierten bzw. subjektorientierten Ansätzen zu. Diese Unterscheidung gelingt nicht in jedem Fall, da sich die Argumente oft nicht eindeutig zuteilen oder aber beiden Aspekten zuordnen lassen. 


\subsubsection{Strukturorientierte Erklärungsansätze}

Bei den strukturorientierten Ansätzen steht der Versuch der Klärung der sozialen Ausgrenzung aufgrund des Geschlechts im Arbeitsmarkt im Zentrum. Dazu wird beispielsweise die historisch bedingte Aufteilung der Geschlechter im Berufsbildungssystem genannt (vgl. Krüger 1986, 1991). Frauen sollten dabei die berufliche Bildung als Vorbereitung auf ihre spätere Familienarbeit sehen, eine Ernährerinnenrolle war nicht vorgesehen. Dieser historische Umstand erklärt jedoch nicht, weshalb die jungen Frauen heute noch in eine frauentypische Berufsbildung einmünden, insbesondere da ihnen vermutlich klar ist, wie gering ihre Verdienst- und Karriereaussichten in diesem Bereich sind (vgl. Brandt/Cornelissen 2004).

Andere Ansätze beleuchten Abschottungs- und Schließungsmechanismen. Der Fokus liegt auf dem Einstellungsverhalten der Ausbildungsbetriebe. Sie beurteilen die Eignung der Bewerbenden durch die Geschlechterbrille, sie vertrauen auf ihre Intuition. Solche Entscheide sind jedoch dahingehend verzerrt, als dass Bekanntes und Vertrautes bevorzugt wird. Ein Mann fühlt sich einem männlichen Jugendlichen näher als einer weiblichen und umgekehrt. Die tradierte Geschlechterordnung wird damit von den Beteiligten unbewusst weitergeschrieben. Dieser Mechanismus wirkt jedoch nicht erst beim Einstellungsentscheid, sondern bereits beim Bewerbungsverhalten, indem Jugendliche ihre geringeren Chancen im geschlechtsuntypischen Berufsumfeld antizipieren und sich dort gar nicht erst bewerben. Das soziale Umfeld verstärkt diese Einschätzung und schürt die Unsicherheit über das Bestehen in einer geschlechtsuntypischen Berufslehre. Was Brandt und Cornelissen (2004: 25) für Frauen formulieren, gilt wohl entsprechend ebenso für Männer: „Gemäß diesem Ansatz wählen Frauen Frauenberufe nicht, sondern sie werden durch antizipierte ungünstigere Chancen in ,geschlechtsfremden Territorien" in die traditionellen Frauenberufe gedrängt." Dass diese antizipierten verminderten Chancen empirische Evidenz haben, konnten Nissen et al. (2003) anhand von erhöhten Arbeitslosenquoten bei Frauen in technikund naturwissenschaftlichen Berufen in den 1990er-Jahren belegen.

Doch könnte mit Heintz et al. (1997) oder Brandt und Cornelissen (2004) argumentiert werden, dass nicht strukturelle Gründe für die segregierte Berufsfindung sprechen, sondern vielmehr kulturelle - dies insofern, als die Vermengung von Geschlechts- und Berufssystem mittels Analogienbildung Territorien (vgl. Hagemann-White 1984) schafft, die entweder von Frauen oder von Männern besetzt werden. Als ein prominentes Beispiel steht die Technik, die als ein Hoheitsgebiet der Männer gilt und deren Beherrschung mit hohem Prestige verbunden ist (vgl. Schmid-Thomae 2012). Sich in einem geschlechtsfremden Territorium zu bewähren, erfordert von Frauen und Männern Selbstbehauptung gegenüber Vorbehalten und Verunsicherungsversuchen seitens des sozialen Umfelds (vgl. Brandt/Cornelissen 2004). Obwohl sich für Männer in frauenreichen Berufen durch die gängigen Klischees im 
Bereich Kompetenzzuschreibung Vorteile ergeben, vermag das Absprechen der Männlichkeit zu verunsichern, vermutlich ebenso wie Unterstellungen, denen Frauen begegnen, sie könnten die Anforderungen weniger gut bewältigen. ,Während es bei Frauen vor allem Selbstzweifel sind, die sie daran hindern, einen Männerberuf zu ergreifen, wird den Männern das Geschlechtslabel des Berufes zum Problem“" (Heintz et al. 1997: 226).

\subsubsection{Subjektorientierte Ansätze}

Dass die Berufsfindung, wie vielfach versucht, als eine rationale Entscheidung bezeichnet werden kann, in der das Individuum Kosten und Nutzen einer Berufsausbildung auf das spätere Leben abschätzt, muss - obwohl stringent formuliert - angezweifelt werden. So ist bei diesem ersten subjektorientierten Ansatz kaum zu erklären, warum sich die Mehrheit der Frauen für Berufe entscheidet, die ihnen ökonomisch kaum Vorteile verschaffen, ihrem schulisch erworbenen Fähigkeitsprofil wenig entsprechen und sie bei der Vereinbarkeit von Familie und Beruf wenig unterstützt (vgl. Brandt/ Cornelissen 2004, Heintz et al. 1997, Nissen et al. 2003, Schmid-Thomae 2012).

Einen zweiten subjektorientierten Ansatz zur Erklärung der geschlechtstypischen Berufsfindung sehen Brandt und Cornelissen (2004) in der biografischen Konstruktion von Geschlecht. Diese wird mit dem Berufskonzept verwoben und hilft Jugendlichen im Aufbau und in der Festigung ihrer Geschlechtsidentität. Eine Orientierung an den tradierten Geschlechterstereotypen führt damit zu gesellschaftlicher Akzeptanz.

Als Motiv geschlechtsspezifischer Berufsorientierung wird einzig der Wunsch unterstellt, den Stereotypen einer geschlechterkodierten Welt zu entsprechen, auf diese Weise einen gesellschaftlich akzeptierten Selbstentwurf vorzulegen und mit einer den Geschlechterstereotypen entsprechenden Berufswahl vielleicht auch die Anstrengungen von Eigensinn und Widerstand zu vermeiden, die bei der Integration in ein ,geschlechtsfremdes“" Territorium nötig wären. (Brandt/Cornelissen 2004: 31)

Diese Erklärung bleibt jedoch die Antwort auf die Frage schuldig, weshalb einige Jugendliche dennoch einen geschlechtsuntypischen Beruf wählen.

Praxistheoretische Ansätze weichen diese eben besprochene enge Anbindung an den Versuch, mittels der Berufsfindung dem Geschlechterstereotyp $\mathrm{zu}$ entsprechen, etwas auf und geben dem Individuum Handlungsspielraum zurück. Die soziale Praxis wird im Sinn von Doing Gender verstanden, als „routinisierte, inkorporierte kulturelle Deutungs- und Handlungsmuster“ (Brandt/Cornelissen 2004: 31). Die Berufsfindung ergibt sich damit aus der Darstellung von Geschlecht und der Absicherung von geschlechtshomogenen sozialen Räumen.

Die genannten Ansätze zur Erklärung der geschlechtsspezifischen Berufsfindung geben Hinweise, wie Jugendliche in einem nach Geschlecht segregierten Berufsumfeld zu einer Entscheidung kommen, die in den meisten 
Fällen dem Vorgefundenen entspricht. Wichtig zu sehen ist dabei, dass Jugendliche ihre Wahl auf dem Hintergrund von vielerlei Einflüssen treffen. Durch ihre Sozialisation sind sie vergesellschaftlicht und vergeschlechtlicht. Sie versuchen auf der Basis ihrer individuellen Eigenschaften einen Weg zu finden. Es ist davon auszugehen, dass gerade Jugendliche mit einem geschlechtsuntypischen Berufswunsch wissen, dass sie damit gegen die zweigeschlechtliche, heteronormative Berufswelt ankämpfen. Anscheinend gewichten sie Interesse oder Prestige/Status stärker als die geschlechtliche Passung und bilden damit eine Ausnahme im Theoriegefüge von Gottfredson (1981). Erweiternd kann noch hinzugefügt werden, dass im Berufsfindungsprozess zusätzlich untergeordnete Kriterien wie das Milieu (z.B. ein eher urbanes Umfeld mit wenig sozialer Kontrolle) und das soziale Bezugssystem (z.B. Familie mit einer liberal-offenen Einstellung) eine Rolle spielen. Doch an einer gelingenden Ausbildung in einer geschlechtsuntypischen Berufslehre sind nicht nur die Jugendlichen mit ihrem jeweiligen familiären Umfeld selbst beteiligt, sondern auch die Lernorte und mit ihnen die betrieblichen Entscheidungsträgerinnen und -träger mit ihrem Anstellungsverhalten.

\subsubsection{Betriebliches Anstellungsverhalten}

In den vorangegangen Abschnitten lag der Fokus der Betrachtung bei den Lehrstellensuchenden und ihren Berufsfindungsprozessen. Die Perspektive wechselt nun zu denjenigen Personen in den Betrieben, die Jugendliche einstellen und ausbilden wollen. Das Anstellungsverhalten von Lehrverantwortlichen in Betrieben ist entscheidend, denn sie bestimmen, an wen eine Lehrstelle vergeben wird. Für Betriebe ist die Ausbildung von Lernenden eine Investition in die Zukunft des Unternehmens. Mit dem Versuch zur Minimierung der Risiken reproduziert sich jedoch die Geschlechterordnung. Diese Mechanismen werden im Folgenden beschrieben.

Die Motivation von Betrieben, sich in der Ausbildung von Lernenden zu engagieren, gründet zu einem erheblichen Teil in ihrer sozialen Verantwortung und der Sicherung des Nachwuchses von Fachkräften (Stalder 1999). Das übergeordnete Ziel des Unternehmens bleibt weiterhin bestehen: die Produktivität zu garantieren und mindestens so viel Geld zu verdienen, dass der Betrieb aufrechterhalten, also Rechnungen und Löhne bezahlt werden können und in die Zukunft des Unternehmens investiert werden kann. Im Durchschnitt ist die Wertschöpfung, die Lernende generieren, etwas höher als die Kosten, die ihre Ausbildung verursacht (Mühlemann et al. 2007). $\mathrm{Zu}$ Beginn der Berufslehre investiert jedoch vor allem das Unternehmen, da Lernende meist noch nicht produktiv eingesetzt werden können. Betriebe sind deshalb daran interessiert, diejenigen Jugendlichen als Lernende $\mathrm{zu}$ gewinnen, die eine hohe Chance haben, die Berufslehre erfolgreich abzu- 
schließen und wenn möglich im Betrieb weiterzuarbeiten. Vorzeitige Lehrvertragsauflösungen (vgl. Abschnitt 1.5.2 und Kapitel 5) bedeuten für die Betriebe neben menschlichen insbesondere finanzielle Verluste (Imdorf 2012a, Wettstein/Gonon 2009).

Mit den Betrieben beteiligt sich die öffentliche Hand an den Ausbildungskosten. Beispielsweise tragen die Kantone die Hauptlast der Berufsfachschulen (Wettstein/Gonon 2009). Für die Betriebe selbst sind primär die Kosten relevant, die die Berufsbildung intern verursacht. Eine Lehrstelle kostet pro Jahr durchschnittlich zwischen 20'000 und 30'000 Franken (Schweri et al. 2003). An diesen Kosten machen je ca. 45 Prozent die Löhne der Ausbildenden und der Lernenden aus. Ca. 10 Prozent entfallen auf Sachwerte wie Einrichtung, Räume und Material. Der Betrieb muss die Mehrkosten für die Ausbildung tragen, die zu Beginn der Berufslehre nicht durch die Produktivität der Lernenden abgedeckt werden. Gegen Ende der Berufslehre ist es umgekehrt: Die Lernenden können nun produktiv eingesetzt werden und generiert mit ihrer Arbeitskraft einen Überschuss. Einige Berufslehren haben höhere Investitionskosten, und diese zahlen sich erst aus, wenn die Jugendlichen nach der Berufslehre noch einige Zeit im Lehrbetrieb weiterarbeiten. Lehrabbrüche und vorzeitige Lehrvertragsauflösungen sind für die Betriebe also mit Verlusten verbunden (Wettstein/Gonon 2009). In den Worten von Wettstein: „Abgebrochene Ausbildungen [...] stellen hingegen ein Verlustgeschäft dar, ganz abgesehen von Ärger und Enttäuschung“" (2008: 10).

Lehrvertragsauflösungen verursachen finanzielle Einbußen, das Fehlen von Fachkräften und viel administrative Arbeit. Ausbildungsverantwortliche wählen deshalb jene Jugendlichen aus, die mit hoher Wahrscheinlichkeit die Berufslehre abschließen werden. Diese antizipierte Einschätzung basiert meist auf heuristischen Überlegungen und führt, wie ich im Folgenden anhand der Arbeit von Imdorf (2012a) darstellen werde, zum Erhalt der geschlechtlichen Segregation im Ausbildungsmarkt und schließlich im Arbeitsmarkt selbst.

Ausbildungsverantwortliche in den Betrieben vergeben ihre Ausbildungsplätze aufgrund von Kriterien wie Fähigkeiten, Interesse und Einsatzbereitschaft. Daneben stützen sich Arbeitgebende auf offensichtliche, sogenannte askriptive Merkmale wie Alter, Nationalität und Geschlecht. Ausbildende in kleineren und mittleren Ausbildungsbetrieben ziehen diese askriptiven Eigenschaften über Lernende heran, um eine Entscheidung bei oft schwer abzusichernden Einschätzungen im Bewerbungsverfahren zu fällen. Doch genau dies führt zur Benachteiligung von Personen, die den vermeintlich relevanten Kriterien nicht entsprechen (Imdorf 2010, 2012a, 2012b, Scherr et al. 2015). Beim askriptiven Merkmal Geschlecht führt dies zu geschlechtstypischen Lehrstellenvergaben: „Sobald die Arbeitgeber Jugendliche bei der Ausbildungsplatzvergabe aufgrund der Geschlechtszugehörigkeit nicht gleichwertig 
beurteilen, tragen Erstere ebenso zur Geschlechtersegregation des Ausbildungssystems bei“ (Imdorf 2012a: 244). Warum dem so ist, stellen folgende Ausführungen dar.

Imdorf (2012a) erklärt die Begründung des Einstellungsverhaltens der Ausbildenden mit der Rechtfertigungstheorie von Boltanski und Thévenot (2007). Diese Rechtfertigungstheorie nennt vier zentrale Koordinations- und Ordnungsprinzipien, sogenannte Welten: die häusliche, die staatsbürgerliche, die industrielle und die marktförmige Welt. 1) Die Argumente aus der ,häuslichen Welt' stellen das Herkömmliche und Bekannte in den Mittelpunkt. Dazu gehören traditionelle Geschlechterverhältnisse, wertkonservative Geschlechterrollen oder heteronormative Paar- und Geschlechterbeziehungen. Lernende des anderen Geschlechts würden demnach die innerbetrieblichen Sozialbeziehungen stören. Dazu gehören Überlegungen seitens der Ausbildenden, dass etwa die rüden Umgangsformen in Werkstätten und auf Baustellen keine geeignete Lernumgebung für eine junge Frau sind oder ein männlicher Jugendlicher zu wenig Einfühlungsvermögen für die Betreuung von Kleinkindern hat. 2) Erklärungsmodelle der ,staatsbürgerlichen Welt ${ }^{\star}$ verweisen auf die Solidarität, auf das Prinzip der Chancengleichheit und darauf, dass Frauen und Männer gleichermaßen für die jeweiligen Tätigkeiten geeignet sind. 3) Rechtfertigungen aus der ,industriellen Welt' betonen die Effizienz in der Produktivität der Unternehmung. Beispielsweise dominiert dabei das Argument - trotz fehlender empirischer Grundlage -, dass Frauen aufgrund ihrer körperlichen Voraussetzungen (Kraft und Belastbarkeit), aber auch aufgrund ihres technischen Verständnisses und ihrer manuellen Fertigkeiten weniger geeignet sind als Männer. 4) Die Rechtfertigungen für Ungleichbehandlung in der , marktförmigen Welt ${ }^{\star}$ beziehen sich auf die Konkurrenz zwischen den Geschlechtern. Den Unternehmen geht es unter anderem darum, Fehlinvestitionen zu vermeiden. Erwartungen können deshalb dazu führen, dass ein Geschlecht bevorzugt wird. Dazu gehören etwa die Annahmen, dass Frauen wegen Schwangerschaft, Mutterschaft und Familienpflichten früher als Männer aus dem Unternehmen austreten oder dass Männer in frauentypischen Berufen die höhere Fluktuation aufweisen. Für die Selektion von Frauen können sich notwenige Investitionen zur Einhaltung gesetzlicher Vorschriften (z.B. geschlechtergetrennte sanitäre Anlagen, Mutterschaftsurlaub) als nachteilig erweisen.

Die Vergabe der Lehrstellen weist Verzerrungen aufgrund der Geschlechtszugehörigkeit auf und wirkt sich verstärkend (evtl. sogar vorwegnehmend) auf die Selbstselektion der Jugendlichen aus. Diese verwenden meist unbewusst das Geschlecht eines Berufs als Auswahlkriterium für die Lehrstelle und setzen die Berufsrolle als Konstruktionshilfe für die Darstellung ihre eigenen Weiblichkeit bzw. Männlichkeit ein (vgl. Unterkapitel 1.1 ,Geschlechtliche Segregation des Arbeitsmarkts'). Die berufliche Segregation nach Geschlecht und die vielschichtigen Verschränkungen von Geschlecht 
mit Familie, Schule und Arbeit schlagen sich in der beruflichen Grundbildung nieder. Der Kontext der Untersuchungen in der vorliegenden Arbeit ist das schweizerische Berufsbildungssystem. Die Besonderheiten dieses Systems werden im folgenden Unterkapitel vorgestellt.

\subsection{Berufliche Grundbildung in der Schweiz}

Die vorliegende Arbeit bezieht Daten des Schweizer Berufsbildungssystems ein. Dieser kurze Überblick stellt die wichtigsten Merkmale der gut ausgebauten und in der Wirtschaft stark verankerten Ausbildungsgänge der dualen Berufsbildung vor.

Die berufliche Grundbildung ist in der Schweiz der am häufigsten gewählte Bildungsgang auf der Sekundarstufe II (vgl. SBFI ${ }^{7}$ ). Zwei Drittel der Jugendlichen über 16 Jahre steigen nach der obligatorischen Schule in eine Berufslehre ein (BFS 2008). In über 230 Berufslehren vermittelt die berufliche Grundbildung die notwendigen Fertigkeiten und Kenntnisse zur Ausübung eines Berufs. Dabei werden zwei Anspruchsniveaus angeboten: Die zweijährige Grundbildung mit, Eidgenössischem Berufsattest EBA` sowie die drei- bzw. vierjährige Grundbildung mit ,Eidgenössischem Fähigkeitszeugnis $E F Z^{\prime}$. In diesem sogenannten dualen Berufsbildungssystem werden die Lernenden an zwei Lernorten unterrichtet: im Lehrbetrieb und in der Berufsfachschule. In den letzten Jahren hat sich der ,überbetriebliche Kurs üK', der meist blockartig in einer bis mehreren Wochen angeboten wird, als dritter Lernort etabliert (vgl. Wettstein 2008). Neuenschwander et al. (2012) regen an, das Lernen zu Hause (in der Familie) als ein vierter Lernort zu betrachten kann und belegen, dass dieser Lernort auf den Erfolg während der Berufsbildung ebenfalls Einfluss ausübt. Dennoch hat sich der Terminus ,duale Berufsbildung' etabliert.

Die Aufteilung der Ausbildungszeit auf die Lernorte wird bei den verschiedenen Berufslehren und gelegentlich auch innerhalb eines Berufs unterschiedlich gehandhabt. Verbreitet ist eine Form von drei bis vier Tagen im Lehrbetrieb und einem bis zwei Tagen in der Berufsfachschule. Ausbildungsmodelle wie das ,Basislehrjahr' ohne Einsatz im Lehrbetrieb (z.B. ,Informatiker/-in EFZ'), saisonaler Rhythmus (,Landwirt/-in EFZ') oder Praktika (,Chemie- und Pharmatechnolog/-in EFZ') sind wenig verbreitet (zur Übersicht über die Ausbildungsmodelle vgl. Neuenschwander et al. 2012). /sbfi/de/home/themen/berufsbildung/berufliche-grundbildung.html; Zugriff: 22.03.2017]. 
Abbildung 1.1: Das Schweizer Berufsbildungssystem

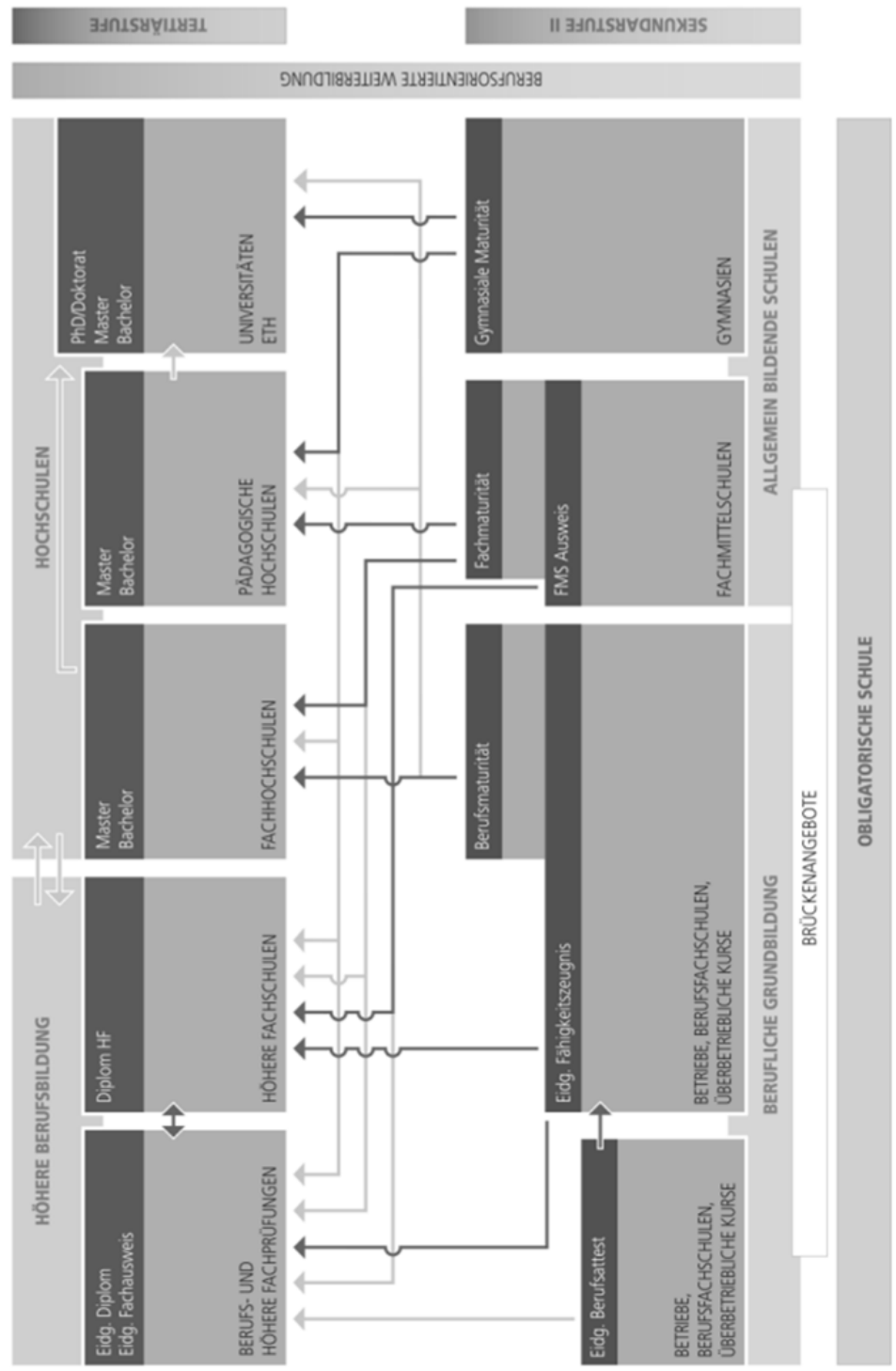

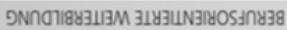

Quelle: Darstellung nach SBFI 2016. 
Das duale Berufsbildungssystem der Schweiz zeichnet sich durch das Prinzip der stetigen Weiterbildungsmöglichkeiten, durch die Durchlässigkeit zwischen den einzelnen Programmen sowie durch die Vermeidung von Sackgassen aus (vgl. Abbildung 1.1). Jedes Jahr beginnen über 70'000 Jugendliche und junge Erwachsene eine Berufsausbildung. 2012 waren rund 230'500 Personen in einer beruflichen Grundbildung (SBFI 2015). Die duale Berufsbildung gilt als Erfolgsmodell. Dies belegt etwa die tiefe Jugendarbeitslosigkeit (15- bis 24-Jährige) in der Schweiz. Ausnahmsweise lag die Jugendarbeitslosigkeit im Juni 2011 mit 2.5 Prozent zum ersten Mal sogar unter der allgemeinen Arbeitslosigkeitsrate von 2.8 Prozent (BFS 2011). Im April 2011 wurden mehr Lehrstellen ausgeschrieben, als es an einer Berufslehre interessierte Jugendliche gab: 81'000 offene Lehrstellen für 77'000 Jugendliche (BFS 2011). Dies hatte die Situation auf dem Lehrstellenmarkt deutlich entspannt. Zur tiefen Jugendarbeitslosigkeitsquote und zum erhöhten Lehrstellenangebot haben sowohl Reformen in der Berufsbildung, die von Wirtschaft, Schule und Verwaltung umgesetzt wurden, als auch die damals gute Wirtschaftslage beigetragen (Strupler/Wolter 2012).

Mit dem Eintritt in die Berufslehre eröffnen sich den Jugendlichen neue Mikrosysteme $^{8}$, in denen sie sich bewähren müssen. Im Mikrosystem ,Lehr-

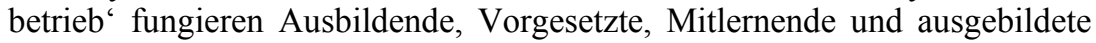
Mitarbeitende als Agenten der berufliche Sozialisation. In den Mikrosystemen ,Berufsfachschule' und ,überbetrieblicher Kurs' sind es Lehrpersonen, Mitschülerinnen und Mitschüler. Auf die spezifischen, für die vorliegende Arbeit relevanten Verhaltensweisen der Personen des sozialen Umfelds in den Mikrosystemen, die einen Einfluss auf die Vermittlung und evtl. Durchsetzung von tradierten Wertvorstellungen haben - insbesondere bei Geschlechtsrollenerwartungen -, gehe ich in Kapitel 4 ein. Zunächst werden im folgenden Unterkapitel Kriterien vorgestellt, nach denen Erfolg und Misserfolg bzw. Schwierigkeiten in der Berufsbildung beurteilt werden können. Diese Kriterien werden im empirischen Teil herangezogen, um Muster zu erkennen, die eine Benachteiligung der Jugendlichen in geschlechtsuntypischen Berufslehren aufdecken könnten.

\subsection{Erfolg und Misserfolg während der Berufsausbildung}

Die erfolgreiche berufliche Laufbahn fußt auf einem Abschluss auf der Sekundarstufe II. Verzögerungen oder Abbrüche können, insbesondere wenn sie mit Arbeitslosigkeit verbunden sind, gravierende Auswirkungen auf die 
berufliche, persönliche und gesundheitliche Zukunft der jungen Menschen haben (vgl. McKee-Ryan et al. 2005). Erfolg bzw. Misserfolg wird in der vorliegenden Arbeit als Indikator dafür verstanden, wie Jugendlichen in geschlechtsuntypischen Berufslehren die Sozialisation gelingt und ob sie dabei wesentliche Hindernisse überwinden müssen. Das Unterkapitel 1.5 beschreibt Erfolgskriterien in der Berufsbildung insbesondere so, wie sie in der vorliegenden Forschungsarbeit operationalisiert werden. Eine Zusammenstellung erfolgt in Abschnitt 1.5.1 anhand von Indikatoren für den Erfolg in der Berufslehre. Im Anschluss werden folgende Erfolgs- und Misserfolgsindikatoren vorgestellt: Lehrvertragsauflösung (Abschnitt 1.5.2), selbstberichtete Kriterien wie Ausbildungszufriedenheit (Abschnitt 1.5.3) und Bestehen der Lehrabschussprüfung (Abschnitt 1.5.4).

Erfolg in der Berufslehre kann beispielsweise bedeuten, Lerninhalte zu verinnerlichen, Handfertigkeiten zur Zufriedenheit anderer auszuführen, sich einen beruflichen Habitus anzueignen oder die Lehrabschlussprüfung $\mathrm{zu}$ bestehen. Wie vielschichtig die Einflussfaktoren spezifisch für den Erfolg in der Berufslehre sind, führen die unterschiedlichen Konzepte von allgemeinem ,Berufserfolg' aus. Die Aufzählungen von Erfolgsfaktoren sind zwar oft abhängig vom jeweiligen Kontext oder von der Operationalisierung von Berufserfolg im Forschungsumfeld (Dette et al. 2004), dennoch lassen sich daraus Determinanten ableiten. Die am häufigsten untersuchten Einflussfaktoren, die sich auf den Laufbahnerfolg auswirken, sind nach Tharenou (1997) Humankapital (Ausbildung, Arbeitserfahrung, Schulbildung) und demografische Faktoren (Alter, Geschlecht, Zivilstand, Anzahl Kinder). Des Weiteren erweisen sich bei Judge et al. (1999) Persönlichkeitseigenschaften (etwa Gewissenhaftigkeit positiv und Neurotizismus negativ) sowie Intelligenz als voraussagekräftige Prädiktoren für Laufbahnerfolg. Judge et al. (1999) schlagen aufgrund der vielen Einflussfaktoren vor, zwischen objektiven und subjektiven Aspekten von beruflichem Erfolg zu unterscheiden. Objektive Erfolgsaspekte sind sichtbar und relativ einfach zu beobachten. Zu ihnen gehören Lohn und Anzahl Beförderungen bzw. beruflicher Status. Zu den subjektiven Aspekten beruflichen Erfolgs zählen individuelle Reaktionen auf die eigene Karriere, insbesondere gemessen in Form von Laufbahn- und Arbeitszufriedenheit.

Diese Unterscheidung in subjektive und objektive Kriterien ist nach Dette et al. (2004) unscharf, insbesondere dann, wenn im Sinn der Testtheorie zwischen subjektiven Einschätzungen bei Selbst- und Fremdwahrnehmungen sowie objektivierten Daten differenziert wird. Deshalb unterscheidet das Komponentenmodell des beruflichen Erfolgs nach Dette et al. (2004) drei Ebenen. Die erste dieser Ebenen bildet das Bezugskriterium: 1) spezifische Arbeit vs. 2) globale Laufbahn. Die zweite Ebene unterscheidet drei Datenarten: 1) neutrale Kennzahl, 2) Vergleich mit Bezugsstandards (Zielerreichung, andere Person oder Norm) und 3) Zufriedenheitseinschätzungen. Die dritte 
und letzte Ebene schließlich differenziert drei Datenquellen: 1) Akten/Dokumente, 2) Fremdurteile und 3) Selbstberichte. Diese drei Ebenen bilden eine $2 \times 3 \times 3$-Felder-Tafel, die es ermöglicht, die vielen Einflussfaktoren von Berufserfolg zu ordnen. Das Modell des beruflichen Erfolgs ist theoretisch und metaanalytisch abgesichert (Dette et al. 2004, Dette 2005). Die Tabelle 1.1 stellt das Komponentenmodell ergänzt mit Erfolgskriterien während der Berufsbildung dar. Im Folgenden stelle ich Forschungsdesiderate zu Erfolgen in der Berufsbildung vor und verorte sie anschließend im Komponentenmodell.

\subsubsection{Indikatoren für den Erfolg in der Berufslehre}

Bisher habe ich Erfolgsfaktoren für das Berufsleben im Allgemeinen vorgestellt. Im nächsten Schritt wende ich mich spezifisch den Einflussfaktoren für Erfolg in der Berufsbildung zu. Die OECD ${ }^{9}$ hat strukturelle Erfolgsgrößen bzw. Zielvorgaben für den Übergang von der Schule ins Erwerbsleben definiert (Althoff 1992, Häfeli 2001, OECD 2000). Aus den übergeordneten Zielvorgaben können angepasst an die individuelle Ebene Erfolgskriterien formuliert werden. Die von der OECD (2000) beschriebenen Kriterien sollen auf der strukturellen Ebene von vielen jungen Erwachsenen erfüllt werden. Da die Erfolgskriterien im Komponentenmodell auf der individuellen Ebene angesiedelt sind, habe ich die Formulierung der OECD (2000) leicht angepasst. Lernende sollen demnach die folgenden Kriterien möglichst gut erfüllen: 1) anerkannter Abschluss auf der Sekundarstufe II, der für Arbeit bzw. Studium qualifiziert, 2) hoher Ausbildungsstand am Ende der Ausbildung, 3) keine Arbeits- und Ausbildungslosigkeit, 4) stabiler Übergang an der zweiten Schwelle (gemeint ist eine Arbeitsstelle im Anschluss an die Ausbildung), 5) nur kurze Arbeitslosigkeit, sollte es doch zu einem Unterbruch kommen, 6) stabile und positive Arbeits- und Ausbildungsbiografie in den Jahren nach dem Lehrabschluss und 7) gleiche Chancen für alle Personen, besonders punkto Geschlecht, soziale und regionale Herkunft (vgl. Tabelle 1.1).

Eine Operationalisierung dieser OECD-Erfolgskriterien realisierten Häfeli und Schellenberg (2009a 2009b). Zum einen zählen sie über 50 Faktoren auf, die den Lehrerfolg beeinflussen (Häfeli/Schellenberg 2009b), zum anderen fokussieren sie auf 4 objektive Erfolgskriterien (Häfeli/Schellenberg 2009a). Die umfassendere Aufzählung von über 50 Einflussfaktoren für den Erfolg in der Berufsbildung haben Häfeli und Schellenberg (2009b) auf der Grundlage zahlreicher Studien zusammengetragen. Sie zählen sieben Bereiche auf: 1) Person (Geschlecht, Schulleistungen, Intelligenzquotient, Selbstwert, Substanzmissbrauch, gute Umgangsformen, Sozialkompetenz etc.), 2) Familie 
(Schicht, Familiensituation, Nationalität, Bildungsaspiration, Erziehungsstil, emotionale Unterstützung, Beziehungsnetz etc.), 3) Schule und Lehrpersonen (Schultyp, Berufswahlunterricht, Schulklima, Engagement der Lehrperson, Beziehung, Beratung etc.), 4) Betrieb und Berufsbildende (Anforderungsniveau, Berufsgruppe, Vielseitigkeit, Belastungen, Beziehung etc.), 5) Beratungs- und Interventionsangebote (Zeitpunkt, Niederschwelligkeit, Beziehung, Struktur, Bezug zur Arbeitswelt, Netzwerk etc.), 6) Freizeit und Gleichaltrige (aktive Freizeitgestaltung, vor allem in strukturierten Gruppen, respektvoller Umgang etc.) und 7) Gesellschaft als Demografie, Wirtschaft, Sozialraum, Politik, Verwaltung (Anzahl Schulaustretende, wirtschaftliche Situation, Lehrstellenangebot etc.). Aus dieser Aufzählung wird ersichtlich, dass nicht nur die proximale Variablen der Person, sondern auch distale Einflussfaktoren den Erfolg in der Berufsbildung mitgestalten können. Die Integration aller distalen Einflussfaktoren von Häfeli und Schellenberg (2009b) ins Komponentenmodell nach Dette et al. (2004) wäre der Übersichtlichkeit abträglich. Aufgenommen wurden deshalb lediglich die Variablen zur Person (vgl. Tabelle 1.1). Diese Auswahl wird durch die Ergebnisse der Studie zum Ausbildungserfolg bei Kaufleuten von Schafer und Baeriswyl (2015) gestützt, die individuellen Faktoren, in Abgrenzung zu den kontextuellen, mehr Erklärungskraft zuschreiben.

In Anlehnung an die Einteilung der Erfolgsfaktoren nach Judge et al. (1999) in subjektive und objektive Erfolgsfaktoren zählen Häfeli und Schellenberg (2009a) vier objektive Erfolgsfaktoren in der Berufsausbildung auf: 1) Abschluss eines Lehrvertrags, 2) in der Berufslehre durchhalten bis zum Ende der Ausbildung im Gegensatz zu einer vorzeitigen Lehrvertragsauflösung, 3) Bestehen der Lehrabschlussprüfung und 4) Anstellung im erlernten Beruf. Diese vier Erfolgsfaktoren lassen sich im Komponentenmodell des beruflichen Erfolgs nach Dette et al. (2004) im Bezugskriterium ,globale Laufbahn', als Datenart ,neutrale Kennzahl' und als Datenquellen ,Akten/Dokumente، verorten (vgl. Tabelle 1.1).

Die oben aufgeführten Kriterien und Faktoren für Erfolg in der Berufslehre fanden nun Eingang ins Komponentenmodell von Dette et al. (2004), das im Folgenden beschrieben und vorgestellt wird. Die strukturelle Aufteilung und die Benennung der Komponenten sowie die Erfolgsfaktoren mit einer kleinen, hochgestellten 1 dahinter stammen von Dette et al. (2004). Die Herkunft der Ergänzungen ist in der Legende ausgewiesen. Die Tabelle 1.1 veranschaulicht diese Systematisierung. Auf einer ersten Ebene unterscheidet das Komponentenmodell die zwei Bezugskriterien ,spezifische Arbeit' als charakteristische, zeitlich begrenzte Tätigkeit (z.B. Zufriedenheit mit der Tätigkeit) und ,globale Laufbahn' als übergreifender Prozess bzw. Teil der Berufslaufbahn (z.B. Laufbahnzufriedenheit). Weiter werden drei Datenarten genannt. Dabei beziehen sich ,neutrale Kennzahlen' auf aktenkundiges Material, ,Vergleiche mit Bezugsstandards' auf Gegenüberstellungen mit vorher 
vereinbarten Zielen, Standards, Normen oder mit anderen Personen sowie ,Zufriedenheit' auf die Einschätzung eines affektiv-emotionalen Zustands der Bedürfnisbefriedigung. Schließlich geht es um die Herkunft der Informationen, die drei Datenquellen. Dazu gehören ,Akten und Dokumente' als objektive Daten (relativ unabhängig und unempfindlich gegenüber Verzerrungen) sowie ,Fremdurteil' und ,Selbstbericht', die beide einer gewissen Wahrnehmungsverzerrung unterliegen.

Tabelle 1.1: Komponentenmodell des Erfolgs in beruflicher Grundbildung

\begin{tabular}{|c|c|c|c|c|}
\hline \multirow{2}{*}{$\begin{array}{l}\text { Bezugs- } \\
\text { kriterium }\end{array}$} & \multirow{2}{*}{ Datenart } & \multicolumn{3}{|c|}{ Datenquelle } \\
\hline & & Akten/Dokumente & Fremdurteil & Selbstbericht \\
\hline \multirow{3}{*}{$\begin{array}{l}\text { spezifische } \\
\text { Arbeit }\end{array}$} & $\begin{array}{l}\text { neutrale } \\
\text { Kennzahlen }\end{array}$ & $\begin{array}{l}\text { Lehrabschlussprüfung } \\
\text { Stückzahl }^{3}\end{array}$ & \multirow{3}{*}{$\begin{array}{l}\text { Fähigkeit/Kompetenz }{ }^{2} \\
\text { Lehrabschlussprüfung }^{3,5} \\
\text { Leistungsbeurteilung }^{1,3}\end{array}$} & Stückzahl ${ }^{1}$ \\
\hline & $\begin{array}{l}\text { Vergleich mit } \\
\text { Bezugs- } \\
\text { standards }\end{array}$ & & & $\begin{array}{l}\text { Fähigkeitsselbstkonzept }{ }^{5} \\
\text { Leistungseinschätzung }^{1} \\
\text { Passung }^{5}\end{array}$ \\
\hline & $\begin{array}{l}\text { Zufrieden- } \\
\text { heit }\end{array}$ & & & $\begin{array}{l}\text { Passung }^{5} \\
\text { Zufr. mit der Tätigkeit }^{1}\end{array}$ \\
\hline \multirow{3}{*}{$\begin{array}{l}\text { globale } \\
\text { Laufbahn }\end{array}$} & $\begin{array}{l}\text { neutrale } \\
\text { Kennzahlen }\end{array}$ & 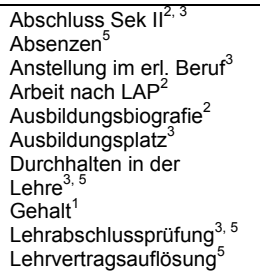 & \multirow{3}{*}{$\begin{array}{l}\text { Ausbildungsstand } \\
\text { Beförderungswürdigkeit }^{2} \\
\text { Geschlecht }^{4} \\
\text { Geschlechtstypisch }^{5} \\
\text { Intelligenz }^{4} \\
\text { Lehrvertragsauflösung }^{5} \\
\text { Schulleistung }^{4} \\
\text { Sozialkompetenz }^{4}\end{array}$} & $\begin{array}{l}\text { Absenzen } \\
\text { Gehalt }^{1}\end{array}$ \\
\hline & $\begin{array}{l}\text { Vergleich } \\
\text { mit Be- } \\
\text { zugsstan- } \\
\text { dards }\end{array}$ & & & $\begin{array}{l}\text { berufliche Sozialisation }^{5} \\
\text { Chancengleichheit }^{2,3,5} \\
\text { Erfolg im Vergleich mit }{ }^{1} \text { Peers }^{1} \\
\text { Passung }^{5} \\
\text { Selbstwert }^{4}\end{array}$ \\
\hline & $\begin{array}{l}\text { Zufrieden- } \\
\text { heit }\end{array}$ & & & $\begin{array}{l}\text { berufliche Sozialisation }^{5} \\
\text { Laufbahnzufriedenheit }^{1} \\
\text { Passung }^{5} \\
\text { psychosomatische } \\
\text { Beschwerden } \\
\text { Ausbildungszufriedenh. }{ }^{5}\end{array}$ \\
\hline
\end{tabular}

Legende: 1 Erfolgsfaktoren nach Dette et al. (2004: 174); 2 Erfolgsfaktoren nach OECD (2000); 3 Erfolgsfaktoren nach Häfeli und Schellenberg (2009); 4 Erfolgsfaktoren nach Häfeli und Schellenberg (2009b); 5 in der vorliegenden Arbeit untersuchte Erfolgsfaktoren. Quelle: Eigene Darstellung.

Dieses Modell des beruflichen Erfolgs dient als Grundlage für die Einordnung der Faktoren des Erfolgs bei der beruflichen Grundbildung. Die Aufzählung ist nicht abschließend, sondern eine Auslegeordnung des relativ breiten Untersuchungsfelds. Aus untersuchungsökonomischen Gründen kann in der vorliegenden Arbeit lediglich auf bestimmte Faktoren im Hinblick auf den Erfolg von Lernenden in geschlechtstypischen und geschlechtsuntypischen Berufslehren eingegangen werden. Die in der Tabelle 1.1 mit der Ziffer 
5 markierten Erfolgsfaktoren werden untersucht. Es ist zu erkennen, dass Daten aus beiden Bezugskriterien, aus allen drei Datenarten und aus allen drei Datenquellen berücksichtig werden. Einige Erfolgskriterien werden mehrfach aufgeführt, da sie aus unterschiedlichen Perspektiven betrachtet werden können. Beispielsweise zählt die Lehrabschlussprüfung sowohl als neutrale Kennzahl für die spezifische Arbeit (Handlungsrückmeldung der Leistung an den Prüfungstagen) wie auch für die globale Laufbahn im Sinn des erfolgreichen Abschlusses der Ausbildung auf der Sekundarstufe II. Es versteht sich, dass die Lehrabschlussprüfung selbst ein Fremdurteil ist, das Vergleiche mit der erbrachten Leistung und Zielen, Standards bzw. den Leistungen anderer Lernender beinhaltet. In den nachfolgenden Abschnitten 1.5.2 bis 1.5.5 werden die in der vorliegenden Arbeit verwendeten Erfolgskriterien genauer erläutert.

\subsubsection{Erfolgsfaktor: Sich während der Berufslehre bewähren}

Ein wichtiger Faktor für den Erfolg in der Berufsausbildung ist ,das Durchhalten in der Lehre“ (Häfeli/Schellenberg 2009: 15). Demnach kann der Lehrabbruch bzw. die Lehrvertragsauflösung ein Misserfolgserlebnis in der Berufsausbildung für die betroffenen Lernenden sein. Dabei gilt es zu berücksichtigen, dass eine Lehrvertragsauflösung, sofern ausreichend Stützund Schutzfaktoren wie Anschlusslösung, fachliche und familiäre Unterstützung vorhanden sind, auch als Auftakt zu einer Verbesserung der persönlichen Situation gewertet werden kann. Im Komponentenmodell (vgl. Tabelle 1.1) ist die Lehrvertragsauflösung als Kriterium der globalen Laufbahn entweder eine neutrale Kennzahl und damit im Bereich der Akten/Dokumente verortet oder als Fremdurteil im Vergleich mit Bezugsstandards, die nicht erreicht werden. In der vorliegenden Arbeit wird sie als neutrale Kennzahl verstanden, da amtliche Populationsdaten vom BFS verwendet werden (vgl. Kapitel 5). Insgesamt müssen 20-25 Prozent der Lernenden eine vorzeitige Beendigung der Berufslehre bewältigen. Warum dies Lehrvertragsauflösung genannt wird, die Gründe, wie es dazu kommen kann, und warum die Berechnung einer Quote einige Schwierigkeiten bereitet, davon handelt dieser Abschnitt.

Ausbildungsvereinbarungen im dualen System bedingen einen sogenannten Lehrvertrag zwischen dem Lehrbetrieb und der/dem Lernenden ${ }^{10}$. Die Lehrverträge und die damit verbundene Bestätigung durch das kantonale Arbeitsamt dienen dem Schutz der Lernenden vor der Ausbeutung als billige Arbeitskräfte und vor der Kündigung bei prekärer Auftragslage im Lehrbetrieb. Der Lehrbetrieb verpflichtet sich mit diesem Vertrag, die Auflagen zur

10 In vielen Fällen unterschreiben die Eltern, da die Jugendlichen noch nicht volljährig sind. 
Ausbildungsberechtigung einzulösen ${ }^{11}$. Tritt eine Partei vor dem vorgesehenen Ende (erfolgreicher Lehrabschluss) von diesem Vertrag zurück, liegt eine Lehrvertragsauflösung vor. Von Lehrvertragsauflösung wird gesprochen, da nicht jede vorzeitige Beendigung des Lehrverhältnisses bedeutet, dass die Ausbildung auf der Sekundarstufe II aufgegeben wird. Eine Typologisierung der zahlreichen Lehrvertragsauflösungen nach Art der Orientierungsphase, also nach dem weiteren Verlauf der Ausbildung, schlagen Süss et al. (1996) vor. Gemäß dieser Einteilung vollziehen drei von vier Lernenden einen unmittelbaren Wechsel in ein höheres oder tieferes Leistungsniveau, einen anderen Betrieb oder einen anderen Beruf. Dies kann mit einer kurzfristigen Unterbrechung der Ausbildung verbunden sein. Den meisten von einer Lehrvertragsauflösung betroffenen Lernenden gelingt längerfristig, innerhalb dreier Jahre, der Wiedereinstieg in eine Ausbildung auf der Sekundarstufe II (Schmid 2010).

Eine Lehrvertragsauflösung ist für gewisse Gruppen von Betroffenen eine kritische Phase. Für die betroffenen Lernenden bedeutet sie eine Unterbrechung der Routine und oft eine Gefährdung des Fähigkeitsselbstkonzepts. Eine längere Unterbrechung der Ausbildung führt zum Verlust von Struktur, Anregung und Teilen des sozialen Netzes (vgl. Neuenschwander et al. 2012). Die Folgen können eine stark erschwerte berufliche Integration und sozialer Abstieg sein, oftmals verbunden mit Substanzmissbrauch und Delinquenz (vgl. Schmid/Stalder 2008). Nicht nur die Jugendlichen selbst, sondern auch ihre Eltern sind stark gefordert. Denn Eltern werden von den Jugendlichen am häufigsten um Unterstützung gebeten (Neuenschwander et al. 2012). Die Familie wird als eine der wichtigsten Ressourcen beansprucht. Umso schwieriger ist es für Jugendliche aus belasteten Familienverhältnissen, diese kritische Phase zu bewältigen. Nicht nur steigt wegen der fehlenden familiären Unterstützung ihr Risiko, die Berufslehre vorzeitig abzubrechen, sondern auch der Wiedereinstieg in die Ausbildung ist erschwert (vgl. Schmid/Stalder 2008, Süss et al. 1996).

Die Gründe für Lehrvertragsauflösungen sind vielfältig. Die häufigsten Ursachen sind ungenügende Leistungen in der Berufsfachschule oder im Lehrbetrieb, verbunden mit Interesseverlust und Konflikten (Stalder/Schmid 2006). Die Ursachen werden von Jugendlichen und Lehrbetrieb zum Teil unterschiedlich wahrgenommen; während für die Lernenden das beeinträchtigte Lernklima zu Interesseverlust und mangelnder Anstrengungsbereitschaft führt, sehen die Ausbildenden die Verkettung eher umgekehrt (Lamamra/Masdonati 2009, Pätzold 2004, Stalder/Schmid 2006). Dass die Ausbildungsqualität sowohl in den Augen der Lernenden wie der Lehrenden fürs Durchhalten in der Berufslehre wichtig ist, konnten Negrini et al. (2015) in einer Studie mit Köchinnen und Köchen sowie Malerinnen und Malern aus 
der Deutschschweiz belegen. Sie bezeichnen die Ausbildungsqualität als eine „Art Schutzfaktor gegen Lehrvertragsauflösungen“ (Negrini et al. 2015: 77).

Die Einstellung zur Lehrvertragsauflösung seitens der Lernenden kann ebenfalls eine Erklärung für die hohe Quote sein. So wird ein Lehrabbruch von Jugendlichen nicht mehr als ein Tabu gesehen, sondern als Bestandteil des Suchprozesses bei der Berufsfindung (vgl. Neuenschwander et al. 2012). Sie verstehen den Beruf und die Berufslehre als selbst gewählten Lebensentwurf und sind eher bereit, Fehlentscheide zu korrigieren (vgl. Bertossa et al. 2008, Weiss 2002).

Des Weiteren sind Lehrvertragsauflösungen nicht nur für die Lernenden und ihre Familien mit persönlichen, sozialen und finanziellen Einbußen verbunden, sondern auch für die Lehrbetriebe. Da Lernende gegen Ende ihrer Ausbildung produktiver sind als am Anfang, verlieren die Betriebe ihre Investitionen in die Produktivität der Lernenden (vgl. Abschnitt 1.3.4). Stalder und Schmid (2006) berechneten, dass Betriebe pro Lehrvertragsauflösung mehrere Tausend Franken verlieren. Ihre Schätzungen für den Kanton Bern ergeben jährliche Kosten für Staat und Betriebe in der Größenordnung von 20 Millionen Franken.

Berechnung der Auflösequoten. Lehrbetriebe sind verpflichtet, eine Lehrvertragsauflösung dem kantonalen Arbeitsamt zu melden ${ }^{12}$. Die Zahl der Lernenden, die pro Jahr von einer Lehrvertragsauflösung betroffen sind, wird von den Ämtern erfasst. Die Berechnung des prozentualen Anteils wird unterschiedlich gehandhabt und bedarf daher einer Erklärung. Da die kantonalen Berufsbildungsämter die Vertragsauflösungen sammeln und nur aggregierte Daten ans BFS weiterleiten, basieren die Auflösequoten auf Berechnungen. Die Daten sind nach Kanton, Jahr, Geschlecht und Beruf geordnet. Eine Lehrvertragsauflösung kann in jedem Ausbildungsjahr der 2-4 Jahre dauernden Berufslehren stattfinden. Für die Berechnung der Quote müssten die Personendaten strenggenommen längsschnittlich erfasst werden, was aber anhand der vorliegenden querschnittlichen Daten nicht möglich ist. Eine Annäherung an die effektive Quote besteht darin, die Anzahl der Lehrvertragsauflösungen in einem Jahr durch die Zahl der im gleichen Jahr neu abgeschlossenen Lehrverträge zu dividieren (vgl. Neuenschwander 1999, Neuenschwander et al. 2012). Dies mag auf den ersten Blick unlogisch erscheinen, da verschiedenartige Grundgesamtheiten (Basis ein Jahrgang vs. Basis alle Lehrjahre) verglichen werden. Die Anzahl neuer Lehrverträge wird deshalb der Anzahl aller Lernenden vorgezogen, da so jeweils die Anzahlen lediglich eines Jahrgangs erfasst werden. Die Berechnung der Quote auf Basis aller Lernenden in einem Jahr ergäbe eine zu tiefe Quote und würde zu einer Unterschätzung der realen Verhältnisse führen. Ungenauigkeiten aufgrund von Schwankungen bei der Anzahl neuer Lehrverträge können durch die Zusam-

12 Bei frühzeitiger Information von einer möglichen Lehrvertragsauflösung, versuchen die Ämter zu vermitteln. 
menfügung von Daten aus mehreren Jahren ausgeglichen werden. Dabei ist zu berücksichtigen, dass Jugendliche, die bereits eine Lehrvertragsauflösung erlebt haben, eine größere Wahrscheinlichkeit haben, erneut von einem Lehrabbruch betroffen zu sein (Stalder/Schmid 2006).

In der vorliegenden Arbeit werden die Quoten der Lehrvertragsauflösungen von Frauen und Männern in den Berufslehren verglichen (vgl. Kapitel 5). Dazu werden die Berufslehren nach ihrem Frauenanteil in Gruppen eingeteilt. Die zu prüfende Hypothese lautet: „Lernende in geschlechtsuntypischen Berufslehren sind häufiger von Lehrvertragsauflösungen betroffen als Lernende in geschlechtstypischen bzw. geschlechtergemischten Berufslehren." Gemäß dem aktuellen Forschungsstand wurde diese Hypothese bisher nicht untersucht. Es besteht demnach Forschungsbedarf zu der Frage, inwieweit sich in der Schweiz die geschlechtliche Minderheitssituation auf das Risiko einer Lehrvertragsauflösung auswirkt.

\subsubsection{Erfolgsfaktoren im Selbstbericht}

Für den oben beschriebenen Erfolgsfaktor ,Durchhalten in der Berufslehre“ gibt es eine Vielzahl von Indikatoren, die herangezogen werden können, um den Misserfolg - die Lehrvertragsauflösung - zu beschreiben. Die Messung dieser Faktoren basiert meist auf Selbsteinschätzungen der Jugendlichen. Für die vorliegende Arbeit konnten Datensätze zweier umfangreicher Langzeitstudien reanalysiert werden. Zum einen handelt es sich um das Forschungsprojekt ,Familie Schule Beruf FASE B` (Neuenschwander et al. 2005, Neuenschwander et al. 2012, vgl. Kapitel 7), andererseits um die gesamtschweizerische Längsschnittstudie ,Transitionen von der Erstausbildung ins Erwerbsleben TREE' (TREE 2013, vgl. Kapitel 8). Beide Projekte haben, neben vielen weiteren Variablen, eine Reihe von Erfolgsfaktoren erhoben, die sich im Komponentenmodell von Dette et al. (2004) bei den Datenquellen beim Selbstbericht einordnen lassen (vgl. Tabelle 1.1). Die Erfolgsfaktoren aus den beiden Projekten werden im Folgenden vorgestellt.

\subsubsection{Faktoren aus dem FASE B-Projekt}

Aus dem Projekt FASE B können insbesondere die ,Sozialisation im Lehrbetrieb', das ,berufliche Fähigkeitsselbstkonzept", die ,wahrgenommene Passung zwischen Fähig-/Fertigkeiten und Anforderungen des Berufs' sowie die ,Zufriedenheit mit der Ausbildung' als Faktoren für eine erfolgreiche Integration in die Berufslehre betrachtet werden.

Unter ,Sozialisation im Lehrbetrieb" wird die Meisterung von Herausforderungen verstanden, die die Ausbildung im dualen Berufsbildungssystem mit sich bringen (vgl. Unterkapitel 1.2). Darunter fallen das Verhältnis zu der bzw. dem Ausbildenden, der Umgang mit den anderen Mitarbeitenden, die 
Anwendung von Wissen, die Einhaltung von Regeln, das Übernehmen von Verantwortung, der Leistungsdruck im Lehrbetrieb und in der Berufsfachschule, die Motivation für die Berufslehre oder die Befriedigung durch die Berufsausübung. Diese Aspekte tragen dazu bei, sich mit den Bedingungen und Möglichkeiten auseinanderzusetzen, Werte zu übernehmen und sich Fertigkeiten zu eigen $\mathrm{zu}$ machen, in anderen Worten sich im Umfeld des Berufs zu integrieren und ein Teil davon zu werden, ohne sich selbst dabei aufzugeben.

Der Erfolgsfaktor ,berufliches Fähigkeitsselbstkonzept ${ }^{\star}$ kann als Teilaspekt der beruflichen Sozialisation betrachtet werden. Es geht um das kognitive Selbstbild, das Lernende von ihren fachlichen und methodischen Kompetenzen haben. Nach Marsh und Shavelson (1985) ist das Selbstkonzept ein mehrdimensionales und hierarchisch organisiertes Gefüge von alltäglichen, selbstbezogenen Informationen. Für den Aufbau des beruflichen Fähigkeitsselbstkonzepts finden Vergleichsprozesse der Lernenden mit ihnen selbst zu einem früheren Zeitpunkt oder aber mit anderen Lernenden statt. Spezifisch geht es um die Selbsteinschätzung in der Handhabung von Hilfsmitteln, Geräten und Werkzeugen, um das Planen von Arbeitsschritten und um die Aneignung fachlicher Kenntnisse.

Die ,wahrgenommene Passung zwischen den eigenen Interessen bzw. Fähigkeiten und den Anforderungen der Berufslehre، hängt stark mit dem beruflichen Fähigkeitsselbstkonzept zusammen (vgl. Gerber-Schenk et al. 2010). Es geht dabei um die Einschätzung, ob die gegenwärtige berufliche Tätigkeit mit den persönlichen Interessen übereinstimmt. Nägele und Neuenschwander (2015) können belegen, dass die wahrgenommene Passung einerseits ein Resultat des Berufsfindungsprozesses und andererseits ein Prädiktor für Absicht ist, die Berufslehre abzuschließen. Die Passungswahrnehmung hängt demnach mit der betrieblichen Sozialisation, dem beruflichen Fähigkeitsselbstkonzept und der Zufriedenheit mit der Berufslehre zusammen.

Der Erfolgsfaktor ,Zufriedenheit mit der Ausbildung' kann als globalisierte Aussage darüber verstanden werden, ob die zahlreichen Aspekte der Berufslehre in ihrer Summe ein Wohlgefühl auslösen. Ausbildungs- oder Arbeitszufriedenheit ist ein emotionales Einstellungskonzept mit einer relativ stabilen, erfahrungsbasierten Haltung gegenüber einem Objekt (HupkaBrunner/Kriesi 2013, Rosenstiel 2001, Semmer/Udris 1995).

Ein Teilaspekt beim Erfolg in der Berufsbildung bezieht sich auf die Entwicklung von Fähigkeiten, Fertigkeiten und Berufshabitus. Mit den vier vorgestellten Konstrukten gelingt eine Annäherung an diese Erfolgsbetrachtung. In Kapitel 7 der vorliegenden Arbeit wird überprüft, inwieweit es Unterschiede zwischen Jugendlichen in geschlechtstypischen vs. geschlechtsuntypischen Berufslehren bei den Erfolgsfaktoren Sozialisation, Fähigkeitsselbstkonzept, Passung und Zufriedenheit gibt. Es wird davon ausgegangen, dass aufgrund der Minoritäten-Stress-Theorie (Testa et al. 2015) bzw. des in Kapi- 
tel 4 vorgestellten Modells der geschlechtsuntypischen Berufssozialisation Lernende in geschlechtsuntypischen Berufslehren tiefere Werte in den genannten Erfolgsfaktoren aufweisen als Lernende in geschlechtstypischen Berufslehren.

\subsubsection{Erfolgsfaktoren aus dem TREE-Projekt}

Die untersuchten Faktoren aus dem Projekt TREE beziehen sich auf die Auswirkungen, die sich aufgrund der Minderheitenposition ergeben können. Wie die Minoritäten-Stress-Theorie nach Testa et al. (2015) nahelegt, können Ausgrenzungserfahrungen die Arbeitsbeziehungen gefährden und der damit verbundene Stress die Gesundheit der Lernenden mindern, was zu erhöhten Fehlzeiten führen kann. Die Erfolgs- bzw. Misserfolgsfaktoren ,psychosomatische Beschwerden" und ,Absenzen" nehmen also aufgrund der, Diskriminierungswahrnehmung' $\mathrm{zu}$, und das ,gute Auskommen mit Arbeitskolleginnen und -kollegen' und der, Gefallen am Umgang im Lehrbetrieb' wird beeinträchtigt. Die Variablen, Chancengleichheit im Lehrbetrieb' und ,soziale Unterstützung' dienen als mögliche vermittelnde Variablen.

Die Forschungsfrage lautet, ob es Unterschiede gibt zwischen Jugendlichen in geschlechtstypischen vs. geschlechtsuntypischen Berufslehren. Gemäß der Minoritäten-Stress-Theorie (Testa et al. 2015) ist davon auszugehen, dass die Jugendlichen in geschlechtsuntypischen Berufslehren bei den Gesundheitsaspekten, insbesondere bei ,psychosomatischen Beschwerden " wie Magenschmerzen, Appetitlosigkeit, Konzentrationsschwäche, Schwindel, Einschlafschwierigkeiten, Nervosität/Unruhe, ungewohnter Müdigkeit oder sehr starken Kopfschmerzen stärker in Mitleidenschaft gezogen werden als ihre Kolleginnen und Kollegen. Diese Beschwerden könnten zu vermehrten Fehlzeiten führen. ,Absenzen" werden als globaler Hinweis betrachtet, dass für diese Lernende etwas im Lehrbetrieb nicht stimmt.

Zur ,Zufriedenheit im Lehrbetrieb' gehören zwei wesentliche Aspekte, die unmittelbar mit dem typisch/untypisch Sein zusammenhängen. Einerseits gehört dazu das Verhältnis zu den Mitarbeitenden im Lehrbetrieb, andererseits die Einschätzung, wie diese Personen einander behandeln. Die Mitarbeitenden in den Betrieben sind über längere Zeit mit den Jugendlichen zusammen, jedoch nicht so sehr an der Aufrechterhaltung einer guten Beziehung interessiert wie etwa nahe Familienangehörige oder von Berufs wegen zur Unterstützung verpflichtete Personen wie Ausbildende oder Lehrerinnen und Lehrer an den Berufsfachschulen. Insbesondere interessant ist die Frage, ob es dem Betrieb und seinen Mitarbeitenden gelingt, Frauen und Männer gleich $\mathrm{zu}$ behandeln. Als Hinweis auf Diskriminierung im Sinn von Andersbehandlung von Frauen und Männern dient das Konstrukt, Chancengleichheit im Lehrbetrieb'.

Unter, sozialer Unterstützung' wird die emotionale Teilhabe in Form von Interesse von bedeutsamen Personen aus dem persönlichen Umfeld der Ler- 
nenden verstanden. Dazu gehören in erster Linie die Eltern und Familienmitglieder. Weiter sind feste Freundin / fester Freund, beste Schulkolleginnen/kollegen, beste Arbeitskolleginnen/-kollegen im Betrieb, Ausbildner/-in und Klassenlehrer/-in wichtige Personen, die einen entscheidenden Einfluss auf die Wahrnehmung der Jugendlichen haben, ob sie von ihrem Umfeld verstanden werden. Neben der Anteilnahme ist die tatkräftige Unterstützung insbesondere bei auftretenden Problemen entscheidend für den erfolgreichen weiteren Verlauf der Ausbildung.

Nachdem Erfolgskriterien während der Berufsbildung vorgestellt wurden, bleibt der Abschluss der Ausbildung - die Lehrabschlussprüfung - zu untersuchen. Im Nachfolgenden Abschnitt wird dargestellt, wie sich die Situation in der geschlechtlichen Minderheit zu sein auf das Bestehen der Abschlussprüfung auswirkt.

\subsubsection{Erfolgsfaktor: Lehrabschlussprüfung bestehen}

Die erfolgreiche Absolvierung der Berufslehre beinhaltet das Bestehen der Prüfungen am Ende der Ausbildung (Althoff 1992, Häfeli/Schellenberg 2009a, 2009b, OECD 2000). Wie weiter oben erläutert kann die Lehrabschlussprüfung im Komponentenmodell (vgl. Tabelle 1.1) an unterschiedlichen Stellen verortet werden: als Fremdurteil bzw. Akte/Dokument der spezifischen Arbeit am Tag der Prüfung oder als Akte/Dokument der globalen Laufbahn, als Summe aller Tätigkeiten während der Berufslehre.

Ein beruflicher Titel ist ein Zeugnis des kulturellen Kapitals und der berufliche Werdegang ein komplexer Prozess von Kapitalumwandlungen (Bourdieu 1983). Dabei vollzieht sich eine Transformation von inkorporiertem Kulturkapital (gelerntes Wissen) in institutionalisiertes Kulturkapital (Zertifikat). Dieser Umwandlungsprozess erfolgt in Abhängigkeit vom sozialen Kapital. Das kann sich etwa in den Möglichkeiten ausdrücken, die die Gruppenzugehörigkeit (wohlhabende Familie, leistungsstarkes Niveau in der Schule oder angesehener Beruf) eröffnen. Die Überführung ist nicht linear, sondern eher unscharf. Dies verdeutlicht sich bei Abschlussprüfungen im vergleichsweise geringen Abstand zwischen den Schwächsten der Erfolgreichen und den Stärksten der Nicht-Erfolgreichen. Eine Prüfung zu bestehen, erfolgt nach dem Alles-oder-nichts-Prinzip (Imdorf 2005). Eine genaue Betrachtung der Entstehung dieser Bewertung (Prüfungsresultat) ist außerdem eine Überprüfung der Chancengerechtigkeit des Systems (Berufsbildung). So sind die Auswertungen in Kapitel $6 \mathrm{zu}$ den Lehrabschlussprüfungen eine Überprüfung der Chancengleichheit zwischen Lernenden in geschlechtstypischen und -untypischen Berufslehren.

Gemäß Settelmeyer und Tschirner (2002) fällt die Entscheidung über den Prüfungserfolg weitgehend vor und nicht während der Prüfung. Innerhalb der 
Lehr- und Ausbildungszeit erweisen sich viele Faktoren als einflussreich für den Lernerfolg (Granato et al. 2002). Besondere Bedeutung für das Bestehen der Lehrabschlussprüfung haben die schulischen Leistungen, die kontinuierliche Betreuung durch Ausbildende und Berufsschullehrpersonen, die vollständige Vermittlung der Lehrinhalte sowie die Prüfungsvorbereitung. Diese Faktoren wirken zusammen und in einem Geflecht mit weiteren, in der Abbildung 1.2 dargestellten Aspekten. So sind im Betrieb beispielsweise Rekrutierung, Ausbildungsplan, Betriebsklima und die wirtschaftliche Situation einflussreich, während sich in der Berufsfachschule die Unterrichtsorganisation, die Vermittlung von Lerninhalten, die Praxisrelevanz und weitere mehr als bedeutsam erweisen. Zusammenhänge mit dem Erfolg an der Lehrabschlussprüfung weisen bei den Lernenden zusätzlich $\mathrm{zu}$ den schulischen Voraussetzungen der Werdegang, der Berufsfindungsprozess, das Interesse am Beruf und am Unterricht sowie die Motivation und die Prüfungsvorbereitungen auf.

\section{Abbildung 1.1: Faktoren des Prüfungserfolgs}

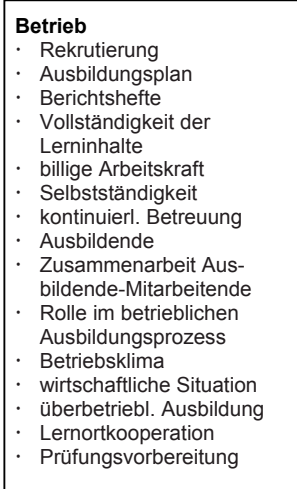

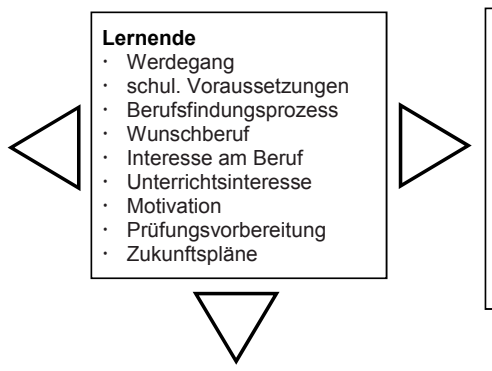

Berufsfachschule

Unterrichtsorganisation

Vermittlung von Lerninhal-

ten

ausbildungsbegl. Hilfen

Verhältnis von Lehrperso-

nen und Lernenden

Praxisrelevanz

Lernortkooperation

Prüfungsvorbereitung

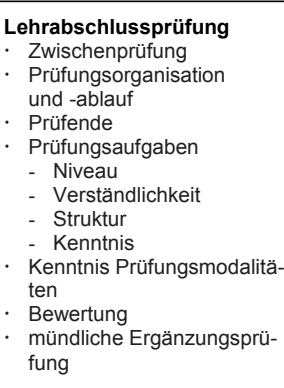

Quelle: Darstellung nach Settelmeyer und Tschirner (2002: 43).

Sowohl Settelmeyer und Tschirner (2002) als auch Häfeli und Schellenberg (2009a) beschreiben neben individuellen auch strukturelle und institutionelle Einflussfaktoren für das Bestehen der Lehrabschlussprüfung. Im Vorder- 
grund stehen die unmittelbar Involvierten, nämlich die Jugendlichen in ihrer Rolle als Lernende, die Lehrpersonen in den Berufsfachschulen und die Ausbildenden in den Lehrbetrieben. Demnach entscheiden einerseits individuelle Faktoren wie Schulleistungen als inkorporiertes Kapital (vgl. Bourdieu 1983), vermittelt durch Betrieb und Schule (vgl. Settelmeyer/Tschirner 2002), und andererseits strukturelle Bedingungen, wie sie bei Häfeli und Schellenberg (2009a) unter Gesellschaft (Abschnitt 1.5.1) aufgeführt werden, über Erfolg und Misserfolg bei der Lehrabschlussprüfung.

Diese Auswirkungen der strukturellen Bedingungen auf den Erfolg an der Lehrabschlussprüfung werden in der vorliegenden Arbeit als Indizien für Chancengleichheit oder eben Chancenungleichheit verstanden. Mit Chancen ist die statistische Wahrscheinlichkeit gemeint, die Lehrabschlussprüfung erfolgreich bewältigen zu können. Chancenungleichheiten wirken sich nicht erst auf das Bestehen aus, sondern bereits im Rahmen der Berufsfindung. So können Chancenungleichheiten - in Form von gesellschaftlichen Zwängen bei Jugendlichen Willensbildungsprozesse beeinflussen (Imdorf 2005), etwa indem Berufslehren von Jugendlichen nicht als Option in Erwägung gezogen werden, weil sie befürchten, die Lehrabschlussprüfung nicht zu bestehen, oder indem aufgrund der hohen Misserfolgswahrscheinlichkeit die Motivation, auf die Lehrabschlussprüfung zu lernen, unterminiert wird.

In Kapitel 6 gehe ich der Frage nach, ob sich die geschlechtsuntypische Situation auf den Erfolg an der Lehrabschlussprüfung auswirkt. Ist die allgemeine Misserfolgsquote hoch, steigt das individuelle Risiko, durchzufallen. Obwohl in einigen Berufslehren die Misserfolgsquote auffallend hoch ist, sind lediglich wenige systematische Untersuchungen dazu publiziert worden. Ihre Befunde belegen, dass sich Misserfolgsquoten zwischen den Berufslehren stark unterscheiden (Amos et al. 2003a, 2003b, Klaghofer et al. 1992, 1994). Einige Berufslehren, wie etwa ,Gipser/-in EFZ', weisen eine viel höhere Misserfolgsquote auf (ca. 40 Prozent) als andere, wie etwa ,Elektroinstallateur/-in EFZ' mit etwas über 1 Prozent (vgl. Daten des BFS 2009). Die Datengrundlage unterscheidet nicht zwischen erstem, zweitem oder drittem Versuch an der Lehrabschlussprüfung.

Eine Varianzanalyse von Amos et al. (2003a) belegt, dass die Faktoren Kanton und Beruf 4.5 Prozent der Varianz der Erfolgsquote erklären können. Daneben erklären Geschlecht, Nationalität, Art der Ausbildung, Alter, soziale Herkunft und Repetition der Lehrabschlussprüfung nochmals etwa gleich viel Varianz. Folgende Personengruppen haben eine geringere Wahrscheinlichkeit, die Lehrabschlussprüfung zu bestehen: Männer, Ausländerinnen und Ausländer, sozial Schwache, Schulschwache sowie Repetierende.

Männer haben über alle Berufslehren gesehen eine höhere Misserfolgsquote als Frauen. Nach Amos et al. (2003a) hängt das damit zusammen, dass Männer öfter in gewerblich-industriellen Berufslehren sind, die eine höhere Misserfolgsquote aufweisen als etwa Berufslehren im Gesundheitswesen 
oder im Handel. Dies können Neuenschwander et al. (2012) für einige Ausbildungsfelder 2008 belegen. Das Argument ist insofern zu relativieren, als in gewerblich-industriellen Berufslehren die Frauen ebenfalls die höhere Erfolgsquote haben als Männer, wie die Auswertungen von Neuenschwander et al. (2012) hervorheben.

Anknüpfend an diese Befunde zu den Unterschieden zwischen Frauen und Männern in den segregierten Berufen überprüfe ich, ob Lernende in geschlechtsuntypischen Berufslehren eine höhere Wahrscheinlichkeit haben, an der Lehrabschlussprüfung Misserfolg zu haben, als Lernende in geschlechtstypischen oder geschlechtergemischten Berufslehren. Die Bestätigung dieser Hypothese gäbe Hinweise darauf, dass es Personen in geschlechtsuntypischen Berufslehren weniger gut gelingt, ihr inkorporiertes Kulturkapital in institutionalisiertes Kulturkapital überzuführen.

In Kapitel 1 ,Beruf und berufliche Grundbildung ' habe ich beschrieben, dass die Arbeits- und Berufsbildungswelt geschlechtlich segregiert ist, wie Berufe Jugendliche sozialisieren, wie angehende Lernende unter dem Aspekt des Geschlechts ihre Berufslehre auswählen bzw. ausgewählt werden und wie sich Erfolg in der Berufslehre manifestiert. Der Beruf bildet die eine Seite der geschlechtsuntypischen Berufssozialisation. In Kapitel 2 ,Geschlecht' beschreibe ich die beiden vermeintlich so klar umrissenen Kategorien von Geschlechtlichkeit: männlich und weiblich. Die Betrachtungen zu Geschlecht, seinen Wahrnehmungen und Definitionen, Erklärungsansätze für die Unterschiede zwischen den Geschlechtern sowie die Auswirkungen auf die Entwicklung der Geschlechtsidentität, der Stereotype und Rollenvorstellungen demonstrieren, wie vorgefasste Meinungen den Blick verstellen können. 


\section{Geschlecht}

Das Wort ,Geschlecht' stammt vom mittel- bzw. althochdeutschen ,schlagen“ (geslehte bzw. gislahti) und bezieht sich auf das, was in dieselbe Richtung schlägt, also von übereinstimmender Art ist. Anfänglich bezog sich Geschlecht auf die familiäre Abstammung und auf die Gesamtheit der gleichzeitig lebenden Menschen ${ }^{13}$. In der vorliegenden Arbeit wird ,Geschlecht ${ }^{6}$ als Überbegriff für das biologische Geschlecht (sex, sexus) und das soziale Geschlecht (gender) verwendet, was im Folgenden erläutert wird.

Die Einteilung von Menschen in weibliche und männliche Individuen ist verbunden mit der Zuschreibung von mehr oder weniger ausprägten Eigenschaften zu den jeweiligen Polen. Das Phänomen der Polarisierung hilft, das Geschlecht im Gegenüber zu erkennen, und wird von beiden Seiten - Sendenden und Empfangenden - verstärkt. Gegenseitig bedingen sich der Wunsch, das Geschlecht im Gegenüber zu erkennen, und das Bedürfnis, sein eigenes Geschlecht erkennbar zu machen. Durch diese Polarisierung der Geschlechter besteht die Gefahr, dass Gemeinsamkeiten, Überschneidungen sowie die Vielfalt von Eigenschaften, Fähigkeiten und Ausdrucksformen, die Individuen - Frauen wie Männer - haben, verschwinden.

Am Beispiel der körperlichen Stärke lässt sich demonstrieren, wie sich geringe Unterschiede zu wirksamen Rollenerwartungen entwickeln. Die Fähigkeit, Lasten zu heben, dürfte normalverteilt sein. Durchschnittlich können Männer aufgrund ihres Körperbaus und Muskelwachstums größere Lasten tragen: $35 \mathrm{~kg}$ vs. $23 \mathrm{~kg}$ (Bischof-Köhler 2004). Der Unterschied beträgt etwa 40 Prozent (SUVA 2010). Ebenso gibt es bei den Extremwerten Unterschiede zwischen Frauen und Männern: Der Weltrekord im Gewichtstoßen beträgt bei den Frauen $193 \mathrm{~kg}$ (2014) und bei den Männern $263 \mathrm{~kg}(2004)^{14}$. Verallgemeinert für eine Vielzahl von Eigenschaften ist dies in der obersten Verteilungskurve in der Abbildung 2.1 dargestellt. Der Mittelwert der Männer liegt in dieser Darstellung über demjenigen der Frauen. Für andere Eigenschaften kann es umgekehrt sein. Es wird zusätzlich sichtbar, dass es Frauen gibt, die höhere Ausprägungen haben als viele Männer. Die Überlappung der Fähigkeitsausprägungen von Frauen und Männern ist erheblich. In anderen Worten: Der Unterschied zwischen den Mittelwerten der beiden Geschlechter ist geringer als die Verteilungsspanne innerhalb eines Geschlechts. Menschen sind es gewohnt, in Kategorien zu denken und Unterschiede verstärkt wahrzunehmen (Bischof-Köhler 2004). Wenn nun das eine Merkmal positiv dargestellt wird, erfährt das andere Merkmal eine Abwertung. Aus einem empirischen Mehr-oder-weniger wird ein normatives Alles-oder-nichts. Die Ab-

13 Vgl. Duden, Das Herkunftswörterbuch (1989: 235), Stichwort Geschlecht.

14 Wikipedia [http://de.wikipedia.org/wiki/Liste_der_Weltrekorde_im_Gewichtheben\#cite_ note-1kiloregel-3; Zugriff: 22.03.2017]. 
bildung 2.1 verdeutlicht die gesellschaftliche Transformation einer überlappenden empirischen Verteilung geschlechtstypischer Verhaltensmerkmale (oben), die Akzentuierung der gelebten Unterschiede durch die normative Dichotomie der Rollen (Mitte) und die Höherbewertung der männlichen bzw. die Abwertung der weiblichen Verhaltensmuster (unten).

Abbildung 2.1: Verteilung und Wertung von Geschlechtsmerkmalen

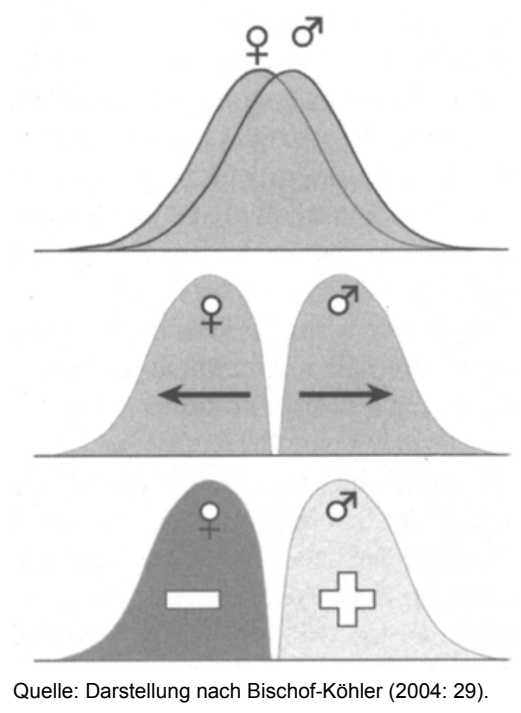

Für das Verständnis von Personen, die sich aufgrund ihrer Minderheitsposition im beruflichen Umfeld diese grundlegenden mentalen Prozesse nicht zunutze machen können, lohnt sich eine eingehende Betrachtung einiger fundamentaler Facetten des Geschlechts. Eine erste Frage ist, ob die dichotomen Kategorien des Geschlechts so eindeutig sind, wie sie uns erscheinen. In Unterkapitel 2.1,Biologisches und soziales Geschlecht' relativiert sich die vermeintliche Eindeutigkeit. Nichtsdestoweniger lassen sich Unterschiede zwischen den beiden Geschlechtern beschreiben, und diese verlangen nach Erklärungen. Die vorliegende Arbeit beschreibt exemplarisch vier wichtige ,Erklärungsansätze der Geschlechtsunterschiede‘ (Unterkapitel 2.2). Eine Rekonstruktion der Entwicklung der allgemein positiveren Bewertung der männlichen Eigenschaften bzw. der männlichen Dominanz wird in Unterkapitel 2.3 ,Ursprünge der männlichen Hegemonie' dargelegt. Nach diesem historischen Exkurs rückt die individuelle ,Entwicklung der Geschlechtsidentität' (Unterkapitel 2.4) ins Zentrum der Betrachtung. Gerade weil das eigene Geschlecht zu typischen Interaktionen mit der Umwelt führt und damit eine 
zentrale Rolle im Aufbau einer Geschlechtsidentität spielt, können die Betrachtungen in den Unterkapiteln 2.5 ,Geschlecht als soziale Konstruktion" sowie 2.6 ,Geschlechterstereotype und Geschlechtsrollen' Aufschluss darüber bieten, womit Lernende konfrontiert werden, wenn sie in geschlechtsuntypischen Berufslehren gegen den Strom schwimmen.

\subsection{Biologisches und soziales Geschlecht}

Im deutschsprachigen Raum existiert die Unterscheidung zwischen biologischem (engl. ,sex') und sozialem Geschlecht (engl. ,gender') im alltäglichen Sprachgebrauch nicht. In den Sozialwissenschaften wurde versucht, den beiden Ebenen von biologischem Geschlecht und Geschlechterrollen - das biologisch determinierte und das gesellschaftlich-kulturell geprägte Geschlecht - zu unterscheiden (Rubin 1975).

Das ,biologische Geschlecht' bezieht sich auf den Genotypus XX für Frauen und XY für Männer, der die Ausschüttung von Geschlechtshormonen (wie Östrogen, Testosteron, Noradrenalin etc.) steuert, die körperlichen Geschlechtsdifferenzen vom Embryostadium an definiert und gemäß dem genetischen Bauplan einen weiblichen oder einen männlichen Körper mit dem entsprechenden Phänotyp ausbildet (Bischof-Köhler 2004, Bosinski 2000). Eine interessante Sichtweise in der Umkehrung der althergebrachten Begründung der Überlegenheit des Mannes bietet Borneman (1981), indem er die Unvollständigkeit des Y-Chromosoms und die damit verbundene höhere Prävalenz bestimmter Erbkrankheiten wie Farbenblindheit oder Bluterkrankheit als Schwäche des Mannes darstellt.

Was den normalen Mann von der Frau unterscheidet, ist also nicht die Anwesenheit seines Y-Chromosoms; sondern die Abwesenheit des zweiten X. In diesem Sinne ist der Mann auch nur eine unfertige Frau, eine biologische Entgleisung, die in zahllosen biologischen Aspekten benachteiligt ist. (Borneman 1981: 514)

Mit ,Gender' wird der Unterschied zwischen dem sozialen bzw. psychologischen Geschlecht einer Person und dem biologischen Geschlecht verdeutlicht. Der Terminus stammt von John Money, der ihn im Kontext seiner Forschungsarbeiten zu Intersexualität einführte, weil das körperliche Geschlecht nicht eindeutig war, Geschlechtsidentität und Geschlechtsrollenpräsentation hingegen eindeutig waren. Bei Money (1955) wird der Terminus Geschlechtsrolle benutzt, um all jene Ausdrucksformen zu beschreiben, die eine Person benutzt, um sich selbst den Status Frau oder Mädchen bzw. Mann oder Junge zu geben. $\mathrm{Zu}$ diesen Ausdrucksformen gehören Sprache, Tätigkeiten, Verhaltensweisen, Kleidung, Vorlieben, Beruf, Freizeitbeschäftigungen usw. 
In Sinn von Butlers (1991) Geschlechterkritik ist die Trennung zwischen ,Sex ' und ,Gender' auf den kartesianischen Dualismus zurückzuführen, also auf die Unabhängigkeit von Körper und Geist. So bestehe der Mensch einerseits aus seinem biologischen, unhinterfragbaren, natürlich gegebenen Körper (Sex) und andererseits aus seinem vom Körper unabhängig quasi frei wählbaren Geschlecht (Gender). Nach Butler (1991) erscheint aber nicht nur das soziale Geschlecht als Konstruktion, sondern auch das biologische Geschlecht als hinterfragbare Wahrheit oder als eine kulturelle Interpretation des Körperlichen.

Wenn man den unveränderlichen Charakter des Geschlechts bestreitet, erweist sich dieses Konstrukt namens ,Geschlecht" vielleicht als ebenso kulturell hervorgebracht wie die Geschlechtsidentität. Ja, möglicherweise ist das Geschlecht (sex) immer schon Geschlechtsidentität (gender) gewesen, so dass sich herausstellt, dass die Unterscheidung zwischen Geschlecht und Geschlechtsidentität letztlich gar keine Unterscheidung ist. (Butler 1991: 24; Herv. i. Original)

Das biologische Geschlecht als Konstrukt zu verstehen, bedeutet nun nicht, dass es seine Relevanz verliert oder wegdiskutiert werden könnte, sondern damit kommt zum Ausdruck, dass die in der Alltagsvorstellung unumstößlich scheinende Tatsache, wie Frauen und Männer sind, eine innerpsychische, jedoch interindividuell geteilte Vorstellung von Geschlecht ist. Die Geschlechtsorgane mit ihren reproduktiven Funktionen sind für den Konstruktionsprozess prägend. Die deterministische Dichotomie und viele damit verbundene Zuschreibungen sind jedoch ein Produkt der Sozialisation.

Die terminologische Unterscheidung von ,Sex ${ }^{6}$ und ,Gender' wurde breit diskutiert (z.B. Butler 1991, Deaux/LaFrance 1998, Schössler 2008). Das Erklärungspotenzial ist jedoch gering, insbesondere da bei der Mehrheit der Personen körperlich-biologisches Geschlecht und soziales Geschlecht übereinstimmen. Im Folgenden wird in der vorliegenden Arbeit ausschließlich das deutsche Wort ,Geschlecht' verwendet, im Bewusstsein, dass das Geschlecht eine starke biologische Komponente hat (genetisches, genitales, hormonelles, phänotypisches Geschlecht), aber auch eine ebenso einflussreiche soziale Konstruktion ist (Rollen, Erwartungen, soziales Geschlecht, Verhalten, Identität). Mit dem Geschlecht verbunden sind Zuschreibungen von Merkmalen, die vermeintlich oder manifest bei Frauen und Männer in unterschiedlicher Ausprägung auftreten. Wie sich Geschlechtsunterschiede erklären lassen, damit befasst sich das nachfolgende Unterkapitel.

\subsection{Erklärungsansätze der Geschlechtsunterschiede}

Die Beschäftigung mit Geschlechtsunterschieden birgt die Gefahr, dass die Polarisierung der Geschlechter verstärkt wird. Mit den nachfolgenden Aus- 
führungen wird dem entgegengehalten. Bevor nun vier Erklärungsansätze für die Unterschiede zwischen Frauen und Männern vorgestellt werden, soll noch einmal betont werden, dass diese Unterscheidung die Entfaltungsmöglichkeiten von Individuen einschränken kann und die Überschneidungen größer sind als oft wahrgenommen.

Geschlecht kann als soziales Konstrukt (vgl. Unterkapitel 2.5) verstanden werden. Dennoch gibt es einen starken biologischen Bezug. Menschen kommen nicht als fertige Wesen zur Welt, sondern sie entwickeln sich körperlich und geistig aus dem genetischen Erbe und werden vom Moment der Zeugung bis zu ihrem Tod von ihrer Umwelt beeinflusst. Doch der Mensch nimmt die äußeren Einflüsse nicht einfach auf, sondern er reagiert darauf. In diesen Interaktionen prägen sich Mensch und Umwelt gegenseitig. Es findet eine Sozialisation statt. Der genetische wie der soziokulturelle, beide Einflussbereiche haben einen historischen Hintergrund. Grundsätzlich haben Menschen, Frauen und Männer, mehr gemeinsam, als sie unterscheidet. Die Betrachtung der Unterschiede verdeckt Überlappungen, Graubereiche und die Tatsache, dass die Trennlinie nie genau zwischen den Geschlechtern verläuft, sondern dass die Trennung eine kulturelle Leistung ist.

Obwohl in der dualistischen Sichtweise ein Kind entweder Mädchen oder Junge, eine erwachsene Person Frau oder Mann ist, verhalten sich Kinder und Erwachsene sowohl feminin wie maskulin. Dabei unterscheiden sich Kinder und Erwachsene im Grad ihrer Weiblichkeit und Männlichkeit. Trautner (2008) unterscheidet bei der Beschreibung von Geschlechtsunterschieden zwischen geschlechtsspezifischen und geschlechtstypischen Merkmalen. Als geschlechtsspezifische Merkmale gelten jene, die jeweils nur ein Geschlecht betreffen. Sie sind unmittelbar mit den spezifischen Funktionen der Geschlechter im Prozess der biologischen Reproduktion verbunden (Bosinski 2000). Bei der Frau sind dies beispielsweise Menstruation, Schwangerschaft und Stillen von Kindern, beim Mann die Zeugungsfähigkeit. Da diese Merkmale im Normalfall entweder weiblich oder männlich vorkommen, sind die Geschlechtsdifferenzen bipolar-dichotom geteilt. Fließende Übergänge sind zwar möglich, gehen aber meist mit der Beeinträchtigung der Reproduktionsfunktion einher (Bosinski 2000). Wenn bei der Mehrheit der Frauen bzw. Männer ein Merkmal besonders ausgeprägt ist, spricht Trautner (2008) von geschlechtstypischen Merkmalen. Sie ergeben sich aus dem Vergleich von Geschlechtergruppen aufgrund statistisch-deskriptiver Beschreibungen. Geschlechtstypische Merkmale treten häufiger oder intensiver innerhalb einer Geschlechtergruppe auf. Meistens ist der Mittelwertsunterschied der Geschlechtergruppen jedoch geringer als die Variationsbreite innerhalb der Geschlechter.

Die Abweichung vom Mittelwert und die Überlappung mit der Verteilung der Funktion, Eigenschaft etc. innerhalb der anderen Geschlechtergruppe ist konstituierend für diese Art von Unterschieden, somit nicht krankhaft, sondern die Regel. (Bosinski 2000: 99) 
Für die meisten Menschen ist die individuelle Wahrnehmung der Geschlechtszugehörigkeit bzw. die Geschlechtsidentität (vgl. Unterkapitel 2.4) eindeutig einem Geschlecht zugeordnet. Dabei ist wichtig zu sehen, dass sie von anderen Dimensionen wie Interessen, Eigenschaften, Vorlieben, Verhaltensweisen und sogar sexueller Orientierung unabhängig ist. Obwohl oft ein Zusammenhang hergestellt wird, verstehen sich Frauen als Frauen, Männer als Männer, gleichgültig ob ihre sexuelle Orientierung hetero-, homo-, bioder pansexuell ist und ob sie beispielsweise das Bedürfnis haben, sich wie Angehörige des anderen Geschlechts zu kleiden oder zu frisieren. „Auch die Ausübung eines geschlechtsuntypischen Berufs oder der Besitz einzelner geschlechtsuntypischer Eigenschaften stellt nicht zwangsläufig die (biologische und soziale) Geschlechtszugehörigkeit in Frage" (Trautner 2008: 631). ${ }^{15}$

Die Unterschiede zwischen Frauen und Männern sind virulent und beschäftigen die Personen im Alltag. Welche davon empirisch nachzuweisen sind, woher sie kommen und wie sie erklärt werden können, davon wird im Folgenden berichtet. Vier Erklärungsansätze für Geschlechtsunterschiede werden überblicksmäßig und exemplarisch vorgestellt: ,Evolutionspsychologische Erklärungsansätze“ (Abschnitt 2.2.1), ,Gene und Sexualhormone“ (Abschnitt 2.2.2), ,Hirnbeschaffenheit und Ausprägung der Hemisphären “ (Abschnitt 2.2.3) sowie ,Sozialisation der Geschlechtsunterschiede' (Abschnitt 2.2.4).

\subsubsection{Evolutionspsychologische Erklärungsansätze}

Die Erklärungsversuche der Evolutionspsychologie beginnen ihre Ausführungen in einer fernen Vergangenheit. Gemäß diesen Beschreibungen entwickelte sich vor etwa einer halben Milliarde Jahren bei den frühen Lebensformen unseres Stammbaums die innere Befruchtung, was zur Folge hatte, dass die Weibchen nicht mehr ableichten und die Brut anschließend sich selbst überließen, sondern zu Empfängerinnen und Trägerinnen der neuen Generation wurden. Die parentale Investition ist seither bei den meisten landlebenden Wirbeltieren ungleich verteilt. Aufgrund dieser Aufteilung entwickelten sich unterschiedliche körperliche Voraussetzungen und Verhaltensweisen sowohl in der Brunst- und Balzzeit als auch bei der Aufzucht der Jungtiere (Bischof-Köhler 2004).

Diese Variante der Fortpflanzung hatte sich gemäß Bischof-Köhler (2004) bewährt und setzte sich bei den Hominiden und schließlich beim Homo sapiens fort. Während der Steinzeit begannen die Menschen, Jagd auf größere Tiere zu machen. Für diese Tätigkeit war laut Bischof-Köhler (2004) der größere und kräftigere Körper der Männer besser geeignet als jener der

15 Für die spezifischen Aspekte für Inter- oder Trans-Menschen sei auf folgende Literatur hingewiesen: Groß et al. (2008). 
Frauen. Frauen ihrerseits waren aufgrund ihrer enormen parentalen Investition - während der wenigen Wochen vor und nach der Niederkunft - weniger in der Lage, weite Strecken zurückzulegen. Mit diesem evolutionspsychologischen Blick ließen sich eine Reihe von Differenzen zwischen den Geschlechtern erklären, etwa die besseren räumlich-visuellen Fähigkeiten der Männer aufgrund der Jagd und des Erkundens unbekannter Gelände (Bischof-Köhler 2004), Überlebensvorteile (Survival of the fittest) für jene Männer, die sich gut orientieren können und den Heimweg wiederfinden. So plausibel diese evolutionstheoretischen Erklärungen scheinen, bestehen doch Zweifel an ihrer Folgerichtigkeit und Unausweichlichkeit. So beschreibt Halpern (1997) einen Umstand, den Evolutionstheorien gerne ausblenden, nämlich dass Frauen - obwohl allenfalls weniger an der Jagd beteiligt - ebenfalls mit Aufgaben betraut waren, die räumlich-visuelle Fähigkeiten besonders förderten, so etwa mit dem Herstellen von Kleidern, Unterkünften, Körben etc. Es lasse sich anhand von Evolutionstheorien schwer erklären, wie die Differenzen bei den räumlichen Rotationsaufgaben zustande kämen oder was diese Unterschiede mit der Fähigkeit des Wiederfindens des Aufenthaltsorts der Familie in einer steinzeitlichen Umgebung zu tun hätten (Halpern 1997).

Obwohl der evolutionspsychologische Erklärungsansatz mit der ungleichen parentalen Investition ein interessantes Phänomen beschreibt, sind die Zusammenhänge zu den heute gefundenen Geschlechterungleichheiten eher spekulativ und widersprüchlich. Vielleicht lohnt sich der Blick auf die Bausteine der körperlichen Entwicklung, nämlich die Gene und mit ihnen die Sexualhormone.

\subsubsection{Gene und Sexualhormone}

Die Gene spielen eine wichtige Rolle bei der körperlichen Reifung. So bestimmen bei der menschlichen Entwicklung die Chromosomen X und Y, ob sich ein Mädchen (Chromosomenpaar XX) oder ein Junge (Chromosomenpaar XY) entwickelt. Im Falle der weiblichen Entwicklung werden Ovarien gebildet. Diese nehmen ungefähr in der 12. Schwangerschaftswoche ihre Funktion auf und produzieren Östrogen und Progestin. Im Falle einer männlichen Entwicklung stoßen die gerade neu gebildeten Geschlechtszellen ab der 5. bis 7. Schwangerschaftswoche männliche Sexualhormone, sogenannte Androgene (hauptsächlich Testosteron und Dihydrotestosteron) aus (Bosinski 2000). Die genannten Hormone finden sich sowohl im weiblichen als auch im männlichen Körper, jedoch in unterschiedlichen Konzentrationen. Diese Hormone sind nicht nur wichtig bei der Aktivierung der Bildung der Geschlechtsorgane, sondern auch bei der Ausbildung des Gehirns (Halpern 1997). Dieser Befund führte zu einer Reihe von Hypothesen und Erklärungsversuchen für Unterschiede zwischen Frauen und Männern bei mentalen und 
körperlichen Fähigkeiten, Vorlieben, Verhaltensweisen und nicht zuletzt den Berufen (Bischof-Köhler 2004).

Im Folgenden illustrieren ein paar dieser Befunde exemplarisch, was damit gemeint ist. Männliche Geschlechtshormone können mit dem verlangsamten Wachstum der linken Hirnhemisphäre in Verbindung gebracht werden, wodurch sich die rechte schneller entwickeln soll und ,infolgedessen beim männlichen Geschlecht die dominierende Rolle übernimmt" (BischofKöhler 2004: 242). Weibliche Geschlechtshormone sind einem monatlichen Rhythmus unterworfen, was zu schwankenden Leistungen führen kann. So schneiden Frauen im Untertest, Verbale Flüssigkeit' eines Intelligenztests besser $a b$, wenn der Östrogenspiegel hoch ist. Während der Menstruation, wenn sich das Östradiol am Tiefpunkt befindet, erreichen Frauen hingegen im Untertest ,Räumliches Denken' desselben Intelligenztests bessere Ergebnisse (Aiken 1996, Bischof-Köhler 2004). Männer sind ebenso zyklischen Hormonschwankungen unterworfen. So ist der Testosteronspiegel am Morgen höher als am Abend. Bei US-Amerikanern ist er im Herbst höher als im Frühling (Halpern 1997). Ein weiterer Befund, der für den Einfluss der Hormone auf die intellektuellen Fähigkeiten spricht, tritt in der Tatsache in Erscheinung, dass Frau-zu-Mann-Transsexuelle nach einer Hormonbehandlung ihre Leistungen in visuell-räumlichen Fertigkeiten verbessern und tiefere Werte in Tests zur verbalen Flüssigkeit zeigen (Halpern 1997). Hormone wirken sich also auf die Entwicklung des Gehirns aus und beeinflussen damit das Denken. Weitere neurologische Forschungsbefunde werden im folgenden Abschnitt vorgestellt.

\subsubsection{Hirnbeschaffenheit und Ausprägung der Hemisphären}

Das menschliche Gehirn besteht aus etwa 100 Milliarden Nervenzellen und hat die erstaunliche Eigenschaft, über das ganze Leben plastisch zu bleiben und sich den jeweiligen Gegebenheiten anpassen zu können. Wird es gefordert, baut es seine Vernetzung weiter aus, fallen einzelne Hirnzellen oder Nervenzentren aus, können in günstigen Fällen andere Hirnareale ihre Funktionen übernehmen (Goldstein et al. 1997).

Bei Jungen lässt sich bereits im Alter von 6 Jahren bei Aufgaben, die sie mit der rechten bzw. mit der linken Hand lösen sollen, ein deutlicher Unterschied in der motorischen Fertigkeit der rechten bzw. der linken Hand erkennen. Bei Mädchen konnte dieser Unterschied nicht gefunden werden (Aiken 1996). Daraus könne abgeleitet werden, dass Mädchen und Frauen vermehrt beide Hemisphären zum Lösen von Aufgaben einsetzten, bei Jungen und Männern hingegen eine Lateralisierung zugunsten der rechten Hemisphäre eintrete (Aiken 1996). Die linke Hemisphäre ist in erster Linie für analytische Fähigkeiten, schlussfolgerndes Denken und für alle sprachgebundenen Leis- 
tungen verantwortlich. Die rechte Hemisphäre dagegen ist eher für die globalganzheitliche Erfassung und für nicht-verbale Leistungen wie anschauliches Vorstellen, raumbezogene Fähigkeiten und Musikalität zuständig (Bischof-Köhler 2004).

Es gibt weitere hirnphysiologische Unterschiede, von denen einige noch kontrovers diskutiert werden. Lynn (1994) folgert aufgrund eines Zusammenzugs zahlreicher Studien, Männer hätten ein größeres Gehirn und würden deshalb in Intelligenztests besser abschneiden. Doch mit der Größe des Gehirns ist noch nichts über die Dichte der Neuronen im Großhirn gesagt. Halpern (1997) führt dazu aus, dass das weibliche Gehirn sich in der Form vom männlichen unterscheide und eine größere Oberfläche mit mehr Wölbungen aufweise und dementsprechend mehr graue Hirnzellen habe. Zählungen von Packenberg und Gunderson (1997) beweisen in einer Studie, dass keine Dichteunterschiede bestehen: Männliche Großhirne hatten in dieser Untersuchung durchschnittlich 22.8 Milliarden und weibliche 19.3 Milliarden Neuronen. Beide Geschlechter hatten die gleiche Dichte an Hirnzellen. Wenn die Männer die zusätzlich vorhandenen Neuronen gleichermaßen nutzen könnten, dann wäre das eine Erklärung für die besseren Leistungen in aufwendigen räumlich-visuellen Aufgaben, denn beim räumlich-visuellen Denken sind mehr Hirnanteile involviert als etwa beim verbalen Schlussfolgern (Lynn 1994).

Dieser Abschnitt hat die Unterschiede im Aufbau von weiblichen und männlichen Gehirnen beschrieben. Diese beeinflussen zwar die Wahrnehmung und das Denken, doch sind die Zusammenhänge mit dem unterschiedlichen Verhalten von Frauen und Männern nicht direkt erkennbar. Eine wesentliche Eigenschaft des menschlichen Gehirns ist seine Plastizität und damit die Lernfähigkeit. Neuronale Strukturen bilden sich bei Gebrauch um. Wie sie benutzt werden, das entscheiden nicht allein Hormone und Gehirnstrukturen, sondern die Umwelt und ihre Reize, die das denkende Gehirn aufnimmt und verarbeitet. Insbesondere das soziale Umfeld sozialisiert weibliche und männliche Individuen unterschiedlich.

\subsubsection{Sozialisation der Geschlechtsunterschiede}

Menschen sind nicht nur durch ihre genetische Veranlagung und durch das Wirken der Hormone in ihrem Körper determiniert, sondern interagieren ebenso mit ihrer Umwelt, werden von dieser geprägt. Individuen werden von ihrer Umgebung sozialisiert und sozialisieren sich selbst. Dabei sind Erwartungen und Stereotype besonders geschlechterdivergierend. Kinder werden je nach Geschlecht mit abweichenden Botschaften, Modellen, Belohnungen und Bestrafungen konfrontiert (Halpern 1997). Ob diese geschlechtsspezifischen 
Erfahrungen so unmittelbar das Verhalten bzw. Fähigkeiten prägen, ist durchaus umstritten.

Beispielsweise lösen Jungen bei Intelligenztests die Aufgabe der mentalen Rotation von Würfeln durchschnittlich besser als Mädchen (BischofKöhler 2004, Lubinski 2004). Holling et al. (2004) argumentierten, Jungen erhielten mehr Spielzeuge, die räumliche Fähigkeiten förderten (etwa Bauklötze, Lego ${ }^{\circledR}$ oder STOKYS), und dass ihnen mehr Bewegungsfreiheit zugestanden werde. Dies könnten Gründe für die relativ konsistenten Unterschiede im Bereich des räumlichen Denkens zwischen den Geschlechtern sein. Obwohl die Unterschiede nicht nur aus dem (Spiel-)Verhalten entstehen, so verstärkt es doch die allfälligen Dispositionen. Ein weiteres Indiz für die Erklärung der Unterscheide zwischen den Geschlechtern liefert die Beobachtung, dass Frauen mit weniger ausgeprägten Geschlechtsrollenstereotypen in räumlichen Aufgaben besser abschnitten als Frauen mit stärker ausgeprägten (Krasnoff et al. 1989). Je wirksamer die Stereotypen das Denken beeinflussen, desto größer sind die Unterscheide zwischen den Geschlechtern.

Die beiden Geschlechter werden mit geschlechtsspezifischen Einflüssen konfrontiert und entwickeln eventuell gerade deshalb unterschiedliche Interessen. Laut einer Befragung von Studierenden an einem College verbrachten die Männer viel mehr Zeit mit Sport, Feiern, Fernsehen und Computerspielen als ihre Kommilitoninnen (Halpern 1997). Beispielsweise beschäftigten sich 37 Prozent dieser jungen Männer mehr als eine Stunde pro Woche mit Videospielen, während dies nur gerade 7 Prozent der Frauen taten. Frauen in dieser Altersgruppe verbrachten viel mehr Zeit mit Haushalt, Kinderpflege, Lesen zum Vergnügen, Lernen und Freiwilligenarbeit. Halpern (1997: 1096) bringt die Befunde folgendermaßen auf den Punkt: „On average, women and men live systematically different lives.“

Wie sich diese unterschiedlich stark ausgeprägten Interessen und Lebensweisen ausbilden, könnte mehrere Gründe haben. Es ist zu erwarten, dass mehrere Faktoren in die gleiche Richtung wirken. Neben genetischen Prädispositionen tragen Modelle und Erwartungen der Umwelt zusätzlich dazu bei, bestimmte Aktivitäten und Fähigkeiten auszuführen und zu fördern. Schließlich werden jene Tätigkeiten beibehalten, die von der Umwelt positiv bewertet und damit verstärkt werden.

Wie stark sich Erwartungshaltungen auf die Performanz auswirken können, demonstriert eine weitere Studie von Halpern (1997). Der Hälfte der teilnehmenden Frauen wurde gesagt, dass der Test, den sie gleich lösen sollten, Geschlechterunterschiede aufweise. Die Gruppe mit dieser Bedingung erreichte eine signifikant schlechtere Leistung als wenn gesagt wurde, derselbe Test sei geschlechtergerecht. Diese Ergebnisse belegen, wie die Aktivierung eines negativen Stereotyps die Leistung beeinflussen kann.

Ein Ziel der Koedukation in der Schule, also des gemeinsamen Unterrichtens von Mädchen und Jungen, war die Gleichstellung von Schülerinnen und 
Schülern und somit die Verbesserung der Chancengleichheit von Frau und Mann. Auf die umfangreichen Forschungen in den 1980er-Jahren folgte entgegen allen Erwartungen keine substanzielle Nivellierung zwischen den Geschlechtern. Pointiert zusammengefasst lässt sich sagen: Mädchen werden in der Schule gescheit und Jungen selbstbewusst (z.B. Rustemeyer 1988, Rustemeyer/Jubel 1996). Den Vorsprung in den schulischen Leistungen konnten die Mädchen jedoch nicht ins Berufsleben mitnehmen.

Der Erfolg, den sie [die Mädchen, Anm. des Autors] hinsichtlich der erzielten Abschlüsse und Noten verzeichnen können, steht in erheblicher Diskrepanz zu dem im Vergleich zu den Jungen weit weniger ausgeprägten Selbstvertrauen, das sie während der Schulzeit aufbauen können. (Horstkemper 1995: 218)

Als Erklärungsansatz für diesen Befund ist einerseits der Attributionsstil zu nennen, wonach Mädchen und Frauen die Tendenz haben, Misserfolg auf selbstwertgefährdende Art Ursachen zuzuschreiben, nämlich internal, global und stabil (Horstkemper 1995). Das heißt, sie suchen Erklärungen für ihre weniger guten Leistungen in der eigenen (Un-)Fähigkeit (internal) statt in der Schwierigkeit der Aufgabe (external). Sie beziehen die negative Rückmeldung aus einer einzelnen Situation eher auf andere (global), statt sie als einmaligen Ausrutscher einzuordnen (lokal). Sie werten Misserfolg häufiger als immerwährendes Versagen (stabil) statt als einmaliges Ereignis (variabel). Zusätzlich bekommen Mädchen und Jungen vonseiten der Lehrkräfte unterschiedliche Rückmeldungen, sogar wenn diese noch so sehr darauf achten, weder das eine noch das andere Geschlecht zu bevorzugen.

Abbildung 2.2: Geschlechtstypische Verteilung von Lob und Tadel

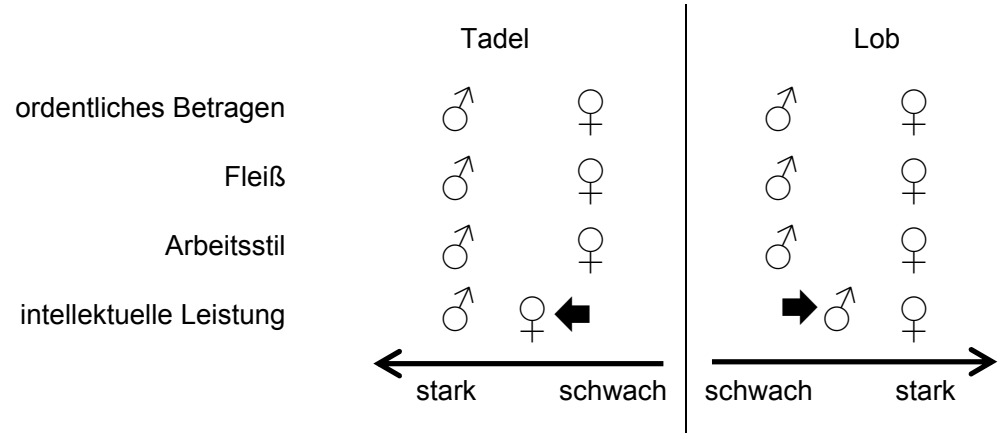

Quelle: Darstellung nach Bischof-Köhler (2004: 282).

In einer Studie von Dweck et al. (1978) ergab sich in den vier untersuchten Bereichen ,ordentliches Betragen', ,Fleiß ', ,Arbeitsstil' und ,intellektuelle Leistung' ein einheitliches Bild: Jungen werden öfter getadelt und Mädchen öfter gelobt. Einzig im Bereich der, intellektuellen Leistung' werden die 
Jungen etwas mehr bestätigt als in den anderen Bereichen, die Mädchen hingegen etwas öfter korrigiert als in anderen Bereichen (Dweck et al. 1978, vgl. Abbildung 2.2). Bischof-Köhler (2004) beschreibt die Tatsache, wonach Mädchen trotz verstärkten Lobens über ein geringeres Selbstvertrauen verfügen, folgendermaßen:

\begin{abstract}
Wenn die Mädchen also überhaupt getadelt wurden, dann in erster Linie für intellektuelles Versagen, und das ,fuhr' ihnen natürlich ,ein'. Wenn man nun berücksichtigt, dass sich Mädchen generell Tadel mehr zu Herzen nehmen, dann ist leicht abzusehen, dass diese Verstärkungspraxis das Vertrauen in das eigene intellektuelle Können kaum fördert; das Lob in diesem Bereich ging ja bei der allgemeinen Lob-,Inflation' unter. (Bischof-Köhler 2004: 282)
\end{abstract}

In diesem Unterkapitel über Geschlechtsunterschiede stellte ich dar, dass es durchaus naturbedingte Unterschiede zwischen den Geschlechtern gibt, doch dass zusätzlich starke kulturelle Phänomene dazu beitragen, die tatsächlichen und die vermeintlichen Unterschiede manifest zu machen. Ob sich die Arbeitsteilung zwischen den Geschlechtern auf die parentale Investition zurückführen lässt, erscheint ebenso fraglich wie ob und inwiefern die zahlreichen Befunde aus der Hirnforschung relevant sind, wenn es darum geht, sich zwischen komplexen beruflichen Tätigkeiten zu entscheiden.

Insgesamt durchlaufen Mädchen und Jungen, Frauen und Männer eine ähnliche Entwicklung, die geprägt ist von vielen gleichen Voraussetzungen. Unterschiede treten dann in Erscheinung, wenn sie das Fortbestehen der Menschheit (der eigenen Art bzw. der Weitergabe der Gene) tangieren oder zwingend notwendig sind. Einige vermeintliche Tatsachen, was die Geschlechterdifferenzierung anbelangt, erscheinen willkürlich, ja künstlich und ungerechtfertigt.

In dieser Arbeit stehen Personen mit einem geschlechtsuntypischen Beruf im Mittelpunkt. Dafür ist zum einen von Belang, wie sich die Segregation der Geschlechter im Leben und in der Berufswelt etablieren konnte, und zum andern, wie sich die Vorherrschaft des männlichen Geschlechts entwickeln konnte. Das folgende Unterkapitel gibt einen Einblick in diese Thematik.

\title{
2.3 Ursprünge der männlichen Hegemonie
}

Das berufliche Umfeld weist eine starke Segregation auf (vgl. Unterkapitel 1.1). Es scheint, als gäbe es einen Bereich für Frauen und einen für Männer. Begründet und unterstützt wird diese Segregation durch das größere Interesse der Frauen für Personen und der Männer für Sachen (Heintz et al. 1997, Su et al. 2009, Trauttmansdorff 2003). Wie einseitig diese Wahrnehmung ist, zeigt sich darin, dass Technik vor allem dann als Technik bezeichnet wird, wenn sie im männlichen Bereich erscheint (Schmid-Thomae 2012). Beispielsweise 
werden Arbeiten an Nähmaschinen (vermeintlich weiblich) nicht mit Technik assoziiert, Tätigkeiten mit Laubsägemaschinen (vermeintlich männlich) hingegen schon, obwohl - technisch betrachtet - die erste Maschine komplexer konstruiert ist als die zweite. Im Folgenden wird ein Erklärungsansatz beschrieben, der darstellt, wie sich die Segregation entwickelt haben könnte. Die folgenden Überlegungen basieren auf Borneman (1981) und widersprechen zum Teil den evolutionspsychologischen Erklärungsansätzen (vgl. Abschnitt 2.2.1). Obwohl Borneman verschiedentlich kritisiert wurde (vgl. Siegfried 2015), halte ich mit Bammer (1979), der in einer Würdigung des Buches ,Das Patriarchat - Ursprung und Zukunft unseres Gesellschaftssystems ${ }^{6}$ schrieb, dagegen:

Das Vermächtnis der Antike in unserer heutigen Gesellschaft wird nicht von jedermann als Beglückung, sondern von manchen als Bedrückung empfunden. Viele dieser in der Antike entstanden Zwänge wirken heute versteckt, oftmals werden heutige unter der Berufung auf eine glorifizierte Antike ganz offen verteidigt. Hier ist B.s Buch ein Markstein. Denn [...] werden antike Mechanismen als das freigelegt, was sie manchmal sind: starke, aber vielleicht doch überwindbare Belastungen. (Bammer 1979: 158)

Aus den umfangreichen Ausführungen Bornemans (1981) lässt sich Folgendes zusammenfassen: Die frühen altsteinzeitlichen Lebensgemeinschaften der Menschen kannten keine Arbeitsteilung. Frauen und Männer sammelten Nahrung und gingen auf die Jagd. Knochenfunden zufolge waren die Frauen den Männern körperlich ähnlicher als heute. Die Schwangerschaft und die Geburt von Kindern schränkten Frauen (Mütter) nicht dermaßen ein, wie wir uns das aus heutiger Sicht vorstellen und wie es in evolutionspsychologischen Erklärungsansätzen der Geschlechtsunterschiede als Argument verwendet wird (vgl. Abschnitt 2.2.1). Diese mutterrechtlichen Stammesgesellschaften waren frei von geschlechtlicher Unterdrückung und geprägt von einem egalitären Verhältnis der Geschlechter. Es war nicht so wichtig, das Geschlecht durch Abgrenzungshandlungen darzustellen. „Im Gegensatz zum Patriarchat mit seiner extremen Polarisierung der Geschlechter, die sich z.B. in der Kleidung niederschlägt, war auch in allen matrilinearen Gesellschaftsordnungen die Kleidung der Geschlechter außerordentlich ähnlich“ (Borneman 1981: 521). In patriarchal geprägten Gesellschaftsformen gilt es als unsittlich, wenn Frauen männliche Kleidung oder Männer weibliche tragen (vgl. die Ausführungen von Bourdieu (1997 2012) zu den Bergbauern in der Kabylei).

Mit der Entdeckung des Ackerbaus und der Erfindung zahlreicher Gerätschaften, Werkzeuge und der Lagerung von Lebensmitteln konnten mehr Menschen versorgt werden als zuvor. Es bildeten sich Sippen, Schwesterund Bruderschaften sowie Stämme. Die Menschen wurden sesshaft, die Arbeit aufgeteilt und Eigenbesitz, Leistungsdenken und Vererbung wurden wichtig. Nach Borneman (1981) sind die ersten technischen Errungenschaf- 
ten weibliche Erfindungen, weil sie näher an den Aufgaben der Frauen waren:

\begin{abstract}
Diese Entdeckung [die Töpferei, Anm. BR] führte zu der Vermutung, die sich mittlerweile bestätigt hat, dass ein großer Teil der vorgeschichtlichen Werkzeuge von Frauen erfunden worden ist. Im Widerspruch zu der unter heutigen Männern herrschenden Ansicht, dass der Mann ,von Natur' größere technische Begabung als die Frau besitze und die technische Entwicklung der Menschheit deshalb allein das Werk der Männer sei, stellt es sich immer mehr heraus, dass gerade diejenigen Werkzeuge der Vorzeit, auf denen sich Jahrtausende später das industrielle Zeitalter aufbauen sollte, Erfindungen der Frau waren. (Borneman 1981: 72)
\end{abstract}

$\mathrm{Zu}$ diesen weiblichen Erfindungen gehören zum Beispiel Gerätschaften wie Grabstock, Hacke, Spinnwirtel, Spinnrad und Webstuhl oder die Kultivierung von Flachs und Baumwolle. So manche männliche Errungenschaft der industriellen Entwicklung geht auf diese Grundtechniken zurück, die Borneman (1981) den Frauen zuschreibt.

Demnach sei die Zuteilung von Arbeit zu einem bestimmten Geschlecht ein gesellschaftliches, kein natürliches, gegebenes oder zwangsläufiges Phänomen. Die Hierarchisierung der Geschlechter ist durch die Jahrtausende der Männerherrschaft und damit der Unterdrückung von Menschengruppen weiterhin fest verankert (vgl. frühere Sklavenhaltung und Leibeigenschaft oder heutige wirtschaftliche und militärische Macht westlicher Länder etc.). Sowohl die Segregation als auch die Hierarchisierung sind unter diesem Gesichtspunkt eine Konstruktion.

Diese Konstruktion ist deshalb so erfolgreich, weil zwei Gruppen an den etablierten Strukturen festhalten: diejenigen, die ihre Privilegien (Macht, Reichtum) verlieren könnten, und jene, die zwar benachteiligt sind, aber hoffen, eines Tages in der Rolle der Privilegierten aufsteigen zu können. Die Hoffnung, einmal nicht mehr zu den Unterdrückten, sondern zu den Unterdrückern zu gehören, ist stärker als die Freiheit. Simone de Beauvoir (1956) beschreibt, dass der Mechanismus der Machterhaltung sogar von der Seite der Frauen unterstützt wurde. Die Frauen hätten sich darum so lange nicht gewehrt, weil die Frau eher mit ihrem Mann solidarisch ist und so ihren Status sichern kann, als sich mit anderen Frauen zusammenzuschließen. Dieser Umstand äußerte sich beispielweise darin, dass sich in der Schweiz bürgerliche und gut situierte Frauen gegen die Einführung des Frauenstimmrechts stark machten. Vergleichbare Prozesse wurden ebenso bei der Arbeiter- und der Antiapartheidbewegung beobachtet.

Diese männliche Vorherrschaft fußt schließlich auf der spezifischen physischen Überlegenheit. Männer können zwar kurzfristig größere Kräfte mobilisieren, ermüden jedoch früher. Bei langandauernden Anstrengungen, die unter besonders schwierigen Umständen wie zum Beispiel bei Kälte stattfinden, können sich Frauen mit Männern messen. „Deshalb halten Frauen auch alle Langschwimmrekorde; unter den Ärmelkanalschwimmern befindet sich kein einziger Mann, der auch nur entfernt an die Zeiten der Frauen herange- 
kommen ist" (Borneman 1981: 514). Nicht um die Leistung dieser ersten Kanalschwimmerinnen zu schmälern, sondern der Vollständigkeit halber sei hier erwähnt, dass seit 2007 ein Mann den Geschwindigkeitsrekord innehat. Am häufigsten (43 Mal) überquerte allerdings mit der Britin Alison Streeter eine Frau schwimmend den Ärmelkanal ${ }^{16}$.

Borneman (1981) verweist einige Behauptungen der männlichen Hegemonie ins Reich der Fantasie. Das weibliche Gehirn sei proportional zur Körperlänge größer als das männliche, im Gegensatz zu der patriarchalischen Legende von der weiblichen intellektuellen Unterlegenheit. Ebenso seien Stirnlappen und Kleinhirn der Frauen im Durchschnitt größer als jene der Männer. Aufgrund des größeren Durchmessers der Halsschlagader und der Arterien sei das weibliche Gehirn besser durchblutet und deshalb leistungsfähiger. Biologische bzw. körperliche Begründungen wurden immer wieder bemüht, um die Unterschiede zwischen Frauen und Männern zu begründen. Gemäß Martschukat und Stieglitz (2005) sind zentrale Merkmale moderner hegemonialer Männlichkeit die Biologisierung von Geschlechterunterschieden sowie die heterosexuelle Dominanz. Das Konzept werde dabei einerseits von der Institution Ehe und andererseits von homosozialen Männerbünden gestützt.

Die Behauptung der ,natürlichen' Überlegenheit des Mannes über die Frau entbehrt jeglicher nachvollziehbaren Grundlage. Dennoch verfügen die Männer nach Schössler (2008) über mehr symbolisches und ökonomisches Kapital in einer Gesellschaft als Frauen. Die männliche Macht ergibt sich aus der Unterdrückung von Frauen und anderen Männern. Macht bedingt damit immer die Ohnmacht von anderen. Nach Martschukat und Stieglitz (2005: 55) speist sich ,männliche Macht [...] mithin durch die systematische Ausgrenzung weiblicher Reproduktionskapazitäten [...] entlang von vier institutionellen Achsen [...]: Zwangsheterosexualität und Vaterschaft in einem eher privaten, wissenschaftliche und staatliche Reproduktionskontrolle in einem öffentlichen Rahmen.“

Wie Borneman (1981) selbst schreibt, war das Ziel seiner akribischen Recherchetätigkeit, $\mathrm{zu}$ beweisen, wie das patriarchale Unterdrückungsregime funktioniert. So ist vieles, was von der Gesellschaft als unumstößliche Tatsache wahrgenommen wird, Teil einer Rechtfertigung für die vermeintliche Überlegenheit der Herrschenden. Personen, die sich dem Geschlechterkodex widersetzen und zum Beispiel einen geschlechtsuntypischen Beruf erlernen oder ausüben, heben sich demnach nicht nur von der aktuellen gesellschaftlichen Norm ab, sondern lehnen sich gegen eine jahrtausendalte Tradition auf. Alle Menschen müssen sich auf die eine oder andere Weise mit den Normen ihrer Gesellschaft auseinandersetzen. Im Falle von Jugendlichen, die sich in einem geschlechtsuntypischen Berufsumfeld bewegen, gilt dies verstärkt.

16 Wikipedia [http://de.wikipedia.org/wiki/Kanalschwimmen\#Offizielle_Rekorde; Zugriff: 22.03.2017]. 
Auf der Individualebene wird im nächsten Unterkapitel beschrieben, wie sich die eben vorgestellten Machtgefüge reproduzieren. Zunächst wird erklärt, wie sich die Geschlechtsidentität bei Kindern entwickelt.

\subsection{Geschlechtsidentität}

Die Voraussetzung, um am gesellschaftlichen Leben teilnehmen zu können, ist eine stabile Geschlechtsidentität (Blank-Mathieu 2001). Mit Blick auf die Lernenden in geschlechtsuntypischen Berufslehren werden in diesem Unterkapitel zuerst Definitionen präsentiert und es wird auf die Entwicklung der Geschlechtsidentität eingegangen, bevor einschlägige Erklärungsansätze aus dem Bereich der Kognitions- und der Lerntheorie für die Entwicklung der Geschlechtsidentität vorgestellt werden. In Abgrenzung zu anderen Erklärungsansätzen wie etwa dem biologischen Erklärungsansatz, der gemäß Trautner (2008) eher auf der Unterschiedlichkeit der Geschlechter als auf Unterschieden innerhalb der Geschlechter beruht, fokussieren die ausgewählten Ansätze auf das Lernen im sozialen Kontext.

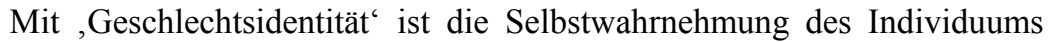
als weiblich oder männlich gemeint (Hartmann/Trautner 2009). Eine der ersten Definitionen geht auf Money zurück (vgl. Unterkapitel 2.1 zum Thema Gender): Geschlechtsidentität ist ,die überdauernde Erfahrung der eigenen Individualität, des eigenen Verhaltens und der eigenen Erlebnisweisen als eindeutig und uneingeschränkte männlich, als eindeutig und uneingeschränkt weiblich oder als in größerem bzw. kleinerem Grad ambivalent" (Money/Ehrhardt 1975: 16). Während ältere Theorien von einer eindimensionalen Eigenschaft mit zwei Ausprägungen, maskulin vs. feminin, ausgehen (vgl. Bem 1974), setzen sich heute mehrdimensionale Ansätze von Geschlechtsidentität durch (Egan/Perry 2001, Hartmann/Trautner 2009). Beispielsweise hat die Geschlechtsidentität nach Tobin et al. (2010) fünf Dimensionen: 1) Wissen um die eigene Gruppenzugehörigkeit (,Ich bin ein Mädchen bzw. ein Junge'); 2) Geschlechtszentralität verstanden als die Wichtigkeit von Geschlecht gegenüber anderen Identitäten wie etwa ethnischer Identität; 3) Geschlechtszufriedenheit (insbesondere die Wahrnehmung von untypischen Persönlichkeitseigenschaften, Interessen oder sexueller Orientierung bei sich selbst geht vermehrt einher mit der Unzufriedenheit mit dem eigenen Geschlecht); 4) gefühlter Druck zur Geschlechtskonformität (wahrgenommener Druck, Geschlechterstereotypen zu entsprechen) und 5) gefühlte geschlechtliche Passung (wahrgenommene Übereinstimmung mit gleichgeschlechtlichen anderen). In einer Studie von Hartmann und Trautner (2009) erhärtete sich, dass die Jungen im Vergleich zu den Mädchen einerseits zufriedener mit ihrem Geschlecht sind, andererseits von mehr Anpassungsdruck berichten 
und sich selbst geschlechtstypischer einstufen. Jungen setzen sich dabei oft selbst unter Druck, berichten jedoch zusätzlich über mehr Druck von ihrer Peergruppe.

Die Entwicklung der Geschlechtsidentität beginnt im Kindesalter (Maccoby 1998, Ruble/Martin 1998) und bekommt mit Eintritt in die Pubertät einen zusätzlichen Schub. Sie verläuft vielschichtig und basiert auf biologischen, sozialen und kognitiven Voraussetzungen (Lohaus/Vierhaus 2015). Als Basis gilt die Unterscheidung von weiblich und männlich konnotierten Eigenschaften. Dies beginnt schon früh: Bereits wenige Monate nach der Geburt können Säuglinge weibliche und männliche Stimmen unterscheiden (Bischof-Köhler 2004). Im 1. Lebensjahr bildet sich eine Präferenz für das eigene Geschlecht. Allerdings können Kinder ihre Geschlechtszugehörigkeit erst mit zweieinhalb bis drei Jahren zuverlässig auf Nachfragen angeben (vgl. Trautner 2008). Zwischen dem 3. und dem 6. Lebensjahr lernen Kinder, dass das Geschlecht eine unveränderliche Eigenschaft aller Menschen ist, sie bauen Geschlechterstereotype auf und haben vielfältige Assoziationen zu Weiblichkeit und Männlichkeit (Perry/Pauletti 2011). In diesem Alter verbinden Kinder bestimmte metaphorische Eigenschaften mit den beiden Geschlechtern, obwohl es dafür keine objektiven Belege gibt. So werden Gegenstände, die groß, dunkel, spitz oder rau sind, Jungen zugeordnet. Beispielsweise bringen Kinder Stofftiere mit langen und gefährlich aussehenden Zähnen wie Haie oder Gorillas eher mit Männlichkeit in Verbindung und im Gegensatz dazu weiche, glatte, abgerundete, kleine oder zerbrechlich aussehende Objekte wie Schmetterlinge mit Weiblichkeit (Leinbach et al. 1997). Sogar einen Gesichtsausdruck nehmen Kinder geschlechtlich konnotiert wahr: eine zornige Mimik als männlich und eine fröhliche als weiblich (Leinbach et al. 1997).

In der Adoleszenz gewinnt durch stärkere Beschäftigung mit dem Selbst die Geschlechtsidentität an Wichtigkeit und Vielschichtigkeit (Trautner 1996). Am Anfang steht die Akzeptanz des eigenen geschlechterkonnotierten Körpers. Was so selbstverständlich klingt, führt häufig zu Verunsicherung bezüglich der Wahrnehmung durch die anderen und zu erstaunlichen Deklarationshandlungen sowie mehr oder weniger theatralischen Inszenierungen von Geschlecht (Flammer/Alsaker 2002). Die Entwicklungsaufgabe ,Aufbau einer sexuellen Orientierung ' wird besonders dann zur kritischen Herausforderung, wenn nicht der breit vorgespurten Heteronormativität entsprochen werden kann. Außerdem gilt es für die jungen Erwachsenen nach der Pubertät eine Reihe von Entwicklungsaufgaben erfolgreich zu bewältigen (Trautner 1996). Für beide Geschlechter unabhängig von der sexuellen Orientierung bergen das Eingehen neuer Beziehung zu Gleichaltrigen, die Auseinandersetzung mit den gesellschaftlich vorgegebenen Geschlechterrollen und die Ausbildung schulischer und beruflicher Interessen weitere Entwicklungsaufgaben. Zusammengefasst können diese als Vorbereitung auf die üblicherweise 
geschlechtstypisch verteilten familiären und beruflichen Rollen beschrieben werden ${ }^{17}$.

Gemäß Banduras (1986) sozialer Kognitionstheorie ist das frühe kindliche Lernen ein hoch komplexer Prozess mit vielen Faktoren. Drei Einflussbereiche spielen dabei eine wichtige Rolle: Verhalten (Aktivitätsmuster), Person (Erwartungen, Absichten, Ziele) und Umgebung (Verstärkungen). Die soziale Wahrnehmung beeinflusst das Verhalten, wobei das Kind durch das beobachtende Lernen eine aktive Rolle zugeschrieben bekommt. Es richtet seine Aufmerksamkeit auf bestimmte Ereignisse oder Personen, ordnet, kombiniert und imitiert das beobachtete Verhalten, entscheidet, wann es dieses Verhalten zeigt, und erfasst die Wirkung des neuen Verhaltens. An der Entwicklung der Geschlechtsidentität sind individuelle kognitive Prozesse beteiligt, die soziale Umwelteindrücke verarbeiten und eine wichtige Rolle in der jeweiligen Ausgestaltung der Geschlechtsidentität spielen. Das Kind übernimmt nicht einfach stereotype Informationen von außen. Stereotype entstehen nicht ausschließlich aufgrund stereotyper Verhaltensweisen von Eltern und der Umgebung, die übernommen werden, sondern auch in der Auseinandersetzung mit den Eindrücken aus der Erwachsenenwelt. Diese nimmt Einfluss in Form von Kleidung, Spielzeug, Erwartungen, Sprache etc. und mittels Verstärken von geschlechtskonformem sowie Ignorieren bzw. Sanktionieren von geschlechtsuntypischem Verhalten.

Das Selbstkonzept des Kindes, sich selbst als weiblich bzw. männlich zu erkennen, ermöglicht es dem Kind erst, Geschlechterrollen zu entdecken und zu bestätigen. Ein Mädchen, das sich als Mädchen erkennt, möchte Mädchensachen machen. Entsprechendes gilt für Jungen. Es handelt sich demnach um Selbstsozialisation (vgl. Kohlberg 1966). Obwohl es in der Umgebung genug Informationen gibt, also die Bandbreite an Geschlechtsrollen vorhanden ist, suchen sich die Kinder die für sie relevanten Aktivitäten aus, organisieren die notwenigen Informationen und verhalten sich entsprechend der neu gewonnenen Informationsstruktur. Dieser Erklärungsansatz geht über ein rein durch Verstärkung oder Nachmachen provoziertes geschlechtertypisches Verhalten hinaus.

In Anlehnung an Piagets kognitive Entwicklungsstadien postulierte Kohlberg (1966) seine Theorie der Entwicklung der Geschlechtsidentität. Entsprechen der kognitiven Entwicklung unterteilte er die Entwicklung in drei Stadien. Die drei Stadien werden ohne Auslassung in dieser Reihenfolge durchlaufen. Das Alter kann stark variieren.

1. Stadium Kennzeichnung $(2 ; 4-5 ; 2)$ : Mittels wahrgenommenen Ähnlichkeiten von Charakteristiken mit sich selbst und geschlechtstypischen Merkmalen anderer können Kinder sich und andere als Frauen/Mädchen und Männer/Jungen identifizieren, wobei Geschlecht noch nicht als stabil angese- 
hen wird. Für Kinder in diesem Alter sind Haarlänge und Kleider für die Zuteilung relevant, nicht die primären und sekundären Geschlechtsmerkmale.

2. Stadium Stabilität $(3 ; 0-5 ; 8)$ : Kinder erkennen, dass das Geschlecht sich stabil entwickelt und Mädchen zu Frauen heranwachsen, Jungen zu Männern. Dabei wird trotzdem noch angenommen, dass Geschlecht aufgrund der oberflächlichen Erscheinung oder der Wahl einer bestimmten Aktivität attestiert wird und deshalb veränderlich ist. Je stärker die Kinder die Stabilität des Geschlechts wahrnehmen können, desto stärker wird ihre Suche nach geschlechtsbezogenen Informationen. Konforme Informationen werden positiv bewertet. Die Übereinstimmung mit geschlechtsangemessenem Verhalten wird zur treibenden Kraft der Selbstregulation (vgl. Trautner 2008).

3. Stadium Konsistenz (3;5-5;7): Kinder erkennen, dass das Geschlecht über die Zeit und über Situationsbedingungen konstant bleibt. Die Kinder identifizieren sich damit selektiv mit gleichgeschlechtlichen Modellen und imitieren diese stärker als gegengeschlechtliche (Trautner 2008). Das Erreichen des dritten Stadiums ermöglicht es dem Kind, sich in der nach Geschlecht segregierten Umwelt geschlechtskonform zu verhalten. „Child's gender identity can provide a stable organizer of the child's psychosexual attitudes only when he is categorically certain of its unchangeability" (Kohlberg 1966: 95).

Gemäß Bussey und Bandura (1999) spielen Kinder, bevor sie Geschlechterkonsistenz erreichen, lieber mit Spielsachen, die traditionell mit ihrem Geschlecht in Verbindung gebracht werden. Zudem ahmen sie eher gleichgeschlechtliche Modelle nach und drängen Gleichaltrige dazu, sich geschlechtstypisch zu verhalten. Eine Schlüsselrolle für die frühen Kognitionsprozesse ist die Fähigkeit der Kinder, sich als Mädchen oder Junge zu bezeichnen (Martin et al. 2002). Das Auftreten von Stereotypen in der Kindheit ist damit nicht eine einseitige Folge aus den Einflüssen der sozialen Umwelt, sondern eher eine Konsequenz der kindlichen Informationsprozesse. Stereotype auszubilden ist so ein effizienter Weg, mit einer großen Informationsmenge umzugehen und Voraussagen für Verhalten zu treffen.

Bosinski (2000) versteht die Entwicklung der Geschlechtsidentität als einen durch interagierende biologische, innerpsychische und soziokulturelle Faktoren bestimmten Entwicklungsprozess. Dieser Prozess nimmt im frühen Kindesalter seinen Anfang und wird nie ganz vollendet. Mit dem Ende der Adoleszenz kommt er jedoch zu einem relativen Abschluss. Die drei Faktoren ergänzen und bedingen sich gegenseitig, das heißt sie schließen sich nicht aus. Pränatal interagieren Natur und Kultur in Form des stammesgeschichtlich geprägten, genetischen Bauplans und der Bedingungen im Mutterleib. Nach der Geburt prägen Kinder mit ihrem Temperament die Reaktionen ihrer Umwelt, vor allem die der Eltern und naher Bezugspersonen. Die Kultur mit ihren gesellschaftlichen Stereotypen wirkt durch die Eltern und ihre Erwartungen erheblich darauf ein, wie sich ein Mädchen bzw. ein Junge zu verhal- 
ten hat: „Das Kind organisiert seine Umwelt und wird zugleich von ihr organisiert" (Bosinski 2000: 132). Die Entwicklung zur Frau bzw. zum Mann lässt sich nicht durch einzelne Faktoren erklären, sondern durch mehrere Einflussfaktoren, die aufeinander wirken und in einem zeitlichen Verlauf stehen. Je stärker ein Faktor in diesem komplexen biopsychosozialen Bedingungsgefüge ist, desto größer ist seine Auswirkung (vgl. Bosinski 2000).

Wie in Abschnitt 2.2.2 ,Gene und Sexualhormone' dargelegt, wird die Entwicklung der Geschlechtsidentität von den körperlichen Einflüssen gesteuert. Die genetischen Anlagen ermöglichen einerseits die Kontaktaufnahme mit der Umwelt, sind jedoch von ihr abhängig. Umwelteinflüsse stimulieren oder hindern bestimmte körperliche Abläufe. Neugeborene kommen demnach nicht mit einem vollendeten Entwicklungsplan zur Welt. Sie lernen aufgrund von Wahrnehmungen, die nicht nur wichtig, sondern - in Form von körperlichem Kontakt und emotionaler Zuwendung - sogar überlebenswichtig sind (Bauer 2010). Ein besonders gut untersuchbares und vermutlich entscheidendes Prinzip von Aneignung ist das Beobachten. Bandura (1977b) nannte es Lernen am Modell.

Eine behavioristische Erklärungsebene bezieht sich auf die Bekräftigungstheorie. Dabei wird davon ausgegangen, dass das Umfeld, hier vor allem nahe Bezugspersonen wie Eltern, Geschwister, Großeltern usw., erwünschtes Verhalten bekräftigen und unerwünschtes Verhalten entweder ignorieren oder tadeln. Ein Mädchen also, das sich trotz Herumtollens im Freien nicht schmutzig gemacht hat, erhält positive Beachtung, ebenso wie der Junge, der mit schmutzigen Kleidern zurückkommt. Bei rollenkonträrem Verhalten können sich Erwachsene kaum verkneifen, diese Kinder zum Beispiel als ,Ruäch' (Dialektwort, im engeren Sinn ,rohe Person', in diesem Zusammenhang für wilde Mädchen verwendet) bzw. ,Finöggeli‘ (Dialektwort, im engeren Sinn ,feines, zartes Mädchen', hier für sensible Jungen gebraucht) zu betiteln. Differenzielle Erwartungen provozieren differenzielle Reaktionen (Trautner 2008).

Zur Imitationstheorie (Trautner 1996) gehören drei Teilthesen: 1) Für Kinder gibt es mehr gleichgeschlechtliche Beobachtungen als gegengeschlechtliche (Differenzielle Beobachtungshäufigkeit), da oft in gleichgeschlechtlichen Spielgruppen agiert wird, 2) gleichgeschlechtliche Modelle werden häufiger imitiert (Selektive Nachahmung), 3) der gleichgeschlechtliche Elternteil ist das bevorzugt nachgeahmte Modell (Elternidentifikation). Doch alle drei Teilthesen lassen sich kaum bestätigen, und es gibt Hinweise, die das Gegenteil oder ein ausgeglichenes Geschlechterverhältnis vermuten lassen (vgl. Trautner 2008). Insgesamt erscheint bei der Entwicklung der Geschlechtsidentität fraglich, ob die Richtung des Zusammenhangs immer so eindeutig ist. Der Einfluss der Person selbst - im Sinn von Temperament, Kognition, Verarbeitungskapazität, Selbstsozialisation und anderen individu- 
ellen Voraussetzungen -, der Gleichaltrigen sowie der vorgefundenen materiellen Verhältnisse ist nicht geklärt (vgl. Trautner 2008).

Zusammenfassend lässt sich festhalten, dass die Geschlechtsidentität sich aus wechselseitigen biologischen, sozialen und kognitiven Beeinflussungen entwickelt. Dabei ist das Individuum stark durch die eigene bewusste und unbewusste Mitgestaltung beteiligt. Dies verdeutlicht sich besonders gut in den fünf Dimensionen der Geschlechtsidentität nach Tobin et al. (2010). Ergiebig für die Betrachtung der Situation von Jugendlichen in geschlechtsuntypischen Berufslehren sind vor allem die Dimensionen Geschlechtszufriedenheit, gefühlter Druck und gefühlte geschlechtliche Passung (vgl. Hartmann/Trautner 2009). Unter Umständen kann sich die Zufriedenheit mit dem eigenen Geschlecht aufgrund der Wahrnehmung gegengeschlechtlicher Interessen oder Fähigkeiten verschlechtern. Nach Tobin et al. (2010) sind dabei die individuelle Wahrnehmung und die Gewichtung von erlebtem Anpassungsdruck zentral. Individuen können durchaus unterschiedlich auf gleiche Ausgangsbedingungen reagieren. Während es einige zu Höchstleistungen anspornt, sich gegen gesellschaftliche Beeinflussungsversuche zu stemmen, neigen andere dazu, negative Reaktionen vorwegnehmend und entgegen ihren eigenen Interessen sich geschlechtskonform zu verhalten. Diese individuellen Unterschiede weisen auf eine weitere wichtige Bedingung von Geschlecht hin, nämlich auf die Sichtweise, dass Geschlecht nicht nur biologisch determiniert ist, sondern ein bedeutender Teil der Auswirkungen dieser Biologie sich als soziales Konstrukt erweist.

\subsection{Geschlecht als soziale Konstruktion}

Im Alltag gelingt die Zuordnung von Menschen zu einer der beiden Kategorien ,weiblich 'bzw. ,männlich“ so schnell und mühelos, dass die hierfür notwenigen Voraussetzungen selten bewusst sind, ja nicht einmal als Voraussetzungen erkannt werden. Der Mechanismus läuft so reibungslos und automatisch, dass er naturgegeben und eindeutig erscheint. Das Alltagswissen über die Zweigeschlechtlichkeit beruht nach Wetterer (2010) auf folgenden Pfeilern: 1) Es gibt zwei und nur zwei Geschlechter. 2) Man gehört entweder dem einen oder dem anderen Geschlecht an. 3) Mit der Geburt bzw. schon vorher ist das Geschlecht festgelegt. 4) Das Geschlecht ist nicht veränderbar. 5) Aufgrund der Genitalien ist das Geschlecht eindeutig bestimmbar. Entgegen dieser verfestigten Annahme ist die Variationsbreite auf der körperlichen Ebene bedeutend und die Einteilung in weiblich oder männlich keineswegs eindeutig. Die Ausprägungen der Eigenschaft deuten auf ein Kontinuum mit zwei Polen hin. Hagemann-White (1988) geht davon aus, dass die Zweigeschlechtlichkeit und damit das Geschlecht keineswegs notwendig oder natur- 
haft vorgeschrieben sind, sondern sozialgeteilte kulturelle Konstruktionen sind. Diese Erkenntnis, dass das Geschlecht uns ausschließlich binär erscheint und wir die Welt durch diese Brille betrachten, ist ebenso eine zentrale Aussage bei Wittig (1981):

\begin{abstract}
Das Geschlecht wird als ,unmittelbar Gegebenes', als ,sinnlich Gegebenes', als ,physikalische Merkmale‘ aufgefasst, die zu einer natürlichen Ordnung gehören. Aber was wir für einen physische, unmittelbare Wahrnehmung halten, ist nur eine kulturell erzeugte, raffinierte und mythische Konstruktion, eine ,imaginäre Formation', die die physikalischen Merkmale (die an sich ebenso neutral sind wie andere, aber durch ein Gesellschaftssystem markiert werden) durch das Netz der Beziehungen reinterpretiert, in dem sie wahrgenommen werden. (Wittig 1981: 48, in Butler 1991: 170)
\end{abstract}

Wenn das Geschlecht als soziales Konstrukt gedacht wird, heißt dies keineswegs, dass damit Geschlecht beliebig wandelbar wird. Es bedeutet vielmehr, dass die innere Repräsentation von Geschlecht auf Alltagswissen aufbaut, immer wieder neu hergestellt und von anderen bestätigt werden muss. „Man hat ein Geschlecht erst dann, wenn man es für andere hat" (Hirschauer 1993: 53). Dabei wird der soziale Prozess ,Doing Gender" wichtig, der das Geschlecht als folgenreiche Polarisierung hervorbringt und die tradierten Strukturen reproduziert (vgl. Gildemeister 2010, West/Zimmermann 1987). Doing Gender oder ,Geschlecht herstellen' meint, dass Personen in Aussagen und Handlungen einerseits ihr eigenes Geschlecht betonen und darstellen und andererseits das Geschlecht des Gegenübers wahrnehmen und spiegeln (Gildemeister 2010). Geschlecht ist demnach nicht einfach da, sondern es wird vielschichtig präsentiert und repräsentiert. Nach Wetterer (2002) wird Geschlechtszugehörigkeit in einem reflexiven Interaktionsprozess zugleich vorausgesetzt und hervorgebracht, indem Personen ihr eigenes Geschlecht darstellen und dasjenige des Gegenübers anerkennen: „Jede(r) ist nicht nur dafür verantwortlich, ein Geschlecht zu ,haben' (und es zu ,zeigen'), sondern auch dafür, den anderen ihr Geschlecht zu ,geben', deren Geschlechtszugehörigkeit zu validieren“ (Wetterer 2002: 120). Da viele Berufe eine geschlechtliche Konnotation aufweisen, werden sie mitunter zum Katalysator von Doing Gender. Sie unterstützen Wahrnehmung und Darstellung im Fall von Kongruenz zwischen dem Geschlecht der Person und dem des Berufs.

Frauen und Männer müssen sich unterscheiden, weshalb sie nicht die gleichen Tätigkeiten ausführen dürfen. Rubin (1975) nennt dieses Phänomen ,Gleichheitstabu'. Nach ihr dürfen Frauen und Männer tun, was ihnen beliebt, solange es nicht etwas ist, was das andere Geschlecht tut. In den meisten von ihr untersuchten Gesellschaften bestehen Tabus, wonach Tätigkeiten, Bereiche oder Kleider dem einen oder dem anderen Geschlecht vorbehalten sind. Das Tabu wirkt sich ebenso auf die beruflichen Tätigkeiten aus. Durch die Segregation der Berufswelt wird das Gleichheitstabu gestärkt, was wiederum dazu führt, dass die Segregation erhalten bleibt. Mittels des Gleichheitstabus manifestieren sich die biologischen Differenzen zwischen 
den Geschlechtern und reichen darüber hinaus. Abgesehen von den geschlechtsspezifischen Merkmalen unterscheiden sich Frauen und Männer nicht wesentlich voneinander. Rubin (1975) karikiert die von der Gesellschaft inszenierte Dualität der geschlechtlichen Unterschiede:

Men and women are, of course, different. But they are not as different as day and night, earth and sky, yin and yang, life and death. In fact, from the standpoint of nature, men and women are closer to each other than either is to anything else - for instance, mountains, kangaroos, or coconut palms. (Rubin 1975: 179)

Mit dem Gleichheitstabu verstärken sich die Verschiedenheiten von Frau und Mann, und zusätzlich reduzieren sich Ungleichheiten (vgl. Wetterer 2002). Mit der Geschlechtertrennung werden sowohl Persönlichkeitszüge von allen - Frauen und Männern - unterdrückt als auch die typischen Verhaltensweisen erlernt (vgl. Doing Gender). Alle müssen sich den Rollenerwartungen fügen, die von der Gesellschaft als Ganzes und durch ihre Agenten (also alle) eingefordert werden (vgl. Rubin 1975). Die Segregation in der Arbeitswelt ermöglicht es, die biologischen Differenzen zwischen den Geschlechtern zu vergröBern und in zwei vermeintlich exklusive Kategorien zu teilen. So findet denn berufliche Sozialisation in einer segregierten Arbeitswelt statt.

Zur Sozialisation im konstruktivistischen Sinn gehören nach Paseka (2008) drei Wirkebenen: 1) das Verständnis der strukturellen Rahmenbedingungen (soziale Erwartungen und Ziele sowie materielle Ausstattung), 2) die aktive Eigenleistung des Subjekts und 3) interaktive Prozesse (Aneignungs-, Internalisierungs- und Inkorporierungsprozess). Im Verlauf ihrer Entwicklung werden Kinder zu Mädchen und Jungen sozialisiert. Nach Paseka (2008) lernen Kinder im gesellschaftlichen Kontext folgende Normen in Bezug auf das Geschlecht: die Norm der Zweigeschlechtlichkeit, die Norm der Eindeutigkeit, die Norm der Unveränderbarkeit, die Norm der Naturhaftigkeit und die Norm der Heterosexualität. Zudem wird den Kindern schon früh bewusst, dass die Achtung der beiden Geschlechter nicht gleich ist, sondern dass eine Asymmetrie besteht, besonders wenn gesellschaftlichen Konventionen nicht Folge geleistet wird. Dabei werden Mädchen und Jungen durchaus anders behandelt. Wenn Mädchen sich wie Jungen kleiden, mit männlich konnotiertem Spielzeug spielen oder einen männlichen Beruf erlernen wollen, erzeugt dies in dieser Entwicklungsphase Wohlwollen. Jungen, die weiblich konnotiertes Verhalten zeigen, werden hingegen eher eingeschränkt oder sanktioniert (vgl. Epstein 1992). Im Allgemeinen scheint es, als stünden die Männer unter einem größeren Identitätszwang als Frauen (Knapp 1995). Der Verlust der Anerkennung wird durch die unterstellte Verweiblichung erreicht (vgl. Schössler 2008).

Die Geschlechtszugehörigkeit und die Zweigeschlechtlichkeitsnorm (oder Zweigeschlechterordnung) sind nach Wetterer (2010: 126) so stark mit der menschlichen Alltagswahrnehmung verwoben, dass sie uns als ,fraglose und nicht weiter begründungsbedürftige Selbstverständlichkeiten“" erscheinen. 
Die Zweigeschlechtlichkeitsnorm ist eine starke soziale Norm, die uns weitgehend unbewusst ist. Wetterer (2010) lehnt sich dabei an Hagemann-White (1984) an und formuliert die Zweigeschlechtlichkeitsnorm folgendermaßen:

Dass es zwei und nur zwei Geschlechter gibt; dass jeder Mensch entweder das eine oder das andere Geschlecht hat; dass die Geschlechtszugehörigkeit von Geburt an feststeht und sich weder verändert noch verschwindet; dass sie anband der Genitalien zweifelsfrei erkannt werden kann und deshalb ein natürlicher, biologisch eindeutig bestimmbarer Tatbestand ist, auf den wir keinen Einfluss haben - all das sind Basisregeln unserer ,Alltagstheorie der Zweigeschlechtlichkeit‘ (Hagemann-White 1984), die ebenso unbezweifelbar richtig scheinen wie die Annahme, dass dies zu allen Zeiten so war und auch in anderen Kulturen nicht anders ist. (Wetterer 2010: 126)

Hier spiegelt sich die rigide Vorstellung von Kindern in der Phase der Geschlechtsidentitätsentwicklung wider. Kinder wissen mit etwa 2-3 Jahren, dass es zwei Geschlechter gibt und dass sie einem davon angehören (Geschlechtsidentität), dass aus Mädchen Frauen und aus Jungen Männer werden (Geschlechtsstabilität) und dass äußere Veränderungen wie Kleider oder Haarlänge das Geschlecht nicht ändern (Geschlechtskonstanz; vgl. Kohlberg 1966; Unterkapitel 2.4). Bis etwa zum achten Lebensjahr sind die stereotypen Zuschreibungen rigid und erlauben wenig Spielraum. Mit der Zeit weichen sich die Geschlechtsrollenerwartungen auf. Sie werden differenzierter und weniger stereotyp. Dennoch bleiben die Geschlechtsrollenerwartungen im Erwachsenenalter bestehen, obschon - meist aufgrund bewusster Kognitionen - den Individuen mehr Freiraum zugestanden wird (Lohaus/Vierhaus 2015). Beispielsweise dürfen Männer Emotionen wie Trauer ausdrücken, oder Frauen wird zugestanden, harte Entscheidungen in ihrer Führungsrolle zu treffen.

Die Ungleichheit zwischen den Geschlechtern (Geschlechterhierarchie) ist heute etwas weniger stark als früher. „Die heutigen Frauen sind in weit höherem Maße den Männern gleichgestellt, als es deren Mütter und Großmütter waren. Die Diskriminierung der Frauen dauert indes an" (Wobbe/Nunner-Winkler 2007: 301). Nach wie vor stellen Männer in den meisten privaten und öffentlichen Gremien die Mehrheit, besetzen die einflussreicheren Posten, verdienen mehr und bilden beinahe gänzlich ohne Frauen das staatliche Gewaltmonopol von Militär und Polizei usw. (vgl. Wobbe/Nummer-Winkler 2007).

Eine geschlechtsuntypische berufliche Tätigkeit stellt damit eine Abweichung von den üblichen Erwartungen dar und damit nach Rubin (1975) eine Übertretung des Gleichheitstabus. Die Konstruktion und Rekonstruktion von Geschlecht läuft nicht mehr von selbst. Das System erfährt eine Irritation, es kommt zu Geschlechtsrollenkonflikten. Im folgenden Unterkapitel werden diese Konflikte anhand von Geschlechterstereotypen und Geschlechtsrollenerwartungen beschrieben. 


\subsection{Geschlechterstereotype und Geschlechtsrollen}

,Geschlechterstereotype“ sind kognitive Strukturen, die sozial geteiltes Wissen über Fähigkeiten, Wesensmerkmale und Verhaltensweisen von Menschen enthalten, die damit als weiblich bzw. männlich wahrgenommen werden (Eckes 1997 2008). „Geschlechterstereotype sind Erwartungen oder Überzeugungen darüber, welche Merkmale weibliche und männliche Personen wahrscheinlich besitzen“ (Hannover 2006: 467). Dazu gehören soziale Rollen und typische Personenmerkmale der beiden Geschlechter. Insbesondere einflussreich sind in der Berufslehre all jene stereotypisierten Zuschreibungen, die mit berufskompetenzenrelevanten Fähigkeiten und Fertigkeiten zu tun haben. Diese werden Frauen bzw. Männern zu- bzw. abgesprochenen, wie technisches Verständnis oder Einfühlsamkeit. Geschlechterstereotype haben beschreibende und zuschreibende Anteile. „Gender stereotypes are people's belief about how the sexes differ (descriptive stereotypes) or should differ (prescriptive stereotypes)“ (Perry/Pauletti 2011: 67). Stereotype beeinflussen somit zahlreiche psychologische Funktionen, einschließlich Aufmerksamkeit, Gedächtnis, Wahrnehmung, Interesse, Werte, Sozialverhalten, Reaktionen auf das eigene Verhalten und das von anderen sowie wahrgenommenen Kompetenzen (Ruble et al. 2006). Zu den beschreibenden Anteilen von Geschlechterstereotypen gehören tradierte Annahmen über das Wesen von Frauen und Männern, ihre Eigenschaften und Verhaltensweisen. Nach Eckes (1997) werden die Konzepte ,Wärme' und ,Expressivität ${ }^{`}$ eher Frauen als Männern zugeschrieben sowie ,Kompetenz' und ,Instrumentalität ${ }^{\text {‘ }}$ eher Männern als Frauen. Werden Frauen nicht als verständnisvoll und emotional bzw. Männer nicht als dominant und zielstrebig wahrgenommen, reagiert das Umfeld oft überrascht. Werden hingegen statt der deskriptiven die präskriptiven Annahmen (wie Frauen und Männer sein sollen) verletzt, ,resultiert in der Regel Ablehnung und Bestrafung“ (Eckes 2008: 178). Lernende in geschlechtsuntypischen Berufslehren sind somit besonders gefährdet, auf negative Reaktionen zu treffen. Die Irritationen, die die negativen Reaktionen auslösen, scheinen fortzudauern, denn nach Prentice und Carranza (2003) sind Veränderungen von Geschlechterstereotypen durch ihre Verletzung kaum zu erwarten.

Stereotypisierungen wie im vorherigen Abschnitt beschrieben können aufgrund einer illusorischen Korrelation entstehen (Hamilton/Grifford 1976). Diese illusorische Korrelation geht davon aus, dass seltene Ereignisse hoch salient sind und die Aufmerksamkeit auf sich ziehen. So entsteht eine Korrelation zwischen dem ungewohnten Verhalten und der Minderheit. „Gepaarte Distinktheit von negativen Eigenschaften und Mitgliedern kleiner Gruppen lässt den Eindruck entstehen, dass negative Eigenschaften häufiger bei Minoritäts- als bei Majoritätsmitgliedern zu finden sind“ (Erb/Bohner 2006: 496). 
In der Situation von Lernenden in geschlechtsuntypischen Berufslehren heißt das überspitzt dargestellt: Frauen sind körperlich nicht stark genug, und Männern fehlt es an Empathie.

Verhaltenserwartungen, nach denen sich Individuen aufgrund des sozial zugeschriebenen Geschlechts zu richten haben, nennt man ,Geschlechtsrollen'. Obwohl Lempert (2002) ebenso beschreibt, dass Verletzungen von normativen Rollenerwartungen - gerade mit Blick auf Lernende und Erwerbstätige - zu Sanktionen unterschiedlicher Härte führen können, hält er die Möglichkeit für wahrscheinlich, dass Rollenkonflikte Neudefinitionen auslösen und ein Antrieb zur moralischen Weiterentwicklung sein können. Dies steht im Gegensatz zu den Veränderungen bei Geschlechterstereotypen (vgl. Prentice/Carranza 2003). Dass Lempert (2002) die Reaktionen aufgrund von Überschreitungen von Geschlechtsrollenerwartungen als alles andere als harmlos einstuft, ergibt sich aus seiner Beschreibung der Sanktionen. Sie reichen „,vom leichten Stirnrunzeln bis hin zu juristischen Strafmaßnahmen, ja sogar, wenn sich die Gesellschaft zu Gewalttaten hinreißen lässt, Lynchjustiz" (Lempert 2002: 185).

Nach der sozialen Geschlechtsrollentheorie von Eagly (1987) werden Frauen mit expressiven (hilfsbereit, sensibel, unterstützend und mitfühlend) und Männer mit instrumentellen (dominant, durchsetzungsfähig, abenteuerlustig und mutig) Eigenschaften beschrieben. In gleichen sozialen Rollen aber werden Frauen und Männer als ähnlich in ihren Persönlichkeitseigenschaften beurteilt. Das heißt geschlechtsstereotype Persönlichkeitsbeurteilung entstehen aufgrund unterschiedlicher sozialer Rollen (Berufs-, Familienrollen). Geschlechtsrollen haben durchdringende Effekte, weil das Geschlecht eine starke Kategorisierungsform darstellt und weil Stereotype über Frauen und Männer so leicht und automatisch aktiviert werden, vor allem im Vergleich mit anderen Stereotypen wie Rasse, Alter und Beruf (Eagly/Karau 2002).

In den Augen von Kindern haben Geschlechterrollen den Charakter eines Naturgesetzes oder eines moralischen Prinzips. Vorschulkinder glauben fest daran, dass sich Personen an die Geschlechterrolle halten sollten, und reagieren rigide auf Verstöße (Perry/Pauletti 2011). Mit 7-11 Jahren nimmt die Rigidität gegenüber den Erwartungen an die Verhaltensweise bezogen auf das Geschlecht ab. Es entwickelt sich ein Verständnis für Diversität und Toleranz bezüglich Geschlechtsrollenerwartungen, insbesondere gegenüber weiblichen (Trautner et al. 1988). Jedoch bleiben die Vorstellungen vor allem gegenüber Erwachsenenrollen, maskulinen Attributen und Aktivitäten stark stereotypisiert. Während Freiheiten für das eigene individuelle Geschlechtsrollenbild zunehmen, verharren gerade Spielsachen und Berufsrollen in klischierten Rollenbildern. Dass die Geschlechterrollen auf sozialen Konventionen beruhen, ist jedoch sogar vielen Erwachsenen kaum bewusst. 
In einer Langzeitstudie mit 12'000 Teilnehmenden über die ganzen USA untersuchten Judge und Livingston (2008) Geschlechtsrollenorientierungen. Das Ziel bestand darin, die Unterschiede beim Einkommen zwischen Frauen und Männern zu erklären. Die Resultate belegen, dass Männer, die eine traditionellere Geschlechtsrollenorientierung haben, gegenüber Männern mit egalitärerer Geschlechtsrollenorientierung mehr verdienen. Doch sogar diese Männer haben immer noch ein höheres Einkommen als Frauen. Die egalitär eingestellten Frauen verdienen nur leicht besser als Frauen mit traditioneller Geschlechtsrollenorientierung. Die Resultate weisen zusätzlich auf eine Veränderung der Geschlechtsrollenorientierung zu mehr egalitärer Orientierung hin. "The results showed that men's gender role orientations have changed more rapidly over time than have women's" (Judge/Livingston 2008: 1008). Dennoch haben Verheiratete eine konservativere Orientierung. Personen mit höherer Intelligenz und Ausbildung denken hingegen egalitärer.

Treten Abweichungen zwischen der eigenen Wahrnehmung und den Erwartungen anderer auf, etwa im beruflichen Umfeld, können Geschlechtsrollenkonflikte entstehen. Gemäß der Theorie der Geschlechtsrollenkonflikte können Restriktionen der Geschlechtsrollen die psychische Gesundheit beeinträchtigen (O’Neil 2008). Weiter können sich Geschlechtsrollenkonflikte auf das Verhalten, die Leistungen und die Motivation negativ auswirken. Geschlechtsrollenkonflikte im Beruf können die Zufriedenheit gefährden, den Wunsch zum Berufswechsel sowie die Absenzen erhöhen. Frauen empfinden Geschlechtsrollenkonflikte stärker als Männer (Rustemeyer/Thrien 2001).

Was weiblich und was männlich ist, ist eine normative Setzung. Den Berufen wird ein Geschlecht zugeschrieben, das im Alltag als gegeben erscheint, einer näheren Betrachtung jedoch nicht standhält. Berufe und Geschlecht werden konstruiert, einzelne Tätigkeiten werden, entsprechend dem Geschlecht, dem sie zugeschrieben werden sollen, hervorgehoben. Es ist nicht plausibel, dass Frauen nicht mit Technik umgehen können. Erstens ist die Definition von Technik nach Geschlecht verzogen und zweitens nutzen beide Geschlechter Technik. Ebenso wenig plausibel ist die Zuschreibung der körperlichen Kraft auf die Ausübung einer bestimmten Tätigkeit. In vielen Berufen unterstützen Maschinen die auszuführenden Tätigkeiten. In den beiden vorangegangen Kapiteln wurden Berufe und Geschlecht aus unterschiedlichen Blickwinkeln betrachtet. Dabei hat sich erwiesen, dass Jugendliche vor dem Hintergrund einer geschlechtlich segregierten Arbeitswelt sozialisiert werden und sich Jugendliche in geschlechtsuntypischen Berufslehren mit divergierenden Ansprüchen auseinandersetzen müssen. Im folgenden Kapitel rücken Sozialisationsbedingungen in geschlechtsuntypischen Berufen ins Zentrum der Betrachtung. 


\section{Sozialisation in geschlechtsuntypischen Berufen}

Wie stark einige Berufe mit einem bestimmten Geschlecht verknüpft sind, manifestiert sich an der benötigten kognitiven Anstrengung, wenn man zum Beispiel nach der weiblichen Form von ,Zimmermann“ oder der männlichen Form von ,Hebamme ${ }^{6}$ sucht $^{18}$. Selten sind die Situationen, in der eine der beiden Minderheitsformen gebraucht wird. Wie und warum die Berufe zum Teil stark nach Geschlecht segregiert sind, habe ich in Unterkapitel 1.1 dargestellt. Die Berufsbezeichnung einer Person vermittelt anderen neben Informationen über eine spezifische fachliche Fähigkeit auch Bezüge zur sozialen und gesellschaftlichen Rolle. Der Beruf kann helfen, die Geschlechtsrolle zu stärken, da er meist mit einem der beiden Geschlechter eng assoziiert wird (vgl. Unterkapitel 2.6). Damit ist es attraktiv, einen geschlechtstypischen Beruf zu wählen, weil sich Individuen dadurch als Frau bzw. als Mann präsentieren können. Diesem Blick auf Geschlecht liegt eine determinierende Dualität zugrunde. Die Herausforderung besteht darin, dass das Geschlecht theoretisch als Kontinuum und soziales Konstrukt verstanden werden kann (vgl. Unterkapitel 2.5), der Alltag jedoch durch eine doppelte Dichotomie geprägt ist: Frau/Mann und frauen-/männertypischer Beruf. Mit dieser Vorannahme der doppelten Dichotomie verringert sich der kognitive Aufwand in vielen Situationen. Damit werden jedoch die tradierten Geschlechtsund Berufsvorstellungen verfestigt. Obwohl dieses Dilemma nie ganz aufzulösen ist, kann es hilfreich sein, die Mechanismen zu verstehen.

In diesem Kapitel werden Forschungsbefunde und Theorien vorgestellt, die ein tieferes Verständnis für die Situation der Lernenden in geschlechtsuntypischen Berufslehren ermöglichen. Die beiden vorangegangenen Kapitel zu Beruf und Geschlecht werden mit dem Fokus auf das Geschlechtsuntypische verbunden. Dazu setze ich mich mit der Beschreibung der Situation von Jugendlichen in geschlechtsuntypischen Berufslehren (Unterkapitel 3.1), den Auswirkungen dieser geschlechtsuntypischen Situation (Unterkapitel 3.2), der sozialen Unterstützung durch die Eltern (Unterkapitel 3.3) sowie Theorien und Modellen (Unterkapitel 3.4) auseinander, die zu meinem Modell der geschlechtsuntypischen Berufssozialisation in Kapitel 4 führen werden. 


\subsection{Beschreibung der geschlechtsuntypischen Berufssituation}

Wenn Jugendliche einen geschlechtsuntypischen Beruf erlernen und damit nicht vom Vorteil der automatisierten Geschlechtszuschreibung profitieren, könnten folgende Eigenschaften diesen Entscheid begünstigen: Diese Jugendlichen verfügen über ein starkes Selbstbewusstsein, einen hohen Selbstwert, eine hohe Selbstwirksamkeit und ein gesundes Bewusstsein über die eigene Weiblichkeit bzw. Männlichkeit, das keiner zusätzlichen Stärkung durch die gesellschaftskonforme Berufsausübung bedarf (vgl. Heppner/Heppner 2009). Diese Eigenschaften sind insbesondere wichtig, um mit negativen Reaktionen des sozialen Umfelds umzugehen, wie ich in diesem und im nachfolgenden Kapitel 4 darlegen werde. Doch für den Entscheid, einen geschlechtsuntypischen Beruf zu erlernen, sind weitere Bedingungen ebenfalls zentral, wenngleich es unterschiedliche Gewichtungen im individuellen Berufsfindungsprozess gibt. Wie die einschlägigen Befunde in diesem Kapitel belegen werden, kann ein starkes berufliches Interesse oder eine ausgeprägte berufliche Ambition einen nicht geschlechtskonformen Berufsbildungswunsch ebenso begünstigen wie ein gleichgeschlechtliches Vorbild, Transgenderthematik, sexuelle Orientierung oder Unkenntnis über die gesellschaftlichen Zuschreibungen. Manchmal führen äußere Gründe wie Lehrstellenmangel oder Perspektiveneinschränkung Jugendliche in eine geschlechtsuntypische Berufslehre (vgl. Kapitel 9).

Der Überblick über den Forschungsstand zu Personen in geschlechtsuntypischen Berufen ist in drei Bereiche eingeteilt. In Abschnitt 3.1.1 werden Befunde zur geschlechtsuntypischen Berufsfindungsphase vorgestellt. Es folgen Abschnitt 3.1.2 mit Befunden zu Frauen in männerreichen Berufen und Abschnitt 3.1.3 mit Befunden zu Männern in frauenreichen Berufen.

\subsubsection{Befunde zur geschlechtsuntypischen Berufsfindungsphase}

Berufsinteressen sind bei beiden Geschlechtern bereits im Schulalter unterschiedlich ausgeprägt (Faulstich-Wieland/Scholand 2016, Flammer/Alsa-ker 2002). Daneben sind jedoch aufseiten der Ausbildungsanbietenden innerbetriebliche Barrieren und persönliche Einstellungen bzw. Vorurteile der Ausbildenden für die geschlechtsspezifische Berufsfindung verantwortlich (Thege et al. 1996). Es halten sich beispielsweise mehr junge Frauen als Männer für geeignet für sogenannt traditionelle Frauenberufe wie Dentalhygienikerin, Lehrerin, Physiotherapeutin oder Sekretärin (Flammer/Alsaker 2002). Umgekehrt fanden mehr junge Männer als Frauen, dass Berufe wie Buchhalter, Ingenieur, Mathematiker oder Polizist zu ihnen passen. 
Berufswünsche von Jugendlichen entwickeln sich oft als Ergebnis vielschichtiger und unvorhersehbarer Prozesse (Bright/Pryor 2005, Hirschi 2013). Zum Beispiel können Freizeitaktivitäten oder Begegnungen mit Personen, die begeistert über ihren Beruf erzählen, zur Festigung eines Berufswunsches beitragen. Mit zunehmendem Alter immer deutlicher sind die Berufswünsche moderiert durch die Kompetenzerfahrung in der Schule (Grimm 2009). Bei guten Leistungen werden anspruchsvollere Berufe angestrebt, als wenn Schulschwierigkeiten und Leistungsprobleme vorhanden sind. Die Schule trägt mit ihren Fächern und Themen wesentlich dazu bei, dass Schülerinnen und Schüler eigene Interessen und Fähigkeiten kennenlernen und weiterführen.

Bereits vor mehr als 20 Jahren legte Horstkemper (1995) dar, dass Mädchen in der Schule ihr Wissen stärker steigern als Jungen, während Jungen ihren Selbstwert stärker erhöhen als Mädchen. Sie forderte eine kritische Auseinandersetzung mit der Berufs- und Lebensplanung der Mädchen und Jungen sowie Unterstützung vonseiten der Schule bei der realistischeren Einschätzung ihrer Möglichkeiten. Geschlechtsspezifische Disparitäten sollen aufgezeigt werden und Hilfestellungen bei der Suche nach Verhaltensmustern gegeben werden, um die bestehenden Geschlechtsrollen zu verändern und zu überschreiten.

Schon beim Übertritt in die Sekundarstufe I werden systematische Verzerrungen zum Nachteil der Jungen manifest (Haeberlin et al. 2004a, 2004b), indem weniger Jungen als Mädchen in eine Niveau mit erweiterten Ansprüchen eintreten. Mädchen und Jungen orientieren sich in ihren Berufswünschen an tradierten Geschlechtsrollenbildern und ergreifen Berufe im entsprechenden Bereich. Nach Gottfredson (1981, vgl. Abschnitt 1.3.2) müssen Jugendliche Kompromisse eingehen, um eine Lehrstelle zu erhalten.

\subsubsection{Befunde zu Frauen in männerreichen Berufen}

Strukturelle Benachteiligungen von jungen Frauen basieren auf einem kleineren Angebot an Berufslehren und Lehrstellen sowie auf höheren schulischen Anforderungen in frauenreichen Berufsbildungsgängen (Hirschi 2009). Junge Frauen gewichteten in einer amerikanischen Langzeitstudie berufliche Opfer zugunsten der Familie und der Möglichkeit, neben dem Beruf anderen zu helfen und Wertvolles für die Gesellschaft zu tun, höher als junge Männer (Eccles et al. 1999). Demgegenüber war es jungen Männern wichtiger, berühmt zu werden, viel Geld zu verdienen, sich Herausforderungen zu stellen und eine Arbeit zu verrichten, die mit Mathematik und Computern verbunden ist.

Gründe für die geschlechtsstereotype Berufsfindung wurden in der Wahl von Mathematik- und Sprachniveau in der Sekundarstufe I (Watt 2008), in 
der Kompatibilität von Familie und Beruf, im unterschiedlichen Interesse an Mathematik und Physik (Frome et al. 2008), in den Überzeugungen und Erwartungen der Eltern (Chhin et al. 2008) sowie in der Höhe des pränatalen Androgens (Berenbaum/Korman Bryk 2008) identifiziert. Einen Teil zur Segregation der Geschlechter im beruflichen Umfeld trägt die Geschlechtsrollensozialisation bei (Sheridan 1997). Demnach wählen Frauen frauenreiche Berufe, weil sie hineinsozialisiert werden. Wohingegen Mädchen, deren Mütter in männerreichen Berufen tätig sind, häufiger männerreiche Berufe wählen. Mädchen mit starker Arbeitsorientierung verlassen männerreiche Berufe seltener, und Mädchen mit hohen Arbeitsstatuserwartungen verlassen frauenreiche Berufe häufiger. Daneben spielt der Stellenmarkt eine bedeutende Rolle, denn ist der Markt ausgetrocknet, sind Frauen öfter in männerreichen Berufen anzutreffen (Sheridan 1997). Gesellschaftliche Dimensionen lassen sich unter anderem darin erkennen, dass die Geschlechtsspezifität in ostdeutschen Bundesländern weniger stark ausgeprägt ist als in westdeutschen (Christmas-Best/Schmitt-Rodermund 2001), oder in den Befunden von Küskü et al. (2007), die in der Türkei grundlegende Vorurteile gegenüber Ingenieur-Studentinnen nachweisen konnten, die auf vielfältige Weise in Familien und Schulen geschürt würden. Trotz gegenteiliger Erwartungen werden gemäß Bradley (1993) durch die Personen in geschlechtsuntypischen Berufen das System der beruflichen Segregation oder der darunterliegende Sexismus nicht unterwandert, da Frauen oder Männer lediglich wegen einer Krise oder wegen industriellen Wandels in einem gegengeschlechtlichen Beruf arbeiten. Das System der Geschlechterhierarchie bleibt jedoch intakt.

Damit stehen für Ihsen (2010) Ingenieurinnen in Deutschland vor größeren Herausforderungen als Ingenieure. So stellt sie fest, dass die Vereinbarkeit von Beruf und Familie nicht gewährleistet ist, Ingenieurinnen gegenüber ihren männlichen Kollegen einen geringeren Grad an beruflicher Identifikation aufweisen, häufiger arbeitslos und in der Wissenschaft untervertreten sind. Was den Frauen nicht gelinge, sei die Entfaltung eines konformen beruflichen Habitus, der eine erfolgreiche Laufbahn begünstige. Eine unreflektierte Reproduktion von offiziellen und inoffiziellen Regeln (heimlicher Lehrplan), führe dazu, dass Frauen aus dem Rahmen fielen. Ihsen (2010) zufolge reagierten die Ingenieurinnen, je nachdem wie sie ihre Situation interpretierten, mit verstärkten individuelle Anpassungsbemühungen, mit innerer Emigration, mit konstruktiver Auseinandersetzung mit dem System oder mit dem Verlassen des Berufs. Dass es sich nicht um eine „Leaky pipeline“ handle, indem die Ingenieurinnen mit der Zeit verloren gingen, sondern dass sie erst gar nicht mit der Ausbildung anfangen, belegen Bieri Buschor et al. (2012: 30). Frauen, die die Absicht hätten, Ingenieurin zu werden, zögen dies auch durch. Doch gibt es im Alter von 15 Jahren relativ wenige junge Frauen, die 
die Absicht haben, einen MINT-Beruf ${ }^{19} \mathrm{zu}$ ergreifen (vgl. Makarova et al. 2016). Frauen mit einem intrinsischen Interesse an Mathematik, Informatik und Naturwissenschaft hätten gute Chancen, einen technischen Beruf erlernen und ausüben zu können (Bieri Buschor et al. 2012).

Das geschlechtskonforme Desinteresse von Mädchen und jungen Frauen an technischen Fragen lässt sich z.B. in der Untervertretung von Mädchen in berufsbildenden Kursen der Sekundarstufe I zu technischen Berufsfeldern belegen (Eardley/Manvell 2006). Obwohl in vielen US-amerikanischen Bundesstaaten rechtliche Antidiskriminierungsbestimmungen bestehen, würden diese Verordnungen kaum aufgegriffen. Aufgrund der erheblichen Lohndifferenzen zwischen technischen und betreuenden/pflegenden Berufen bzw. zwischen männer- und frauenreichen Berufen sei es wichtig, dass Mädchen ebenfalls technische Kurse in der Schule belegten und nicht davon abgehalten würden.

\subsubsection{Befunde zu Männern in frauenreichen Berufen}

Die Motivation für eine geschlechtsuntypische Berufsorientierung ist bei Jungen im Gegensatz zu Mädchen bislang wenig untersucht. Beim Übergang in die Berufsbildung schneiden nach Imdorf (2005) männliche Jugendliche (meist in männerreichen bzw. geschlechtsneutralen Berufslehren) häufig erfolgreicher ab als weibliche. Mädchen können demnach ihren schulischen Vorsprung nicht, wie es im Gymnasium gelingt, in die nachobligatorische berufliche Bildung überführen. Denn männliche Jugendliche ergreifen meist besser bezahlte und karriereorientierte Berufe. Berufsbezeichnungen wie ,-pflege“ oder ,-helfer(in)/Hilfs-' sind für Jungen weniger interessant als Berufe mit Teilnamen ,-fachkraft' oder ,-macher' (Krewerth et al. 2004). Vermutlich blockiert unter anderem eine tradierte Geschlechterauffassung von Jungen die Wahl von Berufszweigen wie Gesundheit, Gastronomie oder Erziehung. Ein nicht unerheblicher Grund für die wenigen Männer in frauenreichen Berufen ist nach England und Herbert (1993) das tiefe Lohnniveau in frauenreichen Berufen. Da Männer in männerreichen Berufen ebenfalls mehr verdienen als Frauen, ist der Lohnausfall für Männer in frauenreichen Berufen größer als für Frauen in männerreichen Berufen. Dem halten Budde et al. (2008) entgegen, dass weniger der tiefere Lohn als vielmehr mangelndes Prestige sowie an der Ernährerrolle und althergebrachten Geschlechterstereotypen orientierte Männlichkeitsnormen ein stärkeres Engagement von jungen Männern verhindern. Dennoch entscheiden sich gelegentlich männliche Jugendliche für einen frauenreichen Beruf. Soweit ich die Befundlage überblicke, wurden Entscheidungsgrundlagen und Erfahrungen mit den Reaktionen 
des Umfelds von männlichen Jugendlichen bislang für den deutschsprachigen Raum selten untersucht.

Jacobs (1993) verglich das Mobilitätsmuster von Männern in frauenreichen Berufen mit einer Drehtür. Ihre kurze Verweildauer in geschlechtsuntypischen Berufen (Eintritt und kurz danach Austritt), wurde bekannt als Drehtür-Effekt. Je weniger Männer den Beruf ausüben, desto weniger wollen ihn erlernen und desto kürzer ist die Verweildauer in den Berufen. Das System der lebenslangen sozialen Kontrolle verstärkt den Drehtür-Effekt und die Tatsache, dass Männer schnell in einen Beruf tendieren, der geschlechtstypischer ist (Williams 1993, vgl. Abschnitt 3.4.3).

Männer in frauenreichen Berufen zeigen weniger geschlechtsrollentypische Erwartungen, mehr Hausarbeitsverantwortlichkeit, größere emotionale Sensibilität, größere weibliche Beeinflussung bei der Berufsfindung, gehören eher einer sozialen Minderheit an oder kommen aus tieferen sozioökonomischen Schichten (Lemkau 1984). Können diese Männer den Glauben an ihre Überlegenheit bewahren, kommen sie mit Frauen in ihrem Arbeitsumfeld zurecht (Swerdlow 1989). Männer in frauenreichen Berufen haben ähnliche Eigenschaften wie Frauen im gleichen Beruf (Chusmir 1990, Rustemeyer/Thrien 2001). Im Gegensatz zum Stereotyp fühlen sie sich wohl in ihrer männlichen Sexualität, obwohl ihre Geschlechtsrollen-Identitätswerte nach dem Bem'schen Geschlechtsrolleninventar etwas tiefer sind als bei Männern in traditionellen Berufen (Chusmir 1990). Obschon Koberg und Chusmir (1991) keine Differenzen bei Geschlechtsrollenkonflikten von Frauen und Männern in geschlechtsuntypischen vs. -typischen Berufen finden konnten, werden Männer in frauenreichen Berufen mit Vorurteilen zu ihnen fehlenden männlichen Eigenschaften und dem Verdacht auf Homosexualität konfrontiert (Jome et al. 2005). Ein Befragung von Rochlen et al. (2009) mit 174 USamerikanischen Pflegern hat ergeben, dass sich Männer in diesem frauenreichen Beruf insgesamt wohlfühlen, zufrieden mit ihrer Rolle und ihrem Leben sind, wenige Arbeitsbeschränkungen erleben, wenig Arbeit-FamilieKonflikte haben, soziale Unterstützung durch ihnen wichtige Personen erleben und mit ihrer körperlichen und emotionalen Ausdrucksmöglichkeiten gegenüber anderen Männern weniger hadern. Es gibt demnach neben den Nachteilen auch Argumente für einen geschlechtsuntypischen Beruf.

Dennoch sind die heteronormativen Vorstellungen bei der Mehrheit der Männer in typischen Berufen vorhanden. Diese haben im Vergleich zu Männern in geschlechtsuntypischen Berufen mehr homophobe Einstellungen und weniger soziale Interessen (Jome et al. 2005), beharren auf stärker auf traditionellen männlichen Werten und haben mehr Geschlechtsrollenkonflikte (Jome/Tokar 1998). Männer in geschlechtsuntypischen Berufen werden als weniger kompetent eingeschätzt und kämpfen, wenn sie mit kleinen Kindern arbeiten, gegen den Verdacht der Pädosexualität (Sargent 2004). Insbesondere Männer, denen weibliche Eigenschaften unterstellt werden oder die sich 
emotional und affektiert verhalten, werden von der Mehrheit mit Ablehnung bestraft (Glick et al. 2015). Beispielsweise seien Geburtshelfer im Konstruktionsprozess ihrer Identitätsbildung stark gefordert: „Being a man in a nontraditional occupation generates multiple social and psychological conflicts [...]“ (Cottard 2014: 108).

Zur Begründung ihrer geschlechtsuntypischer Berufsfindung nennen Männer folgende Punkte: Selbstverwirklichung, mehr Möglichkeiten für Karriere, Zusammenarbeit mit Frauen (Hayes 1986, 1989), persönliche Einflüsse (Hintergrund, Einstellung, Werte, intrinsische Bedürfnisse), familiäre Einflüsse (Einstellung zu Familienrollen, Beziehung mit Kindern, Mutterstatus), soziale Einflüsse bei Geschlechtsrolle und Normen (Chusmir 1990), Führungsbesterben (Williams 1993), Altruismus, Arbeitszufriedenheit (Bartlett et al. 1999), Alter (Chusmir 1990), sexuelle Orientierung (Chung/Harmon 1994), Werdegang, Einstellung gegenüber Kindern und Familienarbeit (Simpson 2005). Trotz all dieser positiven Befunde, die für die Wahl eines geschlechtsuntypischen Berufs sprechen, existieren soziale und persönliche Barrieren, die die freie Wahl einschränken. Um die Nachteile abzuschwächen, werden nach Simpson (2005) folgende Strategien angewandt: Spezialisierung in eine geschlechtsrollenkonforme berufliche Nische, KarriereEntwicklung und Übernahme von Führungsverantwortung, Definition oder Hervorheben männlich konnotierter Aspekte in der frauentypischen Tätigkeit.

Lupton (2006) schlägt zwei Ansätze zur Erklärung vor, weshalb Männer in frauenreiche Berufe wechseln: 1) individuelle Eigenschaften und Präferenzen wie berufliches Interesse, Karriere machen, weniger männliche Eigenschaften, tieferer Testosteronspiegel oder Partnersuche und 2) soziale Muster wie Drehtür- vs. Falltür-Effekte, späte Entscheidung oder Kombination von Umständen. Dabei wurde ein Paradox festgestellt. In Großbritannien lehnen demnach Männer aus ethnischen Minderheiten oder tieferen sozialen Schichten Frauenarbeit zwar stärker ab, sind jedoch häufiger in frauenreichen Berufen anzutreffen. Dieser Befund findet sich ebenso bei ausländischen Jungen in der Schweiz (Haeberlin et al. 2004b). Diese weichen vermehrt auf Ausbildungen in frauenreichen Berufen aus als einheimische Jungen. Ein Hinweis dafür, dass die Umstände eine wichtige Rolle spielen, die einen Mann in einen frauenreichen Beruf drängen, dokumentiert die Feststellung, dass Männer bei sich bietender Gelegenheit in eine geschlechtsrollenadäquatere Beschäftigung wechseln (Simpson 2005). Das Vermeiden von geschlechtsuntypischen Berufslehren ist jedoch nicht nur bei Jungen, sondern auch bei Mädchen vorhanden und hat vermutlich mit der klaren geschlechtsnormierten Forderung der Gesellschaft zu tun. Daraus können sich diskriminierende Verhaltensweisen ergeben, die Personen in geschlechtsuntypischen Berufen so erfahren. Im folgenden Unterkapitel gehe ich auf mögliche Auswirkungen ein, die die geschlechtsuntypische Berufssituation mit sich bringen kann. 


\subsection{Auswirkungen der geschlechtsuntypischen Berufssituation}

Der Minderheitsstatus aufgrund des untypischen Geschlechts führt einerseits dazu, dass die Individuen in einen Geschlechtsrollenkonflikt (O’Neil 2015) geraten. Dieser Konflikt läuft vor allem innerpsychisch ab, weil die Erwartungen und die Ansprüche des Berufs und des Geschlechts einander diametral widersprechen. So soll etwa ein Krankenpfleger als Mann Härte demonstrieren und als Pfleger einfühlsam sein. Nach Evans und Steptoe (2002) sind es jedoch die Stigmatisierungserfahrungen, die dazu führen können, dass vermehrt gesundheitliche Probleme bei Personen in geschlechtsuntypischen Berufen auftreten. „In combination, these findings suggest that men and women working in jobs in which they are in a minority, and where the culture is dominated by the opposite sex, may be especially vulnerable to stressrelated problems" (Evans/Steptoe 2002: 490). Die Restriktionen der Geschlechtsrolle wirken sich nicht nur negativ auf die psychische und physische Gesundheit aus, sondern auch auf das Verhalten, die Leistungen und die Motivation (O’Neil 2008). Speziell ist die Situation von Hausmännern, die die Kinderbetreuung übernehmen. So berichtet mehr als die Hälfte der Teilnehmer an einer Online-Studie von diskriminierenden und stigmatisierenden Erfahrungen (Rochlen et al. 2010). In dieser Studie wurden für diese negativen Reaktionen der sozialen Umgebung fehlende Vertrautheit mit der Rolle der Hausmänner, religiöse Überzeugungen, gegensätzliche Einstellungen zu Geschlechterrollen und Ignoranz verantwortlich gemacht.

Negative Stereotype gegenüber Frauen in geschlechtsuntypischen Berufen sind nach wie vor vorhanden (z.B. Shewring 2009). Trotz oder vielleicht nicht zuletzt wegen ihren Erfolgen im geschlechtsuntypischen Beruf sind diese Frauen weniger beliebt und werden insbesondere dann seltener befördert als Männer, wenn das berufliche Umfeld klar männlich konnotiert ist (Heilman et al. 2004). Plicht und Schreyer (2002) kommen zum Schluss, dass Ingenieurinnen und Informatikerinnen gegenüber ihren Fachkollegen deutlich benachteiligt sind. Sie bekleiden häufiger Stellen auf unteren und mittleren, seltener auf höheren betrieblichen Positionen. Ihre Arbeitsverträge sind öfter befristet und ihre Arbeitslosigkeit ist etwa doppelt so hoch wie diejenige ihrer ehemaligen Kommilitonen. Nach Plicht und Schreyer (2002) tun deshalb Reformen nicht nur in Schule und Studium Not, sondern es müssten zusätzlich die Arbeitsmarktchancen und die Beschäftigungsbedingungen zugunsten der Frauen in geschlechtsuntypischen Berufen verbessert werden. Das hier vor allem ein fundamentales Umdenken aufseiten der geschlechtlichen Mehrheit stattfinden muss, verdeutlichen die Befunden von Swerdlow (1989). In ihrer Langzeitstudie über vier Jahre mit teilnehmenden Beobachtungen und Interviews kommt sie zur pointierten Aussage, dass Männer den 
Glauben an ihre Überlegenheit bewahren können müssten, um mit Frauen in ihrem Arbeitsumfeld zurechtzukommen. Die vor über 30 Jahren gesammelten Daten zu den Ausgrenzungserfahrungen von Frauen in männerreichen Arbeiterberufen belegen, wie hart das Umfeld damals war. Wir dürfen davon ausgehen, dass sich nicht nur bei den involvierten Männergruppen, sondern in der gesamten Gesellschaft seither einiges zum Besseren verändert hat. Hinweise dafür lassen sich bei der elterlichen Unterstützung finden, die Jugendliche in geschlechtsuntypischen Berufslehren erleben dürfen, wie im anschließenden Unterkapitel 3.3 dargestellt wird.

\subsection{Einfluss der Herkunftsfamilie}

Kürzlich erschienene Studien im deutschen Sprachraum zeichnen den elterlichen Einfluss auf die geschlechtstypische und -untypische Berufsfindung auf mehreren Ebenen nach. Vor allem die geschlechtsstereotypen Begabungszuschreibungen (Mädchen gut in Sprachen; Jungen gut in Mathematik) von Eltern führen zu einer geschlechtstypischen Berufsfindung (Buchmann/Kriesi 2012). Obwohl der Einfluss des Elternhauses geschlechtsspezifisch ist, wählen Mädchen aus oberen Sozialschichten aufgrund der höheren beruflichen Aspirationen eher einen männertypischen Beruf als ihre gleichaltrigen Kolleginnen aus tieferen sozialen Schichten (Helbig/Leuze 2012). Bei Jungen hat die Schichtzugehörigkeit keinen Einfluss auf die Entscheidung für eine geschlechtsuntypische Berufslehre.

Fähigkeitszuschreibungen durch Eltern vollziehen sich gemäß Buchmann und Kriesi (2012) auf drei Ebenen: 1) direkt in Gesprächen, Unterstützungsleistungen und Ratschlägen, 2) indirekt über die frühe Sozialisation in der Familie und das Rollenverhalten der Erwachsenen sowie 3) indem Eltern als Rollenmodelle und Vorbilder dienen. Aufgrund der Dynamik in der Berufswelt werden Vorbildfunktionen zunehmend schwierig. Dennoch weisen die Befunde in einer breit angelegten Studie von Makarova und Herzog (2014) in die Richtung, dass Mütter und Väter, die einen männertypischen oder geschlechtsneutralen Beruf ausübten, als Vorbilder von ihren Töchtern gesehen werden. Die Wahrscheinlichkeit, einen frauenuntypischen Berufs zu wählen, war bei diesen Mädchen erhöht.

Zwar hat die soziale Schicht - wie oben beschrieben - Einfluss auf die Berufsfindung, doch sind weniger die Strukturmerkmale der Familie oder die persönlichen Merkmale der Eltern ausschlaggeben als vielmehr deren Handeln (Ignaczewska 2014). Buchmann und Kriesi (2012) beschreiben dies als unmittelbaren Einfluss der Fähigkeitszuschreibung. Diese elterlichen Hilfestellungen und ihre Gesprächsführung sind stark durch den Geschlechtskontext geprägt. ,Zudem haben die elterlichen Einstellungen als Prozessmerkma- 
le der Familie, die sich durch Unterstützung und Bestärkung der jungen Frauen in ihren Fähigkeiten äußern, eine zentrale Bedeutung bei der geschlechtsuntypischen Studienwahl“" (Ignaczewska 2014: 173). Den Einfluss geschlechtsspezifischen Verhaltens seitens der Eltern belegten Buchmann und Kriesi (2012). Dieses sei nach den beruflichen Aspirationen und Werten der Jugendlichen der zweitwichtigste Einflussfaktor. So könnte hier ein Zusammenhang bestehen, dass Jungen mit guten Schulleistungen eher einer anspruchsvollen intellektuell-technischen Berufslehre zugewiesen werden als einer handwerklichen. Bei Mädchen fand sich dieser Effekt nicht. Sie wählen, obwohl ihre Schulleistungen gut waren, anspruchsvolle (administrative/verwaltende) genauso wie weniger anspruchsvolle haushaltsnahe Berufe.

Die oben vorgestellten Befunde führen den starken und vielschichtigen Einfluss der Herkunftsfamilie auf den Berufsfindungsprozess vor Augen. Die zitierten Studien haben den Fokus darauf, was Eltern zur geschlechterstereotypen Berufsfindung ihrer Kinder beitragen, doch impliziert sich gerade darin die verstärkende Wirkung. Wenn Eltern auf die Berufsaspirationen ihrer Kinder eingehen und sie in ihrem Wunsch unterstützen, einen geschlechtsuntypischen Beruf zu erlernen, können sie entscheidende Ressourcen sein, die helfen, Widerständen zu begegnen. In den im Folgenden beschriebenen Modellen und Theorien ist der familiäre Einfluss ebenfalls eine wichtige Komponente.

\subsection{Modelle und Theorien zu geschlechtsuntypischen Berufen}

Im Bereich der geschlechtsuntypischen Berufssozialisation gibt es nur wenige Modelle oder Theorien, die empirisch überprüft wurden. In diesem Unterkapitel stelle ich zwei Modelle zur geschlechtsuntypischen Berufsfindung und zwei Theorien vor, die sich durch eine hohe Plausibilität auszeichnen, empirisch absichert sind und fruchtbar für die weitere Untersuchung eingesetzt werden konnten. Im ersten vorgestellten Modell von Flores et al. (2006) werden Faktoren zur geschlechtsuntypischen Berufsfindung vorgestellt. Dabei untermauern insbesondere familiäre und individuelle Faktoren ihren Einfluss (Abschnitt 3.4.1). Das zweite Modell ist ein Rahmenmodell von Chusmir (1990) und führt Faktoren auf, die eine geschlechtsuntypische Berufsfindung bei Männern unterstützen, obwohl Geschlechtsrollenkonflikt- und Tokenismus-Theorie umgekehrte Ergebnisse erwarten ließen (Abschnitt 3.4.2). Die beiden Theorien, die ich in diesem Unterkapitel vorstelle, erheben ihren Gültigkeitsanspruch jeweils für lediglich das Geschlecht in der Minderheitsposition. Kanter (1977) entwickelte aus ihren Untersuchungen von Frauen in Führungspositionen die Tokenismus-Theorie (Abschnitt 3.4.3), während 
Williams (1993) Männer in frauenreichen Berufen befragte und so ihre Glaslift-Theorie bestätigen konnte (Abschnitt 3.4.4).

\subsubsection{Modell der geschlechtsuntypischen Berufsfindung}

Verglichen mit der Vielzahl an Berufsfindungsmodellen (z.B. Holland, Gottfredson, Hackett, vgl. Unterkapitel 1.3), die Geschlecht zum Teil als einflussreiche Variable betrachten, gibt es nur wenige Modelle, die die geschlechtsuntypische Berufsfindung zu erklären versuchen. Ich stelle ein Modell vor, das einer empirischen Überprüfung unterzogen worden ist.

Abbildung 3.1: Modell der geschlechtsuntypischen Berufsfindung

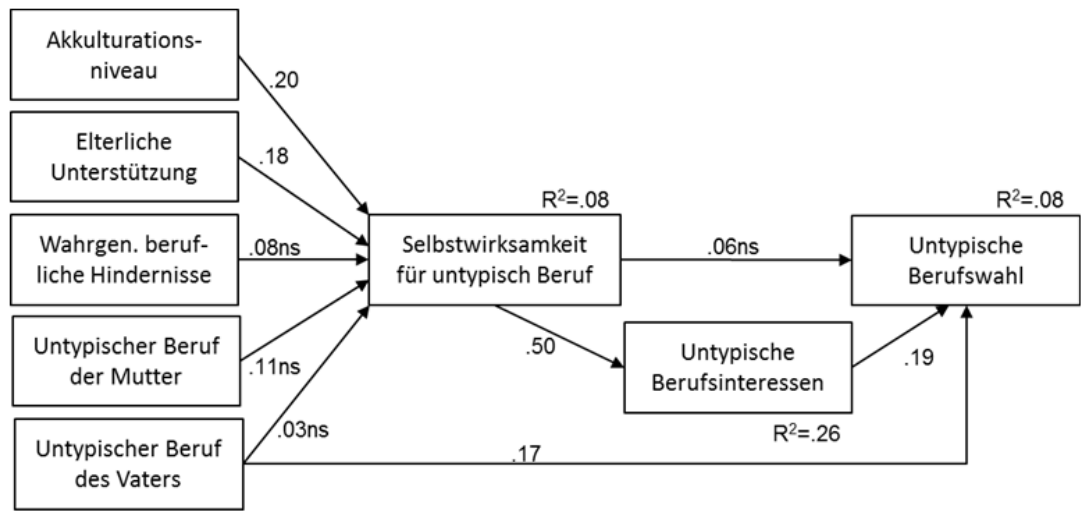

Quelle: Darstellung nach Flores et al. (2006: 226).

Das Modell der geschlechtsuntypischen Berufsfindung entstand in Anlehnung an das Berufsfindungsmodell von Lent und Brown (1996). Flores et al. (2006) entwickelten ein Modell, das die geschlechtsuntypische Berufsfindung anhand von familiär gesteuerten Sozialisationsprozessen (,Akkulturationsniveau'), der ,elterlichen Unterstützung', der ,wahrgenommenen beruflichen Hindernisse sowie der, geschlechtsuntypischen Berufe von Mutter und Vater $^{\natural}$ zu erklären versucht (vgl. Abbildung 3.1). Diese Einflussfaktoren wirken sich auf die ,Selbstwirksamkeit der Jugendlichen bezüglich ihrer geschlechtsuntypischen Berufsfindung aus und begünstigen bzw. reduzieren die Wahrscheinlichkeit einer, geschlechtsuntypischen Berufswahl' über das Interesse an einem solchen Beruf. Die Testung des Modells an Mexikoamerikanischen Jugendlichen erreichte gute bis hinreichende Testwerte $\left(C h i^{2}=25.37, d f=9, p<.01, C F I=.91, R M S E A=.08, N=302\right)$. Die Vorbildfunktion des gleichgeschlechtlichen Elternteils (hier des Vaters) kam 
deutlich zum Ausdruck. Im Modell musste ein direkter Pfad vom geschlechtsuntypischen Beruf des Vaters zur untypischen Berufsfindung der Jugendlichen eingefügt werden. Dieser Befund des Vorbildcharakters für geschlechtsuntypische Berufsfindung konnte für Frauen in der Schweiz ebenfalls gefunden werden (Buchmann/Kriesi 2012, Makarova/Herzog 2014). In Ergänzung zum ersten Modell von Flores et al. (2006), das die geschlechtsuntypische Berufsfindung aus der familiäre Perspektive erklärt, benennt das nachfolgend vorgestellte Modell von Chusmir (1990) zusätzlich neben familiären auch soziale Faktoren zur Entscheidung für oder gegen eine Laufbahn im gegengeschlechtlichen Bereich.

\subsubsection{Rahmenmodell zur geschlechtsuntypischen Laufbahn}

Das Rahmenmodell zur Laufbahnentscheidung entwickelte Chusmir (1990, vgl. Abbildung 3.2) für Männer, die einen geschlechtsuntypischen Beruf anstreben. Am Anfang stehen, individuellen Faktoren', die von ,externalen Einflüssen" modifiziert werden und nach dem ,Berufsentscheid' in Erfolg oder Misserfolg im geschlechtsuntypischen Beruf münden. Der individuelle Einfluss setzt sich zusammen aus ,Hintergrund", Einstellungen und Werten sowie ,Motiven'. Mit ,Hintergrund' ist gemeint, dass Männer in geschlechtsuntypischen Berufen älter sind, eher einer Minderheit angehören, öfter aus traditionellen Familien der Mittel- und Arbeitsklasse stammen, und eher besser ausgebildet sind. Bei den,Einstellungen und Werten ${ }^{6}$ unterscheiden sich Männer in geschlechtsuntypischen Berufen von anderen Männern in folgenden Aspekten: ihnen ist Soziales (Liebe, Altruismus, Erziehung) und Ästhetik wichtiger als Wirtschaft und Politik. Sie sind liberaler und emotional sensibler, jedoch weniger kompetitiv. Für die ,Motive gilt, dass Männer, die sich für einen geschlechtsuntypischen Beruf entscheiden, ein tieferes Anschlussmotiv als ihre Kolleginnen sowie ein tieferes Statusmotiv als andere Männer haben, dafür über mehr intrinsische Motivation für ihren Beruf berichten.

Diese individuellen Einflüsse werden von externalen (familialen und sozialen) Einflüssen verändert. Die ,familialen Einflüsse sind geprägt von den liberalen Einstellungen der Männer in frauenreichen Berufen: zu Familie, Geschlechtsrollen, Kinderbetreuung und der Erwerbsarbeit der Mutter. Mit ,sozialem Einfluss' ist ein breites Spektrum gemeint, das von Gleichaltrigen und Medien über Geschlechtsrollenerwartungen bis zur Unterstützung durch Eltern, Lehrpersonen und anderem reicht.

Lease (2003) bestätigte in einer Untersuchung mit über 350 Studierenden in männer- und frauenreichen Berufen die individuellen und sozialen Einflusskomponenten von Chusmirs Modell. Abschlussaspiration, soziale Einstellungen, sozioökonomischer Status, Fähigkeiten und prestigereiche Karrie- 
re sagten eine geschlechtstypische Karriere voraus. Chusmir (1990) resümiert, dass der Entscheid für einen geschlechtsuntypischen Beruf bei Männern öfter zu einem positiven Ergebnis kommt als zu einem negativen, wie es die Theorie der Rollenkonflikte oder die Tokenismus-Theorie (Kanter 1977) erwarten ließen.

Abbildung 3.2: Faktoren zur geschlechtsuntypischen Laufbahn

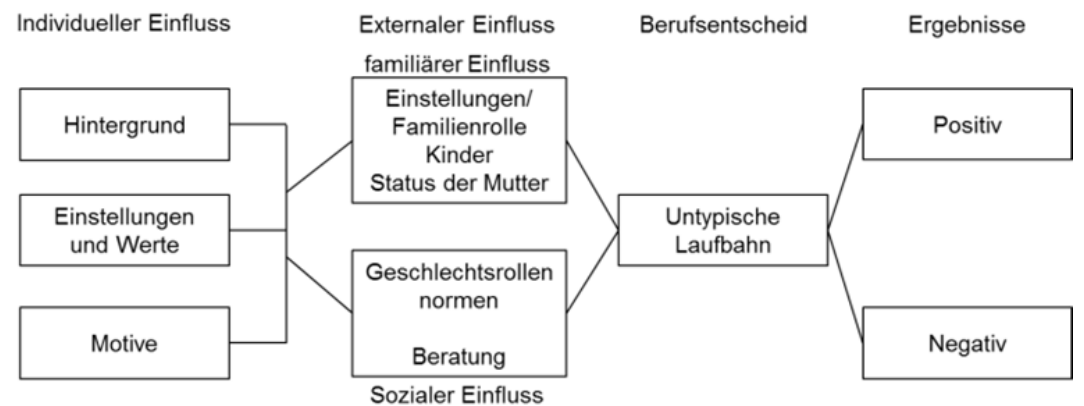

Quelle: Darstellung nach Chusmir (1990: 12).

Die Überprüfung des Modells erfolgte mittels einer Langzeitstudie mit 354 Collegestudenten, die 1984 und 1990 an einer großangelegten Studie mit über 4'400 Teilnehmenden befragt wurden. Die individuellen und die externalen Komponenten von Chusmirs Modell konnten bestätigt werden, da Aspiration, soziale Einstellung, sozioökonomischer Status, Fähigkeiten und Prestige die geschlechtsuntypische Berufsfindung voraussagen konnten. Da die erklärte Varianz lediglich 17 Prozent beträgt, ist davon auszugehen, dass weitere eventuell berufsfindungsnähere Prädiktoren am Berufsentscheid beteiligt sind.

Im Anschluss an die beiden eben beschriebenen Modelle folgen nun die Theorien von Kanter zu Frauen und von Williams zu Männern in geschlechtsuntypischen Berufen. Beide Theorien erwiesen sich bei der Interpretation der Ergebnisse der vorliegenden Arbeit als ergiebig.

\subsubsection{Williams Glaslift-Theorie}

Gemäß Christine L. Williams $(1989,1993)$ entscheiden sich Männer selten für frauenreiche Berufe, weil soziale und ökonomische Kräfte dagegenwirken. Ihr zufolge besagen konservative Theorien, dass Männer und Frauen besser in bestimmte Berufe passen, weil sie unterschiedlich sozialisiert wurden, z.B. interessieren sich Männer für Sachen und Frauen für Personen ( $\mathrm{Su}$ et al. 2009). Radikale Theorien wiederum begründeten die Segregation mit 
der Männerherrschaft (Eisenstein 1979). Nach Williams (1993) sind beide Ansätze falsch, da Berufe sich passend zur Geschlechterhierarchie entwickelt haben. In der Medizin beispielsweise sind Arzt und Krankenschwester eng verbunden. Berufe wurden für Frauen entwickelt, basierend auf der kulturellen Annahme der weiblichen Natur und ihres ,richtigen' Platzes in der Gesellschaft. Überschreitet ein Mann diese Linie, indem er einen frauenreichen Beruf ausübt, stört er diese Annahme. Ihm wird unterstellt, kein richtiger Mann, irgendwie falsch, schwul, verweiblicht oder faul zu sein (Williams 1993).

Männer, die diesen Vorurteilen dennoch trotzen, werden im beruflichen Umfeld für ihre Andersartigkeit belohnt, Frauen hingegen bestraft. Männer in geschlechtsuntypischen Berufen nehmen oft leitende Positionen ein, werden besser bezahlt als Frauen. Williams (1992) nennt dies den Glaslift-Effekt (glass escalator). Anstatt wie bei den Frauen durch eine unsichtbare gläserne Decke (glass ceiling: Cotter et al. 2001) am beruflichen Aufstieg gehindert zu werden, werden Männer scheinbar ohne eigenes Dazutun die Karriereleiter hinaufbefördert. Kommen diese Vorteile nicht zum Tragen, bleiben Männer gemäß Williams (1992) nicht lange in frauenreichen Berufen, sondern wechseln in einen männerreicheren Bereich. Im Anschluss an den Blick auf die Situation von Männern in frauenreichen Berufen wechselt die Perspektive zu Befunden von Frauen in männerreichen Berufen, denen Kanter (1977) einen allgemeingültigen Anspruch beimaß.

\subsubsection{Kanters Tokenismus-Theorie}

Elizabeth Moss Kanter hat in den 70er-Jahren die Situation von Frauen in Firmen untersucht und folgende Schlussfolgerungen gezogen. Bei Personen, die in irgendeiner Form einer Minderheit angehören, das heißt die mit einer seltenen und auffälligen Ausprägung stark untervertreten sind, laufen vergleichbare Prozesse ab: etwa bei einer einzelnen Frau unter Männern, einzelnen Fremdsprachigen unter Einheimischen, einer einzelnen Glarnerin unter Bernerinnen.

At the same time, they also echoed the experiences of people of any kind who are rare and scarce: the lone black among whites, the lone man among women, the few foreigners among natives. Any situation where proportions of significant types of people are highly skewed can produce similar themes and processes. It was rarity and scarcity, rather than femaleness per se, that shaped the environment for women in the parts of Indsco [Name der untersuchten Unternehmung, Anm. BR] mostly populated by men. (Kanter 1977: 207)

Personen, die einer kleinen Minderheit angehören, werden von der Mehrheit sofort wahrgenommen und fühlen sich aufgrund ihres ,Minderheitsstatus exponiert. Kanter (1977) nennt diese Personen ,Token', was wörtlich übersetzt Quoten- oder Alibiperson heißt. Diese Übersetzungen sind im Deut- 
schen negativ konnotiert, weshalb ich nur im Zusammenhang mit dieser Theorie den Terminus, Token' verwende. Kanters (1977) Untersuchungen basieren auf Frauen in Karrieresituationen, die nicht wegen, sondern trotz ihres Geschlechts in einer Führungsposition sind. Der Terminus Quotenfrau wäre demnach unzutreffend. Der Umstand, dass die Personen die einzigen mit einem wichtigen und auffälligen Merkmal sind, macht sie zu Token, mehr als die jeweilige Eigenschaft an sich. Für die Untersuchung der Prozesse bei Minderheiten im Geschlechterverhältnis identifiziert Kanter (1977) vier Populationsgruppen: 1) uniforme Gruppen, geschlechtshomogen, lediglich eines der beiden Geschlechter geht dieser Tätigkeit nach, 2) extrem verzerrte Gruppen, die Minderheitsgruppe, die sogenannten Token, zählt maximal 15 Prozent, 85 Prozent gehören dem zahlenmäßig dominierenden Geschlecht an, 3) schräge Gruppen, 15-40 Prozent bilden die Minderheit, 60-85 Prozent die Mehrheit und 4) ausgeglichene Gruppen, das Geschlechterverhältnis liegt zwischen 40 und 60 Prozent.

Bei den ausgeglichenen Gruppen ergeben sich oft Untergruppen, die dann ihrerseits Verzerrungen aufweisen und der uniformen, extrem verzerrten oder schrägen Gruppe zuzuordnen wären.

Token sind sichtbar, salient und erhalten für eine so kleine Gruppe übermäßig viel Aufmerksamkeit. Kanter (1977) beschreibt drei Wahrnehmungstendenzen, die auftreten, sobald eine Person mit dem andern Geschlecht zu einer uniformen Gruppe hinzukommt: (1) ,Polarisierung': Uniforme Gruppen merken gar nicht, was sie verbindet, erst wenn ein Token erscheint, wird dieses spezifische Merkmal wahrgenommen, es bilden sich Gegensätze, und die Dominanten schließen Token oft aus. (2) ,Anpassung': Token sind einfacher zu stereotypisieren als Personen in größeren Populationen. Ironischerweise sind Token hoch sichtbar und verschieden von der Mehrheit, aber ohne die Erlaubnis, individuell zu sein. Sie werden in hohem Maß stereotypisiert. (3) ,Leistungsdruck': Token stehen im Rampenlicht und sind damit dem zweischneidigen Schwert der Bekanntheit ausgesetzt. Sie müssen nichts tun, um wahrgenommen zu werden, jedoch umso mehr, um Anerkennung für ihre Leistung zu erhalten. „The token does not have to work hard to have her presence noticed, but she does have to work hard to have her achievements noticed" (Kanter 1977: 216).

Der Fokus von Kanter (1977) lag auf Frauen in männerreichen Berufen. Männer drängen Frauen in Tokensituationen in die Rolle der ,Mutter', der ,Verführerin' oder des ,Lieblings'. Wenn sich eine Frau keiner der drei Rollen zuschreiben lässt, wird ihr das Etikett ,Eiserne Jungfrau“ verpasst. Sie wird demnach als knallhart und gefährlich wahrgenommen.

The ,iron maiden' is a contemporary variation of the stereotypical roles into which strong women are placed. Women who failed to fall into any of the first three roles and, in fact, resisted overtures that would trap them in a role (such as flirtation) might consequently be responded to as ,tough' or dangerous. (Kanter 1977: 236) 
Während Kanter (1977) in ihrer Theorie die Andersbehandlung der Frauen in geschlechtsuntypischen Positionen mit benachteiligenden Konsequenzen beschreibt, profitieren in der Glaslift-Theorie die Männer von ihrem Alleinstellungsmerkmal. Bei Männern konnte Kanters (1977) TokenismusVorhersage trotz großer Stichprobe (5'734 Männer) nicht bestätigt werden, Williams Glaslift-Modell hingegen schon (vgl. Williams 1992). Die Männer in frauenreichen Berufen erleiden weniger Nachteile, sie profitieren eher von ihrer Situation (Cognard-Black 2004). Im folgenden letzten Theoriekapitel werden die Ausführungen zum Beruf und zum Geschlecht zu einem Modell der geschlechtsuntypischen Berufssozialisation verdichtet. 


\section{Modell der geschlechtsuntypischen Berufssozialisation}

In Kapitel 4 erkläre ich das spezifische Umfeld bei der Sozialisation von Lernenden in geschlechtsuntypischen Berufslehren anhand des Modells der geschlechtsuntypischen Berufssozialisation. Hierfür beschreibe ich das Modell und definiere die zentralen Termini. Das Modell der geschlechtsuntypischen Berufssozialisation entwickelte ich auf der Basis der in den vorangegangenen Kapiteln beschriebenen Befunde und Literatur.

Jugendliche, die eine Berufslehre absolvieren, erlernen nicht nur berufliche Fertigkeiten, sondern werden auch in ein berufliches Milieu hineinsozialisiert (Kammeyer-Mueller/Wanberg 2003, Neuenschwander/Gerber 2014). Sie übernehmen dabei teilweise bewusst und teilweise unbewusst Werthaltungen, Meinungen, Ansichten und Handlungsroutinen. Die Jugendlichen bringen sich selbst ein, interagieren mit ihrer Umwelt und beeinflussen ihrerseits das berufliche Umfeld. Dabei spielt eine wechselseitige Beeinflussung der Erwartungen, die an die Jugendlichen gestellt werden, und von Zielen, die sich die Jugendlichen selbst gesetzt haben, eine wichtige Rolle.

Die Basis, auf der die Sozialisation im beruflichen Milieu stattfindet, hat vielfältige Charakteristiken. Für die vorliegende Arbeit ist die geschlechtliche Segregation der Arbeitswelt das entscheidende Merkmal (vgl. Unterkapitel 1.1). Sobald eine Person im für ihr Geschlecht untypischen Beruf im relativ kleinräumigen beruflichen Kontext (etwa Werkstatt, Garage, Salon oder Laden) mitarbeitet, werden spezifische geschlechtliche Zuschreibungen erkennbar. Weiblichen und männlichen Eigenschaften, die vorher für die Beteiligten weder sichtbar noch relevant waren, polarisieren (Kanter 1977, vgl. Abschnitt 3.4.4). Die Person im geschlechtsuntypischen Beruf ist salient, und dies führt dazu, dass das Herkömmliche und Bekannte infrage gestellt wird. Diese spezielle Situation von Lernenden in geschlechtsuntypischen Berufslehren und ihre Auswirkungen auf die betroffenen Jugendlichen beschreibt das von mir für die vorliegende Arbeit entwickelte Modell der geschlechtsuntypischen Berufssozialisation. Das Modell entstand mit der Intention, die Situation von Jugendlichen in geschlechtsuntypischen Berufslehren besser zu verstehen und ihren Erfolg bzw. Misserfolg während der Berufsausbildung zu erklären.

Das Modell der geschlechtsuntypischen Berufssozialisation greift auf die im Kapitel 3 vorgestellten Theorien zurück und versucht, den empirischen Befunden einen Rahmen zu geben. Die Blickwinkel, die dabei eingenommen werden, lassen sich der Soziologie, den Genderstudies, der Sozial-, Arbeitsund Entwicklungspsychologie sowie der Pädagogik zuordnen. Damit öffnen sich unterschiedliche Denkebenen. Während Soziologie und Genderstudies Prozesse auf der Makroebene thematisieren (z.B. berufliche Segregation nach 
Geschlecht, Schließungsmechanismen), fokussieren die sozial-, arbeits- und entwicklungspsychologischen Blickwinkel auf Prozesse der intra- und interindividuellen Ebene (z.B. Berufsfindung, Diskriminierung, Ressourcen und Belastungen). Die Berufslehre als eine stark strukturierte Ausbildung schließt damit zusätzlich pädagogische Überlegungen mit ein. In Bezug auf die Sozialisation ergibt sich im Kontext der Berufsbildung wegen des Ausbildungscharakters eine besondere Konstellation: In der hierarchischen Dyade von Ausbildenden und Lernenden findet Sozialisation gelenkt statt. Im Gegensatz dazu ist die informelle Sozialisation in der Gruppe der Gleichaltrigen ohne institutionalisierte Hierarchie und erzieherische Planung zu verstehen (Heyer et al. 2012).

Das Modell der geschlechtsuntypischen Berufssozialisation ist also im Dreieck Pädagogik, Soziologie und Psychologie angesiedelt und bedingt auf der empirischen Ebene unterschiedliches methodisches Vorgehen (vgl. Kapitel 5 bis 9). So werden auf der Makroebene einerseits Strukturdaten zu Lehrvertragsauflösung und Lehrabschlussprüfung analysiert und andererseits individualpsychologische Bedingungen $\mathrm{zu}$ den Wirkungszusammenhängen auf der Mikroebene geprüft. Der empirische Teil dient als Beleg für das postulierte Modell. Im vorliegenden Kapitel 4 werden zentrale Termini definiert (Unterkapitel 4.1) und das Modell der geschlechtsuntypischen Berufssozialisation vorgestellt (Unterkapitel 4.2). Das Kapitel endet mit der Fragestellung und den in der vorliegenden Arbeit zu prüfenden Hypothesen (Unterkapitel 4.3).

\subsection{Definitionen grundlegender Begriffe des Modells}

In den vorangehenden Kapiteln 1 bis 3 wurden einschlägige Theorien zu Segregation, beruflicher Sozialisation, Berufsfindung, Geschlecht u.a. beschrieben. Im Folgenden werden diejenigen Termini definiert und erläutert, die zum Verständnis des Modells der geschlechtsuntypischen Berufssozialisation geklärt werden müssen, da sie in der Literatur oft unterschiedlich angewandt werden. Das Ziel dabei ist nicht, den Diskurs wieder aufzunehmen und weiterzuführen. Vielmehr wird fokussiert und zusammengefasst dargestellt, wie die Begriffe im Modell der geschlechtsuntypischen Berufssozialisation $\mathrm{zu}$ verstehen sind. In Abschnitten 4.1.1 bis 4.1.6 werden folgende zentrale Termini definiert: ,Berufliche Sozialisation', ,Jugendliche und Lernende', ,Beruf und Berufslehre', ,Geschlecht und Geschlechtsrolle', ,berufliche Segregation nach Geschlecht' und ,Lernende in geschlechtsuntypischen Berufslehren'. Für eine bessere Verständlichkeit werden die folgenden zentralen Begriffe zusammen mit der Beschreibung des Modells (Unterkapitel 
4.2) erläutert: ,soziales Umfeld‘, ,Diskriminierung‘, ,Bewältigung‘, ,Ressourcen' sowie ,Belastung'.

\subsubsection{Berufliche Sozialisation}

Sozialisation ist ein andauernder Prozess der Akkulturation, Enkulturation, Personalisation und Vergesellschaftung, in dem sich das Individuum aktiv mit seiner Umwelt auseinandersetzt. In der beruflichen Sozialisation (vgl. Unterkapitel 1.2) entfalten, verfestigen und verändern Jugendliche ihre individuellen Persönlichkeitsstrukturen in Interaktion mit den Charakteristiken der beruflichen und betrieblichen Strukturen (Lempert 2002). Diese Strukturen können dabei reproduziert und transformiert werden.

Für die vorliegende Arbeit steht - neben dem Sozialisationsmerkmal, geschlechtliche Segregation' - vor allem die berufliche Sozialisation im Zentrum der Betrachtung. Heinz (1997) versteht darunter den Prozess, durch Imitation und Auseinandersetzung beruflichen Habitus (vgl. Bourdieu 1997b) zu erwerben:

Der berufliche Habitus ist ein stabiles System verinnerlichter Handlungsregeln, die nicht nur der Anpassung an die Arbeitsanforderungen, sondern auch der Selbstinterpretation und der Deutung gesellschaftlicher Verhältnisse dienen. Es sind insbesondere die sozialen Anforderungen beim Erlernen und Ausüben eines Berufs, wie z.B. Umgangsformen und Sprachstil, die Personen mit einem gleichen Habitus, also mit gemeinsamen Denk- und Beurteilungsmustern sowie Handlungsschemata hervorbringen. (Heinz 1995: 51)

Nach Heinz (1995) versteht Bourdieu unter Habitus ein System verinnerlichter Handlungsmuster, die aus Sozialisationsprozessen in Familie sowie schulischen und beruflichen Lernorten hervorgegangen sind. $\mathrm{Zu}$ den sozialen Anforderungen gehören geschlechtlich konnotierte Eigenschaften, die die Entwicklung des beruflichen Habitus und die berufliche Sozialisation für Lernende in geschlechtsuntypischen Berufslehren erschweren.

\subsubsection{Jugendliche und Lernende}

Das Jugendalter als Entwicklungsphase zwischen Kindheit und Erwachsenenalter wird je nach Kontext verschieden definiert. Nach Oerter und Dreher (1998) schließt an das Jugendalter (11-17 Jahre) die späte Adoleszenz (1821 Jahre) an. Die meisten der in der vorliegenden Arbeit untersuchten Jugendlichen sind zwischen 15 und 20 Jahre alt. Da Berufslehren gelegentlich von jungen Erwachsenen (ab 21 Jahren) absolviert werden, können diese vereinzelt im Datenmaterial vorkommen.

Mit ,Lernenden' sind in der vorliegenden Arbeit Jugendliche gemeint, die einen vom SBFI zertifizierten dualen Berufslehrgang absolvieren. Bezeich- 
nungen wie ,Lehrtochter' und ,Lehrling' oder in Deutschland ,Auszubildende' bzw. ,Azubi' sind veraltet und werden daher nicht verwendet (vgl. SBFIWortgebrauch). Lernende werden von Berufsbildenden (Ausbildende oder veraltet Lehrmeisterin, Lehrmeister) im Lehrbetrieb angeleitet und in Berufsfachschulen von Lehrpersonen unterrichtet.

\subsubsection{Beruf und Berufslehre}

Der Beruf (vgl. Kapitel 1) ist nach Duden ${ }^{20}$ die erlernte Arbeit bzw. Tätigkeit, mit der jemand sein Geld verdient, im engeren Sinn die Erwerbstätigkeit. Beruf ist die im Rahmen einer arbeitsteiligen Wirtschaftsordnung aufgrund besonderer Eignung und Neigung systematisch erlernte und mit Qualifikationsnachweis versehene, dauerhaft gegen Entgelt ausgeübte spezialisierte Betätigung eines Menschen. Eine Berufslehre ist eine Ausbildung im dualen Berufsausbildungssystem. Mit ,dual' wird eine Ausbildung bezeichnet, bei der in einem Lehrbetrieb gearbeitet und die Berufsfachschule besucht wird. In der Schweiz werden die Berufslehren von Organisationen der Arbeitswelt (Privatfirmen, Verwaltungen, Spitäler etc.) und Berufsfachschulen angeboten (vgl. Wettstein/Gonon 2009). Jede Ausbildung endet mit einem Zertifikat wie dem eidgenössischen Berufsattest EBA oder dem eidgenössischen Fähigkeitszeugnis EFZ (SBFI 2015).

\subsubsection{Geschlecht und Geschlechtsrolle}

In der vorliegenden Arbeit wird das Geschlecht als Kontinuum mit den zwei Polen weiblich und männlich betrachtet (vgl. Kapitel 2). Das Geschlecht, verstanden als soziales Konstrukt, hat sowohl körperliche Komponenten (engl. sex) als auch gesellschaftliche (engl. gender). Die wechselseitige Konstruktion von Geschlecht drückt sich darin aus, dass Geschlecht einerseits vom Individuum produziert (Doing Gender) und andererseits von der Gesellschaft anerkannt wird (vgl. Unterkapitel 2.5). Das Geschlecht stellt eine der stärksten sozialen Kategorisierungen im Alltag dar, was zu mehr oder weniger rigiden Erwartungen an das Verhalten der Individuen führt (vgl. Unterkapitel 2.6).

Um die Abläufe im Modell der geschlechtsuntypischen Berufssozialisation zu verstehen, ist es wichtig, einige gesellschaftliche Prozesse im Zusammenhang mit Geschlecht zu betrachten. Die in der Literatur beschriebenen Mechanismen Doing Gender, Zweigeschlechtlichkeitsnorm, Geschlechterstereotype, Geschlechterhierarchie und Gleichheitstabu sind in Kapitel 2 eingeführt worden. Distale Konstrukte wie Heteronormativität (vgl. Unterkapitel 
2.5) und patriarchale Strukturen (vgl. Unterkapitel 2.3) können sich im Einzelfall in der Praxis - ähnlich wie die anderen Konstrukte - ebenfalls als wirkungsvoll erweisen.

An Frauen und Männer werden unterschiedliche Geschlechtsrollenerwartungen gestellt: Wie sie sich verhalten, kleiden oder sprechen sollen bzw. welche (beruflichen) Tätigkeiten zu ihnen passen. Diese impliziten Verhaltensauflagen wirken sich auf die beruflichen Tätigkeiten aus, denn „unter Geschlechterrollen wird ein Set von Erwartungen verstanden, die Menschen gegenüber einer Person hegen, die eine bestimmte Position oder Eigenschaft besitzt" (Hannover 2006: 465). Klischeehafte Bilder von Berufen wie Arzt und Krankenschwester, Chef und Sekretärin, Handwerker oder Flugbegleiterin sind Muster, die in moderner Form und trotz geschlechtsneutraler Berufsbezeichnung in der nach Geschlecht segregierten Arbeitswelt weiterleben (vgl. Williams 1993).

\subsubsection{Berufliche Segregation nach Geschlecht}

Mit Segregation ist hier die Aufteilung der Berufe in männer- und frauentypische Berufe gemeint (vgl. Unterkapitel 1.1). Die berufliche Segregation nach Geschlecht ist nicht komplett oder ausnahmslos zu verstehen, die Verteilung in den jeweiligen Berufen kann ausgeglichen bis sehr stark zu einem Geschlecht verschoben sein. Es gibt eine Reihe von Berufen, die heute von beiden Geschlechtern ausgeführt werden. Das darf jedoch nicht darüber hinwegtäuschen, dass sogar in Berufen mit ausgeglichenem bzw. ausgeglichenerem Geschlechterverhältnis geschlechtstypische Nischen besetzt und zugeschrieben werden. So bleiben tradierte Rollenvorstellungen und Berufsbilder bestehen.

Die Aufteilung in weiblich und männlich konnotierte Berufe wird einerseits durch das wiederholte Praktizieren von Zuschreibungen und die Klassifikation in Geschlechter hergestellt und andererseits durch diese bedingt, unterstützt und gefördert. Das führt zu einem sich selbst aufrechterhaltender Kreislauf. Eine Mehrheit der jungen Frauen in der Schweiz wählt zum Beispiel einen Beruf aus den Bereichen Dienstleistung, Service, Pflege und Betreuung (vgl. Unterkapitel 1.1). Damit können sie dem weiblichen Geschlechterstereotyp entsprechen. Junge Männer wählen ebenso mehrheitlich einen Beruf, der mit den Geschlechtsrollenerwartungen einhergeht, vor allem im technischen und baulichen Bereich (Abraham/Arpagaus 2008, Charles/Grusky 2004, Gauthier/Gianettoni 2013, Leemann/Keck 2005). Damit ist die geschlechtliche Segregation ein wichtiges Merkmal der beruflichen Sozialisation. 


\subsubsection{Lernende in geschlechtsuntypischen Berufslehren}

Mit Lernenden in geschlechtsuntypischen Berufslehren sind Jugendliche gemeint, die sich in einem beruflichen Umfeld bewegen, in dem die Mehrheit der Lernenden im gleichen Beruf dem anderen Geschlecht angehört. Die Begriffspaare ,Frauen-/Männerberuf ‘ und ,frauen-/ männerdominiert' für Berufe, die starke geschlechtliche Mehrheitsverhältnisse aufweisen, sind unpräzis und verleiten zu Fehlschlüssen. So schließt das Begriffspaar ,Frauen-/Männerberuf ' das jeweils andere Geschlecht aus, was den Tatsachen widerspricht. Die verbreiteten Adjektive ,frauen-/männerdominiert' können den Eindruck erwecken, dass die Mehrheit die Minderheit dominiert, was insbesondere in frauenreichen Berufen deshalb nicht der Fall ist, weil die Kaderstellen oft von Männern besetzt sind (Lupton 2006). Darum werden in dieser Arbeit die Adjektive ,frauen-/männerreich` eingeführt. Damit können die Mehrheits- und Minderheitsverhältnisse benannt werden, ohne negative Konnotationen auszulösen oder Machtverhältnisse verzerrt darzustellen. Als Synonyme werden zusätzlich ,frauen-/männertypisch ‘ verwendet.

\subsection{Beschreibung des Modells der geschlechtsuntypischen Berufssozialisation}

Das Modell der geschlechtsuntypischen Berufssozialisation soll erklären, unter welchen Vorzeichen Lernende in geschlechtsuntypischen Berufslehren sozialisiert werden. Diese Lernenden haben zusätzliche Herausforderungen zu bewältigen, denen Jugendliche in geschlechtstypischen bzw. gemischtgeschlechtlichen Berufslehren nicht begegnen (vgl. Abbildung 4.1). Das Modell ist eine hypothetische Annahme. Sieben Teilbereiche des Modells versuche ich in den empirischen Kapiteln zu beweisen (vgl. Unterkapitel 4.3). Bevor das Modell im Detail und Schritt für Schritt in den Abschnitten 4.2.1 bis 4.2.6 erklärt und begründet wird, bietet eine Kurzbeschreibung des Modells einen ersten Überblick. Das Beispiel einer jungen Frau, die Maurerin werden möchte, illustriert anschließend die folgende Beschreibung.

Das Modell der geschlechtsuntypischen Berufssozialisation hat drei Ebenen: 1) ,Gesellschaft; Arbeitswelt', 2), soziales Umfeld des Individuums ' und 3) ,Individuum ‘. Auf der Makroebene ,Gesellschaft; Arbeitswelt ' bilden zwei Prämissen die Grundlage des Modells. Erstens sind der Arbeitsmarkt und der Berufsbildungsmarkt nach Geschlecht segregiert (vgl. Unterkapitel 1.1). Zweitens werden an Frauen und Männer unterschiedliche Erwartungen an ihre Geschlechtsrolle gestellt, sogenannte Geschlechtsrollenerwartungen (vgl. Unterkapitel 2.5). Von Frauen erwartet die Gesellschaft, dass sie einem frau- 
entypischen Beruf nachgehen, während Männer einen männertypischen Beruf ausüben sollen. Dies führt dazu, dass die Berufe nach Geschlecht segregiert sind. Die Segregation nach Geschlecht wiederum teilt Berufe in frauen- bzw. männertypische Berufe ein und erzeugt geschlechtsbezogene Rollenerwartungen. Die ,berufliche Segregation nach Geschlecht ${ }^{\star}$ und ,Geschlechtsrollenerwartungen " erzeugen und stützen sich gegenseitig. Sie bilden einen Kreislauf, der sich selbst erhält.

Abbildung 4.1: Modell der geschlechtsuntypischen Berufssozialisation

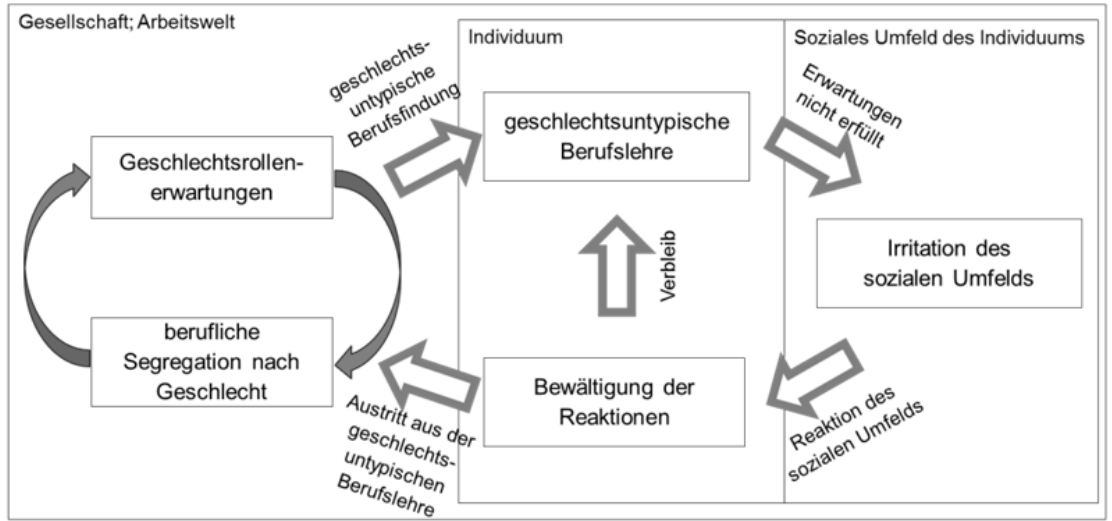

Quelle: Eigene Darstellung.

Dieser Kreislauf auf der Makroebene wirkt sich auf die Dynamiken auf der Mikroebene zwischen dem Individuum und seinem jeweiligen sozialen Umfeld aus. Sobald nun ein ,Individuum ' (hier eine Lernende bzw. ein Lernender) eine ,geschlechtsuntypische Berufsfindung' durchlebt und eine ,geschlechtsuntypische Berufslehre beginnt, verlässt es den Kreislauf ,berufliche Segregation nach Geschlecht - Geschlechtsrollenerwartungen". Der Kreislauf bleibt jedoch aktiv, obwohl das Individuum diese gesellschaftlichen Erwartungen nicht mehr erfüllt. Personen des ,sozialen Umfelds ${ }^{\varsigma}$ des Individuums haben dem Kreislauf entsprechend Erwartungen an das Geschlechtsrollenverhalten: Eine Frau hat einen frauentypischen und ein Mann einen männertypischen Beruf zu ergreifen bzw. auszuüben. Wegen der geschlechtsuntypischen Berufsfindung des Individuums werden bei diesen Personen die ,Erwartungen nicht erfüllt ${ }^{\star}$ und es erfolgt eine ,Irritation des sozialen Umfelds'. Diese Irritation löst entweder Zustimmung oder Ablehnung aus. Es erfolgt die ,Reaktion des sozialen Umfelds ${ }^{\star}$ gegenüber dem Individuum in der geschlechtsuntypischen Berufslehre. Das Individuum versucht mit der Reaktion des sozialen Umfelds umzugehen: ,Bewältigung der 
Reaktion'. Reichen die Ressourcen aus, führt das Individuum den geschlechtsuntypischen Beruf fort: ,Verbleib'. Weil die Geschlechtsrollenerwartungen des sozialen Umfelds weiterhin nicht erfüllt werden, halten die Irritationen und Reaktionen an und bilden so einen zweiten Kreislauf. Jedoch können sich die Personen des sozialen Umfelds an die geschlechtsuntypische Situation des Individuums gewöhnen und die Irritationen sich damit abschwächen. Aufgrund der positiven Erfahrungen können sich die Geschlechtsrollenerwartungen modifizieren und die negativen Reaktionen einstellen. Oder aber das Individuum stößt weiterhin auf Ablehnung und muss persönliche Stärke zeigen und Anstrengung für die Bewältigung der negativen Reaktionen aufbringen. Diese Prozesse bilden also den zweiten Kreislauf, der so lange erhalten bleibt, wie das Individuum in der geschlechtsuntypischen Berufslehre bleibt. Übersteigen die Belastungen jedoch die Ressourcen, verlässt das Individuum diesen zweiten Kreislauf. Es kommt zum ,Austritt aus der geschlechtsuntypischen Berufslehre'. Das Individuum beteiligt sich wieder am ersten Kreislauf , berufliche Segregation nach Geschlecht Geschlechtsrollenerwartungen'.

Das nun folgende Beispiel von Lara, die Maurerin lernen möchte, veranschaulicht die Stationen und die Dynamiken im Modell der geschlechtsuntypischen Berufssozialisation. Es ist eine Konstruktion aus verschiedenen Aussagen, die Lernende in geschlechtsuntypischen Berufslehren in den Interviews (vgl. Kapitel 9) gemacht haben. Die bildhafte Sprache des Beispiels spiegelt teilweise die klischeehaften Schilderungen der Interviewten wider.

Mit der Entscheidung, Maurerin zu werden, widerspricht Lara den Geschlechtsrollenerwartungen ihres sozialen Umfelds. Die Arbeit auf einer Baustelle wird vom sozialen Umfeld entsprechend dem männlichen Stereotyp mit körperlicher Arbeit, Lärm, Schmutz und rüden Umgangsformen in Verbindung gebracht. In einer nach Geschlecht segregierten Arbeitswelt löst Laras Äußerung, Maurerin werden zu wollen, Reaktionen bei Eltern, Lehrpersonen und Mitschülerinnen aus. Laras Mutter hat wegen der körperlichen Arbeit Angst um ihre Tochter. Ihr Vater sieht sie all den rauen Umgangsformen auf der Baustelle ausgesetzt. Ihre Freundinnen finden Laras Entscheidung mutig, äußern aber Bedenken wegen des Schmutzes und der Schwielen an den Händen. Sogar ihre Lehrerin will sie überzeugen, doch Schreinerin zu lernen, weil dieser Beruf in einer Werkstatt ausgeübt wird. Doch Lara bleibt bei ihrer Entscheidung, da sie davon träumt, einmal Kranführerin zu werden und den ganzen Bauprozess von oben beobachten zu können.

Körperliche Arbeit und despektierliche Sprüche verunsichern Lara nicht. Auf der Baustelle ist Lara als einzige Frau unter vielen Männern sofort zu erkennen. Kommt sie auf eine neue Baustelle, auf der sie noch unbekannt ist, schleppt sie manchmal demonstrativ schwere Gegenstände herum, was ihr meist Anerkennung bei den Männern einbringt. Was sie jedoch immer mehr ärgert, ist, dass ihr niemand zutraut, ihr Fachgebiet zu kennen. Sie spürt, dass 
sie anders behandelt wird. Immer wieder hört sie den einleitenden Kommentar: „Du als Frau ..... Die männlichen Lernenden in ihrem Alter werden selbstverständlich für alle anfallenden Arbeiten eingeteilt, während für sie immer etwas Spezielles gesucht wird. Kunden oder Arbeiter anderer Firmen wenden sich, wenn sie etwas wissen wollen, nicht an sie, sondern an ihre Mitarbeiter. Im ersten Lehrjahr war ihr klar, dass sie noch viel lernen musste. Aber auch jetzt, kurz vor der Lehrabschlussprüfung und obwohl sie eine der besten in ihrer Klasse an der Berufsfachschule ist, traut ihr Vorgesetzter ihr wenig zu.

Zwei andere junge Frauen haben mit Lara die gleiche Klasse der Berufsfachschule besucht. Doch sie haben schon im ersten Ausbildungsjahr die Berufslehre abgebrochen. Mittlerweile denkt Lara oft darüber nach, ob es nicht besser gewesen wäre, ebenfalls einen geschlechtstypischen Beruf zu lernen. Sie fragt sich, ob ihr je die verantwortungsvolle Aufgabe auf einem Baukran anvertraut wird.

Das Beispiel veranschaulicht, wie unterschiedlich - und zum Teil widersprüchlich - die Reaktionen des sozialen Umfelds sein können. Die Lernenden bleiben im geschlechtsuntypischen Beruf, solange die Ressourcen reichen und der Beruf Spaß macht. Doch die Reaktionen hören nicht plötzlich auf, obwohl sich einige Personen des sozialen Umfelds an die Situation gewöhnen. Manche Jugendliche setzen sich den Reaktionen nicht aus und lernen einen geschlechtstypischen Beruf (vgl. Williams 1997) oder geben mit der Zeit auf (vgl. Drehtür-Effekt). Die Details dieses Modells der geschlechtsuntypischen Berufssozialisation werden nun in Teilprozessen beschrieben: ,Geschlechtsrollenerwartungen - berufliche Segregation nach Geschlecht' (Abschnitt 4.2.1), ,Geschlechtsuntypische Berufslehre' (Abschnitt 4.2.2), ,Irritation des sozialen Umfelds' (Abschnitt 4.2.3), ,Reaktion und Bewältigung' (Abschnitt 4.2.4), ,Verbleib in der geschlechtsuntypischen Berufslehre" (Abschnitt 4.2.5) und ,Austritt aus der geschlechtsuntypischen Berufslehre' (Abschnitt 4.2.6).

\subsubsection{Geschlechtsrollenerwartungen - berufliche Segregation nach Geschlecht}

Zwei Annahmen bilden diesen ersten Kreislauf, Geschlechtsrollenerwartungen - berufliche Segregation nach Geschlecht (vgl. Abbildung 4.1). Erstens ist die Arbeitswelt nach Geschlecht aufgeteilt. Viele Berufe werden mehrheitlich von nur einem der beiden Geschlechter ausgeführt oder werden einem Geschlecht zugeordnet. Zweitens gibt es nach wie vor relativ strikte Geschlechtsrollenerwartungen. Der erste Kreislauf postuliert die Dynamik, dass die Geschlechtsrollenerwartung und die geschlechtliche Segregation der Arbeitswelt sich gegenseitig stützen. Sie produzieren und reproduzieren die 
tradierten Verhältnisse. Das bedeutet, dass einerseits die Ursachen dafür, wie sich die beiden Geschlechter verhalten, stark durch die Erwerbstätigkeit und die darin enthaltene Aufteilung der Arbeit nach Geschlecht bestimmt und definiert sind. Andererseits wählen die meisten Personen aufgrund der Geschlechtsrollenerwartungen, die sie seit ihrer Kindheit verinnerlichen, ihre berufliche Tätigkeit aus. Dieser erste Kreislauf wirkt stabilisierend auf die Erwartungen an das Geschlechtsrollenverhalten und auf die beruflichen Ambitionen. Er symbolisiert die gesellschaftlichen Phänomene in Bezug auf das Geschlecht und auf das Geschlechterverhältnis. Die Ausführungen in Kapitel 2 prägnant zusammenfassend können dazu fünf zentrale Dynamiken festgehalten werden: 1) Die Zweigeschlechtlichkeitsnorm verfestigt die dichotome und unflexible Sicht auf das Geschlecht. 2) Durch das Doing Gender wird Geschlecht produziert und reproduziert. 3) Die Zuschreibungen von Eigenschaften und Fähigkeiten äußern sich in Geschlechterstereotypen. 4) Es besteht nach wie vor in manchen, vor allem in beruflichen Bereichen eine Hierarchie zwischen den Geschlechtern: Frauen und Männer werden nicht gleichwertig behandelt. 5) Es besteht ein Gleichheitstabu: Frauen und Männer sollen sich unterscheiden und etwa unterschiedlichen Berufen nachgehen. Diese gesellschaftlichen Prozesse oder Phänomene stützen und erhalten die geschlechtliche Segregation und damit die Möglichkeit geschlechtsuntypischen Berufs. Damit wird deutlich, dass die Sozialisation von Lernenden in geschlechtsuntypischen Berufslehren unter anderen Prämissen stattfindet als diejenige von Lernenden in geschlechtstypischen Berufslehren. Mit der geschlechtsuntypischen Berufsfindung handelt ein Individuum entgegen den gesellschaftlichen Geschlechtsrollenerwartungen und tritt quasi aus dem ersten Kreislauf aus, ohne dass dieser deshalb seinen Einfluss auf das Individuum oder sein soziales Umfeld verlöre.

\subsubsection{Geschlechtsuntypische Berufslehre}

Die Berufsfindung (vgl. Unterkapitel 1.3) wird als Prozess verstanden, der in der Familie und in der Schule vorbereitet wird. Die Berufsfindungstheorie von Gottfredson (1981) besagt, dass das Geschlecht neben dem Interesse und der Eignung ebenfalls eine Entscheidungsgrundlage bietet, ob sich eine Person für oder gegen einen Beruf entscheidet (vgl. Busshoff 2009, Imdorf 2005, Junk/Armstrong 2010). Interessiert sich ein Individuum für einen Beruf, der öfter von Personen des andern Geschlechts ausgeführt wird, dann tritt es aus dem Kreislauf, Geschlechtsrollenerwartungen - berufliche Segregation nach Geschlecht ${ }^{\star}$ aus. Die Unterschiede zwischen Berufen mit der Konnotation als frauentypisch bzw. männertypisch sind erheblich und ihre Wahrnehmung entspricht nicht immer den tatsächlichen Verhältnissen. So kann sich das Geschlechterverhältnis bei Berufen ändern, wie bei Lehrpersonen oder Ärz- 
tinnen/Ärzten, oder der Beruf wird zwar von vielen Frauen ausgeübt, wurde traditionell aber von Männern ausgeführt und bleibt deshalb männlich konnotiert, wie bei den Berufen Tramchauffeur/-chauffeuse oder Maler/-in.

Jugendliche in geschlechtsuntypischen Berufslehren sind eine Minorität. Erb und Bohner (2006) formulieren drei Definitionen für Minoritäten, von denen zwei hier relevant sind: 1) Minoritäten sind gesellschaftliche Subgruppen, die sich in einem oder mehreren Merkmalen von der Majorität unterscheiden, als minderwertig angesehen und darum abgelehnt werden (Randgruppen). Sie können entweder zahlenmäßig in der Minderheit sein (etwa Homosexuelle in der Gesamtbevölkerung oder Juden außerhalb von Israel) oder zu Gruppen zählen, die zwar arithmetisch ähnlich groß sind (z.B. Frauen), aber mit weniger Machtressourcen ausgestattet sind. Minoritäten haben im Vergleich zur Majorität einen niedrigeren Status, geringere Attraktivität, einen Mangel an Macht und weniger ökonomische Ressourcen. 2) Minoritäten können in der gleichen Gruppe wie die Majorität sein, aber eine abweichende Meinung (z.B. linker Parteiflügel) oder eine deviante Einstellung haben. 3) Minoritäten, die tatsächlich eine numerisch kleine Gruppe sind. Als seltene Exemplare haben diese Minderheiten hohe Distinktheit und hohe Salienz. Einer Minderheit anzugehören, die einem negativen Stigma unterworfen ist, ist für das Individuum subjektiv negativer, als Teil einer negativ bewerteten Majorität zu sein: „Die Bewertung der Eigenschaften einer Minorität ist extremer als die Bewertung der Eigenschaften einer Majorität“ (Erb/Bohner 2006: 498).

Die Definitionen, ab welchem Geschlechterverhältnis von einem typischen bzw. untypischen Beruf gesprochen werden kann, werden unterschiedlich gehandhabt (vgl. Kapitel 3). Die subjektive Wahrnehmung der Minderheitsposition ist von der lokalen Gegebenheit geprägt: Einerseits spielen Merkmale des Berufs und seiner Umgebung (etwa Elektroninstallateurin auf der Baustelle bzw. Florist im Blumenladen) eine Rolle, andererseits die Verhältnisse im jeweiligen Lehrbetrieb und das individuelle Verhalten der beteiligten Personen. Für die Reaktion des sozialen Umfelds (in diesem Fall der Majorität) sind jedoch nicht nur die spezifischen lokalen Gegebenheiten ausschlaggebend, sondern zusätzlich die Bilder, Muster und Vorstellungen, die die involvierten Personen haben.

\subsubsection{Irritation des sozialen Umfelds}

Die Erwartungen des sozialen Umfelds an das Rollenverhalten werden dann nicht erfüllt, wenn ein Individuum dem sich selbst erhaltenden ersten Kreislauf ,Geschlechtsrollenerwartungen - berufliche Segregation nach Geschlecht nicht entspricht, indem es eine geschlechtsuntypische Berufslehre absolviert. Das soziale Umfeld umfasst Menschen und Personengruppen, die 
mit dem Individuum, hier Lernende in geschlechtsuntypischen Berufslehren, zu tun haben. Dazu gehören Personen aus dem privaten (Familie, Verwandte, Bekannte, Freundinnen und Freunde und deren Familienangehörige etc.), dem schulischen (Schulkolleginnen und -kollegen, Lehrpersonen, Berufsberatende etc.) und dem beruflichen Umfeld (Ausbildende, Vorgesetzte, Mitarbeitende, Mitlernende, Kundinnen und Kunden, Klientinnen und Klienten, Bewohnende und ihre Angehörigen, Mitarbeitende anderer Unternehmungen wie etwa auf Baustellen, Passantinnen und Passanten wie z.B. bei Straßenarbeitenden etc.). Je näher und intensiver der Kontakt zwischen dem Individuum und einzelnen Vertretenden des sozialen Umfelds ist, desto stärker dürfte der Einfluss auf die Wahrnehmung der Situation durch das Individuum sein. Die Reaktionen des sozialen Umfelds können unterstützend und wohlwollend sein, oft sind sie jedoch ablehnend. Beide Reaktionen, die positiven wie die negativen, sind Folgen des Bruchs mit der Rollenerwartung und der damit verbundene Irritation.

\subsubsection{Reaktion und Bewältigung}

Weil die Bewertung der Eigenschaften einer Minderheit nach Erb und Bohner (2006) verzerrter ist als die Bewertung der Eigenschaften von Mehrheiten, aktiviert der Minoritätsstatus Stereotype (vgl. Hamilton/Gifford 1976, Unterkapitel 2.6). Denn wenn das Geschlecht im jeweiligen Kontext relevant ist, werden geschlechterspezifische Stereotype eher abgerufen: „Je stärker Geschlecht in einer konkreten Interaktionssituation hervorgehoben ist, desto wahrscheinlicher sind die Personen von Geschlechterstereotypen beeinflusst" (Hannover 2006: 469).

Für Frauen und Männer fallen die Reaktionen unterschiedlich aus. Frauen in einem männerreichen beruflichen Umfeld kann unter Umständen die Kompetenz, diese Arbeit gut auszuführen, abgesprochen werden (z.B. Kanter 1977 2006). Eher Anerkennung und Bewunderung erhalten sie hingegen durch ihre Peergruppe oder allgemein von Personen außerhalb des beruflichen Umfelds (Faulstich-Wieland 2016). Bei den Männern in frauenreichen Berufen verhält es sich gerade umgekehrt: Bei ihnen wird weniger die berufliche Kompetenz in Frage gestellt als vielmehr ihre Männlichkeit angezweifelt (Faulstich-Wieland 2016, Simpson 2005). Williams (1993) beschreibt die Situation von Männern in geschlechtsuntypischen Berufen so:

The man who crosses over into a female-dominated occupation upsets these gender assumptions embedded in the work. Almost immediately, he is suspected of not being a ,real man': There must be something wrong with him (,Is he gay? Effeminate? Lazy?') for him to be interested in this kind of work. If these popular prejudices are not enough to push him out of his occupation, they will certainly affect how he manages his gender identity on a daily basis. (Williams 1993: 3) 
Männer in geschlechtsuntypischen Berufen müssen mit selbstwertgefährdenden Vorurteilen rechnen. Beispielsweise wird ihnen unterstellt, verweichlicht, faul oder homosexuell zu sein (Williams 1993). Vorurteile sind insbesondere in Berufen mit kleinen Kindern vorhanden (Buschmeyer 2013, Fellmann 2014).

Die negativen Reaktionen des sozialen Umfelds können in Form von Diskriminierung auftreten. Die meisten Definitionen von Diskriminierung beinhalten die Herabwürdigung und die Benachteiligung aufgrund der Zugehörigkeit zu einer sozialen Kategorie. Einschlägig ist die klassische Definition von Allport (1971). Er thematisiert die Relevanz für die Benachteiligten und die Unzulänglichkeit der Kriterien, aufgrund derer die Diskriminierung entsteht: „Diskriminierung liegt vor, wenn einzelnen oder Gruppen von Menschen die Gleichheit der Behandlung vorenthalten wird, die sie sich wünschen" (Allport 1971: 64). Dabei sind jedoch Ungleichbehandlungen aufgrund von individuellen Eigenschaften oder Verhaltensweisen ausgenommen, z.B. im Fall von Verbrechen. Allport (1971: 64) stützt sich dabei auf ein UN-Memorandum, das besagt: „Diskriminierung umfasst alles Verhalten, das auf Unterschieden sozialer oder natürlicher Art beruht, die keine Beziehung $\mathrm{zu}$ individuellen Fähigkeiten oder Verdiensten haben noch zu dem wirklichen Verhalten der individuellen Person."

Die Einteilung in eine soziale Kategorie wie z.B. Alter, Geschlecht, Nationalität, Herkunft, Ethnie, sexuelle Orientierung etc. ist an sich noch keine Diskriminierung, sondern erst die ungerechtfertigte Benachteiligung aufgrund dieser.

\footnotetext{
Diskriminierung wird als Handlung verstanden, als eine registrierbare Folge individuellen Handelns, die eingetreten ist, weil Akteure andere Akteure aufgrund wahrgenommener sozialer oder ethnischer Merkmale als ungleiche bzw. minderwertige Partner angesehen und, im Vergleich zu den Angehörigen des eigenen Kollektivs, entsprechend abwertend behandelt haben. (Markefka 1995: 43)
}

Die Diskriminierung hat viele Erscheinungsformen: bewusste und unbewusste, unmittelbare und mittelbare, alltägliche und strukturelle sowie individuelle und institutionelle Diskriminierung. Mit institutioneller Diskriminierung ist die Benachteiligung durch Organisationen wegen überindividueller Sachverhalte gemeint (Hasse/Schmidt 2012). In Abgrenzung dazu ist die soziale Diskriminierung eine Benachteiligung von Personen aufgrund einer ablehnenden Beurteilung (Wagner et al. 1990) und bezieht sich auf die Benachteiligung einer Person aufgrund ihrer Gruppenzugehörigkeit (Van Dick 2006).

Das Individuum kann gar nicht anders, als auf die Reaktionen des sozialen Umfelds zu reagieren. Sogar das Ignorieren diskriminierender Äußerungen ist eine Reaktion. Durch Gespräche und Begegnungen können sich aufseiten der Majorität Verständnis oder Gewöhnung entwickeln, sodass die negativen Reaktionen mit der Zeit abnehmen oder sogar positiv werden. Die Qualität und den Verlauf dieser Interaktionen können die Individuen beein- 
flussen. Dies fällt ihnen umso leichter, je mehr Ressourcen sie dafür zur Verfügung haben.

\subsubsection{Verbleib in der geschlechtsuntypischen Berufslehre}

Das Modell der geschlechtsuntypischen Berufssozialisation besagt, dass das Individuum so lange in der geschlechtsuntypischen Situation bleibt, wie die Ressourcen des Individuums zur Bewältigung der negativen Reaktionen des sozialen Umfelds ausreichen. Das bedeutet wiederum, dass das Individuum weiterhin mit den negativen Reaktionen im sozialen Umfeld umgehen muss. Da sich Lernende in geschlechtsuntypischen Berufslehren permanent mit den Diskriminierungen aufgrund des Geschlechts auseinandersetzen müssen und die Bewältigung zusätzliche Kraft kostet, sind sie gegenüber Lernenden in geschlechtstypischen oder geschlechtergemischten Berufslehren im Nachteil. Nach Imdorf (2012a) benötigen junge Frauen folgende Ressourcen, um in einer männerreichen Berufslehre im Autogewerbe Erfolg zu haben:

[...] Bewusstsein für die ,Männerwelt‘ im Betrieb; Wille, sich in diese zu integrieren;
Durchsetzungsvermögen; psychische Stabilität und Belastbarkeit; überdurchschnittliche
Leistungsfähigkeit; und die Unterstützungsbereitschaft der Eltern. Eine wichtige Res-
source ist zudem ein für die Geschlechterfrage sensibilisierter Ausbildner, der die jun-
gen Frauen vor den Zumutungen männlicher Mitarbeiter zu schützen weiß. (Imdorf
2012a: 260)

Allgemein gesprochen sind Ressourcen Erfahrungen, Überzeugungen, Einstellungen, Interpretationen, Ereignisse, Verhaltensweisen und zwischenmenschliche Gewohnheiten, die zu einer positiven Wahrnehmung des eigenen Lebens beitragen (Ray/Keeney 1993). Ressourcen unterstützen eine Person dabei, ihre Ziele zu erreichen, und haben eine ,potenzielle positive Wirkung“ (Alpers 2005: 335). Ressourcen wie soziale Unterstützung oder mentale Fähigkeiten können als spezifische Bewältigungsstrategien operationalisiert werden (vgl. Alpers 2005). Eine pädagogische Sichtweise brachten bereits Cronbach und Snow (1977) ein, indem sie bei der Förderung von Schülerinnen und Schülern gezielt auf den Aufbau von bereits vorhandenen Stärken und Fähigkeiten verwiesen.

Alpers (2005) unterscheidet Ressourcen, die sich in der Person finden, von solchen in der Umwelt. Zu den Ressourcen in der Person zählen etwa Selbstwert, Intelligenz, Optimismus, Flexibilität, emotionale Offenheit, Belastbarkeit, Selbstwirksamkeitserwartung und Zielstrebigkeit. Zu den Ressourcen in der Umwelt gehören soziökonomischer Status, stabile soziale Netzwerke, emotionale und soziale Unterstützung.

Bandura (1997) beschreibt die Selbstwirksamkeitserwartung als die Überzeugung, Erfolg in einer persönlich wichtigen Tätigkeit zu haben. Spezifisch für die vorliegende Arbeit wäre demnach die Einschätzung, sich im berufli- 
chen Umfeld bewähren zu können. Bei der sozialen Unterstützung spielt vor allem die eigene Wahrnehmung der Unterstützung durch das soziale Umfeld eine wichtige Rolle (Sarason et al. 1987), denn sie führt dazu, dass Stress weniger stark erlebt wird und dass weniger Stresshormone ausgeschüttet werden (Heinrichs et al. 2003).

Im Jugendalter bildet die soziale Unterstützung eine der wichtigsten Ressourcen, da die Unsicherheiten aufgrund von biografischen und körperlichen Veränderungen in dieser Lebensphase besonders bedeutsam sind, ebenso die Abhängigkeit von Urteilen aus dem sozialen Umfeld. Laireiter (1993, 2006) unterscheidet zwei Klassen von sozialer Unterstützung: 1) Zur psychologischen Unterstützung zählen Zugehörigkeit, Rückhalt, kognitive Unterstützung, Verlass und Selbstwertunterstützung. 2) Die instrumentelle Unterstützung enthält Information, Ratschläge, tatkräftige Hilfe, Intervention und finanzielle Unterstützung. Soziale Unterstützung beeinflusst das allgemeine Wohlbefinden und die psychische Gesundheit (Cohen/Wills 1985). Darum wird in Kapitel 8 der vorliegenden Arbeit untersucht, ob sich Lernende in geschlechtsuntypischen Berufslehren von anderen Lernenden bei den psychosomatischen Beschwerden unterscheiden.

Die soziale Unterstützung wirkt sich für Lernende in geschlechtsuntypischen Berufslehren auf mehreren Ebenen positiv aus. Insgesamt dürfte es ihnen leichter fallen, die Diskriminierungserfahrungen $\mathrm{zu}$ verarbeiten und sich auf die Ausbildung zu konzentrieren, wenn sie sich auf die Hilfe und Unterstützung ihres sozialen Umfelds verlassen können. Die Ressourcen für die Bewältigung der Diskriminierungserfahrungen können zwar ausreichen, um mit der geschlechtsuntypischen Berufslehre weiterzufahren und damit die eigenen beruflichen Interessen umzusetzen, jedoch könnten sie dann an anderen Orten fehlen. Beispielsweise könnte die berufliche Integration erschwert sein, da sich das Individuum nicht wirklich zugehörig oder gar abgelehnt fühlt (vgl. beruflicher Erfolg im Selbstbericht in Kapitel 7), beim Lernen können als Konsequenz die Leistungen sinken (vgl. Lehrvertragsauflösung in Kapitel 5 bzw. Lehrabschlussprüfung in Kapitel 6), oder es kann die Gesundheit darunter leiden (vgl. Hormel 2010, psychosomatische Beschwerden und Absenzen in Kapitel 8).

\subsubsection{Austritt aus der geschlechtsuntypischen Berufslehre}

Nach dem Modell der geschlechtsuntypischen Berufssozialisation kommt es zur Aufgabe des Berufs bzw. zu einer vorzeitigen Auflösung des Lehrvertrags, wenn der Druck aufgrund von Diskriminierungen durch das soziale Umfeld die Ressourcen des Individuums übersteigt. Das Individuum wechselt z.B. in eine geschlechtstypische Berufslehre, verhält sich damit wieder den Geschlechtsrollenerwartungen entsprechend und beteiligt sich wieder am 
Kreislauf ,Geschlechtsrollenerwartungen - berufliche Segregation nach Geschlecht'.

Das Modell der geschlechtsuntypischen Berufssozialisation postuliert, dass sich die negativen Reaktionen des sozialen Umfelds auf die Verweildauer der Lernenden in ihren Berufslehren auswirken. Jugendliche geben ihre geschlechtsuntypischen beruflichen Ambitionen eher auf als andere Gleichaltrige ihr geschlechtstypischen (vgl. Gottfredson 1981, Kapitel 5). Wie in Kapitel 3 beschrieben, wechseln Erwachsene in geschlechtsuntypischen Berufen ebenfalls häufig nach kurzer Zeit wieder in geschlechtstypische Berufe (Williams 1993, Simpson 2005). Diese Phänomene nennt Williams (1992, 1993) Drehtür- bzw. Glaslift-Effekt. Der Drehtür-Effekt (,revolving door pattern of mobility“, vgl. Jacobs 1993: 50) besagt, dass Personen zwar geschlechtsuntypische Berufe beginnen, sie aber in kurzer Zeit wieder aufgeben. Der Glaslift-Effekt (,glass escalator effect“, vgl. Williams 1992: 227) kommt lediglich bei Männern in geschlechtsuntypischen Berufen vor und bezeichnet - in Anlehnung an den ,glass ceiling effect“, wonach eine gläserne Decke Frauen daran hindert, in der Karriere weiterzukommen - die Situation, dass Männer wie in einem unsichtbaren Lift befördert werden, da die Vorgesetztenposition eher dem männlichen Stereotyp entspricht.

\subsection{Forschungsfrage und Hypothesen}

Im Bereich der geschlechtsuntypischen Berufsbildung gibt es im englischen Sprachraum zurzeit nur wenige quantitative Forschungsarbeiten (u.a. Flores et al. 2006, Jome et al. 2005, Junk/Armstrong 2010, McLean/Kalin 1994, Rochlen et al. 2010). Das ist wohl auf die jeweils geringen Fallzahlen von geschlechtsuntypischen Berufsleuten und die geringe Verbreitung von dualen Berufsausbildungen zurückzuführen. Für den deutschen Sprachraum - und insbesondere für die Schweiz - sind vor allem zwei Forschungsarbeiten zu erwähnen, die zu den ersten publizierten gehören und sich mit diesen Fragen befassten: Der Befund von Althoff (1992) zur erhöhten Lehrvertragsauflösung bei Jugendlichen in geschlechtsuntypischen Berufslehren und die Forschungsarbeiten von Borkowsky (2000) zur starken Segregation in der Schweizer Berufsbildung. Im letzten Jahrzehnt erschien eine Reihe von quantitativen und qualitativen Studien mit Schweizer Daten zu diesem Thema ${ }^{21}$ : Bieri Buschor et al. 2012, Buchmann/Kriesi 2012, Gauthier/Gianettoni 2013, Gianettoni et al. 2010, Hadjar/Aeschlimann 2015, Hupka-Brunner/Kriesi 2013, Ignaczewska 2014, Imdorf 2012a, Lamamra 2011, Lamam-

21 Die Auflistung ist nicht abschließend; sie ist eine Zusammenstellung der in der vorliegenden Arbeit zitierten Literatur. 
ra/Masdonati 2009, Maihofer et al. 2012, 2013, Maihofer/Schwiter 2013, Makarova/Herzog 2014, Schwiter et al. 2011, Schwiter et al. 2014, Wehner et al. 2016.

Im zweiten Teil der vorliegenden Arbeit (Kapitel 5 bis 9) werde ich Teile des in den Unterkapiteln 4.1 und 4.2 vorgestellten theoretischen Modells quantitativ und qualitativ überprüfen. Im quantitativen Teil werden Auswertungen dargestellt, wie erfolgreich Lernende in geschlechtsuntypischen Berufslehren sind, ob sie häufiger ihre Ausbildung vorzeitig beenden (Lehrvertragsauflösung), ob sie mit einer größeren Wahrscheinlichkeit von Misserfolg an der Lehrabschlussprüfung betroffen sind, ob es Unterschiede bei der Sozialisation sowie gesundheitlichen Aspekten gibt. Dazu werden Auswertungen mit Populationsdaten des BFS und zwei bestehenden Datensätzen aus den Projekten ,Familie Schule Beruf FASE B' und ,Transitionen von der Erstausbildung ins Erwerbsleben TREE' reanalysiert. Die Forschungsfrage der vorliegenden Arbeit ,Inwiefern sind Lernende in geschlechtsuntypischen Berufslehren benachteiligt?' wird anhand von vier Hypothesen, die sich aus dem Modell der geschlechtsuntypischen Berufssozialisation ergeben, mittels quantitativen Verfahren getestet.

Hypothese 1: Lehrvertragsauflösung: Die Lernenden in geschlechtsuntypischen Berufslehren müssen mit dem Alleinstellungsmerkmal umgehen und die damit verbundenen positiven und negativen Reaktionen des sozialen Umfelds bewältigen. Reichen ihre Ressourcen dafür nicht aus, beenden sie ihre Ausbildung vorzeitig und suchen sich etwas anders. Wenn dem so ist, dann lösen mehr Lernende in geschlechtsuntypischen Berufslehren ihre Lehrverträge auf als andere Lernende. Die Hypothese 1 prüft den Pfad ,Austritt aus der geschlechtsuntypischen Berufslehre', wird in Kapitel 5 bearbeitet und lautet: Lernende in geschlechtsuntypischen Berufslehren sind häufiger von Lehrvertragsauflösung betroffen als Lernende in gemischtgeschlechtlichen bzw. geschlechtstypischen Berufslehren.

Hypothese 2: Lehrabschlussprüfung: Eine weitere Auswirkung der negativen Reaktionen besteht darin, dass die Ressourcen für ihre Bewältigung bei der Vorbereitung der Lehrabschlussprüfung fehlen und dass die Selbstwirksamkeit untergraben wird. Die Hypothese 2 prüft die ,Bewältigung der Reaktionen" in Kapitel 6. Sie heißt: Lernende in geschlechtsuntypischen Berufslehren scheitern häufiger an der Lehrabschlussprüfung als Personen in gemischtgeschlechtlichen bzw. geschlechtstypischen Berufslehren.

Hypothese 3: Selbstberichtete Kriterien des Erfolgs in der Berufslehre: Der Hypothese 3 wird in Kapitel 7 nachgegangen, sie prüft ebenfalls die Auswirkungen der ,Bewältigung der Reaktionen ' des sozialen Umfelds. Die Vergleiche von Erfolgskriterien aus der selbstberichteten Einschätzung der Lernenden sollen Differenzen zwischen Lernenden in typischen und untypischen Berufslehren ausweisen. Die Hypothese 3 ist: Lernende in geschlechts- 
untypischen Berufslehren schätzen sich selbst als weniger erfolgreich ein als Lernende in geschlechtstypischen Berufslehren.

Hypothese 4: Einfluss des sozialen Umfelds: Die Reaktion des sozialen Umfelds ruft Bewältigungsreaktionen hervor. Diese werden durch das Verhalten des sozialen Umfelds mediiert. Es wird der Mediationseffekt durch den Pfad über die ,Irritation des sozialen Umfelds' untersucht. Die Hypothese 4 wird in Kapitel 8 getestet. Sie lautet: Der Einfluss der geschlechtsuntypischen Berufslehre auf die Bewältigung wird durch die Wahrnehmung von Diskriminierung und/oder sozialer Unterstützung vermittelt.

Hypothese 5: Psychische und körperliche Auswirkungen: Die Hypothese 5 überprüft ebenfalls Auswirkungen der negativen Reaktionen des sozialen Umfelds. Diskriminierungserfahrungen beeinträchtigen die Gesundheit der Lernenden in geschlechtsuntypischen Berufslehren. Sie überprüft die ,Bewältigung der Reaktionen" in Kapitel 8: Lernende in geschlechtsuntypischen Berufslehren haben mehr psychosomatische Beschwerden und eine erhöhte Absenzenquote im Vergleich zu anderen Lernenden.

Im qualitativen Teil werden Interviews mit Lernenden in geschlechtsuntypischen Berufslehren analysiert, um herauszufinden, was sie über die Themen Berufsfindung, Belastungen, Ressourcen und Doing Gender denken. In Kapitel 9 werden ausgehend von der globalen Fragestellung vier Fragen untersucht. Die globale Fragestellung lautet: Berichten Lernende in geschlechtsuntypischen Berufslehren über spezifische Schwierigkeiten und Herausforderung während der Berufslehre?

Frage A befasst sich mit dem Pfad, geschlechtsuntypische Berufsfindung und lautet: Wie verlief der Berufsfindungsprozess im Hinblick auf das untypische Geschlecht?

Frage B untersucht die Belastungen aufgrund der, Reaktionen des sozialen Umfelds'. Sie heißt: Von welchen Belastungen in Zusammenhang mit dem untypischen Geschlecht berichten sie?

Frage $\mathbf{C}$ thematisiert die Ressourcen, die Lernende in geschlechtsuntypischen Berufslehren benötigen, um die Berufslehre zu beenden. Sie untersucht den Pfad ,Verbleib' und heißt: Über welche Ressourcen verfügen die Jugendlichen, die ihnen das Bestehen in der geschlechtsuntypischen Berufslehre ermöglichen?

Frage D befasst sich mit einem Teil des ersten Kreislaufs, Geschlechtsrollenerwartungen - berufliche Segregation nach Geschlecht ${ }^{\circ}$. Geschlecht wird durch einen Interaktionsprozess hergestellt (vgl. Unterkapitel 2.5), genannt Doing Gender. Die Frage lautet: Wie stellen Lernende in geschlechtsuntypischen Berufslehren ihr Geschlecht dar? 


\section{Empirischer Teil}

Im empirischen Teil wird die übergeordnete Forschungsfrage mit der Reanalyse von vier Datensätzen und einer Interviewstudie aufgearbeitet, indem der berufliche Erfolg von Lernenden in geschlechtsuntypischen Berufslehren mit demjenigen in geschlechtergemischten und geschlechtstypischen verglichen wird. Der berufliche Erfolg von Jugendlichen wird vorwiegend während der Berufslehre betrachtet (vgl. Unterkapitel 1.5).

$\mathrm{Zu}$ den Kriterien des beruflichen Erfolgs während der Berufslehre mit einer Datengrundlage Akten/Dokumente können Absentismus, Lehrvertragsauflösung und Erfolg bei der Lehrabschlussprüfung gezählt werden (vgl. Tabelle 1.1). In Kapitel 5 werden die Lehrvertragsauflösungen in der Schweiz im Zeitraum von 2008 bis 2012 untersucht. Die Hypothese 1 prüft dazu den Pfad ,Austritt aus der geschlechtsuntypischen Berufslehre' im Modell der geschlechtsuntypischen Berufssozialisation. Die Populationsdaten stammen vom BFS.

Ein weiteres Erfolgskriterium für die Berufslehre ist das Bestehen der Lehrabschlussprüfung. Wie bei den Lehrvertragsauflösungen bilden die BFSPopulationsdaten des Zeitraums von 2008 bis 2012 die Grundlage für diese Analysen (vgl. Hypothese 2, Kapitel 6). Die Absenzen sind Bestandteil der Auswertungen in Kapitel 8 (Hypothese 4, die mit Daten des Forschungsprojekts TREE (TREE 2008) berechnet werden. Beide Erfolgskriterien sind im Modell der geschlechtsuntypischen Berufssozialisation bei der ,Bewältigung der Reaktionen' angesiedelt.

$\mathrm{Zu}$ den Erfolgskriterien, die das Fremdurteil bzw. den Selbstbericht mit Datenquellen untersuchen, zählen latente psychische Konstrukte wie Ausbildungszufriedenheit, Passungswahrnehmung, Selbstwert, Selbstkonzept, Sozialkompetenz und psychosomatische Beschwerden. Auf diese Aspekte wird in Kapitel 7 (Hypothese 3) mit dem Datensatz aus der Studie FASE B (Neuenschwander et al. 2012) und anhand von insgesamt vier latenten Konstrukten im Längs- und Querschnitt Bezug genommen.

Die psychosomatischen Beschwerden von Lernenden in geschlechtsuntypischen und -typischen Berufslehren werden mithilfe der TREE-Daten verglichen. Diese sind ebenfalls Indizien für die Bewältigungshandlung (vgl. Hypothese 4). Die Analysen dazu werden in Kapitel 8 präsentiert. Im gleichen Kapitel wird der Einfluss des sozialen Umfelds auf das Erleben der Sozialisation von Jugendlichen in geschlechtsuntypischen Berufslehren anhand des Modells der geschlechtsuntypischen Berufssozialisation überprüft (Hypothese 5).

In Kapitel 9 folgen schließlich die Ergebnisse der Interviewstudie mit 14 Lernenden in geschlechtsuntypischen Berufslehren. Im Zentrum stehen die Erfahrungen und Erlebnisberichte der Jugendlichen im Lehrbetrieb, in der Berufsfachschule und im privaten. 


\section{Analyse I: Lehrvertragsauflösungen (LVA)}

Jeden Sommer beginnen nach der BFS-Statistik der beruflichen Grundbildung rund 70'000 Jugendliche eine Berufslehre mit Eidgenössischem Fähigkeitszeugnis EFZ (BFS 2008, 2011). Die Ausbildungszusammenarbeit zwischen dem Lehrbetrieb und der Lernenden, dem Lernenden wird in einem Vertrag ${ }^{22}$ geregelt, der zum Schutz der Lernenden von der zuständigen kantonalen Behörde bestätigt wird. Lehrverträge können lediglich mit der formalen Zustimmung des kantonalen Berufsbildungsamtes geändert oder aufgelöst werden (SDBB ${ }^{23}$ 2013). Wie in Abschnitt 1.5.2 beschrieben, wird im Durchschnitt jeder vierte Lehrvertrag vorzeitig aufgelöst (z.B. Neuenschwander et al. 2012, Stalder/Schmid 2006, Süss et al. 1996). Die hohen Auflösequoten erstaunen. Bohlinger (2002) betrachtet die zahlreichen LVA als Bestandteil des noch nicht abgeschlossenen Berufsfindungsprozesses. Dabei ist ein Teil der Auflösungen auf Umstufungen in Berufslehren anderer Niveaus zurückzuführen. Dies könnte etwa ein Niveauwechsel von ,AutomobilMechatroniker/-in EFZ' zu ,Autofachmann/-frau EBA ${ }^{624}$ sein. Berufs- und Niveauwechsel haben immer eine LVA zur Folge. Ein erheblicher Anteil der LVA jedoch erfolgt ohne Anschlussbestätigung, was einem Lehrabbruch bzw. Lehrunterbruch gleichkommt (Neuenschwander 1999).

Im vorliegenden Kapitel werden die Analysen und Ergebnisse vorzeitiger LVA dargestellt. Im Zentrum steht die Frage, ob Jugendliche in geschlechtsuntypischen Berufslehren weniger erfolgreich sind und häufiger von LVA betroffen sind als Lernende in geschlechtergemischten oder geschlechtstypischen Berufslehren. Das Kapitel ist gegliedert in ,Fragestellung der Analyse und Hypothesen“ (Unterkapitel 5.1), ,Methoden“ (Unterkapitel 5.2), ,Ergebnisse‘ (Unterkapitel 5.3) sowie ,Diskussion‘ (Unterkapitel 5.4).

\subsection{Fragestellung der Analyse und Hypothesen}

Gemäß dem der vorliegenden Arbeit zugrunde liegenden Modell der geschlechtsuntypischen Berufssozialisation (vgl. Unterkapitel 4.2) erfüllen Lernende in geschlechtsuntypischen Berufslehren die Geschlechtsrollenerwartungen der segregierten Arbeitswelt nicht. Während einige Personen des

22 Der Lehrvertrag ist ein befristeter Einzelarbeitsvertrag. Er muss in schriftlicher Form Regelungen zu Art und Dauer der beruflichen Bildung, Lohn, Probezeit, Arbeitszeit sowie Ferien festhalten (vgl. SDBB, 2012).

23 SDBB Schweizerisches Dienstleistungszentrum Berufsbildung, Berufs-, Studien- und Laufbahnberatung [http://www.sdbb.ch;Zugriff: 22.03.2017].

24 Beschreibung der unterschiedlichen Berufslehrformen in Kapitel 1.4. 
jeweiligen sozialen Umfelds positiv und unterstützend auf diese Jugendlichen zugehen, reagieren andere mit Irritation. Diese Irritation aufgrund nicht erfüllter Erwartungen findet ihren Ausdruck beispielsweise in mehr oder weniger unterschwelligen und abwertenden Bemerkungen. Die Bewältigung der Reaktionen durch die Lernenden auf diese Formen der Ablehnung kann je nach Charaktereigenschaften und Ressourcen unterschiedlich ausfallen: Ärger, Wut, Herausforderung zu verstärktem Ansporn oder aber Interessensverlust, Leistungsabfall und Konflikte bei der Arbeit. Überwiegen die negativen Emotionen, folgt oft der Ausstieg aus der geschlechtsuntypischen Situation. Damit kann die Hoffnung verbunden sein, in einem geschlechtsrollenkonformeren Beruf mehr beruflichen und vor allem mehr sozialen Erfolg zu erreichen. Im Modell der geschlechtsuntypischen Berufssozialisation wird dazu der Pfad ,Austritt aus der geschlechtsuntypischen Berufslehre ' geprüft (vgl. Abbildung 4.1).

Werden Lehrverträge von Frauen in männerreichen und von Männern in frauenreichen Berufslehren häufiger vorzeitig aufgelöst als von anderen Gruppen von Lernenden? Das Bestreben, diese Fragen zu klären, stößt auf zwei strukturelle Schwierigkeiten: Erstens gibt es beachtliche Unterschiede in der Anzahl der Lernenden in den ca. 230 Berufslehren, von einzelnen bis hin $\mathrm{zu}$ beinahe 10'000 Lernenden pro Jahrgang. Zweitens gehören Lernende in geschlechtsuntypischen Berufslehren definitionsgemäß einer kleinen Minderheit an. Aus diesen beiden Gründen werden die Populationsdaten zu LVA des BFS aus den fünf Jahren 2008 bis 2012 zusammengefasst. Anhand zweier Hypothesen wird überprüft, ob diese Zusammenfassung gelingt, ohne dass Effekte übersehen werden. Die erste Prüfung gilt der Stabilität des Frauenanteils in den Berufslehren. Dafür wird für jede Kombination von Jahren die Stärke der Korrelation der Frauenanteile in allen Berufslehren ermittelt. Die Hypothese 1a lautet: Die Frauenanteile der fünf untersuchten Jahre korrelieren stark und bleiben damit konstant.

Die LVA-Quote gibt den prozentualen Anteil aller von LVA betroffenen Lernenden an allen Lernenden mit Lehrbeginn im Erhebungsjahr an. Die ausführliche Beschreibung dieser Berechnungsgrundlage befindet sich in Abschnitt 1.5.2. Da die LVA-Quoten in den Berufslehren unterschiedlich sind, thematisiert die Hypothese $1 b$ deren Stabilität: Die LVA-Quoten der fünf Jahre hängen stark zusammen und bleiben damit konstant.

Können die Hypothesen $1 \mathrm{a}$ und $1 \mathrm{~b}$ bestätigt werden, womit die Stabilität von Frauenanteil und LVA-Quoten gegeben sind, können die Datensätze aus den fünf Jahren zusammengefasst und die Hypothese 1c angegeben werden. Hypothese 1c lautet: Lernende in geschlechtsuntypischen Berufsbildungen sind häufiger von LVA betroffen als Lernende in gemischtgeschlechtlichen und geschlechtstypischen Berufslehren. 


\subsection{Methode}

Das Datenmaterial der Populationsdaten für die Schweiz zu LVA stammt vom $\mathrm{BFS}^{25}$. Darin enthalten sind die Meldungen der kantonalen Berufsbildungsämter zu LVA der Jahre 2008 bis 2012, aufgeschlüsselt nach den Variablen Berufslehren, Vertragstypen und Geschlecht. Zunächst werden die Datenstruktur (Abschnitt 5.2.1) und anschließend die Auswertungsmethoden (Abschnitt 5.2.2) beschrieben.

\subsubsection{Beschreibung der Datenstruktur}

Anders als in den meisten sozialwissenschaftlichen Analysen werden die verwendeten Daten nicht auf Individualebene analysiert, sondern als aggregierte Daten auf Berufsebene. Es sind dies Häufigkeiten von neu abgeschlossenen Lehrverträgen und die Anzahl der Auflösungen pro Berufslehre. Die Tabelle 5.1 zeigt ein fiktives Beispiel mit Anzahl und Quoten für beide Geschlechter, den Frauenanteil sowie den ,Gender Gap ${ }^{26}$.

Tabelle 5.1: Fiktives Beispiel zur Demonstration der Datenrepräsentation

\begin{tabular}{|c|c|c|c|c|c|c|c|c|c|c|c|}
\hline \multirow{2}{*}{$\begin{array}{l}\text { Berufs- } \\
\text { lehre }\end{array}$} & \multicolumn{3}{|c|}{ Anzahl neue Lehrverträge } & \multicolumn{3}{|c|}{ Anzahl LVA } & \multicolumn{3}{|c|}{ LVA-Quote in \% } & \multirow{2}{*}{$\begin{array}{c}\text { Frauen- } \\
\text { anteil } \\
\text { in } \%\end{array}$} & \multirow{2}{*}{$\begin{array}{c}\text { Gender } \\
\text { Gap } \\
\text { in } \%\end{array}$} \\
\hline & Total & Männer & Frauen & Total & Männer & Frauen & Total & Männer & Frauen & & \\
\hline 1 & 200 & 100 & 100 & 40 & 20 & 20 & 20.0 & 20.0 & 20.0 & 50.0 & 0.0 \\
\hline 2 & 200 & 10 & 190 & 24 & 5 & 19 & 12.0 & 50.0 & 10.0 & 95.0 & 40.0 \\
\hline
\end{tabular}

Quelle: Eigene Darstellung.

Die Lehrverträge werden in 67 Lehrvertragstypen unterteilt; beispielsweise EFZ, EBA, Verkürzung, Verlängerung, Kettenlehrvertrag, Lehrbetriebsverbund, Saisonvertrag, Lehrfortsetzung usw. Für die hier durchgeführte Auswertung ist der Regelfall ausschlaggebend, um von ihm die Besonderheit der Lernenden in geschlechtsuntypischen Berufslehren zu unterscheiden. Von den 67 Lehrvertragstypen wurde lediglich der Vertragstyp ,Berufliche Grundbildung 3 oder 4 Jahre mit EFZ' in die Auswertung einbezogen. Mit

25 Dem Bundesamt für Statistik BFS - speziell Herrn Anton Rudin - danke ich für die auf Anfrage zusammengestellten Daten und die Erlaubnis zur Publikation der Ergebnisse.

26 Der Terminus ,Gender Gap “ ist den Wirtschafts- und Sozialwissenschaften entlehnt und bezeichnet dort die Kluft zwischen Frauen und Männern, vor allem in Bezug auf systematische Lohndifferenzen (vgl. Goldin, 1990). Er wird in Diversity-Schriften verwendet, um die Vielfalt unterschiedlicher Geschlechter und Geschlechtsidentitäten darzustellen (Bäcker_in). Er ist als Alternative zum Binnen-I (z.B. BäckerIn) zu verstehen, um den impliziten Vorstellungen der Zweigeschlechtlichkeit entgegenzuwirken. In der vorliegenden Arbeit bezeichnet Gender Gap die Differenz der LVA- und LAP-Quoten. 
296 '783 neuen Lehrverträgen in den Jahren 2008 bis 2012 entspricht dies einem Anteil von 77.3 Prozent aller Lehrverträge.

Im Datensatz sind Berufslehren mit alten und neuen Berufsbezeichnungen $^{27}$ getrennt aufgeführt. Um ausreichend große Häufigkeiten zu erhalten, wurden diese Angaben zusammengefasst.

Einige Berufslehren gliedern sich in Fachrichtungen. In den drei Berufslehren ,Gärtner/-in EFZ', ,Detailhandelsfachmann/-frau EFZ“ und ,Kaufmann/-frau EFZ' wurden innerhalb der Fachrichtungen geschlechtliche Segregationseffekte festgestellt. Da genügend Lernende pro Fachrichtung vorhanden waren, werden diese Fachrichtungen auf die Ebene Berufslehren gehoben und ausgewertet. Die Berufslehre ,Gärtner/-in EFZ' hat vier Fachrichtungen: ,Baumschule (Frauenanteil FA $=17.9 \%$ ) ,Garten- und Landschaftsbau' $(\mathrm{FA}=7.7 \%)$,Stauden " $(\mathrm{FA}=68.5 \%)$ und ,Zierpflanzen" $(\mathrm{FA}=$ 69.1\%). Im Detailhandel und bei den Kaufleuten ist die Situation speziell, da es jeweils zwei Berufslehren mit unterschiedlichem Anforderungsniveau gibt und zwischen 22 und 28 Fachrichtungen unterschieden werden. Es gibt zudem überdurchschnittlich viele Lernende in diesen vier Berufslehren (über $80^{`} 000$ in den fünf untersuchten Jahren; vgl. Online-Anhang II). Da sich die geschlechtliche Segregation durch die Fachrichtungen zieht und weniger durch das Anforderungsniveau, wurden Berufslehren derselben Fachrichtung zusammengefasst. Die Bezeichnung der Fachrichtung wird neu auf der Ebene der Berufslehren geführt und als solche in die Berechnungen einbezogen.

Mittels der oben aufgeführten Datenbereinigungsschritte kommt ein Datensatz mit 228 EFZ-Berufslehren zustande (vgl. Online-Anhang III). In 15 dieser Berufslehren waren in den untersuchten 5 Jahren weniger als 10 neue Lehrverträge unterschrieben worden (insgesamt 49 Lehrverträge). Da die Berechnung des Frauenanteils in diesen 15 Berufslehren starke Verzerrungen aufwies, wurden sie ausgeschlossen. Es handelt sich dabei um Berufslehren wie ,Fotolaborant/-in', ,Zementer/-in' oder, Theatermaler/-in EFZ'.

Die LVA-Quoten belegen, dass über 23 Prozent aller Lehrverträge aufgelöst wurden. Bei den Frauen ist es etwas mehr als ein Fünftel, bei Männern sogar etwas mehr als ein Viertel. Der Unterschied zwischen den Geschlechtern (Gender Gap) beträgt rund 4 Prozentpunkte. Dieser Gender Gap dient als Kriterium für die Beurteilung, ob sich bei Lernenden in geschlechtsuntypischen Berufslehren die LVA-Quote von derjenigen anderer Gruppen von Lernenden unterscheidet. Die LVA-Quoten in den verwendeten 213 Berufslehren sind etwas tiefer als bei allen Vertragstypen zusammen bzw. bei allen EFZ-Vertragstypen. Die Unterschiede in den LVA-Quoten sind gering, tendenziell jedoch in den ausgeschlossenen Vertragstypen höher.

27 Mit der Einführung des neuen Berufsbildungsgesetzes 2004 veränderten sich zahlreiche Berufsbezeichnungen. (Dokument ,Übersicht $\mathrm{zu}$ den neuen Berufsbezeichnungen“ [http://www.nbbg.bl.ch/fileadmin/user_upload/AA_Redaktor_Red/Umsetzung_BL_BS/Ber ufsbezeichnungen_alphabetisch.pdf; Zugriff: 22.03.2017]. 


\begin{tabular}{|c|c|c|c|c|c|c|c|c|}
\hline Berufslehre & 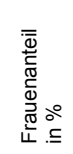 & 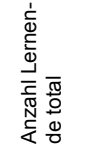 & 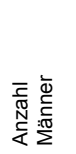 & 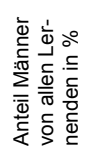 & 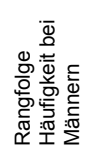 & 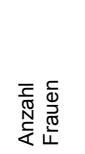 & 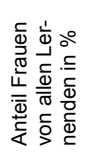 & 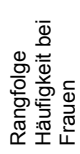 \\
\hline $\begin{array}{l}\text { Kaufmann/-frau EFZ - Dienstleis- } \\
\text { tung \& Administration }\end{array}$ & 69.4 & $18^{\prime} 347$ & $5^{\prime} 611$ & 3.4 & 7 & $12 ' 736$ & 9.9 & 1 \\
\hline Fachmann/-frau Gesundheit EFZ & 90.0 & $12 ' 421$ & & & & $11 ' 178$ & 8.6 & 2 \\
\hline Elektroinstallateur/-in EFZ & 1.7 & 9 '829 & $9^{\prime} 661$ & 5.8 & 1 & & & \\
\hline Koch/Köchin EFZ & 36.3 & $9^{\prime} 809$ & $6 ' 245$ & 3.7 & 4 & 3'564 & 2.8 & 11 \\
\hline $\begin{array}{l}\text { Kaufmann/-frau EFZ - Öffentliche } \\
\text { Verwaltung }\end{array}$ & 69.9 & $9^{\prime} 162$ & $2 ' 760$ & 1.6 & 19 & $66^{\prime} 402$ & 5.0 & 5 \\
\hline Fachmann/-frau Betreuung EFZ & 87.2 & $8^{\prime} 686$ & & & & $7 ' 576$ & 5.9 & 3 \\
\hline Polymechaniker/-in EFZ & 3.3 & $7 ' 827$ & 7'569 & 4.5 & 2 & & & \\
\hline Coiffeur/-euse EFZ & 91.7 & 7'346 & & & & 6'735 & 5.2 & 4 \\
\hline Zeichner/-in EFZ & 32.7 & $7^{\prime} 028$ & $4^{\prime} 733$ & 2.8 & 11 & $2^{\prime} 295$ & 1.8 & 15 \\
\hline Automobil-Fachmann/-frau EFZ & 3.5 & $66^{\prime} 632$ & $66^{\prime} 400$ & 3.8 & 3 & & & \\
\hline Logistiker/-in EFZ & 13.3 & 6'529 & $5^{\prime} 663$ & 3.4 & 6 & & & \\
\hline Schreiner/-in & 9.6 & $66^{\prime} 503$ & $5 ' 882$ & 3.5 & 5 & & & \\
\hline Kaufmann/-frau EFZ - Bank & 51.0 & $66^{\prime} 485$ & $3 ' 176$ & 1.9 & 14 & 3'309 & 2.6 & 12 \\
\hline $\begin{array}{l}\text { Detailh.fachmann/-frau EFZ - } \\
\text { Nahrungs- u. Genussm. }\end{array}$ & 66.4 & 6'054 & & & & 4'017 & 3.1 & 9 \\
\hline Informatiker/-in EFZ & 7.9 & 5 '850 & 5 '386 & 3.2 & 9 & & & \\
\hline Maurer/-in EFZ & 0.8 & $55^{\prime} 546$ & $5^{\prime} 504$ & 3.3 & 8 & & & \\
\hline Maler/-in & 36.7 & 4'912 & 3'108 & 1.9 & 16 & $1 ' 804$ & 1.4 & 17 \\
\hline Dentalassistent/-in EFZ & 99.3 & 4'842 & & & & 4'809 & 3.7 & 6 \\
\hline Zimmermann/Zimmerin & 1.0 & $4^{\prime} 821$ & $4^{\prime} 771$ & 2.8 & 10 & & & \\
\hline $\begin{array}{l}\text { Detailhandelsfachmann/-frau } \\
\text { EFZ - Textil }\end{array}$ & 83.7 & 4'521 & & & & 3'786 & 2.9 & 10 \\
\hline Pharma-Assistent/-in EFZ & 96.9 & 4'513 & & & & 4'375 & 3.4 & 7 \\
\hline $\begin{array}{l}\text { Bäcker/-in-Konditor/-in- } \\
\text { Confiseur/-in EFZ }\end{array}$ & 58.6 & $4^{\prime} 374$ & & & & 2'564 & 2.0 & 14 \\
\hline $\begin{array}{l}\text { Gärtner/-in EFZ - Garten- und } \\
\text { Landschaftsbau }\end{array}$ & 7.7 & $4 ' 332$ & $4^{\prime} 000$ & 2.4 & 13 & & & \\
\hline Sanitärinstallateur/-in EFZ & 1.4 & $4^{\prime} 212$ & $4^{\prime} 155$ & 2.5 & 12 & & & \\
\hline $\begin{array}{l}\text { Medizinische/r Praxisassistent/- } \\
\text { in EFZ }\end{array}$ & 99.2 & $4^{\prime} 055$ & & & & $4^{\prime} 023$ & 3.1 & 8 \\
\hline Restaurationsfachmann/-frau EFZ & 75.7 & 3'840 & & & & 2'907 & 2.2 & 13 \\
\hline Automobil-Mechatroniker/-in EFZ & 3.4 & 3'255 & 3'143 & 1.9 & 15 & & & \\
\hline $\begin{array}{l}\text { Fachmann/-frau Betriebsunterhalt } \\
\text { EFZ }\end{array}$ & 7.9 & 3'236 & $2^{\prime} 981$ & 1.8 & 18 & & & \\
\hline Automatiker/-in EFZ & 2.0 & 3'110 & 3'047 & 1.8 & 17 & & & \\
\hline Carrossier/-in EFZ & 12.0 & 3'101 & $2 ' 728$ & 1.6 & 20 & & & \\
\hline $\begin{array}{l}\text { Kaufmann/-frau EFZ - Masch.-, } \\
\text { Elektro- u. Metallindustrie }\end{array}$ & 66.0 & 2'963 & & & & 1'956 & 1.5 & 16 \\
\hline $\begin{array}{l}\text { Kaufmann/-frau EFZ - Treuhand/ } \\
\text { Immobilientreuhand }\end{array}$ & 59.4 & $2 ' 702$ & & & & $1 ' 605$ & 1.2 & 20 \\
\hline Hotelfachmann/-frau EFZ & 92.1 & $1 ' 924$ & & & & $1 ' 771$ & 1.4 & 18 \\
\hline Fachmann/-frau Hauswirtsch. EFZ & 95.9 & $1 ' 699$ & & & & $1^{\prime} 630$ & 1.3 & 19 \\
\hline
\end{tabular}

Legende: Anzahlen der Jahre 2008-2012 zusammengezählt; graue Schattierung: bei beiden Geschlechtern in den Top 20; fett: nur bei Frauen in den Top 20; kursiv: nur bei Männern in den Top 20; 30\%-Kriterium für typisch/untypisch; Reihenfolge: Anzahl Lernende Total. Quelle: Eigene Darstellung. 
Die Tabelle 5.2 erlaubt einen Überblick über die geschlechtliche Segregation in der Berufsbildung. Lediglich 6 Berufslehren kommen sowohl bei den Frauen als auch bei den Männern in den 20 am häufigsten absolvierten Berufslehren vor (vgl. grau markierte Berufslehren). Am beliebtesten ist ,Kaufmann/-frau EFZ - Dienstleistung und Administration" bei beiden Geschlechtern, vor ,Fachfrau Gesundheit EFZ' bei den Frauen und ,Elektroinstallateur $E F Z^{`}$ bei den Männern. In den am häufigsten gewählten Berufslehren mit einem hohen Männeranteil ist der Anteil an Frauen in 8 Fällen unter 4 Prozent, im umgekehrten Fall lediglich in 3 Fällen.

\subsubsection{Auswertungsmethoden}

Das Verhältnis der Geschlechter bestimmt den Frauenanteil bei den neu abgeschlossenen Lehrverträgen. Je höher der Frauenanteil, desto mehr Frauen erlernen im Verhältnis zu den Männern diesen Beruf. Die LVA-Quoten der Berufslehren wurden insgesamt und nach Geschlecht berechnet. Die Stabilitäten des Frauenanteils und der LVA-Quote wurden mit PearsonKorrelationen unter paarweisem Ausschluss verglichen. Die Analysen erfolgen mit den Statistik-Computerprogrammen SPSS 22 und Microsoft Excel 2010.

Die Differenz zwischen der LVA-Quote der Frauen und jener der Männer wird mit der Höhe des Gender Gaps ausgedrückt (LVA-Quote der Männer minus LVA-Quote der Frauen). Positive Werte beim Gender Gap bedeuten, dass Männer stärker von LVA betroffen sind als Frauen, und negative Zahlen, dass Frauen häufiger den Lehrvertrag auflösen als Männer.

Der Gender Gap bildet eine Suchheuristik, um Trends für allfällige Benachteiligungen von Lernenden in geschlechtsuntypischen Berufslehren aufzudecken. Da durchschnittlich 25.1 Prozent der männlichen und 21.0 Prozent der weiblichen Lernenden von LVA betroffen sind, wird diese Differenz, also der Gender Gap von 4.1 Prozent, als kritischer Wert betrachtet. Interpretiert werden demnach die Abweichungen vom durchschnittlichen Gender Gap. Zur Veranschaulichung wird bei der grafischen Darstellung der Nullpunkt um 4.1 Prozent verschoben. Mit Schmid und Stalder (2007) kann folgendermaßen über die Erklärungsansätze für die Differenz spekuliert werden: Einerseits würden sich Frauen eher weniger zutrauen und darum eher Berufslehren mit tieferem Anforderungsniveau wählen. Zusätzlich könnten die Berufsbildenden diesen Selbstselektionseffekt mittels einer geschlechtsspezifischen Lehrstellenvergabe verstärken. Andererseits seien Frauen möglicherweise eher bereit, die in der Berufsfachschule geforderten Leistungen zu erbringen. 


\subsection{Ergebnisse}

Die Präsentation der Ergebnisse erfolgt in drei Abschnitten. Im Abschnitt 5.3.1 werden die Stabilitäten des Frauenanteils und der LVA-Quoten dargestellt und die Hypothesen 1a und $1 \mathrm{~b}$ geprüft. In den Abschnitten 5.3.2 und 5.3.3 werden die beiden Hypothesen 1c und 1d zum Gender Gap bei der LVA und zu den Gründen für LVA geklärt.

\subsubsection{Stabilitäten der Frauenanteile und der LVA-Quoten}

Die Stabilitäten des Frauenanteils und der LVA-Quote wurden mittels Korrelationsanalysen überprüft. Für die Berechnung wurden Berufslehren, die in fünf Jahren weniger als 100 Lehranfängerinnen und -anfänger aufwiesen, ausgeschlossen. Nahezu perfekt korrelieren die Frauenanteile in den Berufslehren während dieser Zeit: $r=.97$ bis .98 (vgl. Online-Anhang IV). Die LVA-Quoten für die Berufslehren in den fünf untersuchten Jahren 2008 bis 2012 sind mit höchst signifikanten Korrelationen zwischen $r=.56$ und .75 ebenfalls stabil. Die Ergebnisse bestätigen die beiden ersten Hypothesen 1a und $1 b$.

Mit dem Nachweis der Stabilität des Frauenanteils und der Misserfolgsquoten ist das Zusammenführen der fünf Datensätze aus den Jahren 2008 bis 2012 möglich. Diese Zusammenfassung erlaubt sogar Berufslehren zu untersuchen, die jährlich von wenigen Personen des jeweils untervertretenen Geschlechts absolviert werden. Der nächste Schritt besteht in der Prüfung von Hypothese 1c: Lernende in geschlechtsuntypischen Berufsausbildungen sind häufiger von LVA betroffen als Lernende in geschlechtstypischen bzw. gemischtgeschlechtlichen Berufsausbildungen.

\subsubsection{LVA-Quoten}

Zur Beurteilung der Hypothese 1c wurden die Berufslehren einerseits in 5 und andererseits in 22 Gruppen eingeteilt. Die Einteilung in 5 Gruppen erfolgte anhand des 30-Prozent-Kriteriums ${ }^{28}$ bei den Geschlechteranteilen. Die detailliertere Einteilung in 22 Gruppen basiert auf 5-Prozent-Schritten des Frauenanteils. Innerhalb dieser neu gebildeten Gruppen werden die Häufig-

28 Die Grenze von 30:70\% reiht sich in die Tradition der aktuellen Forschungsarbeiten in der Schweiz zu Fragestellungen zur Geschlechtstypik ein: z.B. Gianettoni et al. (2010), Makarova/Herzog (2014), Schwiter et al. (2014), Wehner et al. (2016). Andere Einteilungen verwendeten z.B. Kanter (1977, USA): $85: 15 \%$ und 65:35\%; Althoff (1992, D): 85:15\% und 30:70\%; Sax/Bryant (2006, USA): 2/3:1/3; Helbig/Leuze (2012, D): 40:60\% oder Abraham/Arpagaus $(2008, \mathrm{CH}): 75: 25 \%$. 
keiten der neuen Lehrverträge und der LVA zusammengezählt und die LVAQuoten sowie der Gender Gap neu berechnet.

\subsubsection{30-Prozent-Gruppen (5 Gruppen)}

In der ersten und der fünften Gruppe gibt es lediglich Lernende eines Geschlechts. Sie sind der Vollständigkeit wegen aufgeführt. Von Interesse sind die drei mittleren Gruppen. Bei den 94 Berufslehren, die die Gruppe der männerreichen Berufslehren bilden (vgl. Tabelle 5.3), hat die Frauenminderheit eine LVA-Quote von 28.4 Prozent, also eine höhere als die der Männer in dieser Gruppe (25.2 Prozent). Wird die Frauenquote von der Männerquote abgezogen, ergibt sich der Gender Gap von -3.2 Prozent. Die Frauen in männerreichen Berufslehren sind damit stärker von LVA betroffen als die Männer in dieser Gruppe. In der Gruppe der frauenreichen Berufslehren ergibt sich ein analoges Bild. Die Männer weisen eine LVA-Quote von 31.7 Prozent auf und die Frauen eine um einiges tiefere von 21.8 Prozent. Der Gender Gap in der Gruppe der frauenreichen Berufslehren beträgt damit 9.9 Prozent zuungunsten der Männer in geschlechtsuntypischen Berufslehren. In der Gruppe der gemischtgeschlechtlichen Berufslehren sind ebenfalls die Männer häufiger von LVA betroffen.

Tabelle 5.3: LVA-Quoten und Gender Gap in den 30 Prozent-Gruppen

\begin{tabular}{|c|c|c|c|c|c|c|c|c|c|c|c|}
\hline \multirow{2}{*}{$\begin{array}{l}\text { Gruppen } \\
\text { FA 30\% }\end{array}$} & \multirow{2}{*}{$\begin{array}{l}\text { Anzahl } \\
\text { Berufs- } \\
\text { lehren }\end{array}$} & \multicolumn{3}{|c|}{ Anzahl neue Lehrverträge } & \multicolumn{3}{|c|}{ Anzahl LVA } & \multicolumn{3}{|c|}{ LVA-Quote in \% } & \multirow{2}{*}{$\begin{array}{c}\text { Gender } \\
\text { Gap } \\
\text { in } \%\end{array}$} \\
\hline & & Total & Männer & Frauen & Total & Männer & Frauen & Total & Männer & Frauen & \\
\hline nur Männer & 4 & 172 & 172 & 0 & 42 & 42 & 0 & 24.4 & 24.4 & - & - \\
\hline männerreich & 94 & $123^{\prime} 881$ & $115^{\prime} 714$ & $8^{\prime} 167$ & $31^{\prime} 447$ & $29 ' 131$ & 2'316 & 25.4 & 25.2 & 28.4 & -3.2 \\
\hline gemischt & 70 & $97^{\prime} 628$ & $43^{\prime} 522$ & $54 ' 106$ & $20^{\prime} 611$ & $10^{\prime} 365$ & $10^{\prime} 246$ & 21.1 & 23.8 & 18.9 & 4.9 \\
\hline frauenreich & 43 & $74 ' 910$ & $8^{\prime} 030$ & $66 ' 880$ & $17^{\prime} 115$ & $2^{\prime} 544$ & $14^{\prime} 571$ & 22.9 & 31.7 & 21.8 & 9.9 \\
\hline nur Frauen & 2 & 143 & 0 & 143 & 37 & 0 & 36 & 25.9 & - & 25.2 & - \\
\hline Total & 213 & $29^{\prime} 6734$ & $167^{\prime} 438$ & $129 ' 296$ & $69^{\prime} 252$ & $42 ' 082$ & $27^{\prime} 169$ & 23.3 & 25.1 & 21.0 & 4.1 \\
\hline
\end{tabular}

Legende: Jahre 2008-2012; FA: Frauenanteil; fett gedruckt: hohe LVA-Quoten; hellgraue Markierung: zum Nachteil der Frauen, wenn der Gender Gap kleiner ist als 4.1\%; dunkelgraue Markierung: zum Nachteil der Männer, wenn der Gender Gap größer ist als 4.1\%. Quelle: Eigene Darstellung.

Wie in Unterkapitel 5.2 beschrieben, besteht eine durchschnittliche Differenz zwischen der LVA-Quote der Männer und derjenigen der Frauen von 4.1 Prozent. Dieser mittlere Gender Gap wird zur besseren Übersicht vom jeweiligen Gender Gap der Gruppe abgezogen. Diese Abweichungen vom mittleren Gender Gap ergeben ein deutliches Bild: In der Gruppe der männerreichen Berufslehren weicht der Gender Gap um -7.3 Prozent vom Durchschnitt ab (-3.2\% minus 4.1\% ergibt $-7.3 \%)$. Die Abbildung 5.1 zeigt diese Abweichungen vom mittleren Gender Gap für die drei relevanten Gruppen. Es bestätigt sich, dass Frauen in männerreichen und Männer in frauenreichen Berufslehren häufiger von LVA betroffen sind. Das ist ein starker Beleg für die 
Richtigkeit der Hypothese 1c. Im zweiten Schritt mit detaillierteren Gruppen wird sich der Trend bestätigen.

\section{Abbildung 5.1: LVA-Abweichungen in 30-Prozent-Gruppen}

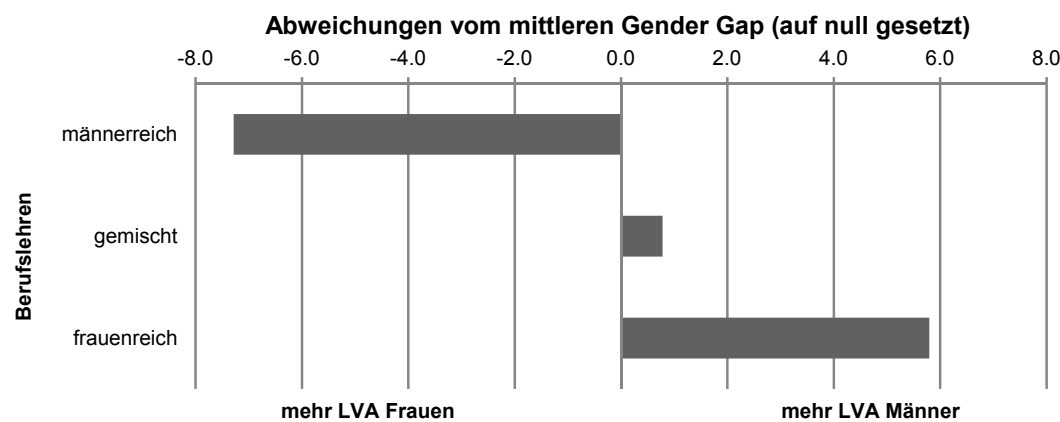

Legende: Gruppen zu 30 Prozent Frauenanteil. Quelle: Eigene Darstellung.

Tabelle 5.4: LVA-Quoten und Gender Gap in den 5-Prozent-Gruppen

\begin{tabular}{|c|c|c|c|c|c|c|c|c|c|c|c|c|}
\hline \multirow{2}{*}{\multicolumn{2}{|c|}{$\begin{array}{l}\text { Gruppe } \\
\text { FA 30\% }\end{array}$}} & \multirow{2}{*}{$\begin{array}{l}\text { Anzahl } \\
\text { Berufs- } \\
\text { lehren }\end{array}$} & \multicolumn{3}{|c|}{ Anzahl neue Lehrverträge } & \multicolumn{3}{|c|}{ Anzahl LVA } & \multicolumn{3}{|c|}{ LVA-Quote in \% } & \multirow{2}{*}{$\begin{array}{l}\text { Gende } \\
\text { Gap } \\
\text { in } \%\end{array}$} \\
\hline & & & Total & Männer & Frauen & Total & Männer & Frauen & Total & Männer & Frauen & \\
\hline & 0 & 4 & 172 & 172 & 0 & 42 & 42 & 0 & 24.4 & 24.4 & - & - \\
\hline \multirow{6}{*}{ 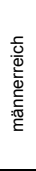 } & $0.1-5$ & 40 & $70^{\prime} 333$ & $68^{\prime} 811$ & 1'522 & $18^{\prime} 603$ & $18^{\prime} 007$ & 596 & 26.5 & 26.2 & 39.2 & -13.0 \\
\hline & $5-10$ & 16 & $24^{\prime} 057$ & $22^{\prime} 094$ & $1^{\prime \prime} 963$ & $6^{\prime} 250$ & $5^{\prime} 635$ & 615 & 26.0 & 25.5 & 31.3 & -5.8 \\
\hline & $10-15$ & 13 & $17^{\prime} 204$ & $15^{\prime} 069$ & $2^{\prime} 135$ & $3^{\prime} 679$ & $3^{\prime} 161$ & 518 & 21.4 & 21.0 & 24.3 & -3.3 \\
\hline & $15-20$ & 16 & $7^{\prime} 441$ & $6^{\prime} 091$ & $1^{\prime} 350$ & $1 ' 891$ & $1^{\prime} 543$ & 348 & 25.4 & 25.3 & 25.8 & -0.5 \\
\hline & $20-25$ & 8 & $3^{\prime} 727$ & $2^{\prime} 852$ & 875 & 757 & 590 & 167 & 20.3 & 20.7 & 19.1 & 1.6 \\
\hline & $25-30$ & 1 & 1'119 & 797 & 322 & 267 & 195 & 72 & 23.9 & 24.5 & 22.4 & 2.1 \\
\hline \multirow{8}{*}{ 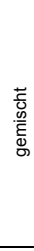 } & $30-35$ & 7 & $8^{\prime} 969$ & $6^{\prime} 040$ & $2^{\prime} 929$ & $1^{\prime} 818$ & 1'172 & 646 & 20.3 & 19.4 & 22.1 & -2.7 \\
\hline & $35-40$ & 6 & $15^{\prime} 479$ & 9'837 & $5^{\prime} 642$ & 5'316 & $3^{\prime} 600$ & $1 ' 716$ & 34.3 & 36.6 & 30.4 & 6.2 \\
\hline & $40-45$ & 6 & $2^{\prime} 735$ & $1^{\prime} 599$ & $1^{\prime} 136$ & 590 & 367 & 223 & 21.6 & 23.0 & 19.6 & 3.3 \\
\hline & $45-50$ & 9 & $3^{\prime} 821$ & $2^{\prime} 032$ & $1^{\prime} 789$ & 613 & 332 & 281 & 16.0 & 16.3 & 15.7 & 0.6 \\
\hline & $50-55$ & 8 & 9'225 & $4^{\prime} 456$ & $4^{\prime} 769$ & $1^{\prime} 054$ & 525 & 529 & 11.4 & 11.8 & 11.1 & 0.7 \\
\hline & $55-60$ & 9 & $11^{\prime} 833$ & $4^{\prime} 923$ & $6^{\prime} 910$ & 2'577 & $1^{\prime 2} 237$ & 1'340 & 21.8 & 25.1 & 19.4 & 5.7 \\
\hline & $60-65$ & 11 & 6'105 & 2'298 & 3'807 & 1'175 & 451 & 724 & 19.3 & 19.6 & 19.0 & 0.6 \\
\hline & $65-70$ & 14 & $39^{\prime} 461$ & $12^{\prime} 337$ & $27^{\prime} 124$ & $7^{\prime} 468$ & 2'681 & $4^{\prime} 787$ & 18.9 & 21.7 & 17.7 & 4.1 \\
\hline \multirow{7}{*}{ 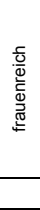 } & $70-75$ & 7 & $4^{\prime} 606$ & 1'308 & $3^{\prime} 298$ & 878 & 313 & 565 & 19.1 & 23.9 & 17.1 & 6.8 \\
\hline & $75-80$ & 8 & 7'858 & $1^{\prime} 826$ & $6^{\prime} 032$ & 2'705 & 666 & 2'039 & 34.4 & 36.5 & 33.8 & 2.7 \\
\hline & $80-85$ & 3 & $5^{\prime} 529$ & 906 & $4^{\prime} 623$ & $1^{\prime} 384$ & 281 & $1 ' 103$ & 25.0 & 31.0 & 23.9 & 7.2 \\
\hline & $85-90$ & 5 & $22^{\prime} 246$ & $2^{\prime} 480$ & $19^{\prime} 766$ & $3^{\prime} 380$ & 603 & 2'777 & 15.2 & 24.3 & 14.1 & 10.3 \\
\hline & $90-95$ & 8 & $12^{\prime} 912$ & $1 ' 056$ & $11 ' 856$ & $4^{\prime} 217$ & 490 & $3^{\prime} 727$ & 32.7 & 46.4 & 31.4 & 15.0 \\
\hline & 95-99.9 & 12 & $21^{\prime} 759$ & 454 & 21'305 & 4'551 & 191 & 4'360 & 20.9 & 42.1 & 20.5 & 21.6 \\
\hline & 100 & 2 & 143 & 0 & 143 & 37 & 0 & 36 & 25.9 & - & 25.2 & - \\
\hline \multicolumn{2}{|c|}{ Total } & 213 & $2966^{\prime} 734$ & $167^{\prime} 438$ & $129^{\prime} 296$ & 69'252 & $42^{\prime} 082$ & $27^{\prime} 169$ & 23.3 & 25.1 & 21.0 & 4.1 \\
\hline
\end{tabular}

Legende: Jahre 2008-2012; FA: Frauenanteil; fett gedruckt: hohe LVA-Quoten; hellgraue Markierung: zum Nachteil der Frauen, wenn der Gender Gap kleiner ist als 4.1\%; dunkelgraue Markierung: zum Nachteil der Männer, wenn der Gender Gap größer ist als 4.1\%. Quelle: Eigene Darstellung. 


\subsubsection{5-Prozent-Gruppen (22 Gruppen)}

Das Vorgehen ist wie oben. Anhand der Aufteilung in 22 Gruppen gelingt ein detaillierterer Blick auf das Verhältnis von Lernenden in geschlechtsuntypischen Berufslehren. Auffällig in Tabelle 5.4 sind die beiden Gruppen mit dem kleinsten und dem größten Frauenanteil. Die 1'522 Frauen in den 40 männerreichsten Berufslehren (FA 0.1-5\%) haben eine LVA-Quote von beinahe 40 Prozent. Dies ist die höchste LVA-Quote für Frauen überhaupt. Ähnlich hoch ist die LVA-Quote für die 454 Männer in den 12 frauenreichsten Berufslehren (FA 95-99.9\%), mit 42.1 Prozent die zweithöchste. Diese wird lediglich übertroffen von der Gruppe der Männer in Berufslehren mit einem Frauenanteil von 90-95 Prozent.

Tabelle 5.5: LVA-Quoten 2008-2012 in männerreichen Berufslehren

\begin{tabular}{|c|c|c|c|c|c|c|c|c|c|c|c|}
\hline \multirow{2}{*}{$\begin{array}{l}\text { Berufslehren mit } \\
\text { einem Frauenanteil } \\
\text { von } 0.1-5 \%\end{array}$} & \multicolumn{3}{|c|}{ Anzahl neue Lehrverträge } & \multicolumn{3}{|c|}{ Anzahl LVA } & \multicolumn{3}{|c|}{ LVA-Quote in $\%$} & \multirow{2}{*}{$\begin{array}{l}\text { Frauen- } \\
\text { anteil } \\
\text { in \% }\end{array}$} & \multirow{2}{*}{$\begin{array}{r}\text { Gender } \\
\text { Gap in \% }\end{array}$} \\
\hline & Total & Männer & Frauen & Total & Männer & Frauen & Total & Männer & Frauen & & \\
\hline Forstwart/-in EFZ & $1^{\prime} 537$ & 1'517 & 20 & 220 & 211 & 9 & 14.3 & 13.9 & 45.0 & 1.3 & -31.1 \\
\hline $\begin{array}{l}\text { Automobil- } \\
\text { Mechatroniker/-in EFZ }\end{array}$ & $3^{\prime} 255$ & $3^{\prime} 143$ & 112 & $1 ' 050$ & 981 & 69 & 32.3 & 31.2 & 61.6 & 3.4 & -30.4 \\
\hline $\begin{array}{l}\text { Montage-Elektriker/-in } \\
\text { EFZ }\end{array}$ & $2^{\prime} 481$ & $2^{\prime} 449$ & 32 & 674 & 657 & 17 & 27.2 & 26.8 & 53.1 & 1.3 & -26.3 \\
\hline $\begin{array}{l}\text { Boden-Parkettleger/-in } \\
\text { EFZ }\end{array}$ & 967 & 927 & 40 & 298 & 278 & 20 & 30.8 & 30.0 & 50.0 & 4.1 & -20.0 \\
\hline Automatiker/-in EFZ & $3^{\prime} 110$ & $33^{\prime} 047$ & 63 & 535 & 512 & 23 & 17.2 & 16.8 & 36.5 & 2.0 & -19.7 \\
\hline $\begin{array}{l}\text { Automobil-Fachmann/ } \\
\text {-frau EFZ }\end{array}$ & $6^{\prime} 632$ & $6^{\prime} 400$ & 232 & $2 ' 174$ & $2^{\prime} 064$ & 110 & 32.8 & 32.3 & 47.4 & 3.5 & -15.2 \\
\hline $\begin{array}{l}\text { Produktionsmechani- } \\
\text { ker/-in EFZ }\end{array}$ & 1'694 & $1^{\prime} 623$ & 71 & 407 & 380 & 27 & 24.0 & 23.4 & 38.0 & 4.2 & -14.6 \\
\hline $\begin{array}{l}\text { Elektroinstallateur/-in } \\
\text { EFZ }\end{array}$ & $9^{\prime} 829$ & $9^{\prime} 661$ & 168 & 3'177 & 3'103 & 74 & 32.3 & 32.1 & 44.1 & 1.7 & -11.9 \\
\hline Metallbauer/-in EFZ & $2 ' 710$ & $2^{\prime} 663$ & 47 & 778 & 759 & 19 & 28.7 & 28.5 & 40.4 & 1.7 & -11.9 \\
\hline $\begin{array}{l}\text { Polymechaniker/-in } \\
\text { EFZ }\end{array}$ & $77^{\prime} 827$ & 7'569 & 258 & $1^{\prime} 310$ & $1^{\prime} 240$ & 70 & 16.7 & 16.4 & 27.1 & 3.3 & -10.8 \\
\hline $\begin{array}{l}\text { Sanitärinstallateur/-in } \\
\text { EFZ }\end{array}$ & $4^{\prime} 212$ & $4^{\prime} 155$ & 57 & 1'109 & $1^{\prime} 088$ & 21 & 26.3 & 26.2 & 36.8 & 1.4 & -10.7 \\
\hline Elektroniker/-in EFZ & $1^{\prime} 499$ & $1^{\prime} 425$ & 74 & 243 & 225 & 18 & 16.2 & 15.8 & 24.3 & 4.9 & -8.5 \\
\hline Spengler/-in EFZ & $1^{\prime} 368$ & $1^{\prime} 330$ & 38 & 524 & 507 & 17 & 38.3 & 38.1 & 44.7 & 2.8 & -6.6 \\
\hline $\begin{array}{l}\text { Multimediaelektroni- } \\
\text { ker/-in }\end{array}$ & 758 & 736 & 22 & 268 & 259 & 9 & 35.4 & 35.2 & 40.9 & 2.9 & -5.7 \\
\hline $\begin{array}{l}\text { Zimmer- } \\
\text { mann/Zimmerin } \\
\text { Anlagen- und }\end{array}$ & $4 ' 821$ & $4^{\prime} 771$ & 50 & 1'003 & 990 & 13 & 20.8 & 20.8 & 26.0 & 1.0 & -5.3 \\
\hline $\begin{array}{l}\text { Apparatebauer/-in } \\
\text { EFZ }\end{array}$ & $1^{\prime} 271$ & $1^{\prime} 243$ & 28 & 283 & 276 & 7 & 22.3 & 22.2 & 25.0 & 2.2 & -2.8 \\
\hline Maurer/-in EFZ & $5^{\prime} 546$ & $5 ' 504$ & 42 & $1 ' 706$ & $1 ' 692$ & 14 & 30.8 & 30.7 & 33.3 & 0.8 & -2.6 \\
\hline Polybauer/-in EFZ & $1^{\prime} 299$ & $1 ' 272$ & 27 & 358 & 350 & 8 & 27.6 & 27.5 & 29.6 & 2.1 & -2.1 \\
\hline Gipser/-in & 963 & 935 & 28 & 444 & 433 & 11 & 46.1 & 46.3 & 39.3 & 2.9 & 7.0 \\
\hline
\end{tabular}

Legende: fett gedruckt: hohe LVA-Quoten; hellgraue Markierung: zum Nachteil der Frauen, wenn der Gender Gap kleiner ist als $4.1 \%$; dunkelgraue Markierung: zum Nachteil der Männer, wenn der Gender Gap über 4.1\% ist. Quelle: Eigene Darstellung.

Der Befund von oben wird mittels der Darstellung in Abbildung 5.2 verdeutlicht. Es lässt sich ein Trend erkennen: Je kleiner die geschlechtliche Minderheit in einer Gruppe von Berufslehren ist, desto häufiger ist sie von LVA betroffen. Diese Auswertung bestätigt ebenfalls die Hypothese 1c. Die Gruppe mit einem Frauenanteil von 30-35 Prozent weicht vom allgemeinen Trend leicht ab. Die weitaus größte Berufslehre in dieser Gruppe ist mit über 7'000 
Lernenden ,Zeichner/-in EFZ' (vgl. Online-Anhang I), ein handwerklicher, ehemals männerreicher Beruf. In den letzten Jahrzehnten hat sich der Frauenanteil von 2 Prozent (1955) über 19 Prozent (1970) auf 34 Prozent (2006) erhöht (Zihlmann 2009). Obwohl dieser Beruf wegen des hohen Frauenanteils nicht mehr zu den männerreichen Berufslehren zählt, verweist seine LVA-Quote auf seine Vergangenheit mit den Charakteristiken eines typischen Männerberufs.

Abbildung 5.2: LVA-Abweichungen 5-Prozent-Gruppen

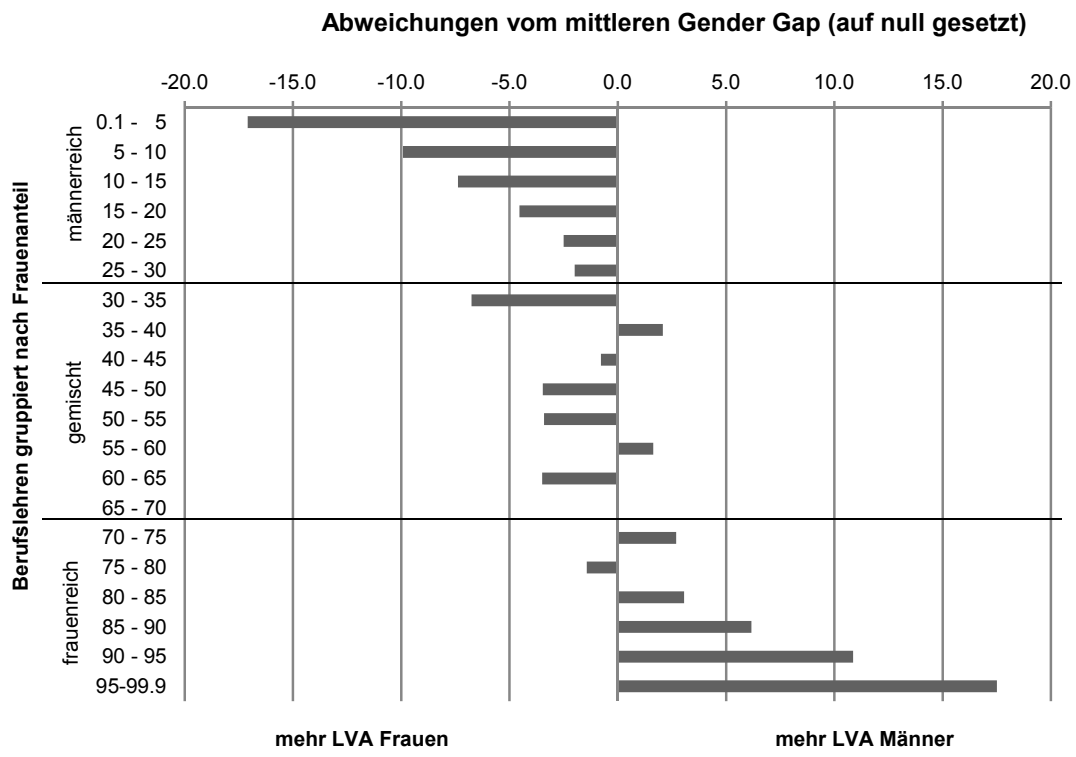

Legende: Gruppen zu 30 Prozent Frauenanteil. Quelle: Eigene Darstellung.

In den Auswertungen in Tabelle 5.4 und Abbildung 5.2 wurden die Berufslehren als strukturgebende Einheit bisher wenig berücksichtigt. Jede Berufslehre hat ihr spezifisches Umfeld, wie etwa Arztpraxis, Kindertagesstätte, Garage, Werkstatt, Baustelle, Gemüserayon im Großverteiler, Büro etc. Für die folgenden Auswertungen gelten die Berufslehren als Analyseeinheit. In den Berufslehren mit einem Anteil an geschlechtsuntypischen Lernenden von maximal 30 Prozent ist der gleiche Trend wie oben nachweisbar (vgl. Tabelle 5.6). Für die Auswertung wurden lediglich Berufslehren berücksichtigt, bei denen während der 5 Jahre mindestens 20 Lernende, die der Minderheit angehören, eine Berufslehre beginnen. Ausgewertet wurde, ob bei der Berufslehre die Frauen oder die Männer vom mittleren Gender Gap abweichen. 


\section{Tabelle 5.6: LVA-Quoten 2008-2012 in frauenreichen Berufslehren}

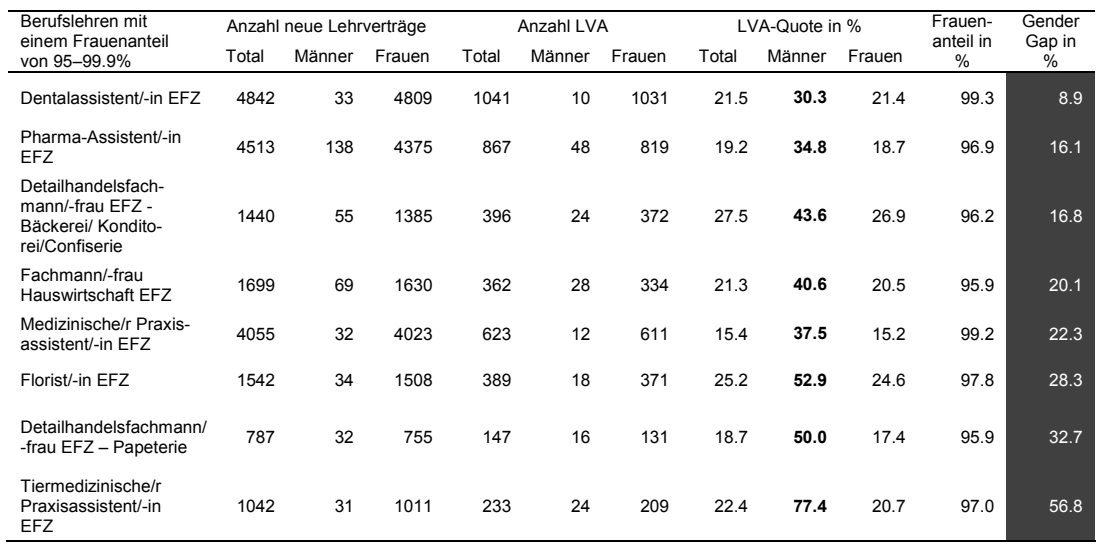

Legende: fett gedruckt: hohe LVA-Quoten; hellgraue Markierung: zum Nachteil der Frauen, wenn der Gender Gap kleiner ist als $4.1 \%$; dunkelgraue Markierung: zum Nachteil der Männer, wenn der Gender Gap über 4.1\% ist. Quelle: Eigene Darstellung.

\section{Abbildung 5.3: LVA-Abweichungen in Berufslehren}

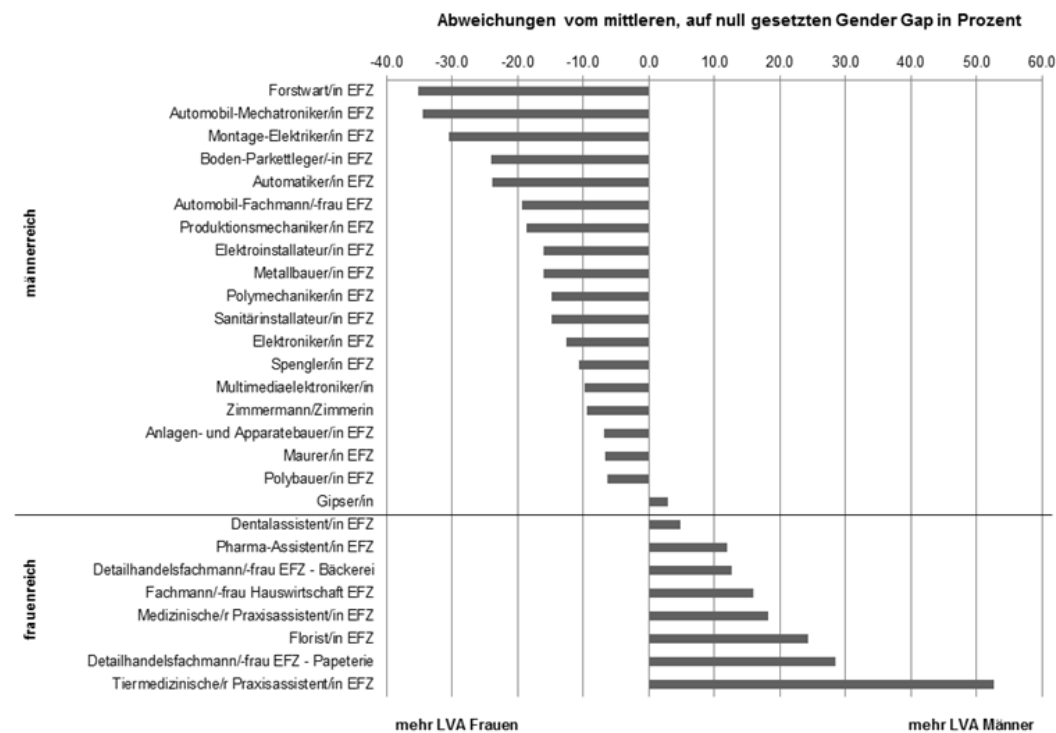

Quelle: Eigene Darstellung. 
Wie in der Tabelle 5.5 dargestellt, lösen in 18 von 19 männerreichen Berufslehren die Frauen öfter ihre Lehrverträge vorzeitig auf. Zum Teil ist ihre LVA-Quote über 50 Prozent. Stark betroffen sind etwa die ,AutomobilMechatronikerinnen EFZ' (LVA-Quote $=61.6 \%$ ), die ,Montage-Elektrikerinnen EFZ' (53.1\%) und die ,Boden-Parkettlegerinnen EFZ' (50.0\%). Lediglich die ,Gipser ' lösen ihren Lehrvertrag häufiger auf als die ,Gipserinnen'.

Männer in frauenreichen Berufslehren sind ebenfalls häufiger von LVA betroffen. Überdurchschnittlich hohe LVA-Quoten haben die ,Tiermedizinischen Praxisassistenten EFZ' (LVA-Quote $=77.4 \%$ ), die ,Floristen EFZ (52.9\%) und die ,Detailhandelsfachmänner EFZ - Papeterie $(50.0 \%)$. Zu berücksichtigen ist, dass die Anzahlen bei den Männern eher gering sind und die Prozentwerte deshalb bei wenigen Vertragsauflösungen hoch werden können. Dennoch bestätigt sich der Trend aus den oben stehenden Auswertungen (vgl. Tabelle 5.6 und Abbildung 5.3).

Der Effekt ist deutlich: Lernende in geschlechtsuntypischen Berufslehren sind häufiger von LVA betroffen als diejenigen in geschlechtergemischten bzw. -typischen Berufslehren.

\subsection{Diskussion}

In Kapitel 5 wurde überprüft, ob ein Drehtür-Effekt (vgl. Abschnitt 3.1.3) bei Lernenden in geschlechtsuntypischen Berufslehren nachzuweisen ist, sie also häufiger den Lehrvertrag vorzeitig auflösen, den Beruf verlassen und sich damit als weniger erfolgreich erweisen als Lernende in geschlechtstypischen oder gemischtgeschlechtlichen Berufslehren (vgl. Abschnitt 1.5.2). Dies wurde mittels Populationsdaten des BFS zu LVA überprüft. Die Daten der Jahre 2008 bis 2012 wurden zusammengefasst und ausgewertet. Bedenken, mit der Zusammenfassung könnten wichtige Effekte übersehen werden, konnten mit der Bestätigung der Hypothese 1a: ,Stabilität des Frauenanteils“ und der Hypothese 1b: ,Stabilität der LVA-Quoten` ausgeräumt werden.

Zur Klärung der Hypothese 1c ,Lernende in geschlechtsuntypischen Berufslehren sind häufiger von LVA betroffen" wurden die Differenzen zwischen den LVA-Quoten von Frauen und Männern in frauenreichen, geschlechtergemischten und männerreichen Berufslehren verglichen. In Berufslehren mit starker Segregation lösen Lernende der geschlechtlichen Minderheit überproportional häufig ihre Lehrverträge vorzeitig auf. Anhand des Gender Gaps konnte der Trend sichtbar gemacht werden, dass je stärker die geschlechtliche Segregation ist, desto größer der Anteil der LVA für Personen in geschlechtsuntypischen Berufslehren wird. In einigen Berufslehren ist dieser Effekt besonders erheblich (etwa ,Forstwart/-in EFZ' oder ,Tiermedizinische/-r Praxisassistent/-in EFZ'). 
Die LVA lassen sich ins Komponentenmodell für Erfolgsfaktoren während der Berufslehre nach Dette et al. (2004) einteilen und gehören gemessen anhand der BFS-Daten zu den globalen Laufbahnindikatoren, neutralen Kennzahlen und Akten/Dokumente. Das beweist, dass Lernende in geschlechtsuntypischen Berufslehren bei den LVA benachteiligt sind. Die Wahrscheinlichkeit, von einer LVA betroffen zu sein, ist bei ihnen erhöht. Je kleiner die Minderheit ist, desto wahrscheinlicher wird eine vorzeitige Beendigung der Ausbildung. Das ist insofern für die Betroffenen ungünstig, weil sie sich in ihrem Berufswunsch nicht erfüllen konnten, sich das Erlebnis des Scheiterns auf den Selbstwert niederschlagen kann und der Übergang in eine neue Ausbildung nicht in allen Fällen glückt (vgl. Neuenschwander et al. 2012, Schmid/Stalder 2007, Süss et al. 1996). Damit ist auch ein wichtiger Teil des Modells der geschlechtsuntypischen Berufssozialisation bewiesen, nämlich dass diese Lernenden häufiger den Lehrvertrag auflösen und damit die Wahrscheinlichkeit erheblich ist, dass sie in einen anderen Beruf wechseln.

Die kantonalen Berufsbildungsämter erfassen jeweils einen Grund für die LVA. Dies ist für die Unterscheidung wichtig, ob es sich um einen technischen Grund, etwa eine formale Anpassung aufgrund von Änderungen der Berufsbezeichnung, oder um andere Gründe handelt. Für eine genaue Ursachenabklärung im Spezialfall der Lernenden in geschlechtsuntypischen Berufsbildungssituationen sind diese Erfassungen weniger geeignet, da häufig mehrere Gründe zu einer LVA führen. Befragungen von Neuenschwander (1999) oder Stalder/Schmid (2006) belegen, dass für Lernende vielfach Konflikte am Lernort Ursachen für Unwillen, Interessensverlust, Demotivation oder Leistungseinbrüche die Ursache für LVA und damit für eine Wiederaufnahme des Berufsfindungsprozesses sind. In der vorliegenden Arbeit konnte einzig festgestellt werden, dass Frauen in männerreichen Berufslehren häufiger aus gesundheitlichen Gründen und Männer in frauenreichen Berufslehren häufiger wegen Pflichtverletzungen von LVA betroffen sind (vgl. OnlineAnhang V). Mögliche Erklärungen für den Effekt bei den Frauen lassen sich aus dem geforderten Körpereinsatz in männerreichen Berufslehren ableiten, denn der weibliche Körper reagiert weniger schnell mit Muskelwachstum auf erhöhte Anforderungen (Bischof-Köhler 2004). Dass die Männer in frauenreichen Berufslehren öfter an Pflichtverletzungen scheitern, könnte auf die erforderliche Achtsamkeit beim Umgang mit zu betreuenden oder zu pflegenden Personen zurückzuführen sein. Da in den BFS-Daten lediglich eine Ursache für LVA erfasst wurde, ist die Aussagekraft dieser Auswertung begrenzt.

Einschränkend muss festgehalten werden, dass die vorliegenden Daten eine Annäherung an die effektiven LVA-Quoten sind, da das Verhältnis der LVA-Zahlen in einem Jahr zu den Anzahlen neu abgeschlossener Lehrverträgen berechnet wurde. Die effektiven Quoten könnten mittels einer Langzeit- 
verfolgung auf Personenebene erfasst werden. Die eben beschriebenen Auswertungen verweisen insgesamt auf einen Trend. Wie genau die Ursachen dafür zu deuten sind, erfordert weitere und spezifischere Untersuchungen.

Diese Befunde belegen zwar die erhöhte LVA-Quote bei Lernenden in geschlechtsuntypischen Berufslehren, können jedoch den im Modell der geschlechtsuntypischen Berufssozialisation (vgl. Kapitel 4) postulierten Effekt, dass Lernende in geschlechtsuntypischen Berufslehren aufgrund gesellschaftlichen Drucks häufiger ihren Lehrvertrag vorzeitig auflösen, nicht beweisen. Dies ließe sich mit der Tokenismus-Theorie von Kanter (1977, vgl. Abschnitt 3.4.4) interpretieren. Ob Lernende in geschlechtsuntypischen Berufslehren vermehrt von Misserfolg bei den Lehrabschlussprüfungen betroffen sind, wird im folgenden Kapitel 0 untersucht. 


\section{Analyse II: Erfolgsquoten bei Lehrabschlussprüfungen (LAP)}

In der Schweiz befinden sich aktuell über 200'000 Jugendliche in einer der ca. 230 vom SBFI anerkannten beruflichen Grundbildungen. Die zwei- bis vierjährige Lehrzeit führt mit dem Bestehen der LAP zum eidgenössischen Fähigkeitszeugnis EFZ bzw. eidgenössischen Berufsattest EBA (vgl. Unterkapitel 1.4). Das vorliegende Kapitel befasst sich mit den Chancen bei der LAP von Lernenden in geschlechtsuntypischen Berufslehren im Vergleich mit Lernenden in geschlechtstypischen bzw. geschlechtergemischten Berufslehren. Die größte Gruppe, die EFZ-Berufslehre mit jährlich über 66'000 Kandidierenden, bildet die Grundlage für die folgenden Betrachtungen. Die Ausgangsdaten dafür stammen vom BFS $^{29}$, das jährlich eine Gesamterhebung für die Schweiz veröffentlicht. Dabei werden die Anzahlen der Kandidierenden und ausgestellten Zertifikate nach Geschlecht und Beruf bzw. nach Geschlecht und Kanton ausgewiesen. Die LAP-Erfolgsquoten unterscheiden sich beträchtlich sowohl zwischen den Berufslehren als auch zwischen den Kantonen (Rottermann 2011). Die Erfolgswahrscheinlichkeit in kleineren Deutschschweizer Kantonen ist höher als in bevölkerungsreichen Kantonen bzw. Kantonen der Westschweiz. Über alle Berufslehren hinweg bestehen 4 Prozent mehr Kandidatinnen als Kandidaten. Details zu berufs- und geschlechtsspezifischen sowie kantonalen Unterschieden belegen Neuenschwander et al. (2012).

In den folgenden Analysen werden die Erfolgswahrscheinlichkeiten von Lernenden untersucht. Scheitert eine Gruppe von Lernenden häufiger als eine andere, sind neben individuellen Bedingungen wie Intelligenz, berufsspezifische Fähig- und Fertigkeiten, Lernmotivation, Einsatzbereitschaft auch äußere Einflussgrößen ausschlaggebend. Bisherige Befunde konnten Benachteiligungen für die Gruppe von Lernenden in geschlechtsuntypischen Berufslehren belegen (vgl. Kapitel 3 und Kapitel 5): beispielsweise Althoff (1992) mit Populationsdaten von 1988 bis 1990 für Deutschland oder Gianettoni et al. (2010) sowie Schwiter et al. (2014) mit Schweizer Stichprobendaten des Forschungsprojekts TREE. Anhand von Auswertungen aktueller Populationsdaten kann dieses Kapitel einen Beitrag zur Klärung der Frage leisten, ob Lernende in geschlechtsuntypischen Berufslehren bei den LAP systematisch benachteiligt sind. Das Kapitel gliedert sich in ,Fragestellung der Analyse und Hypothesen“ (Unterkapitel 6.1), ,Methoden' (Unterkapitel 6.2), ,Ergebnisse“ (Unterkapitel 6.3) sowie ,Diskussion“ (Unterkapitel 6.4). 


\subsection{Fragestellung der Analyse und Hypothesen}

Die Frage, ob Lernende in geschlechtsuntypischen Berufslehren häufiger als ihre Kolleginnen und Kollegen in geschlechtstypischen bzw. geschlechtergemischten Berufslehren von Misserfolg bei der LAP betroffen sind, wird anhand zweier vorbereitenden Hypothesen und einer Haupthypothese bearbeitet. Die beiden vorbereitenden Hypothesen stehen im engen Zusammenhang mit der Art des auszuwertenden Datenmaterials. Zum einen sind die Häufigkeiten der Lernenden in den jeweiligen Berufslehren unterschiedlich: Sie reichen von Einzelpersonen bis zu über 10'000 in einem Jahr. Zum anderen bilden Lernende in geschlechtsuntypischen Berufslehren definitionsgemäß eine kleine Minderheit: Teilweise sind es weniger als 10 Männer bzw. Frauen in einem Jahr und Beruf. Deshalb werden die Häufigkeitsdaten von fünf aufeinanderfolgenden Jahren zusammengezählt. Die beiden vorbereitenden Hypothesen prüfen, ob die Zusammenfassung der fünf Datensätze aus den Jahren 2008 bis 2012 möglich ist. Damit wird vermieden, dass sich Effekte aufgrund der Zusammenführung ausnivellieren. Das Vorgehen ist dasselbe wie in Kapitel 5.

Die Hypothese 2a befasst sich mit dem Geschlechterverhältnis in den Berufslehren. Obwohl die geschlechtliche Segregation in der Berufswelt sich auch durch die Berufslehre zieht (z.B. Abraham/Arpagaus 2008), gilt es zu prüfen, ob der Frauenanteil über die fünf Jahre in den untersuchten Berufslehren stabil bleibt. Dafür wird die Stärke des Zusammenhangs des Frauenanteils jeder Berufslehre und jedes Jahrs ermittelt. Daraus folgt die Hypothese $2 a$ : Die Frauenanteile der fünf untersuchten Jahre hängen stark zusammen und bleiben damit konstant.

Berufslehren weisen unterschiedliche Misserfolgsquoten auf (Rottermann 2011). Die Misserfolgsquote bezeichnet den prozentualen Anteil der Lernenden, die die LAP nicht bestanden haben, an allen LAP-Kandidierenden. Die Stabilität der Misserfolgsquote gibt Auskunft darüber, ob das Bestehen bzw. Scheitern einem hohen Anteil an Zufälligkeit und Variabilität unterworfen ist. Die Stabilität der Misserfolgsquoten innerhalb der Berufslehren wird mit Hypothese $2 b$ geprüft: Die Misserfolgsquoten der fünf Jahre hängen stark zusammen und bleiben damit konstant.

Sind die Frauenanteile und die Misserfolgsquoten stabil, können die Datensätze aus den fünf Jahren zusammengeführt werden. Damit ist der Weg frei, um die zentrale Frage zu bearbeiten. Sind Lernende, wenn sie zur geschlechtlichen Minderheit gehören, überzufällig häufig von einem Misserfolg bei der LAP betroffen? Hypothese 2c: Lernende in geschlechtsuntypischen Berufslehren scheitern häufiger an der LAP als Personen in geschlechtstypischen oder geschlechtergemischten Berufslehren. 
Diese Hypothese prüft im Modell der geschlechtsuntypischen Berufssozialisation, ob sich die negativen Reaktionen des sozialen Umfelds auf die Leistungen an der LAP der Lernenden in geschlechtsuntypischen Berufslehren auswirken. Nach dem Modell könnten höhere Belastungen aufgrund der Minderheitenposition leistungshemmend wirken.

\subsection{Methode}

Die Grundlagen der Auswertungen in diesem Unterkapitel bilden die vom BFS erstellt jährlich Statistiken zur beruflichen Grundbildung aus den Jahren 2008 bis 2012. Auf die Beschreibung der Datenstruktur (Abschnitt 6.2.1) folgen die Ausführungen zu den Auswertungsmethoden (Abschnitt 6.2.2).

\subsubsection{Beschreibung der Datenstruktur}

In den verwendeten BFS-Datensätzen werden für jeden Beruf die Häufigkeiten der Kandidierenden der LAP und der ausgestellten Fähigkeitszeugnisse dargestellt. Häufigkeitsangaben liegen zusätzlich für die beiden Geschlechter vor. Die Tabelle 6.1 zeigt an einem fiktiven Beispiel von zwei Berufslehren die Anzahl der Kandidierenden in einem Jahr im Total und aufgeteilt nach Männern und Frauen. Die Häufigkeiten der ausgestellten Fähigkeitszeugnisse folgen in den nächsten drei Spalten. Wie ich die Misserfolgsquoten, Frauenanteile und Gender Gaps berechnet habe, beschreibe ich in Abschnitt 6.2.2 Auswertungsmethoden.

Tabelle 6.1: Fiktives Beispiel zur Demonstration der Datenrepräsentation

\begin{tabular}{|c|c|c|c|c|c|c|c|c|c|c|c|}
\hline \multirow{2}{*}{$\begin{array}{l}\text { Berufs- } \\
\text { lehre }\end{array}$} & \multicolumn{3}{|c|}{$\begin{array}{c}\text { Anzahl } \\
\text { Kandidierende an LAP }\end{array}$} & \multicolumn{3}{|c|}{$\begin{array}{c}\text { Anzahl } \\
\text { Fähigkeitszeugnisse }\end{array}$} & \multicolumn{3}{|c|}{$\begin{array}{l}\text { Misserfolgsquote } \\
\text { in } \%\end{array}$} & \multirow{2}{*}{$\begin{array}{l}\text { Frauen- } \\
\text { anteil } \\
\text { in \% }\end{array}$} & \multirow{2}{*}{$\begin{array}{l}\text { Gende } \\
\text { Gap } \\
\text { in \% }\end{array}$} \\
\hline & Total & Männer & Frauen & Total & Männer & Frauen & Total & Männer & Frauen & & \\
\hline 1 & 200 & 100 & 100 & 160 & 80 & 80 & 20.0 & 20.0 & 20.0 & 50.0 & 0.0 \\
\hline 2 & 200 & 180 & 20 & 176 & 162 & 14 & 10.0 & 30.0 & 10.0 & 10.0 & -20.0 \\
\hline
\end{tabular}

Quelle: Eigene Darstellung.

In die Auswertung wurden 200 EFZ-Berufslehren aufgenommen (vgl. Tabelle 6.2). In den fünf untersuchten Jahren traten 321'495 Lernende zur LAP an. Prüfungswiederholende sind in den Zahlen enthalten (1., 2. und 3. Versuch). Beinahe 10 Prozent scheitern an der LAP. Eine Mehrheit tritt im Folgejahr noch einmal an, ihre Erfolgschancen sind geringer als bei Erstkandidierenden (Amos et al. 2003). 9 Berufslehren wurden ausgeschlossen, da keine Häufigkeitsangaben für sie vorhanden waren. In den meisten Fällen handelte es sich 
um auslaufende Lehrgänge. Bei den 3 Berufslehren „Detailhandelsfachmann/-frau EFZ“, „Gärtner/-in EFZ“ und „Kaufmann/-frau EFZ“ gingen Werte der Fachbereiche auf der Ebene der Berufslehren in die Auswertung ein. Einerseits ist die Geschlechterzusammensetzung auf Ebene Fachbereich in diesen Berufslehren unterschiedlich, andererseits gibt es genügend Lernende, sodass sich eine Aufteilung anbietet. Eine Liste mit allen 200 Berufslehren und ihren Misserfolgsquoten ist im Online-Anhang VII zu finden.

Für die Korrelationsanalysen wurden lediglich diejenigen Berufslehren einbezogen, die über mehr als 100 Kandidierende verfügten. Die selten besuchten Berufslehren wurden ausgeschlossen, um Verzerrungen aufgrund kleiner Fallzahlen, die sich stark auf die Prozentzahlen auswirken würden, zu vermeiden.

\subsubsection{Auswertungsmethoden}

Zur Ermittlung der Verhältnisse der Geschlechter wurden die Frauenanteile berechnet. Je höher der Frauenanteil, desto mehr Frauen erlernen diesen Beruf. Die Misserfolgsquoten der Berufslehren wurden insgesamt und nach Geschlecht berechnet. Die Stabilität wurde mit Korrelationen nach Pearson mit paarweisem Ausschluss bestimmt. Für die Korrelationsberechnungen wurden die Jahren 2008 bis 2012 jeweils paarweise verglichen.

Die Differenz zwischen der Misserfolgsquote der Frauen und jener der Männer wird mit der Höhe des Gender Gaps ${ }^{30}$ ausgedrückt. Der Gender Gap ergibt sich aus der Differenz der Misserfolgsquoten zwischen den Geschlechtern. Positive Werte beim Gender Gap bedeuten, dass mehr Frauen als Männer die LAP bestehen, und negative Zahlen, dass Frauen häufiger als Männer scheitern.

Der Gender Gap bildet eine Suchheuristik, um Trends für allfällige Benachteiligungen von Lernenden in geschlechtsuntypischen Berufslehren aufzudecken. Da durchschnittlich 11.2 Prozent männliche und 7.0 Prozent der weiblichen Kandidierenden die LAP nicht bestanden haben, wird diese Differenz, also der Gender Gap von 4.2 Prozent, als kritischer Wert betrachtet (vgl. Tabelle 6.2). Die Differenz kann dadurch erklärt werden, dass Frauen generell häufiger metakognitive Lernstrategien verwenden (Ziegler/Dresel 2006) und mehr Zeit fürs Lernen investieren als Männer (Lehmann et al. 2001), Männer auf der anderen Seite ihre Fähigkeiten tendenziell eher überschätzen (Baumert/Lehmann 1997, Bischof-Köhler 2004, Horstkemper 1995).

Interpretiert werden also die Abweichungen vom durchschnittlichen Gender Gap. Um die Interpretation der Resultate zu verdeutlichen, werden bei der grafischen Darstellung die Abweichungen vom durchschnittlichen Gender

Vgl. Abschnitt 5.2.1, Fußnote 26. 
Gap dargestellt. Zur Veranschaulichung wird der Nullpunkt um 4.2 Prozentpunkte verschoben.

\subsection{Ergebnisse}

Die Befunde zu den Stabilitäten der Kennwerte Frauenanteil und Misserfolgsquote werden in Abschnitt 6.3.1 behandelt. Im darauffolgenden Abschnitt 6.3.2 werden die Misserfolgsquoten und die Gender Gaps dargestellt.

\subsubsection{Stabilitäten der Frauenanteile und der Misserfolgsquoten}

Hypothese 2a. Die Frauenanteile der fünf untersuchten Jahre hängen stark zusammen und bleiben damit konstant. Die Korrelationskoeffizienten für die Frauenanteile der Jahre 2008 bis 2012 betragen $r=.98-.99(p<.001)$. Die höchst signifikanten Korrelationen belegen eine starke Stabilität der Frauenanteile über die fünf untersuchten Jahre.

Hypothese $2 b$. Die Misserfolgsquoten der fünf Jahre hängen ebenfalls stark zusammen und bleiben damit konstant. Sie korrelieren höchst signifikant $(r=.67-.80, p<.001$, vgl. Online-Anhang VI).

Mit dem Nachweis der Stabilität der Frauenanteile und der Misserfolgsquoten ist das Zusammenführen der fünf Datensätze aus den Jahren 2008 bis 2012 möglich. Diese Zusammenfassung ermöglicht, dass sogar Berufslehren untersucht werden können, die lediglich von wenigen Personen des jeweils untervertretenen Geschlechts absolviert werden. Der nächste Schritt besteht darin, die Hypothese $2 c$ zu prüfen: Lernende in geschlechtsuntypischen Berufslehren scheitern häufiger an der LAP als Lernende in geschlechtstypischen bzw. gemischtgeschlechtlichen Berufslehren.

\subsubsection{Misserfolgsquote}

In den folgenden Darstellungen wurden alle EFZ-Berufslehren der Jahrgänge 2008 bis 2012 berücksichtigt. Der Datensatz umfasst 321'495 Lernende in 200 Berufslehren. Zu beachten ist, dass die Fallzahlen in den Berufslehren stark variieren. Die Einteilung der Berufslehren entsprechend des Frauenanteils in 22 Gruppen macht die Effekte sichtbar, ohne dass die unterschiedlichen Fallzahlen durch Verzerrungen eine Interpretation verunmöglichen. Die Anzahlen der Kandidierenden und der ausgestellten Fähigkeitszeugnisse wurden für jede Kategorie zusammengezählt. Anschließend wurden die 
Misserfolgsquote, der Frauenanteil und der Gender Gap auf dieser Grundlage neu berechnet.

Tabelle 6.2: LAP-Misserfolgsquoten und Gender Gap

\begin{tabular}{|c|c|c|c|c|c|c|c|c|c|c|c|c|}
\hline \multirow{2}{*}{\multicolumn{2}{|c|}{$\begin{array}{l}\text { Gruppen nach } \\
\text { Frauenanteil } \\
\text { in \% }\end{array}$}} & \multirow{2}{*}{$\begin{array}{l}\text { Anzahl } \\
\text { Berufs- } \\
\text { lehren }\end{array}$} & \multicolumn{3}{|c|}{ Kandidierende } & \multicolumn{3}{|c|}{ Fähigkeitszeugnisse } & \multicolumn{3}{|c|}{ Misserfolgsquoten in \% } & \multirow{2}{*}{$\begin{array}{c}\text { Gender } \\
\text { Gap } \\
\text { in } \% \\
\end{array}$} \\
\hline & & & Total & Männer & Frauen & Total & Männer & Frauen & Total & Männer & Frauen & \\
\hline \multirow{7}{*}{ 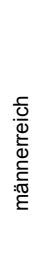 } & 0 & 7 & 848 & 848 & 0 & 739 & 739 & 0 & 12.9 & 12.9 & & \\
\hline & $0.1-5$ & 37 & $80^{\prime} 183$ & $78 ' 508$ & $1^{\prime} 675$ & $700^{\prime} 087$ & $68^{\prime} 608$ & $1^{\prime} 479$ & 12.6 & 12.6 & 11.7 & 0.9 \\
\hline & $5-10$ & 25 & $29 ' 604$ & $27^{\prime} 088$ & 2'516 & $266^{\prime} 240$ & $23 ' 936$ & 2'304 & 11.4 & 11.6 & 8.4 & 3.2 \\
\hline & $10-15$ & 11 & $10^{\prime} 919$ & $9^{\prime} 608$ & 1'311 & $10^{\prime} 090$ & $8^{\prime} 867$ & $1 ' 223$ & 7.6 & 7.7 & 6.7 & 1.0 \\
\hline & $15-20$ & 12 & $12^{\prime} 007$ & $10^{\prime} 042$ & 1'965 & $11 ' 170$ & $9^{\prime} 286$ & $1 ' 884$ & 7.0 & 7.5 & 4.1 & 3.4 \\
\hline & $20-25$ & 8 & $5^{\prime} 073$ & 3'919 & 1'154 & $4^{\prime} 624$ & 3'555 & $1^{\prime} 069$ & 8.9 & 9.3 & 7.4 & 1.9 \\
\hline & $25-30$ & 5 & 7'569 & $5 ' 338$ & 2'231 & 6'930 & 4'884 & $2^{\prime} 046$ & 8.4 & 8.5 & 8.3 & 0.2 \\
\hline \multirow{8}{*}{ 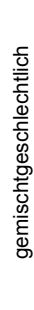 } & $30-35$ & 5 & 2'170 & $1^{\prime} 468$ & 702 & 1'980 & 1'333 & 647 & 8.8 & 9.2 & 7.8 & 1.1 \\
\hline & $35-40$ & 8 & $15^{\prime} 533$ & 9'807 & $5^{\prime} 726$ & $13^{\prime} 133$ & 7'921 & $5^{\prime} 212$ & 15.5 & 19.2 & 9.0 & 10.3 \\
\hline & $40-45$ & 6 & $5^{\prime} 729$ & 3'285 & $2^{\prime} 444$ & 5'377 & $3^{\prime} 057$ & $2^{\prime} 320$ & 6.1 & 6.9 & 5.1 & 1.9 \\
\hline & $45-50$ & 7 & 1'735 & 921 & 814 & 1'567 & 835 & 732 & 9.7 & 9.3 & 10.1 & -0.7 \\
\hline & $50-55$ & 3 & 7'121 & $3^{\prime} 459$ & $3^{\prime} 662$ & 6'876 & 3'336 & 3'540 & 3.4 & 3.6 & 3.3 & 0.2 \\
\hline & $55-60$ & 13 & $12^{\prime} 281$ & $5^{\prime} 005$ & $7^{\prime} 276$ & 11'391 & 4'555 & 6'836 & 7.2 & 9.0 & 6.0 & 2.9 \\
\hline & $60-65$ & 4 & $5^{\prime} 979$ & 2'217 & $3^{\prime} 762$ & $5^{\prime} 527$ & $2^{\prime} 019$ & 3'508 & 7.6 & 8.9 & 6.8 & 2.2 \\
\hline & $65-70$ & 4 & $32 ' 650$ & $10^{\prime} 705$ & 21'945 & $29 ' 646$ & $9^{\prime} 679$ & $19 ' 967$ & 9.2 & 9.6 & 9.0 & 0.6 \\
\hline \multirow{7}{*}{ 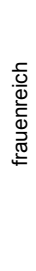 } & $70-75$ & 7 & $17^{\prime} 063$ & $4^{\prime} 943$ & $12^{\prime} 120$ & $15^{\prime} 678$ & $4^{\prime} 445$ & $11 ' 233$ & 8.1 & 10.1 & 7.3 & 2.8 \\
\hline & $75-80$ & 7 & $8^{\prime} 809$ & 1'996 & 6'813 & $8^{\prime} 116$ & $1 ' 780$ & 6'336 & 7.9 & 10.8 & 7.0 & 3.8 \\
\hline & $80-85$ & 3 & 1'389 & 250 & 1'139 & 1'271 & 238 & $1^{\prime} 033$ & 8.5 & 4.8 & 9.3 & -4.5 \\
\hline & $85-90$ & 5 & $14^{\prime} 051$ & 1'837 & $12^{\prime} 214$ & $13^{\prime} 350$ & $1 ' 727$ & $11 ' 623$ & 5.0 & 6.0 & 4.8 & 1.1 \\
\hline & $90-95$ & 8 & $26 ' 891$ & 2'167 & $24 ' 724$ & $25^{\prime} 440$ & 1'975 & $23^{\prime} 465$ & 5.4 & 8.9 & 5.1 & 3.8 \\
\hline & 95-99.9 & 12 & $23 ' 712$ & 368 & $23^{\prime} 344$ & $21^{\prime} 737$ & 303 & $21^{\prime} 434$ & 8.3 & 17.7 & 8.2 & 9.5 \\
\hline & 100 & 3 & 179 & 0 & 179 & 162 & 0 & 162 & 9.5 & & 9.5 & \\
\hline \multicolumn{2}{|c|}{ Total } & 200 & $321 ' 495$ & $183^{\prime} 779$ & $137^{\prime} 716$ & 291'131 & $163^{\prime} 078$ & $128^{\prime} 053$ & 9.4 & 11.3 & 7.0 & 4.2 \\
\hline
\end{tabular}

Legende: Jahre 2008-2012; fett gedruckt: hohe Misserfolgsquoten; hellgraue Markierung: zum Nachteil der Frauen, wenn der Gender Gap kleiner ist als 4.2\%; dunkelgraue Markierung: zum Nachteil der Männer, wenn der Gender Gap über $4.2 \%$ ist. Quelle: Eigene Darstellung.

Mit 11.7 Prozent haben Frauen in männerreichen Berufslehren der Gruppen mit einem Frauenanteil von 0.1-5 Prozent die höchste Misserfolgsquote von allen Frauengruppen (vgl. Tabelle 6.2). In dieser Gruppe befinden sich 37 Berufslehren und über 80'000 Lernende. Es ist mit Abstand die größte Gruppe, und die Misserfolgsquote ist sowohl bei den Frauen wie bei den Männern $(12.6 \%)$ hoch. In der frauenreichen Gruppe mit einem Frauenanteil von 9599.9 Prozent ist die Misserfolgsquote für Männer mit 17.7 Prozent am zweithöchsten. Der Gender Gap ist in dieser Gruppe ebenfalls der zweithöchste. Die höchste Misserfolgsquote bei den Männern und beim Total für beide 
Geschlechter hat mit 19.2 Prozent bzw. 15.5 Prozent die Gruppe mit einem Frauenanteil von 35-40 Prozent. In dieser Gruppe sind die beiden Berufslehren ,Koch/Köchin EFZ' und ,Maler/-in EFZ' diejenigen mit den meisten Lernenden. Beide Berufslehren haben überdurchschnittlich hohe Misserfolgsquoten. Hier findet sich auch der größte Unterschied zwischen den Geschlechtern mit einem Gender Gap von 10.3 Prozentpunkten.

Abbildung 6.1: LAP-Abweichungen in 5-Prozent-Gruppen

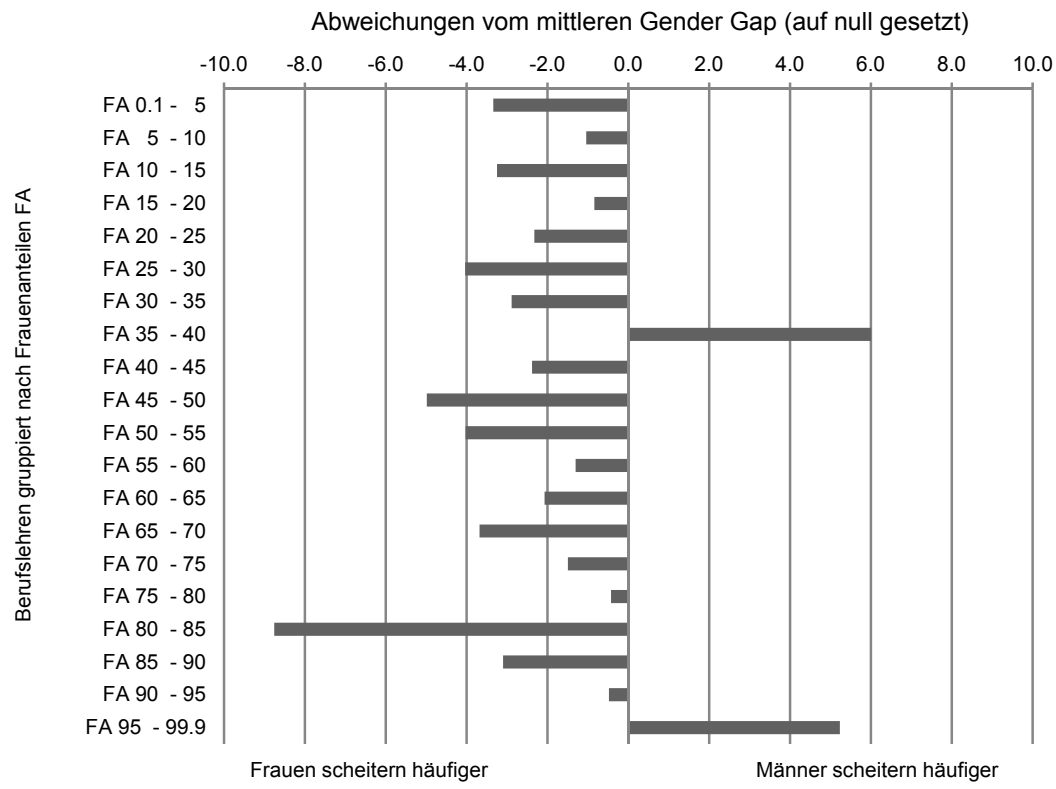

Quelle: Eigene Darstellung.

Es fällt auf, dass Frauen in männerreichen Berufslehren mit einer Misserfolgsquote von 11.7 Prozent weit über der mittleren Misserfolgsquote von 7.0 Prozent liegen. Der gleiche Effekt erscheint bei Männern in frauenreichen Berufslehren. Sie haben eine deutlich erhöhte Misserfolgsquote von 17.7 Prozent im Vergleich zu der mittleren Quote von 11.3 Prozent. Diese Effekte verschwinden bei Vergleichen mit drei Gruppen (männerreiche Berufslehren mit einem FA bis 30\%, geschlechtergemischte Berufslehren [FA $=30-70 \%$ ] und frauenreiche Berufslehren mit einem FA ab 70\%). Diese Befunde lassen vermuten, dass sich zwar keine generelle Diskriminierung von Lernenden in geschlechtsuntypischen Berufslehren bei der LAP nachweisen lässt, jedoch in den stark geschlechtersegregierten Berufslehren ein erhöhtes Risiko für die 
Minderheiten besteht, die LAP nicht zu bestehen. Eine eingehende Betrachtung auf Berufsebene verspricht hier weitere Erkenntnisse.

In der eher kleinen Gruppe mit einem Frauenanteil von 80-85 Prozent sind die drei Berufslehren ,Polydesigner/-in 3D EFZ', ,Buchhändler/-in $E F Z^{`}$ und ,Keramiker/-in EFZ' zusammengefasst. In allen dreien sind die Männer erfolgreicher (vgl. Online-Anhang VII).

Tabelle 6.3: LAP-Misserfolgsquoten in männerreichen Berufslehren

\begin{tabular}{|c|c|c|c|c|c|c|c|c|c|c|c|}
\hline \multirow{2}{*}{$\begin{array}{l}\text { Berufslehren } \\
\text { FA } 0.1-5 \%\end{array}$} & \multicolumn{3}{|c|}{ Kandidierende } & \multicolumn{3}{|c|}{ Fähigkeitszeugnisse } & \multicolumn{3}{|c|}{ Misserfolgsquote in \% } & \multirow{2}{*}{$\begin{array}{c}\text { Frauen- } \\
\text { anteil } \\
\text { in } \% \\
\end{array}$} & \multirow{2}{*}{$\begin{array}{l}\text { Gender } \\
\text { Gap } \\
\text { in \% }\end{array}$} \\
\hline & Total & Männer & Frauen & Total & Männer & Frauen & Total & Männer & Frauen & & \\
\hline Polybauer/-in EFZ & $1 ' 595$ & $1 ' 560$ & 35 & 1'313 & $1 ' 289$ & 24 & 17.7 & 17.4 & 31.4 & 2.2 & -14.1 \\
\hline Forstwart/-in EFZ & $1 ' 644$ & $1^{\prime} 621$ & 23 & $1 ' 438$ & $1^{\prime} 421$ & 17 & 12.5 & 12.3 & 26.1 & 1.4 & -13.7 \\
\hline Spengler/-in EFZ & $1 ' 826$ & $1 ' 782$ & 44 & $1 ' 519$ & $1^{\prime} 485$ & 34 & 16.8 & 16.7 & 22.7 & 2.4 & -6.1 \\
\hline $\begin{array}{l}\text { Automobil-Mecha- } \\
\text { troniker/-in EFZ }\end{array}$ & 6'209 & 6'027 & 182 & 5'151 & $5^{\prime} 010$ & 141 & 17.0 & 16.9 & 22.5 & 2.9 & -5.7 \\
\hline $\begin{array}{l}\text { Multimediaelektroni- } \\
\text { ker/-in }\end{array}$ & $1 ' 074$ & 1'046 & 28 & 850 & 829 & 21 & 20.9 & 20.7 & 25.0 & 2.6 & -4.3 \\
\hline Gipser/-in & 814 & 777 & 37 & 561 & 536 & 25 & 31.1 & 31.0 & 32.4 & 4.5 & -1.4 \\
\hline $\begin{array}{l}\text { Automobil- } \\
\text { Fachmann/-frau EFZ }\end{array}$ & $5^{\prime} 763$ & $5^{\prime} 577$ & 186 & 4'995 & 4'836 & 159 & 13.3 & 13.3 & 14.5 & 3.2 & -1.2 \\
\hline Elektroniker/-in EFZ & $4^{\prime} 522$ & $4^{\prime} 373$ & 149 & 4'368 & $4^{\prime} 225$ & 143 & 3.4 & 3.4 & 4.0 & 3.3 & -0.6 \\
\hline Metallbauer/-in EFZ & 2'598 & $2 ' 551$ & 47 & $2 ' 377$ & 2'334 & 43 & 8.5 & 8.5 & 8.5 & 1.8 & 0.0 \\
\hline $\begin{array}{l}\text { Polymechaniker/-in } \\
\text { EFZ }\end{array}$ & $8^{\prime} 671$ & $8^{\prime} 390$ & 281 & $8^{\prime} 579$ & 8'301 & 278 & 1.1 & 1.1 & 1.1 & 3.2 & 0.0 \\
\hline Maurer/-in EFZ & $6 ' 320$ & $6^{\prime} 277$ & 43 & $5^{\prime} 427$ & $5 ' 390$ & 37 & 14.1 & 14.1 & 14.0 & 0.7 & 0.2 \\
\hline $\begin{array}{l}\text { Zimmermann/Zim- } \\
\text { merin }\end{array}$ & 4'854 & $4^{\prime} 789$ & 65 & 4'324 & 4'266 & 58 & 10.9 & 10.9 & 10.8 & 1.3 & 0.2 \\
\hline $\begin{array}{l}\text { Montage-Elektriker/- } \\
\text { in EFZ }\end{array}$ & $2 ' 859$ & $2^{\prime} 823$ & 36 & 2'297 & $2^{\prime} 268$ & 29 & 19.7 & 19.7 & 19.4 & 1.3 & 0.2 \\
\hline $\begin{array}{l}\text { Sanitärinstallateur/-in } \\
\text { EFZ }\end{array}$ & $4^{\prime} 684$ & $4^{\prime} 619$ & 65 & $3^{\prime} 757$ & $3^{\prime} 704$ & 53 & 19.8 & 19.8 & 18.5 & 1.4 & 1.3 \\
\hline $\begin{array}{l}\text { Produktionsmechani- } \\
\text { ker/-in EFZ }\end{array}$ & $1 ' 871$ & $1 ' 809$ & 62 & $1 ' 784$ & $1^{\prime} 724$ & 60 & 4.6 & 4.7 & 3.2 & 3.3 & 1.5 \\
\hline $\begin{array}{l}\text { Straßenbauer/-in } \\
\text { EFZ }\end{array}$ & 1'575 & $1 ' 548$ & 27 & $1 ' 479$ & $1^{\prime} 453$ & 26 & 6.1 & 6.1 & 3.7 & 1.7 & 2.4 \\
\hline Automatiker/-in EFZ & $3^{\prime} 511$ & $3^{\prime} 418$ & 93 & $3^{\prime} 413$ & 3'320 & 93 & 2.8 & 2.9 & 0.0 & 2.6 & 2.9 \\
\hline $\begin{array}{l}\text { Elektroinstallateur/-in } \\
\text { EFZ }\end{array}$ & $10^{\prime} 220$ & $10^{\prime} 054$ & 166 & $8^{\prime} 222$ & $8^{\prime} 078$ & 144 & 19.5 & 19.7 & 13.3 & 1.6 & 6.4 \\
\hline $\begin{array}{l}\text { Carrossier/-in } \\
\text { Spenglerei EFZ }\end{array}$ & 1'268 & 1'247 & 21 & $1 ' 030$ & 1'011 & 19 & 18.8 & 18.9 & 9.5 & 1.7 & 9.4 \\
\hline
\end{tabular}

Legende: Jahre 2008-2012; fett gedruckt: hohe Misserfolgsquoten; hellgraue Markierung: zum Nachteil der Frauen, wenn der Gender Gap kleiner ist als $4.2 \%$; dunkelgraue Markierung: zum Nachteil der Männer, wenn der Gender Gap über $4.2 \%$ ist. Quelle: Eigene Darstellung.

Für die Darstellung dieser Resultate wurde ein spezielles Verfahren gewählt. In der Abbildung 6.1 stellen die Balken die Abweichung vom mittleren Gender Gap von 4.2 Prozent dar. Beispielsweise scheitern Frauen in der männerreichen Gruppe (Frauenanteil 0.1-5\%) häufiger an der LAP. Ihre Misserfolgsquote ist um 3.3 Prozent tiefer als der durchschnittliche Gender Gap von 4.2 Prozent (Gender Gap der Gruppe minus mittlerer Gender Gap: 0.9\% $4.2 \%=-3.3 \%$ ). Diese Darstellung bildet damit eine Suchheuristik zur Erken- 
nung von Trends. Im Durchschnitt scheitern die Männer häufiger. In zwei Gruppen scheitern die Männer noch deutlich häufiger: Gruppen mit FA = 3540 Prozent und FA = 95-99.9 Prozent. In der Gruppe mit einem Frauenanteil von 80-85 Prozent scheitern die Frauen auffällig häufig.

Tabelle 6.4: LAP-Misserfolgsquoten in frauenreichen Berufslehren

\begin{tabular}{|c|c|c|c|c|c|c|c|c|c|c|c|}
\hline \multirow{2}{*}{$\begin{array}{l}\text { Berufslehren } \\
\text { FA 95-99.9\% }\end{array}$} & \multicolumn{3}{|c|}{ Kandidierende } & \multicolumn{3}{|c|}{ Fähigkeitszeugnisse } & \multicolumn{3}{|c|}{ Misserfolgsquote in \% } & \multirow{2}{*}{$\begin{array}{c}\text { Frau- } \\
\text { en- } \\
\text { anteil } \\
\text { in } \% \\
\end{array}$} & \multirow{2}{*}{$\begin{array}{c}\text { Gen- } \\
\text { der } \\
\text { Gap in } \\
\%\end{array}$} \\
\hline & Total & Männer & Frauen & Total & Männer & Frauen & Total & Männer & Frauen & & \\
\hline $\begin{array}{l}\text { Detailhandelsfach- } \\
\text { mann/-frau EFZ - } \\
\text { Papeterie }\end{array}$ & 801 & 24 & 777 & 783 & 23 & 760 & 2.2 & 4.2 & 2.2 & 97.0 & 2.0 \\
\hline $\begin{array}{l}\text { Bekleidungsgestal- } \\
\text { ter/-in EFZ }\end{array}$ & $1^{\prime} 528$ & 55 & $1^{\prime} 473$ & $1^{\prime} 462$ & 51 & $1^{\prime} 411$ & 4.3 & 7.3 & 4.2 & 96.4 & 3.1 \\
\hline Florist/-in EFZ & $1^{\prime} 820$ & 39 & $1^{\prime} 781$ & $1^{\prime} 736$ & 35 & $1^{\prime} 701$ & 4.6 & 10.3 & 4.5 & 97.9 & 5.8 \\
\hline $\begin{array}{l}\text { Detailhandelsfach- } \\
\text { mann/-frau EFZ - } \\
\text { Bäckerei u.ä. }\end{array}$ & $1^{\prime 2} 258$ & 32 & 1'226 & 1'160 & 25 & '135 & 7.8 & 21.9 & 7.4 & 97.5 & 14.5 \\
\hline $\begin{array}{l}\text { Pharma-Assistent/-in } \\
\text { EFZ }\end{array}$ & $4^{\prime} 224$ & 79 & 4'145 & $3^{\prime} 804$ & 56 & $3^{\prime} 748$ & 9.9 & 29.1 & 9.6 & 98.1 & 19.5 \\
\hline $\begin{array}{l}\text { Fachmann/-frau } \\
\text { Hauswirtschaft EFZ }\end{array}$ & $2^{\prime} 180$ & 60 & $2^{\prime} 120$ & $1^{\prime} 964$ & 40 & $1^{\prime} 924$ & 9.9 & 33.3 & 9.2 & 97.2 & 24.1 \\
\hline
\end{tabular}

Legende: fett gedruckt: hohe Misserfolgsquoten; hellgraue Markierung: zum Nachteil der Frauen, wenn der Gender Gap kleiner ist als 4.2\%; dunkelgraue Markierung: zum Nachteil der Männer, wenn der Gender Gap über $4.2 \%$ ist. Quelle: Eigene Darstellung.

Zusammenfassend sind die gefundenen Effekte nicht so deutlich, wie in der Hypothese formuliert. Die beiden Extremgruppen jedoch bestätigen die Annahme, dass das marginalisierte Geschlecht öfter an der LAP scheitert als erwartet.

Die Befunde sind noch deutlicher, wenn Berufslehren mit 5 Prozent des jeweils andern Geschlechts untersucht werden. In den Tabellen 6.3 und 6.4 werden Berufslehren mit einseitigen Geschlechterverhältnissen dargestellt (FA $=0.1-5 \%$ bzw. 90-99.9\%). Die Berufslehren sind in der Reihenfolge des Gender Gaps aufgeführt, angefangen beim höchsten. In den 19 männerreichsten Berufslehren haben Frauen meistens erhöhte Misserfolgsquoten. In 17 davon wird der Erwartungswert des Gender Gaps unterschritten. Mehr Frauen als erwartet scheitern demnach bei der LAP. In lediglich 2 Berufslehren ergibt sich ein umgekehrtes Bild: Die ,Elektroinstallateurinnen EFZ' und die ,Carrossierinnen $E F Z^{\prime}$ reüssieren häufiger als ihre männlichen Kollegen (vgl. Abbildung 6.2).

Im Vergleich zu ihren Kolleginnen scheitern die Männer in 4 der 6 frauenreichen Berufslehren mit einem Frauenanteil von über 95 Prozent häufiger als erwartet: Es sind dies ,Floristen EFZ', ,Detailhandelsfachmänner EFZ Bäckerei/Konditorei/Confiserie“, ,Pharma-Assistenten EFZ' und ,Fachmänner Hauswirtschaft EFZ' (vgl. Tabelle 6.4). Die ,Detailhandelsfachmänner EFZ - Papeterie ' und die ,Bekleidungsgestalter EFZ' scheitern weniger häufig an der LAP als der Durchschnitt. Dieser Trend ist deutlich in der Abbil- 
dung 6.2 ersichtlich. Es werden wiederum die Abweichungen vom mittleren Gender Gap dargestellt.

Abbildung 6.2: LAP-Abweichungen in Berufslehren

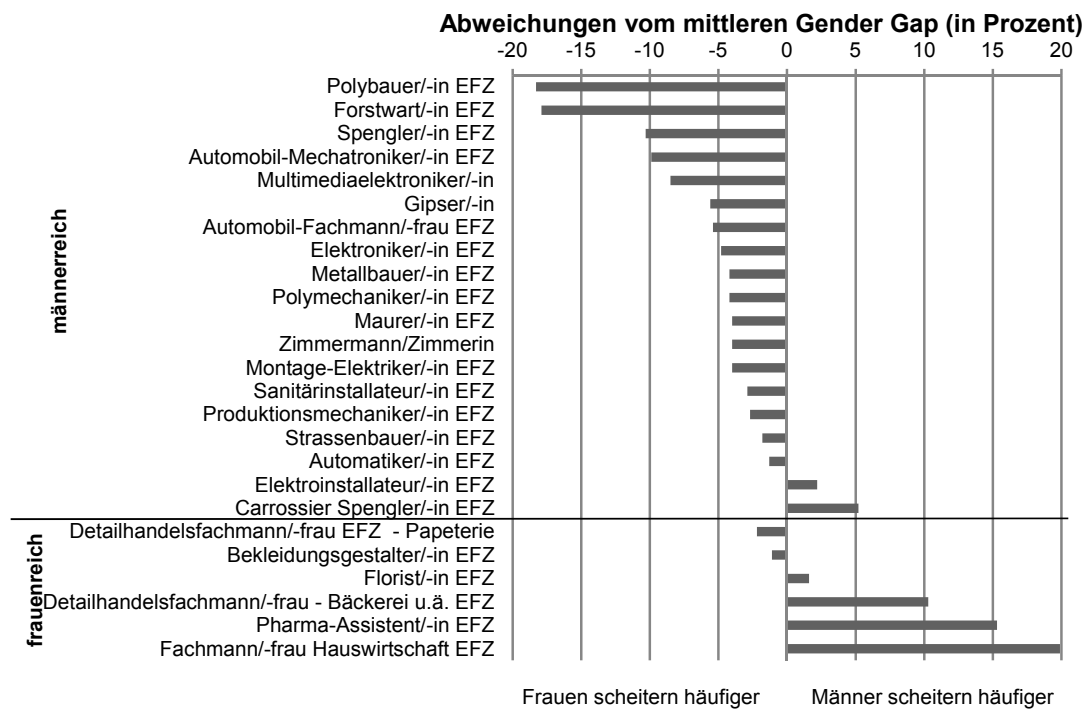

Legende: Berufslehren mit maximal 5 Prozent Lernenden in geschlechtsuntypischen Berufslehren. Quelle: Eigene Darstellung.

\subsection{Diskussion}

Mit den oben dargestellten Auswertungen wurde die Frage bearbeitet, ob Lernende in geschlechtsuntypischen Berufslehren bei der LAP einen Nachteil erfahren. Dazu wurden zwei vorbereitende Hypothesen aufgestellt, um zu prüfen, ob eine Zusammenfassung des verwendeten Datenmaterials über die fünf Jahre opportun ist. Das Datenmaterial stützt die beiden Hypothesen 2a und $2 \mathrm{~b}$. Da in den untersuchten Jahren hohe Korrelationskoeffizienten festgestellt wurden, konnten die fünf Jahre zusammengefasst werden, ohne dass befürchtet werden musste, divergierende oder sich ausgleichendende Effekte zu übersehen. Die Frauenanteile und Misserfolgsquoten der Berufslehren bleiben über die fünf Jahre konstant. 
Die Hypothese 2c - Lernende in geschlechtsuntypischen Berufslehren scheitern an der LAP häufiger als Lernende in geschlechtstypischen bzw. gemischtgeschlechtlichen Berufslehren - erwies sich teilweise als korrekt. Die Befundlage ist heterogener als die Ergebnisse zu den Lehrvertragsauflösungen. Insbesondere in Berufslehren mit einem stark verzogenen Geschlechterverhältnis weisen die Ergebnisse einer Mehrheit von Berufslehren eine systematische Benachteiligung der Frauen im Sinn von Kanters TokenismusTheorie (1977) auf. Zwar bestehen in einigen Berufslehren Vorteile für Männer in frauenreichen Berufslehren, wie dies Williams Glaslift-Theorie (1993) postuliert, in anderen ist es jedoch genau umgekehrt. Auffälligkeiten sind an folgenden Stellen aufgetreten: In männerreichen Berufslehren fallen die Frauen etwas häufiger durch die LAP als in frauenreichen und geschlechtergemischten Berufslehren (,Polybauerinnen EFZ', ,Forstwartinnen EFZ', ,Spenglerinnen EFZ', ,Automobil-Mechatronikerinnen EFZ', ,Multimediaelektronikerinnen' und ,Gipserinnen', vgl. Abbildung 6.2). Bei den Männern in frauenreichen Berufslehren fallen die wenigen Männer öfter durch als erwartet (,Floristen EFZ', ,Detailhandelsfachmänner EFZ - Bäckerei/Konditorei/Confiserie', ,Pharma-Assistenten EFZ' und ,Fachmänner Hauswirtschaft EFZ', vgl. Tabelle 6.4). Jedoch gibt es daneben Gegenbeispiele, wie die ,Bekleidungsgestalter EFZ' und ,Detailhandelsfachmänner $E F Z$ - Papeterie ${ }^{6}$, die häufiger als erwartet reüssieren. Dies könnte ein Hinweis darauf sein, dass die geschlechtsuntypischen Effekte dann auftreten, wenn die Betroffenen kaum je auf eine Kollegin bzw. einen Kollegen des gleichen Geschlechts treffen (vgl. Abbildung 6.2). Das Gefühl, die einzige Frau bzw. der einzige Mann zu sein, könnte dazu beitragen, dass sich diese Personen besonders mit ihrer Geschlechterrolle auseinandersetzen bzw. von außen auf das vermeintlich nicht passende Geschlecht angesprochen werden. Dies könnte die Selbstwirksamkeit untergraben (vgl. Flores et al. 2006) und dazu führen, dass sie sich nicht zutrauen, die anstehenden Arbeiten gut zu erledigen, gerade weil sie nicht dem angeblich prädestinierten Geschlecht angehören.

Das Modell der geschlechtsuntypischen Berufssozialisation sagt voraus, dass Lernende die negativen Reaktionen des sozialen Umfelds bewältigen und dafür zusätzliche Ressourcen aufbringen müssen, die ihnen dann bei der Vorbereitung der LAP fehlen. Für einige Berufslehren konnten Hinweise für diesen Effekt gefunden werden. Da er sich jedoch bei anderen Berufslehren nicht fand oder Lernende in geschlechtsuntypischen Berufslehren sogar mehr Erfolg hatten als die anderen Lernenden, kann nicht von einem generalisierten Effekt ausgegangen werden.

Es kann also nicht davon gesprochen werden, dass im Schweizer Berufsbildungssystem Personen, die eine geschlechtsuntypische Berufslehre absolvieren, generell häufiger an der LAP scheitern. Dennoch gibt es Hinweise auf spezifische Schwierigkeiten von Lernenden in geschlechtsuntypischen Be- 
rufslehren, wie die Ergebnisse in den Berufslehren mit weniger als 5 Prozent geschlechtsuntypischen Lernenden belegen. Die hohe Stabilität und die Auffälligkeiten in einzelnen Berufslehren geben Anlass, die Befunde im Einzelnen zu prüfen. Die Misserfolgsquoten in manchen betrieblichen oder handwerklichen Berufslehren sind bedenklich hoch. Als Beispiele dafür sind folgende fünf Berufslehren mit den höchsten Misserfolgsquoten und mit je über 1'000 Lernenden in fünf Jahren zu nennen: ,Plattenleger/-in EFZ' $(32 \%)$, ,Boden-Parkettleger/-in EFZ' (28\%), ,Gärtner/-in EFZ - Garten- und Landschaftsbau' (22\%), ,Maler/-in EFZ' (21\%) und ,Multimediaelektroniker/-in' (21\%). Da in der Gruppe der Lernenden in geschlechtsuntypischen Berufslehren schon vor der LAP eine (Selbst-)Selektion mittels vorzeitiger Lehrvertragsauflösung stattgefunden hat (vgl. Kapitel 5), gilt zu berücksichtigen, dass die wenigen Lernenden in geschlechtsuntypischen Berufslehren, die es bis zur LAP schaffen, sich als besonders durchhaltewillig in ihrem Beruf erwiesen haben.

Die Befunde in diesem Kapitel sind deshalb beachtenswert, weil sie auf Populationswerten und nicht auf Hochrechnungen oder Inferenzstatistik beruhen. Erfreulicherweise ist der Effekt, wonach aufgrund des Geschlechts stark marginalisierte Lernende ein erhöhtes Misserfolgsrisiko bei der LAP aufweisen, in etwas geschlechtergemischteren Berufslehren nicht so stark.

Weitere in diesem Kapitel nicht dargestellte Analysen mit einem strengeren Ausschlusskriterium ergeben noch eindeutigere Stabilitäten für die Frauenanteile und Misserfolgsquoten. Zur Variation der Misserfolgsquoten tragen also vor allem die Berufslehren mit tiefen Fallzahlen bei. In Anbetracht der unterschiedlichen Misserfolgsquoten von nahezu 0 Prozent (etwa ,Konstrukteur/-in EFZ') bis über 30 Prozent (etwa ,Plattenleger/-in EFZ') ist dies ein umso erstaunlicheres Ergebnis. Es scheint bei der Durchführung der Prüfungen in Abhängigkeit vom Schwierigkeitsgrad und von den Fähigkeiten bzw. vom Lernaufwand der Lernenden einen Grundsatz zu geben, wonach eine vorgängig bestimmte bzw. stillschweigend angestrebte Erfolgsquote erreicht werden soll. $\mathrm{Ob}$ mit dem Einhalten von Erfolgsquoten die vermutlich von den Berufsverbänden angestrebte Qualitätssicherung erreicht werden kann, sollte diskutiert werden. Die Ergebnisse lassen zudem die Frage aufkommen, ob die Lernenden allenfalls zusätzlich zu stofflicher bzw. inhaltlicher Ausbildung in Lernorganisation und -motivation unterstützt werden können.

Die belegte Heterogenität der Abschlussquoten und die unterschiedlich hohen Fallzahlen der Berufslehren relativieren die Interpretation der Ergebnisse. In beiden Geschlechterkategorien sind unterschiedliche Berufslehren mit divergierenden Misserfolgsquoten und Fallzahlen von einzelnen bis mehreren Tausend Kandidierenden $\mathrm{zu}$ finden. Obwohl lediglich EFZ-Berufslehren in die Auswertung einbezogen wurden, konnte nicht vermieden werden, dass Berufslehren mit unterschiedlichem Anspruchsniveau zusammengefasst wurden. Zumal eingestanden werden muss, dass das Anspruchsniveau 
der Berufslehren Auswirkungen auf die Zusammensetzung der Lernenden und auf deren Lernmotivation sowie mentale Möglichkeiten hat. Im Datenmaterial sind zudem Lernende enthalten, die zum zweiten oder dritten Mal zur LAP antraten. Laut Amos et al. (2003a) haben Wiederholende schlechtere Chancen als Erstkandidierende. Vor allem bei Berufslehren mit hohen Misserfolgsquoten kann das Doppelt- oder Dreifachzählen von Wiederholenden zu einer zusätzlichen Erhöhung der Misserfolgsquoten führen.

Abschließend ist anzumerken, dass es sich hierbei um eine Querschnittsanalyse handelt. Darum werden in den nachfolgenden Kapiteln 7 und 8 längsschnittlich einige Erfolgsindikatoren von geschlechtstypischen, gemischtgeschlechtlichen und geschlechtsuntypischen Berufslernenden betrachtet. 


\section{Analyse III: Berufserfolge im Selbstbericht - FASE B-Daten}

Die berufliche Grundbildung hat zum Ziel, Jugendlichen das theoretische Fachwissen und die praktischen Fertigkeiten zu vermitteln, um die erforderlichen Tätigkeiten in diesem Beruf ausführen zu können (z.B. Wettstein/Gonon 2009, vgl. Unterkapitel 1.4). Zusätzlich sollen sich die Lernenden mit dem Beruf identifizieren, sich integrieren, sich als kompetent erleben sowie eine Passung zwischen ihren Fähigkeiten/Interessen und den Anforderungen des Berufs wahrnehmen. Dies sind insofern besondere Erfolgskriterien, als sie auf einer subjektiven Einschätzung beruhen. Im Komponentenmodell des Erfolgs in der beruflichen Grundbildung nach Dette et al. (2004, vgl. Tabelle 1.1) lassen sich diese Kriterien den Schnittstellen Selbstbericht/Vergleich mit Bezugsstandards und Selbstbericht/Zufriedenheit zuordnen.

Im vorliegenden Kapitel 6 wird Erfolg während der Berufsbildung längsschnittlich betrachtet. Es wird untersucht, ob sich Lernende in geschlechtsuntypischen Berufslehren von Lernenden in geschlechtstypischen oder geschlechtergemischten Berufslehren unterscheiden. Vier Konstrukte dienen hier als Indikatoren für den Erfolg während der Berufsbildung: 1) Lernen, sich in der Berufslehre zurechtzufinden, und die Anforderungen erfüllen können, 2) Arbeitszufriedenheit, 3) fachliches und methodisches Wissen aufbauen und das Selbstkonzept entsprechend anpassen sowie 4) erleben einer Passung zwischen sich und seinen Fähigkeiten und den beruflichen Herausforderungen.

Das Kapitel führt über die Fragestellung der Analyse und Hypothesen (Unterkapitel 7.1) und Methoden (Unterkapitel 7.2) zu den Ergebnissen (Unterkapitel 7.3) und schließt mit der Diskussion der Befunde (Unterkapitel 7.4). Dabei eröffnet der beschriebene Forschungsprozess der Testung einer plausiblen Hypothese und ihrer Falsifikation eine neue Sichtweise auf Lernende in geschlechtsuntypischen Berufslehren.

\subsection{Fragestellung der Analyse und Hypothesen}

Die beiden vorangegangenen Kapitel 5 und 6 belegen, dass Lernende in geschlechtsuntypischen Berufslehren öfter von Lehrvertragsauflösungen betroffen und in mehreren Berufslehren weniger erfolgreich bei der Lehrabschlussprüfung sind. Nach dem Modell der geschlechtsuntypischen Berufssozialisation ist dies eine Folge (,Bewältigung der Reaktion') von spezifischen Herausforderungen, Diskriminierungen und Herabsetzungen, die diese Jugendli- 
chen bewältigen müssen, um ihre beruflichen Interessen und Wünsche umzusetzen. Könnte es sein, dass durch diese zusätzlichen Anstrengungen und das Bereitstellen der Bewältigungsstrategien ihr subjektiv eingeschätzter beruflicher Erfolg und die Sozialisation in den Beruf gefährdet oder zumindest verzögert werden?

Lernende in geschlechtsuntypischen Berufslehren fallen aufgrund ihres salienten Geschlechts auf (Kanter 1977). Sie lernen einen Beruf, der vermeintlich dem anderen Geschlecht vorbehalten ist (vgl. Gleichheitstabu nach Rubin 1975). Dabei ist es möglich, dass sie Geschlechtsrollenkonflikte erleben (Simpson 2005, O’Neil 2015) und mit Vorurteilen konfrontiert werden (Faulstich-Wieland 2016a). Entsprechend dem Modell der geschlechtsuntypischen Berufssozialisation benötigt die Bewältigung dieser spezifischen Herausforderungen Ressourcen, die bei der beruflichen Sozialisation fehlen könnten. Dies würde die Befunde aus vorangegangen Kapiteln erklären.

Für die Bewältigung der Reaktionen des sozialen Umfelds könnte das heißen, dass diese Lernenden weniger zufrieden sind, da es schwieriger für sie ist als für die Andersgeschlechtlichen, weil sie etwa dumme Sprüche erdulden bzw. parieren müssen. Sie könnten sich unwohl fühlen und sich vermehrt mit Jugendlichen in geschlechtstypischen Berufslehren vergleichen und die Fehler bei sich suchen. Aufgrund von geschlechtsspezifischen Vorurteilen werden ihnen gewisse Fähigkeiten abgesprochen oder gewisse Tätigkeiten nicht anvertraut. Dies könnte dazu führen, dass sie in beruflichen Belangen geringere Fortschritte machen, seltener das Gefühl haben, etwas zu lernen, Kompetenz zu erwerben bzw. kompetent zu sein und den Anforderungen zu genügen. Spezielle Behandlungen wie vermehrtes Kritisieren oder Vorenthalten von Informationen bzw. Tätigkeiten können ihnen das Gefühl vermitteln, dass sie nicht passen. Sie könnten mit Rückzug oder Trotz reagieren, was dazu führen würde, dass sie sich weniger dazugehörig fühlen. Insgesamt findet damit die berufliche Sozialisation unter anderen Vorzeichen statt. Der Erfolg in der Berufslehre wird im vorliegenden Kapitel mit den vier Konstrukten ,Sozialisation im Lehrbetrieb', ,Zufriedenheit mit der Ausbildungssituation', ,berufliches Fähigkeitsselbstkonzept ${ }^{6}$ und ,Passungswahrnehmung zwischen den eigenen Fähigkeiten/Interessen und den Anforderungen des Berufs' operationalisiert.

Aus diesen Überlegungen lässt sich Hypothese 3 ableiten: Lernende in geschlechtsuntypischen Berufslehren schätzen sich selbst in den vier Konstrukten als weniger erfolgreich ein als Lernende in geschlechtstypischen Berufslehren. 


\subsection{Methode}

Die Daten zur Analyse in diesem Kapitel stammen aus dem Forschungsprojekt ,Familie Schule Beruf FASE B`. Das Design dieser Untersuchung wird in Abschnitt 7.2.1 vorgestellt. Darauf folgen die Beschreibungen der Auswertungsstrategie (Abschnitt 7.2.2), der Stichprobe (Abschnitt 7.2.3) und der verwendeten Instrumente (Abschnitt 7.2.4).

\subsubsection{Forschungsprojekt Familie Schule Beruf-FASE B}

Das Forschungsprojekt FASE B ist eine Längsschnittstudie mit vier Erhebungswellen in den Jahren 2002, 2006, 2007 und 2008 (vgl. Neuenschwander et al. 2012). FASE B untersuchte, wie sich Kinder und Jugendliche in Schule und Familie die Ausgangssituationen ihrer beruflichen und persönlichen Laufbahn als Erwachsene erwerben und wie sich die Entwicklung von Jugendlichen in den drei Lebensbereichen Familie, Schule und Beruf vollzieht. Die mittels Leistungstests, standardisierten Fragebogen und halbstrukturierten Interviews erhobenen Daten erlaubten die Rekonstruktion der Transitionsprozesse von der Primarstufe bis zum Abschluss der Berufsbildung oder des Gymnasiums sowie des Beginns des Erwerbslebens. Dabei ergaben sich für Jugendliche, die eine Berufslehre absolvieren, drei hauptsächliche Übergangssituationen: 1) der Übergang in die Sekundarstufe I, 2) der Übergang von der obligatorischen Volksschule nach 9 Schuljahren in die duale Berufsbildung (1. Schwelle) und 3) der Übergang von der dualen Berufsbildung ins Erwerbsleben (2. Schwelle).

Abbildung 7.1: Forschungsdesign FASE B

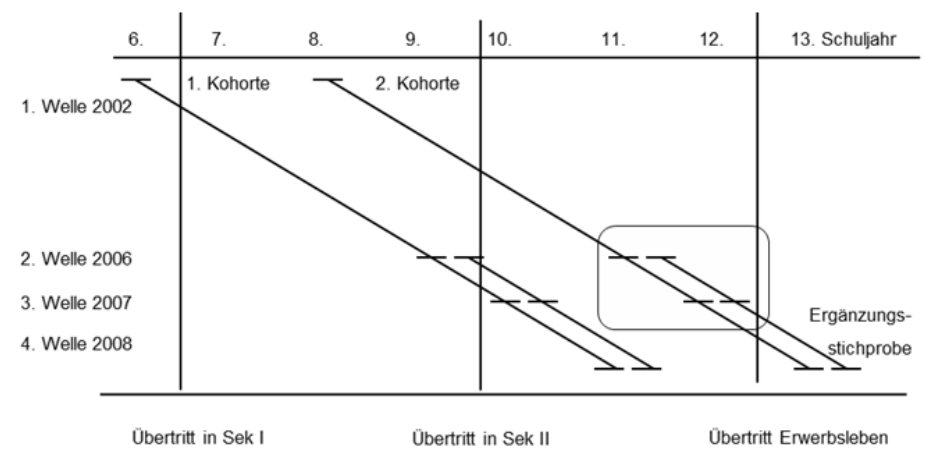

Legende: Rahmen: in der vorliegende Arbeit verwendete Daten. Quelle: Darstellung nach Neuenschwander et al. (2012: 50). 
Die Schülerinnen und Schüler sowie Berufslernenden setzten sich aus zwei Kohorten der Kantone Aargau, Bern und Zürich zusammen. Sie wurden anfänglich klassenweise befragt. Aufgrund der individuellen Bildungsverläufe war diese klassenweise Befragung nicht mehr in allen Fällen möglich und die Jugendlichen wurden, wenn nötig, postalisch oder am Telefon befragt.

In Abbildung 7.1 ist das FASE B-Stichprobendesign dargestellt. Bei der zweiten Befragung im Frühsommer 2006 wurde die Stichprobe um zusätzliche Jugendliche erweitert (Ergänzungsstichprobe) und 2007 sowie 2008 erneut untersucht. Die statistischen Auswertungen in diesem Kapitel beziehen sich auf Daten von Jugendlichen in der beruflichen Grundbildung dieser beiden Stichprobenzeitpunkte der zweiten Kohorte (vgl. Umrahmung in der Abbildung 7.1). Zu diesem Zeitpunkt befanden sich die Lernenden im zweiten und ein Jahr später im dritten Lehrjahr.

\subsubsection{Auswertungsstrategie}

Zum Datensatz von FASE B mit allen vier Wellen (aus den Jahren 2002, 2006, 2007 und 2008) wurden die Frauenanteile aus dem vorangegangenen Kapitel 6 für jede Berufslehre hinzugefügt und daraus und aus dem Geschlecht der Lernenden die geschlechtliche Berufstypik ermittelt. Die geschlechtliche Berufstypik enthält 4 Kategorien ${ }^{31}: 1$ ), Männer typisch ' (Frauenanteil FA der Berufslehre 0-70\%), 2), Männer untypisch (FA 70-100\%), 3) ,Frauen typisch (FA 30-100\%), 4), Frauen untypisch (FA 0-30\%). Aus dem Gesamtdatensatz von über 2'600 Personen wurden diejenigen selektiert, die 2006 und 2007 in einer Berufslehre waren und diese nicht gewechselt hatten. Einige Jugendliche in geschlechtsuntypischen Berufslehren mit fehlenden Werten in einem Jahr wurden dennoch einbezogen. Eine weibliche Person, die von einer geschlechtsuntypischen in eine geschlechtergemischte Berufslehre wechselte, wurde nicht selektioniert. Die neue Stichprobe umfasste 896 Personen und beinhaltet ausschließlich Lernende, die eine berufliche Grundbildung absolvieren, also keine Gymnasiastinnen, Gymnasiasten oder berufstätigen Jugendlichen.

Der umfangreiche Datensatz (über 4'900 Variablen) wurde auf die verwendeten Variablen reduziert. Die fehlenden Werte wurden per multiple Imputation mit der Software IBM SPSS Statistics Version 22 geschätzt. Dabei werden plausible Werte für die fehlenden Daten aufgrund aller verfügbaren Informationen geschätzt. Die multiple Imputation erhöht die Robustheit und Reliabilität der Schätzwerte in Varianzanalysen (Schafer/Olsen 1998, Schafer/Graham 2002). Insgesamt fehlten 3.9 Prozent der Werte. Von 24 Variablen hatten zwei über 5 Prozent fehlende Werte: je 23.3 Prozent beim ,beruflichen Fähigkeitsselbstkonzept 2006' und bei der ,Sozialisation im

31 Gemäß des 30\%-Kriteriums vgl. Abschnitt 5.3.2 und Fußnote 28. 
Lehrbetrieb 2006'. Der Vergleich der Originaldaten mit dem imputierten Datensatz ergab keine signifikanten Unterschiede in den verwendeten Konstrukten.

Die vier Konstrukte wurden 2006 und 2007 erhoben. Anhand zweifaktorieller Varianzanalysen werden Zeit-, Gruppen- und Interaktionseffekte für vier bzw. zwei Gruppen berechnet. Da sich in der Gruppe ,Männer in geschlechtsuntypischen Berufslehren" lediglich 8 Personen befanden, wurden die Varianzanalysen mittels nicht-parametrischer Tests zusätzlich abgesichert.

\subsubsection{Stichprobe}

Da für die Gruppe der geschlechtsuntypischen Lernenden lediglich Daten von 15 Frauen und 8 Männern vorlagen, wurde aus den verbleibenden Teilnehmenden eine geschichtete Zufallsstichprobe mit je 30 weiblichen und männlichen Teilnehmenden gezogen. Die so gewonnene Stichprobe enthält 83 Lernende (45 weibliche und 38 männliche), die 2006 zwischen 15 und 33 Jahre alt waren $(M=18.1, S D=2.3) .56$ von ihnen gaben an, das zweite Lehrjahr $\mathrm{zu}$ besuchen, 4 das erste. Aufgrund der Angaben zur Berufslehre und zum Jahrgang kann davon ausgegangen werden, dass die 23 Lernenden ohne Angaben ebenfalls im zweiten Lehrjahr waren. 85.2 Prozent sind Schweizerinnen und Schweizer. 16.9 Prozent sprachen zu Hause eine andere Sprache als Deutsch. Unter den Lernenden in geschlechtsuntypischen Berufslehren befinden sich etwas mehr Lernende mit Schweizer Nationalität und Deutsch als Erstsprache. Die Lernenden wohnten in den Kantonen Aargau, Bern und Zürich.

Für die Einteilung der Lernenden in Gruppen war der Anteil an Frauen maßgebend: bis 30\% entspricht ,männerreicher Beruf ${ }^{*}, 30-70 \%$,geschlechtergemischter Beruf und über $70 \%$,frauenreicher Beruf'(vgl. OnlineAnhang VIII). Die größere Anzahl der männerreichen Berufe fällt sofort auf und spiegelt das bekannte Phänomen, wonach männliche Jugendliche aus einem größeren Spektrum an Berufslehren auswählen, während weibliche Jugendliche häufig einen von fünf frauenreichen Berufslehren ergreifen (Borkowsky, 2000, Hirschi, 2009). Häufungen bei einzelnen Berufslehren entstanden, da die Ergänzungsstichprobe aus zehn Berufslehren rekrutiert wurde: ,Automonteur/-in EFZ', ,Dentalassistent/-in EFZ', ,Detailhandelsangestellte/-r EFZ, ,Coiffeur/-euse EFZ, ,Fachangestellte/-r Gesundheit EFZ, ,Gärtner/-in EFZ', ,Koch/Köchin EFZ', ,Montageelektriker/-in EFZ', ,Pharma-Assistent/-in EFZ' und ,Sanitärmonteur/-in EFZ'. 


\subsubsection{Instrumente}

Da die Variablen der vier Konstrukte 2006 auf einer sechsstufigen Ratingskala und 2007 auf einer vierstufigen erfasst wurden erfolgte eine Umrechnung der Werte von 2006 in eine vierstufige Skala. Die Items wurden mit Hauptkomponentenanalysen überprüft und die interne Konsistenz der gebildeten Faktoren ermittelt.

Der Faktor ,Sozialisation im Lehrbetrieb“ ist eine Konstruktion von Neuenschwander et al. (2007). Die Lernenden geben auf einer Skala an von 1 ,ein sehr großes Problem' bis 4 bzw. 6 ,überhaupt kein Problem ' an, welches die größten Herausforderungen im Lehrbetrieb in diesem Ausbildungsjahr waren in Bezug auf Umgang mit Mitarbeitenden im Betrieb, mit der/dem Ausbildenden, dem Anwenden von Wissen, dem Einhalten von Regeln, Verantwortung, Leistungsdruck, Motivation und Befriedigung in der Berufsausübung. Der Faktor aus 8 Items erreicht gute interne Konsistenzen in den beiden Durchführungsjahren von $\alpha=.80$ und $\alpha=.78$.

Das Konstrukt ,berufliches Fähigkeitsselbstkonzept ${ }^{6}$ basiert auf Sonntag und Schäfer-Rauser (1993) und besteht aus acht Items. Das berufliche Fähigkeitsselbstkonzept entsteht aus der Selbsteinschätzung der fachlichen und methodischen Kompetenzen der Lernenden. Diese vergleichen sich mit sich selbst zu einem früheren Zeitpunkt oder aber mit jüngeren, gleichaltrigen oder älteren Lernenden bzw. mit Expertinnen oder Experten in ihrem Beruf. Die Art der Selbstzuweisung von Fähigkeit ist für ein stabiles Selbstkonzept wichtig, denn ,nur wer sich Fähigkeit als internal stabile Größe zubilligt, kann sich darauf verlassen, dass eine in Zukunft geforderte Leistung auch mit einiger Sicherheit bewältigen werden kann" (Oerter/Dreher 1998). Beispielitems sind: „Ich kann mit vielen verschiedenen Hilfsmitteln, Geräten und Werkzeugen umgehen“, „Es fällt mir schwer, Arbeitsschritte zu planen und einzuteilen“ oder „Im Lauf meiner Berufsausbildung habe ich mir schon sehr viele fachliche Kenntnisse angeeignet." Die Reliabilität in Form von interner Konsistenz wird mit $\alpha=.76$ für 2006 und $\alpha=.70$ für 2007 angegeben.

Das Konstrukt ,Zufriedenheit mit der Ausbildung ' wurde 2006 mit einem Item erfasst: „Wenn du/Sie auf das letzte halbe Jahr zurückblickst/zurückblicken, wie zufrieden bist du / sind Sie mit der aktuellen Schul- bzw. Ausbildungssituation?" 2007 wurden die drei Faktoren ,intrinsische Arbeitszufriedenheit' (5 Items, $\alpha=.83$ ), ,extrinsische Arbeitszufriedenheit ${ }^{\circ}$ (4 Items, $\alpha=.66$ ) und ,Berufsschulzufriedenheit ${ }^{\circ}$ (6 Items, $\alpha=.87$ ) zu einem Faktor zusammengefasst.

Die ,wahrgenommene Passung zwischen den eigenen Interessen bzw. Fähigkeiten und den Anforderungen der Berufslehre" wurde mittels dreier Items erfasst und zu einem reliablen Faktor zusammengeführt $(\alpha=.77 / .63)$. Ein Beispielitem ist: „Meine momentane berufliche Situation stimmt mit meinen persönlichen Interessen überein.“ Die Passung hängt stark mit dem berufli- 
chen Fähigkeitsselbstkonzept zusammen (vgl. Gerber-Schenk et al. 2010, Neuenschwander et al. 2012).

\subsection{Ergebnisse}

Im Folgenden werden die Ergebnisse zur Hypothese vorgestellt, ob sich Lernende in geschlechtsuntypischen Berufslehren von den anderen Lernenden in der Selbsteinschätzung ihres beruflichen Erfolgs unterscheiden. Dazu wurden die Jugendlichen in eine Gruppe mit Lernenden in geschlechtsuntypischen Berufslehren und in eine zweite Gruppe mit Lernenden in geschlechtstypischen oder geschlechtergemischten Berufslehren eingeteilt. Die Ergebnisse sind in Abschnitt 7.3.1 Zweigruppenvergleiche dargestellt. Da es unklar blieb, ob es zusätzlich Geschlechtereffekte gibt, werden Analysen mit vier Gruppen vorgestellt (Abschnitt 7.3.2 Viergruppenvergleiche).

\subsubsection{Zweigruppenvergleiche}

Die Mittelwerte für die Gruppe der Lernenden in geschlechtsuntypischen Berufslehren sollten weniger stark zunehmen oder gar abnehmen. Erstaunlicherweise sind sie in drei von vier Konstrukten zum ersten Messzeitpunkt sogar höher als bei Lernenden in geschlechtstypischen Berufslehren (vgl. Tabelle 7.1). Erwartungsgemäß steigen sie jedoch nicht stärker an. Sie stagnieren oder sinken sogar. Zur Überprüfung, ob diese Mittelwertveränderungen statistisch signifikant sind, wurden zweifaktorielle Varianzanalysen mit Messwiederholung gerechnet.

Die Ergebnisse der Varianzanalysen ergeben lediglich einen signifikanten Effekt für die zeitliche Veränderung der, Sozialisation im Lehrbetrieb ${ }^{`}$. Beide Gruppen zeigen eine Zunahme und fühlen sich demnach stärker als Berufsleute als ein Jahr zuvor. Die erwarteten Gruppeneffekte und Interaktionseffekte können nicht nachgewiesen werden, die Gruppen unterscheiden sich nicht statistisch bedeutsam (vgl. Tabelle 7.2).

Aufgrund der teilweise nicht erfüllten Voraussetzung der gleichen Gruppengrößen für Varianzanalysen wurden für alle Beziehungen Mann-WhitneyU-Tests für unabhängige Stichproben gerechnet. Dabei ergeben sich signifikante Zunahmen für Lernende in geschlechtstypischen Berufslehren bei der ,beruflichen Sozialisation' und bei der ,Zufrieden mit der Ausbildung', während sie für diejenigen in geschlechtsuntypischen nicht signifikant werden. Alle anderen Effekte sind ebenfalls nicht signifikant. 
Tabelle 7.1: Deskriptive Statistik der 2 Gruppenvergleiche

\begin{tabular}{|c|c|c|c|c|c|c|c|c|c|}
\hline \multirow[b]{3}{*}{ Gruppen } & \multirow[b]{3}{*}{$N$} & \multicolumn{4}{|c|}{$\begin{array}{c}\text { Sozialisation } \\
\text { im Lehrbetrieb }\end{array}$} & \multicolumn{4}{|c|}{$\begin{array}{c}\text { berufliches } \\
\text { Fähigkeitsselbstkonzept }\end{array}$} \\
\hline & & \multicolumn{2}{|c|}{ 2. Lehrjahr } & \multicolumn{2}{|c|}{ 3. Lehrjahr } & \multicolumn{2}{|c|}{ 2. Lehrjahr } & \multicolumn{2}{|c|}{ 3. Lehrjahr } \\
\hline & & $M$ & $S D$ & $M$ & $S D$ & $M$ & $S D$ & $M$ & $S D$ \\
\hline typisch & 60 & 3.23 & .37 & 3.41 & .41 & 3.03 & .26 & 3.15 & .39 \\
\hline untypisch & 23 & 3.14 & .43 & 3.27 & .33 & 3.07 & .34 & 3.05 & .35 \\
\hline \multirow[t]{3}{*}{ gesamt } & 83 & 3.20 & .39 & 3.37 & .39 & 3.04 & .28 & 3.12 & .38 \\
\hline & & \multicolumn{4}{|c|}{$\begin{array}{c}\text { Zufriedenheit } \\
\text { mit der Ausbildung }\end{array}$} & \multicolumn{4}{|c|}{$\begin{array}{l}\text { wahrgenommene } \\
\text { Passung }\end{array}$} \\
\hline & & \multicolumn{2}{|c|}{ 2. Lehrjahr } & \multicolumn{2}{|c|}{ 3. Lehrjahr } & \multicolumn{2}{|c|}{ 2. Lehrjahr } & \multicolumn{2}{|c|}{ 3. Lehrjahr } \\
\hline Gruppen & $N$ & $M$ & $S D$ & $M$ & $S D$ & $M$ & $S D$ & $M$ & $S D$ \\
\hline typisch & 60 & 2.87 & .76 & 2.97 & .49 & 3.32 & .42 & 3.33 & .51 \\
\hline untypisch & 23 & 2.98 & .71 & 2.99 & .43 & 3.37 & .45 & 3.25 & .49 \\
\hline gesamt & 83 & 2.90 & .75 & 2.98 & .47 & 3.33 & .43 & 3.31 & .50 \\
\hline
\end{tabular}

Legende: N: Anzahl Personen, M: Mittelwert, SD: Standardabweichung. Quelle: Eigene Darstellung.

Tabelle 7.2: Zweifaktorielle Varianzanalysen mit 2 Gruppen

\begin{tabular}{|c|c|c|c|c|c|c|c|c|c|c|c|c|}
\hline \multirow[t]{2}{*}{ Konstrukt } & \multicolumn{4}{|c|}{ Zeiteffekt } & \multicolumn{4}{|c|}{ Gruppeneffekt } & \multicolumn{4}{|c|}{ Interaktionseffekt } \\
\hline & $F$ & $d f$ & $p$ & $\eta$ & $F$ & $d f$ & $p$ & $\eta$ & $F$ & $d f$ & $p$ & $\eta$ \\
\hline $\begin{array}{l}\text { Sozialisation im Lehrbe- } \\
\text { trieb }\end{array}$ & $9.4^{* \star *}$ & 1,81 & $<.001$ & .10 & 2.1 & 1,81 & .15 & .03 & 0.2 & 1,81 & .63 & .00 \\
\hline $\begin{array}{l}\text { berufliches Fähigkeits- } \\
\text { selbstkonzept }\end{array}$ & 1.9 & 1,81 & .17 & .02 & 0.2 & 1,81 & .65 & .00 & 3.4 & 1,81 & .07 & .04 \\
\hline $\begin{array}{l}\text { Zufriedenheit mit der } \\
\text { Ausbildung }\end{array}$ & 0.6 & 1,81 & .44 & .01 & 0.3 & 1,81 & .61 & .00 & 0.4 & 1,81 & .54 & .01 \\
\hline wahrgenommene Passung & 1.0 & 1,81 & .31 & .01 & 0.0 & 1,81 & .89 & .00 & 1.8 & 1,81 & .19 & .02 \\
\hline
\end{tabular}

Legende: F: F-Wert, $d f$ : Freiheitsgrade, $p$ : Signifikanzniveau, $\eta$ : Effektstärke partielles eta2, ${ }^{\star \star *}: p<.001$. Vergleiche 2. und 3. Lehrjahr. Quelle: Eigene Darstellung.

\subsubsection{Viergruppenvergleiche}

Die Auswertungen mit vier Gruppen (Männer typisch und untypisch sowie Frauen typisch und untypisch) belegen beim Konstrukt ,Sozialisation im Lehrbetrieb' eine Abnahme für männliche Lernende in geschlechtsuntypischen Berufslehren, während die anderen drei Gruppen eine Zunahme verzeichnen. In den beiden Konstrukten ,berufliches Fähigkeitsselbstkonzept ${ }^{*}$ und ,Zufriedenheit mit der Ausbildung' stagnieren die Mittelwerte der beiden untypischen Gruppen von zweiten zum dritten Lehrjahr, die der typischen hingegen nehmen leicht zu. Die Mittelwerte der ,wahrgenommenen Passung ${ }^{6}$ nehmen außer für die Gruppe der weiblichen Lernenden in geschlechtstypischen Berufslehren ab (vgl. Tabelle 7.3, Online-Anhang IX). 
Tabelle 7.3: Deskriptive Statistik der 4 Gruppenvergleiche

\begin{tabular}{|c|c|c|c|c|c|c|c|c|c|}
\hline & \multirow[b]{3}{*}{$N$} & \multicolumn{4}{|c|}{$\begin{array}{l}\text { Sozialisation } \\
\text { im Lehrbetrieb }\end{array}$} & \multicolumn{4}{|c|}{$\begin{array}{c}\text { berufliches } \\
\text { Fähigkeitsselbstkonzept }\end{array}$} \\
\hline & & \multicolumn{2}{|c|}{ 2. Lehrjahr } & \multicolumn{2}{|c|}{ 3. Lehrjahr } & \multicolumn{2}{|c|}{ 2. Lehrjahr } & \multicolumn{2}{|c|}{ 3. Lehrjahr } \\
\hline & & $M$ & $S D$ & $M$ & $S D$ & $M$ & $S D$ & $M$ & $S D$ \\
\hline Männer typisch & 30 & 3.19 & .32 & 3.41 & .41 & 3.03 & .22 & 3.11 & .40 \\
\hline Männer untypisch & 8 & 3.38 & .43 & 3.21 & .24 & 3.16 & .37 & 3.15 & .47 \\
\hline Frauen typisch & 30 & 3.26 & .41 & 3.41 & .43 & 3.03 & .30 & 3.19 & .38 \\
\hline Frauen untypisch & 15 & 3.01 & .39 & 3.31 & .37 & 3.02 & .33 & 3.00 & .28 \\
\hline \multirow[t]{4}{*}{ gesamt } & 83 & 3.20 & .39 & 3.37 & .39 & 3.04 & .28 & 3.12 & .038 \\
\hline & & \multicolumn{4}{|c|}{$\begin{array}{c}\text { Zufriedenheit } \\
\text { mit der Ausbildung }\end{array}$} & \multicolumn{4}{|c|}{$\begin{array}{l}\text { wahrgenommene } \\
\text { Passung }\end{array}$} \\
\hline & & \multicolumn{2}{|c|}{ 2. Lehrjahr } & \multicolumn{2}{|c|}{ 3. Lehrjahr } & \multicolumn{2}{|c|}{ 2. Lehrjahr } & \multicolumn{2}{|c|}{ 3. Lehrjahr } \\
\hline & $N$ & $M$ & $S D$ & $M$ & $S D$ & $M$ & $S D$ & $M$ & $S D$ \\
\hline Männer typisch & 30 & 2.82 & .85 & 2.97 & .55 & 3.30 & .50 & 3.21 & .57 \\
\hline Männer untypisch & 8 & 3.00 & .85 & 3.01 & .41 & 3.18 & .58 & 3.13 & .47 \\
\hline Frauen typisch & 30 & 2.91 & .68 & 2.97 & .43 & 3.33 & .32 & 3.46 & .42 \\
\hline Frauen untypisch & 15 & 2.97 & .66 & 2.99 & .45 & 3.48 & .34 & 3.31 & .51 \\
\hline gesamt & 30 & 2.90 & .75 & 2.98 & .47 & 3.33 & .43 & 3.31 & .50 \\
\hline
\end{tabular}

Legende: N: Anzahl Personen, M: Mittelwert, SD: Standardabweichung. Quelle: Eigene Darstellung.

Tabelle 7.4: Zweifaktorielle Varianzanalysen mit 4 Gruppen

\begin{tabular}{lcccccccccccc}
\hline Konstrukt & \multicolumn{3}{c}{ Zeiteffekt } & \multicolumn{4}{c}{ Gruppeneffekt } & \multicolumn{4}{c}{ Interaktionseffekt } \\
& $F$ & $d f$ & $p$ & $\eta$ & $F$ & $d f$ & $p$ & $\eta$ & $F$ & $d f$ & $p$ & $\eta$ \\
\hline $\begin{array}{l}\text { Sozialisation im Lehrbe- } \\
\text { trieb }\end{array}$ & $5.6^{*}$ & 1,79 & .02 & .07 & 1.1 & 3,79 & .38 & .04 & 2.5 & 3,79 & .07 & .09 \\
$\begin{array}{l}\text { berufliches Fähigkeits- } \\
\text { selbstkonzept }\end{array}$ & 1.8 & 1,79 & .18 & .02 & 0.6 & 3,79 & .63 & .02 & 1.5 & 3,79 & .23 & .05 \\
$\begin{array}{l}\text { Zufriedenheit mit der } \\
\text { Ausbildung }\end{array}$ & 0.5 & 1,79 & .47 & .01 & 0.1 & 3,79 & .95 & .01 & 0.3 & 3,79 & .86 & .01 \\
wahrgenommene Passung & 0.7 & 1,79 & .41 & .01 & 1.2 & 3,79 & .32 & .04 & 2.0 & 3,79 & .13 & .07 \\
\hline
\end{tabular}

Legende: F: F-Wert, df: Freiheitsgrade, $p$ : Signifikanzniveau, $\eta$ : Effektstärke partielles eta2, *: $p<.05$. Vergleiche 2. und 3. Lehrjahr. Quelle: Eigene Darstellung.

Die Varianzanalysen mit Messwiederholung für vier Gruppen ergeben nur einen signifikanten Zeiteffekt für ,Sozialisation im Lehrbetrieb ${ }^{6}$. Die anderen Mittelwertsunterschiede zwischen den Gruppen sind statistisch nicht bedeutsam (vgl. Tabelle 7.4). Aufgrund der kleinen Anzahl in den beiden Gruppen der Lernenden in geschlechtsuntypischen Berufslehren wurden ebenfalls nicht parametrische Tests (Kruskal-Wallis-Test bei unabhängigen Stichproben) gerechnet. Das Ergebnis ist das Gleiche: Es sind keine Gruppenunterschiede vorhanden. Um die zeitliche Veränderung zwischen dem zweiten und dem dritten Lehrjahr zu testen, wurden Wilcoxon-Tests für verbundene Stichproben berechnet. Online-Anhang $\mathrm{X}$ weist signifikante $p$-Werte für die 
beiden Konstrukte ,Sozialisation im Lehrbetrieb“ und ,berufliches Fähigkeitsselbstkonzept ${ }^{\star}$ aus.

Da es weder signifikante Gruppen- noch Interaktionseffekte gibt, ist die Hypothese 3, wonach sich die Lernenden in geschlechtsuntypischen Berufslehren in ihrem beruflichen Erfolg von geschlechtstypischen bzw. geschlechtergemischten unterscheiden, falsifiziert. Was diese Ergebnisse für das Modell der geschlechtsuntypischen Berufssozialisation bedeuten, diskutiere ich im nachfolgenden Unterkapitel.

\subsection{Diskussion}

Die Auswertungen mit den Daten des Forschungsprojekts FASE B belegen, dass es zwischen den Lernenden in typischen und untypischen Berufslehren nur vereinzelt Unterschiede im Selbstbericht der beruflichen Erfolge gibt. Lernende in geschlechtsuntypischen Berufslehren sehen sich nicht vor größere Herausforderungen gestellt, was ihre berufliche Sozialisation im Lehrbetrieb angeht, als ihre Kolleginnen und Kollegen. Sie schätzen ihr berufliches Fähigkeitsselbstkonzept nicht tiefer ein, sie sind nicht weniger zufrieden mit ihrer Ausbildung und nehmen die Passung zwischen ihren Interessen bzw. Fähigkeiten und den Anforderungen der Berufslehre nicht anders wahr.

Wie lassen sich diese Befunde und die Falsifikation der Hypothese 3 erklären? Nach dem Modell der geschlechtsuntypischen Berufssozialisation (Kapitel 4) sind Lernende in geschlechtsuntypischen Berufslehren vor die Herausforderung gestellt, mit den Reaktionen des sozialen Umfelds - etwa in Form von dummen Sprüchen - umzugehen. Diese Zusatzleistungen führen dazu, dass sie häufiger den Lehrvertrag auflösen als Jugendliche in geschlechtstypischen oder geschlechtergemischten Berufslehren (vgl. Kapitel 5). Die befragten Jugendlichen im FASE B-Datensatz unterscheiden sich im selbstberichteten beruflichen Erfolg jedoch nicht. Dies könnte damit zu tun haben, dass bei diesen Lernenden genügend Ressourcen vorhanden sind. Dafür spricht, dass die Jugendlichen weiterhin an der Studie teilnahmen, wenn sie nicht zu stark beansprucht wurden, etwa durch eine bevorstehende oder bereits durchgeführte Lehrvertragsauflösung. Die Personenzahl in der Gruppe der Lernenden in geschlechtsuntypischen Berufslehren ist gering, was weitergehende Untersuchungen kaum möglich macht. Unterschiede könnten innerhalb dieser Gruppe etwa in der Höhe der sozialen Unterstützung, der Sozialkompetenz, der positiven Lebenseinstellung oder des körperlichen Wohlbefindens vorkommen.

Obwohl die Hypothese 3 verworfen werden musste, ist die Erkenntnis nicht gleich null. Diese Variablen können nicht wie erhofft die vorzeitigen Lehrvertragsauflösungen erklären. Die Daten legen nahe, dass sich Lernende 
in geschlechtsuntypischen Berufslehren nicht wesentlich von den geschlechtstypischen unterscheiden und ihre berufliche Entwicklung nicht per se gefährdet ist. Für das Modell der geschlechtsuntypischen Berufssozialisation heißt das, dass sich die Bewältigung der Reaktionen des sozialen Umfelds nicht direkt auf die subjektive Lehrerfolgswahrnehmung auswirkt. Die interessante Frage ist: Was haben diese Jugendlichen, das diejenigen nicht haben, die vorzeitig den Lehrvertrag auflösen bzw. die Berufslehre aufgrund von schlechten Leistungen in der Berufsfachschule oder im Lehrbetrieb aufgeben müssen?

Die wesentlichsten Einschränkungen beziehen sich auf die kleine Stichprobe und die Heterogenität der Stichprobe. Die beruflichen Umfelder und damit verbundenen Rollenerwartungen unterscheiden sich stark. Eine weitere Restriktion bezieht sich auf das Konstrukt ,Zufriedenheit mit der Ausbildung', denn dafür waren zu den beiden Erhebungszeitpunkten unterschiedliche Fragen gestellt worden, was die Interpretation erschwert. 


\section{Analyse IV: Einfluss des sozialen Umfelds - TREE-Daten}

Lernende in geschlechtsuntypischen Berufslehren müssen mit Belastungen umgehen, mit denen andere Lernende nicht konfrontiert werden. Insbesondere geht es dabei um individuelle Geschlechtsrollenkonflikte und die Bewältigung der Reaktionen des sozialen Umfelds aufgrund von nicht erfüllten Geschlechtsrollenerwartungen (vgl. Modell der geschlechtsuntypischen Berufssozialisation in Kapitel 4). In der vorliegenden Analyse IV steht der Einfluss des sozialen Umfelds auf die Bewältigungshandlungen im Fokus. Im Modell der geschlechtsuntypischen Berufssozialisation wird beschrieben, wie Personen, die mit diesen Jugendlichen zu tun haben, positiv wohlwollend oder negativ ablehnend auf die Salienz des untypischen Geschlechts reagieren. Die untypische Situation kann auf unterschiedlichen Ebenen angesprochen werden. So könnten Eltern ihre Sorgen äußern, Ausbildende besonders Rücksicht nehmen, Arbeitskolleginnen und -kollegen Sprüche reißen, Kunden ihre Überraschung ausdrücken oder Passanten Bemerkungen beim Vorbeigehen machen. Mit diesen Reaktionen müssen die Lernenden umgehen, sie parieren, ignorieren oder entkräften. Diese Bewältigung beansprucht emotionale Kraft und kognitive Kapazität. Die Auswirkungen davon, speziell wenn die Anforderungen die Ressourcen der Jugendlichen übersteigen, dürften sich in einem verminderten Wohlbefinden äußern.

Für die Untersuchung wird der Datensatz des Forschungsprojekts Transitionen von der Erstausbildung ins Erwerbsleben TREE ${ }^{32}$ analysiert. Im umfangreichen Datenmaterial konnten einschlägige Variablen ermittelt werden. Diese Items und Konstrukte sowie die verwendeten Analysen, die Erkenntnisse und Schlussfolgerungen werden im vorliegenden Kapitel vorgestellt. Die Gliederung des Kapitels erfolgt in ,Fragestellung der Analyse und Hypothesen“ (Unterkapitel 8.1), ,Methode' (Unterkapitel 8.2), ,Ergebnisse' (Unterkapitel 8.3) und ,Diskussion“ (Unterkapitel 8.4).

\subsection{Fragestellung der Analyse und Hypothesen}

Lernende in geschlechtsuntypischen Berufslehren erleben, dass sie aufgrund ihres Geschlechts speziell wahrgenommen und zum Teil anders behandelt,

32 Die Schweizer Jugendlängsschnittstudie Transitionen von der Erstausbildung ins Erwerbsleben TREE läuft seit dem Jahr 2000 und wurde bisher durch den Schweizerischen Nationalfonds, die Universität Basel, die Bundesämter SBFI und BFS sowie die Kantone Bern, Genf und Tessin finanziert [http://www.tree.unibas.ch; Zugriff: 22.03.2017]. 
manchmal sogar diskriminiert werden. Sie müssen diese Erfahrung bewältigen. Diese Bewältigung fällt den Jugendlichen leichter, wenn sie von wichtigen Personen ihres Umfelds unterstützt werden (Alpers 2005, vgl. Kapitel 4). Dabei spielen vor allem die Eltern und weitere Personen aus dem persönlichen oder beruflichen Umfeld eine zentrale Rolle. Befunde von Wehner et al. (2016) belegen, dass Jugendliche in geschlechtsuntypischen Berufslehren über mehr Ressourcen verfügen und mehr Unterstützung seitens Familie und Berufsumfeld erfahren als Gleichaltrige in geschlechtstypischen oder gemischten Berufslehren.

Wie in Kapitel 5 dargestellt, kommen Lehrvertragsauflösungen bei Lernenden in geschlechtsuntypischen Berufslehren häufiger vor als bei Gleichaltrigen in geschlechtstypischen Berufslehren. Neben diesem bereits belegten Ergebnis über Verbleib bzw. Austritt aufgrund des untypischen Geschlechts können psychosomatische Beschwerden oder Absenzen weitere Folgeerscheinungen sein. In der Abbildung 8.1 werden diese mit ,Folgen ' der Bewältigung bezeichnet. Diese Darstellung leitet sich unmittelbar aus dem Modell der geschlechtsuntypischen Berufssozialisation ab (vgl. Unterkapitel 4.2). Die Jugendlichen in geschlechtsuntypischen Berufslehren (,Geschlechtstypik') gehen auf ihre Art mit der Situation um (,Bewältigung'). Je größer die Schwierigkeiten dabei sind und je weniger gut sie damit umgehen können, desto stärker erhöht sich der psychische Druck, der sich in psychosomatischen Beschwerden, Absenzen und schließlich im Austritt aus der Berufslehre ausdrücken kann (,Folgen'). Die Auswirkung der Geschlechtstypik auf die Bewältigung wird durch die ,Reaktion des sozialen Umfelds“ vermittelt. In der Reaktion des sozialen Umfelds sind sowohl negative Reaktionen wie Diskriminierung wie auch positive Reaktionen wie Unterstützung subsummiert.

Abbildung 8.1: Modell der Mediationshypothese

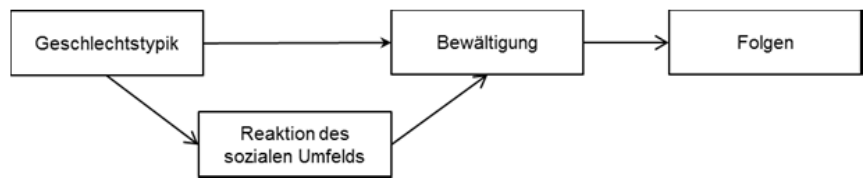

Erklärung: Abhängigkeit der Bewältigung der geschlechtsuntypischen Situation von der Reaktion des sozialen Umfelds. Quelle: Eigene Darstellung.

Die Ressourcen in Form von sozialer Unterstützung stehen den im Modell postulierten Belastungen gegenüber. Unter den Variablen des TREEDatensatzes fanden sich fünf Items bzw. Konstrukte, die Hinweise auf Bewältigungshandeln aufgrund des Minoritätsstatus zuließen:

1) Auskommen mit ihren Arbeitskolleginnen und -kollegen. Es wird angenommen, dass das Verhältnis von Lernenden in geschlechtsuntypischen Be- 
rufslehren zu ihren Arbeitskolleginnen und -kollegen weniger gut ist, da es aufgrund der unerfüllten Rollenerwartungen häufiger zu Irritationen seitens der Arbeitskolleginnen und -kollegen kommt. Rollenkonflikte lösen Gefühle der Verlegenheit, des Unwohlseins oder der Scham aus (Simpson 2005). Sie können sich als intrapersonelle Konflikte wie als negative Reaktionen des Umfelds (interpersonelle Konflikte) manifestieren. Das Verhältnis zu den Arbeitskolleginnen und -kollegen bildet einen Kristallisationspunkt: Erstens wird das Verhältnis mit Beginn der Berufslehre von beiden Seiten (Lernenden und Arbeitskolleginnen/-kollegen) erarbeitet, denn vorher kannten sie sich meist nicht. Zweitens werden die Lernenden durch ihre Arbeitskolleginnen und -kollegen während drei bis vier Tagen pro Woche unmittelbar mit den Anforderungen des Berufs konfrontiert. $\mathrm{Zu}$ diesen Anforderungen gehören Geschlechternormen und Geschlechtsrollenerwartungen, die im geschlechtsuntypischen Fall nicht erfüllt werden können. Wie gut Lernende mit ihren Arbeitskolleginnen und -kollegen auskommen, gibt also Hinweise auf die Art der Bewältigung.

2) Umgang im Lehrbetrieb. Rollenkonflikte bei der Arbeit können Auswirkungen auf die Wahrnehmung des Umgangs im Lehrbetrieb haben: Zeigen Mitarbeitende und insbesondere Vorgesetzte ihre Irritation über das geschlechtsuntypische Verhalten der Lernenden, z.B. in Form von dummen Sprüchen und Neckereien, untergräbt dies das gute Einvernehmen und die Integration der Lernenden in den Lehrbetrieb. Die interne Unternehmenskultur ist maßgeblich vom Verhalten der Mehrheit gegenüber Minderheiten abhängig. Nach Schein (1985) entzieht sich die Unternehmenskultur dem expliziten Wissen und manifestiert sich in Verhaltensroutinen, Werten und Symbolen. Die Einschätzung des Umgangs im Lehrbetrieb ist damit ebenfalls ein Hinweis für Bewältigungshandeln.

3) Chancengleichheit. Ein besonderer Wert, der in einem Betrieb gelebt werden kann, ist die Gleichberechtigung von Frauen und Männern. Die Wahrnehmung der Lernenden in geschlechtsuntypischen Berufslehren ist eine spezielle, da sie qua ihrer Minderheitsstatus und ihrer direkten Betroffenheit sensibler auf Ungleichbehandlung reagieren dürften als ihre Gleichaltrigen in geschlechtstypischen Berufslehren. Personen, die der Mehrheit angehören, sind sich oft gar nicht bewusst, dass sie von dieser Situation profitieren oder dass die Minderheit eine Benachteiligung erfährt (Kimmel 2008). Nehmen Lernende in geschlechtsuntypischen Berufslehren wahr, dass sie anders behandelt werden als Personen, deren Geschlecht in der Mehrheit ist, werden sie diskriminiert. Diskriminierung wäre damit der Grund für die Bewältigungshandlungen und nicht die geschlechtsuntypische Situation als solche.

4) Psychosomatische Beschwerden. Ausgrenzungserfahrungen können zu psychosomatischen Symptomen wie Unwohlsein, Bauch-, Kopf- und Rückenschmerzen führen (De Col et al. 2012). Die im Modell der geschlechts- 
untypischen Berufssozialisation postulierten negativen Reaktionen des sozialen Umfelds und ihre Bewältigung wirken sich in der Folge auf das körperliche und psychische Wohlbefinden der Lernenden aus. Dies manifestiert sich in erhöhten Werten bei psychisch-körperlichen Störungen.

5) Absenzen. Aufgrund von Unwohlsein oder Krankheiten kann es zu Fehlzeiten kommen. In der Sekundarstufe I sind deutliche Korrelationen zwischen der Anzahl unentschuldigter bzw. entschuldigter Fehlzeiten, emotionalen Problemen und Verhaltensauffälligkeiten nachgewiesen (Lenzen et al. 2013). Absentismus könnte dann als weiteres Indiz für die belastenden Herausforderungen gedeutet werden, mit der die Jugendlichen konfrontiert sind.

Im Folgenden werden zwei Forschungsfragen geklärt: Erstens der mediierende Effekt der Reaktion des sozialen Umfelds auf die Bewältigung und zweitens die Folgen dieser Bewältigung. Die Art der Bewältigung der geschlechtsuntypischen Situation ergibt sich aus der Einschätzung des Arbeitsumfelds: Wie wohl fühlen sich die Lernenden? Haben sie ein gutes Einvernehmen mit den Mitarbeitenden vor Ort? Wie schätzen sie das Arbeitsklima ein? Ist das Arbeitsklima von Toleranz und gegenseitiger Unterstützung geprägt? Oder sind abwertende Äußerungen an der Tagesordnung? Operationalisiert wird die Bewältigung mittels zweier Fragen zum Auskommen mit Arbeitskolleginnen und -kollegen sowie zum Umgang im Lehrbetrieb. Die Bewältigung der geschlechtsuntypischen Situation wird von der Reaktion des sozialen Umfelds beeinflusst.

Forschungsfrage Wie wirkt sich die Reaktion des sozialen Umfelds auf die Lernenden in geschlechtsuntypischen Berufslehren aus?

Diese Reaktion des sozialen Umfelds wird in der vorliegenden Untersuchung mit der Wahrnehmung von Diskriminierung und sozialer Unterstützung operationalisiert.

Hypothese 4 Der Einfluss der Geschlechtstypik auf die Bewältigung wird durch die Wahrnehmung von Diskriminierung und/oder sozialer Unterstützung vermittelt.

Die Auswirkungen der Diskriminierung führen zu Problemen. Die Jugendlichen fühlen sich ausgegrenzt, was zu psychischen und körperlichen Reaktion führen kann. Daraus können sich psychosomatische Beschwerden entwickeln und die Absenzen zunehmen.

Forschungsfrage Unterscheiden sich die Lernenden in geschlechtsuntypischen Berufslehren von Lernenden in geschlechtstypischen Berufslehren bei den Folgen von negativen Erlebnissen insbesondere wegen Diskriminierung?

Operationalisiert werden die Folgen in Form der Höhe der psychosomatischen Beschwerden und der Anzahl der Absenzen:

Hypothese $5 a$ Lernende in geschlechtsuntypischen Berufslehren berichten von mehr psychosomatischen Beschwerden als Lernende in geschlechtstypischen Berufslehren. 
Hypothese $5 b \quad$ Lernende in geschlechtsuntypischen Berufslehren haben mehr Absenzen als Lernende in geschlechtstypischen Berufslehren.

\subsection{Methode}

Das methodische Vorgehen erkläre ich in vier Abschnitten. In Abschnitt 8.2.1 stelle ich die Langzeitstudie TREE vor, deren Daten für diese Auswertung verwendet werden. Wie ich mit diesen Daten umgangen bin und welche statistischen Verfahren ich angewendet habe, beschreibe ich in Abschnitt 8.2.2 ,Auswertungsstrategie‘. Die Beschreibung der Stichprobe der 904 in die Auswertung einbezogenen Lernenden erfolgt in Abschnitt 8.2.3, die verwendeten Instrumente aus den TREE-Fragebogen in Abschnitt 8.2.4.

\subsubsection{Forschungsprojekt TREE}

TREE ist die erste schweizerische Längsschnittstudie auf nationaler Ebene, die die Ausbildungsverläufe und den Einstieg ins Berufsleben von Jugendlichen und jungen Erwachsenen untersucht. Über 6'300 Jugendliche, die an der PISA-2000-Befragung (Programme for International Student Assessment) teilnahmen, bilden die Ausgangsstichprobe für Nachbefragungen. Zwischen 2001 und 2015 wurden die Teilnehmenden insgesamt zehnmal befragt, zwischen sogar 2001 und 2007 jährlich. Die Stichprobe ist repräsentativ für die ganze Schweiz mit ihren drei Sprachregionen (vgl. TREE 2008).

Das Ziel des TREE-Forschungsprojekts ist die Untersuchung des Übergangs von der Schule ins Erwerbsleben. Im Zentrum steht die Analyse zahlreicher Transitionen, die Jugendliche in der Schweiz machen. Die PISALese-Kompetenz-Messungen im neunten Schuljahr wurden durch Kontextdaten der Schülerinnen und Schüler sowie der von ihnen besuchten Schulen ergänzt: Persönlichkeitsmerkmale, soziokulturelle und -ökonomische Faktoren, Laufbahnaspirationen sowie Merkmale der Schule vor Abschluss der obligatorischen Schulzeit. Das Längsschnittdesign ermöglicht die Analyse von Veränderungen vieler Einflussgrößen über die Zeit. In der Abbildung 8.2 zum Projekt- und Erhebungsdesign von TREE sind die in der vorliegenden Arbeit verwendeten Ausschnitte als gepunktete Linien dargestellt. Die so entstandenen fünf Quasi-Kohorten werden derart übereinandergelegt, dass die jeweiligen Lehrjahre (eins bis drei) übereinander zu stehen kommen. Dies ermöglicht es, die Veränderungen während der beruflichen Grundbildung zu analysieren. 
Abbildung 8.2: Forschungsdesign TREE

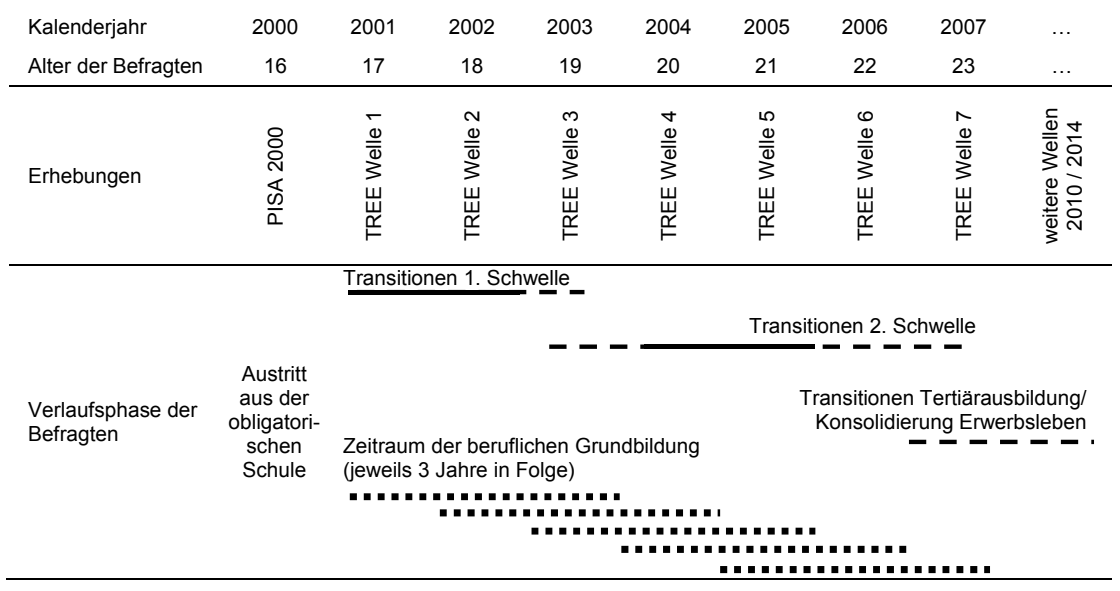

Lebende: Durchgezogene, gestrichelte und gepunktete Linien: Zeiträume der beruflichen Bildung. Quelle: Darstellung nach TREE (2011a: 5).

\subsubsection{Auswertungsstrategie}

Der Datensatz für die vorliegende Arbeit besteht aus dem Schweizer PISA2000-Datensatz, den Datensätzen der sieben TREE-Wellen (2001-2007) sowie den SBFI-Bezeichnungen der Berufslehren und den dazugehörigen Werten des Frauenanteils ${ }^{33}$. Mittels einer Kombination von Geschlechtszugehörigkeit und Frauenanteil der Berufslehre wurde die Gruppeneinteilung der Geschlechtstypik errechnet. Diese Einteilung der vier Gruppen folgt gemäß Kanter (1977, vgl. Abschnitt 3.4.4) der 15 Prozent-Regel: 1) Männer in männerreichen Berufslehren (FA der Berufslehre 0-15\%), 2) Männer in frauenreichen Berufslehren (FA 85-100\%), 3) Frauen in frauenreichen Berufslehren (FA 85-100\%) und 4) Frauen in männerreichen Berufslehren (FA 0-15\%).

Die Daten der Lernenden aus den sieben Wellen wurden so zusammengestellt, dass die drei Messzeitpunkte $t_{1}, t_{2}$ und $t_{3}$ den jeweiligen Ausbildungsjahren entsprachen: $t_{1}=1$. Lehrjahr, $t_{2}=2$. Lehrjahr und $t_{3}=3$. Lehrjahr.

Aufgrund der Selektionsbedingungen (siehe Abschnitt 8.2.3) gab es fehlende Werte bei den Lernenden in geschlechtsuntypischen Berufslehren. Die fehlenden Werte der verwendeten Variablen wurden per multiple Imputation mit der Software IBM SPSS Statistics Version 22 geschätzt. Diese Methode

33 Der Frauenanteil wurde aus allen EFZ-Lernenden der Schweiz aus den Jahren 2008-2012 durch den Autor errechnet. Die detaillierten Angaben dazu sind im Kapitel 5 beschrieben. Eine Übersichtliste befindet sich im Online-Anhang I. 
schätzt plausible Werte für die fehlenden Daten auf der Basis aller verfügbaren Informationen. Im vorliegenden Fall wurden zusätzlich der ,sozioökonomische Status', das ,Geschlecht' und die ,Werte des PISA-Lesetests' einbezogen. Die multiple Imputation erhöht die Robustheit und Reliabilität der Schätzwerte der Inferenzstatistik (Schafer/Olsen 1998, Schafer/Graham 2002).

Mittels Median-Split-Verfahren beim Item ,Absenzen' bzw. beim Konstrukt ,psychosomatische Beschwerden' wurden die Teilnehmenden in zwei Gruppen eingeteilt, jeweils mit hoher vs. tiefer Ausprägung, also viel vs. wenig Absenzen bzw. psychosomatischen Beschwerden. Für jede Person liegt damit zu jedem der drei Messzeitpunkte ein Wert vor, der sie der Gruppe , viel ${ }^{`}$ oder , wenig` zuteilt. Daraus ergaben sich fünf Verlaufstypen. Der Verlaufstyp ,stabil wenig' hat in allen drei Lehrjahren eine tiefe Ausprägung. Dem gegenüber steht der Verlaufstyp ,stabil viel ${ }^{\star}$ mit hoch ausgeprägten Werten zu allen 3 Messzeitpunkten. Der Verlaufstyp ,Verschlechterung ${ }^{6}$ hat im 1. Lehrjahr tiefe, dann im 2. oder im 3. Lehrjahr hohe Ausprägungen (wenig-wenig-viel bzw. wenig-viel-viel). Umgekehrt hat der Verlaufstyp ,Verbesserung' erst hohe, dann tiefe Werte. Der Verlaufstyp ,zwei Wechsel ${ }^{\star}$ verändert sich gleich zweimal (wenig-viel-wenig bzw. viel-wenig-viel).

Die Mediationshypothese 4 wird mittels hierarchischer linearer Regression getestet. Die Mediation wird zusätzlich durch das BootstrappingVerfahren überprüft (Preacher/Hayes 2008). Zur Klärung der Hypothesen 5a und $5 \mathrm{~b}$ werden querschnittlich pro Lehrjahr $C h i^{2}$-Tests gerechnet und längsschnittlich Verlaufsgruppen mit Verfahren ähnlich der Konfigurationsfrequenzanalyse (vgl. Krauth 1993, von Eye 2002) untersucht. So können Unterschiede zwischen Lernenden in typischen und untypischen Berufslehren entdeckt werden.

\subsubsection{Stichprobenbeschreibung}

Insgesamt sind beim TREE-Projekt 6'343 Jugendliche involviert. Die hier verwendeten Daten entstammen den jährlich stattfindenden Nachbefragungen zwischen 2001 und 2007. Die Teilnehmenden unterschieden sich im Zeitpunkt des Lehrbeginns. Die meisten begannen ihre berufliche Grundbildung im Anschluss an die Sekundarstufe I. Die anderen absolvierten erst das 10. Schuljahr, ein Zwischenjahr, mehrere Zwischenjahre oder hatten eine erste Berufslehre vorzeitig abgebrochenen bzw. erfolgreich beendet. Es wurden diejenigen Teilnehmenden selektioniert, die sich an drei aufeinanderfolgenden Jahren im gleichen beruflichen Grundbildungslehrgang befunden und jährlich an den Befragungen teilgenommen hatten. Mit unvollständigen Datensätzen wurden lediglich Lernende in geschlechtsuntypischen Berufslehren einbezogen. Dieses Vorgehen wurde gewählt, um eine genügend große 
Stichprobe für die Minderheitsgruppen zu erhalten. In einer weiteren Selektion wurden lediglich diejenigen Lernenden ausgewählt, die ihre Berufslehre entweder in einem männer- oder frauenreichen Beruf (maximal 15\% Minderheitenanteil) absolvierten.

Die so gewonnene Stichprobe umfasst 904 Lernende (336 Frauen und 568 Männer) aus 23 von 26 Kantonen der Schweiz. Die Kantone Appenzell Innerrhoden und Uri sind bereits in der PISA-Stichprobe nicht vertreten. Die ohnehin wenigen Personen aus dem Kanton Nidwalden wurden aufgrund der oben beschriebenen Selektionskriterien aussortiert. Kantone mit mehr Einwohnerinnen und Einwohnern sind in der Stichprobe häufiger vertreten. Wie die folgenden Berechnungen nahelegen, kann die Stichprobe als repräsentativ betrachtet werden. 59.7 Prozent stammen aus dem deutsch-, 28.1 Prozent aus dem französisch- und 12.2 Prozent aus dem italienischsprachigen Landesteil. Dies entspricht größtenteils der Einwohnerstatistik des BFS für $2013^{34}$, wobei die lateinischen Sprachen etwas stärker vertreten sind. Die Tabelle 8.1 weist die Anzahl Lernende nach Geschlecht und Sprachregion im Überblick aus. Die Verteilung der Lernenden auf Geschlechtstypik und Sprachregion ist unauffällig $\left(C h i^{2}(2)=5.5, p>.05\right)$.

Tabelle 8.1: Anzahl Lernende nach Sprachregion

\begin{tabular}{lrcrrrrr}
\hline \multirow{2}{*}{$\begin{array}{l}\text { Schweizer } \\
\text { Sprachregion }\end{array}$} & \multicolumn{2}{c}{ Frauen } & \multicolumn{2}{c}{ Männer } & \multicolumn{2}{c}{ beide Geschlechter } & Total \\
& typisch & untypisch & typisch & untypisch & typisch & untypisch & \\
\hline Deutsch & 192 & 20 & 317 & 11 & 509 & 31 & 540 \\
Französisch & 70 & 17 & 164 & 3 & 234 & 20 & 254 \\
Italienisch & 27 & 10 & 70 & 3 & 97 & 13 & 110 \\
\hline Total & 289 & 47 & 551 & 17 & 840 & 64 & 904 \\
\hline
\end{tabular}

Legende: Sprachregion und Geschlechtstyp: $C i^{2}(2)=5.5^{\text {n.s. }}$ Quelle: Eigene Darstellung.

Die Anzahl Personen in den vier Gruppen der Geschlechtstypik unterscheiden sich signifikant (Frauen typisch $=289$; Frauen untypisch $=47$; Männer typisch $=551$; Männer untypisch $\left.=17 ; \operatorname{Chi}^{2}(1)=38.8, p<.001\right)$ und widerspiegeln die Situation der Population (vgl. Tabelle 8.1). Die duale Berufslehre ist bei jungen Männern allgemein beliebter als bei jungen Frauen, die rein schulische Ausbildungen bevorzugen (z.B. Borkowsky 2000). Junge Frauen wählen eher einen geschlechtsuntypischen Beruf als junge Männer (z.B. Helbig/Leuze 2012).

Die Teilnehmenden waren im ersten Lehrjahr (erster Messzeitpunkt) zwischen 17 und 24 Jahre alt $(M=17.2, S D=1.2)$. Die Lernenden in geschlechtsuntypischen Berufslehren sind durchschnittlich ein Jahr älter (typisch: $M=17.1, S D=1.1$, untypisch: $M=18.0, S D=2.1$, Mann-Whitney-U- 
Test $U=33325.5, p<.01)$. 91.2 Prozent der Teilnehmenden sind in der Schweiz geboren. Die vier Gruppen der Geschlechtstypik unterscheiden sich nicht zwischen Lernenden mit schweizerischen und nichtschweizerischen Wurzeln $\left(\mathrm{Chi}^{2}(1)=0.1, p>.05\right) .84 .2$ Prozent sprechen die Testsprache zu Hause. Zwischen den vier Gruppen der Geschlechtstypik gibt es ebenfalls keine signifikanten Unterschiede bei der Sprache $\left(C h i^{2}(1)=0.4, p>.05\right)$.

Der Online-Anhang XI weist die Anzahlen der Lernenden in den jeweiligen Berufslehren und die Gruppenzugehörigkeit aus. Für die Berufslehre ,Gärtner/-in EFZ' konnte eine Aufteilung in die Fachrichtungen ,Gartenund Landschaftsbau' sowie ,Zierpflanzen" gemacht werden. Dies bot sich an, da die Geschlechtssegregation in diesen Fachrichtungen ausgeprägt ist und eine genügend große Anzahl von Lernenden vorhanden war. Aufgrund von fehlenden Angaben bei den Fachrichtungen konnten bei den beiden Berufslehren mit den meisten Lernenden, ,Kaufmann/-frau EFZ' und ,Detailhandelsfachmann/-frau EFZ', keine weiteren Unterteilungen in Fachrichtungen vollzogen werden. Damit konnten diese Lernenden nicht selektioniert werden, obschon, wie in Kapitel 5 und 6 dargelegt, eine Differenzierung in diesen Berufslehren notwendig wäre, da die Segregation auf der Ebene der Fachrichtungen stark ausgeprägt ist.

Eine relativ große Gruppe von weiblichen Lernenden $(N=16)$ absolvierte die Berufslehre ,Informatiker/-in EFZ'. Damit machen die Informatikerinnen einen Drittel der Gruppe der Frauen in geschlechtsuntypischen Berufslehren aus. Ebenfalls einen Drittel bilden die ,Fachmänner Gesundheit EFZ' bei den männlichen Lernenden in geschlechtsuntypischen Berufslehren (vgl. OnlineAnhang XI).

\subsubsection{Instrumente}

Zwölf Variablen stammen aus dem PISA-2000-Fragebogen für Schülerinnen und Schüler. Die Variable ,Geschlecht ${ }^{\star}$ hatte zwei Antwortmöglichkeiten: 1) weiblich, 2) männlich. Das ,Alter 6 wurde mittels des Geburtsdatums erfasst und in Monate umgerechnet. Der ,sozioökonomische Status' (Highest International Socio-Economic Index HISEI) basiert auf den Berufen der Mutter bzw. des Vaters. Die Klassifikation der Berufe erfolgte via der internationalen Berufsklassifizierung (International Standard Classification of Occupation ISCO-88). Anschließend wurde der höhere ISCO-88-Wert weiterverwendet und in die HISEI-Skala (Skalenbreite 16-90) transferiert. Zusätzlich werden Angaben zu ,Kanton“, ,Sprachregion“, ,Geburtsland" und ,Hauptsprache zu Hause sowie die fünf plausiblen Werte für die ,PISA-Lesekompetenz verwendet (für detaillierte Informationen wird auf die PISA-Dokumentation verwiesen: OECD 2002). Die im Folgenden dargestellten Konstrukte und Variablen entstammen den TREE-Fragebogen. Wenn keine zusätzliche Lite- 
ratur angegeben ist, basieren die Items auf Eigenproduktionen der TREEForschungsgruppe (vgl. TREE 2008).

Die, soziale Unterstützung ' basiert auf der Frage nach dem Interesse von wichtigen Personen des persönlichen Umfelds. Zur Frage „Wie stark interessieren sich folgende Personen für Ihre Ausbildung?" konnten die Lernenden in vier Stufen von 1 , gar nicht $t^{6}$ bis 4, sehr $^{6}$ für folgende Personen antworten: Mutter, Vater, feste Freundin / fester Freund, beste Schulkollegin / bester Schulkollege, beste Arbeitskollegin / bester Arbeitskollege im Betrieb, Ausbildner/-in sowie Klassenlehrer/-in. Die Angaben zu den jeweiligen Personen wurden zum Mittelwert, soziale Unterstützung' zusammengefasst.

Für das Konstrukt ,Bewältigung' werden aus dem Faktor ,Zufriedenheit mit Lehrbetrieb' zwei Items verwendet. Die beiden Aussagen lauten: 1) „Mit den meisten meiner Arbeitskolleg/-innen im Betrieb komme ich sehr gut aus“" (kurz ,Auskommen mit Arbeitskolleginnen und-kollegen“) und 2) „Mir gefällt sehr, wie die Leute in meinem Betrieb miteinander umgehen“ (kurz ,Umgang im Lehrbetrieb'). Die Ratingskala hat vier Stufen von 1 ,stimmt überhaupt nicht ${ }^{\star}$ bis 4 ,stimmt genau'. Die beiden Einschätzungen wurde zum Konstrukt ,Bewältigung ‘ zusammengefasst. Die interne Konsistenz beträgt zum Zeitpunkt $\mathrm{t}_{3} \alpha=.82$.

Die Items zur ,Diskriminierung' entstammen dem Faktor, Chancengleichheit im Lehrbetrieb“. Dabei wurden zwei Fragen gestellt: „In meinem Betrieb werden Männer besser behandelt als Frauen“ bzw. „In meinem Betrieb werden Frauen besser behandelt als Männer". Die Angabe erfolgte vierstufig von 1 ,stimmt überhaupt nicht ${ }^{\circ}$ bis 4 , stimmt genau'. In den vorliegenden Auswertungen werden die Items jeweils zum Geschlecht passend verwendet. Diese Items wurden zum Messzeitpunkt $t_{1}$ nicht erhoben. Da sich ein der Großteil der Teilnehmenden zu diesem Zeitpunkt im ersten Lehrjahr befand, wurden lediglich die Daten des zweiten und dritten Lehrjahrs verwendet.

Das Konstrukt ,psychosomatische Beschwerden" besteht aus acht Items mit den Ausprägungen von 1 ,nie ${ }^{6}$ bis 5 , täglich ${ }^{\star}$ und setzt sich zusammen aus Einschätzungen zur Häufigkeit von Magenschmerzen, Appetitlosigkeit, Konzentrationsschwäche, Schwindel, Einschlafschwierigkeiten, Nervosität/Unruhe, ungewohnter Müdigkeit und sehr starken Kopfschmerzen. Die Items stammen von Grob et al. (1991).

Die Frage zu den ,Fehlzeiten' lautet: „Wie oft haben Sie im vergangenen Monat in der Schule oder im Betrieb gefehlt?" Es wurde die Anzahl der Tage angegeben. 


\subsection{Ergebnisse}

Einen ersten Überblick über die Zusammenhänge der untersuchten Variablen bietet die Interkorrelationsmatrix in der Tabelle 8.2. Die Variable ,Geschlechtstypik korreliert signifikant mit allen anderen Variablen außer der ,sozialen Unterstützung'. Die Korrelationen sind insgesamt eher gering, gehen jedoch in die postulierte Richtung und sind bis auf wenige Ausnahmen statistisch bedeutsam. Die Präsentation der weiteren Ergebnisse umfasst die Befunde zur Mediationshypothese (Abschnitt 8.3.1) und die Analyse zu den Folgen der Diskriminierung (Abschnitt 8.3.2).

Tabelle 8.2: Mittelwerte, Standardabweichungen und Interkorrelationsmatrix

\begin{tabular}{|c|c|c|c|c|c|c|c|c|c|}
\hline$N=904$ & $M$ & $S D$ & 2 & 3 & 4 & 5 & 6 & 7 & 8 \\
\hline 1 Geschlechtstypik $t_{1}$ & 1.07 & .26 & $-.16^{* *+*}$ & $-.17^{4+t+}$ & $.14^{\text {tot }}$ & $.12^{*+*}$ & -.02 & $.09^{* *}$ & $.11^{* *}$ \\
\hline 2 Bewältigung $t_{1}$ & 3.36 & .70 & & $.30^{* * t}$ & $-.11^{* *}$ & $-.14^{+*+t}$ & $.08^{*}$ & $-.21^{* *}$ & $-.11^{* *}$ \\
\hline 3 Bewältigung $t_{3}$ & 2.95 & .91 & & & $-.21^{* * *}$ & $-.18^{*+*}$ & $.11^{* *}$ & $-.15^{*+*}$ & $-.10^{* *}$ \\
\hline 4 Diskriminierung Frauen $t_{2}$ & 1.43 & .79 & & & & $.53^{* *+*}$ & -.05 & $.08^{*}$ & $.14^{n+*}$ \\
\hline 5 Diskriminierung Männer $t_{2}$ & 1.37 & .71 & & & & & $-.08^{*}$ & $.11^{* *}$ & $.10^{* *}$ \\
\hline 6 soziale Unterstützung $\mathrm{t}_{2}$ & 3.15 & .56 & & & & & & -.06 & -.00 \\
\hline $\begin{array}{l}7 \text { psychosomatische Be- } \\
\text { schwerden } \mathrm{t}_{2}\end{array}$ & 1.81 & .64 & & & & & & & $.12^{+* *+}$ \\
\hline 8 Absenzen $t_{2}$ & 4.50 & 7.95 & & & & & & & \\
\hline
\end{tabular}

Legende: M: Mittelwert; SD: Standardabweichung; t1: 1. Lehrjahr; t2: 2. Lehrjahr; t3: 3. Lehrjahr; ${ }^{*} p<.05 ;{ }^{* \star} p<.01$; *** $p<.001$.

1 Geschlechtstypik: Minderheitengruppen max. $15 \%$ (1,typisch'vs. 2 ,untypisch')

2 Bewältigung: 1. Lehrjahr (1, niedrig' bis 4 ,hoch')

3 Bewältigung: 3. Lehrjahr (1 ,niedrig' bis 4 ,hoch')

4 Diskriminierung Frauen: Männer besser behandelt als Frauen (1, stimmt überhaupt nicht' bis 4 ,stimmt genau')

5 Diskriminierung Männer: Frauen besser behandelt als Männer (1, stimmt überhaupt nicht' bis 4 ,stimmt genau')

6 soziale Unterstützung: Mittelwert Interesse an der Ausbildung von 7 Personen/-gruppen (1, gar nicht' bis 4 , sehr')

7 psychosomatische Beschwerden: Mittelwert aus 8 Angaben zum Unwohlsein (1 ,nie' bis 5 ,täglich')

8 Absenzen: (Anzahl Tage).

Quelle: Eigene Darstellung.

\subsubsection{Mediation durch die Reaktion des sozialen Umfelds}

Die Analyse des Mediationseffekts der Beziehung zwischen der ,Geschlechtstypik' und der ,Bewältigung' durch die ,Reaktion des sozialen Umfelds ${ }^{\star}$ erfolgt mittels der hierarchischen linearen Regressionen und Bootstrapping-Verfahren. Diese Analyse kann aufzeigen, welche Erklärungsvariablen die abhängige Variable, Bewältigung' beeinflussen. Schrittweise werden die Prädiktoren von Modell 1 bis 5 hinzugefügt. Aufgrund der Geschlechtsspezifik werden die Analysen für beide Geschlechter getrennt berechnet. 
Tabelle 8.3: Prädiktoren für Bewältigung im 3. Lehrjahr $\mathrm{t}_{3}$

\begin{tabular}{|c|c|c|c|c|c|c|c|c|c|c|}
\hline \multirow[b]{2}{*}{ Prädiktoren } & \multicolumn{5}{|c|}{ Frauen } & \multicolumn{5}{|c|}{ Männer } \\
\hline & Mod. 1 & Mod.2 & Mod. 3 & Mod. 4 & Mod. 5 & Mod. 1 & Mod. 2 & Mod. 3 & Mod. 4 & Mod. 5 \\
\hline Bewältigung $t_{1}$ & $.31^{* \star *}$ & $.28^{* \star \star}$ & $.27^{* * *}$ & $.28^{\star \star \star}$ & $.27^{* * *}$ & $.29^{* \star *}$ & $.28^{* * *}$ & $.26^{* \star *}$ & $.27^{* \star *}$ & $.25^{* * *}$ \\
\hline Geschlechtstypik $t_{1}$ & & $-.15^{* *}$ & $-.11^{*}$ & $-.13^{*}$ & -.09 & & $-.07^{+}$ & -.05 & $-.08^{+}$ & -.05 \\
\hline Diskriminierung $t_{2}$ & & & $-.18^{*+*}$ & & $-.17^{* *}$ & & & $-.15^{* * *+}$ & & $-.14^{* * *}$ \\
\hline soziale Unterstützung & & & & $.17^{* *}$ & $.15^{*}$ & & & & $.07^{+}$ & $.07^{+}$ \\
\hline$F, p$ & $34.6^{* * *}$ & $21.4^{* * *}$ & $18.9^{* * t}$ & $18.2^{* * *}$ & $16.8^{*+* t}$ & $51.2^{*+* t}$ & $27.2^{* * *}$ & $22.9^{*+* t}$ & $19.2^{* * *}$ & $17.9^{* * * t}$ \\
\hline$d f_{1}, d f_{2}$ & 1,334 & 2,333 & 3,332 & 3,332 & 4,331 & 1,566 & 2,565 & 3,564 & 3,564 & 4,563 \\
\hline$R_{\text {korr }}^{2}$ & $9.1 \%$ & $10.9 \%$ & $13.8 \%$ & $13.3 \%$ & $15.9 \%$ & $8.1 \%$ & $8.5 \%$ & $10.4 \%$ & $8.8 \%$ & $10.7 \%$ \\
\hline \multicolumn{11}{|c|}{ Mediation der Reaktion des sozialen Umfelds (1000 Bootstrapping-Stichproben; KI=95\%) } \\
\hline Total & & & & \multicolumn{2}{|c|}{-.30 bis -.04} & & & & -.22 bis & .02 \\
\hline \multicolumn{3}{|l|}{ Diskriminierung $t_{2}$} & & \multicolumn{2}{|c|}{-.22 bis -.03} & & & & \multirow{2}{*}{$\begin{array}{l}-.25 \text { bis } \\
-.00 \text { bis }\end{array}$} & .02 \\
\hline soziale Unterstützung & & & & $-.14 \mathrm{bis}$ & .01 & & & & & .10 \\
\hline
\end{tabular}

Legende: Standardisierte Beta-Koeffizienten der linearen Regressionsanalyse. Mod.: Modell; $t_{1}: 1$. Lehrjahr; $t_{2}: 2$. Lehrjahr; $t_{3}$ : 3. Lehrjahr; $d f_{1}, d f_{2}$ :Anzahl der Freiheitsgrade; $R^{2}{ }_{\text {korr }}$ : korrigierter R-Quadrat-Wert, ${ }^{+} p<.1 ;{ }^{\star} p<.05 ;{ }^{\star *}$ $p<.01 ;{ }^{* *} p<.001$; Geschlechtstypik $\mathrm{t}_{1}\left(1\right.$, typisch $^{\prime} ; 2$, untypisch $\left.{ }^{*}\right)$. Quelle: Eigene Darstellung.

Ergebnisse für die Gruppe der Frauen: Modell 1 kontrolliert den Einfluss der abhängigen Variable, Bewältigung'. Lernende, die im dritten Lehrjahr gut mit der Situation am Lernort zurechtkommen, dürften dies bereits im ersten Lehrjahr getan haben $(\beta=.31, p<.001 ; \mathrm{vgl}$. Tabelle 8.3). Die weiteren postulierten Effekte müssen sich also gegenüber der kontrollierten Variablen durchsetzen, wenn sie Einfluss haben sollten. Im Modell 2 wurde die Variable ,Geschlechtstypik $k^{6}$ eingefügt. Der Effekt ist signifikant $(\beta=-.15, p<.01)$. Ein negativer Beta-Index erklärt einen indirekten Zusammenhang: Für Lernende in geschlechtstypischen Berufslehren ist die Wahrscheinlichkeit gröBer, mit den Arbeitskolleginnen und -kollegen gut auszukommen und den Umgang im Lehrbetrieb positiv einzuschätzen. Im Modell 3 kommt die erste Mediationsvariable des sozialen Umfelds, die ,Diskriminierung', dazu. Eine Mediation findet dann statt, wenn der Effekt der Variable ,Geschlechtstyp “ abnimmt bzw. nicht mehr signifikant wird. Der Effekt der ,Geschlechtstypik ${ }^{6}$ nimmt mit der Einführung der Variable ,Diskriminierung ${ }^{6}(\beta=-.18$, $p<.001) \mathrm{ab}$, bleibt aber signifikant $(\beta=-.11, p<.05)$. Im Modell 4 wird als Zweites der Effekt der Variable, soziale Unterstützung' getestet. Die, soziale Unterstützung' hat einen signifikanten, positiven Einfluss auf die ,Bewältigung $^{6}(\beta=.17, p<.01)$ und schwächt den Einfluss der Variable ,Geschlechtstypik $k^{6}(\beta=-.13, p<.05)$ ab. Ihr Einfluss bleibt jedoch nach wie vor signifikant. Die vollständige Vermittlung der Variable ,Geschlechtstypik ergibt sich im Modell 5, wenn beide Variablen des sozialen Umfelds einbezogen werden: ,Diskriminierung' $(\beta=-.17, p<.01)$ und ,soziale Unterstützung $^{`}(\beta=.15, p<.05)$. Der Effekt der ,Geschlechtstypik ${ }^{`}$ ist nicht mehr 
signifikant $(\beta=-.09, \quad p>.05)$. Die aufgeklärte Varianz liegt bei $\mathrm{R}_{\text {korr }}^{2}=15.9 \%$.

Mittels des Bootstrapping-Verfahrens nach Preacher und Hayes (2008) werden der direkte Effekt der, Geschlechtstypik' und die indirekten Effekte der ,Diskriminierung' und der, sozialen Unterstützung' auf ihre Signifikanz geprüft. Es wird nachgewiesen, dass die ,Geschlechtstypik ${ }^{6}$ die ,Bewältigung ${ }^{6}$ signifikant beeinflusst (totaler Effekt c-Pfad: Coeff $=-.40, p<.05$ ). Dieser Effekt wird jedoch in Übereinstimmung mit Hypothese 4 nach Einbezug der beiden Variablen ,Diskriminierung' und ,soziale Unterstützung' im Gegensatz zu oben vollständig mediiert, d.h. er wird, im Gegensatz zu oben, nicht mehr signifikant (direkter Effekt c'-Pfad: Coeff $=-.26, p>.05$ ). Während die Konfidenzintervalle vom Total und von der Variable ,Diskriminierung von null verschieden sind (signifikante Mediation), ist die Mediation der Variable ,soziale Unterstützung' nicht signifikant, weil das Intervall null enthält (indirekte Effekte [Konfidenzintervalle KI $=95 \%$ ]: Total -.30 bis -.04 ; ,Diskriminierung ${ }^{6}-.22$ bis -.03 ; , soziale Unterstützung ${ }^{6}-.14$ bis .01 ).

Bei der Gruppe der Männer resultiert ein ähnliches Bild wie bei den Frauen (vgl. Tabelle 8.3). Die Kontrolle der erklärten Variable ,Bewältigung“ im Modell 1 ist signifikant $(\beta=.29, p<.001)$. Die erklärende Variable ,Geschlechtstypik ${ }^{6}$ im Modell 2 ist tendenziell signifikant $(\beta=-.07, p<.1)$ und ihr Einfluss schwächt sich im Modell 3 durch die Mediationsvariable ,Diskriminierung $^{6}(\beta=-.15, p<.001)$ ab. Der Effekt der, sozialen Unterstützung ${ }^{6}$ im Modell 4 ist teilweise signifikant $(\beta=.07, p<.1)$ und mediiert die Variable ,Geschlechtstypik $k^{\star}$ nicht. Modell 5 mit beiden Mediationsvariablen ,Diskriminierung' $(\beta=-.14, p<.01)$ und ,soziale Unterstützung' $(\beta=.07, p<.1)$ ergibt eine vollständige Mediation der Variable ,Geschlechtstypik'. Diese ist nicht mehr signifikant $(\beta=-.05, p>.05)$. Die aufgeklärte Varianz liegt bei $\mathrm{R}_{\text {korr }}^{2}=10.7$ Prozent.

Im Bootstrapping-Verfahren bei den Männern verdeutlicht sich ebenfalls die Mediation mit der Abnahme des totalen Effekts (c-Pfad) der Variable ,Geschlechtstypik' auf ,Bewältigung ${ }^{6}$ von Coeff $=-.36, p<.1$ auf Coeff $=-$ $.28, p>.05$ (c'-Pfad). Die korrigierten Konfidenzintervalle der indirekten Effekte sind im Total (-.22 bis .02) und bei der ,sozialen Unterstützung' (.00 bis .10) zwar nicht, hingegen bei der ,Diskriminierung ${ }^{\circ}(-.25$ bis -.02) schon von null verschieden. Der Mediationseffekt wird durch die Variable ,Diskriminierung 'verursacht und nicht durch die Variable ,soziale Unterstützung'.

Während also bei den Frauen die ,Diskriminierung' im Zusammenspiel mit der ,sozialen Unterstützung ' die Mediation hervorruft, ist es bei den Männer nur die Wahrnehmung der ,Diskriminierung'. Bei beiden Geschlechtern sind die fünf Modelle statistisch signifikant und die erklärte Varianz steigt von Modell zu Modell an. Die Hypothese 4 ist damit bestätigt: Der Einfluss der Geschlechtstypik auf die Bewältigung wird durch die Reaktio- 
nen des sozialen Umfelds vermittelt, jedoch für Frauen und Männer unterschiedlich.

\subsubsection{Verlaufsanalysen}

Die Verlaufsanalysen sollen belegen, dass Jugendliche in geschlechtsuntypischen Berufslehren stärker belastet sind und in der Folge von mehr , psychosomatischen Beschwerden' berichten und mehr ,Absenzen' haben. Die Präsentation der Ergebnisse erfolgt in drei Schritten: 1) querschnittliche Analysen in Kreuztabellen mit $\mathrm{Chi}^{2}$-Tests, 2) deskriptive Beschreibung der Verlaufsgruppen und 3) längsschnittliche Verlaufsgruppenanalyse mittels Konfigurationsfrequenzanalyse.

\subsubsection{Psychosomatische Beschwerden}

Im ersten Schritt werden die Häufigkeiten von Lernenden in geschlechtstypischen und -untypischen Berufslehren verglichen (vgl. Tabelle 8.4). Die Einteilung in zwei Gruppen erfolgte aufgrund der Abweichungen zur Normalverteilung durch die Median-Split-Methode. Die Bezeichnung, wenig' bzw. , viel' ist relativ zur jeweils anderen Gruppe zu verstehen. Im ersten Lehrjahr berichten lediglich 45 Prozent der Lernenden in geschlechtstypischen Berufslehren über relativ viele psychosomatische Beschwerden, hingegen 58 Prozent der Lernenden in geschlechtsuntypischen Berufslehren. Diese erhöhten Werte bei den Untypischen akzentuieren sich in den beiden folgenden Lehrjahren. Die Unterschiede sind in allen drei Lehrjahren signifikant (vgl. Chi ${ }^{2}$ Werte in der Tabelle 8.4). Lernende in geschlechtsuntypischen Berufslehren berichten in allen drei Lehrjahren häufiger als erwartet über psychosomatische Beschwerden.

Tabelle 8.4: Psychosomatische Beschwerden pro Lehrjahr

\begin{tabular}{|c|c|c|c|c|c|c|c|c|c|}
\hline & \multicolumn{3}{|c|}{ 1. Lehrjahr } & \multicolumn{3}{|c|}{ 2. Lehrjahr } & \multicolumn{3}{|c|}{ 3. Lehrjahr } \\
\hline & wenig & viel & Total & wenig & viel & Total & wenig & viel & Total \\
\hline \multirow{2}{*}{ typisch } & 462 & 378 & 840 & 470 & 370 & 840 & 472 & 368 & 840 \\
\hline & $55.0 \%$ & $45.0 \%$ & $100.0 \%$ & $56.0 \%$ & $44.0 \%$ & $100.0 \%$ & $56.2 \%$ & $43.8 \%$ & $100.0 \%$ \\
\hline \multirow{2}{*}{ untypisch } & 27 & 37 & 64 & 23 & 41 & 64 & 24 & 40 & 64 \\
\hline & $42.0 \%$ & $58.0 \%$ & $100.0 \%$ & $35.9 \%$ & $64.1 \%$ & $100.0 \%$ & $37.5 \%$ & $62.5 \%$ & $100.0 \%$ \\
\hline \multirow{3}{*}{ Total } & 489 & 415 & 904 & 493 & 411 & 904 & 496 & 408 & 904 \\
\hline & $54.0 \%$ & $46.0 \%$ & $100.0 \%$ & $54.5 \%$ & $45.5 \%$ & $100.0 \%$ & $54.9 \%$ & $45.1 \%$ & $100.0 \%$ \\
\hline & \multicolumn{3}{|c|}{$\operatorname{Chi}^{2}(1)=3.9^{*}$} & \multicolumn{3}{|c|}{$\operatorname{Chi}^{2}(1)=9.6^{* *}$} & \multicolumn{3}{|c|}{$\operatorname{Chi}^{2}(1)=8.4^{* *}$} \\
\hline
\end{tabular}

Legende: ${ }^{*} p<.05 ;{ }^{* *} p<.01$. Quelle: Eigene Darstellung. 
Im zweiten Schritt wurden die Verlaufsgruppen verglichen. Abbildung 8.3 präsentiert diese Verläufe vom ersten zum dritten Lehrjahr. Im ersten Lehrjahr berichten 378 Lernende in typischen Berufslehren über relativ viele Beschwerden, im zweiten Lehrjahr 370. Von den 370 Lernenden hatten schon 266 zuvor viele Beschwerden, 104 jedoch wenig. Im dritten Lehrjahr sind es noch 368, wovon 212 stabil viele Beschwerden hatten (Gruppe ,stabil viel $\left.^{\circ}\right)$. Die Gruppe ,Verschlechterung' setzt sich zusammen aus den 58 Lernenden, die im dritten Lehrjahr über viele Beschwerden beklagten, im ersten Lehrjahr wenig und im zweiten viel, sowie den 60 Lernenden, die in beiden vorangegangenen Lehrjahren wenige Beschwerden hatten. Die weiteren Gruppen setzen sich entsprechend ihren Verläufen zusammen: ,Verbesserung' $^{\circ} 4+74=128 ;$, stabil wenig' $289 ;,$ zwei Wechsel $38+46=84$.

Abbildung 8.3: Verlaufsgruppen psychosomatische Beschwerden

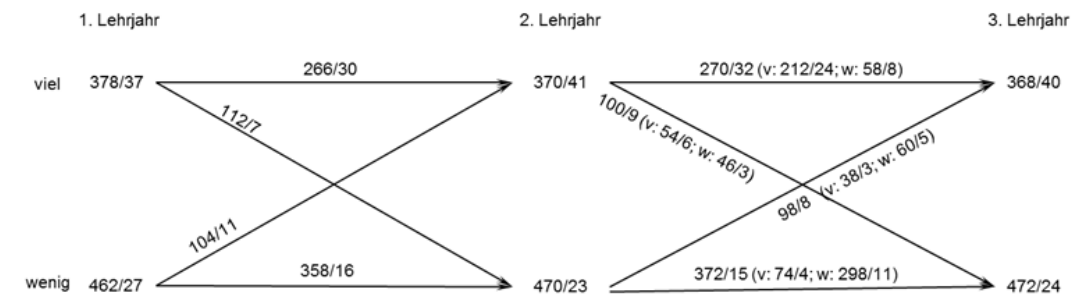

Legende: vordere Zahl: Anzahl Lernende in geschlechtstypischen Berufslehren; hintere Zahl: Anzahl Lernende in geschlechtsuntypischen Berufslehren, v: viel; w: wenig; $N=904$. Quelle: Eigene Darstellung.

Tabelle 8.5: Psychosomatische Beschwerden nach Verlaufstypen

\begin{tabular}{lcccccc}
\hline & \multicolumn{2}{c}{ typisch } & \multicolumn{2}{c}{ untypisch } & \multicolumn{2}{c}{ Total } \\
Verlaufstypen & Anzahl & in Prozent & Anzahl & in Prozent & Anzahl & in Prozent \\
\hline stabil wenig & 298 & $35.5 \%$ & $11^{* *}$ & $17.2 \%$ & 309 & $34.2 \%$ \\
Verschlechterung & 118 & $14.0 \%$ & 13 & $20.3 \%$ & 131 & $14.5 \%$ \\
Verbesserung & 128 & $15.2 \%$ & 10 & $15.6 \%$ & 138 & $15.3 \%$ \\
stabil viel & 212 & $25.2 \%$ & $24^{*}$ & $37.5 \%$ & 236 & $26.1 \%$ \\
zwei Wechsel & 84 & $10.0 \%$ & 6 & $9.4 \%$ & 90 & $10.0 \%$ \\
\hline Total & 840 & $100.0 \%$ & 64 & $100.0 \%$ & 904 & $100.0 \%$ \\
\hline
\end{tabular}

Legende: Anzahlen, Prozente und Chi2-Werte. Chi $^{2}(4)=10.9^{*} ;{ }^{*} p<.05 ;{ }^{* \star} p<.01$. Quelle: Eigene Darstellung.

Im dritten Schritt werden die Ergebnisse mit einem Verfahren analysiert, das sich an die Konfigurationsfrequenzanalyse anlehnt. Diese Analyse testet, ob sich die beobachteten Werte von den erwarteten Werten unterscheiden, und wenn ja, in welchen Gruppen dies der Fall ist (vgl. Tabelle 8.5). Die Ergebnisse belegen, dass es Unterschiede bei den Verlaufstypen gibt. Von den 64 Lernenden in geschlechtsuntypischen Berufslehren berichten 11 konstant 
über wenige psychosomatische Beschwerden $\left(N_{\text {erwartet }}=21.9, \operatorname{Chi}^{2}(1)=5.4\right.$, $p<.01)$ und 24 über viel $\left(N_{\text {erwartet }}=16.7, C h i^{2}(1)=3.2, p<.05\right)$. Die Hypothese 5a ist damit bestätigt: Lernende in geschlechtsuntypischen Berufslehren berichten von mehr psychosomatischen Beschwerden als Lernende in geschlechtstypischen Berufslehren.

\subsubsection{Absenzen}

Das Vorgehen für die Analyse der Absenzen erfolgt ebenfalls in den oben angewandten drei Schritten. Im ersten Analyseschritt ergibt sich im ersten Lehrjahr kein Unterschied zwischen den Lernenden in geschlechtstypischen und -untypischen Berufslehren. Im zweiten und dritten Lehrjahr jedoch haben Lernende in untypischen Berufslehren signifikant mehr Absenzen (vgl. Tabelle 8.6). Auf der deskriptiven Ebene nehmen die Absenzen bei den Lernenden in geschlechtstypischen Berufslehren ab, während sie bei den Lernenden in geschlechtsuntypischen Berufslehren steigen.

Tabelle 8.6: Absenzen pro Lehrjahr von Lernenden

\begin{tabular}{|c|c|c|c|c|c|c|c|c|c|}
\hline & \multicolumn{3}{|c|}{ 1. Lehrjahr } & \multicolumn{3}{|c|}{ 2. Lehrjahr } & \multicolumn{3}{|c|}{ 3. Lehrjahr } \\
\hline & wenig & viel & Total & wenig & viel & Total & wenig & viel & Total \\
\hline \multirow{2}{*}{ typisch } & 479 & 361 & 840 & 536 & 304 & 840 & 540 & 300 & 840 \\
\hline & $57.0 \%$ & $43.0 \%$ & $100.0 \%$ & $63.8 \%$ & $36.2 \%$ & $100.0 \%$ & $64.3 \%$ & $35.7 \%$ & $100.0 \%$ \\
\hline \multirow{2}{*}{ untypisch } & 31 & 33 & 64 & 30 & 34 & 64 & 25 & 39 & 64 \\
\hline & $48.4 \%$ & $51.6 \%$ & $100.0 \%$ & $46.9 \%$ & $53.1 \%$ & $100.0 \%$ & $39.1 \%$ & $60.9 \%$ & $100.0 \%$ \\
\hline \multirow{3}{*}{ Total } & 510 & 394 & 904 & 566 & 338 & 904 & 565 & 339 & 904 \\
\hline & $56.4 \%$ & $43.6 \%$ & $100.0 \%$ & $62.6 \%$ & $37.4 \%$ & $100.0 \%$ & $62.5 \%$ & $37.5 \%$ & $100.0 \%$ \\
\hline & \multicolumn{3}{|c|}{$C h i^{2}(1)=1.8^{\text {n.s. }}$} & \multicolumn{3}{|c|}{$\operatorname{Chi}^{2}(1)=7.3^{* *}$} & \multicolumn{3}{|c|}{$C h i^{2}(1)=16.2^{* * *}$} \\
\hline
\end{tabular}

Legende: n.s. $=$ nicht signifikant; ${ }^{* *} p<.01 ;{ }^{* * *} p<.001$. Quelle: Eigene Darstellung.

Abbildung 8.4: Verlaufsgruppen Absenzen

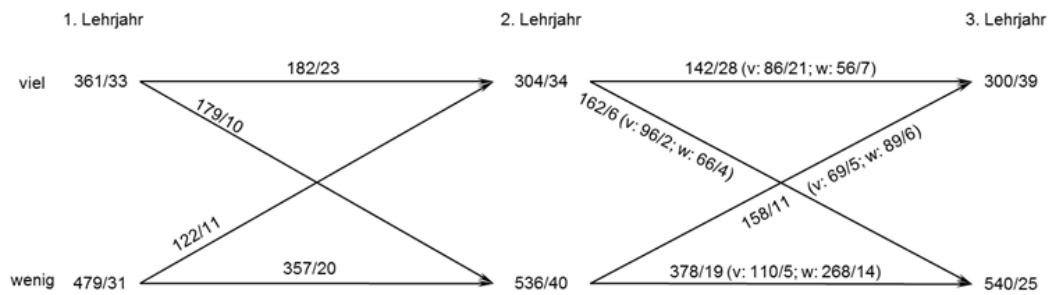

Legende: vordere Zahl: Anzahl Lernende in geschlechtstypischen Berufslehren; hintere Zahl: Anzahl Lernende in geschlechtsuntypischen Berufslehren, v: viel; w: wenig; $N=904$. Quelle: Eigene Darstellung. 
Schritt zwei zeigt diese Verläufe der Absenzen im Detail (vgl. Abbildung 8.4). Es gibt Unterschiede bei den Verlaufstypen $\left(\mathrm{Chi}^{2}(4)=32.6, p<.001\right)$, wie der dritte Schritt in Tabelle 8.7 verdeutlicht. Von den 64 Lernenden in geschlechtsuntypischen Berufslehren berichten 14 konstant über wenige Absenzen $\left(N_{\text {erwartet }}=20.0, C h i^{2}(1)=1.8, p<.1\right), 21$ über konstant viele $\mathrm{Ab}$ senzen $\left(N_{\text {erwartet }}=7.6, \mathrm{Chi}^{2}(1)=23.6, p<.001\right)$ und lediglich 7 Lernende berichten über eine Abnahme der Absenzen $\left(N_{\text {erwartet }}=17.5, C h i^{2}(1)=4.3\right.$, $p<.05$; vgl. Tabelle 8.7).

Tabelle 8.7: Absenzen nach Verlaufstypen

\begin{tabular}{lcccccc}
\hline & \multicolumn{2}{c}{ typisch } & \multicolumn{2}{c}{ untypisch } & \multicolumn{2}{c}{ Total } \\
Verlaufstypen & Anzahl & in Prozent & Anzahl & in Prozent & Anzahl & in Prozent \\
\hline stabil wenig & 268 & $31.9 \%$ & $14^{+}$ & $21.9 \%$ & 282 & $31.2 \%$ \\
Zunahme & 145 & $17.3 \%$ & 13 & $20.3 \%$ & 158 & $17.5 \%$ \\
Abnahme & 206 & $24.5 \%$ & $7^{*}$ & $10.9 \%$ & 213 & $23.6 \%$ \\
stabil viel & 86 & $10.2 \%$ & $21^{* * *}$ & $32.8 \%$ & 107 & $11.8 \%$ \\
zwei Wechsel & 135 & $16.1 \%$ & 9 & $14.1 \%$ & 144 & $15.9 \%$ \\
\hline Total & 840 & $100.0 \%$ & 64 & $100.0 \%$ & 904 & $100.0 \%$ \\
\hline
\end{tabular}

Legende: Anzahlen, Prozente und Chi2-Werte; $\operatorname{Chi}^{2}(4)=32.6{ }^{\star \star *} ;+p<.1 ;{ }^{*} p<.05 ;{ }^{* \star} p<.01 ;{ }^{\star \star *} p<.001$.

Die Hypothese $5 \mathrm{~b}$ ist damit ebenfalls bestätigt: Lernende in geschlechtsuntypischen Berufslehren weisen signifikant mehr Absenzen auf als Lernende in geschlechtstypischen Berufslehren.

\subsection{Diskussion}

Die Befunde der Analyse IV belegen den Einfluss des sozialen Umfelds auf die Jugendlichen in geschlechtsuntypischen Berufslehren. Wie im Modell der geschlechtsuntypischen Berufssozialisation (vgl. Kapitel 4) vorgestellt, benötigen Lernende in geschlechtsuntypischen Berufslehren besondere Ressourcen, um das diskriminierende Verhalten von Teilen ihrer sozialen Umgebung bewältigen zu können. Es ist gelungen, Indikatoren zu überprüfen, die Belastungen bzw. Ressourcen seitens dieser Lernenden aufzeigen. Das Verhältnis zwischen den Lernenden und den Mitarbeitenden sowie das Arbeitsklima im Lehrbetrieb wurden dabei als Manifestation der Bewältigungshandlung der geschlechtsuntypischen Situation herangezogen. Die wahrgenommene Diskriminierung und die soziale Unterstützung vermitteln den Effekt der Geschlechtstypik. Je mehr Diskriminierung Jugendliche in geschlechtsuntypischen Berufslehren erfahren, desto schlechter ist ihr Verhältnis zu den Ar- 
beitskolleginnen und -kollegen und desto tiefer schätzen sie das Arbeitsklima am Lernort ein. Die Wahrscheinlichkeit, dass sich aufgrund dieser Erfahrungen psychosomatische Beschwerden entwickeln und die Fehlzeiten steigen, ist erhöht. Jugendliche in geschlechtsuntypischen Berufslehren berichten von mehr sozialer Unterstützung, die ihnen vermutlich dabei hilft, mit den spezifischen Belastungen umzugehen. Soziale Unterstützung hat nicht nur positive Auswirkungen auf das Wohlbefinden und die psychische Gesundheit (Cohen/Wills 1985), sondern kann Belastungen puffern (Perrez et al. 2005).

Die Auswirkungen der Minderheitssituation manifestieren sich in der erschwerten Integration im Lehrbetrieb. So schätzen Lernende in geschlechtsuntypischen Berufslehren ihr persönliches Arbeitsumfeld - insbesondere das Verhältnis zu Kolleginnen und Kollegen, die Einschätzung des Umgangs im Lehrbetrieb sowie die wahrgenommene Chancengleichheit zwischen Frauen und Männern - weniger positiv ein als Jugendliche in geschlechtstypischen Berufslehren. Die erhöhten Belastungen führten der Theorie folgend zu höheren körperlich-psychischen Beschwerden und zu häufigeren Fehlzeiten (vgl. De Col et al. 2012).

Einige Einschränkungen der Ergebnisse gilt es zu benennen. Erstens ist die Anzahl der Lernenden aus geschlechtsuntypischen Berufslehren gering. Zweitens ist der Mediationseffekt bei den Männern weniger deutlich als bei den Frauen. Männer scheinen auf soziale Unterstützung in Form von Interesse wichtiger Bezugspersonen an ihrer Ausbildung weniger stark zu reagieren als Frauen. Drittens ist bei den Auswertungen zu den psychosomatischen Beschwerden eine Konfundierung durch einen Geschlechtereffekt nicht gänzlich auszuschließen, da weibliche Jugendliche aufgrund der körperlichen Entwicklung stärker von psychosomatischen Beschwerden betroffen sind als männliche (vgl. Langness et al. 2003). Dieser Zusammenhang ist in den vorliegenden Auswertungen ebenso vorhanden: Frauen berichten über mehr psychosomatische Beschwerden als Männer $\left(\right.$ Frauen $_{\text {stabil viel }}=44.3 \%$ vs. Männer $_{\text {stabil viel }}=15.3 \%$ ). Deskriptive Unterschiede gibt es sowohl zwischen den typischen und untypischen Frauen als auch zwischen den typischen und untypischen Männern, jeweils zuungunsten der Untypischen. Da die Voraussetzung des $C h i^{2}$-Tests in den nach Geschlecht aufgeteilten Teilstichproben verletzt wird (mehr als 20 Prozent der Zellen mit Erwartungswert kleiner als 5), lässt diese Zusatzauswertung keine Signifikanzprüfung zu. Die Konfundierung durch Geschlechtsrollenverhalten dürfte jedoch aufgrund der kleinen Unterschiede zwischen den typischen und untypischen Frauen gering sein. Viertens werden durch das Median-Split-Verfahren viele Informationen zusammengefasst. Die Frage bleibt daher zu klären, ab welcher Ausprägung psychosomatische Beschwerden und Absenzen sich negativ auswirken und allenfalls zu einer Lehrvertragsauflösung führen. Eine letzte Einschränkung ergibt sich aus der eher kleinen Stichprobe und der Heterogenität des beruflichen Umfelds bezüglich der Geschlechtsrollenerwartungen gegenüber Frauen 
und Männern. Insbesondere bei Berufslehren mit einer starken geschlechtlichen Segregation auf der Ebene der Fachrichtungen (vgl. ,Kaufmann/-frau $E F Z^{\prime}$ und ,Detailhandelsfachmann/-frau EFZ') wäre die Erfassung der Fachrichtungen hilfreich. In weiterführenden Untersuchen müssten genügend und ähnlich große Stichprobengruppen spezifisch für die Fragestellung ausgewählt und einschlägige Konstrukte bestehend aus mehreren Items formuliert werden.

Wie die Jugendlichen in geschlechtsuntypischen Berufslehren die Situation erleben, welche Ressourcen ihnen zur Verfügung stehen und welche Belastungen sie bewältigen müssen, wird im folgenden Kapitel behandelt. Nach vier Kapiteln mit quantitativen Auswertungsmethoden wendet sich das letzte Ergebniskapitel qualitativen Aspekten zu. In Interviews mit Lernenden, die zum Befragungszeitpunkt in einer geschlechtsuntypischen Berufslehre waren, werden Berufsfindungsprozess, Ressourcen und Belastungen beleuchtet. 


\section{Interviewstudie: Belastungen und Ressourcen}

Die quantitativen Analysen in den vorangegangen Kapiteln belegen, dass Lernende in geschlechtsuntypischen Berufslehren stärker von vorzeitiger Lehrvertragsauflösung betroffen sind, in manchen Berufslehren häufiger durch die Lehrabschlussprüfung fallen und vermehrt von psychosomatischen Beschwerden und Absenzen berichten. Die im vorliegenden Kapitel präsentierte Interviewstudie fokussiert auf die spezifische Situation von Lernenden, die zur Zeit der Befragung eine geschlechtsuntypische Berufslehre absolvieren. Anhand der Auswertung der berichteten Belastungen infolge von Diskriminierungen (vgl. Abschnitt 4.2.4) und vorhandenen Ressourcen (vgl. Abschnitt 4.2.5) lege ich dar, wie es diesen Jugendlichen gelingt, die geschlechtsuntypische Situation zu meistern. Die Forschungsfrage lautet:

Berichten Lernende in geschlechtsuntypischen Berufslehren über spezifische Schwierigkeiten und Herausforderung während der Berufslehre?

In diesem Kapitel werden die Ergebnisse der Interviews mit 14 Lernenden in geschlechtsuntypischen Berufslehren in der Stadt Zürich und in ihrer weiteren Umgebung vorgestellt. Das Kapitel beginnt mit der Fragestellung (Unterkapitel 9.1), legt anschließend die verwendeten Methoden dar (Unterkapitel 9.2), stellt die Ergebnisse vor (Unterkapitel 9.3) und schließt mit der Diskussion zu den Ergebnissen (Unterkapitel 9.4).

\subsection{Fragestellung der Analyse}

Im Modell der geschlechtsuntypischen Berufssozialisation (vgl. Unterkapitel 4.2) wird postuliert, dass Jugendliche aufgrund der geschlechtsuntypischen Berufsfindung ablehnende Reaktionen im persönlichen und beruflichen Umfeld provozieren und lernen müssen, mit der darauf folgenden Reaktion des sozialen Umfelds umzugehen. Diese Herausforderung führt zu einer erschwerten beruflichen Integration bzw. Sozialisation in den Beruf (vgl. Unterkapitel 1.2). In ungünstigen Fällen, wenn sich keine Normalisierung oder Gewöhnung einfindet, können Lehrvertragsauflösungen, schlechte Leistungen (Misserfolg bei der Lehrabschlussprüfung) und/oder psychosomatische Beschwerden die Folge sein. Die Interviewstudie hat zum Ziel, die Situation von Lernenden in geschlechtsuntypischen Berufslehren besser zu verstehen. Im Interview mit den Jugendlichen werden ihre Erfahrungen rekonstruiert. Die Jugendlichen werden gebeten, auf die Erlebnisse im familiären und schulischen Umfeld während des Berufsfindungsprozesses zurückzublicken, die gegenwärtige Situation im beruflichen Alltag wiederzugeben und ihre Ge- 
danken zu den Zukunftsaussichten zu äußern. Dabei sollen Antworten auf vier Fragen gefunden werden:

Frage A Wie verlief der Berufsfindungsprozess im Hinblick auf die geschlechtsuntypische Situation?

Frage B Von welchen Belastungen in Zusammenhang mit der geschlechtsuntypischen Situation berichten die Lernenden?

Frage C Welche Ressourcen erleichtern ihnen das Bestehen in der geschlechtsuntypischen Berufslehre?

Frage D Wie stellen Lernende in geschlechtsuntypischen Berufslehren ihr Geschlecht dar?

\subsection{Forschungsvorgehen und Methode}

Die Forschungsfragen wurden mittels eines leitfadengestützten Interviews und der Inhaltsanalyse nach Mayring (2003) bearbeitet. Im Folgenden wird das methodische Vorgehen beschrieben, indem der Interviewleitfaden vorgestellt (Abschnitt 9.2.1), die Interview-Teilnehmenden beschrieben (Abschnitt 9.2.2) und die Auswertungsstrategie (Abschnitt 9.2.3) mit dem Kategoriensystem (Abschnitt 9.2.4) erläutert werden.

\subsubsection{Interviewleitfaden}

Die Konzeption des Leitfadens für die halbstrukturierten Interviews mit offenen Fragen erfolgte theoriegeleitet im Sinn einer Ableitung von Schwerpunkten aus dem Modell der geschlechtsuntypischen Berufssozialisation. Der Interviewleitfaden (vgl. Online-Anhang XII) wurde mit zwei Lernenden getestet und minimal überarbeitet. Als Einstieg zum Interview wurden einige Informationen zum familiären Hintergrund der Person, wie Beruf der Eltern und Ausbildungssituation der Geschwister, schriftlich erfragt. Der Interviewleitfaden umfasste folgende Themenbereiche: 1) Der erste Teil diente dem Vertrautwerden mit der Interviewsituation und sollte den Teilnehmenden ermöglichen, sich als Person mit unterschiedlichen Facetten darzustellen. Dabei beschrieben sie sich selbst, ihre familiäre Situation, ihre Berufslehre und ihre Freizeitbeschäftigungen. 2) Im zweiten Teil fokussierten die offenen Fragen auf den Bereich Beruf und Geschlecht. Dabei wurde speziell das Erleben der marginalisierten Konstellation mit den Reaktionen des sozialen Umfelds erfragt, sofern es nicht von den Teilnehmenden selbst angesprochen wurde. 3) Anhand zweier Zeitstrahlen auf je einem A3-Bogen wurde versucht, den Berufsfindungsprozess bzw. den Beginn der Berufsausbildung zu 
rekonstruieren, um Hinweise auf Reaktionen des sozialen Umfelds zu erhalten. Dabei standen unterstützende Personen und andere Ressourcen im Zentrum. Negative Reaktionen wie Abwertungen und Absprechen von Kompetenzen bzw. Weiblichkeit/Männlichkeit waren ebenso von Interesse. 4) Zum Schluss gab es offene Fragen zur aktuellen Situation und zu Zukunftsplänen sowie zur sexuellen Orientierung. Da Homosexuelle häufiger geschlechtsuntypische Berufe ausüben als Heterosexuelle (Chung/ Harmon 1994), könnte die sexuelle Orientierung gerade bei Diskriminierungsfragen für die Interpretation relevant sein. Insbesondere ein Coming-out, das oft im Alter der Teilnehmenden stattfindet (Ross 1989) und zu erheblichen Verunsicherungen führen kann (FRA 2013, Meyer et al. 2015), wäre eine zusätzliche Herausforderung, die die geschlechtsuntypische Situation überlagern könnte. Alle Interviewten bezeichnen sich selbst als heterosexuell.

\subsubsection{Teilnehmende}

Aufgrund der kleinen Zahl an geschlechtsuntypischen Lernenden entwickelte sich die Suche nach freiwilligen Interviewteilnehmenden in Wellen. Die Schwierigkeiten bei der Suche nach Teilnehmenden zeigten sich darin, dass Vermittlungspersonen zurückhaltend mit der Weitergabe von Informationen wie Namen und Kontaktdaten waren. Beispielsweise lehnten die meisten angefragten Berufsfachschulen aus Datenschutzgründen die Unterstützung bei der Anfrage ab. Einzige Ausnahme war die Zürcher Berufsfachschule der Automobil-Mechatroniker/-innen. Spontan haben sich vier Frauen auf meine briefliche Anfrage gemeldet. Unterstützt wurde ich ebenfalls von der Verwaltung der Stadt Zürich, die mir die Kontakte zu den entsprechenden Ausbildungsverantwortlichen mit Lernenden in geschlechtsuntypischen Berufslehren vermittelte. Bei direkten Anfragen, etwa in Blumenläden oder in Coiffeursalons, haben oft die Ausbildenden nicht eingewilligt oder dann die Lernenden selbst die Teilnahme am Interview abgelehnt.

Insgesamt konnte ich mit 14 Personen Interviews durchführen. Entgegen des Plans, aus jedem Beruf nur eine Vertreterin bzw. einen Vertreter zu interviewen, habe ich anfangs aufgrund der schwierigen Rekrutierungslage Interviews auch mit mehreren Personen des gleichen Berufs geführt. Die Ziffer in Klammer ist die Anzahl der interviewten Personen, wenn sie von 1 abweicht. Folgende Berufe werden von den Frauen gelernt: Automobil-Fachfrau, Automobil-Mechatronikerinnen (3), Informatikerinnen (2), Landwirtin, Logistikerin und Spenglerin. Die Männer absolvieren folgende Berufslehren: Coiffeur, Dentalassistent, Fachmann Betreuung, Fachmann Gesundheit und Fachmann Hauswirtschaft.

Die 9 Frauen und 5 Männer waren zum Zeitpunkt des Interviews zwischen 17 und 30 Jahre alt. Von den 14 Personen waren 9 im ersten, 2 im 
zweiten, 1 im dritten und 2 im vierten Lehrjahr. 12 Lernende haben die Schweizer Nationalität, davon waren 2 Doppelbürger/-innen. Die beiden ausländischen Interviewten gingen nicht in der Schweiz zur Schule und wuchsen in der Dominkanischen Republik bzw. in Deutschland auf. Bis auf eine Person sprechen alle fließend Deutsch und reden mehrheitlich zu Hause Deutsch. Von lediglich 2 Frauen und 1 Mann gehen die Mütter einem geschlechtsuntypischen Beruf nach (z.B. Bus- und LKW-Fahrerin). Die Väter der Teilnehmenden sind alle in geschlechtstypischem Berufsumfeld tätig. Alle Interviewten haben Geschwister. Die Schwester einer Interviewten lernt Polymechanikerin, alle andern sind in geschlechtstypischen Berufen tätig oder gehen noch zur Schule.

\subsubsection{Datenaufbereitung und-auswertung}

Die Interviews dauerten 40-50 Minuten und haben zwischen Juli 2012 und Juli 2014 an ruhigen Orten (im Lehrbetrieb, in der Berufsfachschule, in Restaurants/Cafés oder bei den Teilnehmenden zu Hause) stattgefunden. Sie wurden digital aufgenommen, transkribiert und gemäß der Inhaltsanalyse nach Mayring (2003) ausgewertet.

Die digitalen Audio-Aufnahmen wurden im Programm F4 Version 4.2 vollständig transkribiert. Während die schweizerdeutsche Mundart in Schriftdeutsch erfasst wurde, blieben die Satzstrukturen dialektnah. Einige Wörter aus der Umgangssprache wurden übernommen, wenn das Originalwort im Kontext relevant war. Es wurde nur die gesprochene Interaktion transkribiert. Nonverbales oder Paraverbales wie Gestik, Mimik, Lachen, Räuspern, Stocken etc. wurde nicht festgehalten. In einem zweiten Arbeitsschritt wurden in Anlehnung an Gropengiesser (2005) Füllwörter, die den Inhalt nicht verändern (z.B. „also“, ,ja“) gestrichen, grammatikalisch und schriftsprachlich geglättet sowie Satzbaufehler behoben, um ein flüssiges Lesen zu gewährleisten.

Die Interviews wurden mit der qualitativen Inhaltsanalyse nach an Mayring (2003) ausgewertet. Charakteristisch für diese Methode ist die systematische Analyse von Texten. Am Material wird schrittweise und theoriegeleitet ein Kategoriensystem entwickelt. Nach Mayring (2003) sind die Grundformen der Auswertung Zusammenfassung, Kategorienbildung, Explikation und Strukturierung. In der vorliegenden Analyse kommt vor allem die ,inhaltliche Strukturierung“ (vgl. Mayring 2003: 89) zur Anwendung. Damit wird das Ziel verfolgt, die vier Themenfelder ,Berufsfindung', ,Belastungen', ,Ressourcen' und ,Doing Gender' aus dem Material herauszufiltern und zusammenzufassen. 


\subsubsection{Kategoriensystem}

Das Grundgerüst des Kategoriensystems entstand deduktiv, aufbauend auf dem Modell der geschlechtsuntypischen Berufssozialisation. Aufgrund von theoretischen Überlegungen bildeten sich vier Themenfelder heraus: Berufsfindung, Belastungen, Ressourcen und Doing Gender. Das Themenfeld ,Berufsfindung' beschreibt den Weg, wie Lernende zu ihrer aktuellen Berufslehre gefunden haben, die erfahrene Unterstützung, die überwundenen Hindernisse und die Auseinandersetzung zwischen ihren Wünschen und den bestehenden arbeitsweltlichen Angeboten. Aus der Theorie ist bekannt, dass Kinder und Jugendliche geschlechtliche Passung als Selektionskriterium früh in die Berufsauswahl miteinbeziehen (vgl. Faulstich-Wieland 2016a, Gottfredson 2005, Hartung et al. 2005, Jungo 2009). In ihrer Studie zu Männern in geschlechtsuntypischen Berufslehren fand Simpson (2005) drei Arten, wie diese zu ihrer aktuellen beruflichen Tätigkeit kamen: 1) Die ,Sucher' haben aktiv einen geschlechtsuntypischen Beruf gewählt, 2) die ,Finder' sind eher zufällig in einen untypischen Beruf geraten, und 3) die ,Siedler' fanden nach negativen Erfahrungen in einem geschlechtstypischen Umfeld zum geschlechtsuntypischen Beruf. Inwieweit diese Muster genauso bei männlichen und weiblichen Jugendlichen, die am Anfang ihrer beruflichen Laufbahn stehen, gefunden werden können, bleibt ebenfalls zu klären.

Gemäß dem Minderheiten-Stress-Modell nach Meyer et al. (2015) erleben Personen in benachteiligten sozialen Positionen alltägliche Diskriminierung und Mikroaggressionen. Diese Erfahrungen erhöhen das Stressniveau und führen zu gesundheitlichen Beeinträchtigungen. Das Themenfeld ,Belastungen' soll spezifisch für Lernende in geschlechtsuntypischen Berufslehren die Herausforderungen aufdecken, denen diese Jugendlichen ausgesetzt sind und die sie bewältigen müssen. Die negativen Reaktionen des sozialen Umfelds müssen von den Lernenden in geschlechtsuntypischen Berufslehren bewältigt werden.

Als Gegenstück zu den Belastungen helfen Schutzfaktoren im Themenfeld ,Ressourcen', den erlebten Stress zu minieren. Ressourcen tragen zu einer positiven Wahrnehmung des eigenen Lebens bei (Ray/Keeney 1993) und unterstützen eine Person bei der Umsetzung ihrer Ziele (Alpers 2005). Imdorf (2012a) konnte folgende Eigenschaften als Ressourcen bei weiblichen Lernenden in Autogaragen benennen: Durchsetzungsvermögen, psychische Stabilität, Leistungsfähigkeit und die Unterstützungsbereitschaft der Eltern. Bei der sozialen Unterstützung spielt vor allem die eigene Wahrnehmung von emotionaler Anteilnahme und tatkräftiger Hilfe eine wichtige Rolle (Sarason et al. 1987) und führt dazu, dass Stress weniger stark erlebt wird und weniger Stresshormone gemessen werden können (Heinrichs et al. 2003). Die Ressourcen helfen Lernenden in geschlechtsuntypischen Berufslehren bei der Bewältigung von negativen Reaktionen des sozialen Umfelds. Dies ermög- 
licht ihnen, die Berufslehre fortzusetzen, und macht sie resistent gegenüber Lehrvertragsauflösungen.

Das Themenfeld ,Doing Gender' zeichnet sich insbesondere dadurch aus, dass Geschlecht nicht einfach gegeben ist, sondern von allen Beteiligten stetig hergestellt werden muss (Bischof-Köhler 2004, Gildemeister 2010). Nach Wetterer (2002) wird die Geschlechtszugehörigkeit in einem reflexiven Interaktionsprozess sowohl selbst produziert als auch von anderen attestiert. Gerade weil Lernende in geschlechtsuntypischen Berufslehren ihren Beruf nicht zur Untermauerung ihres Geschlechts heranziehen können, können ihre Äußerungen zum Prozess des Doing Gender aufschlussreich sein. Dem Modell der geschlechtsuntypischen Berufssozialisation liegt die Annahme zugrunde, dass sich die Geschlechtsrollenerwartungen und die berufliche Segregation nach Geschlecht gegenseitig stützen. Diesem Kreislauf können sich auch Lernende in geschlechtsuntypischen Berufslehren nicht entziehen. Einen entscheidenden Bereich dieses Kreislaufs bildet die Geschlechtsperformanz.

Tabelle 9.1: Übersicht Kategoriensystem

\begin{tabular}{|c|c|c|}
\hline Themenfelder & \multicolumn{2}{|c|}{ Kategorien } \\
\hline Berufsfindung & $\begin{array}{l}\text { a) } \\
\text { b) } \\
\text { c) }\end{array}$ & $\begin{array}{l}\text { Wunschberuf } \\
\text { Zwischenstation } \\
\text { Zufall/Notlösung }\end{array}$ \\
\hline Belastungen & $\begin{array}{l}\text { b) } \\
\text { c) } \\
\text { d) } \\
\text { e) }\end{array}$ & $\begin{array}{l}\text { Diskriminierung } \\
\text { Unterkategorien: Abwertung; Frauenfeindlichkeit; seltsames Gefühl; Kompetenz abspre- } \\
\text { chen; Körperkontakt; Sexualisierung; soziale Ausgrenzung; dumme Sprüche; technische } \\
\text { Gründe; Verwunderung; Vorurteil } \\
\text { Lehrvertragsauflösungen von Gleichaltrigen } \\
\text { Reaktionen von Gleichaltrigen } \\
\text { Reaktionen des Berufsumfelds } \\
\text { belastende Berufsinhalte }\end{array}$ \\
\hline Ressourcen & $\begin{array}{l}\text { b) } \\
\text { c) } \\
\text { d) } \\
\text { e) } \\
\text { f) } \\
\text { g) } \\
\text { h) } \\
\text { i) } \\
\text { j) }\end{array}$ & $\begin{array}{l}\text { Persönlichkeit bzw. Person } \\
\text { Unterkategorien: Gefallen an der eigenen Exklusivität; gute Leistungen; Herausforderung; } \\
\text { Humor; Nische suchen; Orientierung am anderen Geschlecht; Offenheit; Schlagfertigkeit; } \\
\text { Selbstvertrauen; Selbstverwirklichung } \\
\text { Interesse am Beruf und an Berufsinhalten } \\
\text { familiäre Unterstützung } \\
\text { Unterstützung durch Gleichaltrige } \\
\text { Ausbildende und Vorgesetzte } \\
\text { Personen im beruflichen Umfeld } \\
\text { Personen während der Sekundarstufe I } \\
\text { Kundinnen und Kunden } \\
\text { gute Erfahrungen } \\
\text { Vorteile des Geschlechts }\end{array}$ \\
\hline Doing Gender & & $\begin{array}{l}\text { Geschlecht produzieren } \\
\text { Klischees bestätigen }\end{array}$ \\
\hline
\end{tabular}

Legende: Das Kategoriensystem mit Definitionen und Ankerbeispielen ist im Online-Anhang XIII zu finden. Quelle: Eigene Darstellung.

Die Themenfelder erfuhren eine induktive Verfeinerung anhand der Datenlage des Interviewmaterials. Das gesamt Kategoriensystem mit Definitionen der Kategorien und Ankerbeispielen befindet sich im Online-Anhang XIII. Eine Übersicht über die Kategorien der vier Themenfelder wird in der Tabel- 
le 9.1 vorgestellt. Aussagen der Interviewten wurden den vier Themenbereichen zugeordnet. Anschließend wurden die Aussagen systematisiert und eine Überschrift gesucht. In zwei Fällen wurden die Kategorien in weitere Unterkategorien aufgeteilt: bei den ,Belastungen“ in der Kategorie ,Diskriminierung' sowie bei den ,Ressourcen' in der Kategorie ,Persönlichkeit'. Die Erweiterung in Unterkategorien bot sich einerseits aufgrund der Menge an Aussagen an, die im Datenmaterial vorlagen, andererseits geben diese inhaltlich einen differenzierten Einblick in die Herausforderungen, die Lernende in geschlechtsuntypischen Berufslehren zu meistern haben, und wie ihnen dabei selbstwertdienliche Persönlichkeitseigenschaften helfen.

\subsection{Ergebnisse}

Die Darstellung und Interpretation der Ergebnisse gliedert sich entlang der vier deduktiv entwickelten Themenfelder ,Berufsfindung' (Abschnitt 9.3.1), ,Belastungen“ (Abschnitt 9.3.2), ,Ressourcen“ (Abschnitt 9.3.3) und ,Doing Gender" (Abschnitt 9.3.4).

\subsubsection{Berufsfindung}

Der Berufsfindungsprozess (vgl. Unterkapitel 1.3) beinhaltet eine längere Vorbereitungszeit, wird von mehreren Akteurinnen und Akteuren beeinflusst und findet mit der Entscheidung für den Ausbildungsberuf seinen zwischenzeitlichen Höhepunkt. Bei den Jugendlichen, die in der vorliegenden Arbeit portraitiert werden, steht die Entscheidung für eine Ausbildung in der Berufsbildung im Fokus. Dabei fanden sich rückblickend und verallgemeinernd drei Kategorien: Wunschberuf, Zwischenstation und Notlösung/Zufall.

\subsubsection{Wunschberuf}

Dieser Kategorie wurden Aussagen zugeordnet, die einen klaren, starken Berufswunsch ausdrückten, insbesondere dann, wenn die Jugendlichen diesen gegen etwaige Widerstände durchsetzen konnten. Die Lernenden schildern, dass sie bereits während der Kindheit Tätigkeiten aus dem Bereich ihres Traumberufs verrichteten. Zwei Interviewausschnitte belegen dies:

Weil ich schon seit klein Coiffeur werden möchte. Ich ging immer mit der Mutter und der Großmutter zum Coiffeur. Ich föhnte auch immer ihre Haare, bis sie nicht mehr hinhalten wollten. Dann konzentrierte ich mich auf die Schule. Ich machte ab und zu meiner Kollegin ein Zöpfli. (Person N; Abschnitt 23) 
Weil ich sehr viel Freude hatte, mit den Kindern zu arbeiten. Schon sehr früh, durch meine Schwestern, hatte ich mit Kindern zu tun. Ich hütete gern. Ich war auf einer Privatschule mit einem Kindergarten. Dort hatte ich viel Kontakt mit den kleineren Kindern, und sie [die Kindergärtnerinnen, Anmerkung BR] sagten, dass ich es sehr gut mache, dass sie [die Kinder, Anmerkung BR] Freude an mir hätten. $(\mathrm{K} ; 21)$

Während Person N gleich nach der obligatorischen Schule mit der Coiffeurlehre anfing, lernte Person K erst Maurer und entschied sich wegen körperlicher Probleme für eine zweite Berufslehre, die seine positiven Erlebnisse mit Kinderbetreuung berücksichtigte. In den beiden Aussagen wird deutlich, dass Bezüge zu berufsnahen Tätigkeiten bereits in der Kindheit gemacht wurden. Die damit verbundenen positiven Emotionen dienen dazu, die Berufsfindung zu begründen, einerseits vor sich selbst und andererseits vor anderen Personen. Diese Verbindung zu einer naiven, quasi unschuldigen Kindheitserinnerung könnte die Jugendlichen stärken, trotz anderslautender Geschlechtsrollenerwartungen, sich für einen geschlechtsuntypischen Beruf zu entscheiden. Denn ein Wunsch, der so tief in einem drinsteckt, bzw. so früh angelegt ist, stünde über den später dazugekommenen gesellschaftlichen Vorstellungen.

\subsubsection{Zwischenstation}

Die Jugendlichen, deren Aussagen zur Kategorie Zwischenstation zählen, verspüren ebenfalls einen starken Berufswunsch. Sie sehen die gegenwärtige berufliche Tätigkeit als Zwischenschritt auf dem Weg zum Zielberuf. Andere sehen in der aktuellen Ausbildungssituation einen Schritt zu einem größeren Ausbildungsziel. So wählte etwa Person $M$ die Berufslehre als Dentalassistent (mit Berufsmatur), um seinem Traum - Zahnarzt zu werden - näher zu kommen. Er zog diesen Ausbildungsweg mit mehr praktischen und berufsrelevanten Tätigkeiten dem Gymnasium vor. Mit beiden Ausbildungswegen gelangen Absolventen zur Matura, die eine Voraussetzung für die Aufnahme eines Medizinstudiums ist. Entsprechend antwortet er auf die Frage, warum er die Berufslehre als Dentalassistent absolviert:

Weil ich später Zahnarzt studieren möchte. (M; 21)

Person $\mathrm{F}$ dagegen hat sich nicht für eine so tätigkeitsnahe Ausbildung entschieden, denn für die Zulassung zur ihrem Wunschberuf - Polizistin - wird eine abgeschlossene Berufslehre verlangt. Für sie erscheint die Berufslehre als Logistikerin eher als gangbare Zwischenstation mit mäßiger Begeisterung für den Beruf an sich.

Ach, ich werde nicht mehr auf diesem Beruf arbeiten. Nicht, weil es mir nicht gefällt. Ich werde sicher zur Polizei gehen. Und je nach dem gehe ich in die RS, ins Militär. (F; 9)

Die Aussagen der beiden Interviewten machen deutlich, dass die geschlechtsuntypische Situation in Kauf genommen wurde, um einen entscheidenden Schritt näher an den Wunschberuf zu kommen. Bemerkenswert ist 
vor allem die Entscheidung von Person M, sich in ein Feld zu wagen, das beinahe ausschließlich von Frauen gelernt wird (Frauenanteil: 99.3\%, vgl. Online-Anhang I). Die Möglichkeit, bereits jetzt als Dentalassistent nahe am Zielberuf zu sein, war ausschlaggebend. Aufschlussreich ist die Bemerkung von Person M nach dem Interview, als das Aufnahmegerät nicht mehr lief, dass in seinem Umfeld Dentalassistent/-in nicht als typischer Frauenberuf wahrgenommen werde.

\subsubsection{Zufall/Notlösung}

Jugendliche der dritten Kategorie ,Zufall/Notlösung ' wissen nicht, was sie in ihrem Leben werden wollen. Sie lassen sich stark von äußeren Umständen leiten. Die Entscheidung für einen Beruf bzw. die Berufslehre kommt von außen, meist von den Eltern und nicht von ihnen selbst. Dabei scheint der Zufall eine gewisse Rolle zu spielen. Beispielsweise wird ein Lehrbetrieb gewählt, weil er sich in unmittelbarer Nähe zum Elternhaus befindet und gerade eine Lehrstelle offen hat. Die Empfehlung der Sekundarschullehrperson oder die Abwendung einer unerwünschten Anschlusslösung (Gymnasium, Sprachaufenthalt etc.) können ebenfalls zu einem Berufsentscheid führen, der wenig mit den intrinsischen Bedürfnissen der Jugendlichen zu tun hat. Die Rolle des Zufalls wird ausgeprägter, wenn Jugendliche entweder lange nicht wissen, was sie lernen wollen, oder sich für einen pragmatischen Weg entscheiden. Dies wird von ihnen manchmal als Notlösung beschrieben:

$J a$, das war eine Notlösung. Das war der nächste Betrieb von meinem damaligen Zuhause. (I; 19)

Ich ging einfach mal als Spenglerin schnuppern. Es gab da auch eine Frau. Ich dachte aber noch, das ist nichts für mich. Eine Woche später bot mir mein jetziger Lehrmeister eine Lehrstelle an. (E; 13)

Neben der nahegelegenen Lösung zeigt sich, vor allem bei bedeutsamen schulischen und/oder sprachlichen Defiziten, die Schwierigkeit eine Berufslehre zu finden, die dem gegenwärtigen Leistungsstand und den Interessen der Person entspricht. Person L spricht offen aus, dass sie lieber andere Berufe erlernt hätte und wie es sie Überwindung kostete, ihre Ansprüche ihren Möglichkeiten anzupassen.

$J a$, ich hätte gerne Automech gemacht. Auch als Koch schnupperte ich, es hatte mir sehr gut gefallen. Sie sagten, ich arbeite gut, nur mir fehlten viele Begriffe für die Küche. Es war einfach zu wenig. Diesen Beruf hätte ich gerne gemacht. Ich konnte mich damals nicht so unterhalten, wie jetzt mit Ihnen. Sie müssen sich vorstellen, ich war nur vier Jahre zur Schule gegangen, es war nicht so einfach. Deutsch war vor allem sehr schwierig. 2008 entschied ich mich, eine zweijährige Lehre als Hauswirtschaftspraktiker zu machen. Das war wichtig für mich. Ich konnte so langsam stapeln. Berufserfahrungen sammeln, damit ich mich weiterbilden konnte, so wie jetzt. (L; 7) 
Mittlerweile hat Person L nach bestandener Attestlehre, Hauswirtschaftspraktiker EBA' und mehrjähriger Berufserfahrung die Berufslehre zum ,Fachmann Hauswirtschaft EFZ' begonnen.

Manchmal muten Berufsentscheidungen eher wie eine Flucht vor einer nicht gewollten Tätigkeit an:

Am Anfang wusste ich gar nicht, was ich wollte. Bin durch meine Eltern zum Psycholo-
gischen gekommen. Wollte auch Psychologie studieren. Dann rutschte ich in die Astro-
logie ab. Ich wusste nie richtig, was, und machte mir auch keine Gedanken darüber. In
der Sekundarschule umging ich das Thema, ich sagte immer: nicht KV [Kaufmann/-
frau, Anmerkung BR]. (G; 15)

Person G macht eine anspruchsvolle Berufslehre zur Informatikerin Fachrichtung Systemtechnik. Sie wollte weder die Berufslehre zur Kauffrau machen noch mit Frauen zusammenarbeiten. Als sie die Berufslehre begann, war sie die einzige Frau. Mittlerweile hat sie eine jüngere Mitlernende, bekommen, mit der sie sich gut versteht.

\subsubsection{Fazit}

Während die Jugendlichen, deren Aussagen den Kategorien ,Wunschberuf $f^{\circ}$ oder ,Zwischenstation“ zugeordnet wurden, ihre Begründung für die Berufsfindung knapp und klar darstellen können, brauchen Jugendliche der Kategorie ,Zufall/Notlösung' länger, um ihren Berufsentscheid zu erklären, so als schiene die Begründung für den Beruf ungünstig auf sie zurückzufallen. Bei Ersteren hingegen erweist sich die Berufsfindung als Bestärkung ihrer Person. Sie wollen diesen Beruf lernen und können ihr Ziel in die Tat umsetzen. In diesem Fall kann die Berufsfindung zusätzlich als Ressource betrachtet werden. Bevor nun die Ressourcen betrachtet werden, geht es im folgenden Abschnitt um die Belastungen.

\subsubsection{Belastungen}

Gemäß dem Modell der geschlechtsuntypischen Berufssozialisation sind Jugendliche in geschlechtsuntypischen Berufslehren in einer herausfordernden Situation, weil sie aufgrund ihres Geschlechts salient sind und von der Gesellschaft mit diesem Umstand immer wieder konfrontiert werden. Daraus entwickeln sich Belastungen, die unterschiedliche Dimensionen einnehmen können. In diesem Abschnitt beschreibe ich die Belastungen, die von Beobachtungen häufigerer Lehrvertragsauflösungen bei anderen Lernenden in geschlechtsuntypischen Berufslehren bis zu persönlichen Anfeindungen und Diskriminierungen gehen. Neben den beiden genannten können Gleichaltrige, das berufliche Umfeld und sogar die beruflichen Tätigkeiten zu einer Erschwerung führen. 


\subsubsection{Diskriminierung}

Fröhlich (2000: 128) versteht unter Diskriminierung die „Einstellungen oder Verhaltensweisen, die einen anderen Menschen oder eine Gruppe herabsetzen und in ein schlechtes Licht rücken“. Die Jugendliche erzählen, dass sie abwertende Bemerkungen gegen ihre Person erlebt haben. Die Aussagen der Jugendlichen zu diskriminierenden Situationen wurden thematisch sortiert. Dabei werden sowohl direkte wie indirekte Handlungen von anderen Personen genannt. Als indirekt sind etwa technischen Gründe zu verstehen. Die Aufzählungen belegen die spezifischen Diskriminierungen. Während weibliche Lernende mit allgemeiner Frauenfeindlichkeit und Sexualisierung konfrontiert werden und ihnen die beruflichen Kompetenzen abgesprochen werden, fühlen sich männliche Lernende unwohl, wenn sie sich in einem frauenreichen Umfeld bewegen und etwa Situationen mit Körperkontakt zu anderen Männern als schwierig erleben. Beide Geschlechter erleben soziale Ausgrenzung, werden zum Ziel von dummen Sprüchen oder lösen Verwunderung aus und werden mit Vorurteilen konfrontiert. Neben den sozialen sind es strukturelle oder technische Diskriminierungen, die den Jugendlichen zusätzlich zu schaffen machen. Die Aussagen der Jugendlichen zur erlebten Diskriminierung wurden induktiv in Unterkategorien zusammengefasst und folgen nun in alphabetischer Reihenfolge: Abwertung, Frauenfeindlichkeit, seltsames Gefühl, Kompetenz absprechen, Körperkontakt, Sexualisierung, soziale Ausgrenzung, dumme Sprüche, technische Gründe, Verwunderung und Vorurteil.

\section{Abwertung}

Sie haben mich abgestempelt. Frau macht Männerjob, irgendwas stimmt mit ihr nicht. Ich verstand nicht, warum es die Jungs akzeptieren konnten und die Frauen ein Drama daraus machten und es mir unter die Nase rieben. Das tat mir sehr weh. $(\mathrm{B} ; 67)$

Nein, aber in meinem Kollegenkreis wurde das angesprochen. „Hey, was ist passiert, bist du jetzt schwul geworden, oder so? " Aber ich habe mit allen Männern hier zu tun und weiß von keinem, ob er schwul ist. Und auf mich trifft das auch nicht $z u$. $(\mathrm{K} ; 89)$

Dass einen die Kunden blöd ansprechen. Dass sie sagen: ,Was macht ein Mann in diesem Beruf? Das ist doch ein Frauenberuf." (M; 45)

Coiffeur sei ein Beruf für Frauen. Und jeder Mann, der das macht, ist schwul. (N; 39)

Es ist so, ich wohne schon lange nicht mehr bei meiner Mutter und ihrem Freund, seit 2009. Sie hatte noch Kontakt zu meinem Vater. Und alle machten mich fertig. Wegen des Berufs sagten sie: „Aus dir kann so eh nichts werden, und Coiffeur machen nur Schwule, und du bist nicht schwul. " (N; 59)

\section{Frauenfeindlichkeit}

Da sagte man mir immer: „Du bist eine Frau, du hast keine Ahnung. “ Das weckte in mir: "Ich bin eine Frau und ich krieg die Ahnung. " Es entwickelte sich Ärger in mir. (A; 19)

Es gibt Männer die sagen: ,,Frauen gehören nicht in die Werkstatt. “(A; 35) 
Ein Oberstift [älterer Mitlernender, Anmerkung BR] muss immer zeigen, dass ich eine Frau bin und es nicht kann. (B; 45)

Da sah ich die schlechte Seite einer Garage. Die Männer waren grässliche Rüpel. Frauen werden nicht akzeptiert. (B; 55)

Ich hatte einen sehr ekligen Lehrer. Ich dachte immer, er hätte uns drei Frauen nicht so gern. (D; 53)

Einer macht im Auto immer Frauensprüche, dass sie nicht Auto fahren könnten. (E; 19)

Man hört oft: „Frauen haben nichts auf dem Bau verloren. Sie gehören an den Herd. Frauen können nicht arbeiten. “ (E; 29)

Es gibt Sprüche in der Pause, weniger dass ich eine Frau bin, als über Frauen im Allgemeinen. $(\mathrm{F} ; 13)$

\section{Seltsames Gefühl}

Ich habe nur ein Problem, wenn ich nur Frauen sehe, dann fühle ich mich etwas komisch. Es macht mir nichts aus, wenn noch mehrere Männer da sind. Bei den üK [überbetriebliche Kurse; Anmerkung BR] sind etwa 100 Frauen, und ich muss Ihnen ehrlich sagen, ich bin der einzige Mann, und dort fühl ich mich komisch. Es ist ein komisches Gefühl. Dann frage ich mich, warum mache ich nicht KV, Automech oder Chauffeur. Ich habe immer so Gedanken, warum habe ich das nicht gemacht. Das ist ein Frauenberuf. $(\mathrm{L} ; 17)$

Es war etwas komisch, weil ich sehr früh dort war. Dann musste ich warten. Es ist ja ein Frauenschulhaus. Da schauten mich alle komisch an. (M; 101)

\section{Kompetenz absprechen}

Eine Kundin sagte, sie komme wieder, wenn der Chef hier sei. Das war einmal. Ich dachte, das ist ja egal, auch wenn sie ihr Auto später bringt, ich mache die Arbeit ja sowieso. $(\mathrm{D} ; 19)$

Ich fand es schade, dass ich es nicht machen konnte. Z.B. durfte ich erst nach einem halben Jahr auf Steildächer. Ich musste erst mit dem Chef reden. (E; 33)

$J a$, weiß nicht, sie beachten einen gar nicht. Sie denken, man sei in einem Jahr eh nicht mehr in der Ausbildung als Informatikerin, als Frau. (G; 73)

Ich hatte die ersten drei Jahre immer Probleme, das Informatikzeug zu lernen. Weil man es mir auch nicht zutraute. Und darum bin ich jetzt auch nicht wirklich gut darin. Ich weiß aber nicht, ob man es mir nicht zutraute, weil ich eine Frau bin, oder wegen mir als Person. (G; 93)

Wir hatten einen Lehrer in diesem ersten Semester. Ich hatte das Gefühl, er sei zu den Frauen ein bisschen anders als zu den Männern. Für mich war es immer ein Anliegen, die gleichen Aufgaben zu bekommen wie die Jungs. Dass er uns die gleichen Erklärungen gibt und an uns die gleichen Erwartungen stellt. Für mich macht es keinen Unterschied, ob ich eine Frau oder ein Mann bin in diesem Beruf. Ich möchte eine gleichwertige Ausbildung haben wie meine Kollegen. (H; 93)

$J a$, die einen Reaktionen waren sicher: Eine Frau in diesem Beruf, das ist zu streng, auch von den Arbeitszeiten und den Belastungen. $(\mathrm{J} ; 13)$ 


\section{Körperkontakt}

Es gibt Ausnahmen, wo Frauen sich nur von Frauen pflegen lassen, weil sie es nicht anders kennen und kennen wollen. Aber das ist völlig akzeptabel für mich. Jedem das Seine. Ich würde auch akzeptieren, wenn ein Mann sich nur von einem Mann pflegen lassen möchte. Wie es in Teilen von Kulturen vorkommt. Ich muss aber feststellen, dass es eher selten der Fall ist. Erstaunlicherweise. In den vier, fünf Jahren, die ich hier bin, kam es erstaunlich wenig vor, dass Bewohnende fanden: „Nein, einen Mann möchte ich nicht." (I; 53)

Es gibt nur eine Situation, wo man es so richtig merkt. Im Geschäft, wenn ich einem Herrn die Haare waschen sollte. Die Kopfmassage wird oft abgelehnt. (N; 17)

\section{Sexualisierung}

Die Arbeiter auf der Baustelle merken es schnell. Die meisten schauen nur. Als ich einmal alleine auf einer Baustelle war, schauten die anderen Arbeiter in der Pause und begannen mit mir zu reden. Aber wenn ich mit Kollegen bin, dann schauen sie nur blöd. Sprüche höre ich nicht viel. Nur wenn man alleine unterwegs ist. Ich schüttle dann den Kopf, es ist mir aber nicht unangenehm. (E; 15)

Einmal trug ich Parfüm, da sagten sie was von wegen griechischer Sexgöttin. (F; 15)

Sie lassen mich in Ruhe. Es kommen schon amigs chli Sprüch und so. Aber nicht so sexistische Sachen. Eher so, willst du mit mir gehen und so Sachen, ja, es wird schon auf Sex bezogen. Es sind halt alle in meinem Alter, oder, dann haben sie halt andere Sachen im Kopf. (F; 91)

\section{Soziale Ausgrenzung}

In unserer Schule hat es noch Augenoptikerinnen, schöne Frauen. Wir haben schöne Jungs in unserer Klasse, das kann ich sagen. Diese Frauen zieht es zu den Männern. Dann regen sie sich auf, weil ich da bin. Was wollen die mit ihr? Die ist Automech, und wir sind Optikerinnen. Dann keifen sie mich an, obwohl ich gar nichts gemacht habe. Ein Riesendurcheinander in der Schule. Die Jungs stehen zu mir und denken, sie gehört zu uns. Die andern regen sich auf, was soll die Frau in einem Männerjob. Das hat mich mächtig verwirt in der Schule. $(\mathrm{B} ; 65)$

Schon eher im Alltag, aber konkret wüsste ich nicht, ob es ein Problem ist, um soziale Kontakte zu knüpfen. Ich denke, es ist ein Problem, da ich nicht so Kontakte knüpfe. Männer untereinander finden sich eher als Mann und Frau. $(\mathrm{H} ; 129)$

\section{Dumme Sprüche}

Aber man muss sich schon oft doofe Sprüche anhören. (A; 69)

In der Berufsfachschule. Ich bin die einzige Frau und höre öfter Sprüche. Die Jungs meinen das zwar nicht bös. Einer sagte, ich sei selber schuld, dass ich jetzt hier die einzige sei. $(\mathrm{E} ; 27)$

Man muss lernen, mit den Sprüchen, Frauenwitzen und Neckereien umzugehen. (G; 53)

Es fallen schon mal Sprüche, vor allem bei der Berufskunde, auch für die Männer gilt das. Es fällt ab und zu mal ein Spruch, aber es ist nicht irgendwie ein großes Thema. (I; 79) 


\section{Technische Gründe}

Ich verstand an Anfang nicht, warum ich so viele Absagen bekam. Das war bei kleinen Garagen wegen der sanitären Anlagen. Es braucht eine extra Umkleide, WC und Dusche. Das muss gewährleistet sein, sonst darf eine Frau nicht im Betrieb anfangen. (A; 51)

Ich schrieb Bewerbungen und fand keine Stelle, weil ich wollte in Schaffhausen oder in der Nähe im Kanton Zürich. Entweder hatte es keine Stelle, oder es kam eine Reaktion wie: , Wir nehmen keine Frauen. “( $\mathrm{J} ; 49)$

\section{Verwunderung}

Wenn du mit jemandem sprichst und er fragt: „Was machst du? “ Ist die Reaktion: „Nein, wirklich, im Ernst was machst du? " Frauen finden es auch noch cool für sie selbst, aber sie kämen nie darauf. (B; 35)

Wir mussten schon in der zweiten Oberstufe einen Vortrag an einem Elternabend halten. Ich stellte dort schon Automobil-Mechatroniker vor. Die Eltern schauten mich so an: , Was, die? “ (B; 63)

Die [Klassenkameradinnen und -kameraden, Anm. BR] waren ziemlich verwundert. (C; 47)

Am Anfang war es ziemlich absurd, dass ich diesen Beruf mache, auch vom Umfeld her. Alle waren verwundert und sagten: ,Was, wirklich?" (E; 11)

Die einen sagten: ,,Was, das ist ein Männerberuf! Du lernst einen Männerberuf, ist das nicht mega streng? " $(\mathrm{J} ; 69)$

\section{Vorurteil}

Viele denken, dass weibliche Automech fett und hässlich sind. Wenn ich jemandem erzähle, was ich mache, wird mir oft nicht geglaubt. Alle haben eine männliche Frau im Kopf. Ich kenne einige, die dem nicht entsprechen. (C; 21)

Meine Mutter war erst gar nicht einverstanden. Mit all den Männern auf dem Bau! Ich war ja auch nicht so der Typ dazu. (E; 47)

Sie sagten, sie hätten viele Muslime und diese würden Frauen nicht wirklich respektieren. $(\mathrm{F} ; 63)$

Er [Mein Vater, Anmerkung BR] sagte, es sei ein Männerberuf und ich müsse unbedingt einen Frauenberuf machen, weil ich eine Frau sei. $(\mathrm{G} ; 31)$

Sie [Meine Ausbildnerin, Anmerkung BR] muss sich schon durchkämpfen, wenn es um ein Projekt geht, und sie möchte das Projekt weiterführen, und ein anderer Mann macht das auch, dann ist sicher der Mann bevorzugt. Also wenn es eine Wahl geben würde. So in dem Sinn, weil man sagt ja immer Frauen und Technik. Mit diesen Vorurteilen. (G; 67)

Ein Nachteil sind die Schlagzeilen in den Zeitungen. Ein Mann machte etwas mit den Kindern. Das kann schon das Vertrauen der Eltern mindern. Es gibt die, die sagen: „,Wir haben Männer, und wenn es euch nicht passt, dann müsst ihr wieder gehen. " Es gibt welche, die keine Männer nehmen genau deswegen. (K; 37)

Man hört schon: „Das kannst du nicht verstehen, du bist ein Mann. “ Aber es ist weniger bei beruflichen Themen der Fall. (N; 29) 
Das kenne ich zu wenig, da ich ja keine Frau bin. Aber ich denke, man wird von den Kunden mit mehr Respekt angeschaut. Weil es unbekannt ich, dass ein Mann nebenan ist, und dann schauen sie einen teilweise so schräg an. Und denken: ,,Uh, wer ist jetzt das?" $(\mathrm{M} ; 41)$

\subsubsection{Lehrvertragsauflösung von Gleichaltrigen}

Bei den weiblichen Jugendlichen löst jede Fünfte und bei männlichen gar jeder Vierte den Lehrvertrag vorzeitig auf. Wie in Kapitel 5 bewiesen werden konnte, ist die Lehrvertragsauflösung bei Jugendlichen in geschlechtsuntypischen Berufslehren häufiger. Entsprechend kommt dies in den Interviews zum Ausdruck. Die Lernenden erkennen dies aufgrund der Veränderungen der Zusammensetzung ihrer Berufsfachschulklassen. Sie freuen sich anfänglich, dass es in ihrer Klasse oder wenigstens in ihrem Jahrgang an der Schule noch jemanden wie sie gibt. Doch schon nach kurzer Zeit sind sie alleine. Einerseits könnte diese Erfahrung die Lernenden stärken, indem sie sich sagen, ich bin besser, und ich kann durchhalten. Andererseits zeigt ihnen die Lehrvertragsauflösung von Kolleginnen bzw. Kollegen, wie hart die Situation ist und dass es einen gangbaren Ausweg gibt. Nicht zwingend entsteht eine freundschaftliche Verbindung zwischen den geschlechtsuntypischen Lernenden. Der Konkurrenzdruck und die Vergleiche von außen wurden bereits von Kanter (1977) beschrieben.

Die fünf Frauen wurden mit Absicht in eine Klasse gesteckt. Ich bin inzwischen die einzige, die noch lebt. Die eine sahen wir nie, die andere ging nach zwei Wochen. Eine Kollegin musste inzwischen wegen der Schulter aufhören. Und die Vierte hörte jetzt auch auf. Sie war ständig krank, sie fängt nochmals von vorne an. (A; 43)

Es fing noch ein Mann mit mir an, aber es gab Probleme, und er hörte auf. (N; 73)

\subsubsection{Reaktionen von Gleichaltrigen}

Junge Frauen bekommen eher zu hören, dass Frauen im Allgemeinen keinen technischen Sachverstand hätten. Während ihnen also die Kompetenz abgesprochen wird, wird bei jungen Männern eher an ihrer Männlichkeit gezweifelt.

Bei den Männern ist es eher zweigeteilt. Es gibt Männer, die kommen gar nicht damit klar, wenn Frauen technisch begabt sind. Die versteht auf einmal, was im Sportmagazin steht. $(\mathrm{A} ; 77)$

Aber die Männer fanden es zum einen cool, anderen fanden, Frauen und Informatik, das ist ja eh Scheiße, die haben keine Ahnung. (G; 37)

Mich nervt einfach, dass ich mich immer damit auseinandersetzen muss, wenn mich jemand fragt. Ich kann nicht einfach sagen, ich bin Automech. Dann ist gleich klar. Ich sage, Fachmann Hauswirtschaft, dann fragen sie immer, was ist das? Dann muss ich erklären. $(\mathrm{L} ; 61)$ 
Im folgenden Zitat verdeutlicht sich exemplarisch der Umstand, wie sich nach einer vorübergehenden Zurückweisung durch die Gleichaltrigen doch wieder Akzeptanz einstellt und wie dieser Jugendliche mit dem Vorurteil ,Coiffeure sind homosexuell' umgeht. Wie weiter unten dargestellt wird, ist ein starkes Selbstvertrauen eine wichtige Ressource, um sich gegen Vorurteile behaupten zu können.

Alle wussten es. Andeutungen kamen schon: „Coiffeur ist doch eh ein schwuler Beruf.“ Aber ich reagierte überhaupt nicht darauf. Schlussendlich sagten alle, sie kämen zu mir zum Haare schneiden. $(\mathrm{N} ; 65)$

\subsubsection{Reaktionen des Berufsumfelds}

Die meiste Zeit der Berufslehre verbringen die Lernenden im Lehrbetrieb. Die vielfach kleineren Arbeitsgemeinschaften, mit denen die Lernenden zusammenarbeiten, bestehen aus Vorgesetzten, ausgelernten Mitarbeitenden und anderen Lernenden. Für diese kann es neu und ungewohnt sein, mit jemandem des anderen Geschlechts die beruflichen Tätigkeiten auszuführen. Dies löst Unsicherheiten aus. Erwartungen müssen mit der Realität abgeglichen werden. Dabei sind nicht negative Handlungen wie oben bei den Diskriminierungen ausschlaggebend, sondern der Umstand, dass es länger dauert, bis sich Normalität eingespielt hat. Die Jugendlichen beschreiben dies so:

Es war am Anfang nicht einfach mit den Jungs. Es war am Anfang etwas komisch. Sie distanzierten sich. $(\mathrm{A} ; 17)$

„Oh, eine Frau, was sucht die hier [in der Garage, Anmerkung BR]? “ (A; 35)

Am Anfang musste ich mich etwas mehr beweisen als meine beiden Mitstifte [Mitlernende, Anmerkung BR]. Ich habe zwei männliche Mitstifte. (A; 41)

Mit dem Beginn der Lehre gab es happige Zeiten. Es war alles mit dem Lehrbeginn gekoppelt. Man war sehr müde. Manchmal ging es einem schon im Kopf rum, wenn man nur noch unter Männern war. Nichts ist ok, wie du es machst. Man zweifelt an sich, man kann machen, was man will. Es gab eine lange Zeit, in der es mir nicht so gut ging. (B; 11)

Auch im Betrieb, ich bin die erste Spenglerin im Betrieb. Sie kannten es gar nicht so, mit Frauen zusammenzuarbeiten. (E; 11)

\subsubsection{Belastende Berufsinhalte}

Umstände, die die berufliche Tätigkeit mit sich bringt, können den Geschlechtsrollenerwartungen widersprechen und werden so umso mehr als schwierig empfunden. Diese werden als innere Konflikte mit den eigenen Erwartungen und Rollenbildern empfunden.

Mich nervt das schon manchmal ein bisschen. Wenn die Fingernägel so schwarz sind.

(A; 37) 
Was ich nicht gerne machte, ist die Reinigung, wenn ich die Toiletten machen musste. Ich sagte: „Nein, das möchte ich nicht machen, am besten breche ich gleich ab. “Ich biss auf die Zähne. Es war sehr unangenehm. (L; 57)

\subsubsection{Fazit}

Lernende in geschlechtsuntypischen Berufslehren berichten von Belastungen und Diskriminierungen, die sie bewältigen müssen, um in der Berufslehre bestehen zu können. Sie müssen im Lehrbetrieb, in der Berufsfachschule und bei ihren Gleichaltrigen aus der Schulzeit beweisen, dass sie durchaus in der Lage sind, die Tätigkeiten, die dieser Beruf erfordert, zu leisten. Dabei erleben sie die Geschlechtsrollenerwartungen und die damit verbundene Reduzierung ihrer Person auf das Geschlecht hautnah. Es kostet sie einiges an Anstrengung, gegen den Strom zu schwimmen. Sie reiben sich an den gesellschaftlichen Strukturen, die von den jeweiligen Repräsentanten aufrechterhalten werden. Diese Prozesse laufen oft unbewusst ab, was sich in der Verwunderung des Umfelds ausdrückt, wenn sie über ihren Berufswunsch erzählen. Die Personen im Umfeld der Lernenden erleben durch diese eine Verunsicherung. Sie werden herausgefordert, ihr Bild von den Geschlechtern anzupassen. Dieser Druck kann dazu führen, dass sie die Norm wiederherstellen wollen und gegen die Person vorgehen, die den Normbruch verursacht hat. An dieser Stelle gilt es nochmals zu betonen, dass nicht das gesamte Umfeld negativ oder gar diskriminierend auf die Jugendlichen einwirkt, aber dass es oft vorkommt, vor allem bei denjenigen, die sich noch in der geschlechtsuntypischen Berufslehre halten. Die hohe Lehrvertragsauflösungsquote gibt deutliche Hinweise darauf, dass diese Situation dazu führen kann, dass die Jugendlichen die geschlechtsuntypische Situation verlassen, um den Anfeindungen nicht länger ausgesetzt zu sein.

Der Umstand, dass es trotz der widrigen Umstände immer noch und eventuell immer mehr Personen gelingt, sich im geschlechtsuntypischen Berufsumfeld zu bewähren, kann damit zusammenhängen, dass sich die Geschlechtsrollenbilder aufweichen und damit eine Wiederherstellung der Norm von immer weniger Personen als notwendig erachtet wird. Auf der Seite der Lernenden in geschlechtsuntypischen Berufslehren gibt es also Ressourcen, die ihnen helfen, die oftmals unangenehmen Situationen auszuhalten und damit erst einen Prozess in Gang zu setzen. Im nächsten Abschnitt sind die Ressourcen dargestellt, über die die Lernenden in geschlechtsuntypischen Berufslehren berichten.

\subsubsection{Ressourcen}

Dieser Abschnitt stellt die Quellen der inneren Kraft bzw. der äußeren Unterstützung der Jugendlichen dar. Darum liegt der Fokus auf den psychischen, 
sozialen und physischen Ressourcen der Lernenden. Die psychischen Ressourcen bilden den Anfang und unterteilen sich in ,Persönlichkeit bzw. Person' und ,Interesse am Beruf und an Berufsinhalten'. Die sozialen Ressourcen sind vielschichtig und lassen die Vernetzung der Jugendlichen mit ihrem Umfeld erkennen. An erster Stelle stehen die primären Bezugspersonen, darauf folgen Personen, die später dazugekommen sind: ,familiäre Unterstützung', ,Unterstützung durch Gleichaltrige', ,Ausbildende und Vorgesetzte', ,Personen im beruflichen Umfeld', ,Personen während der Sekundarstufe I', ,Kundinnen und Kunden' sowie ,gute Erfahrungen‘. Den Abschluss bilden die physischen Ressourcen: ,Vorteile des Geschlechts'.

\subsubsection{Persönlichkeit bzw. Person}

Eine starke Persönlichkeit hilft den Jugendlichen, die geschlechtsuntypische Situation zu bewältigen. Folgende Unterkategorien stehen für eine starke Persönlichkeit: ein ausgeprägtes Selbstvertrauen, ein starker Wille, Durchsetzungsvermögen, eine gute Portion Nonchalance und Humor sowie die Fähigkeit, Kritik bezogen auf die Geschlechtszugehörigkeit zu überhören, nicht ernst zu nehmen oder nicht auf sich selbst zu beziehen.

Die Mehrheit der Interviewten sagen von sich selbst, sie seien extravertiert, selbstbewusst, schlagfertig und offen sowie, dass sie über eine gute Portion Humor verfügen, um mit Bemerkungen des Umfelds umzugehen oder dumme Sprüche zu parieren. Diese Jugendlichen sind eher kämpferisch, mögen die hervorgehobene Situation und die Bewunderung, die sie durch das Geschlecht erfahren. Sie versuchen ihre Träume real werden zu lassen (Selbstverwirklichung). Ihnen helfen gute Leistungen am Lernort und gute Noten in der Berufsfachschule. Einige Jugendliche betonen, dass sie eigenständig sein wollen, sich eine Nische suchten, weil sie introvertiert sind, mit ihren Geschlechtsgenossinnen und -genossen nicht zurechtkommen bzw. zu Personen des anderen Geschlechts einen einfacheren Zugang haben. So weichen sie ihren Geschlechtsgenossinnen bzw. -genossen aus, haben aber dennoch Respekt vor der untypischen Situation. Im Folgenden werden einige Beispiele in alphabetischer Reihenfolge zu den Unterkategorien gegeben:

\section{Gefallen an der eigenen Exklusivität}

Ich fand das cool, ich bin etwas Besonderes und ich mache das. (B; 37)

Gegen den Strom zu schwimmen, reizte mich. (C; 13)

Die [Arbeitskolleginnen, Anmerkung BR] hatten sehr Freude, als sie mich zum ersten Mal sahen. Ich war der erste Mann bei den Babys. Reaktion: Es sind eigentlich alle zufrieden. $(\mathrm{K} ; 77)$ 


\section{Gute Leistungen}

Eigentlich nach dem Qualifikationsgespräch. Da wird uns die üK-Note mitgeteilt. Mein Mitstift [Mitlernender, Anmerkung BR] hatte eine 4.5 und ich eine 5 [Notenskala Schweiz: 1 (tief) bis 6 (hoch); Anmerkung BR]. Er konnte es nicht für sich behalten. Ab dann war der Respekt irgendwie da. Ab da wusste man, die kann doch was. (A; 73)

\section{Herausforderung}

Wenn ich früher gelebt hätte, wo Röcke tragen Pflicht gewesen wäre, hätte ich gesagt: „Tschüss zämä. “(F; 25)

Nein, eher dass ich zeigen kann, Frauen können das auch. (J; 23)

Es waren außer mir nur Jungs. Bei mir war die Angst, ich möchte einfach mithalten können. $(\mathrm{H} ; 107)$

\section{Humor}

Ja, manchmal schüttle ich den Kopf oder lache darüber, was sie anstellen. Es ist eigentlich ein schönes Erlebnis. (E; 17)

Ich lache einfach mit. $(\mathrm{F} ; 13)$

Ja, je nachdem, meistens lache ich mit oder gebe retour auf die lustige Art. (G; 55)

Es ist lustig, es ist im Rahmen. Es findet überhaupt kein Mobbing statt. Das definitiv nicht. (I; 81)

Ich nehme es locker. $(\mathrm{M} ; 17)$

\section{Nische suchen}

Ich wurde seit dem Kindergarten oft gemobbt, meistens von Frauen, und KV ist für mich so ein Frauenberuf und ich wollte schon immer eher einen Männerberuf. Um den Zickereien und Machtkämpfen zu entkommen. (G; 17)

Von der Sekundarschule her, schon in der Primarschule hatte ich nur Probleme, ich war ein Außenseiter. Ich beschäftigte mich oft nur mit mir allein. Ich beschäftigte mich auch deshalb mit dem PC. $(\mathrm{H} ; 55)$

Ich war mir es nach einem Jahr gewohnt. Es kommt auch selten vor. Die Coiffeusen merken es selber, ich mache eher die Damen, dann fühlen sich beide wohler. (N; 41)

\section{Orientierung am anderen Geschlecht}

Ich verstehe mich einfach gut mit Männern. $(\mathrm{D} ; 11)$

Ich bin zufrieden und fühle mich wohl in der Männerwelt. (D; 17)

\section{Offenheit}

Ich bin offen, spontan, kommunikativ vor allem, habe nicht zwei linke Hände, kann schon mit Werkzeug umgehen, kreative Person. (A; 5)

Lustig. Nett, offen, spontan, ja. $(\mathrm{N} ; 15)$

Eigentlich bin ich eine ziemlich offene Person. Ich bin offen für ziemlich alles. Ich vertrete meine eigene Meinung. (B; 5) 


\section{Schlagfertigkeit}

In der Zwischenzeit werde ich akzeptiert und respektiert. Aber das kam auch daher, dass ich meine Meinung offen sage. Ich kam mit meiner großen Klappe schon weiter, weil ich auch schlagfertig bin. (A; 17)

Da sagte ich: ,Wisst ihr, wenn ihr jeweils zu viel habt, sage ich nichts. Aber bei mir sagt ihr etwas. Ist schon etwas komisch, oder? “ (F; 15)

Das gab es schon, dann wehrte ich mich mit dummen Sprüchen zurück und so. (M; 79)

\section{Selbstvertrauen}

Ich habe zwar keine BMS, aber ich gelte als Ausnahmetalent. (A; 15)

\section{Selbstverwirklichung}

Mir selber fiel das nicht groß auf, aber ich lache mehr, bin aufgestellter. Alle nahmen es sehr gut auf. $(\mathrm{K} ; 79)$

Jugendliche in geschlechtsuntypischen Berufslehren haben persönliche und soziale Ressourcen, die ihnen helfen, die saliente Situation selbstwertschützend zu meistern. Sie nehmen ihre Situation als etwas wahr, wogegen sie sich wappnen müssen. Sie treten in dem Bewusstsein in ihre Berufslehre ein, bezogen aufs Geschlecht in der Minderheit zu sein und sich darin behaupten zu müssen. Dabei helfen ihnen eine starke Persönlichkeit, Offenheit und Humor.

\subsubsection{Interesse am Beruf und an Berufsinhalten}

Die meisten Jugendlichen haben ein starkes Interesse an den beruflichen Tätigkeiten in ihrer Berufslehre, das sich bei einigen schon früh in ihrer Kindheit abzeichnete (siehe Abschnitt 9.3.1 ,Berufsfindung'). Das Zusammenarbeiten mit Menschen oder bestimmten Materialien kann ebenfalls Interesse wecken. Eine Automechatronikerin ist fasziniert von der Breite des Wissens, der Technik und dem Design, die in Fahrzeugen vereint werden. Die Leidenschaft für Autos war so außerordentlich, dass sie trotz Rückschlägen im Berufsfindungsprozess schließlich eine passende Lehrstelle fand. Sie schwärmt für die Vielseitigkeit der Ausbildung, die Kraft der Motoren und eine vielsprechende umweltfreundlichere Zukunftsvision des Autos.

Zwei Hauptpunkte: geniale Ausbildung. Wir lernen so viel. Viele verschiedene Fächer: Physik, Chemie, Mechanik, Informatik, nicht nur alles über Autos. Es sind nur vier Stunden über das Auto, der Rest ist Mathe, Physik. Das lockte mich. Das Zweite ist, obwohl ich nicht so Autofan bin, mich fasziniert, wie das alles miteinander funktioniert. Ich finde Sachen toll, die was Großes bewirken. Wenn man sich vorstellt, was ein Auto alles leistet, ohne dass es die meisten merken. Maschinen, die viel machen, ohne dass wir es merken. Ich habe gerne Autos, schöne Autos machen mir Freude. Auch die Neuheiten an der Automesse in Genf. Zusehen und mithelfen, den großen Schritt zu machen, Autos umweltverträglicher zu machen. Neuwertige Autos, die nicht so Stinkbomben 
sind. Auch in der Ausbildung werden wir ausgebildet, Motoren effizienter zu machen. Wir denken oft in die Zukunft. (B; 21)

Bei einer anderen Automechatronikerin steht die Handlung im Zentrum des Interesses, die Fähigkeit, komplexe Maschinen in einer Art Rätsel auseinander- und wieder zusammenzubauen.

Das finde ich faszinierend. Ich brachte noch jedes Auto auseinander und baute es wieder zusammen. Manchmal dauert es ein bisschen länger. Es klappte noch immer. (D; 57)

Zuweilen können die wirklichen Interessen erst nach dem Abschluss einer ersten Berufslehre verfolgt werden. Zwei Männer haben bereits eine Berufsausbildung durchlaufen, einer davon hat sie sogar erfolgreich abgeschlossen. Erst danach ergab sich für die beiden die Möglichkeit, ihren wirklichen Interessen auf beruflicher Ebene nachzugehen und eine zweite Berufslehre in Angriff zu nehmen. Person K ist gelernter Maurer und macht die Ausbildung zum Fachmann Betreuung. Person I hat nach der Berufslehre als Gipser die Ausbildung zum Fachmann Gesundheit begonnen. Beide sind sehr motiviert und freuen sich an der Zusammenarbeit mit Menschen. Beide äußerten sich mehrfach dazu, wie wichtig ihnen die Arbeit im Team und mit Kindern bzw. älteren Menschen ist. Dabei erleben sie viel Freude und Bestätigung.

Es macht einfach mega Spaß mit den Kindern. Es ist ein schönes Gefühlt, wenn man sieht, wie die Kinder wachsen, wie sie ihre Schritte machen. $(\mathrm{K} ; 23)$

Interesse kann sich, wenn nicht schon vorhanden, während der Berufslehre entwickeln und zu einer Ressource werden. Die Möglichkeit, Spenglerin zu werden, bot sich Person E eher zufällig an. Im Verlauf der ersten Wochen hat sich das Interesse an den zu bearbeitenden Materialien und die Begeisterung für die Tätigkeit in luftiger Höhe entwickelt. Insbesondere die Arbeit auf Giebeldächern war für Person E eine Herausforderung und ein Lernprozess für den Lehrbetrieb. Dieser wollte sie anfänglich nicht auf Schrägdächern arbeiten lassen. Person E musste sich hier für die Gleichbehandlung einsetzen. Sie beschreibt ihre Faszination für den Beruf so:

Auf dem Dach herumklettern. Am Anfang hatte ich Höhenangst, vor allem, wenn es schräg ist. Abwechslungsreiches Material (Kupfer, Amalgam, Zink, Chromnickelstahl), viel unterwegs, verschiedene Baustellen. (E; 39)

Eher zufällig in ihrem Beruf gelandet sind neben der Spenglerin eine der beiden Logistikerinnen und der Fachmann Hauswirtschaft. Sie konnten aber ein Interesse für ihre Tätigkeiten während der Berufslehre entwickeln. Zwei weitere Jugendliche haben ein klar anderes Berufsziel als das, in dem sie im Moment sind. Der Dentalassistent möchte Zahnarzt werden, die Logistikerin Polizistin. Während der Dentalassistent inhaltlich nahe am Berufsfeld ist und das erlangte Vorwissen ihn in der späteren Ausbildung unterstützen wird, ist die Logistikerin wenig an den beruflichen Tätigkeiten interessiert, sondern 
sieht in ihnen eine akzeptable Möglichkeit, Berufserfahrungen zu sammeln und die Bedingungen für die Aufnahme in die Polizeischule zu erfüllen.

Das Interesse für den Beruf und die damit verbundenen Tätigkeiten bildet eine wichtige psychische Ressource für Jugendliche in geschlechtsuntypischen Berufslehren. Bei einigen ist es von Anfang an ausschlaggebend für die Aufnahme einer geschlechtsuntypischen Berufslehre. Andere finden über einen geschlechtstypischen Erstberuf zu ihren Interessen. Einige entdecken im Verlauf der Ausbildung und durch die Auseinandersetzung mit den Tätigkeiten und/oder Materialien eine Faszination für den Beruf und somit eine Quelle der Unterstützung. Für eine vierte Gruppe ist weniger ein Interesse an der jetzigen Berufslehre an sich auszumachen als an der Berufslehre als einem Mittel zur Zielerreichung.

\subsubsection{Familiäre Unterstützung}

Die Familie ist die primäre Unterstützungsquelle, während der Jugendzeit ebenso wie im Berufsfindungsprozess (vgl. Neuenschwander 2005). Doch gerade bei der Frage der geschlechtsuntypischen Berufsfindung ist die Unterstützung durch die Familie nicht selbstverständlich. In Form von Rollenmodellen oder explizit ausgesprochenen Erwartungen versuchen Eltern ihre Kinder zu einer aus ihrer Perspektive erfolgreichen Berufsfindung zu führen. Sie wollen das Beste für ihr Kind, damit sie glücklich und selbstständig leben können. Dabei spielt natürlich die Angst vor Misserfolgen oder Ausgrenzung mit. Zum Beispiel sorgen sich Mütter um ihre Töchter, wenn sie an das antizipierte raue Klima auf Baustellen und in Werkstätten denken (vgl. ,Vorurteil' im Abschnitt 9.3.2 Aussage E; 47), und Väter um die Akzeptanz ihrer Söhne in vermeintlich femininen Berufen (vgl. ,Abwertung' im Abschnitt 9.3.2 Aussage N; 39). Die familiale Ressource ist unter diesen Überlegungen nicht selbstverständlich zugänglich für die Jugendlichen. Einerseits braucht es Mut, geschlechtsuntypische Berufswünsche zu äußern, und andererseits gilt es, die Unterstützung dafür von den Eltern zu erhalten. Die von mir interviewten Jugendlichen beschrieben oft solche oder ähnliche Überlegungen. Ihnen gelang es aber sehr schnell, die Eltern oder zumindest einzelne Familienmitglieder als Unterstützung zu gewinnen.

Bei mehreren Jugendlichen spielte der Vater eine entscheidende Rolle. Er wird als wertvolle Unterstützung in der Berufswelt wahrgenommen. Diese Väter zeichnen sich darin aus, dass sie ihre Kinder mit Durchhalteparolen Mut und Hoffnung vermittelten, ihnen bei der Bewerbung und dem Bewerbungsschreiben tatkräftig zur Seite standen oder sie zu Ausbildungsorten (Schnupperlehre, Lehrvertragsunterzeichnung) begleiteten. Hier einige Beispiele:

Aber mein Vater überzeugte mich, nicht aufzugeben. (B; 57) 
Ich nahm meinen Vater zu Hilfe. Er wusste genau, wie machen, und sah die Sachen, die ich vorbereitet hatte, gut an. Er half mir sehr. $(\mathrm{G} ; 29)$

Mein Vater kaufte Bücher darüber. Und ich solle die doch lesen, bevor die Lehre beginnt. $(\mathrm{H} ; 45)$

Mein Vater unterstützte mich sehr viel. $(\mathrm{J} ; 15)$

Mein Vater sagte immer, du musst das machen, was du willst. (E; 47)

Skepsis gibt es vonseiten der Mutter etwa gegenüber dem männlichen (rauen) Arbeitsumfeld, dem sich ihre Töchter aussetzen wollen. Doch die Mütter sind ebenfalls eine Ressource, insbesondere, wenn sie als Beispiel für geschlechtsuntypische Tätigkeiten herangezogen werden können.

Bei uns ist es wie im Blut drin, sagt meine Mama so gern. Meine Großmutter ist schon 7,5-Tönner gefahren. Meine Mutter ist Berufskraftfahrerin, und mein Bruder ist Verkäufer im Motorradzubehör. Schon von klein auf Benzin im Blut. Mein Ex-Freund bastelte auch immer an seinem Roller herum, nachher am Auto. (A; 19)

Ich wurde auch von meiner Mutters Seite her unterstützt. Es war und ist Tradition, dass von jeder Familie (Geschwister der Mutter) jemand Landwirt wird. Eine Cousine und zwei Cousins lernten auch Landwirt. (J; 59)

Wenn Eltern diese Unterstützung nicht zu geben vermögen, können Personen im weiteren Familienumfeld zur tragenden Kraft werden. In den Interviews wurden folgende Personen genannt: Freund, Stiefvater, Geschwister, Großmutter oder gar etwas weiter entfernt der Mann der Patentante.

Mein Freund unterstützt mich. Er lernt mit mir. (A; 79)

Meine erste Schwester machte Polymechanikerin. (B; 51)

Meine Großmutter war die einzige, die mich unterstützte. Am Schluss durfte nur noch ich ihre Haare machen. Sie sagte immer, ich soll das machen, was ich will. Das lernen, was ich möchte, denn ich müsse es den Rest meines Lebens machen. (N; 59)

Der Mann meiner Gotte ist bei der Flughafenpolizei und brachte eine Broschüre mit. Darin steht, was die Aufgaben der Polizei sind und welche Weiterbildungsmöglichkeiten es gibt. (F; 39)

Familienmitglieder beeinflussen Jugendliche in der Wahl ihrer beruflichen Ausbildung. Wenn es den Jugendlichen gelingt, ihr Interesse und ihre Begeisterung für einen geschlechtsuntypischen Beruf den Eltern und nahen Personen zu vermitteln, sind deren Unterstützung wichtige Pfeiler für den Erhalt einer Lehrstelle und für das Durchhalten in schwierigen Phasen vor und während der Berufslehre.

\subsubsection{Unterstützung durch Gleichaltrige}

Lernende in geschlechtsuntypischen Berufslehren erfahren Unterstützung von ihren gleichaltrigen Freundinnen und Freunden in Form von beruflichem Fachwissen, das genutzt werden kann, in Form von Bewunderung, vor allem 
durch weibliche Jugendliche, und in Form von Akzeptanz und Wertschätzung.

\section{Vorteile verschaffen}

Die meisten wollen, dass ich ihnen etwas repariere. Die versuchen das schamlos auszunutzen. Ich sage dann, du kannst gern bei uns vorbeikommen. Wenn es wirklich gute Freunde sind, dann mache ich auch mal was. Die meisten Frauen reagierten recht cool drauf. (A; 77)

\section{Bewunderung}

Die Mitschüler fanden das gut. Meine beste Kollegin liebt mich dafür. (B; 63)

Andere sagten: „Das finde ich gut, weil es eben nicht viele machen. “ $(\mathrm{J} ; 69)$

\section{Emotionale Unterstützung}

Im Kollegenkreis wurde das auch gut aufgenommen, sehr gute Reaktionen, sie merkten auch, dass sobald ich mit den Kindern zu arbeiten begann, ich mich selbst positiv veränderte. $(\mathrm{K} ; 79)$

Sie fanden es mega lässig, dass ich eine Lehre als Dentalassistent mache. (M; 103)

Eine Kollegin fand es super. Sie kam auch zu uns schnuppern. Ich wollte sie überzeugen, dass sie auch Automech wird. Aber es klappte nicht. (C; 47)

\subsubsection{Ausbildende und Vorgesetzte}

Eindrücklich schildern einige Jugendliche, wie wichtig ihnen das gute Einvernehmen mit den Vorgesetzten oder Ausbildenden ist. Besonders in schwierigen Situationen des beruflichen Beginns ist die Unterstützung durch die Chefin, den Chef enorm wichtig: der Wille, Personen des anderen Geschlechts in die Arbeitsgemeinschaft zu integrieren, mit den Mitarbeitenden zu diskutieren, sich schützend vor die Lernenden zu stellen, ihren bedingungslose Unterstützung zu garantieren, ihnen Mut zu machen und das Gefühl zu vermitteln, hier am richtigen Ort zu sein.

Diesmal hieß es, es gibt einen Mann und eine Frau. Viele wollten keine Frau. Er [Vorgesetzter; Anmerkung BR] nahm mich. Ich bin auch die zweite Frau, die er ausbildet. Er kennt das schon. (A; 67)

Er [Vorgesetzter; Anmerkung BR] sagte aber von Anfang an zu mir, sollte mal ein dummer Spruch kommen oder sollte da mal mehr laufen, dann kommst du zu mir, dann wird das geklärt. $(\mathrm{A} ; 69)$

Sie empfingen mich mit offenen Armen. Sie waren froh, dass eine Frau kommt. Auch mein Chef fand es spannend, etwas Neues zu wagen. Er findet, es bringe auch den andern etwas, auch wenn sie es gar nicht merken. Ich bringe eine gewisse Harmonie. Wenn eine Frau da ist, sind gewisse Sachen lockerer und sie sind nicht immer gleich aufgeregt. Sie sind nicht mehr so aggressiv. Ich merkte das auch schon, der Chef merkte es auch schon. Auch darum möchte er mich unterstützen und hilft mir. Auch bei schulischen Problemen kann ich ihn oder einen Mechaniker fragen. (B; 19) 
Wichtig ist mir, dass mein Chef und mein Lehrmeister so vieles für mich machten. (B; 87)

Dort waren sie offen, hatten Freude, dass eine Frau kommt. Sie wollte mich auch nehmen. $(\mathrm{C} ; 43)$

Ich wählte die Stadt [als Arbeitgeber, G konnte sich zwischen zwei Lehrstellen bei der städtischen oder bei der kantonalen Verwaltung entscheiden; Anmerkung BR], weil ich hier zwei Ansprechpersonen haben, Herrn G und Frau A. Das war eben auch Frau und Mann, und je nach Thema kann man besser mit den Personen reden. Im anderen Betrieb wäre nur ein Mann gewesen. (G; 49)

Sie [die Chancen, Anmerkung BR] sind eher höher als bei einer Frau. Mir wurde eine Lehrstelle angeboten, wenn ich nichts finde. Weil er auch unbedingt mal einen männlichen Stift [Mitlernender, Anmerkung BR] haben möchte, weil es etwas Seltenes ist. Darum wird es auch beliebter sein. (M; 59)

\subsubsection{Personen im beruflichen Umfeld}

Wenn Mitarbeitende und andere Lernende in den Betrieben, Mitschülerinnen und Mitschüler aus den Berufsfachschulen sowie Lehrpersonen den Lernenden in geschlechtsuntypischen Berufslehren vermitteln können, dass sie dazugehören oder dass aus ihrer Sicht das Geschlecht keinen Einfluss darauf hat, ob man den Beruf gut ausführen kann, gibt dies den Lernenden in geschlechtsuntypischen Berufslehre Halt. Insbesondere deshalb, weil es sich bei dieser Personengruppe um eine über die Zeit stabile Gemeinschaft handelt.

Die Mechaniker waren offen mir gegenüber. Die hatten kein Problem, mit mir zu arbeiten. Ich spürte nicht gro $\beta$, dass sie sich verstellen mussten. $(\mathrm{B} ; 65)$

Die Aufnahme [Integration bzw. Akzeptanz bei den Mitarbeitenden, Anmerkung BR] gab mir Kraft. Dann konnte ich alles besser meistern. Es ging einfacher, da ich fragen konnte, wenn ich eine Aufgabe nicht konnte, und sie mir halfen. (B; 77)

Mir helfen sie eher als meinem Mitstift [Mitlernenden, Anmerkung BR] und sie sind nicht ganz so streng zu mir. Sie sagen: „Jaja, du bist eine Frau, du darfst das. “ Zum Beispiel, wenn etwas kaputt geht, und es geht viel kaputt in einer Werkstatt. Vor allem beim Würgen: Klammern abreißen, eine Schraube zu stark anziehen. Beim Heben, weniger körperliche Kraft: z.B. eine Türe abmontieren, die kann ich nicht alleine halten. Dafür komme ich an versteckte Orte. (C; 23)

Es wird einem vermehrt geholfen. Am Anfang haben auch Männer Mühe beim Lastenheben. Ich denke, Männer werden weniger unterstützt. Meine Kollegen kommen sogar und bieten Hilfe an. (E; 21)

Bei einem Klassenwechsel, sagte der Lehrer, jetzt haben wir wieder eine neue Bereicherung. Als ich in eine reine Männerklasse kam. (G; 69)

Man wird auf der menschlichen Seite angeschaut. Man kann hier gute Kollegen finden und auch private Themen besprechen. Es ist ein lockerer Umgang, wir sind alle per Du, bei den Chefs. $(\mathrm{H} ; 81)$

Ich muss aber keine Angst haben, weil ich weiß, dass meine Leute hinter mir stehen. Und wenn Anschuldigungen gemacht werden, dann wird das auch richtig behandelt und angeschaut werden. $(\mathrm{K} ; 37)$ 


\subsubsection{Personen aus der Sekundarstufe I}

Bei Personen aus der Sekundarstufe I handelt es sich vor allem um Lehrpersonen und Berufsberatende, die im Berufsfindungsprozess eine einflussreiche Rolle spielen. Sie können emotional unterstützen, Selbstvertrauen und fachbezogenes Interesse vermitteln, Referenzen geben und Türöffner sein.

Für zwei Tage ging ich danach zurück in die Garage, weil ich nicht wusste, was tun. Dort besuchte mich mein Lehrer. Ich lernte gerade schweißen mit einem Kübel mit 1000 verschiedenen kleinen Metallteilchen. Ich sollte einen Schneemann basteln. Es sah schrecklich aus, weil ich nicht wusste, wie schweißen. Ich erschrak, weil plötzlich mein Lehrer vor mir stand. Er hatte so Freude, ich schenkte ihm den Schneemann. Er hat mich sehr unterstützt. $(B ; 53)$

Im Gymnasium: mein Mathe- und mein Franzlehrer. Ich konnte sie als Referenzen angeben. Ich fand es schön, dass sie mich unterstützt haben. (H; 77)

Durch meine Schwestern, den Kindergarten in der Privatschule und die guten Rückmeldungen der Lehrer musste ich sagen, jetzt muss ich das mal anschauen, und schnupperte und bekam einen Praktikumsplatz. (K; 49)

Die berieten mich. Meine Lehrerin war auch da, und sie sagten, ich solle mal schnuppern als Hauswirtschaftspraktiker. Das machte ich und bekam einen Vertrag, und ich unterschrieb sofort. (L; 47)

Vor allem die Berufswahl in der Schule, die Berufsberaterin [Antwort auf die Frage, wer sie unterstützt hat, Anmerkung BR]. (M; 69)

\subsubsection{Kundinnen und Kunden}

Die bisher beschriebenen Personengruppen zeichnen sich dadurch aus, dass sie über längere Zeit mit den Jugendlichen zusammen sind und sich ein stabiles Vertrauensverhältnis aufbauen kann. Bei Personen, die Empfangende einer Dienstleistung sind - Kundinnen/Kunden, Patientinnen/Patienten, Angehörigen von Bewohnerinnen/Bewohner etc. -, ist der Kontakt von einer anderen Qualität, die Interaktionen sind kürzer. Ergänzend ist zu anzuerkennen, dass der Kontakt insbesondere zu Kindern in Tagesstätten oder Bewohnerinnen und Bewohnern von Pflegeeinrichtungen durchaus eine Qualität annehmen kann, die familiäre Züge beinhalten kann. Hier werden jetzt die flüchtigeren Kontakte, die positive Rückmeldungen enthalten, zusammengefasst.

Ältere Kundinnen sagen oft: „Jö, was machst denn du hier? “ Jüngere ignorieren es eher, dass ich hier bin. Männer verlangen manchmal, dass ich ihr Auto mache. An mir haben sie mehr Freude und geben mir auch Trinkgeld (was aber üblich ist). Weil es besonders ist. $(B ; 31)$

Es gibt Kunden, die Freude haben und sagen: „Schön, dass du das machst als Frau. “ Für andere ist es eher speziell. Nach vier Jahren kennen mich die Kunden, und sie kommen zum Teil direkt zu mir und gehen nicht zum Chef. (D; 21) 
Ich wurde sehr geschätzt, engagierte mich sehr, und das kam gut an. Bei Angehörigen und Angestellten, Mitarbeitern. (I; 43)

Erstaunlicherweise werde ich selten als Schüler angeschaut, vermutlich wegen meines Alters. Die Leute kommen gern zu mir und fragen. Wenn es in meiner Kompetenz ist, gebe ich gerne Auskunft. Ich merke schon, dass die Leute gern zu mir kommen. Sie fragen: ,Wie geht es meiner Mutter, was macht sie, kann man da was machen? " Da sehe ich, dass es im Gegensatz zu meinen Kolleginnen und Mitschülerinnen anders ist. (I; 59)

\subsubsection{Gute Erfahrungen}

Die Bestätigung bzw. Bewältigung einer Situation, die Verunsicherung oder Ängste ausgelöst hat, kann sich positiv auf die Selbstwahrnehmung auswirken und deshalb als eine Ressource für zukünftige, ähnlich geartete Momente gebraucht werden.

Es spielt nicht so eine Rolle, ob Mann oder Frau. Viele finden es kein Problem oder eine Bereicherung. (C; 29)

Der erste Schultag: Ich hatte immer die Hoffnung, dass noch Frauen auftauchen. Die Jungs kamen der Reihe nach herein und waren nicht sehr erstaunt. Nachher sagten sie mir schon, dass es ihnen aufgefallen sei. Es ging gut. (E; 57)

Im Großen und Ganzen wurde ich in diesen Jahren als Person nie abgelehnt. (I; 55)

\subsubsection{Vorteile des Geschlechts}

Das untypische Geschlecht bietet neben den Nachteilen Vorteile, die es den Jugendlichen erleichtern, ihren Platz zu finden. Die Sichtbarkeit bringt auch Beachtung, Freude oder gar Bewunderung mit sich. Körperliche Vorteile wie feingliedrige Hände, die besser in verwinkelte Ecken kommen, oder die Muskelkraft werden von den Jugendlichen genannt.

Die einen fanden es gut, weil es nicht viele Frauen machen. $(\mathrm{J} ; 13)$

Es ist sehr angenehm, mit den Frauen zusammenzuarbeiten. Es ist alles sehr harmonisch, und man geht auch nicht unter als Mann, wird respektiert als Mann im Frauenberuf. Es ist sehr angenehm. $(\mathrm{K} ; 15)$

Ich fühle auch, dass ich anders arbeite als Frauen auf diesem Beruf. Wir haben ein lockereres Angehen mit den Kindern und können trotzdem streng sein. Es ist nicht vergleichbar mit den Frauen. Von dem her ist es schon wichtig, als Mann angesehen zu werden. $(\mathrm{K} ; 27)$

$J a$, es ist schon so. Sie hören auch bei den Frauen gut zu, wenn sie sie kennen. Wenn jemand Neues kommt, merkst du schon den Unterschied, wie sie auf einen Mann oder eine Frau reagieren. Wenn sie einen Mann noch nicht so gut kennen, dass sie trotzdem mehr gehorchen. Es ist nicht so verbreitet. Die Kinder hören es nicht so viel, dass eine so tiefe Stimme kommt und etwas sagt. Dann hören sie gespannt zu. $(\mathrm{K} ; 35)$

Ich denke, ich finde recht schnell etwas, es kommt gerade auf, dass Kitas Männer wollen. Sodass sie nicht nur die Mutterseite vertreten haben, sondern auch die Vaterseite, dass eine männliche Person auch da ist und die Kinder einen Bezug nicht nur zu Frau- 
en, sondern auch zu Männern haben. Von daher habe ich schon beim Schnuppern gemerkt, dass sie sehr zufrieden waren und sich freuten, dass endlich mal wieder ein Mann anfragt. $(\mathrm{K} ; 43)$

$J a$, natürlich. Ich bin groß, stark, schnell. Ich kann Fähigkeiten zeigen, die andere nicht haben. Man ergänzt sich gegenseitig. Ich sehe auch, dass Frauen Männer brauchen. Ich werde sehr viel gebraucht. Meine Chefin gibt mir Aufgaben, die die Frauen nicht machen können: schwere Maschinen. Sie könnten, aber es ist zu schwer für sie. (L;29)

Ansonsten habe ich das Gefühl, dass man bevorzugt wird. Die Lehrer kennen es weniger und freuen sich darüber. Sie haben Freude daran, dass es nicht nur Frauen sind. $(\mathrm{M} ; 17)$

\subsubsection{Fazit}

Die interviewten Lernenden können auf eine bedeutende Zahl an unterschiedlichen Ressourcen zurückgreifen. Was eine Ressource ist, ist sehr individuell. Für die eine Person kann eine gute Bemerkung einer nicht besonders nahestehenden Person die Kraft geben, weiterzumachen, während sie bei anderen der Auslöser sein kann, über die eigene spezifische Situation nachzudenken. Die Kraft aus der eigenen Person zu schöpfen, sich stark genug zu fühlen, bilden die größte Quelle, vor sozialer Unterstützung, vor allem durch die Familie sowie Promotorinnen und Promotoren aus dem Bereich Schule und Lehrbetrieb. In weitergehenden Forschungen wäre zu klären, ob die persönlichen und sozialen Ressourcen notwendig sind, um den Belastungen standzuhalten.

\subsubsection{Doing Gender}

Ein geschlechtstypischer Beruf bietet die Möglichkeit, das eigene Geschlecht entsprechend den tradierten Rollenvorstellungen darzustellen. Geschlecht, verstanden als soziale Kategorie, wird von den Individuen als ein Produkt performativer Tätigkeiten hergestellt. Eine Übereinstimmung des Geschlechts der Lernenden mit dem typischen Geschlecht im Beruf erleichtert damit die Wahrnehmung von außen und die Selbstwahrnehmung als Frau bzw. Mann. Diesen Vorteil, den Beruf als Geschlechtercode zu verwenden, haben Lernende in geschlechtsuntypischen Berufslehren nicht. Sie müssen also eine Alternative suchen, wie sie ihre Weiblichkeit bzw. Männlichkeit darstellen können. Die Aussagen der interviewten Jugendlichen lassen sich in zwei Kategorien einordnen: ,Geschlecht produzieren' und ,Klischee bestätigen“.

\subsubsection{Geschlecht produzieren}

Das Geschlecht ist einem Individuum nicht einfach gegeben, wie es im Alltag meist den Eindruck erweckt, sondern das Geschlecht wird dem Individuum von der sozialen Umwelt zugesprochen. Je nachdem, wie stark der Ge- 
schlechtercode - etwa kurze vs. lange Haare - dargestellt wird, reagieren die Personen um das Individuum herum. Während Männer darauf achten, nicht affektiert zu agieren, untermalen Frauen ihre Weiblichkeit durch die Verstärkung äußerer Merkmale wie Kleidung, Frisur oder Schminke. Schmutz wird als männlich wahrgenommen, das bedeutet, ein Mann soll/darf schmutzig sein, eine Frau nicht. Dieser Umstand ist Person A nicht entgangen, und sie versucht den Anforderungen, sich als Frau zu benehmen, nachzukommen und ihre Weiblichkeit zu erhalten bzw. wiederherzustellen.

Manchmal ist es auch das Schminken. Ich gehe selten geschminkt zur Arbeit. Für mich bringt es das einfach nicht, weil ich innerhalb von einer Stunde eh schwarz im Gesicht bin. Aber auch durch die Art, wie ich mich bewege. Ich versuche, einen Fuß vor den andern zu setzen, und nicht so breitbeinig zu laufen. Ich guck schon ein bisschen auf meine Weiblichkeit. (A; 23)

\subsubsection{Klischees bestätigen}

Eine Voraussetzung, damit das Geschlecht entsprechend attestiert wird, ist, dass Geschlechtercodes oder Klischees wahrgenommen werden können. Einige Klischees werden als solche erkannt und weniger ernst genommen, andere sind unterschwellig und schleichen sich in der Wahrnehmung und der Interpretation ein. Die Sammlung von klischierten Aussagen belegt, wie wichtige diese bei der Konstruktion von Geschlecht sind, wie stark sie das Leben der Jugendlichen bestimmen und wie sie als Folie für die Interpretation der Lebenswirklichkeit eingesetzt werden.

\section{Auto}

Aber meistens ist es eher umgekehrt. Irgendwann denkt man, warum hat es keine Männer in der Klasse? Es fehlen einem die Männergespräche, über Autos reden und so. (M; 17)

\section{Keine Frauen}

Es kommen aber auch nicht viele Frauen schnuppern. Das ganze Jahr über kam keine einzige Frau. Wir hatten zwei für einen Ferienjob, Neuwagen putzen und so. (A; 69)

\section{Keine Männer}

Wenn mal ein Schnupperstift [Schüler, der für ein paar Tage Erfahrungen im Lehrbetrieb sammelt, Anmerkung BR] kommt, ist er am Anfang schockiert, dass es nur einen Mann auf neun Frauen gibt. (N; 73)

\section{Klatsch}

Ich vermisse den Austausch und den Klatsch unter Frauen. Es ist immer anders, ob man mit Frauen oder Männern redet, also für mich. (E; 23) 


\section{Konzentration}

Die üK sind happig. Ich sehe, dass ich besser durchhalte und mehr Stoff verarbeiten kann. Die Jungs haben mehr Probleme mit der Konzentration. Es war schon in der Schule so, dass die Mädels besser durchhielten. (B; 73)

\section{Körper}

Ich komme mit meinen kleinen Händen viel besser zu den Elektronikbauteilen, als große Männer mit ihren Pranken. Sie rufen mich dann auch, um ihnen zu helfen. Ich habe auch weniger Verletzungen, weil ich besser an die Sachen herankomme. $(\mathrm{B} ; 25)$

\section{Kraft}

Von der Kraft her. Dort ist man als Frau etwas im Nachteil. (J; 35)

\section{Kraft vs. Kopf}

Meine Oberstifte [ältere Mitlernende, Anmerkung BR] haben die Tendenz, wenn etwas nicht funktioniert, so lange darauf herumzuhämmern. Oder sie sagen: „Ich weiß nicht, was es ist. Fragen wir den Chef. "Ich schreibe mir in solchen Situation auf, was es sein könnte. Als Frau überlege ich mehr. (B; 27)

\section{Männlicher Habitus}

Die Männer sind es halt total gewohnt, dass sie bei der Arbeit reden und über ihre Frauen schimpfen können. Dass sie das so erzählen können, wie sie wollen, und seit ich da bin, benehmen sie sich anständiger. Das sagte auch mein Chef. Seit ich hier arbeite, ist es ruhiger geworden. $(\mathrm{A} ; 71)$

\section{Mannsweib}

Als sie hörten, dass ein Mädchen kommt, dachten sie an ein Mannsweib. (B; 65)

\section{Problem bereden}

Zum Beispiel private Probleme. Da geht eine Frau besser drauf ein. $(\mathrm{H} ; 117)$

\section{Sprache}

Es geht rauer zu und her als hier, in einem Frauenteam. Die Sprache war anders. Das Körperliche stand mehr im Vordergrund als das Soziale, Gespräche führen, ja. (I; 23)

\section{Schlampe}

Als Frau wird man oft als Schlampe dargestellt, wenn man mit Männern unterwegs ist: Freizeit verbringt, draußen ist. Als Mann könnte ich mit meinen Kollegen ungestört draußen sein. $(\mathrm{C} ; 19)$

\section{Schminke vs. Schlabberlook}

Die Art der Männer ist anders, die Frauen sind immer geschminkt, Männer gehen eher mal mit den Jogginghosen raus. Ziehen unpassende, zu große Pullis an. (F; 25)

Der Umgangston macht viel aus. Hier ist es ruhiger und freundlicher, und man bekommt von den Kindern mehr zurück als beim Beruf Maurer. $(\mathrm{K} ; 25)$ 


\section{Zickenkrieg}

Das denke ich schon, z.B. wenn es Zickenkrieg im Geschäft oder in der Schule gibt. Wenn sie so anfangen über unnötige Sachen, die man auch in zwei Minuten geklärt hätte, eine Stunde zu diskutieren. Dann fragt man sich, ob sie jetzt nicht aufhören könnten. Dann wäre man lieber jemand, der mitreden könnte. (M;29)

Mit den meisten Frauen habe ich Zickenkrieg. Ich habe es nicht so gut. Ich finde sie hochnäsig. Männer sind einfacher und unkomplizierter, sie verstehen mehr Spaß. (C; 11)

\subsubsection{Fazit}

Die Auseinandersetzung mit den Auflagen, die die Gesellschaft an Männer und Frauen stellt, findet bei den Lernenden in geschlechtsuntypischen Berufslehren verstärkt statt. Ihnen wird vorgehalten, dass sie sich nicht innerhalb des Geschlechterkodex bewegen. Die Lernenden haben einen reichen Fundus an Klischees, die sie ebenso bedienen, wie sie unter ihnen leiden.

\subsection{Diskussion}

Die leitfadengestützten Interviews mit 14 Lernenden in geschlechtsuntypischen Berufslehren drehten sich um die vier Themenschwerpunkte Berufsfindung, Belastungen, Ressourcen und Doing Gender. Das Ziel der inhaltsanalytischen Auswertung war, die spezielle Situation von Jugendlichen in geschlechtsuntypischen Berufslehren beschreiben zu können. Das Modell der geschlechtsuntypischen Berufssozialisation besagt, dass diese Jugendlichen in ihrer ohnehin verunsichernden Transitionsphase vom Schulkind zur erwerbstätigen Frau bzw. zum erwerbstätigen Mann zusätzlich unter Druck geraten, indem sie sich den Geschlechtsrollenerwartungen widersetzen und deshalb Ablehnung erfahren. Der Fokus der Interviewbefragung lag somit auf den spezifischen Schwierigkeiten und Herausforderungen während der Berufslehre. Dabei ging es insbesondere darum, Belastungen im Zusammenhang mit dem untypischen Geschlecht aufzudecken. Bei der Bewältigung dieser Ablehnungserfahrung stützen sich diese Jugendlichen auf persönliche Stärken und soziale Unterstützungen. Eine ganze Reihe von Ressourcen konnte aufgedeckt werden. Neben Belastungen und Ressourcen hat der Blick auf die Berufsfindung und die Geschlechterperformanz Zeugnis dafür abgelegt, wie sich die Jugendlichen im geschlechtsuntypischen Umfeld behaupten und wie sie sowohl Bestätigung erhalten als auch Befriedigung durch die berufliche Tätigkeit erfahren.

Am Anfang der Auseinandersetzung steht die Berufsfindung. Die Jugendlichen unterscheiden sich darin, wie sie zu ihrem Berufswunsch gekommen 
sind. Ähnlich der Typologie von Simpson (2005) sind die ,Sucher ${ }^{6}$ zu nennen, die aktiv einen untypischen Beruf gesucht haben, ein außerordentliches Interesse für einen beruflichen Gegenstand hegten oder einen starken Wunsch für eine bestimmte berufliche Tätigkeit hatten. Die Faszination für die Kraft von Maschinen oder der Wunsch, sich intensiv um andere Personen kümmern zu können, war größer als die tradierten Rollenerwartungen der sozialen Umwelt, obwohl die Jugendlichen häufig und von unterschiedlicher Seite auf die Geschlechterthematik hingewiesen wurden oder auf Hindernisse stießen, weil Frauen oder Männer nicht üblich sind im gewählten Beruf. Einigen von ihnen gelang es sogar ohne Unterstützung von zu Hause, einen Lehrvertrag zu bekommen. Bei anderen haben sich insbesondere die Väter stark engagiert, wie aus den Antworten zu den Ressourcen zu erfahren war. Diese Jugendlichen bilden die Ausnahme von den Theorien der Kompromissbildung nach Gottfredson (2005), wonach das Geschlecht neben Prestige und Interesse als eines der wichtigsten Selbstselektionskriterien betrachtet wird.

Die Kategorie ,Finder ${ }^{6}$ bei Simpson (2005) und die Kategorie ,Zufall/Notlösung ' weisen Ähnlichkeiten auf: Die Personen in diesen beiden Kategorien haben nicht aktiv nach dem Beruf gesucht, sondern sind durch äußere Umstände $\mathrm{zu}$ dieser geschlechtsuntypischen Berufslehre geraten. Oftmals sind es diejenigen, die sich mit der Berufsbindung besonders schwertun, die dann ein Angebot annehmen, ohne dafür besondere Begeisterung zu hegen. Während die Kategorie ,Siedler ${ }^{6}$ von Simpson (2005) im vorliegenden Material nicht gefunden werden konnten, vermutlich weil diese Jugendlichen erst am Anfang ihrer beruflichen Laufbahn stehen, ist die Kategorie ,Zwischenstation" hingegen spezifisch für die Ausbildungssituation. Sie hat damit zu tun, dass es für höhere Berufsziele, wie hier Zahnarzt bzw. Polizistin, eine Vorbildung braucht, die auf unterschiedlichen Wegen absolviert werden kann. In den Interviews kam stark heraus, dass sich die beiden betreffenden Lernenden nicht so stark auf die Thematik des Geschlechts einließen. Sie hatten dazu eine eher distanzierte Haltung. Vermutlich, da sie den untypischen Beruf nach Abschluss der Ausbildung verlassen wollen, um ihr eigentliches Berufsziel anzupacken.

Die Befragten nennen eine ganze Reihe von Belastungen, die mit ihrer Minderheitensituation zu tun haben und mit denen sie sich auseinandersetzen müssen. Besonders viele Aussagen wurden als Diskriminierung klassifiziert. Ausnahmslos alle interviewten Lernenden berichten von Erfahrungen, die in diesen Bereich fallen. Diese gehen von unterschwelligen Gefühlen des Fremdseins, die männliche Jugendliche in einer reinen Frauenumgebung beschleicht, bis hin zu klar formulierter Ablehnung. Die Ablehnung kann viele Formen annehmen, wie Kompetenzen oder Männlichkeit absprechen, sexistische, frauenfeindliche Bemerkungen, Verweigerung von Dienstleistungen aufgrund des Geschlechts, Ausschließen von der Gruppe, fiese Sprüche, Misstrauen usw. Obwohl sich die Jugendlichen im Interview relativ 
unbekümmert geben und den Eindruck erwecken, dass die diskriminierenden Handlungen nicht wirklich an sie herankommen, sind die Subbotschaften erkennbar. Aufgrund der Aussagen der Lernenden lässt sich schließen, dass es diesen Jugendlichen gelungen ist, die Diskriminierungen auszuhalten. Der Umstand, dass viele Jugendliche sich nicht vorstellen können, einen geschlechtsuntypischen Beruf zu erlernen, und die häufigeren Lehrvertragsauflösungen veranschaulichen, wie viel Selbstbewusstsein und Unterstützung es braucht, sich im geschlechtsuntypischen Umfeld zu bewegen. Die Diskriminierungen greifen die Integrität der Lernenden in geschlechtsuntypischen Berufslehren an. Private Bereiche wie das Körperbewusstsein, die sexuelle Orientierung, der Selbstwert werden problematisiert als etwas, das ungestraft ins Negative gezogen werden kann. Diese alltäglichen Diskriminierungen und Mikroaggressionen können nach Meyer et al. (2015) zu gesundheitlichen Beeinträchtigungen, im Extremfall zu Suizid führen. Die Machtverhältnisse sind klar verteilt. Jugendliche in geschlechtsuntypischen Berufslehren sind in der Minderheit und oft auf sich selbst gestellt. Deshalb sind Verbündete umso wichtiger. Diese bilden einen Großteil der Ressourcen.

Eine der wichtigsten Ressourcen der Jugendlichen in geschlechtsuntypischen Berufslehren ist ihre starke Persönlichkeit. Ein intaktes Selbstvertrauen, Offenheit und Redegewandtheit gespickt mit Humor und Schlagfertigkeit können helfen, für sich selbst einzustehen und sich in einem nicht immer wohlwollenden Umfeld zu behaupten. Sich selbst als aktiv, wirkungsvoll und wehrhaft zu erleben, kann die positive Wahrnehmung des eigenen Lebens (vgl. Ray/Keeney 1993) unterstützen und dazu beitragen, seine Ziele zu erreichen (vgl. Alpers 2005). Alle interviewten Jugendlichen äußerten Stärken auf der persönlichen Ebene. Einige Frauen konnten ihre anfängliche Unsicherheit gerade dadurch abbauen, dass sie sich in einem männlichen Umfeld bewähren konnten und sich nicht mehr mit Ausgrenzungsverhalten durch weibliche Gleichaltrige - wie während der obligatorischen Schulzeit - auseinandersetzen mussten. Die genannten Beispiele verdeutlichen, dass die Reaktionen des sozialen Umfelds nicht nur in eine negative, ablehnende Richtung gehen, sondern dass sich Jugendliche sehr wohl in einem geschlechtsuntypischen Umfeld aufgenommen fühlen und ihre Persönlichkeit stärken können. Dafür spricht insbesondere die Unterstützung, die diese Jugendlichen von vielen Seiten erfahren. Dabei spielt nicht nur die konkrete Unterstützung, die vor allem weibliche Jugendliche von ihren Vätern erfahren, sondern auch die wahrgenommene Unterstützung eine wichtige Rolle (Sarason et al. 1987). Dies führt zu einem Abbau von Stress (Heinrichs et al. 2003). Die Familie ist bei allen Interviewten eine wichtige Stütze.

Interessanterweise lebten fünf der befragten Lernenden zum Erhebungszeitpunkt nicht bei ihrer Herkunftsfamilie. Obwohl dies einerseits damit zu tun hat, dass diese jungen Erwachsenen etwas älter waren als durchschnittliche Lernende, weist dies vor allem auf ein starkes Bedürfnis nach Selbstbe- 
stimmung und Eigenständigkeit hin. Die selbstständige Wohnsituation basierte aber nicht auf einem Bruch mit der Familie, sondern die Verbundenheit war stark oder es gab sogar Kooperation im familieneigenen Betrieb. Neben der Familie kam immer wieder zum Ausdruck, dass die Ausbildenden oder Vorgesetzten sich stark für die Lernenden einsetzen. Zusätzlich unterstützen Personen im weiteren Umkreis wie Gleichaltrige, Mitarbeitende, Kundinnen/Kunden und Lehrpersonen die Lernenden ebenfalls. Nicht erstaunlich ist, dass das Interesse an den beruflichen Arbeitsinhalten, gute Erfahrungen und die Nutzung von Vorteilen, die das untypische Geschlecht mit sich bringt (z.B. beruhigt eine tiefe Stimme Kinder bzw. kommen feingliedrige Hände an versteckte Orte), die Jugendlichen darin bestärkten, den richtigen Beruf gewählt zu haben.

Das Spielen mit den Vorteilen, die das untypische Geschlecht mit sich bringt, ist ,Doing Gender'. Den Jugendlichen ist es sehr wichtig, als Frau bzw. Mann wahrgenommen zu werden. Und sie versuchen Nischen zu finden, wo die eigenen Geschlechtsrollenerwartungen umgesetzt werden können. Weibliche Jugendliche berichten, dass sie ihre Weiblichkeit vor allem außerhalb des Berufs durch Kleidung, Kosmetik und Verhalten unterstreichen. Männlichen Jugendlichen ist eher wichtig, männerkonnotierte Nischen bei der Arbeit zu besetzen. Im Sinn von Wetterer (2002) verdeutlicht sich dabei, wie Geschlecht selbst konstruiert wird, sodass die Wahrscheinlichkeit steigt, als Frau bzw. Mann wahrgenommen zu werden. Die Lernenden in geschlechtsuntypischen Berufslehren versuchen also in anderen Bereichen als dem Beruf den Geschlechtsrollenerwartungen durchaus gerecht zu werden. 


\section{Zusammenführung}

Im dritten Teil der vorliegenden Arbeit führe ich die theoretischen Ansätze und die empirischen Befunde zusammen. Das Modell der geschlechtsuntypischen Berufssozialisation erklärt, warum Lernende in geschlechtsuntypischen Berufslehren durchschnittlich geringeren beruflichen Erfolg haben als ihr Kolleginnen bzw. Kollegen in der gleichen Berufslehre. Bezugnehmend auf die Befunde in den empirischen Kapiteln wird dargestellt, inwieweit das Modell präzisiert werden muss. Im folgenden Kapitel 10 geht es darum, die Grundbedingungen der geschlechtlichen Segregation in der Arbeitswelt und die damit verbundenen Geschlechtsrollenerwartungen als Ausgangslage für die Reaktionen der sozialen Umwelt zu verstehen, mit denen sich die Lernenden in geschlechtsuntypischen Berufslehren konfrontiert sehen. In der Folge steigt die Wahrscheinlichkeit von psychosomatischen Beschwerden, Absenzen, Lehrvertragsauflösungen und teilweise Misserfolg an der Lehrabschlussprüfung. In den Interviews hat sich herausgestellt, dass Jugendliche, die eine geschlechtsuntypische Berufslehre absolvieren, über besondere Ressourcen verfügen. Eine der wichtigsten dabei ist die soziale Unterstützung durch Personen in ihrem unmittelbaren Umfeld: Eltern, Ausbildende und Lehrpersonen. 


\section{Schlussfolgerungen}

Mädchen und Jungen wachsen in einer vergeschlechtlichten Welt auf und bilden unterschiedliche Interessen aus (Makarova/Herzog 2016). Die Mehrheit der Jugendlichen entscheidet sich für eine geschlechtstypische Berufslehre, dies aufgrund von Prägung, Vorbildern, gesellschaftlichen Strukturen, Bedingungen des Arbeitsmarkts, insbesondere der Entlohnung mit all ihren Konsequenzen (Schwiter et al. 2016). Dies führt dazu, dass die Geschlechtsrollenbilder und -stereotype erhalten bleiben. Das Modell der geschlechtsuntypischen Berufssozialisation erklärt, wie Lernende in geschlechtsuntypischen Berufslehren die Irritationen und Reaktionen ihres sozialen Umfelds bewältigen und wie diese Erfahrungen sich auf den Erfolg in der Berufslehre auswirken. Die Ergebnisse des empirischen Teils der vorliegenden Arbeit zur Überprüfung der postulierten Annahmen werden in Unterkapitel $10.1 \mathrm{zu}-$ sammengefasst. Darauf folgt eine reflexive Auseinandersetzung mit den Befunden in Unterkapitel 10.2. Unterkapitel 10.3 zählt Perspektiven für die Berufsfindung und die Berufsbildung im Zusammenhang Lernenden in geschlechtsuntypischen Berufslehren auf. Unterkapitel 10.4 nennt Limitationen und wagt einen Ausblick.

\subsection{Zusammenfassung der empirischen Befunde}

Die Überprüfungen zahlreicher Beziehungen im Modell der geschlechtsuntypischen Berufssozialisation erfolgte über die Reanalyse von vier bestehenden Datensätzen: Lehrvertragsauflösungen (vgl. Kapitel 5 und Abschnitt 10.1.1), Lehrabschlussprüfungen (vgl. Kapitel 6 und Abschnitt 10.1.2), Forschungsprojekt FASE B (vgl. Kapitel 7 und Abschnitt 10.1.3) und Langzeitpanel TREE (vgl. Kapitel 8 und Abschnitt 10.1.4). Zusätzlich wurden Interviews mit 14 Lernenden in geschlechtsuntypischen Berufslehren durchgeführt (vgl. Kapitel 9 und Abschnitt 10.1.5). Im Folgenden werden die empirischen Befunde zusammengefasst dargestellt.

\subsubsection{Lehrvertragsauflösungen}

Die vom Autor zusammengefügten Populationsdaten des BFS der Jahre 2008 bis 2012 belegen, dass 25 Prozent der männlichen und 21 Prozent der weiblichen Jugendlichen ihren Lehrvertrag vorzeitig auflösen. Vergleichbare Ergebnisse haben Neuenschwander et al. (2012), Schmid und Stalder (2008) sowie Stalder und Schmid (2006) publiziert. Eine detaillierte Liste für jede 
Berufslehre befindet sich im Online-Anhang VII. Die Auflösequoten der Frauen und Männer wurden getrennt berechnet und vergleichend mit dem Indikator Gender Gap ausgewiesen. Der Gender Gap ist die Differenz der Auflösequoten der Männer minus derjenigen der Frauen.

Zur Klärung der Hypothese, ob Jugendliche in geschlechtsuntypischen Berufslehren häufiger von Lehrvertragsauflösungen betroffen sind als ihre Kolleginnen und Kollegen in denselben Berufslehren, wurde als Erstes aus den vorliegenden Daten berechnet, wie hoch der Frauenanteil eines Berufs ist. Dabei fällt auf, dass aus den Top-20-Berufslehren der Frauen und jene der Männer lediglich 6 von beiden Geschlechtern stark nachgefragt werden: ,Kaufmann/-frau EFZ - Dienstleistung und Administration', ,Koch/Köchin EFZ', ,Kaufmann/-frau EFZ - Öffentliche Verwaltung', ,Zeichner/-in EFZ', ,Kaufmann/-frau EFZ - Bank und ,Maler/-in'. Die andern 28 Berufslehren sind zum Teil stark nach Geschlecht segregiert. Hier 6 Berufslehren, die häufig gelernt werden und eine hohe Geschlechtersegregation aufweisen: ,Fachmann/-frau Gesundheit EFZ' (Frauenanteil 90\%), Elektroinstallateur/in EFZ' (2\%), ,Fachmann/-frau Betreuung EFZ‘ (87\%), ,Polymechaniker/-in EFZ' (3\%), ,Coiffeur/-euse EFZ' (92\%) und ,Automobil-Fachmann/-frau $E F Z^{\prime}(4 \%)$. Diese deskriptiven Ergebnisse verdeutlichen das stark nach Geschlechtern getrennte Arbeitsumfeld aller Lernenden. Auf diesem Hintergrund wurden die Gender Gaps bei den Lehrvertragsauflösungen der einzelnen Berufslehren verglichen. Die Ergebnisse verdeutlichen, dass Frauen in männerreichen Berufslehren (Frauenanteil bis 30\%) 7.3 Prozentpunkte vom Erwartungswert abweichen und damit häufiger von Lehrvertragsauflösungen betroffen sind als ihre Kollegen in denselben Berufslehren. Das gleiche Bild ergibt sich bei Männern in frauenreichen Berufslehren. Ihr Gender Gap ist 5.8 Prozentpunkte über dem Durchschnittswert. Detaillierte Analysen konnten die Tendenz aufzeigen, dass je stärker eine Berufslehre nach Geschlecht segregiert ist, desto eher sind die Lernenden in der Minderheit von Lehrvertragsauflösungen betroffen (vgl. Abschnitt 5.3.2). Am stärksten ist der Effekt bei folgenden drei männerreichen Berufslehren: ,Forstwart/-in EFZ' (Gender Gap 31\%), ,Automobil-Mechatroniker/-in' (30\%) und ,Montage-Elektriker/in EFZ' (26\%). In frauenreichen Berufslehren sind es: ,Tiermedizinische/-r Praxisassistent/-in EFZ' (57\%), ,Detailhandelsfachmann/-frau EFZ - Papeterie (33\%) und ,Florist/-in EFZ' (28\%). Zu berücksichtigen ist, dass die Anzahlen in den Minderheitenkategorien gering sind und so die Prozentzahlen durch Einzelfälle stark verzerrt sein können. Dennoch ist ein deutlicher Trend zu erkennen: Lernende in geschlechtsuntypischen Berufslehren sind stärker von Lehrvertragsauflösungen betroffen als Lernende in geschlechtstypischen oder geschlechtergemischten Berufslehren und haben damit weniger Erfolg während der Berufslehre. Dieser Befund ist ein deutliches Indiz dafür, dass bei Lernenden in geschlechtsuntypischen Berufslehren andere Prämissen gelten als bei ihren Kolleginnen und Kollegen. Der Pfad im Modell der ge- 
schlechtsuntypischen Berufssozialisation von der Bewältigung der Reaktion des sozialen Umfelds (vgl. Abschnitt 10.1.4) zum Austritt aus der geschlechtsuntypischen Berufslehre ist damit bestätigt.

\subsubsection{Lehrabschlussprüfungen}

Die Daten der Lehrabschlussprüfungen analysierte ich auf dieselbe Art wie die Daten der Lehrvertragsauflösungen. Die Daten der fünf Jahre 2008 bis 2012 wurden zusammengefasst und der Gender Gap berechnet. Rund 91 Prozent aller Lernenden bestehen die Lehrabschlussprüfung in den fünf untersuchten Jahren. Wenn die zweiten und dritten Versuche dazugezählt würden, wären es sogar noch mehr. Die Frauen sind etwas erfolgreicher und haben eine Misserfolgsquote von 7 Prozent, während diejenige der Männer über 11 Prozent beträgt. Der Trend der Benachteiligung von geschlechtlichen Minderheiten zeigt sich bei den Lehrabschlussprüfungen nicht so deutlich wie bei den Lehrvertragsauflösungen. Bei den Berufslehren, in denen die Minderheitsanteile kleiner als 5 Prozent sind, finden sich deutliche Hinweise auf die These, wonach Lernende an der Lehrabschlussprüfung weniger erfolgreich sind. Die höchste Misserfolgsquote bei den Frauen findet sich bei den 37 am stärksten segregierten Berufslehren, sie beträgt hier durchschnittlich knapp 12 Prozent. Von diesen 37 Berufslehren haben folgende 3 bei den Frauen eine deutlich höhere Misserfolgsquote als bei den Männern ,Gipser/in EFZ' (Misserfolgsquote Frauen 32\%), ,Polybauer/-in EFZ' (31\%) und ,Forstwart/-in EFZ' (26\%). Bei den Männern in frauenreichen Berufslehren ist die Misserfolgsquote in 3 Berufslehren sogar dreimal höher als bei den Frauen: ,Fachmann/-frau Hauswirtschaft EFZ' (Misserfolgsquote Männer 33\%), ,Pharma-Assistent/-in EFZ' (29\%) und ,Detailhandelsfachmann/-frau EFZ - Bäckerei/Konditorei/Confiserie ' (22\%). Diese Ergebnisse weisen darauf hin, dass etwas zuungunsten der Lernenden in geschlechtsuntypischen Berufslehren läuft. Es wäre vermessen, aus diesen Daten eine Diskriminierungshaltung der Prüfenden gegenüber Lernenden in geschlechtsuntypischen Berufslehren abzuleiten, vielmehr scheinen diese Lernenden den Anforderungen an der Lehrabschlussprüfung nicht zu genügen. Über den Grund für die ggf. weniger guten Vorbereitungen kann an dieser Stelle lediglich spekuliert werden. Das Modell der geschlechtsuntypischen Berufssozialisation besagt, dass aufgrund von Diskriminierungserfahrungen die Selbstwirksamkeitsüberzeugung und die Motivation leiden könnten (Althof 1992). In Form einer sich selbst erfüllenden Prophezeiung folgt schließlich in einigen Berufslehren der Misserfolg an der Lehrabschlussprüfung. 


\subsubsection{Berufliche Erfolge im Selbstbericht}

Mit den Paneldaten des Forschungsprojekts ,Familie Schule Beruf FASE B (Neuenschwander et al. 2012) wurde längsschnittlich überprüft, ob sich Lernende in geschlechtsuntypischen Berufslehren von Lernenden in geschlechtstypischen Berufslehren beim selbstberichteten Erfolg im Lehrbetrieb unterscheiden. Dazu wurden im zweiten und dritten Lehrjahr die ,Sozialisation im Lehrbetrieb“, das ,berufliche Fähigkeitsselbstkonzept', die ,Zufriedenheit mit der Ausbildung' und die ,wahrgenommene Passung zwischen den eigenen Interessen bzw. Fähigkeiten und den Anforderungen der Berufslehre gemessen. Die Varianzanalysen mit Zwei- und Viergruppenvergleichen ergeben keine signifikanten Interaktionseffekte zwischen den Gruppen. Nach dem Modell der geschlechtsuntypischen Berufssozialisation wäre zu vermuten, dass die Lernenden in geschlechtsuntypischen Berufslehren ihre beruflichen Erfolge tiefer einschätzen würden als andere Lernende und dies zu der erhöhten Lehrvertragsauflösungsquote beiträgt. Diese Hypothese konnte nicht bestätigt werden. Es scheint, dass diese Lernenden, wenn sie es bis ins dritte Lehrjahr erfolgreich schaffen, ihren Kolleginnen und Kollegen punkto Integration in den Lehrbetrieb, bei selbstberichteten Erfolgen und der Erarbeitung eines beruflichen Habitus ebenbürtig sind. Für das Modell der geschlechtsuntypischen Berufssozialisation heißt das, dass sich des untypischen Geschlechts nicht grundsätzlich negativ auf die beruflichen Erfolge auswirkt. Gemäß dem Modell sind für ein erfolgreiches Vorankommen in einer geschlechtsuntypischen Berufslehre ausreichende persönliche und soziale Ressourcen notwendig (vgl. Abschnitt 10.1.5) sowie ein wohlwollendes soziales Umfeld. Beispielsweise berichteten die Befragten in den Interviews von ihren Vorgesetzten, die sich stark für sie eingesetzt hatten.

\subsubsection{Einfluss des sozialen Umfelds}

Das Modell der geschlechtsuntypischen Berufssozialisation postuliert, dass nicht die Geschlechtstypik an sich für den geringeren Erfolg von Lernenden in geschlechtsuntypischen Berufslehren unmittelbar ursächlich ist, sondern die negativen Reaktionen des sozialen Umfelds. Um diese Hypothese zu testen, wurden Daten der für die Schweiz repräsentativen Langzeitstudie ,Transitionen von der Erstausbildung ins Erwerbsleben TREE' (TREE 2008) verwendet. Die verwendeten Angaben stammen von 904 Lernenden (840 typisch vs. 64 untypisch) im ersten, zweiten und dritten Lehrjahr.

Die Regressionsanalysen testeten, ob der Effekt von der Geschlechtstypik auf die Bewältigung durch die negativen und positiven Reaktionen des sozialen Umfelds mediiert wird. Die Analysen wurden nach Geschlecht getrennt durchgeführt. Der Mediationseffekt war bei den Frauen statistisch bedeutsam: 
Der Einfluss der Geschlechtstypik auf die Bewältigung wird durch Diskriminierung und soziale Unterstützung vermittelt. Während die Diskriminierungswahrnehmung sich negativ auf die Bewältigung auswirkte, war der Effekt bei der sozialen Unterstützung positiv. Bei den Männern sind die Ergebnisse tendenziell signifikant und gehen in die gleiche Richtung wie bei den Frauen. Damit ist ein wesentlicher Teil des Modells der geschlechtsuntypischen Berufssozialisation bewiesen. Die Reaktionen des sozialen Umfelds wirken sich auf die Bewältigung aus. Sie verursachen bei negativen Reaktionen Folgen wie Lehrvertragsauflösung (vgl. Abschnitt 10.1.1), teilweise Misserfolg an der Lehrabschlussprüfung (Abschnitt 10.1.2), psychosomatische Beschwerden und erhöhte Absenzen (vgl. unten). Positive Reaktionen wie soziale Unterstützung wirken in die andere Richtung. Sie zählen zu den Ressourcen und ermöglichen Lernenden in geschlechtsuntypischen Berufslehren eine erfolgreiche Integration in den Beruf. Sie verbleiben damit in der geschlechtsuntypischen Berufslehre bis zum Bestehen der Lehrabschlussprüfung.

In einer zweiten Analyse mit denselben Daten wurde untersucht, ob sich die Lernenden in geschlechtsuntypischen Berufslehren von den Lernenden in geschlechtstypischen Berufslehren bei den Folgen aufgrund der Diskriminierung unterscheiden. Als Folgen wurden psychosomatische Beschwerden und Absenzen in querschnittlichen $C h i^{2}$-Tests und längsschnittlich in einer angepassten Konfigurationsfrequenzanalyse untersucht. Die querschnittlichen Ergebnisse belegen, dass Lernende in geschlechtsuntypischen Berufslehren in allen drei Lehrjahren über signifikant mehr psychosomatische Beschwerde klagten sowie im zweiten und dritten Lehrjahr signifikant mehr Absenzen aufwiesen als Lernende in geschlechtstypischen Berufslehren. Die Ergebnisse der Verlaufsanalyse wiesen ebenfalls stabil erhöhte Beschwerden und Absenzen bei den Lernenden in geschlechtsuntypischen Berufslehren hin. Damit konnten alle Hypothesen in diesem Kapitel bestätigt werden. Die Reaktionen des sozialen Umfelds wirken sich auf die Befindlichkeit dieser Lernenden aus. Ich interpretiere dies als starken Hinweis für die Korrektheit der im Modell der geschlechtsuntypischen Berufssozialisation postulierten positiven und negativen Effekte des sozialen Umfelds auf die berufliche Entwicklung von Lernenden in geschlechtsuntypischen Berufslehren.

\subsubsection{Belastungen und Ressourcen}

Zwischen 2012 und 2014 führte ich mit 14 Lernenden in geschlechtsuntypischen Berufslehren leitfadengestützte, halbstrukturierte Interviews durch. Die Tonaufnahmen der Gespräche habe ich transkribiert und inhaltsanalytisch nach Mayring (2003) ausgewertet. Die Lernenden waren zwischen 17 und 30 Jahre alt und befanden sich im 1. bis 4. Lehrjahr. Die Befragten absolvierten 
eine EFZ-Lehre im Kanton Zürich zur Automobil-Fachfrau, AutomobilMechatronikerin, Informatikerin, Landwirtin, Logistikerin oder Spenglerin bzw. zum Coiffeur, Detailassistenten, Fachmann Betreuung, Gesundheit oder Hauswirtschaft.

Die Antworten der Lernenden wurden in vier Themenfelder kategorisiert: Berufsfindung, Doing Gender, Belastungen und Ressourcen. Das Ziel bestand darin, zu erfahren, wie Lernenden ihre spezifische Situation erleben. Schon im Berufsfindungsprozess war Geschlecht bei den meisten ein Thema. Einige verfolgten zielstrebig ihren Wunschberuf, der sich auf Kindheitserinnerungen zurückführen ließ oder weil sie sich schon immer dafür interessierten. Einige wenige sehen den gegenwärtigen Beruf als Zwischenstation, die sie gern in Kauf nehmen, um ihr eigentliches Berufsziel zu erreichen. Für eine dritte Gruppe glich die Berufsfindung eher einer Zufallswahl oder einer Notlösung. Bei den Lernenden dieser Gruppe stand das Thema Geschlecht weniger im Zentrum, z.B. war einem Lernenden nicht bewusst, dass sein Beruf kaum von Männern gelernt oder ausgeübt wird.

Da die interviewten Lernenden sich auf beruflicher Ebene außerhalb der gängigen Geschlechtsrollenbilder bewegten, betonen sie ihr Geschlecht gemäß den tradierten Bildern in anderen Bereichen wie Kleidung, Verhalten oder Sprache. Wie das Modell der geschlechtsuntypischen Berufssozialisation, zahlreiche Befunde (siehe Kapitel 3 und 4) und Theorien (Chusmir 1990, Flores et al. 2006, Kanter 1977, Williams 1993) zu diesem Thema bestätigen, waren die berichteten Belastungen vielfältig, teilweise geschlechtsspezifisch und selbstwertrelevant. Ausnahmslos alle berichten über Diskriminierungserfahrungen aufgrund des Geschlechts wie Abwertungen, sexistische oder homophobe Bemerkungen, Ausgrenzungen und Vorurteile. Hinzu kommen weitere Belastungen wie die Wahrnehmung, dass sie besonders, neu und ungewohnt für ihre Arbeitskolleginnen und -kollegen sind oder dass die manche ihrer wenigen Geschlechtsgenossinnen oder -genossen den Lehrvertrag auflösten. Anderen wiederum machte es zu schaffen, dass sie Arbeiten verrichten mussten, die sie selbst als nicht adäquat für ihr Geschlecht empfanden. Sie erlebten Geschlechtsrollenkonflikte (vgl. Unterkapitel 2.6).

Dass sich diese Lernenden noch in der geschlechtsuntypischen Berufslehre befanden, lässt auf ausreichend Ressourcen schließen. Die Persönlichkeit der Lernenden kann ihnen eine bedeutende Stütze sein, indem sie Widrigkeiten mit Humor, Schlagfertigkeit und Widerstandsfähigkeit begegnen. Ebenso betonten sie, wie wichtig Fürsprechende und handfeste Unterstützung waren. Eltern, Ausbildende und Lehrpersonen, aber auch enge Freundinnen und Freunde, unterstützten sie in ihren beruflichen Ambitionen und ermunterten sie, ihren Weg zu gehen. 


\subsection{Reflexion zur Sozialisation in geschlechtsuntypischen Berufslehren}

Die Entwicklung eines beruflichen Habitus (Schwiter et al. 2014) und damit die berufliche Sozialisation (Lempert 2002) verläuft für Lernende in geschlechtsuntypischen Berufslehren unter speziellen Vorzeichen. Aufgrund des nach wie vor stark segregierten Arbeitsumfelds (Borkowsky 2000) befinden sie sich in einer Minderheit. Einerseits kann bereits die Minderheitsposition an sich zu Stress führen (Meyer et al. 2015), andererseits kommt es in geschlechtstypischen Berufen für die Minderheit zu Geschlechtsrollenkonflikten (Rustemeyer/Thrien 2001, O’Neil 2015).

Seit den 70er-Jahren sind Personen in geschlechtsuntypischen Berufslehren und ihre spezielle Situation wissenschaftlich untersucht worden (vgl. Kapitel 3, Kanter 1977, Simpson 2005, Williams 1993). Ein immer wieder festgestellter Befund beschreibt, dass diese Personen nicht lange im geschlechtsuntypischen Beruf bleiben. Dies nannte Jacobs (1993) den DrehtürEffekt. Hinweise für diesen Effekt fanden sich in dieser Arbeit bei der erhöhten Zahl von Lehrvertragsauflösungen und in den Interviewaussagen, wonach die Befragten anfänglich gleichgeschlechtliche Mitlernende in der Berufsfachschule hatten. Die meisten davon brachen die Berufslehre vorzeitig ab. Aufgrund der Datenlage lässt sich jedoch nicht eruieren, was die Lernenden mit Vertragsauflösungen im Anschluss gemacht haben. Immerhin theoretisch besteht die Möglichkeit, dass sie die geschlechtsuntypische Ausbildung an einem anderen Ort wieder aufgenommen haben.

Ein weiterer Effekt betrifft die Laufbahn. So wurde vielfach das Stagnieren der Karriere von Frauen mit dem Anstoßen an einer gläsernen Decke (z.B. Cotter et al. 2001) umschrieben, während bei Männern das Umgekehrte einsetzte. Williams (1992) nannte das Hinaufbefördern in eine geschlechtsadäquate Position den Glaslift-Effekt. Bestätigung für den Glaslift-Effekt bei männlichen Jugendlichen fand sich bei den Interviewaussagen, wonach sie leicht eine Lehrstelle fanden oder aussichtsreiche Anstellungsmöglichkeiten nach der Berufslehre erwarten. Die Aussagen sind jedoch eher spekulativ und stehen im Gegensatz zu der erhöhten Wahrscheinlichkeit von Lehrvertragsauflösung und Misserfolg an der Lehrabschlussprüfung in einigen Berufslehren.

Die von Kanter (1977) in der Tokenismus-Theorie beschriebene Polarisierung fand sich in Aussagen der Interviewten, die von Überlegungen der Vorgesetzten oder Ausbildenden zu den positiven Veränderungen des Umgangs beispielsweise in Autowerkstätten handelten. Umgangsformen von reinen Männergruppen scheinen sich zu ändern, wenn eine Frau ins Team eintritt. Obwohl dies in der Beschreibung aus Sicht der Lernenden positiv 
verlief, könnte hier eine Ursache für negative Reaktionen liegen. Nicht alle sind bereit, alte Angewohnheiten abzulegen.

Erfolg während der Berufslehre kann auf vielfältig Art gemessen werden: Fähigkeiten, Kompetenzen, Abschlussnoten, Durchhaltewille, Einstellung, Passung, Gehalt, Absenzen etc. (vgl. OECD 2000, Häfeli/Schellenberg 2009a 2009b). Das Komponentenmodell des Erfolgs in der Berufslehre (Dette et al. 2004) ermöglicht eine Systematisierung. Es ist in der vorliegenden Arbeit gelungen, aus allen relevanten Feldern des Komponentenmodells Erfolgsfaktoren $\mathrm{zu}$ überprüfen. Dabei ist wichtig $\mathrm{zu}$ sehen, dass Lernende in geschlechtsuntypischen Berufslehren erfolgreich sein können. Wie die Auswertungen zu den FASE B-Daten eindrücklich belegen, können sich diese Lernenden genau gleich wie ihre andersgeschlechtlichen Kolleginnen und Kollegen in den Beruf integrieren. Sie entwickeln in diesem Sinn einen beruflichen Habitus, der punkto Zufriedenheit, Selbstkompetenz und Passung den anderen in nichts nachsteht. Damit differenziert sich das Modell der geschlechtsuntypischen Berufssozialisation insofern, als sich das untypische Geschlecht nicht auf selbstberichtete Erfolgsvariablen auswirkt. Diese Lernenden berichten, dass sie sich als passend erleben, zufrieden sind und berufliche Kompetenz aufbauen können. Dieser Befund könnte eine Fortführung der im Modell der geschlechtsuntypischen Berufsfindung von Flores et al. (2006) notwendigen Voraussetzungen für die geschlechtsuntypische Berufsfindung sein: Selbstwirksamkeit und Interesse für den geschlechtsuntypische Beruf.

Doch es lassen sich auch die Spuren der Andersbehandlung erkennen. So belegen die Analysen mit den TREE-Daten, dass die Lernenden in geschlechtsuntypischen Berufslehren stärker mit psychosomatischen Beschwerden wie Kopfschmerzen oder Schlafproblemen zu kämpfen haben und evtl. deshalb auch mehr Absenzen aufweisen als Lernende in geschlechtstypischen Berufslehren. Die geschlechtsuntypische Situation und mit ihr die Erfahrung von Ausgrenzung und Andersartigkeit führt wie im Modell der geschlechtsuntypischen Berufssozialisation postuliert zu einer geringeren Verbundenheit mit den Personen im Lehrbetrieb.

Mittels der 5 Dimensionen der Geschlechtsidentität (Zugehörigkeit, Zentralität, Zufriedenheit, Druck und Typikalität) nach Tobin et al. (2010) kann die Situation von Lernenden in geschlechtsuntypischen Berufslehren beschrieben werden. Obwohl sie sich eindeutig einem Geschlecht zugehörig fühlen, kann sich aufgrund der Wahrnehmung von geschlechtsuntypischen Interessen und Fähigkeiten bei ihnen selbst und aufgrund der Wichtigkeit des Geschlechts gegenüber anderen Identitäten Unzufriedenheit einstellen. Sie spüren einen großen Druck, sich geschlechtskonform zu verhalten. Insbesondere für männliche Jugendliche ist der Druck groß, den tradierten Geschlechtsrollenstereotypen zu entsprechen (vgl. Hartmann/Trautner 2009).

Die gesellschaftlichen Ausgrenzungsmechanismen beim Übertreten der tradierten Geschlechtsrollenvorstellungen werden als Spitze des Eisbergs in 
der bis zu viermal höheren Suizidalität von homosexuellen Jugendlichen sichtbar (vgl. Leu 2008, Savin-Williams 2001, Savin-Williams/Ream 2003). Diese spezifischen Befunde für die Situation von homosexuellen Jugendlichen lassen sich nicht direkt auf die Situation der Lernenden in geschlechtsuntypischen Berufslehren übertragen. Dennoch sind bei ihnen ähnliche Mechanismen festzustellen. Sie sind, allenfalls in abgeschwächter Form, als soziale Minderheiten von Stigmatisierung betroffen. Wie in der vorliegenden Arbeit belegt, wirken sich die sozialen Ausgrenzungsmechanismen auf Jugendliche in geschlechtsuntypischen Berufslehren auf psychosomatische Beschwerden, Absenzen, Lehrvertragsauflösungen und Misserfolg bei den Lehrabschlussprüfung aus.

Das Modell der geschlechtsuntypischen Berufssozialisation beschreibt die Mechanismen von Lernenden, die entgegen der Geschlechtsrollenerwartung und der Segregation des Arbeitsmarkts einen geschlechtsuntypischen Beruf erlernen. Sie erfüllen die Erwartungen ihres sozialen Umfelds nicht und bekommen das zu spüren. Die Analyse der Interviews konnte eine breite Palette an Diskriminierungserfahrungen herausarbeiten. Die negativen Reaktionen des sozialen Umfelds müssen bewältigt werden und führen dazu, dass dadurch berufsrelevante Aspekte zu kurz kommen.

Wie die Lernenden die Reaktionen des sozialen Umfelds bewältigen, ist entscheidend. Gelingt es ihnen, diskriminierendes Verhalten anderer ihnen gegenüber abzuwehren, indem sie beispielsweise humorvoll oder schlagfertig reagieren (vgl. Abschnitt 9.3.3), können sie vor den anderen, aber auch vor sich selbst integer bleiben. Für Ausbildende, die mit der Einstellung einer/eines geschlechtsuntypischen Lernenden ein Interesse haben, dass sich die Investitionen auszahlen, gilt es, den Selbstwert und die Selbstwirksamkeit ihrer Lernenden zu erhalten. Wie wichtig dies ist, zeigen Forschungsarbeiten dazu. Zwei Studien zur Selbstbejahung hatten im schulischen Kontext bei marginalisierten Gruppen (Afroamerikaner in den USA) derart durchschlagende Wirkung, dass noch zwei Jahre nach der Intervention bessere Schulleistungen in der Experimentalgruppe zu verzeichnen waren als in der Kontrollgruppe (Cohen et al. 2006, 2009). Es ging zusammengefasst darum, die Selbstabwertungsspirale zu durchbrechen (vgl. Spitzer 2009). Mittels der Vergegenwärtigung dessen, was diese Jugendlichen gerne tun und gut können, wurde Selbstwirksamkeit real erfahren. Die Investition der Jugendlichen hat sich in sichtbaren Fortschritten offenbart. Diese positive Erfahrung haben sie dann selbstständig auf andere Gebiete übertragen. Zusätzlich hat sie der so gestärkte Selbstwert quasi gegen anderslautende Anspielungen anderer imprägniert. Es könnte ihnen daher gelingen, ihre Erfolge und Misserfolge selbstwertdienlich zu attribuieren und so im geschlechtsuntypischen Umfeld zu bestehen. Diese Erkenntnisse könnten direkt in die Unterstützungsleistungen von Ausbildenden einfließen. Ausführlicher geht das folgende Unterka- 
pitel 10.3 auf die Umsetzung der Erkenntnisse aus der vorliegenden Forschungsarbeit ein.

\subsection{Perspektiven geschlechtsuntypischer Sozialisationsprozesse}

Zur Beschreibung der Perspektiven, die sich für die Sozialisation in geschlechtsuntypischen Berufslehren eröffnen, folge ich dem Modell der geschlechtsuntypischen Berufssozialisation. Dabei wird offenbar, dass Veränderungen auf der Makro- und der Mikroebene denkbar sind. Während gesellschaftliche Prozesse langfristig einen Wandel vollziehen können, dabei jedoch nur schwer steuerbar sind, gibt es auf der interindividuellen Ebene Gestaltungsmöglichkeiten.

Das Modell der geschlechtsuntypischen Berufssozialisation beschreibt als Ausgangslage einen sich selbst stützenden Kreislauf von geschlechtlicher Segregation und Geschlechtsrollenerwartungen (vgl. Unterkapitel 4.2), auf dessen Grundlage die Berufsfindungsprozesse ablaufen. Maßnahmen auf der gesellschaftlichen Ebene schlagen beispielsweise Schwiter et al. (2014) vor. Sie folgern aufgrund ihrer Analysen, dass es ,,verstärkte politische Anstrengungen [braucht], Berufe hinsichtlich Ausbildungsweg, Arbeitsorganisation, Arbeitszeiten und Entlohnung so zu gestalten, dass sie ein breites Spektrum an unterschiedlichen Berufs- und Lebensbiografien erlauben - für Frauen und Männer“ (Schwiter et al. 2014: 423). Die langfristigen Maßnahmen bedingen, dass ein politischer und gesellschaftlicher Wille vorhanden ist, die Arbeitsverteilung zwischen Frauen und Männern zu verändern.

Ein erster Ansatzpunkt auf der Individualebene bietet sich beim Berufsfindungsprozess an. Würden sich mehr Jungen und Mädchen für einen traditionell gegengeschlechtlich konnotierten Beruf entscheiden, würden sich die Geschlechterverhältnisse im Laufe der Zeit ändern. Jedoch sind die bisherigen Bemühungen von wenig Erfolg gekrönt, wie Rahn und Hartkopf (2016: 128f) zusammenfassen: „Die berufswahltheoretisch begründete Skepsis gegenüber der Hoffnung, durch einzelne Interventionen die geschlechtsspezifischen Orientierungsmuster aufbrechen $\mathrm{zu}$ können, erweist sich für beide Geschlechter auch empirisch als begründet".

Insbesondere den schulischen Berufsorientierungen gelingt es nach Faulstich-Wieland (2016) nicht oder noch nicht, mit ihren Maßnahmen mehr Jugendliche für einen geschlechtsuntypischen Beruf zu begeistern. Ob dies damit zu tun hat, dass die Genderfrage eher stereotyp angegangen wird, muss sie offen lassen. Nichtsdestoweniger wären ihren Ausführungen zufolge pädagogische Unterstützungen sinnvoll. Scholand (2016) zieht ebenfalls den Schluss, dass die Berufsfindung auf dem Hintergrund von geringen Kenntnis- 
sen abläuft und dass die geschlechtliche Codierung der Berufe im Berufsfindungsprozess, neben Prestige und Anforderungsniveau, eine zentrale Rolle spielt. Sie fordert nicht nur eine Didaktik, die Geschlecht mitdenkt, sondern auch verstärkte Wissensvermittlung als Grundlage für die Berufsfindung: „Neben einer gendersensiblen Didaktik wäre jedoch ein systematischer Wissensaufbau eine wichtige Voraussetzung für ein ,undoing circumscription““ (Scholand 2016: 80).

Während die oben genannten Aspekte vor allem die Einmündung in einen geschlechtsuntypischen Beruf thematisieren, sind im Modell letztlich nicht die gesellschaftlichen Prozesse und Schließmechanismen für die negativen Auswirkungen verantwortlich, sondern die diskriminierenden Erfahrungen im sozialen Umfeld, die Lernende in geschlechtsuntypischen Berufslehren machen. Dies eröffnet pragmatische Perspektiven auf die Verbesserung der Situation von Lernenden in geschlechtsuntypischen Berufslehren. Die Befunde in Kapitel 7 unterstreichen, dass diese Lernenden durchaus erfolgreich den Übergang ins Berufsleben meistern. Die folgenden Überlegungen zielen also insbesondere darauf, die Bewältigungshandlungen zu stärken, die aufgrund der negativen Reaktionen des sozialen Umfelds notwendig werden.

Vorgesetzten und Ausbildenden kommt eine zusätzliche Funktion zu. Damit eine Integration gelingt, ist es nicht nur wichtig, an Lernende in geschlechtsuntypischen Berufslehren die gleichen berufsspezifischen Anforderungen wie an alle Lernenden zu stellen, sondern genauso, ihnen dabei zu helfen, mit den spezifischen Herausforderungen umzugehen. Dazu gehört beispielsweise der Schutz vor dummen Sprüchen, unfairen Bemerkungen und unzulässigen Zuschreibungen. Wie die Interviewten bezeugen, ist die Unterstützung durch die Vorgesetzten und Ausbildenden willkommen und auch hilfreich. Neben dem Stellungbeziehen gegenüber Mitarbeitenden kann das heißen, ins Gespräch mit den Lernenden zu kommen: ihren Erfahrungen Raum geben, versuchen, Erfolg und Misserfolg selbstwertdienlich zu attribuieren, über die ewiggestrigen Sprüche zu lachen, ihnen Mut und Zuversicht zu geben, ihnen mitzuteilen, dass ihnen zugetraut wird, eine hervorragende Berufsfrau, ein hervorragender Berufsmann $\mathrm{zu}$ werden. Insbesondere ist wichtig, bei Schwierigkeiten nicht das Geschlecht an sich, sondern die Verstrickungen mit dem Geschlecht zu besprechen.

Zur Stärkung der Bewältigungshandlungen gehört auch die Aktivierung von Ressourcen. Dazu gehört, neben Kompetenzen und Persönlichkeitseigenschaften der Lernenden selbst, die soziale Unterstützung aus ihrem Umfeld. Sollten Eltern nicht bereits voll hinter ihren Kindern stehen, gilt es vor allem sie als Verbündete zu gewinnen, z.B. indem herausgestellt wird, wie gut ihre Tochter, ihr Sohn sich in den Lehrbetrieb integriert und die Anforderungen erfüllen kann.

Ein Fokus muss auf die unbedachten Äußerungen von anderen gelegt werden. Vielen Personen, die sich scherzhaft gegenüber Personen in ge- 
schlechtsuntypischen Berufen äußern, scheint nicht bewusst zu sein, was die Häufung von lustig gemeinten Verunglimpfungen bewirkt. Auch wenn sie damit die eigene Überraschung oder Verunsicherung überspielen, zementieren sie mit ihrem Handeln das tradierte Geschlechtsrollenbild und damit immer wieder die Aussage: „Du bist falsch hier. Du kannst es nicht, weil du eine Frau / ein Mann bist.“"Eltern, Ausbildende und Lehrpersonen sind gefordert, ihre eigenen Vorstellungen und tradierten Geschlechtsrollenbilder zu überdenken und insbesondere $\mathrm{zu}$ hinterfragen, in welchen Situationen sie diese Vorstellungen mitteilen. Dies reicht von der Verwendung beider Berufsbezeichnungen über die Nennung beider Geschlechter in allen Zusammenhängen bis zum Unterlassen entwertender Hilfsangebote. Nichtsdestoweniger können Lernende in geschlechtsuntypischen Berufslehren darin bestärkt werden, sich zu wehren, sich Kontersprüche auszudenken und einen Weg zu finden, um die Situation durch gemeinsames Lachen zu entschärfen.

Die Erkenntnisse zu den Lehrvertragsauflösungen belegen, dass der Durchhaltewille gestärkt werden könnte. Frühzeitiges Ansprechen von Schwierigkeiten, wenn noch keine unlösbaren Probleme auftreten, kann Lehrvertragsauflösungen vermeiden (Schmid/Stalder 2008). Lernende im Pubertätsalter fällt es schwer, langfristig zu denken. Sie brauchen Anleitung, um ihre Investitionen als ertragreich zu erkennen. Dabei kann es helfen, Konfrontationen mit viel Verständnis und Unterstützung aufzufangen. Wichtig ist es, immer wieder berufliche und private Ziele zu besprechen, ihnen Mut zu machen, ihre Zuversicht und ihren Glauben an sich selbst zu stärken. Maßnahmen sollen an den Stärken ausgerichtet sein und nicht die Schwächen in den Vordergrund stellen (vgl. Cohen et al. 2006, 2009).

Wie die Auswertungen zum Erfolg bei der Lehrabschlussprüfung belegen, sind insbesondere männliche Jugendliche von Misserfolg betroffen. Diese benötigen bei der Vorbereitung der Lehrabschlussprüfung mehr Unterstützung. Möglicherweise lernen sie zu wenig, weil auch sie von Rollenbildern geprägt sind: „Ein Junge/Mann lernt nicht, er kann es, oder es ist nicht wichtig, das zu können." Vielleicht fällt es ihnen einfach schwer, selbstständig den Stoff zu ordnen, Lernstrategien zu entwickeln und rechtzeitig mit den Vorbereitungen zu beginnen. Ausbildende sind am nächsten an den Lernenden und können hier ansetzen. Es obliegt den Lehrpersonen der Berufsfachschulen, den Stoff nicht nur zu vermitteln, sondern auch Strategien zur Erarbeitung von Wissen zu trainieren.

Eine Handlungsebene bietet sich den Organisationen der Arbeitswelt, Berufsverbänden und Berufsfachschulen und betrifft die hohen Lehrvertragsauflösungsquoten der Lernenden in geschlechtsuntypischen Berufslehren und ihre teils hohen Misserfolgsquoten bei der Lehrabschlussprüfung. Die vorliegende Arbeit kann zwar keine konkreten Gründe für die hohen Quoten nennen oder Maßnahmen aufzählen, um diese zu beheben, aber immerhin liegen Zahlen vor, die ausweisen, welche Berufslehren besonders stark betroffen 
sind (vgl. Online-Anhang I und VII). Dies bietet auch die Möglichkeit, Berufslehren für ein Best Practice zu eruieren.

Die Arbeitswelt lässt sich nicht von heute auf morgen umkrempeln. Die geschlechtliche Segregation des Arbeitsmarkts bleibt vermutlich noch lange eine Grundkonstante. Handlungsmöglichkeiten bieten sich hingegen bei der Unterstützung derjenigen, die nicht auf den Pfaden der tradierten Geschlechterrollen gehen wollen. Egal ob Frau oder Mann, wer sich für einen Beruf interessiert und sich dafür geeignet fühlt, soll nicht nur die Gelegenheit bekommen, allen zu zeigen, dass sie oder er die notwenigen Kompetenzen und Fähigkeiten entwickeln kann, sondern auch die volle Unterstützung seines Umfelds erhalten. Es gibt genügend Herausforderungen für diese Jugendlichen, wir müssen es ihnen nicht unnötig schwer machen, sondern sollten sie dabei unterstützen, ihre Ziele zu erreichen und Ambitionen zu stärken.

\subsection{Limitationen und Ausblick}

Einige Restriktionen sind bezüglich der Datengrundlage zu machen. Bei den Auswertungen zu den Lehrvertragsauflösungen und den Lehrabschlussprüfungen konnte auf schweizerische Gesamtpopulationsdaten des BFS zurückgegriffen werden. Diese Daten sind querschnittlich und aggregiert, also nicht mit individuellen Daten verbunden. Sie sind deskriptiv und erlauben keine weitergehenden Interpretationen. Bei der Zusammenführung von mehreren Jahren war unvermeidbar, dass einzelne Personen mehrfach gezählt wurden. Insbesondere bei den Wiederholungen von Lehrabschlussprüfungen sind die Wahrscheinlichkeiten eines erneuten Misserfolgs erhöht (Amos et al. 2003a 2003b). Da die meisten Lehrvertragsauflösungen erfreulicherweise nicht mit einem Abbruch der Ausbildung verbunden sind, sondern meist die Ausbildung in irgendeiner Form wieder aufgenommen wird, ist bei einer Quote von über 20 Prozent die Anzahl der Doppelzählungen hoch.

Bei den Paneldaten der Forschungsprojekte FASE B und TREE ist zu erwähnen, dass die Stichprobenanzahlen in den Minderheitengruppen klein sind. Insbesondere da die Berufsbildung kleinräumig und hoch spezifisch in Berufslehren unterteilt ist, würden sich Mehrebenenanalysen anbieten. Diese sind jedoch nur mit einer erheblich größeren Stichprobe möglich.

Der schweizerische Rahmen könnte bei der Verallgemeinerung der Ergebnisse einflussreich sein, da die Berufsbildung stark ausgebaut und hoch angesehen ist (Wettstein/Gonon 2009). Bei der gesellschaftlichen Öffnung punkto Gleichberechtigung und Arbeitsteilung liegt die Schweiz im westeuropäischen Vergleich jedoch zurück (Buchmann/Kriesi 2009).

In einer verallgemeinerten Betrachtungsweise könnte das Modell der geschlechtsuntypischen Berufssozialisation für weitere Situationen stehen, in 
denen eine Person nicht den gesellschaftlichen Erwartungen entspricht. So läge auf der Hand, den Titel, Modell der Nonkonformität $t^{\star}$ zu verwenden, der an die angloamerikanische Bezeichnung, non-traditional' für geschlechtsuntypisch anschließen würde. Die Überprüfung einer erweiterten Gültigkeit obliegt zukünftigen Forschungsarbeiten. Zusätzlich sollte geprüft werden, ob die Annahmen im Modell in beruflichen Zusammenhängen außerhalb der Berufsbildung bzw. außerhalb der Schweiz ähnlich wirksam sind. Zudem konnte das Modell nicht als Ganzes überprüft werden, sondern lediglich Teilbereiche. Pfadanalysen könnten Hinweise darauf geben, ob sich die Mediation der Geschlechtstypik über die Diskriminierungserfahrung auf die Bewältigung direkt oder indirekt auf die Folgen auswirkt.

Befragung von Jugendlichen in geschlechtstypischen Berufslehren und vor allem von Jugendlichen, die eine Lehrvertragsauflösung oder einen Misserfolg bei der Lehrabschlussprüfung hinter sich haben, könnten weitere Aufschlüsse darüber geben, wie sich das untypische Geschlecht auf die berufliche Sozialisation auswirkt. Zu berücksichtigen ist, dass - insbesondere bei den Lernenden in geschlechtsuntypischen Berufslehren - sowohl in den längsschnittlichen Analysen als auch in den Interviews diejenigen Lernenden untersucht wurden, die die Berufslehre erfolgreich absolvierten und mit den gesellschaftlichen Reaktionen wenig konfrontiert wurden oder einen für sie persönlich bewältigbaren Umgang fanden. 


\section{Literaturverzeichnis}

Abraham, Martin/Arpagaus, Jürg (2008): Wettbewerb, soziales Umfeld oder gezielte Lebensplanung? Determinanten der horizontalen Geschlechtersegregation auf dem Lehrstellenmarkt. Soziale Welt 59, 3, S. 205-225.

Aeschlimann, Belinda (2014): Berufswahl und Geschlecht: Eine Untersuchung zur Bedeutung von schulischen Faktoren für Berufs- und Studienwahlen von Schülerinnen und Schülern der Sekundarstufe II. Bern: Universität Bern.

Aiken, Lewis Roscoe (1996): Assessment of intellectual functioning (2. Aufl.). New York: Plenum Press.

Allport, Gorden W. (1971): Die Natur des Vorurteils. Köln: Kiepenheuer und Witsch.

Alpers, Georg W. (2005): Ressourcenorientierung. In: Petermann, Franz/Reinecke, Hans (Hrsg.): Handbuch der Klinischen Psychologie und Psychotherapie (Bd. 1). Göttingen: Hogrefe. S334-342.

Althoff, Heinrich (1992): Frauen in Männer-, Männer in Frauenberufen. Weibliche und männliche Jugendliche als Minderheiten in Ausbildungsberufen. Berufsbildung in Wissenschaft und Praxis 21, 4, S. 23-30.

Amos, Jacques/Amsler, Felix/Martin, Margret (2003a): Evaluation der Resultate von Abschlussprüfungen der beruflichen Grundausbildung. Basel: Büro für Kommunikation.

Amos, Jacques /Amsler, Felix/Martin, Margret/Metzger, Christoph (2003b): Evaluation von Abschlussprüfungen der beruflichen Grundausbildung Gelap. Berufsübergreifender Bericht. Basel: Bundesamt für Berufsbildung und Technologie BBT.

Bammé, Arno/Holling, Eggert/Lempert, Wolfgang (1983): Berufliche Sozialisation. Ein einführender Studientext. München: Hueber.

Bammer, Anton (1979): Rezession und Erwiderung auf E. Borneman „Das Patriarchat. Ursprung und Zukunft unserer Gesellschaft“. Hephaistos, 1, S. 152-160.

Bandura, Albert (1977a): Self-efficacy: Toward a unifying theory of behavioral change. Psychological Review 2, S. 191-215.

Bandura, Albert (1977b): Social learning theory. Englewood Cliffs, N.J.: Prentice-Hall.

Bandura, Albert (1986): Social foundations of thought and action: A social cognitive theory. Englewood Cliffs, NJ: Prentice-Hall.

Bandura, Albert (1997): Self-efficacy: The exercise of control. New York, NY: Freeman.

Bartlett, Helene P./Hind, Patricia/Taylor, Heidi R. (1999): A comparison of the career aspirations of degree and P2000 diploma graduates from UK nursing programmes. Journal of Nursing Management 7, S. 37-43.

Bauer, Joachim (2010): Das Gedächtnis des Körpers. Wie Beziehungen und Lebensstile unsere Gene steuern. Köln: Eichborn.

Baumert, Jürgen/Lehmann, Rainer (1997): TIMSS - mathematisch-naturwissenschaftlicher Unterricht im internationalen Vergleich. Deskriptive Befunde. Opladen: Leske und Budrich.

Beauvoir, Simone de (1956): Das andere Geschlecht. Sitte und Sexus der Frau. Hamburg: Rowohlt.

Bem, Sandra L. (1974): The Measurement of Psychological androgynous. Journal of Consulting and Clinical Psychology 42, S. 155-162.

Berenbaum, Sheri A./Korman Bryk, Kristina L. (2008): Biological Contributors to Gendered Occupational Outcome: Prenatal Androgen Effects on Predictors of Outcome. In: Watt, Helen M. G./Eccles, Jacquelynne S. (Hrsg.): Gender and Occupational Outcomes. Washington, D.C.: American Psychological Association, S. 235-264.

Bertossa, Luca/Meyer Schweizer, Ruth/Haltiner, Karl W. (2008): Werte und Lebenschancen im Wandel. Eine Trendstudie zu den Lebens-, Bildungs-, Arbeits- und Politikorientierungen junger Erwachsener in der Schweiz. Zürich: Rüegger.

BFS (2008): Statistik der beruflichen Grundbildung 2008. Neuchâtel: Bundesamt für Statistik.

BFS (2011): Statistik der beruflichen Grundbildung 2010. Neuchâtel: Bundesamt für Statistik. 
BFS (2016): Lernende nach Bildungsstufe und Bildungstyp 2013/14. http://www.bfs.admin.ch [Zugriff: 30.05.2016]

Bielby, William/Baron, James N. (1986): Men and Women at Work: Sex Segregation and Statistical Discrimination. American Journal of Sociology 91, 4, S. 759-799.

Bieri Buschor, Christine/Berweger, Simone/Keck Frei, Andrea/Kappler, Christa (2012): „Geschlecht(un-)typische" Studienwahl: Weshalb Frauen Ingenieurwissenschaften studieren und Männer Primarlehrer werden. Projektbericht. Zürich: Pädagogische Hochschule Zürich, Abteilung Forschung und Entwicklung.

Bischof-Köhler, Doris (2004): Von Natur aus anders. Die Psychologie der Geschlechtsunterschiede. Stuttgart: Kohlhammer.

Blank-Mathieu, Margarete (2001): Sozialisation, Selbstkonzept und Entwicklung der Geschlechtsidentität bei Jungen im Vorschulalter. Tübingen: Fakultät für Sozial- und Verhaltenswissenschaften der Eberhard-Karls-Universität Tübingen.

Bohlinger, Sandra (2002): Ausbildungsabbruch - Forschungsstand eines bildungspolitischen Problemfelds. In: Bohlinger, Sandra/Jenewein, Klaus (Hrsg.):Ausbildungsabbrecher - Verlierer der Wissensgesellschaft? Konzepte, Risiken und Chancen aktueller Handlungsansätze aus der Berufsbildungsforschung und -praxis. Bielefeld: Bertelsmann, S. 27-37.

Boltanski, Luc/Thévenot, Laurent (2007): Über die Rechtfertigung eine Soziologie der kritischen Urteilskraft. Hamburg: Hamburger Edition.

Borkowsky, Anna (2000): Frauen und Männer in der Berufsbildung der Schweiz. Schweizerische Zeitschrift für Bildungswissenschaften 22, 2, S. 279-294.

Borneman, Ernest (1981): Das Patriarchat. Ursprung und Zukunft unseres Gesellschaftssystems. Frankfurt a.M.: Fischer.

Bosinski, Hartmut A. G. (2000): Determinanten der Geschlechtsidentität. Neue Befunde zu einem alten Streit. Sexuologie 7, 2/3, S. 96-140.

Bourdieu, Pierre (1983): Ökonomisches Kapital, kulturelles Kapital, soziales Kapital. In: R. Kreckel (Hrsg.):Soziale Ungleichheiten. Göttingen: Schwartz, S. 183-220.

Bourdieu, Pierre (1997): Außer der Reihe. Männliche Herrschaft revisited. Feministische Studien 2, S. 88-99.

Bourdieu, Pierre (2005, 2012): Die männliche Herrschaft. Frankfurt a.M.: Suhrkamp.

Bradley, Harriet (1993): Across the Great Divide: The Entry of Men Into „Women's Jobs”. In: Williams, Christine L. (Hrsg.): Doing „Women's Work”. Men in Nontraditional Occupations. Newbury Park: SAGE, S. 10-27.

Brandt, Oliver/Cornelissen, Waltraud (2004): Berufsfindung in einer geschlechterkodierten Welt. Praxistheoretische Ansätze können der Berufsfindung neue Impulse geben. Zeitschrift für Frauenforschung und Geschlechterstudien 22, 4, S. 21-38.

Bright, Jim E. H./Pryor, Robert G. L. (2005): The Chaos Theory of Careers: A User's Guide. The Career Development Quarterly, 53, S. 291-305.

Bronfenbrenner, Urie (1981): Die Ökologie der menschlichen Entwicklung. Natürliche und geplante Experimente. Stuttgart: Klett.

Brüggemann, Tim/Rahn, Sylvia (2013): Berufsorientierung. Ein Lehr- und Arbeitsbuch. Münster: Waxmann.

Buchmann, Marlis/Kriesi, Irene (2009): Escaping the gender trap. Young women's transition into nontraditional occupations. In: Schoon, Ingrid/Silbereisen, Rainer (Hrsg.): Transitions from school to work: globalization, individualization, and patterns of diversity. Cambridge: Cambridge University Press, S. 193-216.

Buchmann, Marlis/Kriesi, Irene (2012): Geschlechtstypische Berufswahl: Begabungszuschreibung, Aspiration und Institutionen. Kölner Zeitschrift für Soziologie und Sozialpsychologie, Sonderheft, 52, S. 256-280.

Buchmann, Marlis /Kriesi, Irene /Pfeifer, Andrea/Sacchi, Stefan (2002): Halb drinnen - halb draußen: Zur Arbeitsmarktintegration von Frauen in der Schweiz. Zürich: Rüegger. 
Budde, Jürgen/Scholand, Barbara/Faulstich-Wieland, Hannelore (2008): Geschlechtergerechtigkeit in der Schule. Eine Studie zu Chancen, Blockaden und Perspektiven einer gendersensiblen Schulkultur. Weinheim: Juventa.

Bühler, Elisabeth/Heye, Corinna (2005): Fortschritte und Stagnation in der Gleichstellung der Geschlechter 1970-2000. Eidgenössische Volkszählung 2000. Neuchâtel: Bundesamt für Statistik.

Buschmeyer, Anna (2013): Zwischen Vorbild und Verdacht. Wie Männer im Erzieherberuf Männlichkeit konstruieren. Wiesbaden: VS.

Bussey, Kay/Bandura, Albert (1999): Social cognitive theory of gender development and differentiation. Psychological Review, 106, S. 676-713.

Busshoff, Ludger (2009): Berufsberatung als Unterstützung von Übergängen in der beruflichen Entwicklung. In: Zihlmann, René (Hrsg.): Berufswahl in Theorie und Praxis. Konzepte zur Berufswahlvorbereitung und Beratung unter veränderten wirtschaftlichen und gesellschaftlichen Bedingungen. Bern: SDBB, S. 9-77.

Butler, Judith (1991): Das Unbehagen der Geschlechter. Frankfurt a.M.: Suhrkamp.

Charles, Maria/Grusky, David B. (2004): Occupational Ghettos: The World-wide Segregation of Women and Men. Stanford, CA: Stanford University Press.

Charles, Maria (2005): Entwicklung der beruflichen Segregation nach Geschlecht und nach Staatsangehörigkeit in der Schweiz 1970-2000. Neuchâtel: Bundesamt für Statistik.

Chhin, Cristina S./Bleeker, Martha. M./Jacobs, Janis E. (2008): Gender-Typed Occupational Choices: The Long-Term Impact of Parents' Beliefs and Expectations. In: Watt, Helen M. G./Eccles, Jacquelynne S. (Hrsg.): Gender and Occupational Outcomes. Washington, D.C.: American Psychological Association, S. 215-234.

Christmas-Best, Verona E./Schmitt-Rodermund, Eva (2001): Adolescents' Career Choices in East and West Germany After Reunification. American Behavioral Scientist 44, 11, S. 1879-1898.

Chung, Y. Barry/Harmon, Lenore W. (1994): The career interests and aspirations of gay men: How sex role orientation is related. Journal of Vocational Behavior 45, 223-239.

Chusmir, Leonard H. (1990): Men Who Make Nontraditional Career Choice. Journal of Counseling and Development 69, 1, S. 11-16.

Cognard-Black, Andrew J. (2004): Will They Stay, or Will They Go? Sex-Atypical Work among Token Men Who Teach. The Sociological Quarterly 45, 1, S. 113-139.

Cohen, Geoffrey L./Garcia, Julio/Apfel, Nancy/Master, Allison (2006): Reducing the Racial Achievement Gap: A Social-Psychological Intervention. Science 313, S. 1307-1310.

Cohen, Geoffrey L./Garcia, Julio/Purdie-Vaughns, Valerie/Brzustoski, Patricia (2009): Recursive Processes in Self-Affirmation: Intervening to close the Minority Achievement Gap. Science 324, S. 400-403.

Cohen, Sheldon/Wills, Thomas Ashby (1985): Stress, Social Support, and the Buffering Hypothesis. Psychological Bulletin 98, 2, S. 310-357.

Cornelissen, Waldtraud/Gille, Martina (2005): Lebenswünsche junger Menschen und die Bedeutung geschlechterstereotyper Muster. Zeitschrift für Frauenforschung und Geschlechterstudien 23, 4, S. 52-67.

Cornelissen, Waltraud (2009): Die Relevanz von Geschlechterstereotypen für die Berufswahlentscheidungen - eine Herausforderung für die Gleichstellungspolitik. In: Baer Susanne/Smykalla, Sandra/Hildebrandt, Karin (Hrsg.):Schubladen, Schablonen, Schema F. Stereotype als Herausforderung für Gleichstellungspolitik. München: Kleine, S. 67-88.

Cornelissen, Waltraud/Gille, Martina/Knothe, Holger/Meier, Petra/Queisser, Hannelore/Stürzer, Monika (2002): Junge Frauen - junge Männer. Daten zu Lebensführung und Chancengleichheit. Eine sekundäranalytische Auswertung. Opladen: Leske + Budrich.

Cottard, Laura (2014): Homme et sage-femme: un choix, une pratique, une construction identitaire. Revue de Psychosociologie, 17, 97-108.

Cotter, David A./Hermsen, Joan M./Ovadia, Seth/Vanneman, Reeve (2001): The Glass Ceiling Effect. Social Forces 80, 2, S. 655-682. 
Cronbach, Lee Joseph/Snow, Richard E. (1977): Aptitudes and instructional methods. A handbook for research on interactions. New York: Irvington Publishers.

Cyba, Eva (1993): Überlegungen zu einer Theorie geschlechtsspezifischer Ungleichheiten. In: Frerichs, Petra/Steinrücke, Margareta (Hrsg.): Soziale Ungleichheit und Geschlechterverhältnisse. Frankfurt a.M.: Campus, S. 33-49.

De Col, Christine/Seewald, Günther/Meise, Ullrich (2012): Individuelle Bewältigung von Stigmatisierung und Diskriminierung. In: Rössler, Wulf (Hrsg.): Psychiatrische Rehabilitation. Berlin: Springer, S. 861-874.

Deaux, Kay/LaFrance, Marianne (1998): Gender. In: Gilbert, Daniel T./Fiske, Susan T./Gardner, Lindzey (Hrsg.): The Handbook of Social Psychology (4. Aufl.). Boston: McGraw-Hill, S. 788-827.

Dette, Dorothea E. (2005): Berufserfolg und Lebenszufriedenheit. Eine längsschnittliche Analyse der Zusammenhänge. Erlangen: Friedrich-Alexander-Universität Erlangen-Nürnberg.

Dette, Dorothea E./Abele, Andrea E./Renner, Oliver (2004): Zur Definition und Messung von Berufserfolg. Theoretische Überlegungen und metaanalytische Befunde zum Zusammenhang von externen und internen Laufbahnerfolgsmaßen. Zeitschrift für Personalpsychologie 3,4 , S. 170-183.

Durik, Kevin (2002): Entwicklungssozialpsychologie. In: W. Stroebe/K. Jonas/M. Hewstone (Hrsg.): Sozialpsychologie. Eine Einführung. Berlin: Springer, S. 53-80.

Dweck, Carole S./Davidson, William/Nelson, Sharon/Enna, Bradley (1978): Sex differences in learned helplessness: II, The contingencies of evaluative feedback in the classroom and III, An experimental analysis. Developmental Psychology 14, S. 268-276.

Eagly, Alice (1987): Sex differences in social behavior: A social-role interpretation. Hillsdale: Lawrence Erlbaum.

Eagly, Alice H./Karau, Steven J. (2002): Role Congruity Theory of Prejudice Toward Female Leaders. Psychological Review 109, 3, S. 573-598.

Eardley, Ellen/Manvell, Jessica (2006): Legal remedies for girls' under-representation in nontraditional career and technical education. International Journal of Manpower 27, 4, S. 396416.

Eccles, Jacquelynne S./Barber, Bonnie L./Jozefowicz, Debra (1999): Linking gender to education, occupation, and recreational choices: Applying the Eccles et al. model of achievementrelated choices. In: Swann, William B./Langlois, Judith H./Gilbert, Lucia Albino (Hrsg.): Sexism and stereotypes in modern society: The gender science of Janet Taylor Spence. Washington, DC: APA Press, S. 153-192.

Eckes, Thomas (1997): Geschlechterstereotype: Frau und Mann in sozialpsychologischer Sicht. Pfaffenweiler: Centaurus.

Eckes, Thomas (2008): Geschlechterstereotype: Von Rollen, Identitäten und Vorurteilen. In: Becker, Ruth/Kortendiek, Beate (Hrsg.): Handbuch Frauen- und Geschlechterforschung. Theorie, Methoden, Empirie (2 Aufl.). Wiesbaden: VS, S. 171-182.

Egan, Susan K./Perry, David G. (2001): Gender Identity: A Multidimensional Analysis With Implications for Psychosocial Adjustment. Developmental Psychology 37, 4, S. 451-463.

Eisenstein, Zillah R. (1979): Capitalist patriarchy and the case for socialist feminism. New York: Monthly Review Press.

England, Paula/Herbert, Melissa S. (1993): The Pay of Men in „Female” Occupations: Is Comparable Worth Only for Women? In Williams, Christine L. (Hrsg.): Doing „Women's Work". Men in Nontraditional Occupations. Newbury Park: SAGE, S. S. 28-48.

Epstein, Debbie (1997): Boyz' Own Stories: Masculinities and sexualities in schools. Gender and Education 9, 1, S. 105-116.

Erb, Hans-Peter/Bohner, Gerd (2006): Minoritäten. In: Bierhoff, Hans-Werner/Frey, Dieter (Hrsg.): Handbuch der Sozialpsychologie und Kommunikationspsychologie (Bd. 3). Göttingen: Hogrefe, S. 494-503. 
Evans, Olga/Steptoe, Andrew (2002): The contribution of gender-role orientation, work factors and home stressors to psychological well-being and sickness absence in male- and femaledominated occupational groups. Social Science \& Medicine 54, 4, S. 481-492.

Faulstich-Wieland, Hannelore/Scholand, Barbara (2016): Rahmenbedingungen und Umsetzung der schulischen Berufsorientierung in Hamburg. In: Faulstich-Wieland, Hannelore (Hrsg.): Berufsorientierung und Geschlecht. Weinheim: Beltz Juventa, S. 133-146.

Faulstich-Wieland, Hannelore (2016a): Auszubildende in geschlechtsuntypischen Berufen. In: Faulstich-Wieland, Hannelore (Hrsg.): Berufsorientierung und Geschlecht. Weinheim: Beltz Juventa, S. 85-114.

Faulstich-Wieland, Hannelore (2016b): Berufsorientierung und Geschlecht. Eine Einleitung. In: Faulstich-Wieland, Hannelore (Hrsg.): Berufsorientierung und Geschlecht. Weinheim: Beltz Juventa, S. 7-22.

Feij, Jan A. (1998): Work socialization of young people. In: Drenth, Peter J. D./Thierry, Henk/Wolff, Charles J. de (Hrsg.): Handbook of Work and Organizational Psychology (Aufl. 3). Hove: Psychology Press, S. 207-256.

Fellmann, Max (2014): Nicht auf den Schoß nehmen! SZ-Magazin Süddeutsche Zeitung, Heft 22. http://sz-magazin.sueddeutsche.de/texte/anzeigen/41920/1/1. [Zugriff: 22.03.2017]

Flammer, August/Alsaker, Françoise D. (2002): Entwicklungspsychologie der Adoleszenz. Die Erschließung innerer und äußerer Welten im Jugendalter. Bern: Huber.

Flores, Lisa Y./Navarro, Rachel L./Smith, Jamie L./Ploszaj, Ann M. (2006): Testing a Model of Nontraditional Career Choice Goals With Mexican American Adolescent Men. Journal of Career Assessment 14, 2, S. 214-234.

FRA (2013): EU LGBT survey - European Union lesbian, gay, bisexual and transgender survey - Main results. Luxembourg: EU.

Fröhlich, Werner D. (2000): Wörterbuch Psychologie. München: Deutscher TaschenbuchVerlag.

Frome, Pamela M./Alfeld, Corinne J./Eccles, Jacquelynne S./Barber, Bonnie L. (2008): Is the Desire for a Family-Flexible Job Keeping Young Women Out of Male-Dominated Occupations? In Watt, Helen M. G./Eccles, Jacquelynne S. (Hrsg.): Gender and Occupational Outcomes. Washington, D.C.: American Psychological Association, S. 195-214.

Galliker, M./Wagner, F. (1995): Ein Kategoriensystem zur Wahrnehmung und Kodierung sprachlicher Diskriminierung. Javinia (2013): Socialisation séquentielle et identité de genre liées à la transition de la formation professionnelle à l'emploi. Swiss Journal of Sociology 39, 1, S. 33-55.

Gerber-Schenk, Michelle/Rottermann, Benno/Neuenschwander, Markus P. (2010): Passungswahrnehmung, Selbstkonzept und Jugendarbeitslosigkeit. In: Neuenschwander, Markus P. (Hrsg.): Schulübergang und Selektion. Chur: Rüegger, S. 121-130.

Gianettoni, Lavinia/Simon-Vermot, Pierre/Gauthier, Jacques-Antoine (2010): Orientations professionnelles atypiques: transgression des normes de genre et effets identitaires. Revue française de pédagogie 173, S. 41-50.

Gildemeister, Regine (2010): Doing Gender: Soziale Praktiken der Geschlechterunterscheidung. In: Becker, Ruth/Kortendiek, Beate (Hrsg.): Handbuch Frauen- und Geschlechterforschung. Theorie, Methoden, Empirie. Wiesbaden: VS, S. 137-145.

Glick, Peter/Wilkerson, Mariah/Cuffe, Marshall (2015): Masculine Identity, Ambivalent Sexism, and Attitudes Toward Gender Subtypes. Social Psychology 46, 4, S. 210-217.

Goldin, Claudia (1990): Understanding the gender gap. An economic history of American women. New York: Oxford University Press.

Goldstein, E. Bruce/Ritter, Manfred/Herbst, Gabriele (1997): Wahrnehmungspsychologie. Eine Einführung. Heidelberg: Spektrum Akademischer Verlag.

Gottfredson, Linda S. (1981): Circumscription and Compromise: A Developmental Theory of Occupational Aspirations. Journal of Counseling Psychology Monograph 28, 6, S. 545-579. 
Gottfredson, Linda S. (1996): Gottfredson's Theory of Circumscription and Compromise. In: Brown. Duane/Brooks, Linda (Hrsg.): Career Choice and Development. San Francisco: Jossey-Bass, S. 179-232.

Gottfredson, Linda S. (2005): Applying Gottfredson's Theory of Circumscription and Compromise in Career Guidance and Counseling. In: Brown, Steven Douglas/Lent, Robert W. (Hrsg.): Career development and counseling. Putting Theory and Research to Work. Hoboken, N.J.: Wiley, S. 71-100.

Granato, Mona/Hörsch, Karola/Krempe, Marion/Schwerin, Christine/Settelmeyer, Anke/Tschirner, Wolfgang (2002): Faktoren für den Erfolg bei Abschlussprüfungen im dualen System - dargestellt an ausgewählten Beispielen. Bonn: BIBB.

Grimm, Albert (2009): Praxis der Berufswahlvorbereitung in der Schule. In: R. Zihlmann (Hrsg.): Berufswahl in Theorie und Praxis. Konzepte zur Berufswahlvorbereitung und Beratung unter veränderten wirtschaftlichen und gesellschaftlichen Bedingungen. Bern: SDBB, S. 175-239.

Grob, Alexander/Lüthi, Ruth/Kaiser, Florian G./Flammer, August/Mackinnon, Andrew/Wearing, Alex J. (1991): Berner Fragebogen zum Wohlbefinden Jugendlicher (BFW). Diagnostica 37, S. $66-75$.

Gropengießer, Harald (2005): Qualitative Inhaltsanalyse in der fachdidaktischen LehrLernforschung. In: Mayring, Philipp/Gläser-Zirkuda, Michaela (Hrsg.): Die Praxis der Qualitativen Inhaltsanalyse. Weinheim: Beltz, S. 172-189.

Groß, Dominik/Neuschaefer-Rube, Christine/Steinmetzer, Jan (2008): Transsexualität und Intersexualität. Medizinische, ethische, soziale und juristische Aspekte. Berlin: Medizinisch Wissenschaftliche Verlagsgesellschaft.

Hackett, Gail/Betz, Nancy E. (1981): A Self-Efficacy Approach to the Career Development of Woman. Journal of Vocational Behavior 18, 3, S. 326-339.

Hadjar, Andreas/Aeschlimann, Belinda (2015): Gender stereotypes and gendered vocational aspirations among Swiss secondary school students. Educational Research 57, 1, S. 22-42.

Haeberlin, Urs/Imdorf, Christian/Kronig, Winfried (2004a): Chancenungleichheit bei der Lehrstellensuche. Der Einfluss von Schule, Herkunft und Geschlecht. Bern: Schweizerischer Nationalfonds.

Haeberlin, Urs/Imdorf, Christian/Kronig, Winfried (2004b): Von der Schule in die Berufslehre. Untersuchungen zur Benachteiligung von ausländischen und von weiblichen Jugendlichen bei der Lehrstellensuche. Bern: Haupt.

Häfeli, Kurt/Schellenberg, Claudia (2009a): Erfolgsfaktoren in der Berufsbildung bei gefährdeten Jugendlichen. Bern: Schweizerische Konferenz der kantonalen Erziehungsdirektoren (EDK).

Häfeli, Kurt/Schellenberg, Claudia (2009b): Erfolgsfaktoren in der Berufsausbildung bei gefährdeten Jugendlichen. Schlussbericht. Zürich: Interkantonale Hochschule für Heilpädagogik.

Häfeli, Kurt (2001): Von der Schule ins Erwerbsleben: Gute Noten für die Schweiz. Panorama 3, S. 34-35.

Hagemann-White, Carole (1984): Sozialisation: Weiblich - männlich? Opladen: Leske + Budrich.

Hagemann-White, Carole (1988): Wir werden nicht zweigeschlechtlich geboren. In: HagemannWhite, Carole/Rerrich, Maria s. (Hrsg.): FrauenMännerBilder. Männer und Männlichkeit in der feministischen Diskussion. Bielefeld: AJZ, S. 224-235.

Halpern, Diane F. (1997): Sex Differences in Intelligence. American Psychologist 52, 10, S. 1091-1102.

Hamilton, David L./Gifford, Robert K. (1976): Illusory correlation in interpersonal perception: A cognitive basis of stereotypic judgments. Journal of Experimental Social Psychology 12, S. 392-407.

Hannover, Bettina (2006): Geschlechterrollen. In: Bierhoff, Hans-Werner/Frey, Dieter (Hrsg.): Handbuch der Sozialpsychologie und Kommunikationspsychologie. Göttingen: Hogrefe, S. 464-470. 
Hartmann, Petra/Trautner, Hannes M. (2009): Die Bedeutung des Pubertätsstatus und des Entwicklungstempos für die Geschlechtsidentität von Mädchen und Jungen in der Adoleszenz. Zeitschrift für Entwicklungspsychologie und Pädagogische Psychologie 41, 2, S. 63-78.

Hartung, Paul J./Porfeli, Erik J./Vondracek, Fred W. (2005): Child vocational development: A review and reconsideration. Journal of Vocational Behavior 66, 3, S. 385-419.

Hasse, Raimund/Schmidt, Lucia (2012): Institutionelle Diskriminierung. In: Bauer, Ullrich/Bittlingmayer, Uwe H./Scherr, Albert (Hrsg.): Handbuch Bildungs- und Erziehungssoziologie. Wiesbaden: VS, S. 883-899.

Hausmann, Ann-Christin/Kleinert, Corinna (2014): Berufliche Segregation auf dem Arbeitsmarkt. Männer- und Frauendomänen kaum verändert (9. Aufl.: 1-8). Nürnberg: Institut für Arbeitsmarkt- und Berufsforschung IAB der Bundesagentur für Arbeit.

Hayes, Rader (1986): Gender Nontraditional or Sex Atypical or Gender Dominant or ... Research: Are We Measuring the Same Thing? Journal of Vocational Behavior 29, S. 79-88.

Hayes, Rader (1989): Men in female-concentrated occupations. Journal of Organizational Behavior 10, 3, S. 201-212.

Heilman, Madeline E./Wallen, Aaron S./Fuchs, Daniella/Tamkins, Melinda M. (2004): Penalties for success; reactions to women who succeed at male tasks. Journal of Applied Psychology 89, S. 416-427.

Heinrichs, Markus/Baumgartner, Thomas/Kirschbaum, Clemens/Ehlert, Ulrike (2003): Social Support and Oxytocin Interact to Suppress Cortisol and Subjective Responses to Psychosocial Stress. Biological Psychiatry 54, S. 1389-1398.

Heintz, Bettina/Nadai, Eva (1998): Geschlecht und Kontext. De-Institutionalisierungsprozesse und geschlechtliche Differenzierung. Zeitschrift für Soziologie 27, 2, S. 75-93.

Heintz, Bettina/Nadai, Eva/Fischer, Regula/Ummel, Hannes (1997): Ungleich unter Gleichen. Studien zur geschlechtsspezifischen Segregation des Arbeitsmarktes. Frankfurt: Campus.

Heinz, Walter R. (1995): Arbeit, Beruf und Lebenslauf. Eine Einführung in die berufliche Sozialisation. Weinheim: Juventa.

Helbig, Marcel/Leuze, Kathrin (2012): Ich will Feuerwehrmann werden! Wie Eltern, individuelle Leistungen und schulische Fördermaßnahmen geschlechts(un-)typische Berufsaspirationen prägen. Kölner Zeitschrift für Soziologie und Sozialpsychologie 64, S. 91-122.

Helwig, Andrew A. (2001): A test of Gottfredson's theory using a ten-year longitudinal study. Journal of Career Development 28, 2, S. 77-95.

Heppner, Mary J./Heppner, P. Paul (2009): On Men and Work. Taking the Road Less Traveled. Journal of Career Development 36, 1, S. 49-67.

Herzog, Walter/Neuenschwander, Markus P./Wannack, Evelyne (2006): Berufswahlprozess. Wie sich Jugendliche auf ihren Beruf vorbereiten. Bern: Haupt.

Heyer, Robert/Palentien, Christian/Gürlevik, Aydin (2012): Peers. In: Bauer, Ullrich/Bittlingmayer, Uwe H./Scherr, Albert (Hrsg.): Handbuch Bildungs- und Erziehungssoziologie. Wiesbaden: VS, S. 983-999.

Hirschauer, Stefan (1993): Die soziale Konstruktion der Transsexualität. Über die Medizin und den Geschlechtswechsel. Frankfurt a.M.: Suhrkamp.

Hirschi, Andreas (2009): Eine typologische Analyse des schweizerischen Lehrstellenmarktes: Strukturelle Benachteiligung von jungen Frauen. Schweizerische Zeitschrift für Bildungswissenschaften 31, 2, S. 317-333.

Hirschi, Andreas (2013): Berufswahltheorien - Entwicklung und Stand der Diskussion. In: Brüggemann, Tim/Rahn, Sylvia (Hrsg.): Berufsorientierung. Ein Lehr- und Arbeitsbuch. Münster: Waxmann, S. 27-41.

Hoffmeyer-Zlotnik, Jürgen H. P./Geis, Alfons J. (2003): Berufsklassifikation und Messung des beruflichen Status/Prestige. ZUMA-Methodenberichte 52, S. 125-138.

Holling, Heinz/Preckel, Franzis/Vock, Miriam (2004): Intelligenzdiagnostik. Götting: Hogrefe.

Hormel, Ulrike (2010): Diskriminierung. Grundlagen und Forschungsergebnisse. Wiesbaden: VS. 
Horstkemper, Marianne (1995): Schule, Geschlecht und Selbstvertrauen. Eine Längsschnittstudie über Mädchensozialisation in der Schule (3 Aufl.). Weinheim: Juventa.

Hupka-Brunner, Sandra/Kriesi, Irene (2013): Ausbildungsbedingungen und Ausbildungszufriedenheit in der dualen Grundbildung. Die Rolle von Geschlecht und Migrationshintergrund. In: Hadjar, Andreas/Hupka-Brunner, Sandra (Hrsg.): Geschlecht, Migrationshintergrund und Bildungserfolg. Basel: Beltz Juventa, S. 242-269.

Hurrelmann, Klaus (2002): Handbuch der Sozialisationsforschung. Weinheim: Beltz.

Ignaczewska, Julia (2014): Familie, Geschlecht und Berufswahl. Eine Untersuchung der familialen Bedingungen geschlechtsuntypischer Berufs- und Studienwahl bei Jugendlichen. Bern: Universität Bern.

Ihsen, Susanne (2010): Ingenieurinnen: Frauen in einer Männerdomäne. In: Becker, Ruth/Kortendiek, Beate (Hrsg.): Handbuch Frauen- und Geschlechterforschung. Theorie, Methoden, Empirie. Wiesbaden: VS, S. 799-805.

Imdorf, Christian (2004): Geschlechtsspezifische Selektion bei der Ausbildungsplatzvergabe. In: Schweizerische Konferenz der Gleichstellungsbeauftragten (Hrsg.): Achtung Gender: Ausbildungsverhalten von Mädchen und jungen Frauen. Trends und Tipps. Zürich, S. 99-112.

Imdorf, Christian (2005): Schulqualifikation und Berufsfindung. Wie Geschlecht und nationale Herkunft den Übergang in die Berufsbildung strukturieren. Wiesbaden: VS.

Imdorf, Christian (2010): Wie Ausbildungsbetriebe soziale Ungleichheit reproduzieren: Der Ausschluss von Migrantenjugendlichen bei der Lehrlingsselektion. In: Krüger, HeinzHermann (Hrsg.):Bildungsungleichheit revisited. Bildung und soziale Ungleichheit vom Kindergarten bis zur Hochschule. Wiesbaden: VS, S. 259-274.

Imdorf, Christian (2012a): Wenn Ausbildungsbetriebe Geschlecht auswählen. Geschlechtsspezifische Lehrlingsselektion am Beispiel des Autogewerbes. In: Bergman, Manfred Max/Hupka-Brunner, Sandra/Meyer, Thomas/Samuel, Robin (Hrsg.):Bildung-ArbeitErwachsenwerden. Ein interdisziplinärer Blick auf die Transition im Jugend- und jungen Erwachsenenalter. Wiesbaden: VS, S. 243-264.

Imdorf, Christian (2012b): Zu jung oder zu alt für eine Lehre? Altersdiskriminierung bei der Ausbildungsplatzvergabe. Journal for Labour Market Research 45, 1, S. 79-98.

Jacobs, Jerry A. (1993): Men in Female-Dominated Fields: Trends and Turnovers. In: Williams, Christine L. (Hrsg.): Doing „Women's Work”. Men in Nontraditional Occupations. Newbury Park: SAGE, S. 49-63.

Jäger, Ulrike/König, Tomke/Maihofer, Andrea (2015): Pierre Bourdieu: Die Theorie männlicher Herrschaft als Schlussstein einer Gesellschaftstheorie. In: Kahlert, Heike/Weinbach, Christine (Hrsg.): Zeitgenössische Gesellschaftstheorien und Genderforschung. Einladung zum Dialog. Wiesbaden: VS, S. 15-36.

Jome, LaRae M./Tokar, David M. (1998): Dimensions of Masculinity and Major Choice Traditionality. Journal of Vocational Behavior 52, S. 120-134.

Jome, LaRae M./Surething, Nicole A./Taylor, Kari K. (2005): Relationally Oriented Masculinity, Gender Nontraditional Interests, and Occupational Traditionality of Employed Men. Journal of Career Development 32, 2, S. 183-197.

Judge, Timothy A./Livingston, Beth A. (2008): Is the Gap More Than Gender? A Longitudinal Analysis of Gender, Gender Role Orientation, and Earnings. Journal of Applied Psychology 93, 5, S. 994-1012.

Judge, Timothy A./Higgins, Chad A./Thoresen, Carl J./Barrick, Murray R. (1999): The Big Five Personality Traits, General Mental Ability, and Career Success across the Life Span. Personnel Psychology 52, 3, S.621-652.

Jungo, Daniel (2009): Jugendliche im Berufswahlprozess. Berufsinteressen und ihre Spannungsfelder. In: Zihlmann, René (Hrsg.): Berufswahl in Theorie und Praxis. Konzepte zur Berufswahlvorbereitung und Beratung unter veränderten wirtschaftlichen und gesellschaftlichen Bedingungen. Bern: SDBB, S. 79-97.

Junk, Kate E./Armstrong, Patrick Ian (2010): Stability of Career Aspirations: A Longitudinal test of Gottfredson's Theory. Journal of Career Assessment 37, 3, S. 579-598. 
Kammeyer-Mueller, John D./Wanberg, Connie R. (2003): Unwrapping the organizational entry process: Disentangling multiple antecedents and their pathway to adjustment. Journal of Applied Psychology 88, 5, S. 779-794.

Kanter, Rosabeth Moss (1977): Men and women of the corporation. New York, NY: Basic Books.

Kanter, Rosabeth Moss (2006): Some Effects of Proportions on Group Life: Skewed Sex Ratios and Responses to Token Women. In: Levine, John M./Moreland, Richard L. (Hrsg.): Small groups key readings. New York, NY: Psychology Press, S. 37-54.

Kimmel, Michael S. (2008): Guyland. The perilous world where boys become men. New York, NY: Harper Collins.

Klaghofer, Richard/Martin, Margret/Schläfli, André (1992): Vergleich der Resultate von Lehrabschlussprüfungen. Studie A. Erfolgsquoten der Lehrabschlussprüfungen gemäß Lehrvertragsstatistik des Bundesamts für Statistik in den Erhebungsjahren 1984 bis 1990. Zürich: Schweizerische Gesellschaft für Berufsbildungsforschung SGAB.

Klaghofer, Richard/Martin, Margret/Schläfli, André (1994): Vergleich der Resultate von Lehrabschlussprüfungen. Hauptstudie. Ergebnisse der Lehrabschlussprüfungen von drei ausgewählten Berufen in fünf ausgewählten Kantonen 1990 bis 1992. Zürich: Schweizerische Gesellschaft für Berufsbildungsforschung SGAB.

Knapp, Gudrun-Axeli (1995): Unterschiede machen: Zur Sozialpsychologie der Hierarchisierung im Geschlechterverhältnis. In: Becker-Schmid, Regina/ Knapp, Gudrun-Axeli (Hrsg.): Das Geschlechterverhältnis als Gegenstand der Sozialwissenschaften. Frankfurt a.M.: Campus, S. 163-194.

Koberg, Christine S./Chusmir, Leonard H. (1991): Sex role conflict in sex-atypical jobs: A study of female-male differences. Journal of Organizational Behavior 12, S. 461-465.

Kohlberg, Lawrence (1966): A cognitive-developmental analysis of children's sex-role concepts and attitudes. In: Maccoby, Eleanor Emmons (Hrsg.): The development of sex differences. London: Tavistock, S. 82-172.

Krasnoff, Alan G./Walker, James T./Howard, Mary (1989): Early sex-linked activities and interests related to spatial abilities. Personality and Individual Differences 10, 1, S. 81-85.

Krauth, J. (1993): Einführung in die Konfigurationsfrequenzanalyse (KFA). Ein multivariates nichtparametrisches Verfahren zum Nachweis und zur Interpretation von Typen und Syndromen. Weinheim: Beltz.

Krewerth, Andreas/Tschöpe, Tanja/Ulrich, Joachim G./Witzki, Alexander (2004): Berufsbezeichnungen und ihr Einfluss auf die Berufswahl von Jugendlichen: Theoretische Überlegungen und empirische Ergebnisse. Bielefeld: Bertelsmann.

Krüger, Helga (1986): Die Segmentierung des Berufsbildungssystems. Eine bildungspolitische Barriere für Marktpositionen weiblicher Arbeitskräfte. In: Krüger, Helga (Hrsg.): Berufsbiographien im Wandel. Opladen: Leske + Budrich, S. 33-52.

Krüger, Helga (1991): Doing Gender - Geschlecht als Statuszuweisung im Berufsbildungssystem. In: Brock, Ditmar/Hantsche, Brigitte/Kühnlein, Gertrud/Meulemann, Heiner/Schober, Karin (Hrsg.): Übergänge in den Beruf. Zwischenbilanz zum Forschungsstand. Weinheim: DJI, S. 139-169.

Krüger, Helga (2001): Geschlecht, Territorien, Institutionen. Beitrag zu einer Soziologie der Lebenslauf-Relationalität. In: Born, Claudia/Krüger, Helga (Hrsg.): Individualisierung und Verflechtung. Geschlecht und Generation im deutschen Lebenslaufregime. Weinheim: Juventa, S. 257-299.

Küskü, Fatima/Özbilgin, Mustafa/Özkale, Lerzan (2007): Against the Tide: Gendered Prejudice and Disadvantage in Engineering. Gender, Work and Organization 14, 2, S. 109-129.

Laireiter, Anton-Rupert (1993): Soziales Netzwerk und soziale Unterstützung. Konzepte, Methoden und Befunde. Bern: Huber.

Laireiter, Anton-Rupert (2006): Soziale Unterstützung. In: Bierhoff, Hans-Werner/Frey, Dieter (Hrsg.):Handbuch der Sozialpsychologie und Kommunikationspsychologie (Bd. 3). Göttingen: Hogrefe, S. 166-173. 
Lamamra, Nadia/Masdonati, Jonas (2009): Arrêter une formation professionnelle: mots et maux d'apprenti-e-s. Lausanne: Ed. Antipodes.

Lamamra, Nadia (2011): Ce que l'arrêt prématuré de l'apprentissage révèle de la fabrique du genre. Thèse de doctorat. Lausanne: Université de Lausanne.

Langness, Anja/Richter, Matthias/Hurrelmann, Klaus (2003): Zusammenfassung der Ergebnisse und Konsequenzen für eine jugendgerechte Prävention und Gesundheitsförderung. In: Hurrelmann, Klaus/Klocke, Andreas/Melzer, Wolfgang/Ravens-Sieberer, Ulrike (Hrsg.): Jugendgesundheitssurvey. Internationale Vergleichsstudie im Auftrag der Weltgesundheitsorganisation WHO. Weinheim: Juventa, S. 301-333.

Lease, Suzanne H. (2003): Testing a model of men's nontraditional occupational choices. The Career Development Quarterly 51, 3, S. 244-258.

Leemann, Regula Julia/Keck, Andrea (2005): Der Übergang von der Ausbildung in den Beruf. Die Bedeutung von Qualifikation, Generation und Geschlecht. Neuchâtel: Bundesamt für Statistik.

Lehmann, Rainer/Peek, Rainer/Gänsfuß, Rüdiger/Lutkat, Sabine/Mücke, Stephan/Barth, Ingola (2001): QuaSUM. Qualitätsuntersuchung an Schulen zum Unterricht in Mathematik. Ergebnisse einer repräsentativen Untersuchung im Land Brandenburg (Heft 1). Potsdam: Ministerium für Bildung, Jugend und Sport des Landes Brandenburg.

Leinbach, Mary Driver/Hort, Barbara I./Fagot, Beverly I. (1997): Bears are for boys: Metaphorical associations in young children's gender stereotypes. Cognitive Development 12, S. 107130.

Lemkau, Jeanne Parr (1984): Men in Female-Dominated Professions: Distinguishing Personality and Background Features. Journal of Vocational Behavior 24, S. 110-122.

Lempert, Wolfgang (2002): Berufliche Sozialisation oder Was Berufe aus Menschen machen. Eine Einführung (2. überarb. Aufl.). Hohengehren: Schneider.

Lent, Robert W./Brown, Steven D. (1996): Social Cognitive Approach to Career Development: An Overview. The Career Development Quarterly 44, 4, S. 310-321.

Lenzen, Christoph/Fischer, Gloria/Jentzsch, Anika/Kaess, Michael/Parzer, Peter/Carli, Vladimir/Wasserman, Danuta/Resch, Franz/Brunner, Romuald (2013): Schulabsentismus in Deutschland. Die Prävalenz von entschuldigten und unentschuldigten Fehlzeiten und ihre Korrelation mit emotionalen und Verhaltensauffälligkeiten. Praxis der Kinderpsychologie und Kinderpsychiatrie 62, 8, S. 570-582.

Leu, Christian (2008): Sexuelle Orientierung und Suizidalität: Eine Untersuchung der internationalen und nationalen Forschungsergebnisse der Risiko- und Schutzfaktoren für adoleszente Homo- und Bisexuelle. Bern: Fachgruppe Bildung von PINK CROSS, LOS, fels.

Lohaus, Arnold/Vierhaus, Marc (2015): Entwicklungspsychologie des Kindes- und Jugendalters (2., überarb. Aufl.). Berlin: Springer.

Loughrey, Mark (2008): Just how male are male nurses ...? Journal of Clinical Nursing 17, 10, S. $1327-1334$.

Lubinski, David (2004): Introduction to the Special Section on Cognitive Abilities: 100 Years after Spearman's (1904) „'General Intelligence', Objectively Determined and Measured”. Journal of Personality and Social Psychology 86, 1, S. 96-111.

Lupton, Ben (2006): Explaining Men's Entry into Female-Concentrated Occupations: Issues of Masculinity and Social Class. Gender, Work and Organization 13, 2, S. 103-128.

Lynn, Richard (1994): Sex differences in intelligence and brain size: A paradox resolved. Personality and Individual Differences 17, S. 257-271.

Maccoby, Eleanor Emmons (1998): Psychologie der Geschlechter sexuelle Identität in den verschiedenen Lebensphasen. Stuttgart: Klett-Cotta.

Mackert, Jürgen (2004): Die Theorie sozialer Schließung. Tradition, Analysen, Perspektiven. Wiesbaden: VS.

Maihofer, Andrea/Schwiter, Karin (2013): Jugendliche in geschlechtsuntypischen Berufen besitzen oft überdurchschnittliche schulische Kompetenzen. Bern: Schweizerischer Nationalfonds. 
Maihofer, Andrea (2002): Geschlecht und Sozialisation. Erwägen Wissen Ethik 13, 1, S. 13-26.

Maihofer, Andrea/Bergman, Manfred Max/Hupka-Brunner, Sandra/Wehner, Nina/Schwiter, Karin/Huber, Evéline/Kanji, Shireen (2013): Kontinuität und Wandel von Geschlechterungleichheiten in Ausbildungs- und Berufsverläufen junger Erwachsener in der Schweiz. Basel: Schweizerischer Nationalfonds.

Maihofer, Andrea/Schwiter, Karin/Wehner, Nina. (2012): Subtile Mechanismen beeinflussen die Berufswahl. Panorama 5, S. 22-23.

Makarova, Elena/Herzog, Walter (2014): Geschlechtsuntypische Berufswahlen bei jungen Frauen: Muss das Vorbild weiblich sein? Zeitschrift für Soziologie und Sozialisation 34, 1, S. 38-54.

Makarova, Elena/Aeschlimann, Belinda/Herzog, Walter (2016): Wenn Frauen in MINTStudiengängen fehlen. Mathematisch-naturwissenschaftlicher Unterricht und die Studienwahl von jungen Frauen. In: Faulstich-Wieland, Hannelore (Hrsg.): Berufsorientierung und Geschlecht. Weinheim: Beltz Juventa, S. 39-57.

Markefka, Manfred (1995): Vorurteile, Minderheiten, Diskriminierung. Ein Beitrag zum Verständnis sozialer Gegensätze. Neuwied: Luchterhand.

Marsh, Herbert W./Shavelson, Richard J. (1985): Self-Concept: Its Multifaceted, Hierarchical Structure. Educational Psychologist 20, 3, S. 107-123.

Martin, Carol Lynn/Ruble, Diane N./Szkrybalo, Joel (2002): Cognitive theories of early gender development. Psychological Bulletin 128, S. 903-933.

Martschukat, Jürgen/Stieglitz, Olaf (2005): „Es ist ein Junge!“ Einführung in die Geschichte der Männlichkeiten in der Neuzeit. Tübingen: Ed. Diskord.

Mayring, Philipp (2003): Qualitative Inhaltsanalyse. Grundlagen und Techniken (8. Aufl.). Weinheim: Beltz.

McKee-Ryan, Frances M./Song, Zhaoli/Wanberg, Connie R./Kinicki, Angelo J. (2005): Psychological and physical well-being during unemployment: a meta-analytic study. Journal of Applied Psychology 90, 1, S. 53-76.

McLean, Heather M./Kalin, Rudolf (1994): Congruence Between Self-Image and Occupational Stereotypes in Students Entering Gender-Dominated Occupations. Canadian Journal of Behavioural Science 26, 1, S. 142-162.

Meyer, Ilan H./Frost, David M./Nezhad, Sheila (2015): Minority Stress and Suicide in Lesbians, Gay Men, and Bisexuals. In: Goldblum, Peter/Espelage, Dorthy L./Chu, Joyce/Bongar, Bruce (Hrsg.): Youth Suicide and Bullying. Challenges and Strategies for Prevention and Intervention. New York: Oxford University Press, S. 177-187.

Mitchell, Lynda K./Krumboltz, John D. (1994): Die berufliche Entscheidungsfindung als sozialer Lernprozess: Krumboltz' Theorie. In: Brown, Duane/Brooks, Linda (Hrsg.): KarriereEntwicklung. Stuttgart: Klett-Cotta, S. 157-210.

Money, John/Ehrhardt, Anke A. (1975): Männlich-Weiblich: Die Entstehung der Geschlechtsunterschiede. Reinbeck: Rowohlt-TB.

Money, John (1955): Hermaphroditism, gender and precocity in hyperadrenocorticism: psychological findings. Bulletin of Johns Hopkins Hospital 96, S. 253-264.

Montada, Leo (1998): Fragen, Konzepte, Perspektiven. In: Oerter, Rolf/Montada, Leo (Hrsg.): Entwicklungspsychologie (4. Aufl.). München: Beltz, S. 1-83.

Mühlemann, Samuel/Wolter, Stefan C./Fuhrer, Marc/Wüest, Adrian (2007): Lehrlingsausbildung - ökonomisch betrachtet. Ergebnisse der zweiten Kosten-Nutzen-Studie. Zürich: Rüegger.

Müller, Andreas (2006b): Lerncoaching. Grundschule 12, 3, S. 6-15.

Müller, Romano (2006a): Von der Sekundarstufe I zur Sekundarstufe II: Schweizerische und ausländische Jugendliche im Übergang zur Lehre und zum Gymnasium. In: Tanner, Albert/Badertscher, Hans/Holzer, Rita/Schindler, Andreas/Streckeisen, Ursula (Hrsg.): Heterogenität und Integration. Umgang mit Ungleichheit und Differenz in der Schule und Kindergarten. Zürich: Seismo, S. 143-174.

Müller, R. (2009): Berufswahl und Lehre. Berufliche Orientierungs- und Entscheidungsprozesse bei ausländischen und schweizerischen Jugendlichen. Bern: hep. 
Mürner, Beat (2004): Geschlechtsspezifische Berufssegregation. Erklärungsansätze und deren empirische Bedeutung. http://homepage.swissonline.ch/bmuerner/berufswahl/pdfs/. [Zugriff: 09.06.2016]

Nägele, Christoph/Neuenschwander, Markus P. (2015): Passt der Beruf zu mir? Determinanten und Konsequenzen wahrgenommener Passung mit dem Lehrberuf beim Übergang in die Berufsbildung. In: Häfeli, Kurt/Neuenschwander, Markus P./Schumann, Stephan (Hrsg.): Berufliche Passagen im Lebenslauf. Berufsbildungs- und Transitionsforschung in der Schweiz. Wiesbaden: Springer, S. 49-74.

Negrini, Lucio/Forsblom, Lara/Schumann, Stephan/Gurtner, Jean-Luc (2015): Lehrvertragsauflösungen und die Rolle der betrieblichen Ausbildungsqualität. In: Häfeli, Kurt/Neuenschwander, Markus P./Schumann, Stephan (Hrsg.): Berufliche Passagen im Lebenslauf. Berufsbildungs- und Transitionsforschung in der Schweiz. Wiesbaden: Springer, S. $77-100$.

Neuenschwander, Markus P. (1999): Lehrvertragsauflösung im Kanton Zürich. Schlussbericht. Zürich: Impulse.

Neuenschwander, Markus P./Balmer, Thomas/Gasser-Dutroit, Annette/Goltz, Stefanie/Hirt, Ueli/Ryser, Hans/Wartenweiler, Hermann (2005): Schule und Familie. Was sie zum Schulerfolg beitragen. Bern: Haupt.

Neuenschwander, M. P./Gerber, M. (2014): Schulische Vorbereitung auf die berufliche Sozialisation im Lehrbetrieb. Unterrichtswissenschaft 42, 3, S. 244-260.

Neuenschwander, Markus P./Gerber, Michelle/Frank, Nicole/Rottermann, Benno (2012): Schule und Beruf. Wege in die Erwerbstätigkeit. Wiesbaden: VS.

Neuenschwander, Markus P./Schaub, Simone/Angehrn, Franziska (2007): Familiäre Bedingungen von Schülerleistungen. Dokumentation des Schülerfragebogens. Zürich: Jacobs Center for Productive Youth Development, Universität Zürich.

Nissen, Ursula/Keddi, Barbara/Pfeil, Patricia (2003): Berufsfindungsprozesse von Mädchen und jungen Frauen. Erklärungsansätze und empirische Befunde. Opladen: Leske + Budrich.

OECD. (2000): From Initial Education to Working Life. Making Transitions Work. Paris: OECD.

OECD. (2002): PISA 2000 Technical Report. Paris: OECD.

Oerter, Rolf/Dreher, Eva (1998): Jugendalter. In: Oerter, Rolf/Montada, Leo (Hrsg.): Entwicklungspsychologie. Ein Lehrbuch (4. Aufl.). München: Beltz, S. 310-395.

O’Neil, James M. (2015): Men's Gender Role Conflict. Psychological Costs, Consequences, and an Agenda for Change. Washington, DC: American Psychological Association.

Ostendorf, Helga (2005): Steuerung des Geschlechterverhältnisses durch eine politische Institution. Die Mädchenpolitik der Berufsberatung. Opladen: Budrich.

Ostner, Ilona (1990): Das Konzept des weiblichen Arbeitsvermögens. In: Pfau-Effinger, Brigit/Ostner, Ilona (Hrsg.): Erklärungsansätze zur geschlechtsspezifischen Strukturierung des Arbeitsmarktes. Paderborn: Arbeitskreis Sozialwissenschaftliche Arbeitsmarktforschung SAMF, S. 22-39.

Packenberg, Bente/Gunderson, Hans Jørgen G. (1997): Neocortical neurons number in humans: Effects of age and sex. Journal of Comparative Neurology 384, S. 312-320.

Paseka, Angelika (2008): Wie Kinder zu Mädchen und Buben werden. Einige Erkenntnisse aus der Sozialisations- und Geschlechterforschung. In: Buchmayr, Maria (Hrsg.): Geschlecht lernen. Gendersensible Didaktik und Pädagogik. Innsbruck: Studien Verlag, S. 15-31.

Pätzold, Günter (2004): Übergang Schule - Berufsausbildung. In: Helsper, Werner/Böhme, Jeanette (Hrsg.): Handbuch der Schulforschung. Wiesbaden: VS, S. 593-610.

Perrez, Meinrad/Laireiter, Anton-Rupert/Baumann, Urs (2005): In Perrez, Meinrad/Baumann, Urs (Hrsg.): Lehrbuch klinische Psychologie - Psychotherapie (3., vollst. überarb. Aufl.). Bern: Huber, S. 272-304.

Perry, D. G./Pauletti, R. E. (2011): Gender and Adolescent Development. Journal of Research on Adolescence 21, 1, S. 61-74. 
Plicht, Hannelore/Schreyer, Franziska (2002): Ingenieurinnen und Informatikerinnen. Schöne neue Arbeitswelt? Nürnberg: Institut für Arbeitsmarkt- und Berufsforschung der Bundesanstalt für Arbeit.

Polachek, Solomon William (1981): Occupational Self-Selection: A Human Capital Approach to Sex Differences in Occupational Structure. Review of Economics and Statistics 63, 1, S. 60 69.

Prentice, Deborah A./Carranza, Erica (2003): Sustaining Cultural Beliefs in the Face of Their Violation: The Case of Gender Stereotypes. In: Schaller, Marc/Crandall, Christian S. (Hrsg.): The psychological foundation of Culture. Mahwah: Erlbaum, S. 259-280.

Preacher, Kristopher J./Hayes, Andrew F. (2008): Asymptotic and resampling strategies for assessing and comparing indirect effects in multiple mediator models. Behavior Research Methods 40, 3, S. 879-891.

Rahn, Sylvia/Hartkopf, Emanuel (2016): Geschlechtsuntypische Berufswahlen. Beobachtungen aus dem Berufsorientierungspanel. In: Faulstich-Wieland, Hannelore (Hrsg.): Berufsorientierung und Geschlecht. Weinheim: Beltz Juventa, S. 115-132.

Ray, Wendal A./Keeney, Bradford (1993): Resource Focused Therapy. London: Karnac Books.

Rochlen, Aaron B./Good, Glenn E./Carver, Tracy A. (2009): Predictors of Gender-Related Barriers, Work, and Life Satisfaction Among Men in Nursing. Psychology of Men/Masculinity 10, 1, S. 44-56.

Rochlen, Aaron B./McKelley, Ryan A./Whittaker, Tiffany A. (2010): Stay-at-Home Fathers' Reasons for Entering the Role and Stigma Experiences: A Preliminary Report. Psychology of Men/Masculinity 11, 4, S. 279-285.

Rodari, Sophie/Anderführen, Marie (2011): Quelle mixité dans les champs sociaux et sanitaires? FemInfo 24, S. 16-18.

Rosenberg, Morris (1979): Conceiving the self. New York, NY: Basic Books.

Rosenstiel, Lutz von (2001): Die Bedeutung der Arbeit. In: H. Schuler (Hrsg.): Lehrbuch der Personalpsychologie. Göttingen: Hogrefe, S. 15-42.

Ross, Michael W. (1989): Gay Youth in Four Cultures: A Comparative Study. Journal of Homosexuality 17, 3-4, S. 299-314.

Rottermann, Benno (2011): When success is taken out of an individual's hands - wholepopulation data on the Swiss federal vocational education and training diploma examination system. Paper presented at the European conference on Educational Research ECER, Berlin.

Rubin, Gayle (1975): The Traffic in Women: Notes on the „Political Economy” of Sex. In: R. R. Reiter (Hrsg.): Toward an anthropology of women. New York: Monthly Review Press, S. $157-210$.

Ruble, Diane N./Martin, Caorl L. (1998): Gender development. In: W. Damon (Hrsg.): Handbook of child psychology. New York: Wiley, S. 933-1016.

Ruble, Diane N./Martin, Carol L./Berenbaum, Sheri A. (2006): Gender Development. In: W. Damon (Hrsg.): Handbook of child psychology. Hoboken: Wiley, S. 858-932.

Rustemeyer, Ruth/Jubel, Angelica (1996): Geschlechtsspezifische Unterschiede im Unterrichtsfach Mathematik hinsichtlich der Fähigkeitseinschätzung, Leistungserwartung, Attribution sowie im Lernaufwand und im Interesse. Zeitschrift für Pädagogische Psychologie 10, 1, S. $13-25$.

Rustemeyer, R./Thrien: (2001): Das Erleben von Geschlechtsrollenkonflikten in geschlechtstypisierten Berufen. Zeitschrift für Arbeits- und Organisationspsychologie 45, 1, S. 34-39.

Rustemeyer, Ruth (1988): Geschlechtsstereotype und ihre Auswirkungen auf das Sozial- und Leistungsverhalten. Zeitschrift für Sozialisationsforschung und Erziehungssoziologie 8, S. $115-129$.

Sarason, Irwin G./Sarason, Barbara R./Shearin, Edward N./Pierce, Gregory R. (1987): A Brief Measure of Social Support: Practical and Theoretical Implications. Journal of Social and Personal Relationships 4, 4, S. 497-510.

Sargent, Paul (2000): Real Men or Real Teachers? Contradictions in the Lives of Men Elementary Teachers. Men and Masculinities 2, 4, S. 410-433. 
Savin-Williams, Ritch C./Ream, Geoffrey L. (2003): Suicide Attempts among Sexual-Minority Male Youth. Journal of Clinical and Adolescent Psychology 32, 4, S. 509-522.

Savin-Williams, Ritch C. (2001): Suicide Attempts among Sexual Minority Youths: Population and Measurement Issues. Journal of Consulting and Clinical Psychology 69, 6, S. 983-991.

Sax, Linda J./Bryant, Alyssa N. (2006): The impact of college on sex-atypical career choices of men and women. Journal of Vocational Behavior 68, S. 52-63.

SBFI (2015): Berufsbildung in der Schweiz. Zahlen und Fakten 2015. Bern: Staatssekretariat für Bildung, Forschung und Innovation SBFI.

SBFI (2016): Das Schweizer Berufsbildungssystem. http://www.sbfi.admin.ch. [Zugriff: 22.03.2017]

Schafer, Joseph L./Graham, John W. (2002): Missing data: Our view of the state of the art. Psychological Methods 7, 2, S. 147-177.

Schafer, Joseph L./Olsen, Maren K. (1998): Multiple Imputation for Multivariate Missing-Data Problems: A Data Analyst's Perspective. Multivariate Behavioral Research 33, 4, S. 545571.

Schafer, Yves/Baeriswyl, Franz (2015): Erfolg in der Berufsbildung. Faktoren des objektiven Ausbildungserfolgs bei Absolventen/-innen der dualen kaufmännischen Berufslehre. In: Häfeli, Kurt/Neuenschwander, Markus P./Schumann, Stephan (Hrsg.): Berufliche Passagen im Lebenslauf. Berufsbildungs- und Transitionsforschung in der Schweiz. Wiesbaden: Springer, S. 127-160.

Schein, Edgar H. (1985): Organizational culture and leadership. San Francisco: Jossey-Bass.

Scherr, Albert/Janz, Caroline/Müller, Stefan (2015): Diskriminierung in der beruflichen Bildung. Wie migrantische Jugendliche bei der Lehrstellenvergabe benachteiligt werden. Wiesbaden: Springer.

Schmid, Evi/Stalder, Barbara E. (2007): Lehrvertragsauflösung: direkter Wechsel und vorläufiger Ausstieg. Ergebnisse aus dem Projekt LEVA. Bern: Bildungsplanung und Evaluation.

Schmid, Evi/Stalder, Barbara E. (2008): Lehrvertragsauflösung: Chancen und Risiken für den weiteren Ausbildungsweg. Ergebnisse aus dem Projekt LEVA. Bern: Bildungsplanung und Evaluation.

Schmid, Evi (2010): Kritisches Lebensereignis „Lehrvertragsauflösung“. Eine Längsschnittuntersuchung zum Wiedereinstieg und zum subjektiven Wohlbefinden betroffener Jugendlicher. Bern: hep.

Schmid-Thomae, Anja (2012): Berufsfindung und Geschlecht. Mädchen in technischhandwerklichen Projekten. Wiesbaden: Springer.

Scholand, Barbara (2016): Undoing Circumscription? Berufsbezogene Interessen und Kenntnisse von Schülerinnen und Schülern im 8. Jahrgang. In: Faulstich-Wieland, Hannelore (Hrsg.): Berufsorientierung und Geschlecht. Weinheim: Beltz Juventa, S. 58-84.

Schössler, Franziska (2008): Einführung in die Gender Studies. Berlin: Akademie Verlag.

Schweri, Jürg/Mühlemann, Samuel/Pescio, Yasmin/Wolter, Stefan C./Zürcher, Lucas (2003): Kosten und Nutzen der Lehrlingsausbildung aus der Sicht Schweizer Betriebe. Zürich: Rüegger.

Schwiter, Karin (2011): Lebensentwürfe junger Erwachsene im Spannungsfeld zwischen Individualität und Geschlechternormen. Frankfurt am Main: Campus.

Schwiter, Karin/Hupka-Brunner, Sandra/Wehner, Nina/Huber, Evéline/Kanji, Shireen/Maihofer, Andrea/Bergman, Manfred Max (2014): Warum sind Pflegefachmänner und Elektrikerinnen nach wie vor selten? Geschlechtersegregation in Ausbildungs- und Berufsverläufen junger Erwachsener in der Schweiz. Schweizerische Zeitschrift für Soziologie 40, 3, S. 401-428.

Schwiter, Karin/Wehner, Nina/Maihofer, Andrea/Huber, Evéline (2011): Zur Hartnäckigkeit geschlechtssegregierter Ausbildungs- und Berufsverläufe. Konzeptionelle Überlegungen zu einer empirischen Untersuchung. Femina politica 14, 2, S. 20-32.

SDBB. (2012): Wegweiser durch die Berufslehre. Zollikofen: Schweizerisches Dienstleistungszentrum Berufsbildung, Berufs-, Studien- und Laufbahnberatung SDBB. 
SDBB. (2013): Handbuch betriebliche Grundbildung. Zollikofen: Schweizerisches Dienstleistungszentrum Berufsbildung, Berufs-, Studien- und Laufbahnberatung SDBB.

Semmer, Norbert/Udris, Ivars (1995): Bedeutung der Arbeit. In: Schuler, Heinz (Hrsg.): Lehrbuch der Organisationspsychologie. Bern: Huber, S. 133-165.

Settelmeyer, Anke/Tschirner, Wolfgan. (2002): Steigerung des Prüfungserfolgs bei Abschlussprüfungen im Handwerk. BIBB ermittelt Gründe für das Prüfungsversagen. Berufsbildung in Wissenschaft und Praxis 31, 5, S. 42-46.

Sheridan, Jennifer T. (1997): The Effects of the Determinants of Women's Movement Into and Out of Male-dominated Occupations on Occupational Sex Segregation. Paper presented at the Meetings of the Population Association of America, Washington, DC.

Shewring, Fion. (2009): The female 'tradie': Challenging employment perceptions in nontraditional trades for women. Australian Government.

Siegfried, Detlef (2015): Moderne Lüste: Ernest Borneman - Jazzkritiker, Filmemacher, Sexforscher. Göttingen: Wallstein.

Simpson, Ruth (2005): Men in Non-Traditional Occupations: Career Entry, Career Orientation and Experience of Role Strain. Gender, Work and Organization 12, 4, S. 364-380.

Sonntag, Karlheinz/Schäfer-Rauser, Ulrich (1993): Selbsteinschätzung beruflicher Kompetenz bei der Evaluation von Bildungsmaßnahmen. Zeitschrift für Arbeits- und Organisationspsychologie 37, 4, S. 163-171.

Spitzer, Manfred (2009): Ja, ich kann! Selbstbild, Selbstbejahung und nachhaltige Leistungsfähigkeit. Nervenheilkunde 28, 7, S. 425-430.

Stalder, Barbara E./Schmid, Evi (2006): Lehrvertragsauflösungen, ihre Ursachen und Konsequenzen. Ergebnisse aus dem Projekt LEVA. Bern: Erziehungsdirektion des Kantons Bern.

Stalder, Barbara E. (1999): Warum Lehrlinge ausbilden? Ausbildungsbereitschaft, Lehrstellenangebot und Bildungsreformen aus der Sicht von Lehrbetrieben des Kantons Bern. Bern: Amt für Bildungsforschung.

Sternberg, Robert J. (1997): The concept of intelligence and its role in lifelong learning and success. American Psychologist 52, 2, S. 1030-1038.

Strupler, Mirjam/Wolter, Stefan C. (2012): Die duale Lehre eine Erfolgsgeschichte - auch für Betriebe. Ergebnisse der dritten Kosten-Nutzen-Erhebung der Lehrlingsausbildung aus der Sicht der Betriebe. Chur: Rüegger.

Su, Rong/Rounds, James/Armstrong, Patrick Ian (2009): Men and Things, Women and People: A Meta-Analysis of Sex Differences in Interests. Psychological Bulletin 135, 6, S. 859-884.

Super, Donald E. (1992): Toward a comprehensive theory of career development. In: Montross, David H. (Hrsg.): Career Development: Theory and Practice. Springfield: Thomas, S. 3564.

Super, Donald E. (1994): Der Lebenszeit-, Lebensraumansatz der Laufbahnentwicklung. In: Brown, Duane/Brooks, Linda (Hrsg.): Karriere-Entwicklung. Stuttgart: Klett-Cotta, S. 211280.

Süss, Daniel/Neuenschwander, Markus P./Dumont, Jeannine (1996): Lehrabbruch, Gesundheitsprobleme und deviantes Verhalten im Jugendalter. Bern: Universität.

SUVA (2010): Nimm's leicht! Unterrichtspaket zum Heben und Tragen von Lasten. Luzern: SUVA.

Swerdlow, M. (1989): Men's Accommodations to Women Entering a Nontraditional Occupation: A Case of Rapid Transit Operatives. Gender and Society 3, 3, S. 373-387.

Testa, Rylan J./Habarth, Janice/Peta, Jayme/ Balsam, Kimberly/Bockting, Walter (2015): Development of the Gender Minority Stress and Resilience Measure. Psychology of Sexual Orientation and Gender Diversity 2, 1, S. 65-77.

Tharenou, Phyllis (1997): Managerial career advancement. International Review of Industrial and Organizational Psychology 12, S. 39-93.

Thege, Britta/Welpe, Ingelore/Bonnemann-Böhner, Adelheid (1996): Ladies first - Verdrängungsprozesse erwerbstätiger Frauen im Wandel der Wirtschafts- und Arbeitswelt. München: Hampp. 
Tobin, Desiree D./Menon, Meenakshi/Menon, Madhavi/Spatta, Brook C./Hodges, Ernest V. E./Perry, David G. (2010): The Intrapsychics of Gender: A Model of Self-Socialization. Psychological Review, 117(2), 601-622.

Trautner, Hans Martin (1996): Die Bedeutung der Geschlechterkategorie im Jugendalter. In: Schumann-Hengsteler, Ruth (Hrsg.): Entwicklung im Jugendalter. Göttingen: Hogrefe, S. $165-188$.

Trautner, Hans Martin (2008): Entwicklung der Geschlechtsidentität. In: Oerter, Rolf/Montada, Leo (Hrsg.): Entwicklungspsychologie (6., vollst. überarb. Aufl.). Weinheim: Belz, S. 625651.

Trautner, Hans Martin/Helbing, Nicole/Sahm, Winfried Bernhard/Lohaus, Arnold (1988): Unkenntnis - Rigidität - Flexibilität: Ein Entwicklungsmodell der GeschlechtsrollenStereotypisierung. Zeitschrift für Entwicklungspsychologie und Pädagogische Psychologie 20, S. $105-120$.

Trauttmansdorff, Miriam (2003): Geschlechtsspezifische Segregation und Organisationskultur: der organisationale Kontext und sein Einfluss auf die individuellen Karrierewege von Frauen und Männern am Beispiel der Unternehmensberatung. München: Ludwig-Maximilians Universität.

TREE (2008): TREE: Konzepte und Skalen. Befragungswelle 1 bis 7. Bern/Basel: TREE.

TREE (2011a): Projekt-Dokumentation TREE 2000-2010. Basel: TREE.

TREE (2011b): TREE: Konzepte und Skalen. Befragungswelle 1 bis 8. Basel: TREE.

TREE (2013): Projekt-Dokumentation TREE 2000-2012. Basel: TREE.

Van Dick, Rolf (2006): Soziale Diskriminierung. In: Bierhoff, Hans-Werner/Frey, Dieter (Hrsg.): Handbuch der Sozialpsychologie und Kommunikationspsychologie (Bd. 3). Göttingen: Hogrefe, S. 727-734.

von Eye, Alexander (2002): Configural frequency analysis. Methods, models, and applications. Mahwah, NJ: Lawrence Erlbaum Associates.

Wagner, Franc/Huerkamp, Matthias/Jockisch, Heike/Graumann, Carl F. (1990): Sprachlich realisierte soziale Diskriminierungen: empirische Überprüfung eines Modells expliziter Diskriminierung. Heidelberg: Psychologisches Institut der Universität Heidelberg.

Wang, Mo/Zhan, Yujie/McCune, Elizabeth/Truxillo, Donald (2011): Understanding newcomers' adaptability and work-related outcomes; testing the mediating roles of perceived p-e fit variables. Personnel Psychology 64, S. 163-189.

Watt, Helene M. G. (2008): What Motivates Females and Males to Pursue Sex-Stereotyped Careers? In Watt, Helene M. G./Eccles, Jacquelynne S. (Hrsg.): Gender and Occupational Outcomes. Washington, D.C.: American Psychological Association, S. 87-114.

Weber, M./Winckelmann, J. (1985): Wirtschaft und Gesellschaft: Grundriss der verstehenden Soziologie (5., rev. Aufl.). Tübingen: Mohr.

Weber, Max (1980): Wirtschaft und Gesellschaft: Grundriss der verstehenden Soziologie. Tübingen: Mohr.

Wehner, Nina/Schwiter, Karin/Hupka-Brunner, Sandra/Maihofer, Andrea (2016): Geschlechterungleichheiten in Ausbildungs- und Berufsverläufen junger Erwachsener in der Schweiz. Ergebnisse aus einer Mixed-Methods-Studie. In: Faulstich-Wieland, Hannelore (Hrsg.): Berufsorientierung und Geschlecht. Weinheim: Beltz Juventa, S. 23-38.

Weiss, Reinhold (2002): Ausbildungsabbruch - Eine Herausforderung für die betriebliche Berufsausbildung. In: Bohlinger, Sandra/Jenewein, Klaus (Hrsg.): Ausbildungsabbrecher Verlierer der Wissensgesellschaft? Konzepte, Risiken und Chancen aktueller Handlungsansätze aus der Berufsbildungsforschung und -praxis. Bielefeld: Bertelsmann, S. 5-16.

West, Candance/Zimmerman, Don H. (1987): Doing Gender. Gender and Society 1, S. 125-151.

Wetterer, Angelika (1993): Professionalisierung und Geschlechterhierarchie. Vom kollektiven Frauenausschluss zur Integration mit beschränkten Möglichkeiten. Kassel: Jenior und Pressler.

Wetterer, Angelika (2002): Arbeitsteilung und Geschlechterkonstruktion. „Gender at Work“ in theoretischer und historischer Perspektive. Konstanz: UVK. 
Wetterer, Angelika (2010): Konstruktion von Geschlecht: Reproduktionsweisen der Zweigeschlechtlichkeit. In: Becker, Ruth/Kortendiek, Beate (Hrsg.): Handbuch Frauen- und Geschlechterforschung. Theorie, Methoden, Empirie. Wiesbaden: VS, S. 126-136.

Wettstein, Emil/Gonon, Philipp (2009): Berufsbildung in der Schweiz. Bern: hep.

Wettstein, Emil (2008): Berufsbildung der Schweiz - Formen der beruflichen Grundbildung. Unterlagen zum Bildungswesen der Schweiz. Zürich: Berufsbildungsprojekte Wettstein.

Williams, Christine L. (1989): Gender differences at work. Women and men in nontraditional occupations. Berkeley, CA: University of California Press.

Williams, Christine L. (1992): The Glass Escalator: Hidden Advantages for Men in the „Female” Professions. Social Problems 39, S. 227-240.

Williams, Christine L. (Hrsg.). (1993): Doing „Women's Work”. Men in Nontraditional Occupations. Newbury Park: SAGE.

Wilz, Sylvia M. (2004): Für und wider einen weiten Begriff von Schließung. Überlegungen zur Theorie sozialer Schließung am Beispiel von Geschlechterungleichheiten. In: Mackert, Jürgen (Hrsg.): Die Theorie sozialer Schließung. Tradition, Analysen, Perspektiven. Wiesbaden: VS, S. 211-231.

Wittig, M. (1981): One is not born a Woman. Feminist Issues 1, 2, S. 48.

Wobbe, Therese/Nunner-Winkler, Gertrude (2007): Geschlecht und Gesellschaft. In: Joas, Hans (Hrsg.): Lehrbuch der Soziologie (3., überarb. und erw. Aufl.). Frankfurt am Main: Campus, S. 287-312.

Ziegler, Albert/Dresel, Makrus (2006): Lernstrategien: Die Genderproblematik. In: Mandl, Heinz/Friedrich, Helmut Felix (Hrsg.): Handbuch Lernstrategien. Göttingen: Hogrefe, S. 378-389.

Zihlmann, René (2009): Berufswahl. Buch/Kompass 09/10 (9. vollst. überarb. Aufl.). Oberentfelden: Sauerländer. 


\section{Abkürzungen}

BFS Bundesamt für Statistik

BR Benno Rottermann

EBA Eidgenössisches Berufsattest

EFZ Eidgenössisches Fähigkeitszeugnis

FASE B Familie Schule Beruf

HISEI Highest International Socio-Economic Index (sozioökonomische Status)

ISCO International Standard Classification of Occupation (Internationaler Standard der Berufsklassifikation)

LAP Lehrabschlussprüfung

LVA Lehrvertragsauflösung

OECD Organisation for Economic Co-operation and Development (Organisation für Entwicklung und Zusammenarbeit)

PISA Programme for International Student Assessment (Programm zur internationalen Schülerbewertung)

SBFI Staatssekretariat für Bildung Forschung und Innovation

SDBB Schweizerisches Dienstleistungszentrum Berufsbildung, Berufs-, Studienund Laufbahnberatung

TREE Transitionen von der Erstausbildung ins Erwerbsleben

üK überbetrieblicher Kurs, 3. Lernort während der Berufsausbildung

\section{Statistische Abkürzungen}

* $\quad$ Signifikanzniveau $p<.05$ (signifikant $=$ statistisch bedeutsam)

** $\quad$ Signifikanzniveau $p<.01$ (hoch signifikant)

*** Signifikanzniveau $p<.001$ (höchst signifikant)

$+\quad$ Signifikanzniveau $p<.1$ (tendenziell signifikant)

n.s. Signifikanzniveau $p>.05$ (nicht signifikant)

CFI komparativer Anpassungsindex

chi $i^{2} \quad \mathrm{Chi}^{2}$-Wert

Coeff Koeffizient

df $\quad$ Freiheitsgrade

F $\quad$ F-Wert

KI Konfidenzintervall

M arithmetischer Mittelwert

$N \quad$ Anzahl

$p \quad$ Signifikanzniveau

$r \quad$ Korrelationskoeffizient

$R^{2} \quad$ Varianzaufklärung

RSMEA Standardisierte Residualdiskrepanzwurzel

$S D \quad$ Standardabweichung

$\mathrm{t} \quad$ Messzeitpunkt (Time)

$\alpha \quad$ Reliabilität (interner Konsistenz)

$\eta \quad$ Effektstärke partielle eta ${ }^{2}$ 


\section{Unsere Fachzeitschriften auf www.budrich-journals.de}
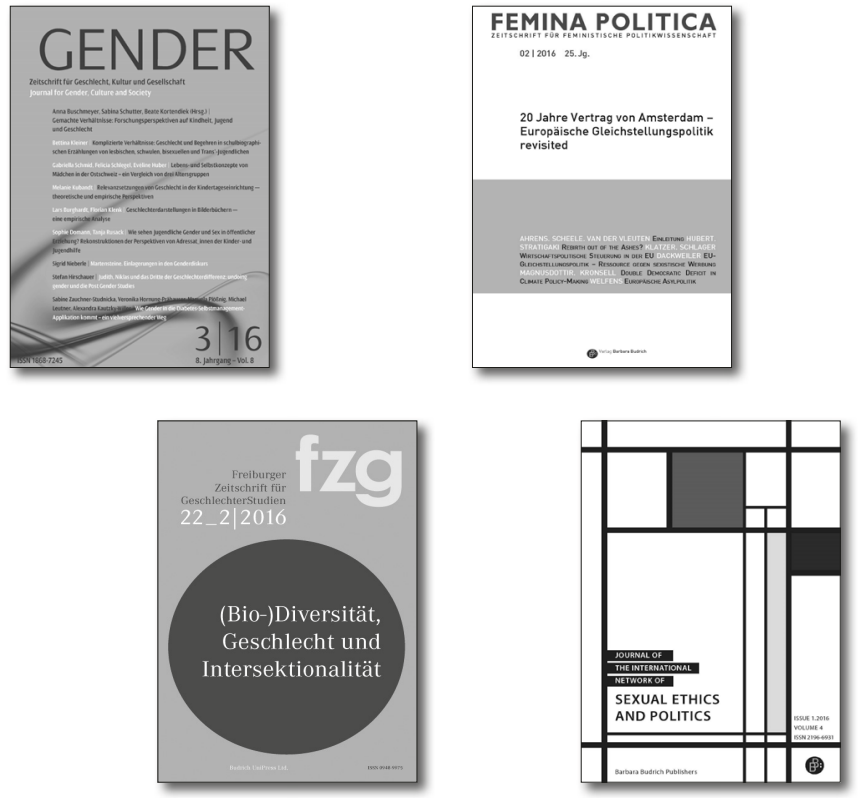

- Einzelbeiträge im Download

- Abonnements (Print + Online)

- Online-Freischaltung für Institutionen über IP

- mit Open Access-Bereichen

Als AbonnentIn mit einem KombiAbo Print + Online bekommen Sie Ihr Heft bequem nach Hause geliefert und Sie haben zusätzlich Zugriff auf das gesamte OnlineArchiv der jeweiligen Zeitschrift.
Verlag Barbara Budrich Barbara Budrich Publishers Stauffenbergstr. 7

D-51379 Leverkusen-Opladen info@budrich-journals.de 